AperTO - Archivio Istituzionale Open Access dell'Università di Torino

\title{
Silica Surface Features and Their Role in the Adsorption of Biomolecules: Computational Modeling and Experiments
}

\section{This is the author's manuscript}

Original Citation:

Availability:

This version is available http://hdl.handle.net/2318/141894

since 2016-08-08T16:37:22Z

Published version:

DOI:10.1021/cr3003054

Terms of use:

Open Access

Anyone can freely access the full text of works made available as "Open Access". Works made available under a Creative Commons license can be used according to the terms and conditions of said license. Use of all other works requires consent of the right holder (author or publisher) if not exempted from copyright protection by the applicable law. 


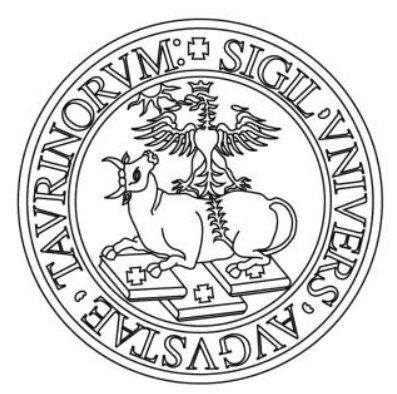

\section{UNIVERSITÀ DEGLI STUDI DI TORINO}

This is an author version of the contribution published on:

Questa è la versione dell'autore dell'opera:

Rimola, A.; Costa, D.; Sodupe, M.; Lambert, J.-F.; Ugliengo, P. Silica Surface

Features and Their Role in the Adsorption of Biomolecules: Computational

Modeling and Experiments. Chemical Reviews 2013, 113, 4216. $10.1021 /$ cr3003054

The definitive version is available at:

La versione definitiva è disponibile alla URL:

http://pubs.acs.org/doi/abs/10.1021/cr3003054?prevSearch=\%255BContrib\%253 A\%2Bugliengo\%255D\&searchHistoryKey= 


\section{Silica surface features and their role in the adsorption of bio-molecules: computational modeling and experiments}

Albert Rimola ${ }^{1}$, Dominique Costa $^{2}$, Mariona Sodupe ${ }^{1}$, Jean-François Lambert ${ }^{3}$ and Piero Ugliengo* 4

${ }^{1}$ Departament de Química, Universitat Autònoma de Barcelona, 08193 Bellaterra (Cerdanyola del Vallès), Spain

${ }^{2}$ Laboratoire de Physico-Chimie des Surfaces, UMR CNRS-ENSCP 7045, Ecole Nationale Supérieure de Chimie de Paris, Chimie-ParisTech, 11, Rue Pierre et Marie Curie, 75005 Paris, France

${ }^{3}$ Laboratoire de Réactivité de Surface, UMR CNRS 7197, Université Pierre et Marie Curie Paris 6, Case 178, 3 Rue Galilée, 94200 Ivry-sur-Seine, France

${ }^{4}$ Università di Torino, Dipartimento di Chimica, and NIS - Nanostructured Interfaces and Surfaces - Centre of Excellence, Via P. Giuria 7, 10125, Torino, Italy

*Corresponding author. E-mail: piero.ugliengo@unito.it 


\section{Contents}

1. Introduction

2. Structure and Polymorphism

2.1. Synthesis of silica samples

2.2. Silica Surfaces - Nomenclature of the surface groups

\section{Experimental Data}

3.1. Fetaures of bare silica surface

3.1.1 Bulk data: TG (thermogravimetry) and silanol surface densities

3.1.2 Bulk data: acid—base titration. How many negatively charged silanolate groups can there be at the interface ?

3.1.3. Vibrational spectroscopies: IR and Raman

3.1.4. NIR, visible and UV

3.1.5. Solid-state NMR

3.1.6. Paramagnetic sites: ESR and related techniques

3.1.7. Adsorption of probe molecules

3.1.8. IRAS, STM, LEED: «flat » silica surfaces

3.1.9. Use of molecular analogues of silica surface groups

3.1.10. Silica in aqueous solutions: existence of a modified water region

3.2. Adsorption of biomolecules on silica

3.2.1. X-Ray diffraction

3.2.2. Bulk adsorption data: geochemical models and adsorption enthalpies

3.2.3. Vibrational spectroscopies

3.2.4. NMR techniques

3.2.5. Flat surface techniques

3.2.6. An overview of experimental studies of small biomolecules/silica systems

3.2.7. From oligopeptides to proteins : adsorption, secondary structure and selectivity

4. Different Approaches to Model Adsorption at Surfaces

4.1. The periodic boundary condition approach

4.2. The cluster approach

4.3. The embedded cluster approach

5. Theoretical Methods and their application to silica surfaces modeling

5.1. Wave function methods

5.2. DFT based methods

5.3. Dispersion corrected methods 
5.4. Basis sets

5.5. Molecular Dynamics simulations

5.6. Approximated methods

5.6.1. Semiempirical methods

5.6.2. SCC-DFTB

5.6.3. Classical force fields

6. Crystalline silica surfaces

6.1. Surface sites

6.2. Surface reconstruction

6.3. Hydroxylated surfaces

7. Amorphous silica surfaces

7.1. The Tielens's model

7.2. The Ugliengo's model

7.3. Other models

7.4. The S2R surface defect

8. Adsorption of water on silica surfaces

8.1. The early story: first principles cluster based calculations

8.2. Water on crystalline hydroxylated silicas

8.2.1. The low coverage case

8.2.2. Intermediate coverages

8.2.3. The case of the water dimer

8.2.4. Water monolayer

8.2.5. Water multilayers

8.2.6. Crystalline Silicas-liquid water interface

8.2.7. Water on hydrophobic crystalline silicas

8.2.8. Adsorption of water on crystalline defects

8.3. Water on amorphous hydroxylated silicas

8.3.1. Hydroxylated amorphous silicas models obtained by classical MD

8.3.2. Hydroxylated amorphous silicas models obtained by ab initio methods

\section{Interaction with Biomolecules}

9.1. Interaction with amino acids

9.1.1. The glycine case 


\subsubsection{Gas-phase interaction}

9.1.1.2. Interaction with the presence of water

9.1.2. Other amino acids

9.1.2.1. Alanine

9.1.2.2. Lysine and Glutamic Acid

9.1.3. General trends for amino acid adsorption on silica surfaces

9.1. 4. Thermal-induced transformations of adsorbed amino acids on silica

surfaces

9.2. Interaction with peptides

9.3. Interaction with Nucleic acid-related biomolecules

9.4. Interaction with drugs and natural products

\section{Summary and perspective}

\section{Acknowledgmnents}

\section{Introduction}

There are many reasons to report on silica interacting with biomolecules. The most obvious one is that, on the Earth's crust, oxygen and silicon are the most abundant atomic species, with percentages of $45.5 \%$ and $27.2 \%$, respectively, which manifests itself in a large variety of silica and silicate minerals so that the contact between living matter and these materials is ubiquitous. ${ }^{1}$ The abundance originates from the stellar evolution of massive stars (>8 solar masses) in which $\mathrm{Mg}$, $\mathrm{Si}$ and $\mathrm{O}$ nuclei are formed in large amounts from the nuclear fusion reactions. ${ }^{2}$ If the star is more massive than 10 solar masses it will explode as a supernova inseminating the deep space with its newly-formed chemical elements. Therefore, silica based minerals in the rocky planets, like Earth, derive from the accretion of interstellar dust grains resulting from chemical reactions between the most electropositive elements and oxygen. More specifically, silica refers to silicon dioxide $\mathrm{SiO}_{2}$, a solid compound present in many different crystalline or amorphous allotropic forms. In nature, it is usually found as quartz, or sand, but is also present in some plants and marine organisms like Radiolarians in which it is metabolized to provide the skeleton structure. The name derives from the Latin Silex and its use is known from the Stone age, as constituent to manufacture flint tools. An enormous literature exists on silica itself and its applications and excellent books and reviews are available on silica properties. In the following, many of the general concepts about silica will be borrowed from the classical book by Iler $^{3}$ and the edited book by Legrand, ${ }^{4}$ the well-known collection of papers on 
adsorption at silica surfaces in the book edited by Papirer ${ }^{5}$ and, in particular, from contributions by El Shafei, ${ }^{6}$ Morrow and Gay ${ }^{7}$ and Davydov. ${ }^{8}$ Another good point of reference is the book by Vansant. $^{9}$

An additional reason to focus on the present topic is that silica surfaces have a key role in many applications dealing with biomolecules interactions, ${ }^{10}$ for instance in chromatography, ${ }^{11}$ just to mention the most common one. Indeed, silica as stationary phase for liquid chromatography system is used, for instance, in the pharmaceutical industry, in the analysis of contaminants, pesticides, bioanalytes, and drug residues in drinks and food samples, medical or environmental tests.

The physico-chemical reasons behind the delicate equilibrium established between the adsorbed and the mobile phases are ultimately dictated by the specific interactions between silica surface functionalities and the adsorbate, resulting in different adsorption constants. The contact between a biomolecule or, more generally, an organic molecule and an inorganic oxide surface is also the key to understand how artificial implants based on bioglasses, a combination of $\mathrm{CaO}$, $\mathrm{Na}_{2} \mathrm{O}$ and $\mathrm{SiO}_{2}$ oxides (of which the latter is the dominant one), perfectly merge with the body bone tissue. ${ }^{12,13}$ Applications of pure silica in health care are found in porous glass which can be designed to encapsulate a specific drug which is then released in situ by a degradation of the silica matrix so as to maximize therapeutic effects and reduce toxicological side effects. ${ }^{14}$ Mesoporous materials are the main example of a "drug delivery systems" (DDS) with two principal aims: the development of systemic-delivery systems, able to release drug at a controlled rate avoiding premature degradation of the active agents, and implantable localdelivery devices, able to release drug as a response to an external stress (for instance the application of a magnetic field) or a change in internal conditions (for instance $\mathrm{pH}$ ). ${ }^{15,16}$ Molecular sieves, particularly MCM-41, have been proposed as DDS starting from $2001^{17}$ and their interaction with ibuprofen, widely employed as anti-inflammatory and analgesic, has been intensively studied. For a DDS to work properly it should show biocompatibility. ${ }^{18}$ Interestingly, the biocompatibility of amorphous silica particles with cellular systems has been proved ${ }^{19,20}$ whereas crystalline silica manifests toxic effects in interaction with cellular systems. The toxicity of crystalline silica, derived from prolonged inhalation of micrometric dust of quartz and its polymorphs of major density, cristobalite and tridymite, that provokes serious lung diseases like pulmonary fibrosis and silicosis, ${ }^{21-23}$ whereas amorphous silicas and silicic acid do not show pathogenic effects. ${ }^{24}$ Many hypotheses have been proposed by scientists to explain the origin of silicosis, all of them involving the role of functionalities exposed at the silica surface in the adsorption of endogenous matter. Adsorption of biomolecules may, in principle, trigger toxicity by modifying their structure and activating the immune system in a sort of autoimmune defense 
mechanism. For instance, the arrangement of silanols and siloxane bridges, which manifests in the presence of hydrophilic or hydrophobic areas, can cause the unfolding of proteins, or the adsorption of phospholipids through the hydrophilic phosphate and may provoke cell membrane rupture. $^{22}$

Less relevant to the present context is the use of silica in heterogeneous catalysis and as a sensing device.The possibility to modify surface silanols to give Si-O-M, where M may be a catalytically active metal as $\mathrm{V}, \mathrm{W}, \mathrm{Mo}, \mathrm{Cr}$ or many other elements, or to host nano sized metal particles at the surface, makes silica an excellent material in catalysis. ${ }^{25}$ Sensing with silica materials is based on surface binding, or encapsulation of sensitive molecules. The change in one or more properties when the silica surface is in contact with the analyte is revealed by the device. An interesting feature of silica as a support for sensors is the possibility to manufacture it in various forms as required, so that doped glasses can be miniaturized and integrated in optics fiber ${ }^{26}$ or arranged in sensor arrays for the detection of multiple analytes. ${ }^{27}$ Furthermore, they can easily be prepared to be used as selective tagged systems. ${ }^{28}$

Most forms of divided silicas are structurally amorphous and, therefore, few techniques can provide atomic resolution information. Exceptions are rare, and only very recently, elegant experiments using scanning tunneling microscopy in ultra-high vacuum conditions ${ }^{29}$ combined with quantum mechanical modeling, revealed the atomic structure of a vitreous thin silica bilayer film grown on $\operatorname{Ru}(0001)$. The difficulty in getting accurate structural information further increases when considering the interaction with biomolecules, despite the fact that for the latter alone, the Protein Data Bank ${ }^{30}$ contains > 82000 biomolecule structures known with atomic accuracy from a variety of diffraction and NMR spectroscopy techniques. It is then clear that experiments alone cannot cope with the complexity of the silica/biomolecule interactions. Indeed, the missing information about atomistic details can be provided by computer simulation techniques of various kinds, which are becoming essential to provide detailed views of the interface regions in which the silica/biomolecule interaction is occurring. Computer experiments can be thought as "virtual microscopes" as they are able to increase our understanding not only providing atomic details but, most importantly, also the energetic features of the adsorption process, vibrational frequencies, NMR chemical shifts, electron density maps, and electrostatic potentials which are essential ingredients for the correct interpretation of the experimental data. Silica functionalities are mainly surface silanol groups $(\mathrm{SiOH})$ and they interact with the polar groups of proteins, nucleic acids or with the phosphate groups of membrane phospholipids through relatively strong H-bonds. To reduce the complexity one can resort to a bottom-up approach, by studying the isolated biopolymer constituents, i.e. amino acids, DNA bases, phosphates etc. As water is also ubiquitously present in the biological context it is then very 
challenging to disentangle the silica/water, water/biomolecule and silica/biomolecule components from the resulting interaction strength. ${ }^{31}$ As it will become clear in this review, a lot of exciting work remain to be done in this field, as many important issues still need to be fully clarified. Just to focus on one of the most common aspects, it is known that silica surface features are sensitive to the thermal treatment suffered by the material as silanol groups are removed from the surface by mutual condensation. What appears to be a rather straightforward process is indeed a very complex phenomenon and neither experiments nor computer simulation have clarified why the surface of an amorphous silica sample heated at high T (>1000 K) will not return to the pristine situation after water adsorption of the thermally treated material, whereas this happens for lower treatment temperatures. ${ }^{32,33}$ Despite its being such a common material, the synthesis of silica, either in gaseous phase as pyrogenic silica or in solution, has only been achieved on the first half of the $20^{\text {th }}$ century, with the development of the sol-gel synthesis technique. ${ }^{13}$ Starting from the 1960s, silica materials have become the subject of intense academic research and industrial application. ${ }^{34}$ Nowadays silica, in all its different forms and possible applications is one of the most studied compounds in the fields of chemistry, physics, material science, biomaterials and engineering. ${ }^{35}$ Indeed, searching for "silica" in the ISI Web of Knowledge, within the 2001-2011 period, a regular increase in the number of entries in article topics and titles is found during the years (see Figure1).
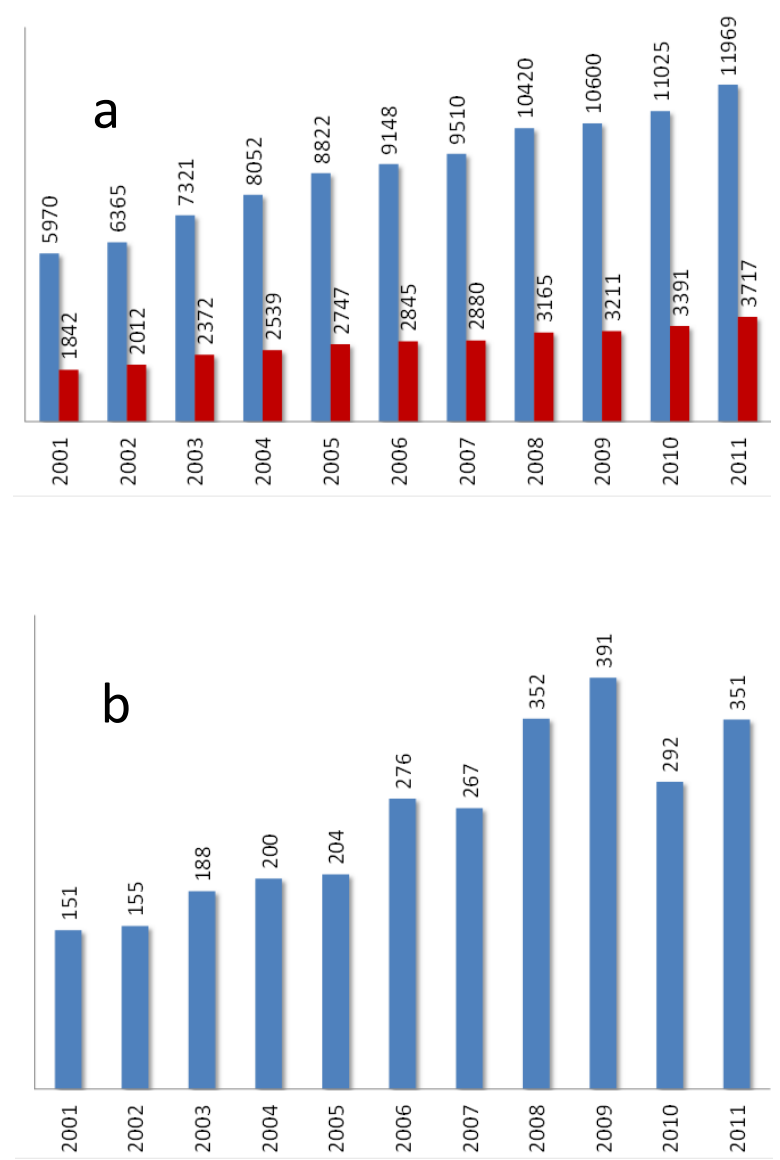
Figure 1. (a) Record count vs publication year (2001-2011) for the word "silica" in article topic (blue bar) and in title (red bar). (b) Record count $v s$ publication year (2001-2011) for "silica" in review's topic. Source: ISI Web of Science®, Databases=SCI-EXPANDED, SSCI, A\&HCI, search date: 07-08-2012.

The name "silica" is also present more than 2500 times in review topics and more than 60 new materials have been patented (silica in patent title) in the same period of time, confirming the importance of silica based materials in a wide range of different fields.

In the present review we only focus on the interaction between silica, either crystalline or amorphous, and biomolecules, restricting our attention to studies reporting a joint use of experiment and modeling techniques. We have also decided to include water as a special case, as $\mathrm{H}_{2} \mathrm{O}$ is the most common biomolecule by election. In the spirit of a bottom-up approach, it resulted that amino acids, small peptides, nucleic acid and a few important drug related molecules have been studied so far in interaction with silica. The situation is similar to other materials like hydroxyapatite ${ }^{36}$ and bioactive glasses. ${ }^{37}$ Specific cases of molecules which are not, strictly speaking, biological are also included when they can play important role in a biochemistry context. The pioneering and elegant work developed for a long time to characterize the interaction of small molecules like, $\mathrm{CO}, \mathrm{CO}_{2}, \mathrm{NH}_{3}$ etc. with silica surfaces from gas/solid adsorption experiments ${ }^{38}$ is not the focus of the present contribution as it has already been reviewed $^{39}$ and it has less relevance in the biological context. It will, however, be briefly reviewed in Section 3 as some fundamental results on simple molecules are also relevant for the case of adsorption of more complex biomolecules.

\section{Structure and polymorphism}

In the following we very briefly summarize the silica surface features common to crystalline as well as amorphous silicas without claiming to be exhaustive (see for instance Ref. ${ }^{40}$ for details). Silica is a solid material with a density between 2 and $3 \mathrm{~g} / \mathrm{cm}^{3}$ and high melting point (ca. $1700^{\circ} \mathrm{C}$ ), whose chemical formula is $\mathrm{SiO}_{2}$. In the structure of most crystalline polymorphs (except for stishovite, vide infra), a silicon atom is bound to four oxygen atoms, and each oxygen to two silicon atoms. The silicon atoms are at the centres of regular tetrahedra of oxygen atoms, $\left[\mathrm{SiO}_{4}\right]$, connected through an oxygen vertex. Already in 1932, Zachariasen suggested that vitreous silica also consists of a continuous random network of corner-sharing $\left[\mathrm{SiO}_{4}\right]$ tetrahedra. ${ }^{41}$ The overall $\mathrm{SiO}_{2}$ structure is neutral because the formal oxidation numbers for silicon and oxygen are (+4) and (-2), respectively, and each oxygen atom in the structure is 
the bridge between two $\left[\mathrm{SiO}_{4}\right]$ tetrahedra, so that the silicon-oxygen ratio is $1: 2$. Thus, the $\left[\mathrm{SiO}_{4}\right]$ tetrahedron represents the building block of almost all silica polymorphs and they only differ in the connectivity of the tetrahedral framework. The different linking patterns for each polymorph translate into different structural, physical and chemical properties.

The Si-O bond shows a predominantly covalent character, as proved by the electron density maps, obtained from X-ray diffraction, and by the bond population analysis obtained from quantum mechanical calculations. Both experimental and computational results assign a charge of ca. +1.4 on silicon atoms and -0.7 on oxygen atoms. ${ }^{42,43}$

Since silicon has empty $d$ orbitals at a relatively low energy it can expand its coordination to become octahedrally coordinated. Stishovite, a high-temperature and high-pressure polymorph of silica, is the only form characterized by a framework in which the silicon atoms are octahedrally coordinated by six oxygen atoms and it was discovered in Meteor Crater, Arizona, due to the favourable high $\mathrm{T}$ and $\mathrm{P}$ caused by the body impact, ${ }^{44}$ and synthesized in the laboratory by Stishov and Popova. ${ }^{45}$ Its X-ray structure revealed a rutile type $\mathrm{SiO}_{2}$ framework. ${ }^{46}$

The $\left[\mathrm{SiO}_{4}\right]$ unit is rigid, as changing the O-Si-O angle from the equilibrium value of $109^{\circ}$ is an energetically expensive process. On the contrary, the $\mathrm{Si}-\mathrm{O}-\mathrm{Si}$ angle that connects two tetrahedra can easily change, for instance as a function of temperature or geometrical constraints. Indeed the flexibility of the Si-O-Si angle is very high, since it costs virtually no energy to change the angle value between $130^{\circ}$ and $180^{\circ}$, as demonstrated in the past in a number of computational studies. ${ }^{47-49}$ For instance, the CISD/TZ+2d potential energy surface of $\mathrm{H}_{3} \mathrm{Si}$-O$\mathrm{SiH}_{3}$ as a cluster model (see Section 3.2) of the siloxane $\mathrm{Si}-\mathrm{O}-\mathrm{Si}$ bond gave a minimum at $142^{\circ}$, while changing the angle from $130^{\circ}$ to $180^{\circ}$, only costs around $6.7 \mathrm{~kJ} / \mathrm{mol}$. $^{47}$ The exact value of the barrier to bring the Si-O-Si to $180^{\circ}$ from the actual minimum was shown to be difficult to compute as Bär and Sauer ${ }^{48}$ showed a large sensitivity of the results to the quality of the adopted Gaussian basis set. For instance, MP2 calculations with basis sets including up to five $d$ and four $f$ polarization functions showed a minimum potential energy at a Si-O-Si angle value larger than $145^{\circ}$, and a linearization barrier of only $1.5 \mathrm{~kJ} / \mathrm{mol}$. Similar results have been reported in an exhaustive work by Tielens et $\mathrm{al}^{50}$ who adopted six different exchange-correlation functionals with very large Gaussian basis sets, confirming the extreme flexibility of the Si-O-Si angle in full agreement with the MP2 results by Bär and Sauer. ${ }^{48}$ All these results are at variance with the case of organic ether in which the C-O-C angle is almost invariably found around $110^{\circ}$ and there is a very high energy barrier to linearization. The Lewis basicity of the Si-O-Si siloxane bridges has also long ago ${ }^{51}$ been proved to be significantly smaller than the corresponding one measured for the C-O-C link found in organic ethers. Reasoning based on the oxygen $p$ orbital back donation through the empty $d$ orbitals of Si have been put forward but more recent quantum 
mechanical calculations did not find any evidence for that and electrostatic interactions have been invoked to explain the relatively short $\mathrm{Si}-\mathrm{O}$ bond length. ${ }^{48}$

The high pliability of the Si-O-Si angle is the reason behind the large number of all-silica materials, envisaging all possible silica polymorphs, from dense crystalline and amorphous structures (quartz or glasses), non-periodic porous systems (aerogels and sponges) to microporous crystalline zeolites and mesoporous materials (see Figure 2).

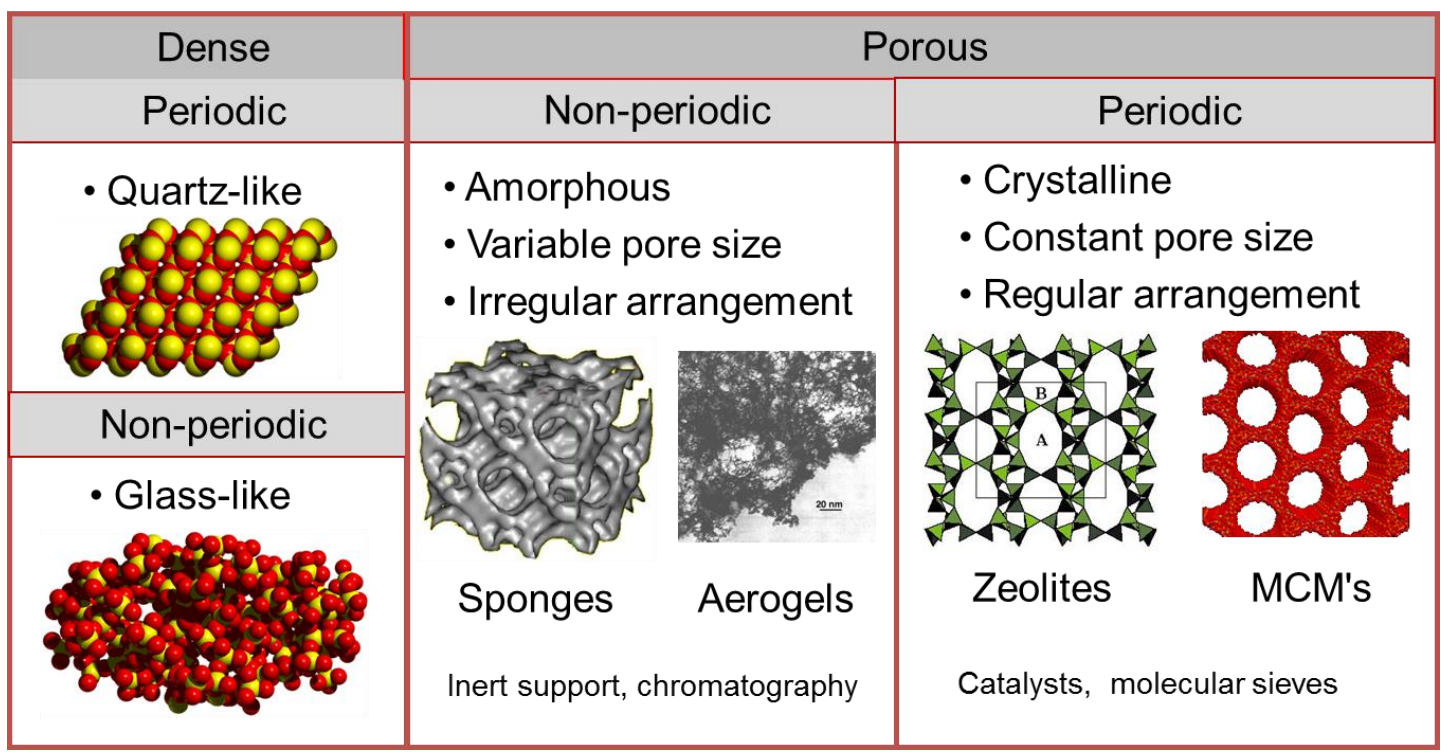

Figure 2. Different silica structures based on corner-sharing $\left[\mathrm{SiO}_{4}\right]$ units.

Just for the limited case of zeolites, the structural database ${ }^{52}$ reports 201 different kind of all-silica frameworks which can be, at least in principle, be synthesized. In practice most of them have only been synthesized as aluminosilicates (zeolites), as a small amount of substitutional aluminium stabilizes the framework. Zeolites are used as catalysts as well as adsorbent in a large number of technological applications. ${ }^{53,54}$

The $\left[\mathrm{SiO}_{4}\right]$ arrangement that determines the formation of different dense polymorphs chiefly depends on the crystallization pressure (P) and temperature (T), and their relative stability change with the values of $\mathrm{P}$ and $\mathrm{T}$. The different polymorphic phases can be present out of their $\mathrm{P}$ and $\mathrm{T}$ stability range because the phase transformation between one polymorph and another is an extremely slow process. A phase that is maintained unaltered outside its stability conditions is named metastable. Zeolites are classical examples of metastable phases and their stability can also depend on the presence of the template molecule. At room temperature and low pressure the most stable silica phase is $\alpha$-quartz followed by $\alpha$-tridymite and $\alpha$-cristobalite. These are the most common high-density crystalline polymorphs. Each one of them can exist also in a phase named $\beta$, which is stable at higher temperature and presents a more symmetric structure. At room conditions $\alpha$-quartz is the most stable phase, and every different crystalline or amorphous, low or high-density silica phase in these conditions is metastable at best. 
The $\left[\mathrm{SiO}_{4}\right]$ building blocks are joined via corner-sharing links in the vast majority of silica materials. Exceptions to this class of structures are stishovite (vide supra) and fibrous silica ${ }^{55,56}$ consisting of chains of edge-sharing $\left[\mathrm{SiO}_{4}\right]$ tetrahedra. The existence of this material is important, as it will be shown later, since two-silicon membered rings $(\mathrm{SiO})_{2}$ (hereafter called S2R) like those constituting the repeating unit in fibrous silica are also formed as isolated defects at the surface of amorphous silica subject to thermal temperature at $\mathrm{T}>800 \mathrm{~K}$, imparting to the surface a reactive character.

\subsection{Synthesis of silica samples}

The formation of colloidal silicas, pyrogenic silica, silica xerogels and precipitated silica has been largely studied in order to synthesize products of technological importance with reproducible properties. The preparation of silica samples can be made in anhydrous vapor phase at high temperature (such samples are trademarked Carbosil $®$ in USA or Aerosil® in Europe), vaporizing $\mathrm{SiO}_{2}$ in an arc or plasma jet and condensing it in a stream of dry inert gas or oxidizing and hydrolyzing silicon compounds $\left(\mathrm{SiH}_{4}, \mathrm{SiCl}_{4}, \mathrm{HSiCl}_{3}\right)$, or in aqueous solution (silica gel or precipitated silica) by condensation of simple monomers $\mathrm{R}_{x} \mathrm{Si}(\mathrm{OH})_{y}(x+y=4)$. Both methods produce porous structures: pyrogenic silica normally presents smaller pores than colloidal silica whereas Aerosil $®$ only exhibits inter-particular porosity. All of these materials have high surface areas as measured by means of the adsorption isotherm obtained with $\mathrm{N}_{2}$ as adsorbate ${ }^{57}$ applying the Brunauer, Emmett and Teller (BET) theory. ${ }^{58}$ Such samples are overall amorphous structures but pyrogenic silica may present crystal-like order in limited regions. This can be explained considering that at the high temperature required by the synthesis the rate of rearrangement of $\left[\mathrm{SiO}_{4}\right]$ tetrahedra is very high, and since configurations with local order similar to those of crystals are the most stable, the tetrahedra tend to arrange locally in ordered networks.

In 1984 Avnir and coworkers ${ }^{59}$ described the hydrolytic polycondensation of silicon alkoxides $\mathrm{Si}(\mathrm{OR})_{4}$ or $\mathrm{R}_{\mathrm{n}} \mathrm{Si}(\mathrm{OR})_{4-\mathrm{n}}$ in solution, to form highly porous organosilicates, where the organofunctional groups R' modifies the network of the product. The reaction was somehow revolutionary because it permits the entrapment of organic and bioinorganic molecules within the structure of glasses at low temperature, something that was not possible before due to the high temperature that the preparation of glasses normally requires.

The porosity of the gel structure changes with the organofunctional R and R' groups and other factors like the drying procedure applied after the synthesis or the use of drying agents like formamide, glycerol, oxalic acid and other organic acids. ${ }^{60}$ For instance, samples dried by natural evaporation (Xerogels) present a minor porosity whereas samples dried in an autoclave (Aerogels), where the solvent is removed above its critical point, present a major porosity and a 
lesser density. The porosity strongly determines the features of the fuctionalities exposed at the surfaces and, thereby, the surface properties of the sample. ${ }^{59,61}$ Clearly, it is not possible within this review to report the innumerable applications of these silica-based hybrids especially since they involve a surface chemistry potentially very different from bare silica.

In 1992, researchers of the Mobile Oil Corporation developed a new family of ordered mesoporous silicas with pore diameters tunable between 15 and $300 \AA$, using liquid crystal surfactants with long n-alkyl chains as templating agents. ${ }^{62,63}$ Such highly porous ordered silicas present pores with amorphous silica walls spatially arranged in periodic patterns. These compounds were named M41S (molecular sieves) and present very high specific surface areas $\left(\sim 700 \mathrm{~m}^{2} / \mathrm{g}\right)$ and pore volumes. The M41S consist of a hexagonal phase (P6m) named MCM-41, a cubic phase (Ia3d), named MCM-48 and a lamellar phase named MCM-50 (see Figure 3).

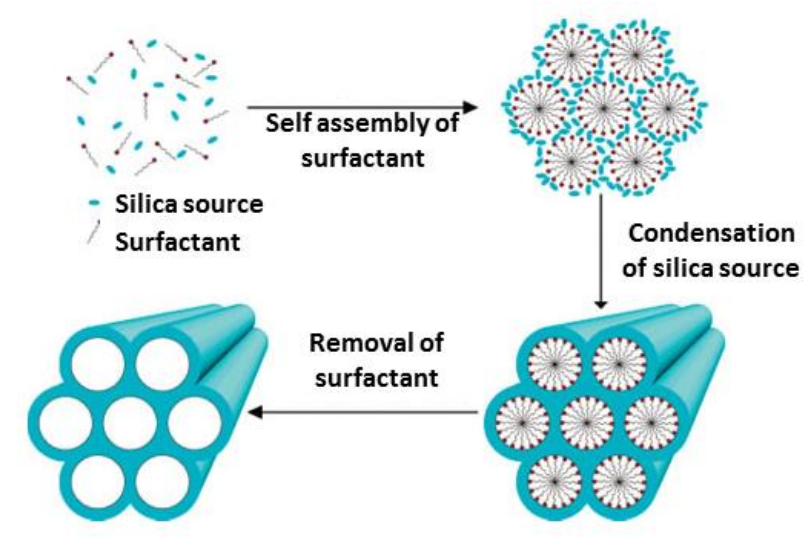

Figure 3. Schematic representation of the process giving rise to molecular sieves. Reprinted with permission from Ref. ${ }^{64}$. Copyright 2005 America Chemical Society.

The pore dimension depends on the length of the alkyl chain and the pore walls are amorphous overall. The pore diameter essentially determines the dimension of the molecules that can be entrapped inside the pores: tuning the pore dimension in a range that varies from a few nanometers to several tens of nanometers, it is possible to host small molecules or macromolecules as proteins. The presence of additives during the synthesis, which modify the functional groups exposed at the wall surfaces, may determine the adsorption properties of the molecular sieves. For this reason such structures present a large number of potential applications.

The list would not be complete without mentioning biogenic silica such as sponge spicules, radiolarian tests, and especially the frustules (silicified cell walls) of diatoms, whose accumulation yields « diatomaceous earth» or kieselguhr and «siliceous ooze » sediments. They have original middle-range organisations such as the regular nanometric spheres of opal, and the micron-size structures of diatom walls are especially spectacular. ${ }^{65}$ Biogenic silicas interact with biomolecules, almost by definition, but in a different way from what is considered in the present paper: specialized biomolecules such as the proteins silaffins and silicateins ${ }^{66}$ pre-exist to the 
siliceous phase and strongly contribute to building it from molecular precursors. This is a fascinating subject but it falls outside of the scope of this review. The question whether biogenic silicas differ from their synthetic counterparts at the molecular level is largely untouched.

\subsection{Silica surfaces - Nomenclature of surface groups}

While an ideal solid, irrespective of its amorphous or crystalline nature is, by definition, infinite, a real solid is always finite and then, characterized by external surfaces. The surfaces represent the interface between a solid bulk and the external environment so that the physical and chemical features characterizing the interaction of (bio)molecules with the silica surfaces should be studied at the atomic level. To achieve that, the first step is to highlight the structural properties of the bare silica surfaces. ${ }^{4,40}$

When silica is fractured in the laboratory under ultra-high vacuum conditions a complex reconstruction of the broken $\mathrm{Si}-\mathrm{O}$ bonds occurs in order to self-heal the dangling bonds at the surface (see also Sections 3.1.6 and 6). This can be attained by the formation of highly strained $\mathrm{Si}-\mathrm{O}$ rings of small nuclearity or unusual defects like $>\mathrm{Si}=\mathrm{O}$ ones (silanones). It has, however, been measured that even a very low water partial pressure (of the order of $10^{-7}$ torr) brings about a complete reconstruction of the silica surface giving rise to surface silanol groups, $\mathrm{SiOH} .{ }^{67}$ Therefore, at the silica surface, in normal laboratory conditions the most common termination is given by two main functional groups: the siloxane links (Si-O-Si) and the silanol groups (Si$\mathrm{OH})$. The siloxanes are also present in the bulk structure, although not accessible, and result from the vertex sharing of the $\left[\mathrm{SiO}_{4}\right]$ tetrahedra. Silanol groups either in the bulk or at the surface are the result of an incomplete condensation during the polymerization that forms the massive solid. The contact between silica and chemicals of various nature may break Si-O bonds at the surface of the material giving rise to unfilled valences. Water is normally present in the environment around the material, so that either protons or hydroxyl groups $(\mathrm{OH})$ are the most suitable candidates to complete the valency of both atoms. Even in cases where the silica derives from combustion process, the final material is, nonetheless, exposed to the moisture. Hydrated surfaces, characterized by high population of surface hydroxyls, are therefore formed. The usual picture of a pure silica surface is, thus, a system composed by regions of siloxane links interrupted by sites exposing silanol groups. Silanols are responsible of the hydrophilic properties of silica, since they can interact with polar groups or molecules via hydrogen bond interactions. $^{8,38}$

In crystalline silica, ideally, the $\left[\mathrm{SiO}_{4}\right]$ tetrahedra are stacked in an ordered design which is repeated regularly, so that a regular distribution of the hydroxyls at the surface has to be expected. In spite of this, experiments evidence that silica samples are quite heterogeneous because of irregular packing and incomplete condensation during the synthesis process. On 
crystalline silica, the upper layers can present an amorphous character with a loss of the expected order. $^{21,68}$ Nevertheless, within the scope of the present review, only regular surfaces have been considered when dealing with crystalline materials so that special surface features are expected due to the repeating nature of the $\mathrm{SiOH}$ groups.

As mentioned before, Si atoms in the bulk silica are mostly bound to four oxygen atoms. This environment, clearly identifiable by $\mathrm{NMR},{ }^{7}$ is labelled as $\mathrm{Q}^{4}$ in which the superscript indicates the number of neighbouring $\left[\mathrm{SiO}_{4}\right]$ groups attached to a given $\mathrm{Si}$. On the other hand, silicon at the surface can bind to one or two hydroxyl groups to complete its valence, according to whether its binding to the bulk involves three or two Si-O-Si bridges: the corresponding environments are therefore denoted as $\left(\mathrm{Q}^{3}\right)$ or $\left(\mathrm{Q}^{2}\right)$, respectively. Surface structures like surface silanone $>\mathrm{Si}=\mathrm{O}$ and $\mathrm{Si}-(\mathrm{OH})_{3}\left(\mathrm{Q}^{1}\right)$, with the silicon bonded to only one bulk oxygen, can exist in principle, but the first one is thermodynamically and kinetically unstable towards water adsorption ${ }^{69-71}$ whereas no evidence of the $Q^{1}$ species has been reported from NMR experiments (see also Section 3). ${ }^{72}$

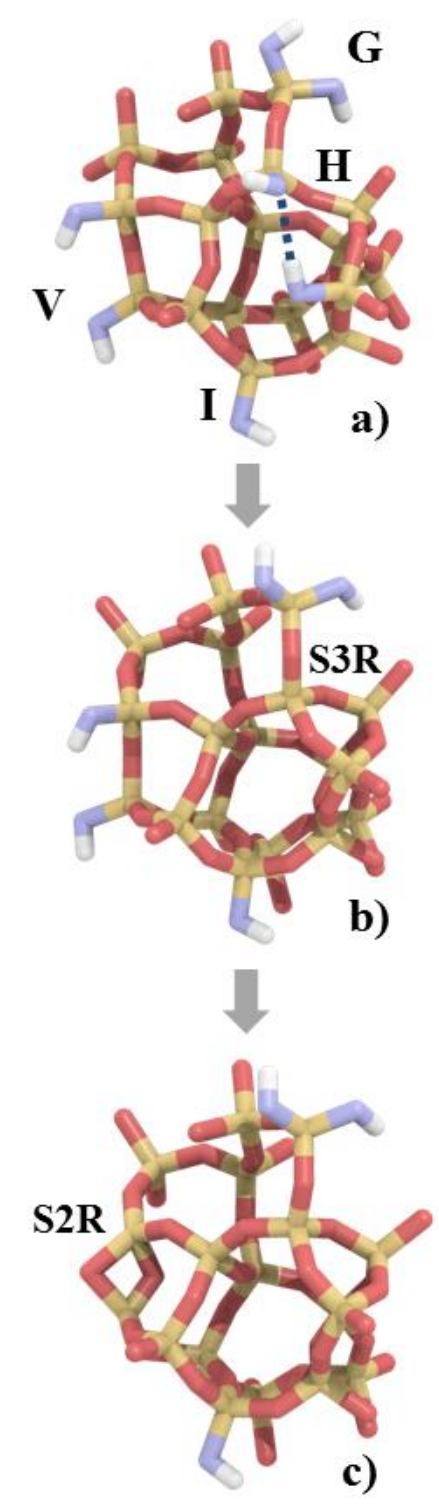


Figure 4. A cartoon of the possible silica surface sites: a) isolated $\mathrm{SiOH}\left(\mathrm{I}, \mathrm{Q}^{3}\right)$, geminal $\mathrm{SiOH}$ $\left(\mathrm{G}, \mathrm{Q}^{2}\right)$, vicinal $\mathrm{SiOH}\left(\mathrm{V}, \mathrm{Q}^{3}\right)$ and $\mathrm{H}$-bonded $\mathrm{SiOH}\left(\mathrm{H}, \mathrm{Q}^{3}\right)$; b) formation of the $(\mathrm{SiO})_{3}$ ring $(\mathrm{S} 3 \mathrm{R})$ by condensation of the $\mathrm{H}$ pair; $\mathrm{c})$ formation of the $(\mathrm{SiO})_{2}$ ring $(\mathrm{S} 2 \mathrm{R})$ by condensation of the $\mathrm{V}$ pair. Evolution from a) to c) by increasing temperature. Colour coding: oxygen (red), oxygen of the $\mathrm{SiOH}$ (blue), hydrogen (white), silicon (yellow). Molecular graphics rendered by Qutemol. ${ }^{73}$

Figure 4a shows a cartoon which helps to visualize different kinds of silanols exposed at silica surfaces. A single or terminal Si-OH group $\left(\mathrm{Q}^{3}\right)$ is called an isolated silanol $\mathrm{SiOH}(\mathbf{I})$ when the distance to the closest $\mathrm{SiOH}$ groups is such that they cannot be involved in H-bond interactions. Silanol groups which are separated by more than $\sim 3.3 \AA$ can be considered as unable to establish mutual hydrogen bond, and therefore as isolated. The isolated silanol groups are then free to establish H-bond interactions with adsorbates as both H-bond donors and acceptors. Pairs of silanols belonging to tetrahedra that share a common oxygen vertex are normally called vicinals $(\mathbf{V})$. The two silicon atoms of vicinals are separated by a single oxygen so that the two hydroxyl groups are separated by less than $3 \AA$, but in spite of that, they are usually not involved in $\mathrm{H}$-bond, at least according to quantum mechanical calculations on cluster models. ${ }^{74,75}$ Nevertheless, local geometrical constraints may force a vicinal site to become weakly H-bonded. Two silanols, that do not belong to directly connected tetrahedra, but nonetheless are closer than $\sim 3.3 \AA$, will establish $\mathrm{H}$-bonds and are called interacting or $\mathrm{H}$ bonded $(\mathbf{H})$. The optimum $\mathrm{O} \cdots \mathrm{O}$ distance between the two $\mathrm{OH}$ involved in a $\mathrm{H}$-bond lies between $\sim 2.5$ and $\sim 2.8 \AA$. It is worth noting that the positions in space of surface $\mathrm{SiOH}$ groups in silica is constrained by the underlying solid structure, so that in many cases, they cannot give rise to strong $\mathrm{H}$-bond interactions as would have occurred in a less constrained environment. Two $\mathrm{OH}$ groups linked to the same surface silicon atom $\left(\mathrm{Q}^{2}\right)$ to give the $\mathrm{Si}-(\mathrm{OH})_{2}$ moiety, are called geminals $(\mathbf{G})$ ) and since the silicon atom is attached in addition to two tetrahedra by Si-O$\mathrm{Si}$ links, a pair of geminals necessarily correspond to a $\mathrm{Q}^{2} \mathrm{Si}$. Even though they are very close, the two geminal $\mathrm{OH}$ are oriented in such a way that they cannot be involved in mutual $\mathrm{H}$-bonds.

When a highly hydrated silica is heated, the main effect is the progressive condensation of pairs of surface silanols involved in H-bond interaction, and the formation of a siloxane bridge, as showed in Figure $4 \mathrm{~b}$. Depending on the pair of $\mathrm{H}$-bonded silanol groups the condensation brings about $(\mathrm{SiO})_{\mathrm{n}}$ rings of distinct nuclearity $\mathrm{n}$. For instance, in Figure $4 \mathrm{~b}$ the condensation of the $\mathbf{H}$ pair gives a three-silicon membered $(\mathrm{SiO})_{3}$ ring $(\mathrm{S} 3 \mathrm{R})$ whereas when vicinal $\mathbf{V}$ pairs are involved a highly strained two-silicon membered $(\mathrm{SiO})_{2} \operatorname{ring}(\mathrm{S} 2 \mathrm{R})$ is formed (Figure $4 \mathrm{c}$ ). Their formation increases the structural tension of the outgassed surface during the heating process. As the $\mathrm{SiOH}$ groups can give rise to $\mathrm{H}$-bond interactions with adsorbates and, in 
particular, with the ubiquitously present water molecules, outgassing silica at high $\mathrm{T}$ decreases its hydrophilic character and increases the hydrophobic one. Thus, for high-temperature treated silicas, the adsorption processes are dominated by hydrophobic interactions, mainly driven by dispersive forces. This means that thermal treatments are a very simple and convenient way to fine-tune the adsorption properties of silica based materials. We will see in Section 3 how infrared spectroscopy can be used to track the dehydroxylation process.

Silica samples prepared in the gas phase, known as Aerosil, are usually less hydrophilic than sol-gel silicas, because the high temperature of the preparation already causes a decrease of the number of $\mathrm{OH}$ groups, which condense during the synthesis process. In this case, it is important to consider the age of the sample to know the degree of surface hydroxylation. ${ }^{6}$ Samples of Aerosil tend, upon aging, to increase the total concentration of silanols, while reducing the concentration of isolated silanols. This is the result of the cleavage of strained siloxane bridges by water molecules in the surrounding environment. ${ }^{76}$ Water breaks Si-O-Si to form a pair of mutually H-bonded silanol groups (apart from the opening of the S2R resulting in a vicinal pair), in the inverse process of that described in Figure 4, so that the total number of silanols increases while the fraction of isolated ones decreases.

Despite the complexity of silica surfaces, Zhuravlev has shown that the average number of $\mathrm{Si}-\mathrm{OH}$ groups per $\mathrm{nm}^{2}$ in a fully hydroxylated surface is a physico-chemical constant amounting to $5 \mathrm{OH} / \mathrm{nm}^{2}$, irrespective of the preparation method for a set of about 100 different silica aerogel samples. ${ }^{77-80}$

More generally, if one considers surfaces that have been thermally activated or are not fully hydroxylated for any reason, the total concentration of silanol terminations (and their distribution between isolated, vicinals and H-bonded, or between terminals and geminals) can vary considerably. ${ }^{8,81,82}$ However, regularities are observable and Zhuravlev $^{78}$ has proposed a "master curve" showing the concentrations of the different types of silanols exposed at the surface as a function of the sample thermal treatment, which is reproduced in Figure 5. 


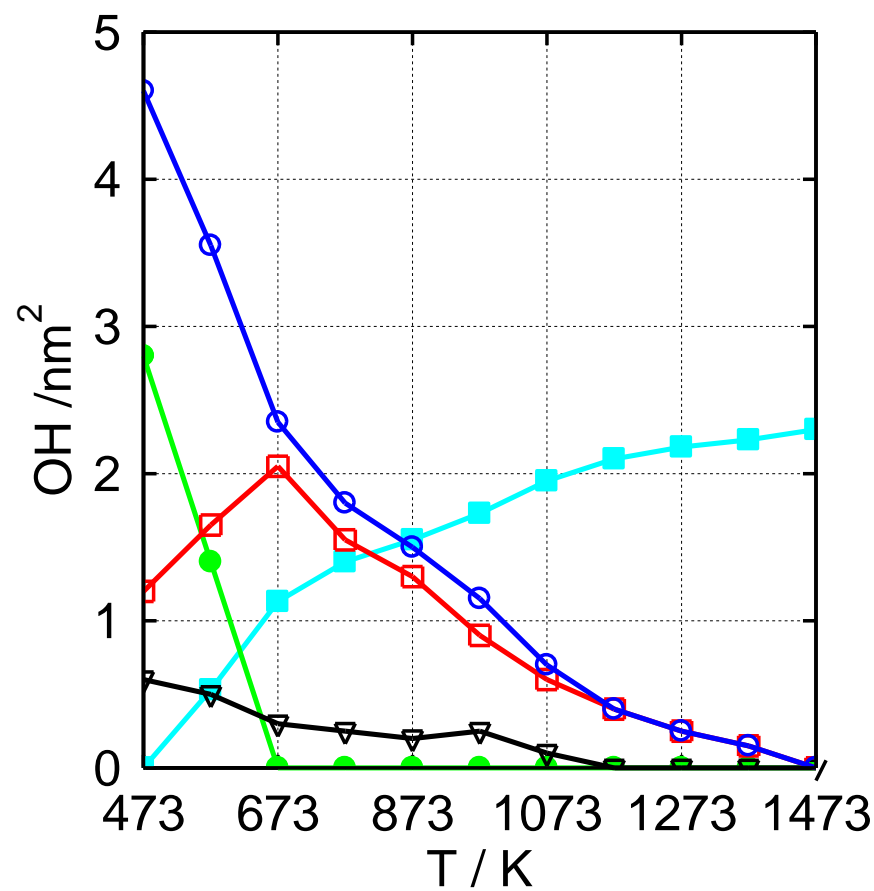

Figure 5. Distribution of the $\mathrm{OH}$ surface group per $\mathrm{nm}^{2}$ as a function of the silica sample treatment temperature. Empty circle: average concentration of total $\mathrm{OH} / \mathrm{nm}^{2}$; filled squares: average concentration $\mathrm{Si}-\mathrm{O}-\mathrm{Si}$; empty squares: average concentration free $\mathrm{OH}$ groups; filled circle: average concentration of $\mathrm{H}$-bonded $\mathrm{OH}$ groups; empty triangles: average concentration of geminal group. Graph drawn by using raw data from Ref. ${ }^{78}$

Thus, for instance, for the total density of $4.6 \mathrm{OH} / \mathrm{nm}^{2}$ on asilica sample fully hydroxylated at $500 \mathrm{~K}$, about $0.6,1.2$ and $2.8 \mathrm{OH} / \mathrm{nm}^{2}$ are due to geminal, isolated and $\mathrm{H}$-bonded ones, respectively. Already at $700 \mathrm{~K}$ all $\mathrm{H}$-bonded silanols have disappeared, while the isolated silanols reach their maximum density of $2 \mathrm{OH} / \mathrm{nm}^{2}$. At about $1000 \mathrm{~K}$ (see also Figure 5) only 1 isolated silanol per $\mathrm{nm}^{2}$ remains, together with a small fraction of geminals, and at about $1500 \mathrm{~K}$ the sample undergoes sintering with the elimination of all surface functionalities. This very last step brings about a complex restructuring of the silica sample making subsequent rehydration by water adsorption an extremely slow process. ${ }^{32,33}$ It is still unclear how it happens and what are the physico-chemical steps controlling that process which implies some sintering of silica.

An interesting aspect of silica surfaces in contact with water solutions of different ionic strength and $\mathrm{pH}$ is silanol deprotonation, which produces negatively charged surface groups $\left(\equiv \mathrm{SiO}^{-}\right)$, with relevant consequences for the adsorption of ions and polar molecules from the solution. What is known about this important point will be detailed in Section 3.1.2.

\section{Experimental Data}

\subsection{Features of bare Silica Surface}


Here we will only be concerned with silica features that can play a role in « realistic » conditions for biomolecules adsorption, i.e., in conditions of high water activity. As discussed in Section 2, this excludes some features such as silanone groups. We will list the experimental techniques that allow the observation and discrimination of silica surface groups and summarize the main results that have been safely established so far.

\subsubsection{Bulk data: TG (thermogravimetry) and silanol surface densities}

Thermogravimetric analysis has been applied to amorphous silicas for a long time. TG traces usually show the elimination of physisorbed water at $\mathrm{T} \leq 100^{\circ} \mathrm{C}$, followed by the condensation of different types of surface silanols up to $1000^{\circ} \mathrm{C}$. A huge amount of data have been analysed in great detail and allowed to devise what is sometimes called 'Zhuravlev's model", ${ }^{78,80}$ whose main feature is an almost constant value of the silanols surface density for maximally hydroxylated silicas, at 4.6 to $4.9 \mathrm{~nm}^{-2}$ based on non-porous silicas such as Aerosils. For mesoporous silicas a figure of $4 \mathrm{~nm}^{-2}$ has been reported by Trébosc et al. ${ }^{83}$ from TG and ${ }^{29} \mathrm{Si}$ NMR (see section 3.1.5); also from TG and ${ }^{29} \mathrm{Si} \mathrm{NMR}$, Landmesser et al. ${ }^{84}$ have evaluated the percentage of $\mathrm{Si}$ atoms bearing $\mathrm{OH}$ groups and while there is some ambiguity regarding the surface area of their material, a figure in the range 4.2 to $4.6 \mathrm{~nm}^{-2}$ has been estimated.

It is generally accepted that precipitated silicas have significantly higher surface silanol densities. For instance, a figure of 7.7 silanols $\mathrm{nm}^{-2}$ (from TG alone) has been reported by Ref. ${ }^{85}$

Conversely, silicas with lower silanol densities ${ }^{86}$ may be prepared if the thermal condensation process is correctly controlled. The only way for silanols to disappear is to condense in pairs, producing one siloxane and one water molecule. This generally results in the formation of strained cycles or rings, which can be observed by vibrational spectroscopy (cf. Section 3.1.3). The evolution of the surface densities of various types of silanols with pretreatment temperatures has been mentioned in Section 2, (Figure 4). Surface silanol densities significantly lower than $4 \mathrm{~nm}^{-2}$ have sometimes been reported for fully hydroxylated silicas without thermal treatment, e.g. 2.2 to $2.7 \mathrm{~nm}^{-2}$ for precipitated silicas. ${ }^{87}$ As it turns out, figures in this range are calculated from $\mathrm{LiAlH}_{4}$ titration of the silanols, ${ }^{76}$ a method which has never been proved to be quantitative.

Note that the (111) face of $\beta$-cristobalite has a silanol density very similar to the average values mentioned above $\left(4.55 \mathrm{~nm}^{-2}\right)$ : this has spurred interest in the use of this surface as a model for that of amorphous silica. ${ }^{88}$ However, the $\beta$-cristobalite (111) surface also has features significantly different from that of amorphous silicas, as the silanols cannot form $\mathrm{H}$-bonds to each other (cf. Section 3.1.3; much more on the topic of model crystalline surfaces will be found in Section 6.3, see especially Table 4 for silanol densities). 
Although TG curves could in principle be used to assess the distribution of surface silanols between populations of different reactivity toward condensation (such as isolated vs. interacting), they are in fact quite featureless and attempts to extract further information from them are not fully convincing. Figure 6 shows the first derivative of the weight loss for a hydrated fumed silica; the first and most intense event peaking at $50^{\circ}$ is due to the desorption of physisorbed water, and it is followed by a very broad feature corresponding to silanols condensation, peaking in the $230-280^{\circ} \mathrm{C}$ range and tailing smoothly up to $1000^{\circ} \mathrm{C}$ at least. DTG traces of mesoporous silicas are only slightly different (Figure 6). For precipitated silicas, sharper peaks in the DTG may sometimes be observed but they are likely due to the elimination of residual organic residues on the surface.
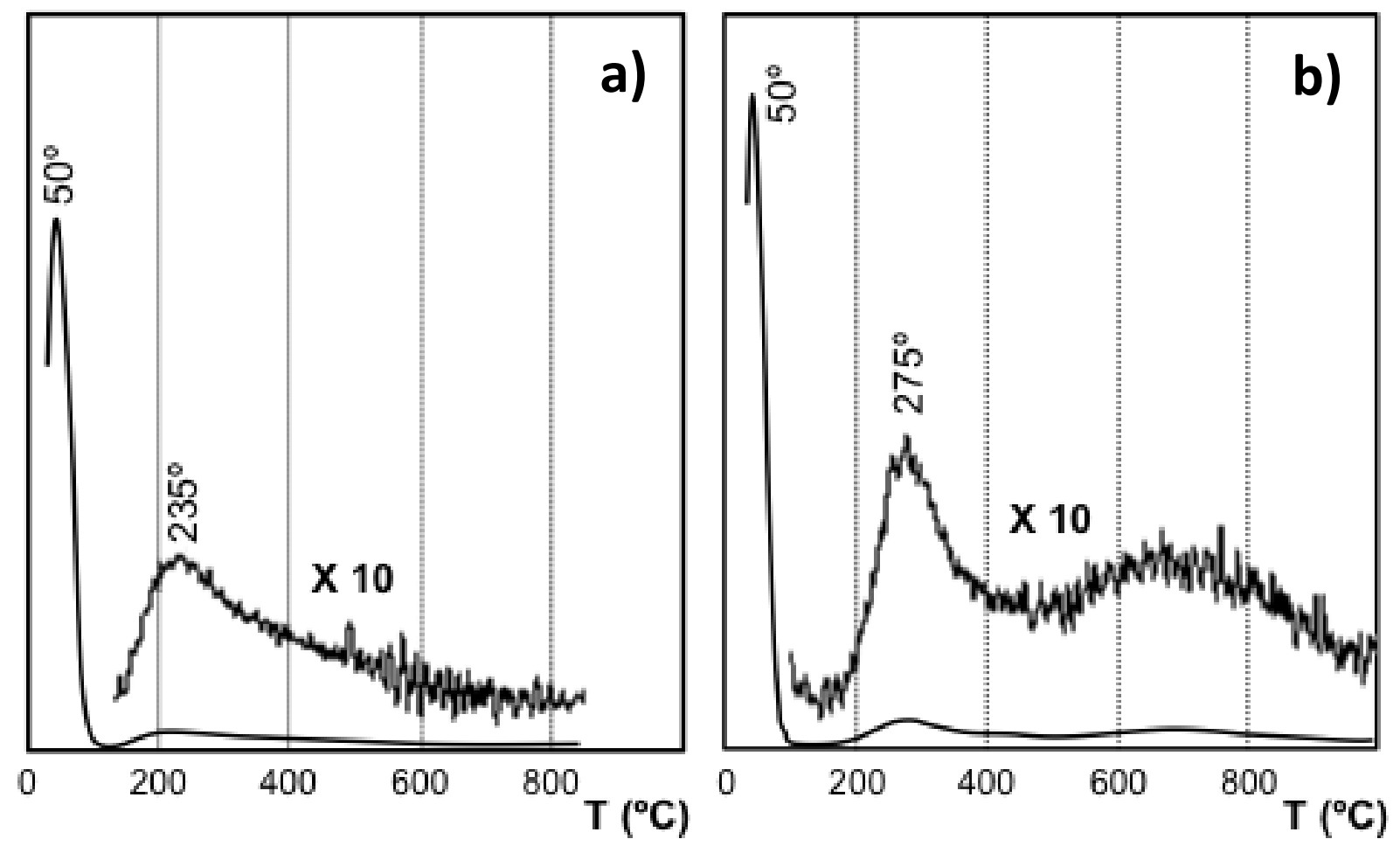

Figure 6. Typical TG traces (presented as first derivative, DTG) of a) a fumed silica, Aerosil 380 (Degussa), b) a mesoporous silica, MCM-41, under dry air flow, $\beta=5^{\circ} \mathrm{C} / \mathrm{min}$.

\subsubsection{Bulk data: acid-base titration. How many negatively charged silanolate groups can there be at the interface?}

There are many ways to differentiate the reactivity of surface silanols, depending on what phase is in contact with the silica surface. If one is dealing with a silica-aqueous solution interface, simple acid-base titration can be useful. Most early studies chiefly aimed at determining the value of the PZC (point of zero charge) of silica, and titration data were thus treated in the "1 site, 2 pK" model. ${ }^{89-91}$ In this model all surface sites are supposed to be 
equivalent, and can be either protonated or deprotonated; thus, the only significant differences would be between protonated silanols $\mathrm{Si}-\mathrm{OH}_{2}{ }^{+}$, neutral silanols $\mathrm{Si}-\mathrm{OH}$ and deprotonated ones $\mathrm{Si}-$ $\mathrm{O}^{-}$. Duval et al. ${ }^{91}$ have analysed their O1s XPS results in this manner. But the appropriateness of the 1 site, 2 pK model may be questioned since only negative surface charges are observed for all tested $\mathrm{pHs},{ }^{92,93}$ besides other techniques amply show the heterogeneity of surface silanols. Furthermore, when the total amphoteric sites density is considered as an adjustable parametre and estimated from the titration curves, it provides values quite different from those estimated from TG. ${ }^{93}$

Therefore it would seem more fruitful to consider that the silica surface exhibits different surface sites, all of them acidic but with different acidity constants, i.e. to use "multisite, $1 \mathrm{pK}$ " models. ${ }^{94}$ Using such models the PAD (proton affinity distribution) could be extracted from acid-base titration curves. In practice, this approach is not widespread and we are not aware of PAD curves for pure silica, although they have been reported for silica-containing mixed oxides. $^{95}$

On the other hand, some authors have observed the evolution of surface ionisation by other means than measuring proton consumption. Ong et al. ${ }^{96}$ followed the surface charging of a quartz surface by SHG (second harmonic generation) observation of polarised interfacial water. This technique does not allow an estimate of the absolute value of the surface charge. However, the existence of two successive jumps separated by $4 \mathrm{pH}$ units is unmistakeable (see Figure 7), and therefore the existence of two silanol populations with intrinsic acidities separated by a factor of 10 000. The first population represents $15-20 \%$ of the total and has a $\mathrm{pK}_{\mathrm{a}}=4.5$ (about the same as for acetic acid), whereas the remaining one accounts for $80-85 \%$ and has a $\mathrm{pK}_{\mathrm{a}}=$ 8.5; very similar conclusions were reached by Ostroverkhov et al. from SFG (sum frequency generation). ${ }^{97}$ 


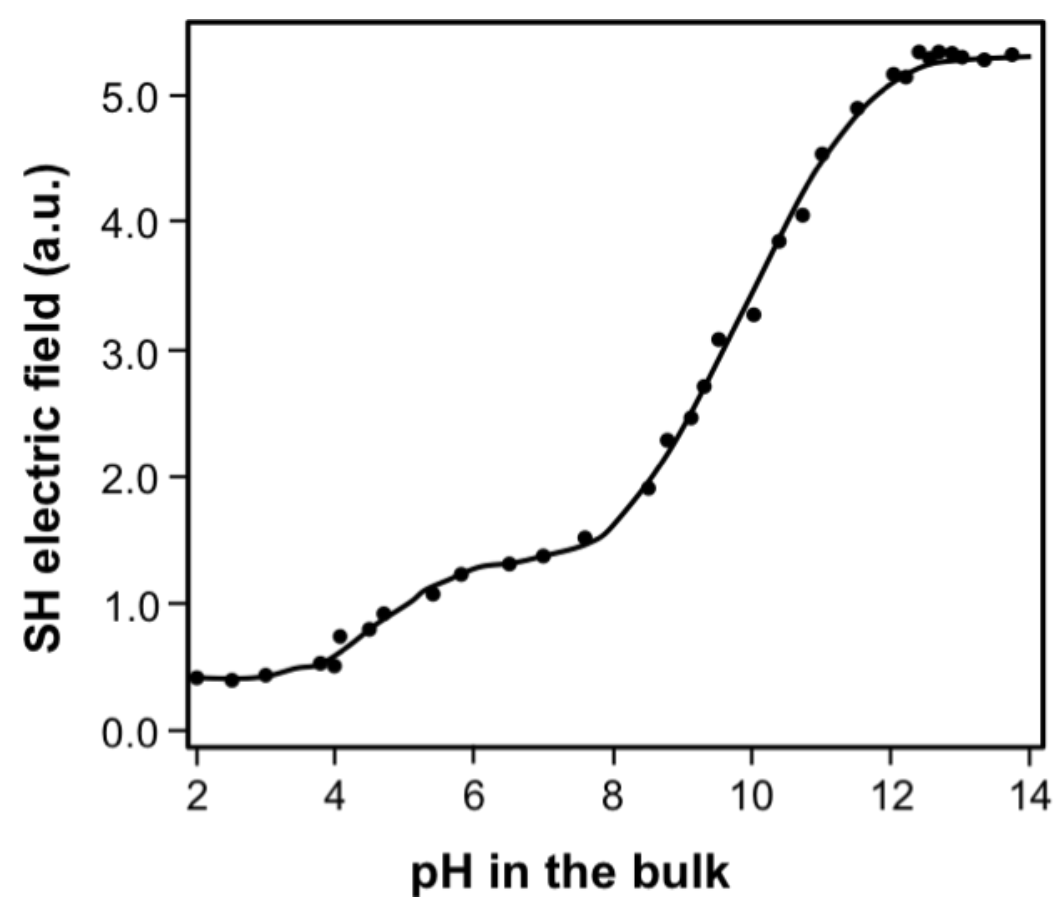

Figure 7. Intensity of the electric field (supposed proportional to the surface charge) at the silica - aqueous $\mathrm{NaCl}$ solution interface as a function of solution $\mathrm{pH}$. Adapted with permission from Ref. ${ }^{96}$. Copyright 1992 Elsevier.

Evanescent wave cavity ring-down spectroscopy is another technique allowing to probe the chemical state of a solid-liquid interface in situ, ${ }^{98}$ and its application to the adsorption of cationic dyes on silica prisms as a function of $\mathrm{pH}$ confirmed the existence of two populations of sites with different acidity in about 3 to 1 ratio. ${ }^{99-101}$ This is an example of using the adsorption of a particular molecule in order to identify a corresponding site on the surface, i.e., it is an instance of probe molecule adsorption at the solid-liquid interface: more will be said on probe molecules in Section 3.1.7.

While the authors of these studies did not provide specific assignments for the two silanol populations, a possibility is that they correspond to geminal and terminal silanols, respectively: the percentage of groups with the smaller $\mathrm{pK}_{\mathrm{a}}$ value (more acidic) is of the same order as the percentage of geminal with respect to total silanols as measured by NMR. Other interpretations of the two populations could be possible, as the state of H-bonding may also influence the acidity of a silanol, and we will propose such an explanation in the conclusions (Section 10). Some simplified models of surface groups, such as the MUSIC (MUltiSIte Complexation) model, and its refinements, have been proposed to theoretically predict these effects, but they seem to do a rather poor job at accounting for silica surfaces. ${ }^{102-104}$ Therefore, the molecular modeling of the surface acidity of silanol groups is currently an active field of research and will be discussed in detail in Section 8.2.6 below. 
Well-defined jumps are not apparent from classic pH-metric data (Figure 7). Whether this difference is simply due to an intrinsically lower resolution for the latter data remains unknown.

A better assignment of $\mathrm{pK}_{\mathrm{a}}$ values observed on amorphous silica would be possible if we knew more about the behavior of silanol groups on crystalline silica faces which should expose silanols in well-known, uniform environments. Churchill et al. ${ }^{105}$ estimated PZCs from AFM measurements at variable $\mathrm{pH}$ and found small but significant differences between the (100) face on the one hand $(\mathrm{PZC}=2.9)$, and the $(101)$ and $(011)$ faces on the other hand $(\mathrm{PZC}=2.7)$. Such studies are still rare.

A question of significance for modeling silica interfaces in the presence of an aqueous solution is the maximum density of negatively charged groups (silanolates, i.e., $\mathrm{Si}^{-} \mathrm{O}^{-}$). If one adopts the 1-site, 2-pK model, the answer should be fairly simple. In this model all surface silanols are supposed to be chemically equivalent, and amphoteric. At a $\mathrm{pH}$ value significantly superior to $\mathrm{pK}_{\mathrm{a} 2}$, virtually all of them should be negatively ionized (dissociation rate $\alpha \approx 1$ ). If one were dealing with a solution of a weak acid, $\alpha$ would be about 0.99 at $\mathrm{pH}=\mathrm{pK}_{\mathrm{a} 2}+2$; in fact for acid groups on a surface, $\alpha$ is smaller due to the adverse energetic effect of accumulating negative charges in close vicinity upon ionization, but it should still tend toward unity at reachable $\mathrm{pH}$ values, i.e., the silanolate density should approach the total silanol density. Now this type of reasoning is not very helpful in practice because there is no independent estimate of the $\mathrm{pK}_{\mathrm{a} 2}$ apart from the proton titration data, and in addition to the 1-site, 2-pK model is probably inadequate to account for the acido-basic behavior of silica surface sites as mentioned above.

What we do obtain from experience with certainty is a value of the surface charge density as a function of $\mathrm{pH}$. To give a few typical examples, for Aerosil300 in $\mathrm{KCl}$ electrolyte, the surface charge varied from about 0.01 to about $0.08 \mathrm{C} . \mathrm{m}^{-2}$ as the $\mathrm{pH}$ was raised from 5 to $8,{ }^{93}$ corresponding respectively to 0.06 and 0.49 silanolates $\left(\equiv \mathrm{SiO}^{-}\right)$per $\mathrm{nm}^{2}$. The earliest study by Bolt ${ }^{89}$ reported a value of about $0.01 \mathrm{C} / \mathrm{m}^{2}$ for a colloidal suspension of silica particles (with a surface area of $\left.180 \mathrm{~m}^{2} / \mathrm{gr}\right)$ marketed as Ludox, ${ }^{106}$ in a $1 \times 10^{-3} \mathrm{M}\left(\mathrm{mol} . \mathrm{L}^{-1}\right) \mathrm{NaCl}$ solution at $\mathrm{pH}=7$. Sonnefeld ${ }^{107}$ reported for spherical silica particles in a solution at $\mathrm{pH}=7$ and ion strength $=5 \times 10^{-3}$ $\mathrm{M}$ a value of $0.02 \mathrm{C} / \mathrm{m}^{2}\left(0.12 \equiv \mathrm{SiO}^{-} / \mathrm{nm}^{2}\right)$. Behrens and Grier ${ }^{108}$ measured a value of 4000 effective negative charges per $\mu \mathrm{m}^{2}$ at $\mathrm{pH}=7$ and ion strength $=5 \times 10^{-6} \mathrm{M}$ for $1 \mu \mathrm{m}$ silica spheres, i.e. only $0.004 \equiv \mathrm{SiO}^{-} / \mathrm{nm}^{2}$. For comparison, the same authors report for a glass plate in contact with a solution at the same $\mathrm{pH}$ and ion strength a value of 3000 effective negative $\equiv \mathrm{SiO}^{-} / \mu \mathrm{m}^{2}$, i.e. $0.003 \equiv \mathrm{SiO}^{-} / \mathrm{nm}^{2}$. Other studies provide silica surface charge data. ${ }^{109-112}$ Figure 8 shows the surface charge density as a function of $\mathrm{pH}$ for three different silicas. ${ }^{93}$ 


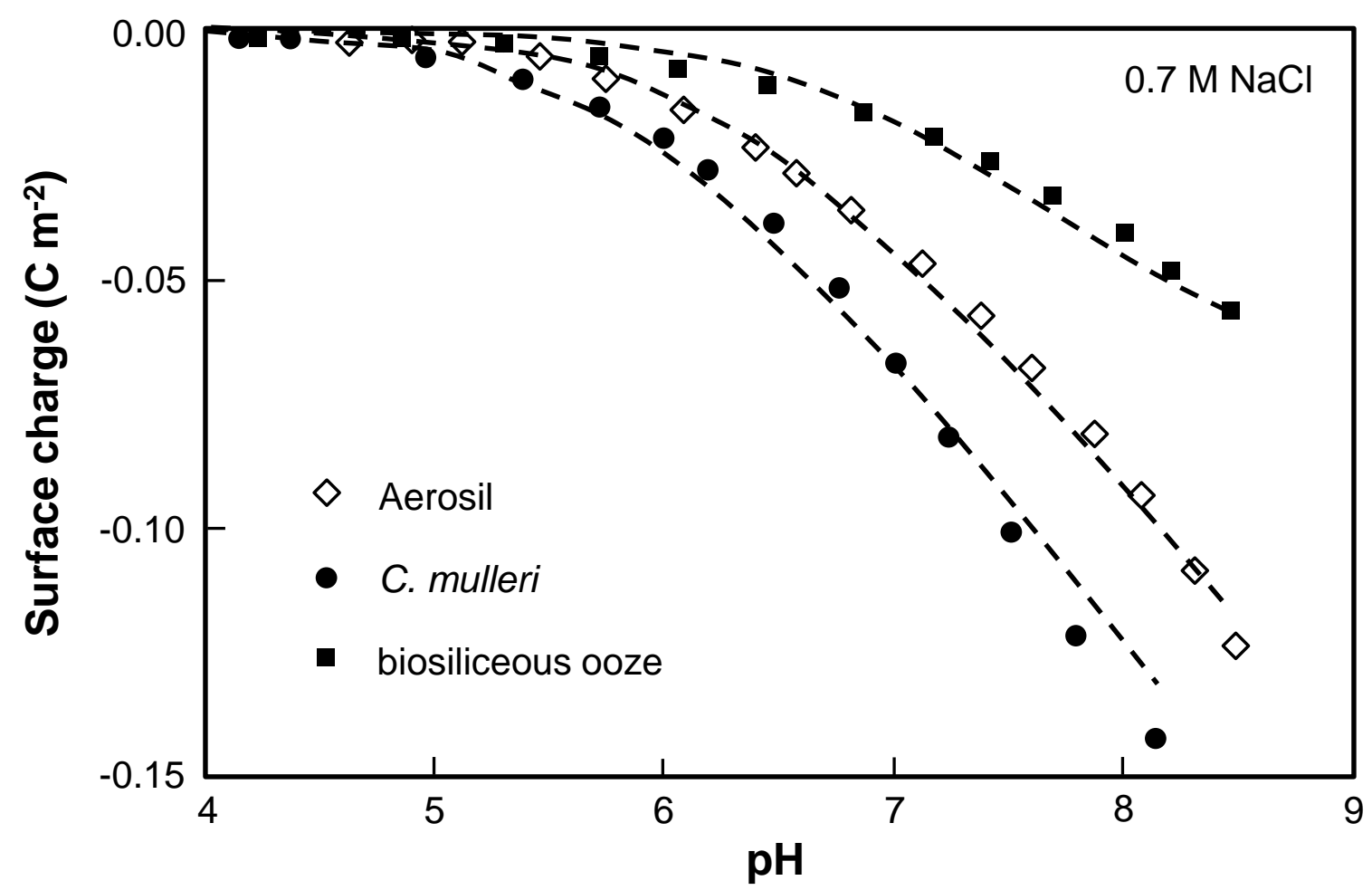

Figure 8. Charge densities from proton titration of three different silicas as a function of $\mathrm{pH}$ : one Aerosil, and two biological silicas. NB: "C. mulleri" refers to a biogenic silica synthesized by the diatom Chaetoceros Mulleri. Adapted with permission from Ref. ${ }^{93}$. Copyright 2002 Elsevier.

These data, while incomplete, show how the actual electric state of the silica surface is crucially determined by the $\mathrm{pH}$ of the solution from which the adsorption is taking place. It also depends on the ion strength, although less sensitively. This has serious consequences if one wishes to model (bio)molecules adsorption from a solution: the surface density of negatively charged silanolate groups introduced in the model will only have a limited range of validity in terms of conditions in the solution. Note that ${ }^{1} \mathrm{H}$ NMR is a possible experimental approach to detect water in the vicinity of a silanolate, which has been reported to resonate at an unusually low chemical shift value. ${ }^{113,114}$

\subsubsection{Vibrational spectroscopies: IR and Raman}

IR spectroscopy has been applied to amorphous silica, in contact with either a gas phase or vacuum, for a long time. A comprehensive review of the literature until 1998 may be found in Ref. $^{40}$ A typical evolution of the IR spectrum of an Aerosil silica in the $2750-3800 \mathrm{~cm}^{-1}$ range is shown in Figure 9. 


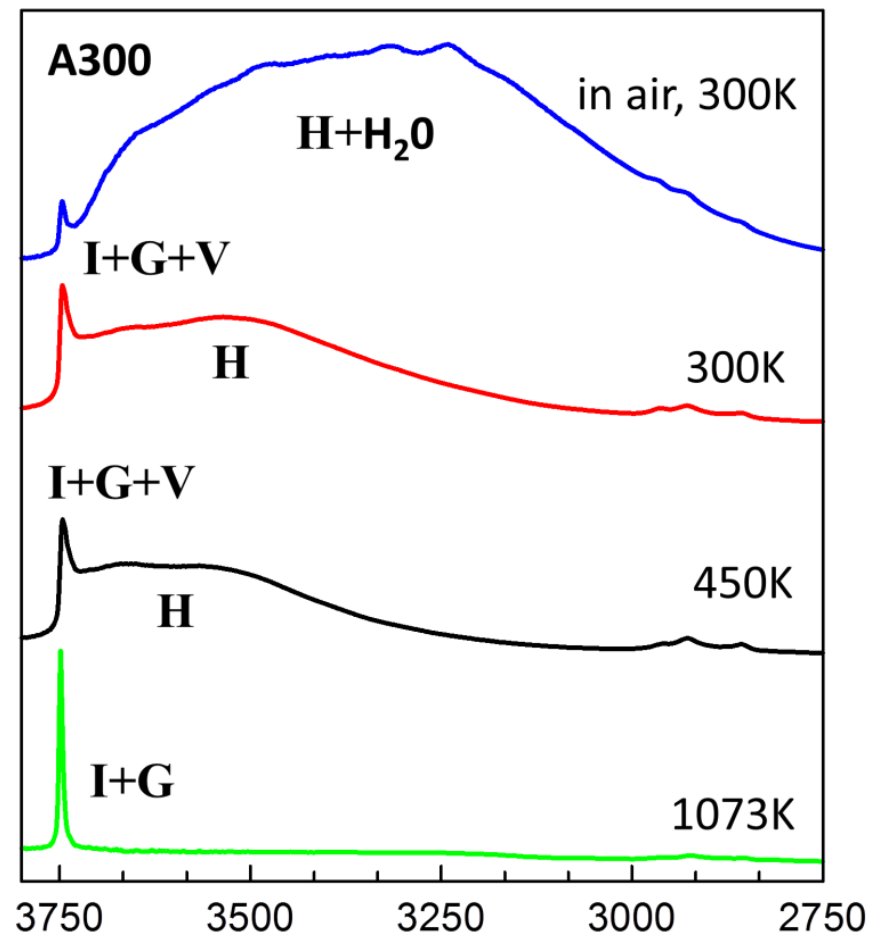

Figure 9. Infrared spectra of a sample of Aerosil 300 (A300, a fumed silica sample characterized by a surface area of $300 \mathrm{~m}^{2} / \mathrm{gr}$ ) after sample pretreatment in vacuum at increasing temperatures. I: isolated, G: geminal, V: vicinal, H: H-bonded (see Section 2.2)

This range contains the stretching vibration of $\mathrm{OH}$ groups $\left(v_{\mathrm{O}-\mathrm{H}}\right)$. If silica is observed under air without any pretreatment, the contribution of the $\mathrm{OH}$ of adsorbed water molecules is dominant. Because water molecules adsorbed in a multilayer are subject to a large range of inhomogeneous $\mathrm{H}$-bond interactions, one observes a large bathochromic shift of the $\mathrm{OH}$ stretching frequency of their $\mathrm{OH}$ groups, together with a heterogeneous broadening. This results in a broad feature extending to about $1000 \mathrm{~cm}^{-1}$ lower than the reference frequency of 3747-3750 $\mathrm{cm}^{-1}$ (labelled $\mathbf{I}+\mathbf{G}+\mathbf{V}$ in Figure 9, since these three types of silanols are indistinguishable, cf. infra). Outgassing at $\mathrm{T}=300 \mathrm{~K}$ already removes the largest fraction of the multilayer adsorbed water, leaving few adsorbed water molecules at the surface so that the absorption is due chiefly to silanol $\mathrm{O}-\mathrm{H}$, and at $450 \mathrm{~K}$ dehydration is probably complete. Even then, this region shows a complex band structure. The narrow band at $3747 \mathrm{~cm}^{-1}$ becomes stronger and more distinct after outgassing; it has been assigned to isolated silanols (denoted I), not interacting through H-bonds. Several red-shifted broad components are observed in addition. They correspond to variously Hbonded silanols. The energy of the stretching vibration is more deeply affected when the O-H group acts as an H-bond donor, when it falls down to about $3520 \mathrm{~cm}^{-1}$; acting as an $\mathrm{H}$-bond acceptor will cause a smaller perturbation, of a few tens of $\mathrm{cm}^{-1}$. It has been claimed that both fumed and precipitated silica surfaces exhibit a density of isolated silanols of about $1.1 \mathrm{~nm}^{-2} .115$ Other studies, however, would indicate that the balance between isolated and H-bonded silanols 
is variable and depends on the geometry of the silica particles, and most notably on their size as the smaller particles have a lesser number of isolated silanols, presumably due to constraints imposed by surface curvature, ${ }^{116}$ whereas on fully hydrated particles with a diameter $>30 \mathrm{~nm}$, only H-bonded silanols were observed.

Sometimes, more precise assignments of the «H-bonded silanol bands » have been proposed but they remain open to question. Quantification of IR absorption results is at best relative: the evolution of the densities of a given type of surface silanol can be followed along a series of treatments, such as thermal activation or chemical functionalisation, but an absolute quantification is difficult due to the high variability of extinction coefficients. In silicalite, which is a kind of microporous silica, several signals including a strong band at $3500 \mathrm{~cm}^{-1}$ and a shoulder at $3450 \mathrm{~cm}^{-1}$ were assigned to small groups of Si-OH mutually interacting in « silanol nests ». ${ }^{117}$ These could in principle form the basis for band assignments on silica (see a similar remark below for ${ }^{1} \mathrm{H}$ NMR results).

Further heating and outgassing at $\mathrm{T}=1073 \mathrm{~K}$ entirely removes the H-bonded pairs leaving on the surface only terminal (isolated and geminal) silanols. IR alone, in contrast with ${ }^{29} \mathrm{Si} \mathrm{NMR}$ (vide infra), cannot discriminate between terminal and geminal (G) groups. ${ }^{118,119}$ Takei et al. ${ }^{120}$ attempted to create de novo geminal groups (corresponding to $\mathrm{Q}^{2} \mathrm{Si}$ ) as well as "triple silanols" $\mathrm{O}-\mathrm{Si}(\mathrm{OH})_{3}$ (corresponding to $\mathrm{Q}^{1} \mathrm{Si}$ ) by grafting of chlorosilanes on the silica surface followed by their hydrolysis; they concluded that the frequencies of such groups are within 2 and $3 \mathrm{~cm}^{-1}$, respectively, of that of isolated terminal silanols $\left(Q^{3} \mathrm{Si}\right)$, which is too small a difference for ordinary spectrometers to resolve.

Silica also shows several diagnostic vibrations outside of the $\mathrm{OH}$ stretching range. ${ }^{121}$ The strong Si-O-Si stretching vibrations in the $1000-1200 \mathrm{~cm}^{-1}$ range, and the bending modes under $800 \mathrm{~cm}^{-1}$, are usually ill-resolved and mostly due to bulk, not to surface groups. An exception can be found in thin silica films obtained by superficial oxidation of Si wafers, where all Si-O-Si groups belong to the surface region. These systems can be observed by a variant of IR spectroscopy, namely GA-ATR (grazing incidence attenuated total reflectance). The asymmetrical Si-O-Si stretch undergoes a phonon splitting between longitudinal optic (LO) and transverse optic (TO) components. It has been shown ${ }^{122,123}$ that in a thin $\mathrm{SiO}_{2}$ film, the TO vibrations are parallel to the film surface while the LO vibrations are perpendicular to it. Both components can be observed in GA-ATR; the band at $1230 \mathrm{~cm}^{-1}$ has been assigned to the LO mode, and the band at $1045 \mathrm{~cm}^{-1}$ to the corresponding TO mode. ${ }^{124-128} 129,130$

More specific vibrational bands can also give information on siloxane ring sizes. Strained rings give rise to breathing modes conspicuous in Raman spectra, at $605-607 \mathrm{~cm}^{-1}$ for 3-rings (S3R), known as D2 defects, ${ }^{131-133}$ and $490 \mathrm{~cm}^{-1}$ for 4-rings (S4R, called D1 defects; the latter is 
more difficult to interprete due to the overlap with another band). 4-rings are only slightly strained. As already mentioned in Section 2, strained rings can be seen in freshly calcined silicas but exposure to water will break the siloxane (Si-O-Si) bond. ${ }^{134}$ The energy gain provided by strain relief seems more than sufficient to cause dissociative chemisorption of water, at least in the case of S3R. This process has been quantified in a thorough study on heat-treated silica gels, ${ }^{135}$ and indeed the disappearance of one D2 defect is accompanied by the appearance of two newly-formed silanols. Conversely, heating at high temperatures causes silanol pairs to condense giving rise to strained ring defects.

3-rings and 4-rings are found in some little-known naturally crystalline silicates which may serve as references (quoted in Ref. ${ }^{136}$ ).

Then there is the important question of 2-rings or S2R which have been mentioned in section $2-\mathrm{S} 2 \mathrm{R}$ are especially important for the present review because they have given rise to a lot of molecular modeling studies. Two bands at $888-891$ and $908-910 \mathrm{~cm}^{-1}$ had been observed to be present in silicas activated at high temperature (fortunately, they lie in a narrow transparent window in the silica spectrum, between Si-O-Si bending and stretching modes) and to disappear upon adsorption of Lewis bases or Lewis acids ${ }^{137}$ (see below, Section 3.1.7). Michalske and Bunker ${ }^{138}$ hypothesized that they were specifically attributable to S2R (which they called « edgeshared defects »), and they were able to substantiate this claim by comparing with the vibrational spectra of molecular disiloxanes ${ }^{139}$ (Figure 10).
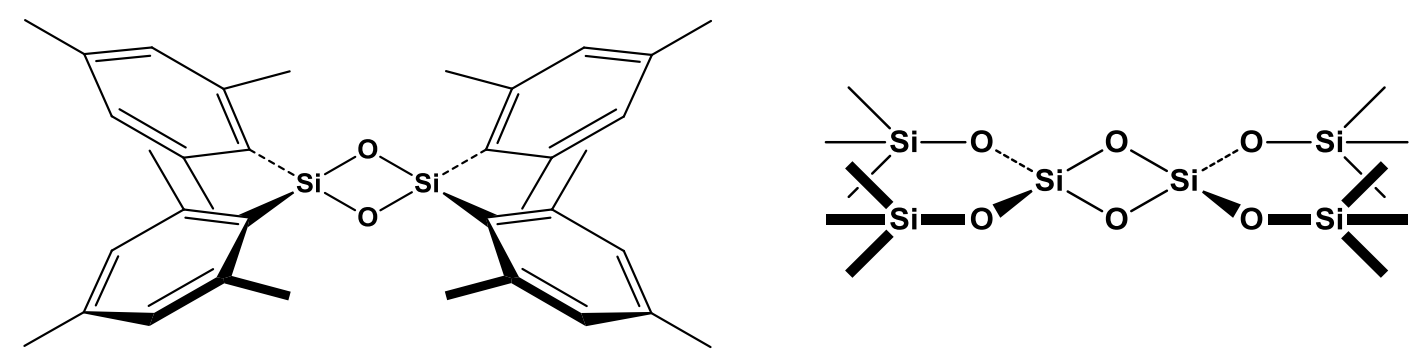

Figure 10. A molecular disiloxane (left), compared with the local structure of the S2R defect on the silica surface (right).

Thus, an experimental basis was available for this assignment, which has first been made before the era of molecular modeling. Some attempts have been made to quantify S2R: Grabbe et al. ${ }^{140}$ reported a figure of 0.2 to $0.4 \mathrm{~nm}^{-2}$ on an Aerosil silica treated at $1075^{\circ} \mathrm{C}$. Of course, less harsh treatments are expected to generate a lesser S2R concentration. Later, the IR bands typical of S2R were also observed on mesoporous silicas pretreated at $600^{\circ} \mathrm{C}{ }^{141}$ and silica thin films pretreated at $1130^{\circ} \mathrm{C}$ in vacuo. ${ }^{142}$

\subsubsection{NIR, visible and $U V$}

The NIR region contains overtones and combinations of stretching and bending modes involving $\mathrm{OH}$ groups of water and silanols. The most specific features associated with silica 
surface groups are bands at 4550 and $7310 \mathrm{~cm}^{-1}$ (about 2200 and $1368 \mathrm{~nm}$, respectively). The first one is a combination band $\left(v_{\mathrm{OH}}+\delta_{\mathrm{SiOH}}\right)$, the second one is the $2 v_{\mathrm{OH}}$ harmonic. ${ }^{40}$ The combination band in particular is useful because it can be observed even in the presence of adsorbed water, as it is energetically well separated from the $\left(v_{\mathrm{OH}}+\delta_{\mathrm{HOH}}\right)$ combination of $\mathrm{H}_{2} \mathrm{O}$. It then shows two or three components that have been attributed to isolated silanols and $\mathrm{H}$ bonded ones: ${ }^{40}$ but some physicists still argue that the $4550 \mathrm{~cm}^{-1}$ band has a more complex origin, ${ }^{143}$ and this spectral region is not widely used to discriminate surface groups.

Silica is essentially transparent in the visible range and exhibits charge transfer bands in the UV (or band gap, depending on whether a localized or a delocalized bond description is adopted), like all solid oxides. It has been claimed ${ }^{144}$ that the $50 \%$ UV cutoff wavelength can provide information on the distribution of siloxane ring sizes, but it is doubtful if precise information can be obtained in this way.

\subsubsection{Solid-state NMR}

Since the first stages of its development, solid-state NMR has been a choice technique in the study of the local structure of silicates and silica polymorphs, ${ }^{72}$ since ${ }^{29} \mathrm{Si}$ is a "good" nucleus for NMR because it has a $1 / 2$ nuclear spin, it provides well-resolved spectra without the complications due to quadrupolar interactions, and ${ }^{29} \mathrm{Si}$ chemical shifts $(\delta)$ are highly sensitive to details of the molecular environment (for instance, a correlation between $\delta$ and the average Si-O$\mathrm{T}$ angle was evidenced early on). Thus, ${ }^{29} \mathrm{Si}$ NMR provides valuable information on the bulk structure. Whether it allows to specifically observe surface groups depends first and foremost on the surface area: if this parametre is too low, surface-specific signals will be overwhelmed by bulk ones. For many amorphous silicas, the surface area reaches values in the order of several hundred $\mathrm{m}^{2} / \mathrm{g}$, and in this case the proportion of total $\mathrm{Si}$ atoms that are exposed on the surface is high enough for their signal to stand out in the spectrum. Bulk Si all belong to $\mathrm{Q}^{4}$ tetrahedra and give rise to a broad signal centred at about $-110 \mathrm{ppm}$. On the other hand, surface Si may belong to either $\mathrm{Q}^{2}, \mathrm{Q}^{3}$ or $\mathrm{Q}^{4}$ tetrahedra, with maxima at about $-90,-100$ and $-110 \mathrm{ppm}$, respectively, according to well-known assignments. ${ }^{145}$ The signals are much broader than e.g. in zeolites because of the large distribution of Si-O-Si angles, but they are well-resolved enough to allow unequivocal quantification (Figure 11). Now this quantification in terms of $\mathrm{Q}^{\mathrm{n}}$ connectivity is correlated with the densities of the different types of silanol groups, since each $\mathrm{Q}^{3}$ Si bears one terminal silanol, while each $\mathrm{Q}^{2} \mathrm{Si}$ bears two geminal ones. This is the most precise way of quantifying surface silanols, and the fact that it correlates well with the completely independent TG data as far as total silanol densities are concerned is encouraging. 


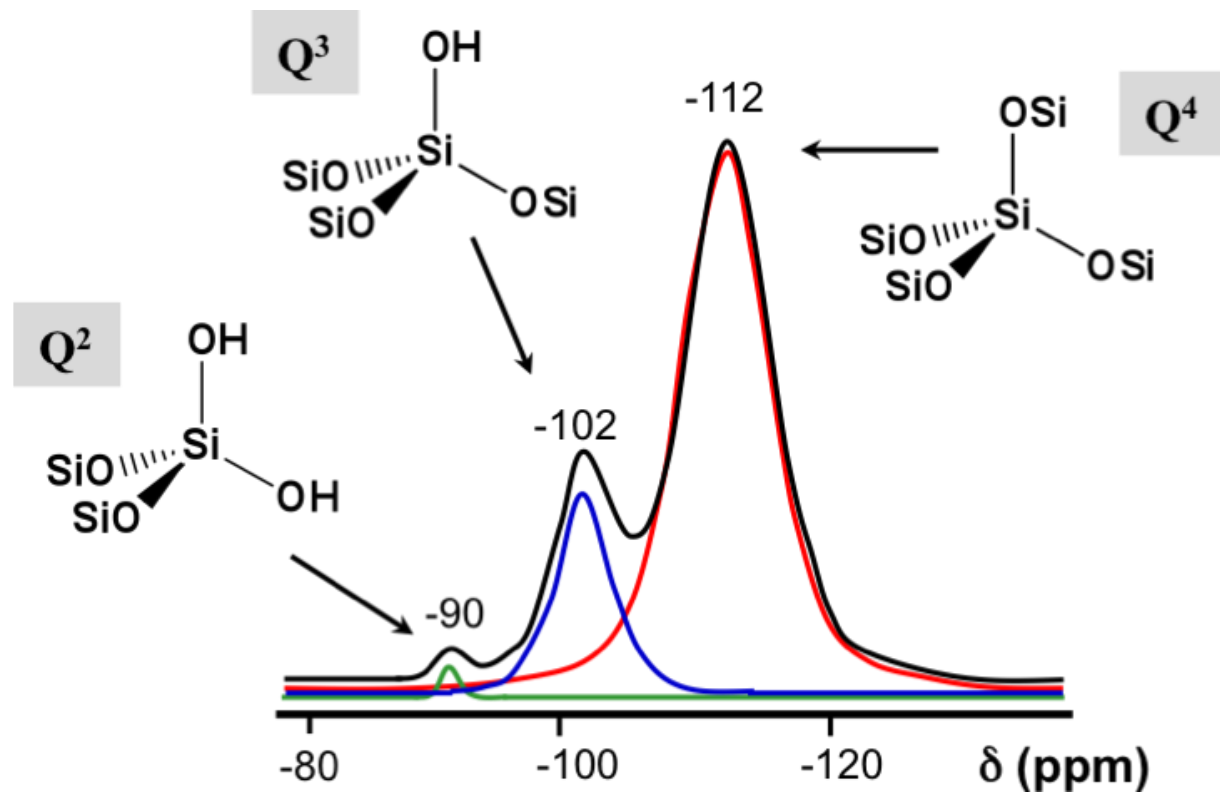

Figure 11. Typical (1-pulse) ${ }^{29} \mathrm{Si}$ MAS-NMR spectrum of a fumed silica (Aerosil 380), with band assignments. Adapted from Ref. ${ }^{146}$

Some authors have proposed that the $\mathrm{Q}^{4}$ peak could be further decomposed into components corresponding to strained and unstrained rings, the former resonating at lower field, but since the hypothesized components of the $\mathrm{Q}^{4}$ peak are never well resolved this probably cannot form the basis of a quantification of different size rings.

It is possible to selectively enhance the NMR signals of surface Si if desired. Detecting the ${ }^{29} \mathrm{Si}$ signals by proton cross-polarisation $\left(\left\{{ }^{1} \mathrm{H}_{-}^{29} \mathrm{Si}\right\} \mathrm{CP}\right)$ ensures that only those $\mathrm{Si}$ atoms in close vicinity of a proton will appear in the spectrum. Since the proton sources are silanols and eventually adsorbed water molecules, this technique is surface-selective (it also has the advantage of being faster than single-pulse NMR); however, quantification of CP results is difficult since $\mathrm{Si}$ atoms in different environments will respond differently, according to the intensity of their coupling with protons which determines the dynamics of polarisation transfer. The most relevant data on this problem are summarised in Ref. ${ }^{147}$ which proposes a CP quantification technique. Recently, an improvement in surface selectivity more spectacular than that observed with CP was shown using dynamic nuclear polarisation (DNP), where a 100-fold enhancement of the surface ${ }^{29} \mathrm{Si}$ signals was observed for a mesoporous silica. ${ }^{148}$ This technique involves contacting the sample with a small amount of radical-containing solution, which is not chemically innocuous, and it is too early to tell what impact it will have on the field.

${ }^{17} \mathrm{O}$ NMR data are much less frequent than ${ }^{29} \mathrm{Si}$ NMR because the natural abundance of the target isotope is lower and makes it difficult to record spectra without isotopic enrichment. Natural abundance ${ }^{17} \mathrm{O}$ spectra were recorded for some silica polymorphs, notably in cristobalite at high temperature. ${ }^{149}$ Potentially, signals specific for surface groups could be even more obvious than in ${ }^{29} \mathrm{Si} \mathrm{NMR}$, because the oxygen in a silanol lies in a very different environment 
from that in a siloxane bridge. A very different chemical shift is therefore expected, and probably also a different quadrupole constant value (a parametre with no analogue for ${ }^{29} \mathrm{Si}$, which determines the line shape). In addition, it may be possible to specifically enrich the surface region by exchange with ${ }^{17} \mathrm{OH}_{2} \cdot{ }^{17} \mathrm{O}$ spectra have indeed been recorded on $45 \%$ enriched sol-gel silicas ${ }^{150}$ but it turned out that the signals originating from silanol groups are difficult to detect due to very short relaxation times. Thus, ${ }^{17} \mathrm{O}$ NMR has not lived up to its promise so far.

${ }^{1} \mathrm{H}$ NMR is of course surface-selective as it « sees » silanol groups, which mostly exist on the surface. However the interpretation of spectra has been complicated by the difficulty to record solid-state NMR spectra on fully dried samples without exposure to room humidity. In most cases protons from adsorbed water molecules are interfering. This problem was specifically discussed in Ref. ${ }^{151}$ On partially dehydrated silica surfaces, two main signals are generally observed. ${ }^{83,152}$ The first one at $1.8 \mathrm{ppm}$ has been assigned to non-interacting silanols and progessively becomes prominent upon dehydration - some authors have hypothesized that these silanols are internal but actually this is not necessary to explain the weak interaction with water. The second signal is observed in the $3-5 \mathrm{ppm}$ range. As the surface is dehydrated it becomes less intense and shifts to lower $\delta$ values. It has been assigned to the protons of silanols in fast exchange with water, and therefore to the $\mathrm{Si}-\mathrm{OH}$ which have the highest affinity for water - as will be mentioned in Section 3.5 they correspond to $\mathrm{H}$-bonded silanols. Thus, the situation is reminiscent of that observed by IR in the $\mathrm{OH}$ stretching region, with the ${ }^{1} \mathrm{H}$ NMR peak at 1.8 ppm evolving like the IR band at $3740 \mathrm{~cm}^{-1}$. It must be underlined that both techniques are basically sensitive to the « isolated vs. associated » silanols dichotomy, while ${ }^{29} \mathrm{Si}$ NMR provides information on the «terminal vs. geminal » distribution.

Different protons may be distinguished on the basis of chemical shifts, but also of spinspin and spin-lattice relaxation times. ${ }^{152}$ Dipolar dephasing experiments allowed Liu and Maciel to conclude that associated silanols are indeed strongly coupled to water but not to isolated silanols. $^{153}$

Some advanced NMR techniques allow «spin counting», i.e. an evaluation of the number of nuclear spins present in an interacting network. ${ }^{154}$ In the solid state, MQ (multiplequantum) methods for spin counting have been developed since $1985 .{ }^{155}$ On silica, proton MQNMR could be used in principle to count the number of silanols interacting in a «silanols cluster ». This technique has been applied to silica catalyst supports ${ }^{156}$ but with rather trivial results, namely that all ${ }^{1} \mathrm{H}$ spins exist in clusters of less than seven silanols. Yet it probably has some potential to identify silanol clusters, as shown by a study on high-silicic zeolite ZSM-12 ${ }^{157}$ where proton MQ-NMR revealed defects that were tentatively identified as 3-silanol clusters. 
Another intriguing possibility is 2-D spin-spin correlation, either between protons or between proton and ${ }^{29} \mathrm{Si}$ (HETCOR, HETeronuclear CORrelation). These techniques have the potential to unravel neighboring relations between target nuclei in detail. They may provide an opportunity to overcome the « short-sightedness » of single-pulse NMR and other spectroscopic methods. In their study focused on MCM-41 mesoporous silica, ${ }^{83}$ Trébosc et al. were led to propose various arrangements involving surface silanols and water molecules bound together by a well-defined lattice of $\mathrm{H}$-bonds. Although their data were not sufficiently precise to discriminate between these arrangements, evidencing such structures is an attainable goal for future research.

\subsubsection{Paramagnetic sites: EPR and related techniques}

In principle this review is not concerned with high-temperature defects which are quenched by exposure to water, as stated in Section 2.2. The question is of course exactly what defects are completely healed, and while silanone groups for instance are rarely claimed to play an important role in silica reactivity, there is abundant evidence that some radical defects are an essential feature in determining the biotoxicity especially of crystalline silica samples such as quartz and cristobalite. ${ }^{158} \mathrm{~A}$ few words should therefore be said on these radical defects, which can be generated by cleavage of a macroscopic crystal, photochemically, ${ }^{159}$ or mecanochemically (by grinding of a powder), especially since they represent a challenge for molecular modeling and are therefore discussed to a small extent in Sections 5.2 and 6.1.

Since they have unpaired electrons, they should be easily observable by EPR (Electron Paramagnetic Resonance, or ESR, Electron Spin Resonance). This technique allows quantifying paramagnetic defects, and can also give qualitative information from the lineshape: signal anisotropy and hyperfine splitting constant (A) allow identifying the chemical nature of the paramagnetic center. Hyperfine splitting can yield valuable information on coupling with neighboring ${ }^{17} \mathrm{O}$ and ${ }^{29} \mathrm{Si}^{160,161}$ For instance, hyperfine splittings were correlated with Si-O-Si in a recent study. ${ }^{162}$

Simple (cw, continuous-wave) EPR is most frequently used. More advanced techniques such as Pulsed EPR, ${ }^{163}$ or ESEEM (Electron Spin-Echo Envelope Modulation) ${ }^{164}$ have occasionally been applied.

Many radical defects exist and have received names based on their luminescence and optical properties. The most relevant ones for the adsorption of biomolecules correspond to $\equiv \mathrm{Si}$ $\mathrm{O} \bullet, \equiv \mathrm{Si} \bullet$, peroxides and species derived from interaction of the latter with dioxygen - belonging to the infamous ROS (reactive oxygen species). The formation of ROS is one consequence of the high reactivity of surface radical species. They are also reactive toward probe molecules and biomolecules themselves, which is the basis for indirect characterization techniques such as spin 
trapping and SDSL (site-directed spin labelling) which are mentioned in the review by Fenoglio. ${ }^{165}$

As regards non-crystalline silicas, Inaki et al. ${ }^{141}$ have observed the EPR spectrum of mesoporous silicas activated at high temperatures in the frame of a study of surface sites active for photometathesis. They interpreted them as stemming from ( $\equiv \mathrm{Si}-\mathrm{O} \bullet)$ defects. They only made use of the quantitative evolution of the signal intensity, without analyzing the signal lineshape. It should be noted that their work led them to promote an unorthodox assignment of the IR bands at 890 and $910 \mathrm{~cm}^{-1}$, mentioned in Section 3.2.4 and generally attributed to S2R; in their view they can also be attributed to $(\equiv \mathrm{Si}-\mathrm{O} \bullet)$.

Mesoporous SBA-15 were also investigated by SQUID magnetometry ${ }^{166}$ and found to exhibit a weak hysteresis at $2 \mathrm{~K}$, indicative of ferromagnetic behavior. This was interpreted as due to the formation of $(\equiv \mathrm{Si}-\mathrm{O} \bullet)$ and/or $(\equiv \mathrm{Si} \bullet)$ defects during the calcination step.

\subsubsection{Adsorption of probe molecules}

The present review exclusively focuses on the interaction between silica and biomolecules and does not intend to provide a general view of adsorption on silica. However, some adsorption studies are specifically aimed at identifying surface sites and probe molecules adsorption can be said to constitute a surface characterisation technique in its own respect. Many important details about the structure of silica "surface active site" have been obtained chiefly or exclusively from these studies; furthermore, they can form a basis for the interpretation of the more complex phenomena resulting from the adsorption of biomolecules.

A probe molecule must have some kind of interactional complementarity with a certain type of surface site, giving rise to site adsorption. The data may be purely macroscopic: at least they must provide quantification of the amount of considered sites, either by volumetry of the adsorbed molecules from the gas phase, or by thermal desorption following the adsorption step; they may also include an estimate of thermodynamic parametres, by calorimetry or from the temperatures of desorption; and in many cases they are complemented by spectroscopic information, providing a molecular-level view of the probe/site interaction. The spectroscopic technique of choice is generally IR.

In view of the importance of the problem for heterogeneous catalysis, ${ }^{167}$ the most common use of probe molecules is to titrate Brönsted acidic sites (also known as Brönsted acidic centres, BACs) and in that case they must of course be Brönsted bases. Pure silica does not have strong Brönsted acidity. It adsorbs $\mathrm{NH}_{3}$ by $\mathrm{H}$-bond formation, without protonation. ${ }^{168,169} \mathrm{CO}$ is another probe molecule that forms $\mathrm{H}$-bonds with BACs, resulting in a blue shift of the $v_{\mathrm{CO}}$ stretching band proportional to the acid strength, and it also reveals only weakly acidic sites. As 
$\mathrm{CO}$ is adsorbed, the $\mathrm{OH}$ stretching band of isolated silanols undergoes a $-79 \mathrm{~cm}^{-1}$ red shift ${ }^{170}$ and they are therefore considered to be the adsorption sites.

Interestingly, in opposition, comparing silicas with different thermal treatments showed that $\mathrm{NH}_{3}$ preferentially adsorbs on H-bonded rather than on isolated silanols. ${ }^{117}$

Pyridine (Py) has been systematically used for many years as a probe of surface acidity for oxides of catalytic interest (e.g. Refs. ${ }^{171,172}$ ), because adsorbed pyridine has several IR bands whose wavenumber is highly responsive to the adsorption mechanism: one can discriminate at the very least between H-bonded Py, Py interacting with BACs, and Py interacting with LACs (Lewis acidic centers). It was actually first observed by Morrow and $\operatorname{Cody}^{137}$ that pyridine binds reversibly to LACs on the surface of silica activated at rather high temperatures, and the nature of these LACs can even be surmised. In fact, the silica bands of «strained siloxane bridges » bands, which were later attributed to S2R (cf. Section 3.1.4). It has been hypothesized that in $\mathrm{S} 2 \mathrm{R}$ sites the $\left(\mathrm{SiO}_{4}\right)$ tetrahedra are strained enough that a $\mathrm{Si}$ atom can make an additional bond to the doublet of pyridine (Figure 12).

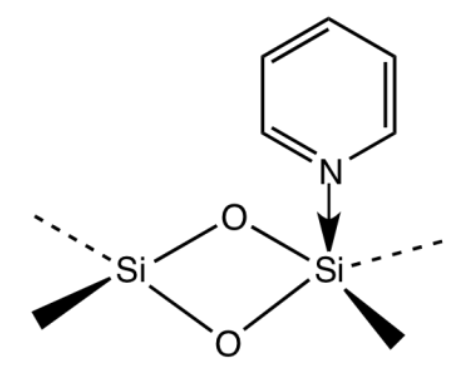

Figure 12. Pyridine bound by a coordinative bond to a S2R site

Water is an intriguing molecule whose interaction with silica surfaces plays a key role for many applications including biomolecules adsorption. Since this will be the topic of Section 8 , there is no need to be exhaustive here. There are very precise works combining water adsorption volumetry and microcalorimetry which provide information on the molar adsorption enthalpy for different silica surfaces. ${ }^{81,82,173}$ An (absolute) value of the molar adsorption enthalpy in excess of the latent heat of liquefaction of water $\left(44 \mathrm{~kJ} \mathrm{~mol}^{-1}\right)$ indicates a specific interaction with the silica surface. In practice, values as high as $170 \mathrm{~kJ} \mathrm{~mol}^{-1}$ have been reported. These data are further discussed in Section 8.1. By combining adsorption isotherms with IR, it is shown that the sites having strong affinity for water, in other words the hydrophilic sites, consist in patches of $\mathrm{H}$ bonded silanols; isolated silanols are not hydrophilic ${ }^{40}$ (the same was observed for $\mathrm{NH}_{3}$ adsorption, see above). This may sound paradoxical in view of their better accessibility to molecules coming from the gas phase. One must realize however that at least the terminal members in a cluster of $\mathrm{H}$-bonded silanols will become a stronger $\mathrm{H}$-bond donor by cooperative effects. 
At room temperature, water physisorption by H-bonding occurs immediately. Hydrolysis of strained bonds, which results in water chemisorption, has both fast and very slow components. They probably correspond respectively to the opening of highly strained S2R, and of somewhat larger rings.

Alcohols share with water the presence of an X-OH moiety. Methanol was one of the first molecules whose adsorption on silica was studied by volumetry, calorimetry and IR. ${ }^{174,175}$ Like water, it can be physisorbed at room temperature by H-bonding, but also opens up strained siloxane rings, yielding easily recognizable methoxy groups (the corresponding reaction has also been reported with ethanol. ${ }^{176,177}$ It is interesting to note that in the latter study, the cooperative effect of several H-bonds resulted in rather strong adsorption, with adsorption enthalpies in the 78-100 $\mathrm{kJ} \mathrm{mol}^{-1}$ range.

The slow chemisorption by siloxane ring opening in S2R involving water or alcohol decomposition can be represented as follows (Figure 13): ${ }^{139,178}$

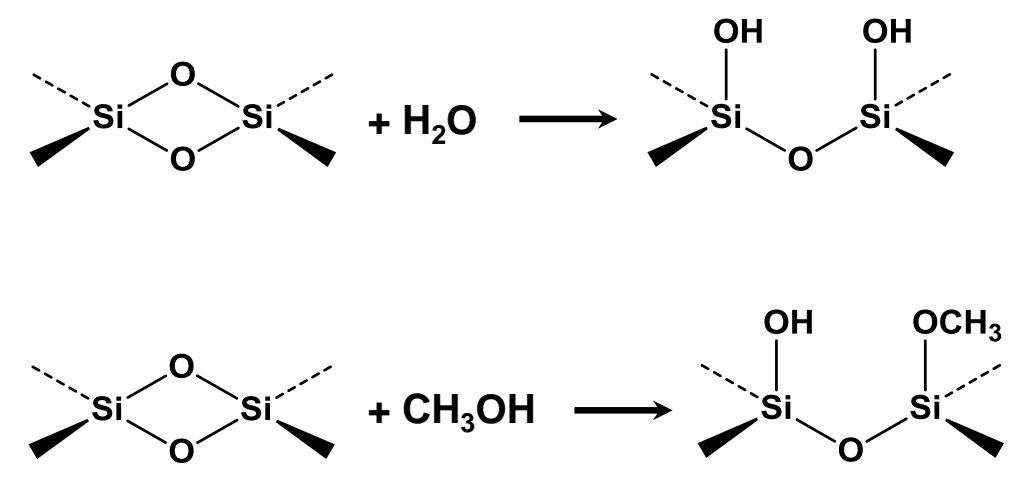

Figure 13. Opening a S2R defect with water (upper) or methanol (lower).

This could in principle happen on other siloxanes, but the dissociation of S2R siloxanes is 5 orders of magnitude faster than that of unstrained siloxanes. ${ }^{178}$

Aldehydes, esters, and ketones adsorption was followed by calorimetry and IR spectroscopy, evidencing H-bond formation with surface silanols: it was reported that upon acceptation of $\mathrm{H}$-bond(s) from silanols, the $\mathrm{C}=\mathrm{O}$ stretching vibration of these molecules could be weakened by several tens of $\mathrm{cm}^{-1}$ (as high as $80 \mathrm{~cm}^{-1}$ in some cases), ${ }^{179}$ which provides a point of comparison for similar studies on biomolecules.

There are a huge number of studies aiming at the modification of silica surfaces through grafting of silanes bearing organic functionalities, or of functional groups based on other metal centers such as $\mathrm{Ti}$ isopropoxyde. Grafting is a form of chemisorption since it involves the formation of covalent bonds, most often with surface silanols, e.g.:

$$
(\mathrm{Si}-\mathrm{OH})_{\text {surf }}+\left(\mathrm{Cl}-\mathrm{SiR}_{3}\right)_{\mathrm{gas}}=\left(\mathrm{Si}-\mathrm{O}-\mathrm{SiR}_{3}\right)_{\text {surf }}+(\mathrm{HCl})_{\mathrm{gas}}
$$


While we are not concerned in this review with the hybrid materials surfaces formed through these procedures, it has been suggested that grafting, as a selective reaction, might be used to obtain information on the specific groups exposed by the starting silica surface. In one study, it was shown that PTMS (propyltrilethoxysilane) preferably reacts with the hydrophobic regions of a mesoporous silica surface, namely those containing only siloxanes and isolated silanols. ${ }^{180}$ Conversely, it seems that Ti isopropoxyde grafting preferentially occurs on H-bonded rather than isolated $\mathrm{SiOH} ;{ }^{181}$ interestingly, to prove the latter point, the authors made use of two silicas, a Stöber microspheric silica which has no isolated silanols, and an Aerosil where previous treatment by $\mathrm{Si}\left(\mathrm{CH}_{3}\right)_{2} \mathrm{Cl}_{2}$ had selectively deactivated the isolated silanols, like in the previous case.

One should not be surprised if some grafting agents exhibited a more stringent selectivity towards silica surface sites than simply prefering isolated vs. H-bonded, or vice-versa. Early on, it was suggested that terminal and geminal silanols may have different reactivities, for instance towards TMS (tetramethylsilane) grafting. ${ }^{182}$ In fact, claims of high specificity may be found in the old literature: some Russian workers ${ }^{183}$ used $\mathrm{VOCl}_{3}$ grafting to titrate groups of two, and of three neighboring silanols. They claimed to be able to separately monitor the evolution of monografted, digrafted and trigrafted $\mathrm{V}$ centers, which followed a logical trend as a function of the total silanols density. Unfortunately this line of thought has not been pursued any further.

Recently, however, interesting data were obtained by grafting of $\mathrm{Ga}\left(\mathrm{CH}_{3}\right)_{3}$ from the gas phase on an Aerosil and a mesoporous silica previously heated at $800^{\circ} \mathrm{C} .{ }^{184}$ The investigators deduced from a precise analysis of Ga K-edge EXAFS (Extended X-Ray Absorption Fine Structure) that gallium atoms were preferentially grafted in pairs, these pairs being connected by two bridging silanolate groups. Integrating these results with bulk mass balance data on the adsorbed $\mathrm{Ga}$ and the methane molecules emitted upon grafting yielded an interesting model of the adsorption site, which would consist in a S2R bearing an additional silanol group, the socalled $\ll \mathrm{d}^{3}$ site $»$ (Figure 14):

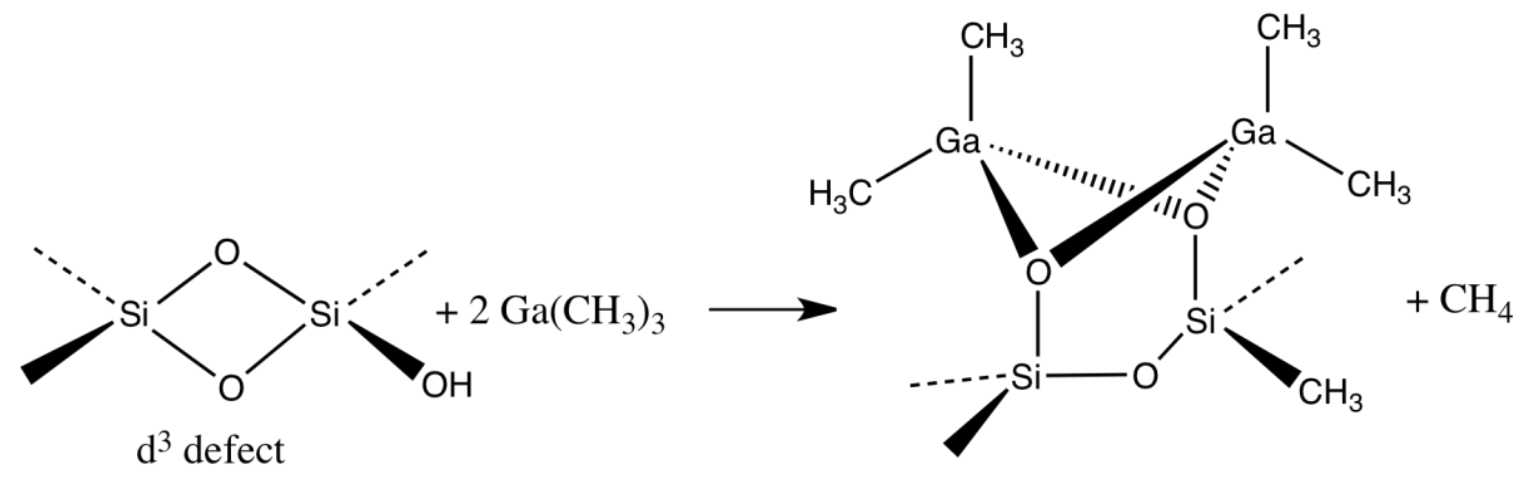

Figure 14. How the reactivity of dimethylgallium allows to evidence the existence of « $\mathrm{d}^{3}$ defects » (S2R bearing one OH group) on the silica surface, according to Ref. ${ }^{184}$ 
If confirmed, the unequivocally evidencing of $\mathrm{d}^{3}$ defects, experimental results would be significant, because it would constitute a step towards the identification of surface defects with more complicated structures, combining several functionalities. Note that the $\mathrm{d}^{3}$ defects would probably be formed at high temperatures from the condensation of $\mathrm{Q}^{2}-\mathrm{Q}^{3}$ vicinal silanol pairs (Figure 15).

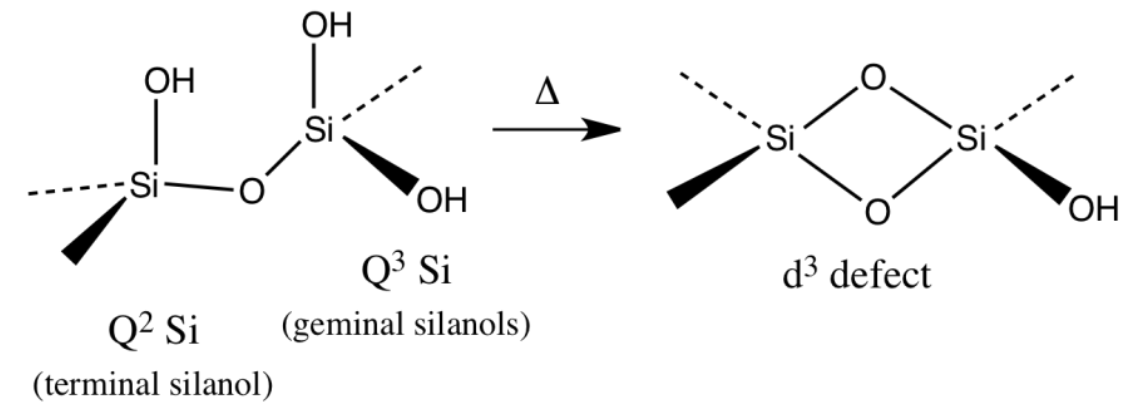

Figure 15. Formation of a $\ll d^{3}$ defect $»$ through high temperature silanol condensation.

It can be inferred from the data that there would be about 0.51 such sites per $\mathrm{nm}^{2}$ on the surface of Aerosil380.

All of the above results refer to adsorption of probe molecules from the gas phase. Adsorption from aqueous solutions could also help identify specific adsorption sites.

Attempts have been made to dose « completely isolated » silanols through the adsorption of a bulky molecule, $\mathrm{CV}$ or « crystal violet ». ${ }^{185}$ A strong adsorption was assigned to $\mathrm{CV}$ cations lying flat on the silica surface, electrostatically bound to a silanolate and unimpaired by interaction with other silanol(ate)s. Since one CV molecule occupies an area of $120 \AA^{2}$, this means that $\mathrm{CV}$ would dose silanols which are separated by more than $10 \AA$ from their nearest neighbors, and there are about 0.11 of them per $\mathrm{nm}^{2}$. This figure may be compared with the value mentioned above for isolated silanols densities, defined as those lying too far apart from other silanols to interact by H-bonding, which is about 10 times higher.

In a study of $\mathrm{Ni}^{\mathrm{II}}$ complexes adsorption (these are significant as precursors of supported catalysts), Boujday et al. ${ }^{186,187}$ measured adsorption isotherms for several polyamine-aqua complexes and observed well-defined and different saturation coverages, which were rationalized by postulating cooperative adsorption sites consisting in 1 to 3 silanols able to act as ligands to the metal ion, together with $\mathrm{H}$-bond accepting groups in close vicinity.

\subsubsection{IRAS, STM, LEED: «flat» silica surfaces}

Thin silica films have been grown on metal single crystal surfaces as model systems for studying the structural properties of $\mathrm{SiO}_{2}$, providing access to surface science techniques such as 
IRAS (Infrared Reflection Attenuation Spectroscopy), STM (Scanning Tunneling Microscopy), LEED (Low Energy Electron Diffraction), etc. Mo(112), ${ }^{188,189,190} \mathrm{Ru}(0001)$ and $\operatorname{Pt}(111){ }^{191}$ have been used as substrates for this purpose, and thin «silica » films were prepared by gas phase Si deposition followed by oxidation. It can be questioned whether these surfaces are really comparable with those of pristine silicas as they are often only a few layers thick, and thus strongly influenced by the substrate so that even the stoichiometry may significantly differ from $\mathrm{SiO}_{2}$. However, spectacular results have recently been obtained for 2-layer thick $\mathrm{SiO}_{\mathrm{x}}$ films on $\mathrm{Ru}(0001)^{29}$ where STM allowed for the first time to visualize in real space the $(\mathrm{SiO})_{\mathrm{n}}$ rings that were so far only indirectly postulated, and thus to obtain experimental histograms of ring size distribution.

On silicon wafers, $\mathrm{SiO}_{\mathrm{x}}$ films can be developped by high-temperature oxidation in an oven but also by «wet» methods involving superficial oxidation e.g. by «piranha» treatment ${ }^{128}$ and observed by ordinary FTIR (Si can be obtained in transparent form) or reflection attenuation IR, ellipsometry, contact angle, ${ }^{192}$ SIMS (secondary ions mass spectroscopy). ${ }^{193,194}$ Work comparing SIMS and XPS and using chemical derivatization has shown that the $\mathrm{m} / \mathrm{e}=45$ $\left(\mathrm{SiOH}^{+}\right)$SIMS peak may be used to quantify total silanol surface densities, which was thus measured at $2.6 \mathrm{~nm}^{-2}$ in Ref. ${ }^{194}$ Reflection attenuation IR also allowed to observe S2R sites. ${ }^{142}$

$\mathrm{SiO}_{\mathrm{x}}$ films on silicon can grow up to at least 1 micrometer thick while still allowing the use of surface techniques such as specular x-ray reflectivity and SANS (small-angle neutron scattering), ${ }^{195}$ and this would be expected to remove the structural influence of the substrate. Here again though, there are indications that the surface of these films may have properties substantially different from that of divided silica. SIMS and XPS Si $2 p$ spectra ${ }^{196}$ of films with $25 \mathrm{~nm}$ thickness indicate a significant amount of Si atoms surrounded by less than 4 oxygens, and they apparently do not belong to the deeply buried $\mathrm{SiO}_{\mathrm{x}} / \mathrm{Si}$ interface since both techniques are surface-sensitive. The reactivity of these systems also has peculiarities; for instance, $\mathrm{SiO}_{\mathrm{x}} / \mathrm{Si}$ was much more reactive towards methanol at RT than a mesoporous silica as decomposition reactions were observed instead of simple adsorption. ${ }^{192}$ In the same way, contacting ethanol with thin films surfaces resulted in the formation of acetaldehyde and ethylene, ${ }^{194}$ which is quite unexpected based on the reactivity of divided silica. Thus, it is possible that flat $\mathrm{SiO}_{\mathrm{x}}$ films present surfaces quite different from divided silicas; at the very least, more work is needed to obtain «normalized» silica adlayers.

\subsubsection{Use of molecular analogues of silica surface groups}

We have already mentioned the role of cyclodisiloxanes in validating the existence of $\mathrm{S} 2 \mathrm{R}$ defects on the silica surface. In fact organometallic chemists have synthesized a large 
variety of molecular compounds containing siloxane and/or silanol groups in well-defined geometries. Many of them could serve as molecular analogues of postulated silica surface groups (see Ref. ${ }^{197}$ for a good review). This line of thought has indeed been explored, but mostly in the field of catalysis, to obtain analogues of silica-supported metal catalysts that are more uniform and energetically homogeneous («homogeneous mimics»), especially from the silsesquioxane family of compounds ${ }^{198,199}$ (Figure 16). The similitude with surface groups of silica, especially as regards the vibrational properties of silanols in well-defined environments, is sometimes put forward. ${ }^{200}$ It must be recognized however that in other respects, such as reactivity and ${ }^{29} \mathrm{Si}$ NMR spectroscopy, the existence of $\mathrm{Si}-\mathrm{C}$ bonds in the silsesquioxane, as opposed to the all-Si-O environment on the surface of silica, will lead to very different properties.
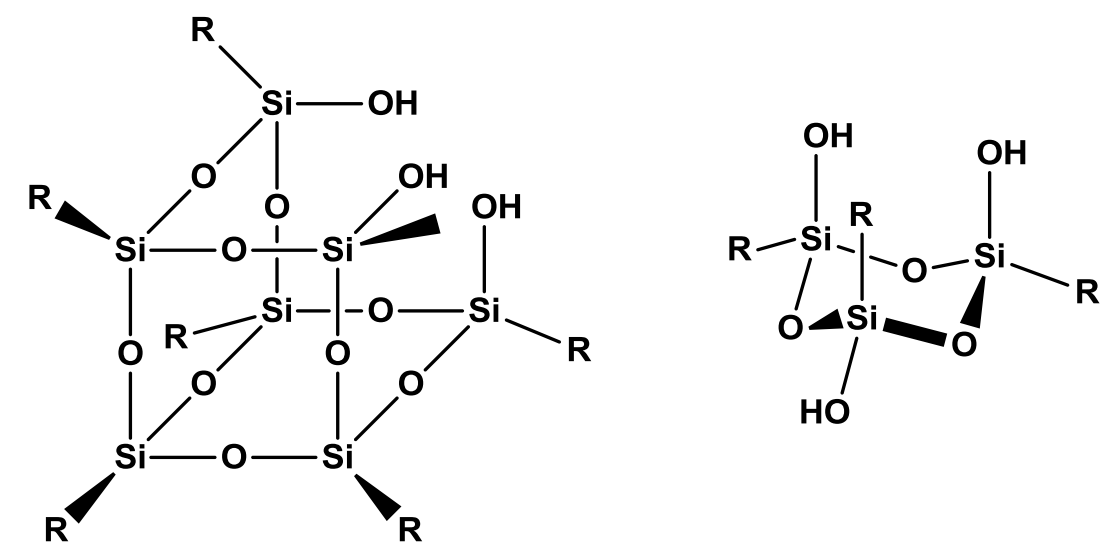

Figure 16. Left, a silsesquioxane bearing a group of 3 silanols in close vicinity. Right, a cyclotrisiloxane that could be used as a molecular mimic of a 3-ring.

\subsubsection{Silica in aqueous solutions: existence of a modified water region}

Physical chemists have long realised that inorganic oxide surfaces are able to induce dramatic changes in the structure of water (Ref. ${ }^{201}$; this is a capability that they share with large biomolecules, incidentally). In the presence of silica, ${ }^{1} \mathrm{H}$ NMR relaxation times were used to evidence surface-induced water structures characterised by a preferred spatial orientation of the water molecules, and a lower molecular density (as compared to bulk water). ${ }^{202}$ These surfaceinduced structures extend at least $8 \mathrm{~nm}$ from the surface. It follows that for a suspension of divided silica with a surface area in the range of several hundred $\mathrm{m}^{2} \mathrm{~g}^{-1}$, the amount of modified water will represent several times the weight of the silica. In fact, it is easy to observe that colloidal silica suspensions tend to form gels when the weight concentration of $\mathrm{SiO}_{2}$ exceeds about $10 \%$ by weight. This has undesired consequences when one tries to study the adsorption of biomolecules - or any molecules for that matter - on divided silica from an aqueous phase. It will not be possible to obtain the pure solid phase by ordinary separation techniques such as 
filtering or centrifugation: instead, it will retain a large amount of solution, containing dissolved molecules that do not directly interact with the surface. If the resulting gel is dried, these molecules will either precipitate as bulk compounds, or establish new interactions with the dry surface, and they will interfere with the molecules that were already adsorbed at the solid/solution interface.

\subsection{Adsorption of biomolecules on silica}

A highly commendable short monograph on the subject is the one of Basiuk, ${ }^{203}$ which asks many of the right questions especially concerning the biomolecule adsorption mechanisms. Other relevant data can be found in several more general reviews, such as Parida et al. ${ }^{204}$ (small organic molecules on silica, including some biomolecules), Lambert ${ }^{205}$ (amino acids on divided oxides, written in a prebiotic perspective) or Fenoglio et al. ${ }^{165}$ (interaction of biomacromolecules with inorganic surfaces).

There are many experimental results from the fields of controlled release/controlled delivery, ${ }^{206}$ chromatography ${ }^{207}$ biosensors and biocatalysts ${ }^{208}$ immobilized enzymes. ${ }^{209}$ Here, as for bare silica surfaces (subsection 3.1), we will first discuss the different techniques that have been used to study the interaction of small biomolecules with silica, and the type of information each one is able to provide; more specific experimental results are discussed in section 9 together with relevant molecular modeling studies. We will also give a (hopefully) exhaustive list of the research papers that have been devoted to the most frequently studied systems, namely, amino acids on silica.

\subsubsection{X-Ray diffraction}

Molecules adsorbed on divided silica, or on divided matter in general, do not in principle give rise to diffraction patterns because the surface of the particles has no 3-dimensional periodicity - this is actually one great difficulty of adsorbed species characterisation. However, performing XRD on biomolecules/divided silica systems is useful at least in a negative way. The amount of biomolecules that can establish a specific interaction with a silica surface is limited and a priori unknown. If the deposition procedure results in retaining a larger amount, excess biomolecules will probably form 3-dimensional crystals. It is necessary to be aware of that if one wishes to interprete correctly the results of other characterisation techniques. For instance, bulk amino acid crystals were evidenced both in $\mathrm{Gly} / \mathrm{SiO}_{2}{ }^{210}$ and $\mathrm{Glu} / \mathrm{SiO}_{2}{ }^{211}$ An interesting phenomenon that will be discussed in Section 9.1.1.2 is that in the case of Gly, excess glycine precipitated as the metastable $\beta$ polymorph, while the $\alpha$ form is normally observed upon precipitation from a clean solution.

In contrast to XRD, LEED (low energy electron diffraction) is a surface-specific diffraction technique which can only be applied to single crystal surfaces. It is possible to obtain 
LEED results on crystalline $\mathrm{SiO}_{2}$ surfaces, notably quartz, ${ }^{212}$ but this is technically difficult. So far, this technique has not been applied to biomolecules on crystalline silica, although on other substrates such as copper it provides unique information, e.g. on the formation of chiral structures $^{213}$ (see also Section 3.2.5).

\subsubsection{Bulk adsorption data: geochemical models and adsorption enthalpies.}

Geochemists have long developed models of adsorption on oxide surfaces from aqueous solutions, such as the double layer and triple layer models. They are most often used to rationalize metal ions adsorption but can be applied to biomolecules as well. The decrease of amino acid concentration in the solution upon contact with e.g. a divided silica is measured by spectrophotometry or HPLC. The evolution of adsorbed quantities as a function of $\mathrm{pH}$ and ion strength can then allow to identify the adsorption mechanism, either physisorption (through $\mathrm{H}$ bond or electrostatics) or chemisorption (grafting). In this way, Vlasova and Golovkova ${ }^{214}$ proposed for basic amino acids (Arg, His, Lys, ornithine) the formation of outer-sphere complexes with silanolates, $\mathrm{Si}-\mathrm{O}^{-\cdots}\left(\mathrm{H}_{2} \mathrm{X}\right)^{+}$, where $\mathrm{X}$ denotes the deprotonated form of the amino acid. In a mesoporous silica, the adsorption of Lys was through a combination of electrostatic and outer-sphere $\mathrm{Si}-\mathrm{O}^{-\cdots}\left(\mathrm{H}_{2} \mathrm{Lys}\right)^{+}$complex formation. ${ }^{215}$

The measurement of adsorption isotherms (adsorbed amount vs. activity in solution, at constant $\mathrm{pH}$ and ion strength) can yield interesting information. If they can be fitted by a Langmuir model (single site adsorption without lateral interaction), two parameters can be extracted from the isotherm, namely the saturation coverage which is equal to the surface density of adsorption sites, and the adsorption equilibrium constant $\mathrm{K}_{\mathrm{ads}}$ which gives access to the adsorption thermodynamics -if isotherms are recorded at different temperatures, the entropic and enthalpic parts can be unraveled by plotting $\ln \left(\mathrm{K}_{\mathrm{ads}}\right)$ against $1 / \mathrm{T}$ (van't Hoff plot). Adsorption isotherms are sometimes recorded (see examples in Table 1) but unfortunately their discussion often remains trivial. Neither are there good instances of the application of other model isotherms, such as the FFG (Frumkin-Fowler-Guggenheim) model which might account for the site adsorption of positively or negatively charged amino acids accumulating charge on the surface.

Thermodynamic data on biomolecule adsorption reactions have been obtained at the silica-water interface however. This was done with the aim to predict separative properties of silicas or modified silicas as chromatographic phases: the retention times of different adsorbates are directly related to the corresponding $K_{a d s}$ values. Free energies of adsorption were estimated by Basiuk ${ }^{207,216}$ for most amino acids and some oligopeptides on a (bare) chromatographic silica. For monomeric amino acids, they are almost always positive $\left(0\right.$ to $\left.+4 \mathrm{~kJ} \mathrm{~mol}^{-1}\right)$ and a compensation effect is observed between $\boldsymbol{\Delta}_{\mathrm{ads}} \mathrm{H}^{\circ}$ and $\boldsymbol{\Delta}_{\mathrm{ads}} \mathrm{S}^{\circ}:{ }^{216}$ while the adsorption of most 
amino acids is exothermic, the entropic contribution is unfavorable. As longer oligopeptides are tested, adsorption becomes more and more favorable. For some series of chemically similar amino acids, a linear correlation of $\Delta_{\mathrm{ads}} \mathrm{H}^{\circ}$ with the number of carbon atoms was observed, which draws the attention to the possible role of dispersion interactions (see section 5.3).

\subsubsection{Vibrational spectroscopies}

Most biomolecules have functional groups with a conspicuous IR signature. However, since they normally represent a minority component in biomolecules/silica systems, only those that fall in a range where the silica support is transparent will be observable. This includes $\mathrm{C}=\mathrm{O}$ stretching and $\mathrm{NH}_{2} / \mathrm{NH}_{3}{ }^{+}$bending vibrations that are present in all amino acids and amide (-CO$\mathrm{NH}-$ ) bands of peptides and proteins. $\mathrm{OH}$ and $\mathrm{NH}$ stretching vibrations are also observable if the surface is dehydrated (otherwise they are lost in the broad background of water $\mathrm{OH}$ stretching). Conventional, transmission IR spectroscopy has indeed been applied to several amino acids/silica systems in the dry state (see Table 1). Technical developments also allow to observe in situ vibrational spectra of amino acids at the aqueous solution/silica interface by $\mathrm{ATR}^{211,217}$ or, for interfaces with planar silica, by SFG. ${ }^{218}$ This is extremely important in view of the importance of water in defining the amino acid-surface interaction, a point which will be discussed in section 9.1.1: the application of a drying step is very likely to modify the state of the adsorbed amino acids.

Raman spectroscopy does not seem to have been applied to amino acids on silica. SERS (Surface Enhanced Raman Scattering) depends on a resonance with electronic plasma oscillation and is not applicable on silica in principle. IRAS, which is adapted to planar reflecting surfaces (e.g. Ref. ${ }^{219}$ for Gly on $\mathrm{Cu}$ ), would be applicable to $\mathrm{SiO}_{2}$ thin films deposited on substrates such as Mo (see Section 3.1.3), but it has not been applied so far to biomolecules adsorption; neither has GA-ATR.

On flat surfaces of fused silica, SFG has recently been used in the hope to detect amino acids at the silica-solution interface. ${ }^{218,220}$ The results are still limited as regards the amino acid itself, but the advantage is that this technique allows water in the interfacial region to be observed in situ as well during the adsorption phenomenon. ${ }^{220}$

Vibrational spectroscopy gives unequivocal results as regards the protonation state of the amino acid because $-\mathrm{COO}^{-}$is rather easily distinguished from $-\mathrm{COOH}$, and $\mathrm{NH}_{2}$ from $\mathrm{NH}_{3}{ }^{+}$. It would be expected that it also provide information on the adsorption mechanism, since even rather weak bonding with a functional group will affect its characteristic vibrations (cf. Section 3.1.7). In fact early studies on amino acids adsorption on silica from the gas phase observed important shifts of the carbonyl band, which were interpreted, after similar studies on kaolin clays, ${ }^{221}$ as meaning that the carboxylic acid terminal had condensed with surface silanols to give 
what was variously referred to as « anhydride with surface hydroxyl groups», «surface acyl» or «surface ester», $\mathrm{Si}-\mathrm{O}-\mathrm{CO}-\mathrm{CH}\left(\mathrm{NH}_{2}\right)-\mathrm{R}$. It is in fact difficult to reach a definite conclusion from IR only, and the conditions of possible formation of such covalently grafted species constitute a testing ground for molecular modeling studies, of which more will be said later. We shall note here that according to Basiuk et al., they only form on thoroughly dehydrated surfaces and may be destroyed when these surfaces are reexposed to water. ${ }^{222}$ In other studies, shifts in the $-\mathrm{NH}_{\mathrm{x}}$ and $\mathrm{CO}$ bands were interpreted as indicative of H-bond formation with the surface. ${ }^{210,223}$

\subsubsection{NMR techniques}

Biomolecules contain several nuclei amenable to NMR study, the most important of which is ${ }^{13} \mathrm{C} .{ }^{1} \mathrm{H},{ }^{2} \mathrm{H}$ and ${ }^{15} \mathrm{~N}$ might also be useful. Multinuclear NMR is a workhorse of organic chemistry and one benefits from a considerable amount of accumulated data to help interpretations, although solid-state NMR spectra are considerably less well-resolved than in the solution. In spite of this, the application of solid-state NMR to understand biomolecules adsorption on silica is recent. ${ }^{211,224-228}$ This is due to the low intrinsic sensitivity of the ${ }^{13} \mathrm{C}$ nucleus. In conjunction with the fact that the biomolecule is a minority species in the samples, it causes NMR signals to be very weak, so that long accumulation times are necessary - unless one uses expensive isotopically enriched biomolecules. The rewards are high however because the ${ }^{13} \mathrm{C}$ isotropic shifts are very sensitive to the molecular environment: for instance it has been demonstrated that the $\mathrm{C}=\mathrm{O}$ signal position responds in a predictable way to the different $\mathrm{H}$ bonding states of $\mathrm{HGly}^{ \pm}$zwitterions in the three polymorphs $\alpha$-, $\beta$ - and $\gamma$-glycine, ${ }^{229}$ and for Gly/Aerosil a specific signal was attributed to glycine zwitterions adsorbed by H-bonding. ${ }^{224} \mathrm{~A}$ word of caution is in order again about the difficulty in quantifying ${ }^{13} \mathrm{C}\left\{{ }^{1} \mathrm{H}\right\} \mathrm{CP}$ spectra (since one-pulse acquisition of ${ }^{13} \mathrm{C}$ spectra would be very slow). If the adsorbed biomolecule is highly mobile, its CP signal may be totally undetectable.

However the potential of NMR techniques goes beyond chemical identification through chemical shifts, as underlined by Ben Shir et al. ${ }^{228}$ These authors used REDOR (Rotationally enhanced double resonance) NMR to evidence neighboring relations between nuclei from the biomolecule and from the silica surface. More precisely, the joint use of ${ }^{29} \mathrm{Si}\left\{{ }^{15} \mathrm{~N}\right\}$ and ${ }^{15} \mathrm{~N}\left\{{ }^{29} \mathrm{Si}\right\}$ REDOR revealed intermolecular interactions between the ammonium group of alanine (Ala) adsorbed on mesoporous silica and three to four predominantly $\mathrm{Q}^{3}$ surface Si species, and $\mathrm{N}-\mathrm{Si}$ distances could even be evaluated. ${ }^{227}$ In addition, ${ }^{15} \mathrm{~N}\left\{{ }^{13} \mathrm{C}\right\}$ REDOR provided information on the state of mobility of adsorbed alanine as a function of temperature, allowing to discriminate between immobile and rapidly reorientating alanine populations. Later on, for the related Gly/mesoporous silica system, the same techniques were applied together with HETCOR, 
a 2-D NMR technique already mentioned for bare silica surfaces in Section 3.1.5 $-{ }^{1} \mathrm{H}\left\{{ }^{13} \mathrm{C}\right\}$, ${ }^{1} \mathrm{H}\left\{{ }^{15} \mathrm{~N}\right\}$ and ${ }^{13} \mathrm{C}\left\{{ }^{15} \mathrm{~N}\right\}$ HETCOR were investigated here in addition to ${ }^{1} \mathrm{H}\left\{{ }^{29} \mathrm{Si}\right\}$, giving access to the corresponding neighboring relations. Here too, two immobile and one freely reorienting glycine populations were evidenced.

The mobility of the functional groups of adsorbed amino acids is an important question, as it will indicate what groups, if any, are strongly bound with the surface. Amitay-Rosen et al. ${ }^{225}$ used deuterium lineshape analysis (or rather, for samples rotating at the magic angle, sidebands analysis) of $\mathrm{CH}_{3}$-deuterated alanine on mesoporous silica, in comparison with ${ }^{1} \mathrm{H}$ spectra. They rationalized their observations on the basis of a 2-site exchange model. Although their conclusions were not fully compatible with those of Ben Shir et al. on the same system, this may be due to a different hydration state.

In spite of these divergences, the studies just mentioned represent a breakthrough in that they started investigating dynamic processes in adsorbed biomolecules systems instead of provinding a static picture -the very strong dependence of the adsorption behavior upon the hydration state of the surface revealed in this way, and the experimental determination of new observables such as dipolar coupling strengths, represent new challenges for molecular modeling.

For adsorbed peptides, NMR can provide rich structural information. Advanced NMR techniques have long been applied to the in-depth study of proteins and peptides and they can be transferred to peptides on surfaces in spite of the additional complexities induced by working in the solid state, or in suspensions. Thus, 2-D NOESY (2-dimensional nuclear Overhauser) experiments based on through-space spin-spin coupling can reveal spatial relations between the different residues of a peptide chain. Mirau et al. ${ }^{230}$ have applied 2-D NOESY to suspensions containing fumed silica particles and specific $\mathrm{SiO}_{2}$-binding peptides. By use of programs making use of distance constraints to determine peptide conformations, they could propose structural models of several peptides adsorbed on silica, which contained common structural elements. As seen in Figure 17, these common elements were observed to fold in very similar ways on the surface, which is a strong argument in favor of the reliability of the results. Note however that the techniques used provide exquisitely detailed information on the adsorbed peptide, but none at all on the silica surface which appears as a featureless background. 


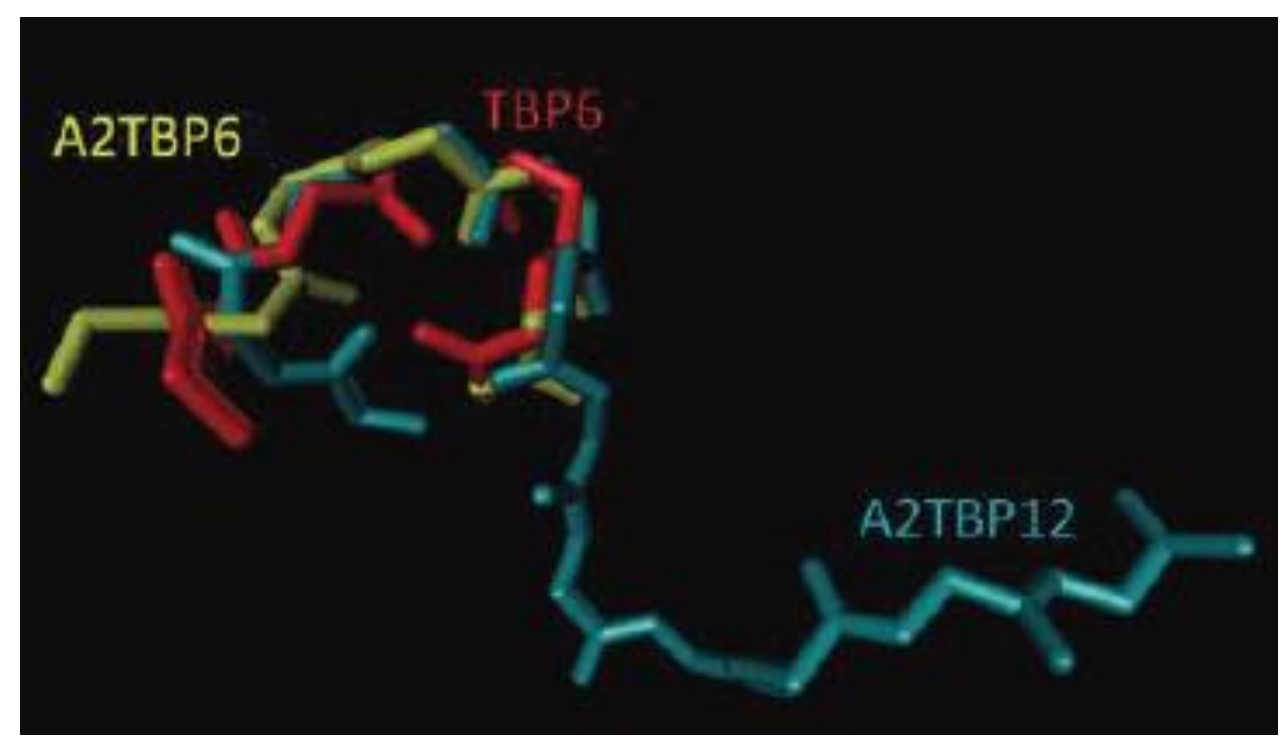

Figure 17. Secondary structures adopted by three related peptides adsorbed on silica. Reprinted with permission from Ref. ${ }^{230}$. Copyright 2011 American Chemical Society.

\subsubsection{Flat surface techniques}

As for bare silica surfaces, one is limited by the non-conducting nature of $\mathrm{SiO}_{2}$. Thus, in keeping with remarks in Section 3.2.1, LEED would be very hard to apply to adsorption on crystalline silica phases. Other surface techniques however can be applied to silica overlayers deposited on a sensing chip.

Thus, QCM (quartz crystal microbalance) allows quantifying adsorption phenomena on flat surfaces and can provide in-situ kinetic data. The quartz crystals may be covered by a layer of amorphous-like silica and the adsorption of «heavy» biomolecules such as proteins may be followed. In this way it was found that streptavidin has a silica adsorption behavior strongly different from avidin or neutravidin, two closely related proteins. ${ }^{231}$ Smaller molecules such as penta- $^{232}$ or heptapeptides, ${ }^{233,234}$ or longer peptides, ${ }^{235}$ have occasionally been studied in this way; application to monomers such as amino acids exist but are still limited on silica so far. ${ }^{220}$ In a significant study, lysine and proline adsorption on flat silica were compared with the adsorption of the corresponding oligomers both quantitatively by QCM, and qualitatively by SFG, which yielded vibrational spectra. ${ }^{236}$

The SPR technique that has allowed in-depth thermodynamic studies of peptides adsorption on $\operatorname{gold}^{237}$ is not easily applicable on silica. To obtain thermodynamic data for peptides adsorption on silica, Thyparambil et al. ${ }^{238}$ developed a protocol using a special AFM (atomic force microscopy) technique: the AFM tip was functionalized with the oligopeptide to be investigated (forming a single-molecule force sensor ${ }^{239}$ ), and the desorption force curve allowed to extract a $\Delta_{\text {ads }} \mathrm{H}^{\circ}$ value, on quartz (100) and fused glass among other surfaces.

One study of protein adsorption on surfaces of varying wettability, including $\mathrm{SiO}_{\mathrm{x}}$ on $\mathrm{Si}$ 
wafers (cf. Section 3.1.8), used contact angle measurements with drops of solutions of the biomolecule to assess the adsorption free energy. ${ }^{240}$ This study underlined again the role of water in adsorption phenomena: on very hydrophilic surfaces, almost no proteins adsorbed because it cost too much energy to displace water molecules.

\subsubsection{An overview of experimental studies of small biomolecules/silica systems}

Table 1 summarizes the most important experimental studies of small biomolecules adsorption on silica surfaces, indicating the type of silica surface, the interface from which adsorption was carried out, and the main characterization techniques that were used. A quick glance at this Table indicates that in spite of the rather large number of reported studies, a lot remains to be done.

Table 1. Most significant experimental studies of small biomolecules/silica adsorption, indicating the main techniques/results used in each case.

\begin{tabular}{|c|c|c|c|c|}
\hline $\begin{array}{l}\text { Oxide } \\
\text { mineral }\end{array}$ & Biomolecules & $\begin{array}{l}\text { From } \\
\text { gas or } \\
\text { solution }\end{array}$ & Main results and/or techniques used & Ref. \\
\hline $\mathrm{SiO}_{2}$ & $\begin{array}{l}\text { Ala, Gly, Ile, Leu, } \\
\text { Met, Phe, Val }\end{array}$ & $\mathrm{G}$ & IR & 241 \\
\hline $\mathrm{SiO}_{2}$ & $\begin{array}{l}\text { Val, Gly, Ala, Leu, } \\
\text { Ile, Pro, Phe, Met, } \\
\text { Thr, Trp }\end{array}$ & G & $\begin{array}{l}\text { Analysis of organic products after } \\
\text { desorption, condensation and analysis } \\
\text { of gaseous products }\end{array}$ & 242 \\
\hline $\mathrm{SiO}_{2}$ & $\begin{array}{l}(\text { Gly })_{2}, \\
\text { Gly-Ala, Gly-Leu, } \\
\text { Gly-Val, Ala-Ala, } \\
\text { Val-Val }\end{array}$ & G & $\begin{array}{l}\text { condensation and analysis of gaseous } \\
\text { products }\end{array}$ & 222 \\
\hline $\mathrm{SiO}_{2}$ & $\begin{array}{l}(\mathrm{Gly})_{2}, \\
\text { Ala-Ala, Leu-Leu, } \\
\text { Val-Val, } \\
\text { Pro-Pro }\end{array}$ & G & $\begin{array}{l}\text { condensation and analysis of gaseous } \\
\text { products }\end{array}$ & 243 \\
\hline $\begin{array}{l}\text { divided } \mathrm{SiO}_{2} \\
\text { (Aerosil380) }\end{array}$ & Gly & G & IR & 223 \\
\hline $\begin{array}{l}\mathrm{SiO}_{2} \text { (quartz) } \\
\text { single crystals }\end{array}$ & $\begin{array}{l}\text { Ala, Asp, Glu, Gly, } \\
\text { Leu, Lys, Tyr }\end{array}$ & $\mathrm{S}$ & AFM (atomic force microscopy) & 105 \\
\hline $\begin{array}{l}\text { Fused silica } \\
\text { prism }\end{array}$ & Asp, Glu, Phe & $\mathrm{S}$ & SFG & 218 \\
\hline
\end{tabular}




\begin{tabular}{|c|c|c|c|c|}
\hline divided $\mathrm{SiO}_{2}$ & Pyroglutamic acid & $S$ & IR & 221 \\
\hline $\begin{array}{l}\text { divided } \mathrm{SiO}_{2} \\
\text { (chromatograp } \\
\text { hic) }\end{array}$ & $\begin{array}{l}\text { dipeptides, cyclic } \\
\text { and linear }\end{array}$ & $S$ & Free energies of adsorption & 242 \\
\hline $\begin{array}{l}\text { divided } \mathrm{SiO}_{2} \\
\text { (chromatograp } \\
\text { hic) }\end{array}$ & $\begin{array}{l}\text { AA, peptides, } \\
\text { Krebs' cycle } \\
\text { molecules, } \\
\text { nucleosides, } \\
\text { nucleotides }\end{array}$ & S & Free energies of adsorption & 244 \\
\hline $\begin{array}{l}\text { divided } \mathrm{SiO}_{2} \\
\text { (chromatograp } \\
\text { hic) }\end{array}$ & $\begin{array}{l}\text { Ala, Asn, Asp, Cys, } \\
\text { Gln, Glu, Gly, Ile, } \\
\text { Leu, Met, Phe, Pro, } \\
\text { Ser, Tyr, Thr, Trp, } \\
\text { Val }\end{array}$ & $\mathrm{S}$ & Free energies of adsorption & 216 \\
\hline $\begin{array}{l}\text { divided } \mathrm{SiO}_{2} \\
\text { (chromatograp } \\
\text { hic) }\end{array}$ & $\begin{array}{l}\text { Ala, Asn, Asp, Cys, } \\
\text { Gln, Glu, Gly, Ser, } \\
\text { Tyr, Thr, Trp, Val }\end{array}$ & S & Free energies of adsorption & 207 \\
\hline $\mathrm{SiO}_{2}$ & Gly, $(\text { Gly })_{2}$ & $\mathrm{~S}$ & Wetting-and-drying cycles & 245 \\
\hline $\mathrm{SiO}_{2}$ & $\begin{array}{l}\text { Ala } \\
(\text { Ala + Gly }) \\
\left.(\text { Ala + (Gly })_{2}\right) \\
(\text { Ala + DKP })\end{array}$ & $S$ & Wetting-and-drying cycles & 245 \\
\hline $\mathrm{SiO}_{2}$ & $\begin{array}{l}\text { Gly, Ala, Pro, Val, } \\
\text { Leu } \\
(\text { Ala + Gly }) \\
\left(\text { Ala + }(\text { Gly })_{2}\right) \\
(\text { Pro+ Gly }) \\
\left(\text { Pro + }(\text { Gly })_{2}\right) \\
(\text { Val + Gly }) \\
\left(\text { Val + }(\text { Gly })_{2}\right) \\
(\text { Leu + Gly }) \\
\left(\text { Leu+ }(\text { Gly })_{2}\right) \\
(\text { Ala + Pro }) \\
(\text { Ala + Val })\end{array}$ & $\mathrm{S}$ & Wetting-and-drying cycles & 246 \\
\hline
\end{tabular}




\begin{tabular}{|c|c|c|c|c|}
\hline $\begin{array}{l}\text { divided } \mathrm{SiO}_{2} \\
\text { (Aerosil380) }\end{array}$ & Gly & $\mathrm{S}$ & IR, macroscopic data & 210 \\
\hline $\begin{array}{l}\text { divided } \mathrm{SiO}_{2} \\
\text { (Kalush A- } \\
300 \text { ) }\end{array}$ & Arg, His, Lys & $\mathrm{S}$ & $\begin{array}{l}\text { macroscopic modeling } \\
\text { of adsorption data }\end{array}$ & 214 \\
\hline $\begin{array}{l}\text { divided } \mathrm{SiO}_{2} \\
\text { (Aerosil380) }\end{array}$ & Gly, Lys & $\mathrm{S}$ & IR, macroscopic data & 247 \\
\hline $\begin{array}{l}\text { Modified } \\
\mathrm{SiO}_{2}\end{array}$ & Gly, Leu, His, Lys & $\mathrm{S}$ & Adsorption isotherms & 248 \\
\hline $\begin{array}{l}\text { Glass, } \\
\text { hydrophilic } \\
\text { and } \\
\text { hydrophobic }\end{array}$ & Leu, Ser & $\mathrm{S}$ & Isotopic labeling & 249 \\
\hline $\begin{array}{l}\text { divided } \mathrm{SiO}_{2} \\
\text { (Kalush A- } \\
300)\end{array}$ & $\begin{array}{l}\text { Ala, Leu, Phe, Trp, } \\
\text { Tyr, Val, co- } \\
\text { adsorption with } \\
\mathrm{Cu}^{2+}\end{array}$ & $\mathrm{S}$ & Macroscopic data, adsorption isotherms & 250 \\
\hline $\begin{array}{l}\text { "chromato- } \\
\text { graphic grade" } \\
\text { divided } \mathrm{SiO}_{2} \\
(\mathrm{SRL})\end{array}$ & His, Trp & $\mathrm{S}$ & $\begin{array}{l}\text { Adsorption isotherms, macroscopic } \\
\text { modeling }\end{array}$ & 251 \\
\hline $\begin{array}{l}\text { Mesoporous } \\
\mathrm{SiO}_{2} \mathrm{MCM}-41\end{array}$ & $(\text { Ala })_{4}$ & $\mathrm{~S}$ & Deuterium NMR & 252 \\
\hline $\begin{array}{l}\text { Mesoporous } \\
\mathrm{SiO}_{2} \mathrm{MCM}-41\end{array}$ & Lys & $\mathrm{S}$ & $\begin{array}{l}\text { Adsorption isotherms, macroscopic } \\
\text { modeling }\end{array}$ & 215 \\
\hline $\begin{array}{l}\text { Mesoporous } \\
\mathrm{SiO}_{2} \text { SBA-15 }\end{array}$ & $\begin{array}{l}\text { Phe, Glu, Arg, Leu, } \\
\text { Ala }\end{array}$ & $\mathrm{S}$ & $\begin{array}{l}\text { Adsorption isotherms, effect of } \mathrm{pH} \text {, ion } \\
\text { strength }\end{array}$ & 253 \\
\hline $\begin{array}{l}\text { divided } \mathrm{SiO}_{2}, \\
615 \mathrm{~m}^{2} / \mathrm{g} \\
\text { (Wakosil } \\
\text { 25SIL) }\end{array}$ & Lys & $\mathrm{S}$ & ATR & 217 \\
\hline $\begin{array}{l}\text { Mesoporous } \\
\mathrm{SiO}_{2} \mathrm{MCM}-41 \\
\text { and SBA15 }\end{array}$ & Ala & $\mathrm{S}$ & ${ }^{2} \mathrm{H}$ NMR on deuterated molecules & 225 \\
\hline Mesoporous & Met (and Ala-Ala) & $\mathrm{S}$ & ${ }^{1} \mathrm{H}$ and ${ }^{2} \mathrm{H}$ MAS NM & 226 \\
\hline
\end{tabular}




\begin{tabular}{|c|c|c|c|c|}
\hline $\mathrm{SiO}_{2} \mathrm{SBA} 15$ & & & & \\
\hline $\begin{array}{l}\text { Mesoporous } \\
\mathrm{SiO}_{2} \text { SBA-15 }\end{array}$ & Ala & $\mathrm{S}$ & NMR: SLF and REDOR & 227 \\
\hline $\begin{array}{l}\text { Mesoporous } \\
\mathrm{SiO}_{2} \mathrm{SBA}-15\end{array}$ & Gly & $\mathrm{S}$ & $\begin{array}{l}\text { NMR : REDOR, TEDOR, SLF, } \\
\text { 2D-HETCOR }\end{array}$ & 228 \\
\hline $\begin{array}{l}\text { Mesoporous } \\
\mathrm{SiO}_{2}, \mathrm{AA}- \\
\text { templated }\end{array}$ & $\begin{array}{l}\text { Pro, Ile, Val, Leu, } \\
\text { Hyp, Phg }\end{array}$ & $\mathrm{S}$ & Macroscopic adsorption data & 254 \\
\hline $\begin{array}{l}\text { Mesoporous } \\
\mathrm{SiO}_{2} \text {, AA- } \\
\text { templated }\end{array}$ & L-Pro, D-Pro & $\mathrm{S}$ & Adsorption isotherms & 255 \\
\hline $\begin{array}{l}\text { Mesoporous } \\
\text { organosilica }\end{array}$ & Gly, Lys, Ile & $\mathrm{S}$ & Adsorption isotherms, effect of $\mathrm{pH}$. & 256 \\
\hline $\begin{array}{l}\text { Mesoporous } \\
\mathrm{SiO}_{2}\end{array}$ & Trp (and proteins) & $\mathrm{S}$ & Adsorption isotherms & 257 \\
\hline $\begin{array}{l}\text { Thin layers on } \\
\text { silicon (111) } \\
\text { wafers }\end{array}$ & Adenine & $\mathrm{S}$ & XPS, NEXAFS & 258 \\
\hline
\end{tabular}

While many studies bear on the essential amino acids (the list of 20 AAs that has been selected by biochemistry for building proteins), far less have been devoted to other small biomolecules. In particular, one would expect to find some investigations on the adsorption of nucleotides, the monomers of RNA or DNA, since rather long DNA and/or RNA sequences have occasionnally been adsorbed on silica for technological reasons. ${ }^{259}$ In fact the only example that we are aware of is a comparative study of nucleotides and nucleosides adsorption with other small molecules possibly involved in prebiotic chemistry, ${ }^{244}$ where the former were found to adsorb more strongly that most AAs at neutral $\mathrm{pH}$. One communication was also devoted to the adsorption on a flat silica surface of adenine, ${ }^{258}$ a nucleobase that is a component of the corresponding nucleotide but is not expected to adsorb in the same way, since they have very different acido-basic speciations (nucleotides, and their polymers, accumulate negative charges due to deprotonation of the polyphosphate groups).

Most amino acids on the other hand have been studied at least once, but comparative studies are rare. For instance, it is not possible to find adsorption isotherms of all natural AAs on silica in controlled conditions, nor systematic studies of $\mathrm{pH}$ effects. The most valuable comparisons are the extensive thermodynamic data of Basiuk et al. (Ref. ${ }^{207}$ and references 
therein). Ref. ${ }^{253}$ considered the hydrophilic/hydrophobic nature of AA; the authors concluded that differences in adsorption parametres could be explained by a combination of electrostatic attraction/repulsion between the AA and the surface, which is $\mathrm{pH}$-dependent, ${ }^{205}$ and hydrophobic effects.

Unsurprisingly, the biomolecule most widely studied in this context is one of the simplest, namely, glycine. It is also for this molecule that the most precise questions start being asked in recent publications, e.g. the identification of $\mathrm{H}$-bonding lattices with the surface.

Other molecules may be worth mentioning which, although they are not biomolecules stricto sensu, share some functional group similarities with e.g. amino acids and can therefore help explain the adsorption behavior of the latter. Such examples are provided by the field of drug delivery, and the most relevant ones will be discussed in Section 9.4.

\subsubsection{From oligopeptides to proteins: adsorption, secondary structure and selectivity}

The preceding paragraphs mentioned a few oligopeptides. When the peptide chain grows longer, the number of spatial conformations accessible to it grows exponentially. It is wellknown that the proper functioning of a protein such as an enzyme depends on its adopting the right tertiary structure, which corresponds to a tiny fraction of the available phase space and whose realization depends on fine details of energetics (interactions between amino acid residues, and between amino acids and solvent molecules). Therefore it is of crucial importance for many biological and biomedical applications to know if the protein structure will be conserved upon adsorption. ${ }^{260,261}$ The conventional answer is negative: adsorption, in particular on silica, changes the folding pattern of the protein chain, in other words it causes denaturation. We cannot discuss here the many results that have been obtained for proteins/silica systems -a good source for these is a recently published, and already mentioned review paper. ${ }^{165}$ The situation is very complex for proteins due to their long chains, and computationally they are only amenable to low-level modeling, mostly by MD. Short-chain peptides on the other hand may achieve some elements of secondary structure such as the $\alpha$-helix or the $\beta$-sheet, and some of them fall within the limits of high-level modeling methods.

Techniques used to determine adsorbed peptide structures include NMR (cf. Section 3.2.4, and Ref. ${ }^{262}$ ), CD (circular dichroism ${ }^{263}$ ) which is applied directly to colloidal suspensions containing the peptide and the silica nanoparticles, IR (analysis of IR spectra in the amide bands region allows to evidence motives such as the $\alpha$-helix ${ }^{264}$ ), or ToF-SIMS (time-of-flight secondary ion mass spectrometry), probably better suited for adsorbed proteins ${ }^{265}$-in this technique, the protein fragments that are eroded first are those that were furthest removed from the silica surface. Ellipsometry and AFM can be used to evidence the formation of larger-scale structures such as fibrils. ${ }^{266}$ 
The effect of adsorption on the secondary structure of adsorbed peptides has been investigated for at least a dozen years. It is reported that in general there is a loss of $\alpha$-helicity (e.g. for the 13-mer DDDDAAAAARRRR ${ }^{267,268}$ ) and concomitant formation of $\beta$-sheets. ${ }^{269,270}$

This is no absolute rule however. Some peptides have been specifically designed that strongly adsorb to silica particles and form helices on the surface, ${ }^{235}$ while they were not structured in the solution. This was achieved by introducing at regular spacings in the polymer chain amino acids with basic side chains such as arginine (R), e.g. YARQQRAEARQQRAHARQKRAEARQQRA. The basic AA are protonated and since the silica surface is negatively charged, the peptide folds in such a way as to direct all arginine chains towards the surface.

In the preceding example, the silica binding peptide was designed from first principles and chemically synthesized, but there is another approach. Biocombinatorial techniques that were developped to select the «best» peptide sequences binding to a particular biochemical target have been applied to choose the best sequences for adsorption on inorganic surfaces. ${ }^{271}$ In this way, quartz-binding peptides have been engineered ${ }^{272}$ and sequences specific for amorphous silica have also been produced. ${ }^{273}$ These studies are quickly generating a database of specific peptide sequences having more or less strong affinity with silica surfaces. There is of course a strong incentive to understand the structural basis for this affinity; at present it seems that many results can be understood by combining an electrostatic interaction between positively charged amino acid side chains and negatively charged silanolates on the silica surface, but also hydrophobic interactions between apolar side chains ${ }^{273}$ and siloxanes, a rationalisation similar to that put forward to explain the silica selectivity of a natural protein which was called a «Sitag». ${ }^{274}$ These explanations are still sketchy: obviously a lot remains to be understood in order to understand the interactions of specific protein moieties with specific silica surface sites, their cooperativity, and by way of consequence their effect on protein structuring. Molecular modeling techniques can play a precious role in unraveling these phenomena.

\section{Different approaches to model adsorption at surfaces}

\subsection{The periodic boundary conditions approach}

In the context of this review we focus on reporting modeling data obtained for surfaces belonging to crystalline and amorphous silica. The starting material, irrespective on its aggregation state, is a solid so that the most natural approach to model its features in a computer is to enforce periodic boundary conditions (PBC) and to solve the corresponding Schrödinger

equation accordingly. ${ }^{275-277}$ For a crystalline bulk material this is a rigorous procedure and more details are provided in Section 6. In essence, besides the level of the adopted theory, to work 
with PBC one has to define a unit cell, a straightforward step for genuine crystalline materials like $\alpha$-quartz, as it is the natural outcome of X-ray or neutron diffraction studies of any crystalline material. Crystallographic databases are available, which contain the unit cell atomic coordinates of the most important silica polymorphs, both dense and microporous. ${ }^{278}$ The atomic positions in the unit cell are related to each other, as a function of the considered system, by symmetry elements dictated by the crystallographic space group. ${ }^{279}$ This, in turn, can be exploited to greatly reduce the computational cost as many components of the unit cell total energy are related by simple symmetry rules and should not be recomputed. For the particular case of silica, one element of difficulty is that silica is also found as an amorphous material. In that case the fine details of its structure remain inaccessible as diffraction techniques only provides averaged information like the radial distribution function. ${ }^{279}$ The way in which the structure of an amorphous bulk silica (but this is in general true for other glass-like materials) is arrived at, in a computer simulation, follows strategies that bring a crystalline starting phase (whose structure is known) towards the amorphous one by decreasing the long-range atomic order. Silica, as already described in Section 2, is made of rigid $\left[\mathrm{SiO}_{4}\right]$ building blocks joined together by very floppy Si-O-Si angle defining the siloxane bond. This means that in the amorphous silica, the local order within the $\left[\mathrm{SiO}_{4}\right]$ building block is preserved whereas the longrange symmetry relationships between $\left[\mathrm{SiO}_{4}\right]$ groups are lost. This kind of disorder can be achieved with a variety of techniques as for instance a Monte Carlo bond-switching ${ }^{280}$ or molecular dynamics carried out at very high $\mathrm{T}$ in order to melt the material followed by a quenching step to kinetically freezes the structure in a glassy state. ${ }^{281-287}$ This procedure can be repeated a convenient number of times until physico-chemical features of the bulk material (density, Si-O-Si ring nuclearity and distribution, radial $\mathrm{Si}-\mathrm{O}$ distribution function, etc) are in good agreement with experiments. Irrespective on the adopted methods, this is a time consuming step which is needed to achieve the proper topology of the material. To be representative of the real amorphous material the simulation should adopt a large enough unit cell to ensure structural richness in the local environment of each $\left[\mathrm{SiO}_{4}\right]$ block. For these reasons, the simulation of amorphous materials is usually much more expensive than that of crystalline ones, particularly when quantum mechanical techniques are adopted. One should also check the dependency of the results on the cell size, a task which is rarely done due to the high cost of the calculation. Nevertheless, results obtained by optimizing at B3LYP a silica bulk derived from the heat/cool procedure gave properties in very good agreement with experiments even for a unit cell content of less than 100 atoms. ${ }^{280,288-290}$

The key point within the present context is how to move from silica bulk to surfaces, as the properties of the latter are the relevant ones when adsorption of (bio)molecules is considered. 
Usually, surfaces are modeled using slabs of finite thickness cut out from the bulk structure along a given Miller $(h k l)$ crystallographic plane. ${ }^{276,291}$ The surface unit cell should then be defined accordingly through a matrix transformation of the original bulk one. At variance with the bulk, the periodic boundary conditions should only be enforced by translation vectors parallel to the surface, as periodicity is now lost in the non-crystallographic direction perpendicular to the slab plane. Technical reasons bound to the adoption of plane waves based methods (vide infra) enforce the adoption of a tridimensional unit cell for slabs as well. Thus, one needs to define a large enough empty space above/below the true slab, to avoid mutual fictitious interactions between the periodically repeated slabsThis is different from periodic quantum mechanical calculations carried out using a Gaussian type basis set, in which the definition of the true bidimensional slab is completely rigorous, i.e. the electrostatic potential above/below the slab decays exponentially with the distance from the slab. ${ }^{276,291}$ In all cases, the adequacy of the adopted slab must be checked by considering convergence of geometry, energy, and electronic properties as a function of the increasing number of atomic layers included in the slab and, for plane-waves based calculations, also of the size of the empty space separating the repeating slabs. For both true oxides and metals, the geometrical cut completely defines a given $(h k l)$ plane that can then be modeled by a variety of techniques. For oxides, extra care should be exerted in order to avoid either charged slabs or slabs with a large dipole moment across the slab itself, as in this case they are electronically unstable. ${ }^{292,293}$ For a covalent material like silica, bond cutting brings about the formation of a variety of surface defects, the most relevant being: the $\mathrm{Q}^{3}(\bullet)$ silyl $\equiv \mathrm{Si}^{\bullet}$, the $\mathrm{Q}^{3}(\mathrm{O} \bullet)$ siloxy $\mathrm{E}^{\prime} \equiv \mathrm{SiO} \bullet$, the $\mathrm{Q}^{2}(:)>\mathrm{Si}$ : and the $\mathrm{Q}^{2}(\mathrm{O})$ silanone $>\mathrm{Si}=\mathrm{O}$. The nature and stability of these defects has been discussed in the literature ${ }^{69-71,294-305}$ showing that all of them, without exception, are thermodynamically unstable with respect to water hydroxylation. As water is ubiquitously present during biomolecules adsorption these defects should not play an important role in that case. In practice, the above defective sites are all "healed" by extraction of $\mathrm{H}$ or $\mathrm{OH}$ groups from the water environment in a real material or by manual addition of $\mathrm{H}$ (to $\equiv \mathrm{SiO} \bullet$ ) and $\mathrm{OH}(\mathrm{to} \equiv \mathrm{Si} \bullet)$ in the simulated one. The "healing" process does not lead, however, to a unique surface termination, as different kind of $\mathrm{SiOH}$ groups are known to exist at the silica surface (see Section 2) as a function of their local environment. For instance, when dealing with surfaces derived from a crystalline silica the height at which the geometrical cut is carried out does indeed matter, as it defines the resulting type of $\mathrm{SiOH}$, i.e. the sole specification of the ( $h k l$ ) crystallographic family plane does not uniquely define the kind of $\mathrm{SiOH}$ termination. 


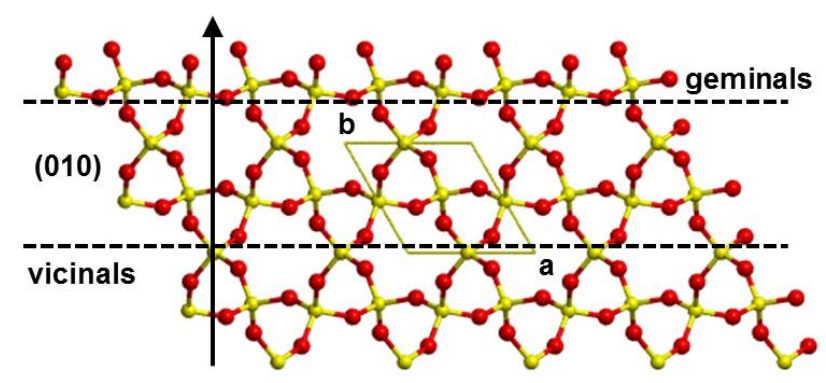

Figure 18. Different geometrical cut planes defining different silica surface terminations for the same (010) crystal plane. Possible cut planes are represented by dotted lines.

As an example, in Figure 18 the same (010) crystallographic face of $\alpha$-quartz can be either terminated by geminal or vicinals $\mathrm{SiOH}$ groups as a function of the cut height along the axis perpendicular to the slab. Therefore, for a crystalline surface, both the $(h k l)$ crystallographic plane and the kind of $\mathrm{SiOH}$ termination chosen should be clearly specified to completely define the system under study. Some subtle effects are possible, however, as for instance, the fully hydroxylated (001) and (010) surfaces of $\alpha$-quartz and the (001) and (110) ones for $\alpha$-cristobalite all envisage geminal terminations, but they are all different from each other, as different $\mathrm{H}$-bond interactions (defining them as "interacting pairs") ${ }^{306}$ are taking place (see Figure 19).

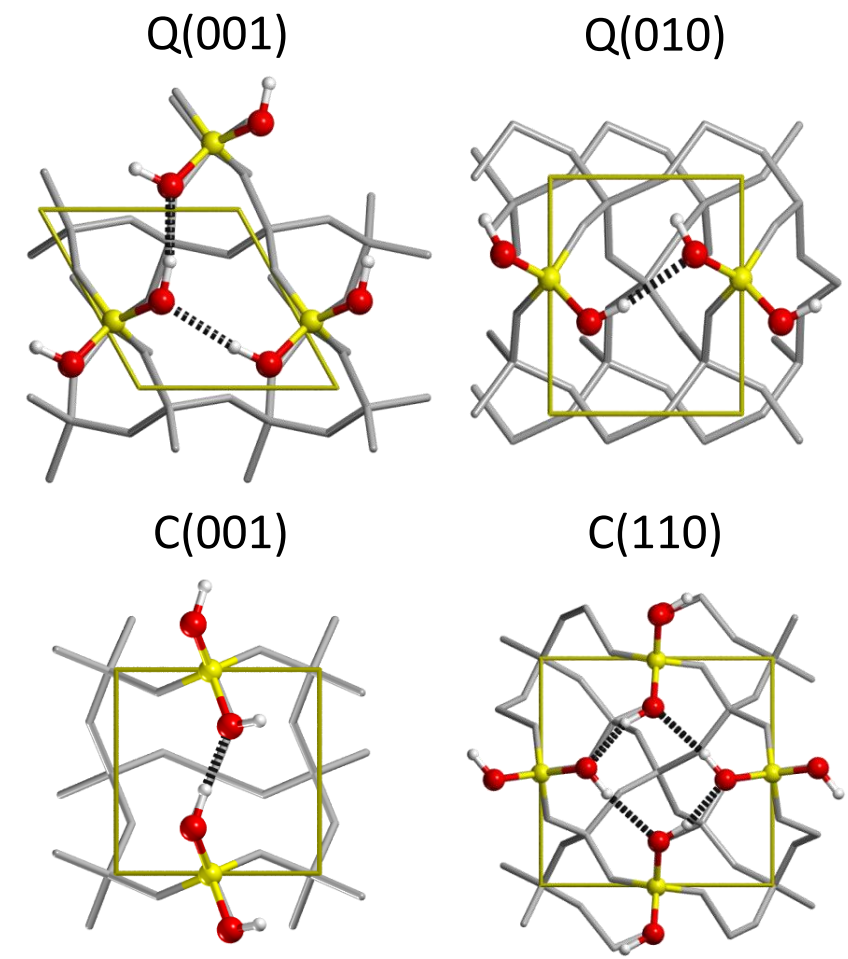

Figure 19. Different H-bond pattern between geminal $\mathrm{SiOH}$ sites at the surfaces of $\alpha$-quartz $(\mathrm{Q}(001)$ and $\mathrm{Q}(010))$ and cristobalite $(\mathrm{C}(001)$ and $\mathrm{C}(110))$.

Therefore, a further step is needed in the calculations to find the best arrangement of the surface $\mathrm{SiOH}$ in order to maximize the $\mathrm{H}$-bond interaction and, consequently, to reduce the 
surface formation energy computed as the energy of reaction of the bulk crystal with water molecules.

The very same approach can be followed to define the surface of an amorphous silica material, starting from an "amorphized" bulk prepared with some of the procedures described above. In this case, the geometrical meaning of a particular crystallographic $(h k l)$ plane is lost due to the amorphous nature of the material and less freedom is left in positioning the cut plane, as electroneutrality should always be enforced. As the unit cell is neutral, this can be automatically accounted for by using slab thickness which are an integer multiple of the unit cell size with geometrical cuts along the three main (100), (010) and (001) crystal planes. It is worth noting that, contrary to crystalline surfaces, the upper face is no longer equivalent to the lower face of the slab after the hydroxylation step due to the amorphous nature of the material. Figure 20 shows the case of the equivalent faces for the (101) cristobalite and the non-equivalent ones for a model of amorphous silica surface recently proposed by Colombi Ciacchi and coworkers. $^{285,307}$
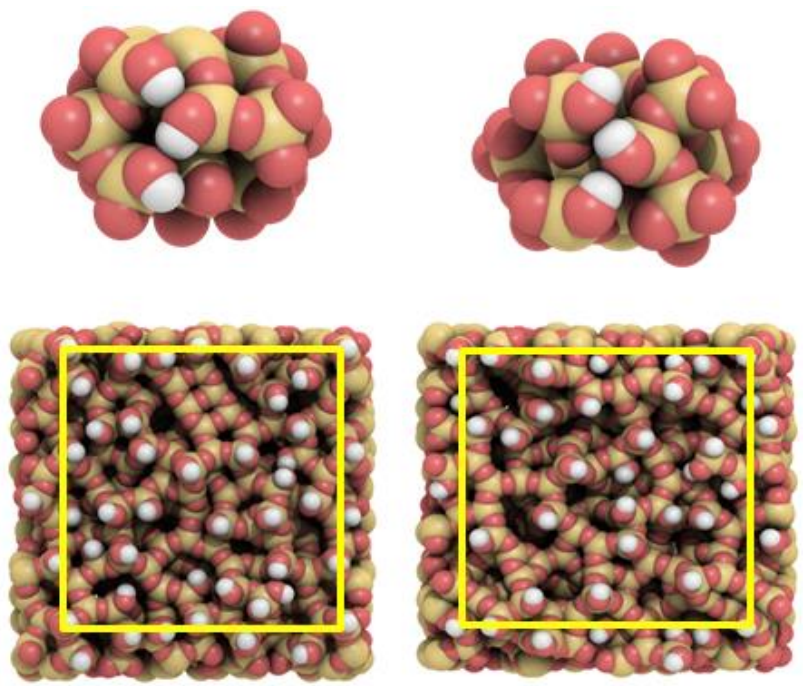

Figure 20. Top: upper/lower fully hydroxylated (101) faces of crystobalite. Bottom: upper/lower fully hydroxylated faces of the silica amorphous surfaces from Ref. ${ }^{285,307}$ Molecular graphics rendered by Qutemol. ${ }^{73}$

This is an advantage for the simulation of amorphous materials as the two different hydroxylated surfaces enrich the variability of the $\mathrm{SiOH}$ surface groups to be studied. For instance, calculation of the $\mathrm{OH}$ vibrational features will be improved due to the increasing variability of the local surrounding of each $\mathrm{OH}$ group at the upper/lower face of the same slab. Also, at variance with what happens for the crystalline silica surfaces, for the amorphous ones different kinds of $\mathrm{SiOH}$ groups are present in the same surface unit cell. 
When dealing with adsorption of (bio)molecules there is one important caveat. The adopted unit cell, irrespective of whether one is dealing with a crystalline or an amorphous silica system, should be large enough to avoid lateral interactions between adsorbates. This forces the surface unit cell to become very large for even small biomolecules like short peptides, preventing the adoption of first principle calculations. For instance Schneider and Colombi Ciacchi adopted a $44 \times 44 \AA^{2}$ unit cell size of a partially oxidized Si surface to study the adsorption of a fully solvated RKLPDA hexapeptide by classical force field molecular mechanics methods. ${ }^{308}$ An even larger unit cell (98x98 $\AA^{2}$ ) was used to simulate the adsorption of the NC1(84-116) domain of collagen VIV on the natively oxidised silicon surface. ${ }^{307}$ Nevertheless, lateral interactions can be easily computed, at least within the local Gaussian basis set approach, and their contribution (either repulsive or attractive) can be subtracted to the total adsorption energy. ${ }^{309-312}$ A number of detailed examples on this point will be discussed in the following sections devoted to adsorption of water and amino acids on silica surface models. Care should also be taken to study not only the adsorption but also the properties of the pristine surfaces, as the adoption of a too small unit cell would impose geometrical constraints biasing the true potential energy surface. An example is the recent study by Sautet and coworkers ${ }^{313}$ on dehydroxylation processes at the surface of crystalline cristobalite as a model of the amorphous silica surface, in which large enough cells have been chosen to ensure enough flexibility in the multi-step simulation of the dehydoxylation process.

In the recent past it has been customary to adopt a specific surface derived from a crystalline silica polymorph to mimic some of the specific features of the hydroxylated amorphous silica surfaces. This originates from the seminal work by Peri ${ }^{314}$ in which the (100) hydroxylated surface of $\beta$-cristobalite was chosen as a model system to interprete the dehydroxylation process occurring at the amorphous silica surface either by heating and outgassing the sample or by specific reactions. At that time, to reference to a crystalline model was a clever idea in order to figure out possible models of the more complex amorphous silica whose modeling was technically unfeasible. In the last twenty years the very same idea was adapted to the computer simulation of amorphous silica surface. ${ }^{315,316}$ As it was recalled in Section 2.2, the real amorphous silica surface exhibits different kinds of surface silanols, a fact which cannot be simulated by a single crystalline surface which exhibits only one kind of $\mathrm{OH}$ group (vide supra). The idea is to consider different surfaces derived from the same crystalline silica polymorph and then merge the results to mimic the more complex behavior of the real amorphous system (see Figure 21). 


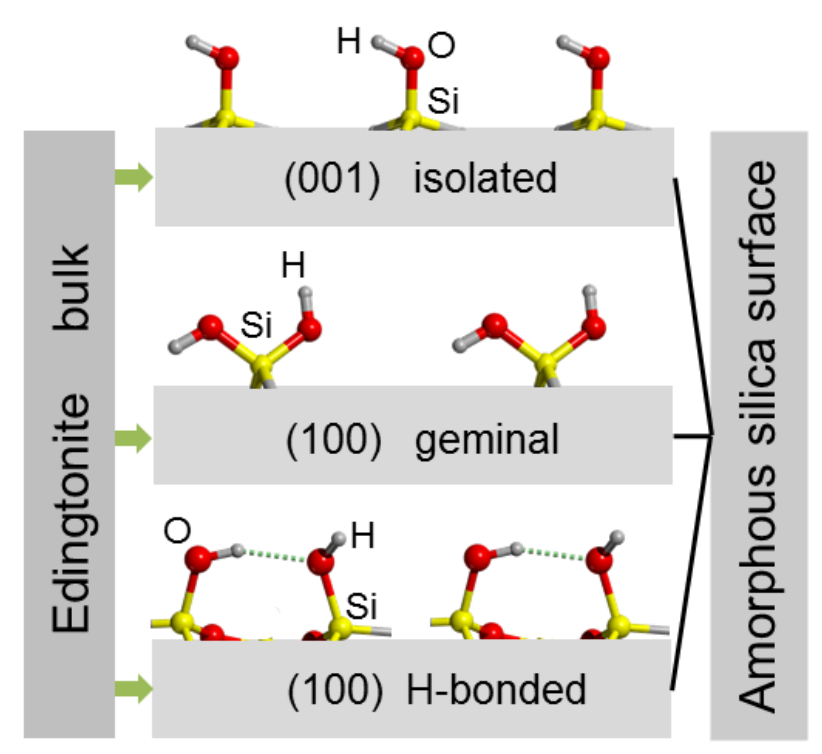

Figure 21. Amorphous silica surface simulated by merging different surface terminations of the edingtonite bulk.

The easiest system to simulate is an amorphous silica outgassed at high temperature in which only isolated silanol groups remain at the surface. In that respect, Civalleri et al. ${ }^{316}$ were the first to propose the (001) surface of edingtonite, a microporous all-silica polymorph particularly suitable to quantum mechanical calculations due to its structural rigidity and simplicity, to model the isolated silanols on an amorphous silica surface. Tosoni et al. ${ }^{317}$ extended the model to include the (100) surface exhibiting either H-bond interacting or geminal sites to model both clean and water interacting surfaces. The infrared spectra were constructed by merging the results of the three independent surfaces obtaining excellent agreement with the experimental ones. The same model has been adopted in a number of studies devoted to study adsorption of simple molecules to silica ${ }^{318-320}$ as well as to study the adsorption of glycine s09,321- $^{3}$ 323 and alanine. ${ }^{324,325}$ This approach has obvious limitations and it is preferable to rely on amorphous silica models, which is now feasible with the development of highly parallelized computer programs ${ }^{326}$ on the modern high performance computing facilities.

In summary, the advantage of the PBC approach is that the extended nature of the material is naturally taken into account at the expense of using a large enough unit cell for modeling amorphous silica or to avoid spurious lateral interactions. The disadvantage is that the maximum level of theoretical method applicable to periodic systems is limited to the DFT approach due to the difficulty of extending the higher correlated methods based on the wave function expansion to solid state treatment. Recent progress has, however, been done both in the direction of the periodic implementation of the MP2 method in its localized definition ${ }^{327}$ in the CRYSCOR program ${ }^{328}$ and methods based on the random phase approximation associated to exact exchange ${ }^{329}$ coded in the VASP program. ${ }^{330}$ In general these approaches are rather 
expensive and the geometries cannot be coherently optimized as the analytical energy gradient has not yet been implemented. Nonetheless, the applicability of MP2 to molecular crystals of chemical interest and medium size has been recently carried out ${ }^{331}$ as well as recent parallelization of the CRYSCOR code ${ }^{332}$ opening the possibility for applications dealing with adsorption of large molecules on silica surfaces.

\subsection{The cluster approach}

The PBC approach focuses, first, on the definition of the bulk structure to reach to a specific hydroxylated silica surface, either crystalline or amorphous. The cluster approach, instead, focuses on the surface first and in particular on the kind of $\mathrm{SiOH}$ groups to be modeled either by: i) cutting out a representative piece of matter around a specific site from an underneath silica material; ii) expanding the size of the cluster around the active site using topologies reminescent of the silica secondary building units found in microporous all-silica zeolites; iii) adopting a tree-like cluster grown around the active site whose extension mimics the underlying solid. In all cases, the $\mathrm{Si}-\mathrm{O}$ bonds at the border of the cluster are saturated by either $\mathrm{H}$ or $\mathrm{OH}$ groups, giving rise to $\mathrm{Si}-\mathrm{H}$ and $\mathrm{Si}-\mathrm{OH}$ terminations, respectively. ${ }^{39}$ Care should be taken to avoid spurious structural and electronic effects of the terminations on the core zone of the cluster where the "active" $\mathrm{SiOH}$ is modeled by enforcing structural constraints at the frontier atoms and bonds. With all dangling bonds properly healed, the cluster becomes a regular molecule so that standard quantum mechanical methods using highly sophisticated quantum chemistry computer programs can be applied to characterize its physico-chemical features. This is a real advantage over the PBC method, as the level of sophistication in the adopted theoretical method is only limited by the available computing resources, so that $\operatorname{CCSD}(\mathrm{T})$, the golden standard for quantum chemical calculations, ${ }^{333}$ can be adopted. Furthermore, solvation methods based on the continuum approach ${ }^{334}$ can also be included as a first approximation to study solvent effects on the adsorptive properties of the surface "active site", which is particularly important in the context of biomolecules adsorption. This is a feature entirely missing from the present computer codes devoted to run periodic calculations. In practice, the cluster method was the only option available in the 90s when computer resources and programs apt to solve the Schrödinger equation for crystalline systems were still in their infancy. The state of this matter until 1994 has already been reviewed. ${ }^{39,335}$ In essence, subminimal and minimal clusters (shell-0 and shell-1 clusters in Ref. ${ }^{39}$ ) were adopted in which only the first neighbour atoms around the $\mathrm{SiOH}$ group were included in the cluster definition. In principle a shell-n cluster, grown in a "tree-like" fashion, would provide a better representation of the local environment of the "active" $\mathrm{SiOH}$ by increasing the $\mathrm{n}$ value. Unfortunately, this approach is impractical, due to the large number of 
geometrical constraints needed to avoid large distorsion of the cluster geometry caused by the interactions between terminus groups. The large number of terminal atoms is also biasing the adsorption of molecules, in particular for those of larger size (see Figure 22).
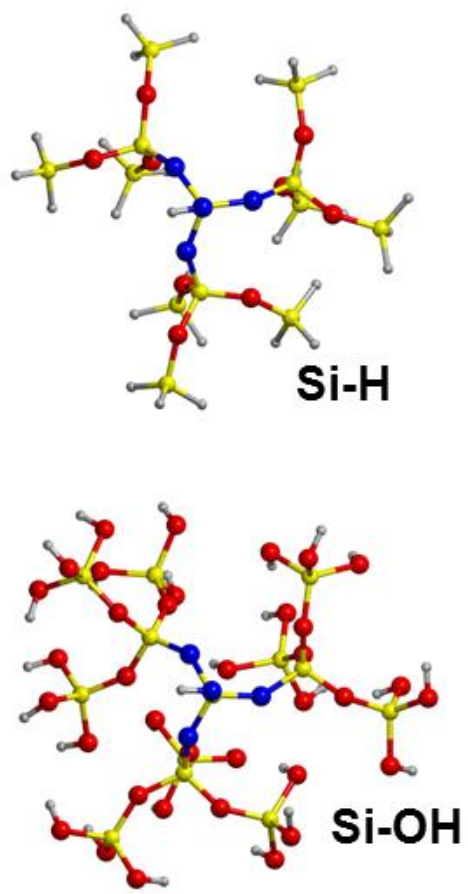

Figure 22. Tree-like models for the isolated $\mathrm{SiOH}$ group at the silica surface terminated by $\mathrm{Si}-\mathrm{H}$ and $\mathrm{Si}-\mathrm{OH}$ groups. The $\mathrm{SiO}_{3} \mathrm{OH}$ moiety representing the surface $\mathrm{SiOH}$ group is depicted in blue.

Later on, clusters based on Si-O secondary building units, the main constituents of silica frameworks, ${ }^{336}$ were proposed $^{337}$ (see Figure 23)
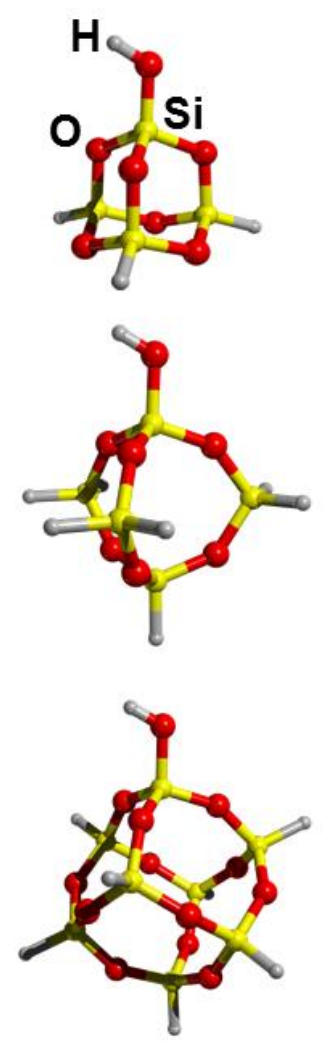
Figure 23. Cage-like cluster models for the isolated $\mathrm{SiOH}$ group at the silica surface.

These studies focused on the dependence of the features of the $\mathrm{SiOH}$ on the cluster ring topology and geometrical tension meant to model the isolated $\mathrm{SiOH}$ at the amorphous silica surface. Specifically, they have been succesful to simulate vibrational features of the $\mathrm{Si}-\mathrm{OH}$ moiety, ${ }^{338}$ the $\delta\left({ }^{1} \mathrm{H}\right)$ and $\delta\left({ }^{29} \mathrm{Si}\right)$ NMR chemical shifts, ${ }^{339}$ the adsorption of $\mathrm{NH}_{3}$ on the isolated ${ }^{340}$ and interacting hydrogarnet $\mathrm{SiOH}$ sites ${ }^{341}$ or to study $\mathrm{H}_{2} \mathrm{O}$ adsorption on external surfaces of zeolite ZSM-5 $5^{342}$ and $\mathrm{CH}_{3} \mathrm{OH}$ on Ca-rich silica surfaces. ${ }^{343}$ The advantage of the "cage-like" models over the "tree-like" ones are their natural structural rigidity which avoids the use of geometrical constraints, and the near absence of spurious effects at the core $\mathrm{SiOH}$ site due to cluster terminations. The isolated cluster method has gradually lost momentum in recent years, the PBC approach being now the most widespread one. Nevertheless, the cluster approach can be extremely useful to check whether the specific level of theory, which is usually limited to DFT in PBC calculations, is adequate by comparing the DFT results with higher levels of theory run on representative clusters cut out from the periodic model. This is particularly important, for instance, for open-shell systems for which it is $k_{n o w n}{ }^{344}$ that functionals based on the generalized gradient approximation (GGA) provide too delocalized spin density with respect to well correlated methods. A recent application to modeling crystalline silica surfaces rich in radical siloxy $\equiv \mathrm{SiO} \bullet$ terminations has adopted this approach to establish the Becke-Half\&Half functional as the best choice by comparing its performance with $\operatorname{CCSD}(\mathrm{T})$ ones run on small clusters. ${ }^{345}$ Another application is for the development and checking of new empirical potentials based on analytical expression. Very recently Colombi Ciacchi and coworkers have run MP2 calculations on silica clusters cut out from a PBC model to develop a new force-field to model the interaction of water with protonated $(\mathrm{SiOH})$ and deprotonated $\left(\mathrm{SiO}^{-}\right)$amorphous silica surfaces. ${ }^{285}$ This is obviously extremely relevant in the present context, as the silica surface is known to become negatively charged at $\mathrm{pH}$ values higher than 6 . It should be clear, however, that the cluster approach remains physically sound only when the properties under study are local in nature and do not depend on long-range interactions or on structural features specifically due to the extended nature of the system (i.e. infinite H-bond chains). Furthermore, collective properties of the adsorption, like water restructuring close to the silica surface compared to bulk liquid water, which are extremely important to our understanding of the wettability of silica based materials, cannot be simulated by the cluster approach. ${ }^{346}$

\subsection{The embedded cluster approach}

In the mid 90', it was realized that it would be interesting to merge the advantages of the cluster approach, in which a high level of theory is applicable, with the geometrical constraints 
naturally imposed by the PBC, but without actually making use of the periodicity. In principle the PBC can be avoided by adopting a large enough cluster to properly mimic the surroundings of the active site. Unfortunately, this approach brings about an exponential growth in the number of atomic orbitals with the cluster size despite the interesting chemistry being localized at one particular active site. A number of methods have been developed to describe with a higher level of theory the chemical relevant part while leaving the outside region to be described by lower (and computationally cheaper) method. Among others, the connecting bond approach by Bakowies and Thiel, ${ }^{347}$ the pseudobond scheme by Zhang and coworkers ${ }^{348}$ and the effective group potential (EGP) by Poteau et al. ${ }^{349,350}$ may be mentioned. Another techniques, inspired by the pseudobond scheme of Zhang et al., ${ }^{348}$ is the transfer Hamiltonian ${ }^{351,352}$ that employs a newly parameterized pseudoatom to describe the short-range interaction. ${ }^{353}$ Special pseudoatoms have been derived to account for the physico-chemical properties of the external chemical part connected to the central site. The pseudo-atoms are then used to cap the central site to arrive at a classical cluster model (see Section 4.2). This has been achieved by defining a pseudo-potential for the link atoms in such a way that the properties computed for the smaller capped cluster are as close as possible to those for a larger model treated with the highest level of available theory $(\operatorname{CCSD}(\mathrm{T}))$. A recent application of the transfer Hamiltonian has been reported ${ }^{87}$ to model the adsorption of $\mathrm{H}_{2} \mathrm{O}, \mathrm{NH}_{3}$ and $\mathrm{NO}$ at the silica isolated, geminal and vicinal surface sites obtaining excellent agreement with the experimental results for both $\mathrm{SiO}-\mathrm{H}$ frequency shifts and adsorption energies. The efficiency of the method allowed the authors to treat the capped cluster at MP2/6-311++G(d,p) level. Interestingly, the results computed for the adsorption of $\mathrm{NH}_{3}$ on isolated silanol groups are in excellent agreement with that computed by Armandi et al. ${ }^{169}$ using periodic boundary condition calculation (see Section 4.1) based on the density functional plus dispersion corrections PBE-D method (see Section 5.3).

A more general solution of the problem has been suggested by Morokuma and coworkers who proposed the "Integrated Molecular Orbital Molecular Mechanics", IMOMM, in which the interesting zone of the cluster was treated at a higher level (quantum mechanics) while adopting a low level (classical force fields) for the external part. ${ }^{354}$ Later on, the method eventually became an internal feature of the Gaussian98 program, ${ }^{355}$ known as the ONIOM2 method in which the lower level of theory was no longer restricted to molecular mechanics but can be any quantum mechanical method. ${ }^{356-358}$ It was immediately clear that this approach was ideal to treat active sites present at the silica surfaces. Particularly relevant in that respect is the QMPot method developed by Sauer and coworkers, which was specifically designed to model silica and zeolite materials. ${ }^{359}$ In brief, the ONIOM2 approach subdivides the system of interest into two parts or layers, each of which is described at a different level of theory. ${ }^{360}$ The most important 
part of the system is the innermost layer, the "model system", which is described at the highest level of theory. Obviously, the values of the computed properties are a function of the size and topology of the model system. The whole system is called the "real system". One delicate step is to define the cut between the model system and the rest of the system. Usually this is attained by adding a set of fictitious atoms, usually hydrogen atoms (called "link atoms"), along the preexisting chemical bonds extending from the model system toward the exterior layer.
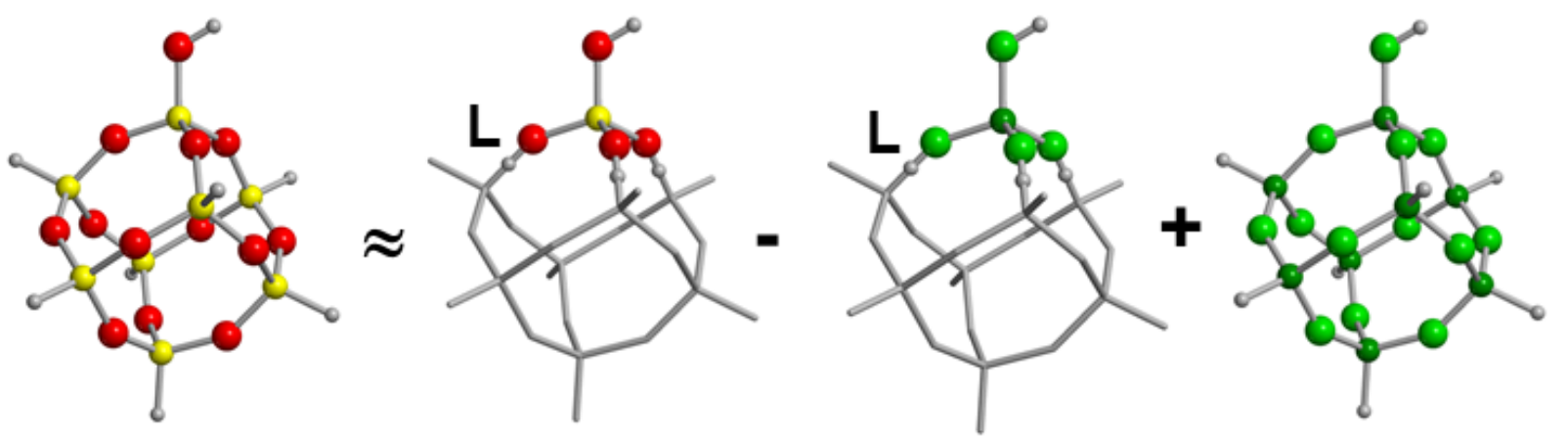

\section{E [High:Low] $\approx \mathrm{E}($ High, Model) $-\mathbf{E}($ Low, Model) + E(Low, Real)}

Figure 24. Definition of the ONIOM2 scheme for a cage cluster model representing the isolated $\mathrm{SiOH}$ group at the silica surface. Color coding identifies the level of calculus. L identifies the link atoms.

In the ONIOM2 approach, the energy E[High:Low] of the system, subdivided into the two layers, is written as (see Figure 24):

$\mathrm{E}[$ High:Low $] \approx \mathrm{E}[$ High:Real $]=\mathrm{E}($ High, Model $)-\mathrm{E}($ Low, Model $)+\mathrm{E}($ Low, Real $)$

in which E(High, Model), E(Low, Model) and E(Low, Real) are the total energies of the model and real systems at the high and low levels of theory. The nice feature about the ONIOM2 formulation is that the method is self-embedding by definition, i.e. if the low level of calculation is increased to reach the highest adopted level or if the model system is enlarged up to the real system, one rigorously gets $\mathrm{E}[\mathrm{High}: \mathrm{High}]=\mathrm{E}(\mathrm{High}, \mathrm{Real})$, for Model $\rightarrow$ Real as well as for Low $\rightarrow$ High.

The first application of the ONIOM2 method to model the adsorption of $\mathrm{NH}_{3}$ at the isolated hydroxyl groups on a highly dehydrated silica surface was discussed in Ref. ${ }^{361}$ The purpose was to establish which level of theory, within the ONIOM2 framework, was optimum to provide internally consistent data for the prediction of a number of physical observables like the $\mathrm{OH}$ vibrational features, the $\mathrm{NMR} \delta\left({ }^{1} \mathrm{H}\right)$ and $\delta\left({ }^{29} \mathrm{Si}\right)$ chemical shifts and to characterize the adsorption of $\mathrm{NH}_{3}$. A cage-like cluster was adopted, which was considered as the real system, 
and subportions of different sizes cut out around the $\mathrm{SiOH}$ group were adopted to define the model system. Results were very satisfactory, as all properties converged quicker than pure cluster calculation to those computed for the real system. Other applications of the ONIOM2 approach to model silica surface features include the adoption of the same cage cluster used to mimic the isolated $\mathrm{SiOH}$ in Ref. ${ }^{361}$ to compare the adsorption energies of $\mathrm{N}_{2}, \mathrm{CO}, \mathrm{H}_{2} \mathrm{O}, \mathrm{NH}_{3}$ and $\mathrm{CH}_{3} \mathrm{CN}$ with clusters representative of Lewis and Brönsted sites in zeolites ${ }^{362}$ and the simulation of the hydrogarnet in all-silica silicalite. ${ }^{117,363}$ In the latter case the silica hydrogarnet in the allsilica silicalite was represented by a $\mathrm{Si}_{56} \mathrm{O}_{90} \mathrm{H}_{52}$ real system and by the $\mathrm{Si}_{4} \mathrm{O}_{16} \mathrm{H}_{4}$ model zone computed with ONIOM[B3LYP/6-31G(d,p):PM3] (Figure 25) to model the fine vibrational features in the $\mathrm{OH}$ stretching region of the hydrogarnet.

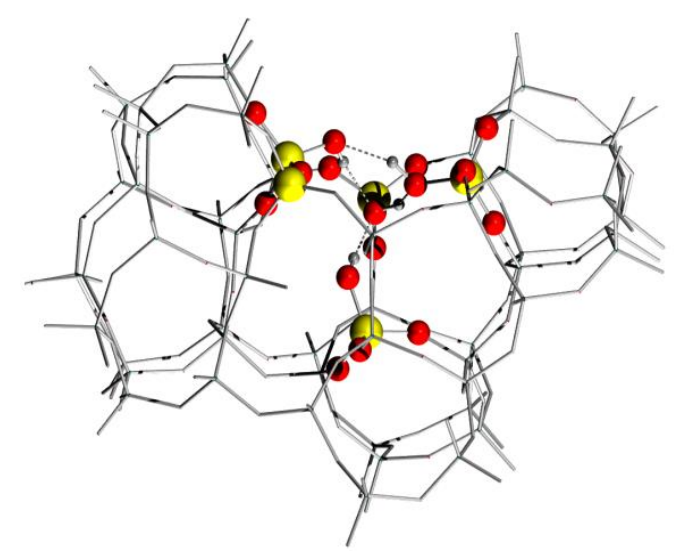

Figure 25. Model of the hydrogarnet in all-silica silicalite structure. The balls identify the model region treated at B3LYP/6-31G(d,p) level.

The mechanical embedding scheme, in its QMPot implementation as proposed by Sauer and coworkers, ${ }^{364}$ has reached a high popularity in the study of Brönsted sites (both acidic and cation exchanged ones) in zeolites. ${ }^{365,366}$ More recently, thanks to the increased computing power, the method has been extended to adopt DFT to treat the real system within the PBC. In the applications, PBE functional and plane waves as implemented in the VASP code have been adopted as the low level of calculus. The model system is usually treated at the MP2 high level with large Gaussian basis set, which ensures that dispersive interactions are also taken into account, at least in the model system. ${ }^{367}$ Recent applications of this modern version of QMPot are the study of $\mathrm{H}_{2}$ adsorption on Metal Organic Framework ${ }^{368}$ or the benzene ethylation catalyzed by the adsorption in H-ZSM-5. ${ }^{369}$ To the best of our knowledge, no applications have been published to date to model adsorption at silica surfaces even if nothing should hinder their success in that respect. In the original implementation of the ONIOM method neither direct charge transfer nor polarization were possible between the exterior layer and the model system. Only indirect effects on the model system occur due to the equilibration of energy gradients 
acting on the link atoms, which force the atoms of the model system to follow those of the exterior layer and vice versa. In the modern implementation of the ONIOM method, the charge distribution of the MM region interacts with the actual charge distribution of the QM region. In this case, the partial charges from the MM region are included in the QM Hamiltonian, which provides a more accurate description of the electrostatic interaction and, in addition, allows the wave function to respond to the charge distribution of the MM region. This approach is referred to as electronic embedding. ${ }^{370}$

\section{Theoretical Methods and their application to silica surfaces modeling}

An accurate description of the properties of silica surfaces and, in particular, their role on adsorption and reactivity processes at the atomic level requires the use of quantum chemistry methods. These methods are based on the resolution of the time-independent, non-relativistic Born-Oppenheimer electronic Schrödinger equation and can be classified into two major groups: methods based on the wave function and those based on the density functional theory. It is not our goal to provide a detailed description of the existing electronic structure methods, which can be found in various textbooks ${ }^{371-373}$ and its current state is nicely reviewed in a recent Chemical Review thematic issue devoted to quantum chemistry. ${ }^{374}$ Instead, we attempt to provide a general overview of the methods that have been and can be applied to study silica surfaces and their interaction with biomolecules, while mentioning their strengths and weaknesses.

\subsection{Wave function based methods}

The simplest ab initio wave function based method, the Hartree-Fock (HF) method, is based on a single Slater determinant. It has limited accuracy since it neglects the electron correlation energy, which can significantly influence the results, particularly when comparing situations with different electron correlations, or when describing radical defects or H-bond and dispersion interactions, aspects that are of great importance to the present topic. Electron correlation can be taken into account by expanding the wave function through the inclusion of excited state configurations. Derived methods are called post Hatree-Fock and include, for instance, the Configuration Interaction (CI), Møller-Plesset (MPn) and Coupled Cluster (CC) methods. ${ }^{371-373}$ Wave function based methods have the advantage that they can be applied hierarchically to obtain increasingly more accurate values converging to the exact solution. Among them, the $\operatorname{CCSD}(\mathrm{T})$ with single, double and triple $\mathrm{T}$ excitations (the latter estimated through perturbation theory) extensively includes electron correlation and, when combined with basis sets extrapolation techniques, is considered the "golden standard" in quantum chemistry. ${ }^{333}$ Applicability of $\operatorname{CCSD}(\mathrm{T})$ is, however, hampered by the size of the modeled system and is 
limited to the cluster approach. Indeed, the main disadvantage of wave function based correlated methods is the high scaling of the computational cost with the size of the system. Local correlation methods, ${ }^{375-383}$ allow exploiting the fundamental local character of dynamical electron correlation, leading to methods that may scale linearly with the size of the system and thus largely reducing the computational costs of post-Hartree-Fock methods. As already anticipated in section 4.1, it is worth mentioning that local-MP2 has been implemented for extended systems by exploiting the translational and point symmetry poperties of crystalline structures $^{384}$ with the periodic boundary conditions as implemented in the CRYSCOR program, ${ }^{328}$ and in the VASP program ${ }^{330}$ through the random phase approximation associated to exact exchange. ${ }^{329}$ These methods are, in general, very expensive when applied to extended systems but the development of codes apt to exploit the power of the high performance computing architectures ${ }^{332}$ will soon make this kind of calculations available to the scientific community.

\subsection{DFT based methods}

Since the mid-90s, approaches based on electron density (see Ref. ${ }^{344}$ for a nice introduction to DFT and for the technical nomenclature) rather than on the wave function have become attractive alternatives because, while including electron correlation, they are computationally cheaper than post-Hartree-Fock methods. In general, DFT methods based on generalized gradient approximation (GGA) functionals such as PBE, PW91, BLYP are usually implemented in codes running periodic calculations based on plane-waves basis set (vide infra). Hybrid functionals like B3LYP and PBE0 contain a fraction of exact exchange in the functional definition, which allows improving the already good performance of GGA functionals for systems where the self-interaction error may become important. ${ }^{385}$ At variance with the GGA case, the implementation of hybrid functionals is easier using localized basis functions (Gaussian type orbitals, vide infra). To show an example of the DFT performance with respect to the present topic, the structural data and relative stabilities of $\alpha$-quartz, $\alpha$-cristobalite and $\alpha$ tridymite $^{386,306}$ are very well reproduced with B3LYP with a 6-31G(d) Guassian basis, as illustrated in Table 2.

Table 2. Structural data and relative stabilities of $\alpha$-quartz, $\alpha$-cristobalite and $\alpha$-tridymite. Cell parameters as a, b and c, cell volume as $\mathrm{V},\langle\mathrm{Si}-\mathrm{O}\rangle$ and $\langle\mathrm{Si}-\mathrm{O}-\mathrm{Si}\rangle$ average values.

\begin{tabular}{lcccccc}
\hline & \multicolumn{2}{c}{$\alpha$-quartz } & \multicolumn{2}{c}{$\alpha$-cristobalite } & \multicolumn{2}{c}{$\alpha$-tridymite } \\
& $\operatorname{Exp}^{\mathrm{a}}$ & B3LYP & $\operatorname{Exp}^{\mathrm{b}}$ & B3LYP & $\operatorname{Exp}^{\mathrm{c}}$ & B3LYP \\
\hline Space group & $\mathrm{P} 3_{1} 2_{1}$ & $\mathrm{P} 3_{1} 2_{1}$ & $\mathrm{P} 4_{1} 2_{1}$ & $\mathrm{P} 4_{1} 2_{1} 2$ & ${\mathrm{C} 222_{1}}$ & $\mathrm{C} 222_{1}$
\end{tabular}




\begin{tabular}{|c|c|c|c|c|c|c|}
\hline $\mathrm{a}(\AA)$ & 4.902 & 4.971 & 4.969 & 5.024 & 8.756 & 8.869 \\
\hline $\mathrm{b}(\AA)$ & 4.902 & 4.971 & 4.969 & 5.024 & 5.024 & 4.968 \\
\hline$c(\AA)$ & 5.399 & 5.467 & 6.926 & 6.989 & 8.213 & 8.267 \\
\hline$V\left(\AA^{3}\right)$ & 112.4 & 117.0 & 171.0 & 176.4 & 45.2 & 45.5 \\
\hline$<\mathrm{Si}-\mathrm{O}>(\AA)$ & 1.612 & 1.632 & 1.603 & 1.629 & 1.559 & 1.626 \\
\hline$<\mathrm{Si}-\mathrm{O}-\mathrm{Si}>\left(^{\circ}\right)$ & 142.6 & 142.7 & 146.7 & 144.5 & 179.7 & 176.8 \\
\hline \multicolumn{7}{|c|}{ Relative stability } \\
\hline & $\operatorname{Exp}^{\mathrm{d}}$ & B3LYP & $\operatorname{Exp}^{d}$ & B3LYP & $\operatorname{Exp}^{d}$ & B3LYP \\
\hline$\Delta \mathrm{H}(\mathrm{kJ} / \mathrm{mol})$ & 0.0 & 0.0 & 10.9 & 10.5 & 12.1 & 12.6 \\
\hline \multicolumn{7}{|c|}{${ }^{\mathrm{a}} \operatorname{Ref}^{387}$ ICSD\#93974. ${ }^{\mathrm{b}} \operatorname{Ref}^{388}$ ICSD\#77452. ${ }^{\mathrm{c}} \operatorname{Ref}^{389}{ }^{38}{ }^{\mathrm{d}} \operatorname{Ref}^{390}$} \\
\hline
\end{tabular}

The agreement between experimental and computational data for the three polymorphs is excellent. Even the B3LYP < $\mathrm{Si}-\mathrm{O}-\mathrm{Si}>$ angle inside the cell, whose value is very sensitive to small perturbations due to its high flexibility, is in good agreement with experimental data, the largest deviation being $2.9^{\circ}$. Calculated $\Delta \mathrm{H}$ values associated to the relative stability of the silica polymorphs are also in excellent agreement with calorimetric data. ${ }^{390}$ The GGA PBE and PW91 functionals provide consistent results, ${ }^{391}$ but with slightly larger expanded lattices due to larger Si-O-Si angles computed at these levels of theory. The hybrid B3LYP functional also appears to be superior to GGA in the calculation of vibrational frequencies of $\alpha$-quartz, ${ }^{392}$ with mean absolute deviations of only $6 \mathrm{~cm}^{-1}$, which validates the B3LYP functional as a reliable method to study silica materials.

Point defects on silica, such as the common non-bridging oxygen hole center $\equiv \mathrm{Si}-\mathrm{O}^{\bullet}$, can also be reasonably described with DFT methods. ${ }^{345,393-396}$ In these cases, however, where radical species are present, the use of hybrid functionals is especially important to get accurate results. GGA functionals tend to delocalize the spin density because these situations are overstabilized $^{397,398}$ due to a bad cancellation of the self-interaction part by the exchange functionals. This can significantly influence the $\equiv \mathrm{Si}-\mathrm{O}^{\circ}$ bond length, the computed EPR data $^{345,393}$ or the optical properties. ${ }^{302,396}$ Indeed, quantitative agreement between measured and computed hyperfine coupling constants and $\mathrm{g}$ factors is only found with hybrid functionals including $50 \%$ of exact exchange. ${ }^{393}$ GGA functionals, such as BLYP, fail in describing these defects. Spin density delocalization also influences the H-bonding in a surface containing radical defects and the kinetics of the $\equiv \mathrm{Si}-\mathrm{O}^{\bullet}+\mathrm{H}_{2} \mathrm{O} \rightarrow \equiv \mathrm{Si}-\mathrm{OH}+\mathrm{OH}^{\bullet}$ reaction. ${ }^{345}$ The energy barrier is significantly smaller with GGA than with hybrid functionals, BH\&HLYP including $50 \%$ of exact exchange being the one that best compares to $\operatorname{CCSD}(\mathrm{T})$ calculations. 
Hydroxylated silica surfaces exhibit H-bonding between surface silanols and with the adsorbed molecules. It is, therefore, important to assess whether DFT methods are capable to properly describe H-bonded interactions of medium strength. ${ }^{399}$ This topic has been largely investigated in the last two decades, the water dimer being probably the most intensively studied hydrogen bonded system. ${ }^{344}$ Among the standard density functionals frequently used in periodic calculations, B3LYP and BLYP have been shown to slightly underestimate the binding energy and the $\mathrm{R}_{\mathrm{O}-\mathrm{O}}$ distance, while both slighthly overestimate the $\mathrm{O}-\mathrm{H}$ stretching frequency shift in an H-donor $\mathrm{OH}$, compared to MP2 and experimental data. ${ }^{400-404}$ The PBE functional, while providing accurate interaction energies, ${ }^{405}$ underestimates the $\mathrm{R}_{\mathrm{O}-\mathrm{O}}$ distance somewhat more, predicts larger frequency shifts ${ }^{401}$ and its reliability is closely connected to the bond directionality. ${ }^{406}$ For the most specific case of hydroxyl groups in ionic crystals like $\beta-\mathrm{Be}(\mathrm{OH})_{2}$ and $\mathrm{Mg}(\mathrm{OH})_{2}$ it was proved ${ }^{407}$ that B3LYP OH frequency shifts are in better agreement with experiments than those resulting from PW91 functional, which were definitely overestimated. In general, it can be safely stated that functionals based on Becke's exchange formulation provide better frequency shifts than those resulting from Perdew's one for bonds acting as H-bond donors. These results show that, on the whole, the properties (binding, geometry and frequency shifts) of systems (molecular and crystalline) with moderately strong H-bonds (dominated by electrostatics ${ }^{399}$ ), are reasonably well described by standard GGA and hybrid functionals.

\subsection{Dispersion corrections}

Standard density functionals are, however, unable to cope with long-range dispersion interactions, ${ }^{408-411}$ which are essential to properly describe the adsorption of biomolecules on silica surfaces (see for instance Refs. ${ }^{321,412,413}$ ). Moreover, accounting for dispersion interactions appears to be important to describe the structure and dynamics of liquid water, ${ }^{414}$ which otherwise becomes overstructured, ${ }^{415,416}$ especially when using GGA functionals. ${ }^{417}$

To account for dispersion, it has been proposed ${ }^{418-421}$ to use an atom-atom additive damped empirical potential of the form $-f(R) C_{6} R^{-6}$ to include long-range dispersion contributions to the computed ab initio DFT total energy and gradients:

$$
E_{D F T-D 2}=E_{D F T}+E_{D I S P}
$$

where $E_{D I S P}$ is the empirical potential:

$$
E_{D I S P}=-S_{6} \sum_{i<j} f\left(R_{i j}\right) \frac{C_{6}^{i j}}{R_{i j}^{6}}
$$


Here, $C_{6}^{i j}$ is the dispersion coefficient for the pair of atoms $i$ and $j, s_{6}$ is a scaling factor that depends on the adopted DFT method and $R_{i j}$ is the interatomic distance between atoms $i$ and $j$ in the molecular system. The damping function $f\left(R_{i j}\right)=\frac{1}{1+e^{-d\left(R_{i j} / R_{v d w}-1\right)}}$ was used to avoid near-singularities and double-counting for small interatomic distances by using the sum of the van der Waals atomic radius of atoms $i$ and $j$ and the the steepness of the damping function as determined by the value of the $d$ parameter. Values of the van der Waals radii, atomic $C_{6}$ coefficients from which the $C_{6}^{i j}$ are computed as geometrical mean, and the $s_{6}$ values for specific functionals are provided in Ref. ${ }^{420}$ The coding of the above formulae for energy and gradient are very simple and the computational cost with respect to a plain DFT calculation is negligible. The above formulae have been promptly extended to correct energy and gradient for crystalline systems as it was reported in Ref. ${ }^{422}$

The beauty of the DFT-D2 approach is that it leaves unaltered the original accuracy of the DFT functional for short-range interactions (bond breaking/making), while it dramatically improves the long-range tail of the interaction dominated by London forces. ${ }^{420,423-425}$ Inclusion of dispersion interactions is mandatory when computing the adsorption energies of biomolecules on silica surfaces, since they can account for up to 40-50\% of the total adsorption energy (see for instance Ref. ${ }^{321}$ ). Moreover, when adsorption is determined by dispersion interactions with siloxane groups and less by $\mathrm{H}$-bonding, such a correction also becomes essential to determine the proper geometry and adsorption energy of the adsorbed adduct. An example of that is the work of Rimola et al. ${ }^{426}$ which studied the interaction of benzene, which can be considered as a partial analogue of the Phe molecule, with a hypothetical hydrophobic silica surface constituted by only $\mathrm{Si}-\mathrm{O}-\mathrm{Si}$ bonds; i.e., with no $\mathrm{SiOH}$ surface groups. Calculations were run adopting periodic boundary conditions (see Section 4.1) using B3LYP and B3LYP-D* methods, the latter inclusive of the dispersion contribution properly reparametrized ${ }^{422}$ to account for the condensed matter nature of the system. At B3LYP level, the calculated BSSE-corrected adsorption energy $\left(\Delta \mathrm{E}^{\mathrm{C}}\right)$ was found to be slightly repulsive $\left(+2 \mathrm{~kJ} \mathrm{~mol}^{-1}\right)$, becoming attractive and as large as $-32 \mathrm{~kJ} \mathrm{~mol}^{-1}$ when dispersion was included at the B3LYP-D* level. Significant changes also resulted in the optimized geometries due to the role of dispersion. That is, at the B3LYP level, the optimum benzene distance from the surface was about $4.5 \AA$, which decreased to $3.3 \AA$ at the B3LYP-D* level (see Figure 26a). When benzene was adsorbed on a highly hydroxylated $\left(5.2 \mathrm{OH} \mathrm{nm}^{-2}\right.$ ) silica surface model (Figure 26b) the optimum structure revealed specific $\mathrm{OH} \bullet \bullet \pi$ interactions. At the B3LYP level, the strength of this interaction, $\Delta \mathrm{E}^{\mathrm{C}}$, was $-10 \mathrm{~kJ} \mathrm{~mol}^{-1}$, which increased (in 
absolute term) to $-48 \mathrm{~kJ} \mathrm{~mol}^{-1}$ at the B3LYP-D* level, i.e. dispersion provides an almost five times increase in the adsorption energy. For this system, the geometry differences between B3LYP and B3LYP-D* were less accentuated than those observed for the hydrophobic silica surface; however, a significant decrease of the $\mathrm{OH} \bullet \bullet \pi$ distance $(\sim 0.3 \AA)$ was observed upon dispersion inclusion.
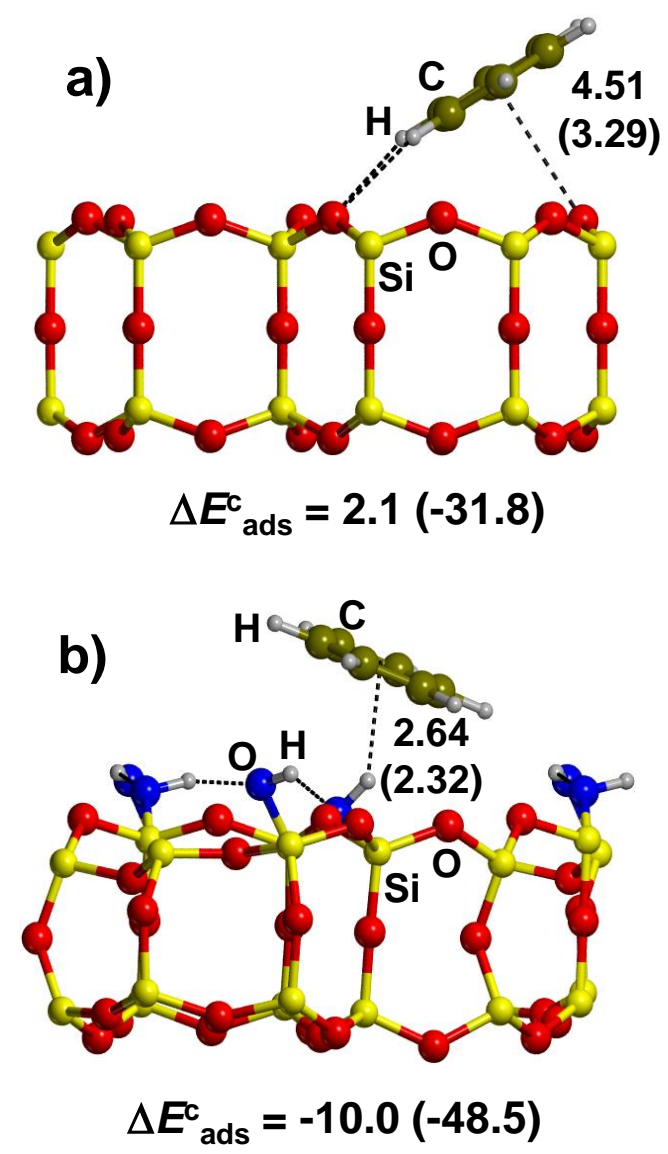

Figure 26. Optimized structures and adsorption energies for benzene adsorbed on a) a hydrophobic and b) a hydrophilic silica surface at the B3LYP (B3LYP-D*) levels of theory. Distances are in Angstroms and energies in $\mathrm{kJ} \mathrm{mol}^{-1}$. Draw with structural and energetic data from Ref. ${ }^{426}$

The DFT-D2 scheme has recently been reformulated as DFT-D3 method ${ }^{421}$ by including atom-pairwise specific dispersion coefficients and cutoff radii that are both computed from first principles. Moreover, a new non-empirical method to obtain $\mathrm{C}_{6}$ coefficients from ground state electron density of a molecule or solid has also been proposed. ${ }^{427}$ Other approaches to account for dispersion corrections have been developed, for instance those based on adding effective atom-centered potentials ${ }^{428-430}$ or explicitly including non-local terms in the correlation functional as in the vdW-DF method. ${ }^{431}$ Alternatively, a new suite of M06 hybrid functionals based on meta-generalized gradient approximation, have been proposed by the Thrular's school $^{432,433}$ and shown to be promising in handling non-covalent interactions. However, these 
functionals represent an improvement only up to medium range contacts and, to the best of our knowledge, are not implemented in codes running periodic calculations.

\subsection{Basis sets}

Besides the errors associated to the chosen functional, one also must also consider the errors due to the basis set expansion of the Kohn Sham orbitals. Two major kinds of basis sets are currently used: localized Gaussian Type Orbitals (GTO) and plane waves (PW). Gaussian orbitals have the advantage that the number of basis functions depends on the number of atoms included in the crystalline cell but not on the dimension or shape of the cell. Moreover, due to the local nature of Gaussian functions, the true dimensionality of the system (0D:molecules, 1D: chains, 2D: slabs and 3D: crystals) is always obeyed. Transferability of GTO basis functions to periodic systems, however, should be done with care, particularly in the case of diffuse functions, to avoid an exponential increase in the number of bi-electronic integrals to be evaluated due to the long-range nature of the tail of diffuse functions. This, in turn, also may lead to linear dependence in the definition of the wave function. On the other hand, the use of a limited number of basis functions entails the so-called basis set superposition error (BSSE); i.e., a spurious stabilizing contribution arising from the improved description of a fragment in a complex due to the assistance of the basis sets of the other fragments. This error is normally corrected a posteriori, at the uncorrected geometries, with the counterpoise method. ${ }^{434}$

Plane wave basis sets are mainly used in solid-state simulations, along with pseudopotentials or projector augmented wave method to account for core electrons. Plane wave calculations are free of BSSE since they are not centered on the atomic nuclei, thereby describing any point in the supercell with the same quality. Moreover, energy and gradient calculations are more efficient than with GTO for GGA functionals. The reverse is true for hybrid functionals as the calculation of the exact exchange using plane waves is very expensive and applications of B3LYP based on plane waves started to appear only very recently ${ }^{435}$ compared to B3LYP with Gaussian type orbitals, which were first run for studying the relative stability of silica polymorphs ${ }^{436}$ already in 1998. This prevents the use of hybrid functionals which, in many ocasions, are more accurate than standard GGA functionals. Another disadvantage of PW basis sets is that calculations are intrinsically periodic in three dimensions (3D), regardless of whether one is dealing with a molecule, a polymer, a slab or a crystal, the 3D box being uniformly filled with plane waves. The quality of PW basis sets is specified by the electronic kinetic energy cutoff $\mathrm{E}_{\mathrm{kin}}$ : the higher $\mathrm{E}_{\mathrm{kin}}$ the better the basis.

With the aim of analyzing the differences arising from using GTO and PW basis sets, for hydrogen bonded systems, the formic acid molecule, its dimers and the molecular crystal were computed with different GGA functionals. This study shows that, irrespective of the functional, 
PW and GTO results converge smoothly as a function of the quality of the basis set. ${ }^{437}$ Moreover, in an attempt to bridge the gap between methods routinely applied in materials science using PW basis sets and quantum chemical methods using local GTO basis sets, the behavior of PBE and PBE0 (including one quarter of exact exchange to PBE) to determine geometries and atomization energies of molecules in the G2-1 data set was assessed using both basis sets. This study shows that discrepancies are very small. Indeed, both approaches yield identical results if convergence is carefully checked and reached for both methods. ${ }^{438}$

\subsection{Molecular Dynamics simulations}

Exploring the potential energy surface; i.e., solving the electronic Schrödinger equation at different nuclei positions, allows determining thermodynamically stable structures (minima) and transition structures (first order saddle points) at 0K. For non-interacting rigid molecules, temperature effects can be reasonably incorporated by assuming the rigid rotor-harmonic oscillator approximation and standard statistical methods from frequency calculations at the minimum energy structure. However, for large molecules, with soft torsional modes, or in solution, thermal effects must be estimated from a representative sampling of the phase space by using simulation techniques such as Monte Carlo and Molecular Dynamics. In Monte Carlo methods the phase space sampling is attempted by random displacements of the relevant coordinate (Cartesian or internal coordinates) with the corresponding energy evaluation. The exploration is usually conditioned through a Boltzmann weighting scheme (Metropolis Monte Carlo) which ensures a reasonable efficiency in the search for local minima. ${ }^{439}$ Many millions steps are, however, needed in order to ensure a statistically meaningful ensemble, as a result of the potential energy sampling.

The Monte Carlo steps cannot be considered as an evolution in time of the system. The latter can be rigorously achieved by a technique known as molecular dynamics. Introduced by Alder and Wainwrigth in $1959^{440}$ and extended by Rahman in $1964,{ }^{441}$ this method allows to study the evolution in time-space phase of the atomic positions subject to the internal forces of chemical nature and to the kinetic energy due to the temperature of the system. To ensure energy conservation, however, the time step used to integrate the Newton equations should be smaller than that associated to the fastest vibration of the system, resulting in values close to $0.1 \mathrm{fs}\left(10^{-16}\right.$ s). Obviously, realistic applications of molecular dynamics imply a huge computational effort to explore the system behavior even for a very short time window. For that reason, molecular dynamics developed to use a description of the internal forces resulting from classical force fields (see Section 5.6.3) which ensures a fast evaluation of energy and forces at the price of lower accuracy. The situation dramatically changed when the Car-Parrinello ${ }^{442}$ (CP) method was introduced in 1985. In this method, forces are evaluated "on the fly" as resulting from a plane 
waves based density functional calculation in which electron density is not computed at each time step but estimated through the time integration of a cleverly defined Lagrangian operator. Since then, the CP method has enjoyed an enormous popularity in many different fields of molecular physics and chemistry. ${ }^{443}$ Among the cases in which molecular dynamics is beneficial are the adsorption of biomolecules on silica surfaces in solution, as this techniques accounts for temperature effects which are essential to understand their chemical behavior. Indeed, several studies presented in the frame of this review (e.g. Section 9.1.1.2) have used molecular dynamics simulations, to study surface reconstruction, water-silica interfaces or biomolecules adsorption. While molecular dynamics (MD) simulations may be essential to afford detailed structural and dynamical insights, they can also become computationally prohibitive for relevant time scales. However, due to the continuous improvement of the power of high performance computer, molecular dynamics simulations with on-the-fly computation of interatomic potentials from electronic structure theory can nowadays reach hundreds of picoseconds whereas classical molecular dynamics, based on predefined classical force fields, derived either from empirical data or from quantum chemical calculations, can extend up to the microsecond time scale. The reliability of these force fields, however, is a critical aspect that should be carefully analyzed, particularly when entering into large time scales (see Section 5.6.3).

\subsection{Approximated methods}

As seen in the previous sections, density functional theory is a formidable and general tool to treat, at least in principle, any kind of molecular or extended system. Limitations are, besides the intrinsic inaccuracy of the DFT itself, due to the rather steep rise in the computational resources needed to simulate systems containing hundreds or thousands of atoms. This is particularly relevant for the present topic as both biopolymers and silica surfaces imply a large number of atoms. For instance, the need for realistic models of amorphous silica surface inevitably implies the adoption of very large surface unit cells without internal symmetry, which prevents the straightforward application of DFT based methods (see Section 7). A further issue is the need to run molecular dynamics calculations when configurational entropy becomes a key ingredient for describing the system, as in all cases in which liquid water plays a key role. Another example in which molecular dynamics is mandatory is when modeling the bulk amorphization process with increasing temperature and reconstructions and relaxation of silica surfaces. To address the above points, a number of approaches have been developed in the literature to reduce the computational burden.

\subsubsection{Semiempirical methods}

A possibility to speed up a calculation, by at least three orders of magnitude with respect to a DFT reference calculation, is to adopt semiempirical methods which are all based on the 
neglect of differential overlap approximation by Pople et al. ${ }^{444}$ The evolution of the earlier CNDO and INDO through the MNDO method ${ }^{445}$ of the early 80 's continued up to the most recent PM6 and PM7 methods available in the MOPAC2012 computer code, ${ }^{446}$ maintained by James Stewart. Basically, no applications of the semiempirical methods to adsorption of molecules/biomolecules on silica have appeared due to the known weakness of these methods in dealing with $\mathrm{H}$-bond and dispersive interactions, which are the dominant components of the adsorption energy for these cases. Nevertheless, the present status of the semiempirical methods can change the matter dramatically, due to important improvements addressed by the PM6$\mathrm{DH} 2^{447,448}$ and PM7 model hamiltonians which allow to model both H-bond interactions and London dispersive forces much more accurately than in the past.

\subsubsection{SCC-DFTB method}

A level of theory which is intermediate between the semiempirical and the full DFT approach is based on the tight-binding approach originally suggested for periodic systems by Koster and Slater. ${ }^{449}$ The modern version is known as self-consistent charge density functional tight-binding method (SCC-DFTB) mainly developed by Elstner's group ${ }^{450-454}$ and is an approximation to density functional theory (DFT), where the Kohn-Sham (KS) total energy is expanded up to the second order in the charge density fluctuations, $\delta$ n, about a reference density, $\mathrm{n}_{0}$. The SCC-DFTB total energy is:

$$
E=\sum_{i}^{o c c}\left\langle\psi_{i}\left|\hat{H}_{0}\right| \psi_{i}\right\rangle+\frac{1}{2} \sum_{\alpha \beta} \gamma_{\alpha \beta} \Delta q_{\alpha} \Delta q_{\beta}+E_{\text {rep }}
$$

where $\Delta q_{\alpha}$ is the Mulliken net charge on atom $\alpha$ and $\gamma_{\alpha \beta}$ is a function that depends on the $\alpha-\beta$ interatomic distance and on the Hubbard parameters $U_{\alpha}$ and $U_{\beta}$, calculated within the generalized gradient approximation with the Perdew-Burke-Ernzerhof (PBE) formalism. $E_{\text {rep }}$ is the repulsion energy approximated as the sum of pairwise repulsive potentials adjusted to DFT calculations on reference systems. SCC-DFTB KS equations are obtained by applying the variational principle to the energy functional by expanding the KS orbitals into a suitable set of localized atomic orbitals, $\psi_{i}=\sum_{v} c_{\nu}^{i} \phi_{v}$. The KS equations are transformed into a set of selfconsistent algebraic equations in which the Hamiltonian and overlap matrix elements are approximated in order to evaluate the calculation of bielectronic integrals. This results in the SCC-DFTB approach being comparable to semiempirical methods as far as computational efficiency is concerned. The energy can also be supplemented by a dispersion correction term ${ }^{448}$ not accounted for by the level of theory. The method has been recently used to study biological 
systems of large complexity. ${ }^{455}$ Specific applications to silica/aminoacids interactions ${ }^{323}$ are reviewed in Section 9.

\subsubsection{Classical force fields}

Another three orders of magnitude with respect to semiempirical and SCC-DFTB methods can be gained by representing the molecular structure by means of "mechanical springs" whose features (length, strength, etc.) mimic the different interactions occurring within the molecule and between different molecules. The set of parameters associated to the springs is known as "force field" and the relaxation of the mechanical energy is the result of a "molecular mechanics calculation". The method has enjoyed an enormous success starting from the first public force field for hydrocarbons, MM2 by Norman Allinger. ${ }^{456}$ The same approach has been extended to inorganic solids and in particular, to the silica bulk. The complexity of inorganic materials and the paucity of experimental data needed to derive the force field parameters forced the adoption of simple two-body potentials for the description of each spring, compared to the most sophisticated terms (three-body and four-body terms) used for molecular cases. For silica, despite the success of the classical two-body potential by van Beest, Kramer and van Santen (BKS), ${ }^{457}$ the accurate description of the highly flexible Si-O-Si bond (see Section 2) required 3body terms involving $\mathrm{O}-\mathrm{Si}-\mathrm{O}$ and $\mathrm{Si}-\mathrm{O}-\mathrm{Si}$ bending modes. Also, the semi-ionic nature of the Si$\mathrm{O}$ bond $^{42,43}$ suggested to adopt a shell-ion model potential in which the electronic charge on the oxygen atoms is split into an inner (core) and an outer (shell) components linked by a mechanical spring ${ }^{458,459}$ to take the electronic polarizability of the oxygen atom into account. Many of the calculations on silica and oxides have been run using the General Utility Lattice Program (GULP) developed by Julian Gale. ${ }^{460}$ Along the above general lines a large number of force fields for modeling the silica bulk have been developed and a separate review would be needed to describe all of them in details. Here we only summarize the most widely used force fields for silica through Table 3, while the interested reader may read the review by Schaible ${ }^{461}$ on silicon and silica force fields for more details. The majority of the studies focused on the silica bulk properties, the surfaces being limited to studies devoted to understand the geometrical relaxation due to the bond cutting with respect to the bulk; water reactions involving the classical $\mathrm{SiOH}$ surface terminations were usually not considered (see Section 7.3 and 7.4). In other words, the state of the fully hydroxylated silica surfaces, either crystalline or amorphous, the latter being the most common for real silica surfaces (see Section 2) has hardly been addressed with these force field (as exceptions see Ref. ${ }^{462-466}$ ), as these studies were not concerned with the description of the silanol groups and their mutual H-bonding interactions. To specifically address the H-bonded features at the silica surface the FFSiOH force field has recently been proposed, 
demonstrating excellent performances for both the bulk and the surface of silica materials as regards structures, energetic and phonon spectra, including the $\mathrm{OH}$ stretching region. ${ }^{467} \mathrm{FFSiOH}$ is based on a shell-ion model potential to which a specific three-body term has been added to describe the H-bonding interaction between $\mathrm{SiOH}$ groups.

Extension of silica force fields to model the interaction with adsorbates is much more difficult due to the complexity of the potential energy surface characterizing the surface/adsorbate interaction. The need for a much more extended set of parameters to deal with the interactions between the biomolecule and the surface has prevented this kind of studies from appearing in the literature. For the silica/water system (see Section 8 for a deeper discussion), Garofalini et $a l^{464,465}$ Singer et $a l^{462,463}$ and $\mathrm{Du}$ et al ${ }^{466}$ have extended the silica force field to include the interaction with water. A recent example of the problems which can be addressed with such an extended force field is the very recent work by Colombi Ciacchi's group who developed a force field for the interaction of $\mathrm{H}_{2} \mathrm{O}$ on a partially deprotonated silica surface. ${ }^{285}$ This is particularly relevant as $\mathrm{SiOH}$ groups at the hydroxylated silica surfaces transform to Si$\mathrm{O}^{-}$in solution as a function of $\mathrm{pH}$ and ionic strength (see Section 3), a kind of situation very common when studying protein adsorption.

The classical force field are not fit for describing chemical reactions as the analytical expressions are not flexible enough to allow changes in the atomic connectivity during chemical reactions with the need of "on the fly update" of the corresponding parameters. Recently, a different approach has been suggested by van Duin and coworkers who developed ReaxFF for hydrocarbons, ${ }^{468}$ a reactive force field which was designed to model equilibrium structures as well as reaction intermediates and activated complexes in chemical reactions. The idea is to correlate bond lengths with bond orders and the latter with bond energies, following old insights from Linus Pauling's work. ${ }^{469}$ The same methodology was then extended to model silicon and silica materials. ${ }^{470}$ At moment, ReaxFF has been very successfully applied to study the reaction of water on a freshly cleaved silica surface ${ }^{471}$ (see also Table 3). While this approach is very powerful and promising, the development of new parameters is a rather cumbersome and a time consuming process as they have to be calibrated against results from quantum mechanical calculations on test systems.

Table 3. Most common classical force fields for modeling silica material.

\begin{tabular}{lclr}
\hline Potential & Year & Description & Ref. \\
\hline BMH & 1988 & Born-Mayer-Huggins 2-body potential plus 3-body terms & 472 \\
& & for O-Si-O and Si-O-Si. \\
TTAM & 1988 & 2-body potential derived from Hartree-Fock calculation & 473
\end{tabular}


on $\mathrm{SiO}_{2}$ model clusters.

BKS

SSLCT

HS

SS96

SS97

ReaxFF

GGMG
1990 2-body potential derived from Hartree-Fock calculations

on $\mathrm{H}_{4} \mathrm{SiO}_{4}$ and supplemented with experimental $\alpha$-quartz elastic constants.

1992 2- and 3-body shell-ion model potential for silica and Alsubstituted zeolites.

1994 Consistent force field from Hartree-Fock calculations on small silica clusters.

1996 Shell-ion model potential for silica and zeolites from Hartree-Fock calculations on small silica clusters.

1997 Shell-ion model potential for silica and zeolites from B3LYP calculations on small silica clusters.

2003 Reactive force field with DFT parametrization based on bond length/bond order and bond order/bond energy concepts.

2008 Shell-ion model potential for silica with a 3-body term for H-bonding from periodic B3LYP calculations on $\alpha$ quartz.

2008 Rigid water modified Guillot-Giussani model potential ${ }^{478}$ merged with specific $\mathrm{SiO}_{2}$ potential to allow bond breaking/making.

\section{Crystalline silica surfaces}

\subsection{Surface sites}

Crystalline silica surfaces are constructed by cutting a slab of a given thickness along a chosen crystallographic plane from the corresponding optimized bulk structure (see Section 4.1). Cutting a silica structure involves breaking, homolytically or heterolytically, highly covalent SiO bonds to form surface sites ${ }^{294}$ (see Figure 27), which are to a large extent responsible for the physico-chemical processes on silica surfaces. In the homolytic cleavage, the pair of electrons of the broken chemical bond is divided evenly between the $\mathrm{Si}$ and $\mathrm{O}$ atoms, leading to the formation of two radicals. The possible surface sites generated by homolytic cleavage of $\mathrm{Q}^{3}$ include the silyl $(\equiv \mathrm{Si})$ and siloxy $\left(\equiv \mathrm{Si}-\mathrm{O}^{\circ}\right)$ radicals. For $\mathrm{Q}^{2}$, this cleavage can lead to silanone $(>\mathrm{Si}=\mathrm{O})$, silylene $\left(>\mathrm{Si}{ }^{*}\right)$ and siladioxirane (cyclo- $\left.>\mathrm{Si}\left(\mathrm{O}_{2}\right)\right)$ sites. In the heterolytic cleavage, the pair of electrons is transferred to the more electronegative oxygen atom, leading to the formation of charged species. The cleavage of $\mathrm{Q}^{3}$ sites produces surfaces with silyl $\left(\equiv \mathrm{Si}^{+}\right)$cations and 
siloxy $\left(\equiv \mathrm{Si}-\mathrm{O}^{-}\right)$anions, whereas that of $\mathrm{Q}^{2}$ would lead to silanone sites, doubly charged silylene cations $\left(>\mathrm{Si}^{++}\right)$and deprotonated geminal diols $\left(>\mathrm{Si}\left(\mathrm{O}^{-}\right)_{2}\right)$.

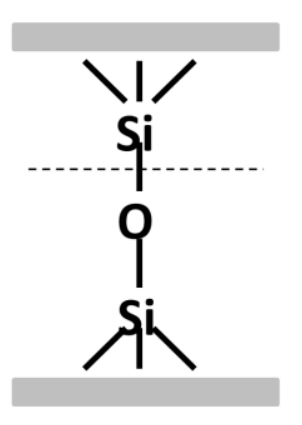

Siloxy radical

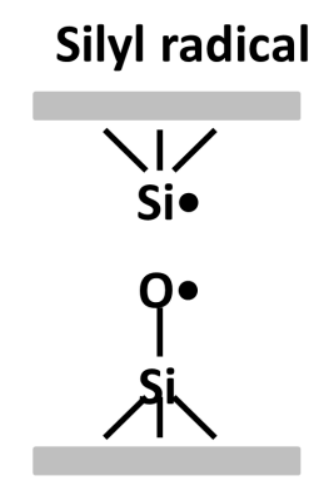

\section{Silyl cation}
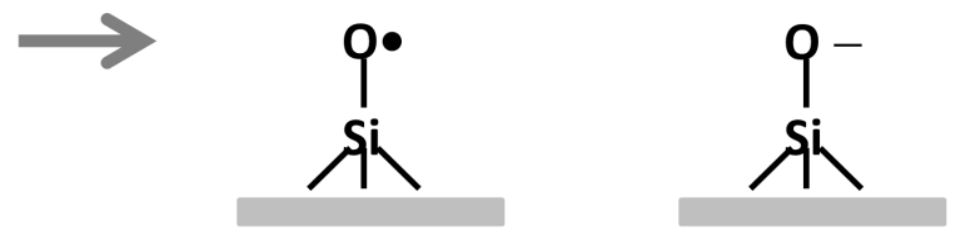

\section{Siloxy anion}

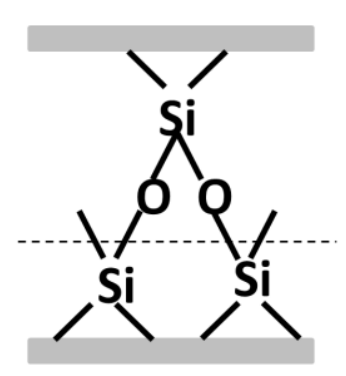

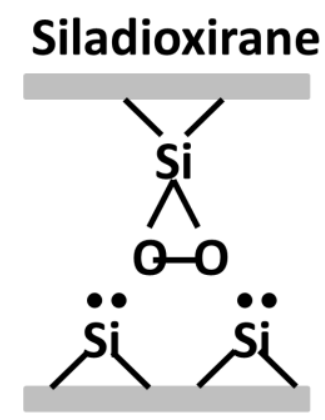

Silylene

\section{Geminal}

Silanolate

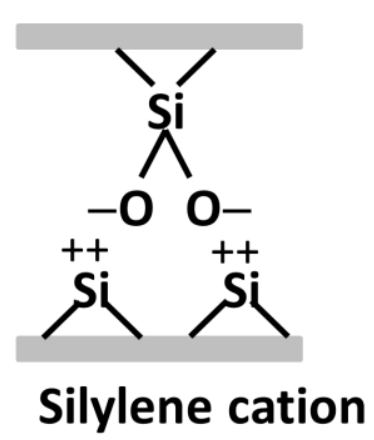

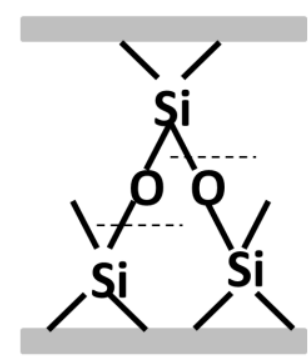

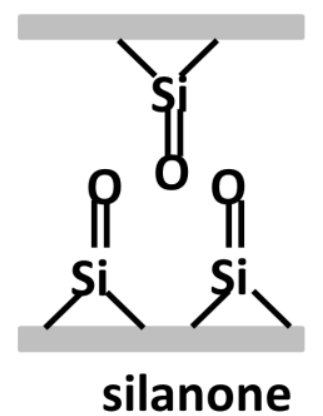

Figure 27. Silica surface sites derived from cleavage. Redraw after Ref. ${ }^{294}$

Silica sites have been investigated with ab initio cluster calculations at the B3LYP level of theory and its bonding patterns characterized by Natural Bond Order analysis. ${ }^{294}$ This work suggests that silanone sites and a pair of silyl/siloxy radicals, resulting from homolytic cleavage, 
are the most stable $\mathrm{Q}^{2}$ and $\mathrm{Q}^{3}$ sites, respectively, on a pristine silica surface. The heterolytic cleavage, entailing charge separation, is significantly less favorable than the homolytic one. ${ }^{294}$

Surface sites can be investigated by different experimental techniques. Radical sites such as the $\equiv_{\mathrm{Si}-\mathrm{O}^{\bullet}}$ centers, also called Non-Bridging Oxygen $(\mathrm{NBO})^{296,479,480}$ are characterized by photoluminescence ${ }^{481-487}$ and Electron Spin Resonance (ESR) spectroscopy. ${ }^{480,488-491}$ From a theoretical point of view, both siloxyl and silyl surface defective centers have been analyzed in different surfaces: edingtonite (100), quartz (0001) and cristobalite (111), using embedded cluster models and the B3LYP functional. ${ }^{394,395}$ DFT (BLYP, B3LYP and BHand HLYP) periodic calculations have also been carried out to analyze the siloxyl defect on three hydroxylated quartz surfaces, ((001), (100) and (011)), in cristobalite (101), and tridymite (001). ${ }^{345}$ For the siloxyl results show that the singly occupied orbital is mainly centered on one of the $2 p$ orbitals of the non-bridging oxygen ${ }^{345,394}$ and that theory is able to reproduce the essential EPR properties of these centers, ${ }^{345,395}$ particularly when using hybrid functionals. Moreover, the overall absorption spectra calculated with time dependent DFT are in qualitative agreement with the experimental data. ${ }^{395}$ On the other hand, results show that computed properties of siloxyl sites are almost independent from the surface structure, ${ }^{345,395}$ confirming the strong local nature of this defect. If embedded in an hydroxylated surface, the surface features that change the most with the presence of this radical defect are the surface H-bonds, which tend to become weaker. ${ }^{345}$ For silyl sites, the unpaired electron is also localized in a $s p^{3}$ orbital of the three coordinated silicon but, the EPR properties, particularly $\mathrm{a}_{\mathrm{iso}}$, are more sensitive to the surface structure, mostly to the $\mathrm{O}-\mathrm{Si}-\mathrm{O}$ angle and the $\mathrm{Si}-\mathrm{O}$ distance. ${ }^{395}$ Remarkably, calculations have also shown that these two well-known paramagnetic centers of silica surface are deep electron traps able to form stable negatively charged surface centers, ${ }^{394}$ which may have important consequences on their physical-chemical properties.

Silanone and siladioxirane surface sites, both diamagnetic, can be identified by optical ${ }^{492}$ and vibrational spectroscopies. ${ }^{296,297}$ Moreover, detailed analysis of the low-lying excitations in these surface sites can be obtained from high level ab initio quantum chemical methods, ${ }^{487,493}$ which can help identifying the bands on photoadsorption and photoluminiscence spectra. For instance, the computed values associated to the lowest fully allowed singlet $\rightarrow$ singlet vertical transitions, found at $\approx 3$ and $\approx 5 \mathrm{eV}$ for siladioxirane and at $\approx 5.7 \mathrm{eV}$ for silanone, are in good agreement with the experimental data $(\approx 3$ and $\approx 5 \mathrm{eV}$ for siladioxirane and $5.65 \mathrm{eV}$ for silanone), thereby providing support to the conclusion that the observed transitions in the optical spectra of the activated silica surface are due to silanone and siladioxirane sites. ${ }^{493}$ The photoluminescence properties of silanone and siladioxirane have also been investigated by quantum chemical methods, ${ }^{302,487}$ both surface sites constituting possible candidates for green- and red-light 
emitters. For silanone, the photoluminescence energies from the two lowest excited singlet states are calculated to appear at 2.3 and $1.6 \mathrm{eV}$, around 3.4-4.0 eV lower than the vertical excitation energies, due to a significant geometry relaxation in the excited states. Indeed, the $\mathrm{Si}=\mathrm{O}$ double bond is highly elongated in the excited state, being transformed into a single bond, which leads to a loss of planarity of the $>\mathrm{Si}=\mathrm{O}$ fragment. For siladioxirane, the excitation initiates the cleavage of the $\mathrm{O}-\mathrm{O}$ bond and radiative transitions contribute to photoluminescence in the ranges $2.1-2.3 \mathrm{eV}$ and $1.7-1.8 \mathrm{eV}$ ranges.

\subsection{Surface reconstruction}

Freshly cleaved surfaces contain under-coordinated (also called "coordinatively unsaturated") reactive sites. To investigate surface properties of fractured silica, models of lowindex unrelaxed surfaces of quartz were constructed and analyzed using periodic density

functional theory calculations. ${ }^{295,494,495}$ Surface energies, defined as $E_{\text {surf }}=\left(E_{\text {slab }}-n E_{\text {bulk }} / 2 A\right)$ with $E_{\text {slab }}$ being the energy of a pristine slab, $E_{\text {bulk }}$ the energy of $\mathrm{SiO}_{2}$ in the bulk, $n$ the number of $\mathrm{SiO}_{2}$ in the supercell and $2 A$ the total surface area (top and bottom), were computed both considering spin paired and spin polarized calculations to describe heterolytic and homolytic cleavage, respectively. Noticeably, in nine out of ten cases, the specific surface energy differences between results of spin-polarized and spin-paired calculations was less than $2 \% .{ }^{495}$ This is in contrast to cluster calculations, ${ }^{294}$ which suggest that the homolytic cleavage of a Si-O bond is energetically more favorable than the heterolytic one. The stabilization of surfaces with charged sites was attributed to intersite electrostatic interactions, ${ }^{295,494}$ which are not taken into account in cluster calculations. From this study it was suggested that cuts with a more uniform distribution of surface sites at the opposite sides of the cleavage plane were associated with lower surface energies. Moreover, cuts with a more uniform distribution of oxygen atoms among the ruptured geminal surface sites, favoring neutral sites over the charged ones, also exhibited lower surface energies. The calculated unrelaxed surface energies of quartz range from 0.161 to $0.200 \mathrm{eV} \AA^{-2}$ (2.6 to 3.2. $\left.\mathrm{J} \mathrm{m}^{-2}\right)^{295,495}$ these are high values, rendering these surfaces very reactive. As a result, they quickly reconstruct or, in the presence of water, become hydrolyzed leading to geminal $\mathrm{Q}^{2}>\mathrm{Si}(\mathrm{OH})_{2}$ and terminal $\mathrm{Q}^{3} \equiv \mathrm{Si}-\mathrm{OH}$ silanol groups.

Several computational studies have been carried out to characterize the major features of surface reconstruction on different silica surfaces. ${ }^{295,496,497}$ First principles DFT (LDA) molecular dynamics simulations for (0001) $\alpha$-quartz, ${ }^{497}$ indicate that the most favourable surface reconstruction presents an unexpected densification of the two uppermost layers of $\mathrm{SiO}_{2}$ tetrahedral units, with three- and six-membered rings, at $300 \mathrm{~K}$. Similar results are obtained with interatomic potential based calculations at $400 \mathrm{~K} .{ }^{496}$ During the reconstruction of the surface, the 
initial under-coordinated silicon and oxygen atoms of the cleaved surface recover full coordination, forming six-membered rings (S6R) parallel to the surface and three-membered rings extending into the bulk (see Figure 28).

a)

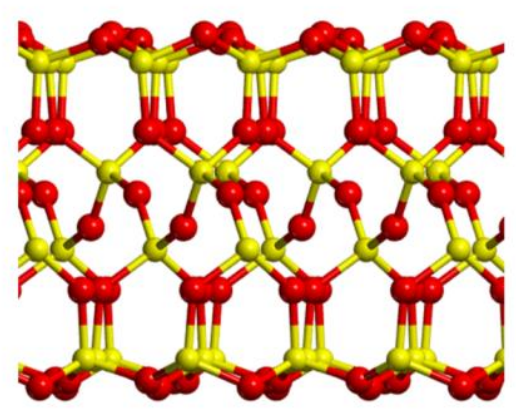

b)

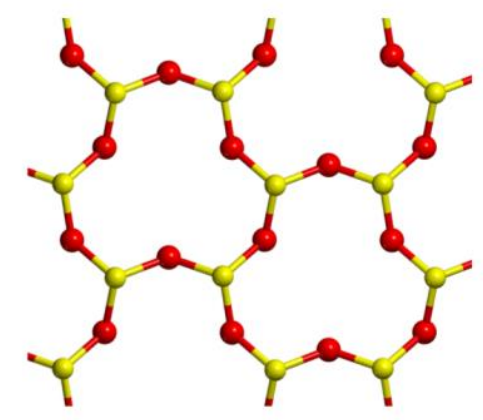

Figure 28. Most stable reconstruction of (001) $\alpha$-quartz. a) side view b) top view. Drawn with data from Ref. ${ }^{391}$

Reconstruction of most common pristine surfaces (101), (102), (112) (100) (111) (110) and (001) of $\alpha$-quartz, was also investigated with periodic DFT(GGA) and plane wave calculations. ${ }^{295}$ Four types of reconstruction were found: a) formation of strained two-membered silicon rings $(\mathrm{S} 2 \mathrm{R})$ from silyl and siloxy sites, b) formation of $(\mathrm{S} 2 \mathrm{R})_{2}{ }^{+}$structures with two side sharing S2R, c) formation of three membered silicon rings (S3R) and d) transformation of silanone sites into siloxane sites. Energies of cleaved unrelaxed surfaces range from 0.161 to $0.200 \mathrm{eV} \AA^{-2}$ (2.6 to $3.2 . \mathrm{J} \mathrm{m}^{-2}$ ), ${ }^{295,495}$ whereas those associated to reconstructed surfaces range from 0.071 to $0.142 \mathrm{eV}^{-2}\left(1.1\right.$ to $\left.2.3 \mathrm{~J} \mathrm{~m}^{-2}\right)$. Relaxation energies are determined by the kind of reconstruction, the formation of $\mathrm{S} 2 \mathrm{R}$ to saturate the valencies of undercoordinated oxygens exhibiting larger values than relaxation of silanone sites. Relaxation energies computed in Ref. ${ }^{295}$ however, are smaller and thus, reconstructed surface energies are higher, than those reported in other studies, ${ }^{391,497}$ due to the fact that in the former reference relaxation was limited to a single silica tetrahedral layer, while in fact it can extend as deep as six $\mathrm{SiO}_{2}$ molecular layers. ${ }^{497}$ Full relaxation, however, can lead to an overstabilization of the slab. Indeed, a recent study on the (001) $\alpha$-quartz surface has revealed the importance of the slab thickness on the reconstructed surface energy, showing that a layer slab made of six $\left[\mathrm{SiO}_{4}\right]$ groups is needed to achieve converged values. $^{391}$

DFT(LDA) molecular dynamics simulations have also been performed recently on the (011) surface of $\alpha$-quartz at room-temperature. ${ }^{498}$ Reconstruction leads to the formation of a fused seven member ring through the reaction of two adjacent $\mathrm{O}^{\circ}$ radicals, followed by linking one of the reactive $\mathrm{O}$ with a nearby silicon. Remarkably, in the newly formed structures some of 
the silicon atoms exhibit a pentacoordinated environment, usually distorted between pure trigonal bipyramid and square pyramid geometries. Moreover, in a few cases, oxygen atoms also became tricoordinated (see Figure 29).

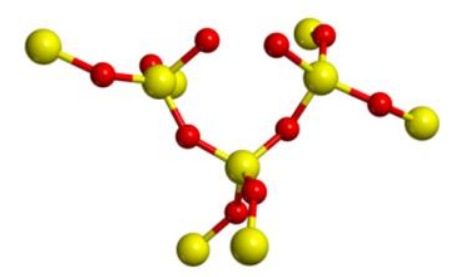

$160 \mathrm{fs}$

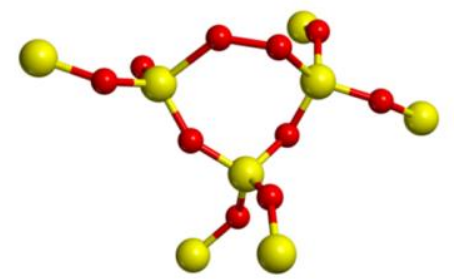

$210 \mathrm{fs}$

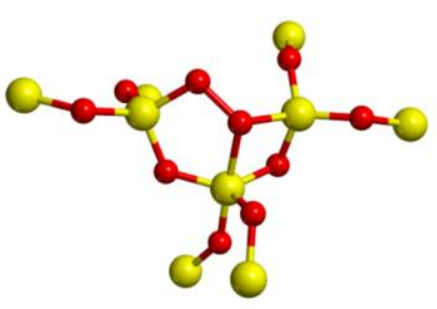

$850 \mathrm{fs}$

Figure 29. Process occurred during reconstruction of the (011) surface of $\alpha$-quartz. Drawn with data from Ref. ${ }^{498}$

\subsection{Hydroxylated surfaces}

Fractured silica is very hydrophilic so that exposure to water results in the hydrolysis of the surface sites with the formation of silanol groups. In principle, hydrolysis can lead to single silanol sites $\left(\mathrm{Q}^{3}(\mathrm{OH})\right)$, to silanediols, i.e., geminal silanols $\left(\mathrm{Q}^{2}(\mathrm{OH})_{2}\right)$, and to silanetriol centers $\left(\mathrm{Q}^{1}(\mathrm{OH})_{3}\right)$. The latter ones, however, are thermally unstable and have not been detected in NMR studies as already mentioned. Previous calculations for (001) $\alpha$-quartz have shown that the cleaved surface reacts exothermically with water until it becomes fully hydroxylated. ${ }^{497,499,500}$ When a water molecule approaches the surface, its oxygen atom binds to an under-coordinated $\mathrm{Si}$ atom, while one of its hydrogen atoms is transferred to a neighboring non-bridging oxygen, leading to the formation of two silanols. (see Figure 30). ${ }^{500}$ Car-Parrinello ab initio molecular dynamics (CP-MD) simulations for the $\alpha$-quartz-water interface at $1000 \mathrm{~K}$ and $0.3 \mathrm{GPa}$ show that dissociation of $\mathrm{H}_{2} \mathrm{O}$ molecules into $\mathrm{H}^{+}$and $\mathrm{OH}^{-}$at the Si-terminated surface takes place on a very short time scale of about 2 ps. ${ }^{501}$ On the other hand, calculations on cluster models ${ }^{502}$ suggest that the energy barrier of this process is only $12-13 \mathrm{~kJ} \mathrm{~mol}^{-1}$ and thus, that it will readily proceed under ambient conditions of temperature and humidity. As the surface becomes hydrated, hydrogen bond chains start appearing. The fully hydroxylated (001) $\alpha$-quartz exhibits zig-zag hydrogen bond networks with alternating strong and weak hydrogen bonds. Remarkably, the dense (reconstructed) surface, characterized by 3-membered and 6 membered-rings with siloxane bonds at the top, ${ }^{497}$ is shown to be hydrophobic, suggesting that surfaces formed by non-strained siloxane bonds are essentially hydrophobic. ${ }^{503}$ 


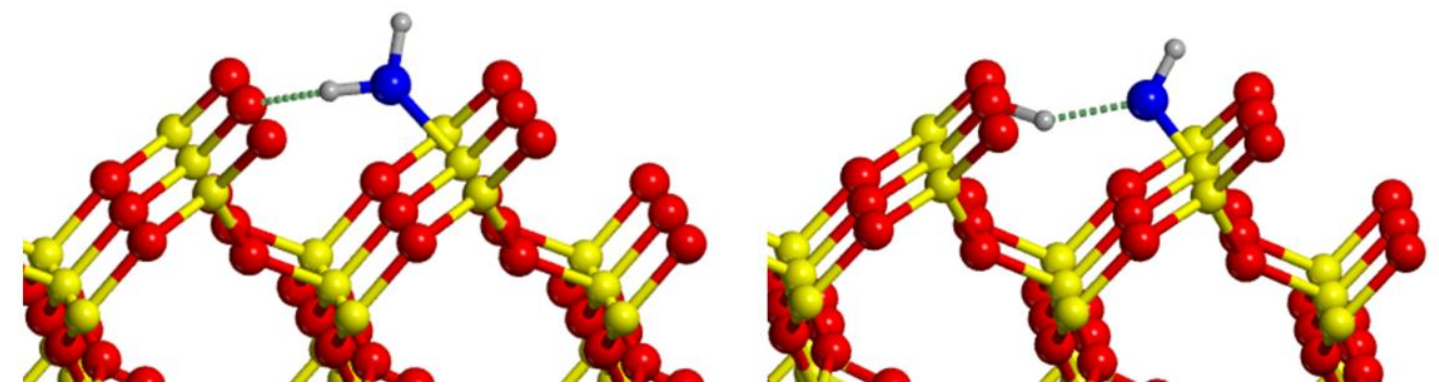

Figure 30. Hydration mechanism for the cleaved (0001) $\alpha$-quartz. Drawn with data from Ref. ${ }^{500}$

The hydrogen bonding features in the hydroxylated surfaces of quartz, ${ }^{295,306,391,499,500,504}$ cristobalite, ${ }^{306,315,492,505,506}$ tridymite $^{306}$ and amorphous silica ${ }^{507-509}$ have been analyzed in several studies with periodic DFT calculations. Among them, the study of Musso et al. ${ }^{306}$ provides a very systematic study of many different hyroxylated surfaces of quartz, cristobalite and tridymite polymorphs. In particular, five surfaces were considered for quartz, $Q(001), Q(100), Q(010)$, $\mathrm{Q}(011)$ and $\mathrm{Q}(101)$, four for cristobalite, $\mathrm{C}(001), \mathrm{C}(100), \mathrm{C}(101)$ and $\mathrm{C}(110)$ and three for tridymite, $\mathrm{T}(001), \mathrm{T}(100)$ and $\mathrm{T}(110)$. Since hydrolysis of freshly cut surfaces proceeds very quickly in the presence of water vapor, surfaces were generated by cutting a slab of a given thickness along the chosen crystallographic plane from the corresponding optimized bulk structure and saturating the unfilled valencies with $\mathrm{H}$ and $\mathrm{OH}$. This leads to different kinds of silanols (vicinal, geminal or interacting, see Section 2.2) and $\mathrm{OH}$ densities depending on the polymorph and crystallographic plane. The thickness of the slab was carefully chosen to avoid an excessive deformation as a result of considering a surface that is too thin. This is important since the slab thickness determines its rigidity and thus, it can largely influence surface H-bonds. ${ }^{306,391}$ The distribution and pattern of $\mathrm{OH}$ groups as well as the $\mathrm{H}$-bond features of each surface are displayed in Figure 31. 

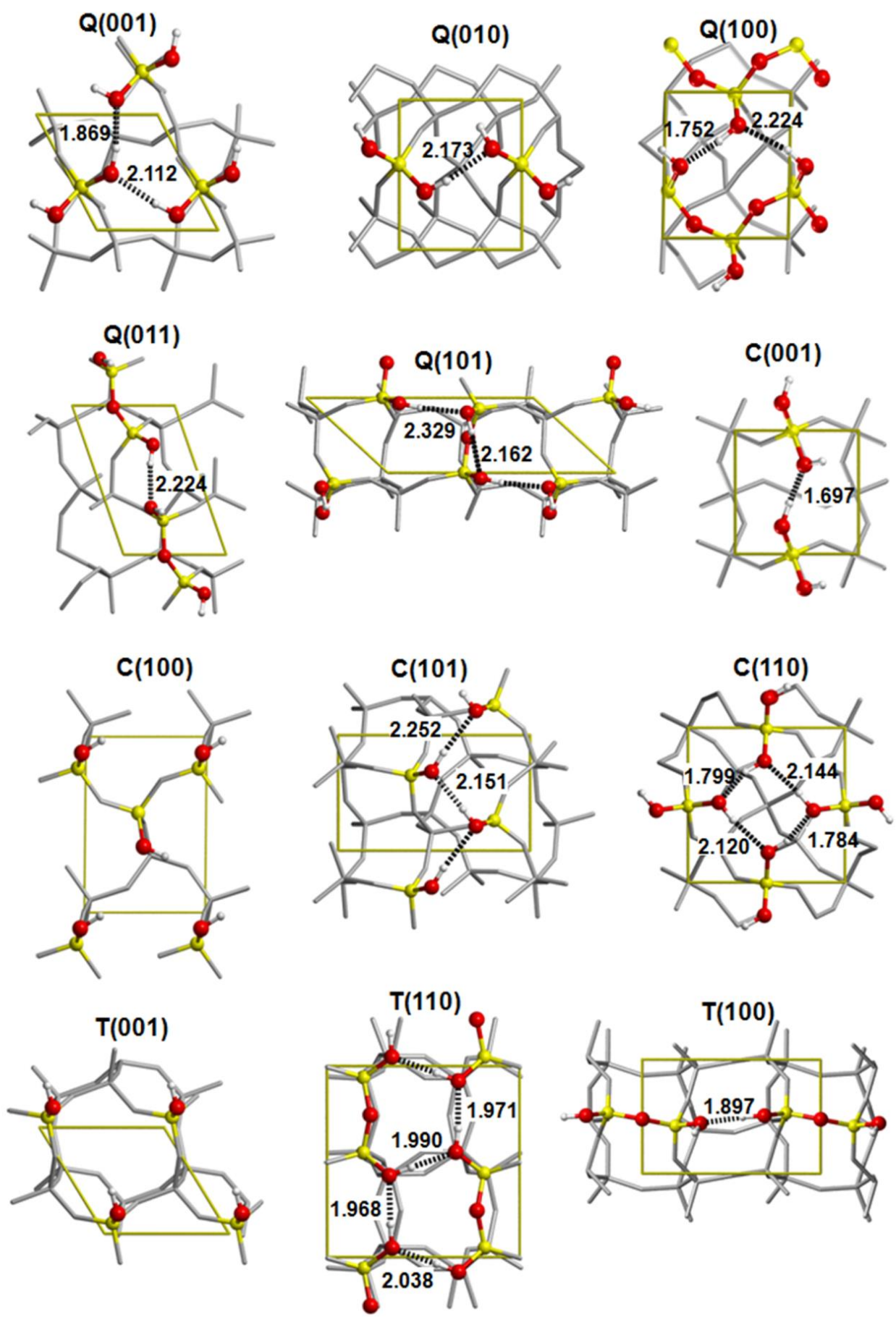

Figure 31. B3LYP optimized structures of fully hydroxylated $\alpha$-quartz, $\alpha$-cristobalite, and $\alpha$ tridymite surfaces (top faces representation). Hydrogen bond distances $(\mathrm{H} \cdots \mathrm{O})$ in $\AA$ as dotted lines. Reprinted with permission from Ref. ${ }^{306}$. Copyright 2009 American Chemical Society.

Optimized structures show different H-bond patterns depending on the distribution of the hydroxyl groups on the surface. $\mathrm{C}(100)$ and $\mathrm{T}(001)$ models, with the highest slab rigidity and the latter with the smallest $\mathrm{OH}$ density as well, only show isolated surface silanols. Q(010), Q(011), $\mathrm{C}(001)$ and $\mathrm{T}(100)$ surfaces exhibit a single H-bond interaction inside the crystalline cell, 
whereas $\mathrm{C}(110)$ shows four silanols interacting through a four-membered H-bond ring. The remaining surfaces, $\mathrm{Q}(001), \mathrm{Q}(100), \mathrm{Q}(101), \mathrm{C}(110), \mathrm{C}(101)$ and $\mathrm{T}(110)$ present infinitely extended H-bond chains.

In order to evaluate the stability of each slab model, the surface energy, was computed according to the following reaction $\mathrm{aB}_{\mathrm{SiO}_{2}}+\mathrm{bH}_{2} \mathrm{O} \rightarrow c \mathrm{~S}_{\mathrm{SiOH}}$, where $\mathrm{B}_{\mathrm{SiO} 2}$ refers to the bulk unit cell and $S_{S i O H}$ to the slab unit cell. The formation energy per unit cell and the surface energy were defined as $\Delta E_{\text {cell }}=c E_{\text {slab }}^{\text {cell }}-\left(a E_{\text {bulk }}^{\text {cell }}+b E_{\mathrm{H}_{2} \mathrm{O}}\right)$ and $\Delta E_{\text {surf }}=\Delta E_{\text {cell }} / 2 A$, respectively, where $A$ is the surface unit cell area. Computed values for each surface, as well as structural data, are given in Table 4. It can be observed that in almost all cases surface energies are negative, resulting in a thermodynamically favored surface formation. Exceptions are observed for $\mathrm{T}(001)$ and $\mathrm{C}(100)$ for which no surface H-bonds are present, thereby suggesting that H-bonding between surface silanols is the key factor that drives the surface energy. Indeed, the surface energy is the result of three different factors: i) the rupture of Si-O-Si bonds of the bulk followed by the formation of surface silanols; ii) the structural relaxation of the interior of the slab and iii) the formation of H-bonds between the surface silanols. Process i) is common to all considered surfaces and can be treated as a fixed contribution per Si-OH group to the surface energy. Its local covalent nature should lead to a near independence of the corresponding value on the considered slab. Processes ii) and iii) are intermingled; however, the fact that the bathochromic shift of the $\mathrm{OH}$ donor stretching frequency, which can be considered a measure of the H-bond strength, exhibits an inverse correlation with the surface energy ${ }^{306}$ (see Figure 32) suggests that $\mathrm{H}$-bonding is probably the most representative contribution to the differences observed.

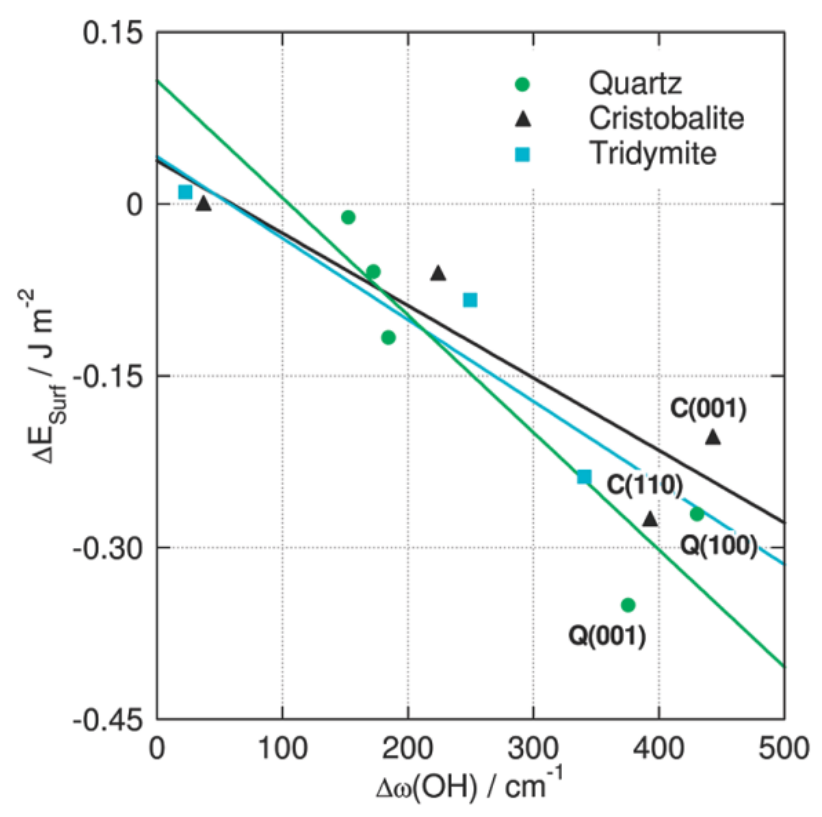


Figure 32. Surface energies $\Delta \mathrm{E}_{\text {Surf }}$ vs the largest bathochromic harmonic $\mathrm{OH}$ frequency shift $\Delta \omega(\mathrm{OH})$ calculated for all polymorph surfaces with respect to the value of $3904 \mathrm{~cm}^{-1}$ for the isolated $\mathrm{OH}$ groups of the $\mathrm{T}(001)$ surface. Reprinted with permission from Ref. ${ }^{306}$. Copyright 2009 American Chemical Society.

Overall, hydroxylated silica surfaces are characterized by different types of $\mathrm{OH}$ groups (geminal, vicinals and isolated) and by different $\mathrm{H}$-bonding patterns that result from the interaction between hydroxyl groups. The H-bond pattern strongly depends on the disposition and on the density of the $\mathrm{OH}$ groups at the surface. Although the majority of the crystalline models exhibit infinitely connected $\mathrm{H}$-bond chains, small local $\mathrm{H}$-bonded rings or isolated pairs through completely free $\mathrm{OH}$ groups have also been found, depending on the $\mathrm{OH}$ density and the rigidity of the silica framework. 
Table 4. Structural data for quartz $(\mathbf{Q})$, cristobalite $(\mathbf{C})$ and tridymite $(\mathbf{T})$ slabs. Cell parameters $(\mathbf{a}, \mathbf{b}, \boldsymbol{\gamma})$, cell area $(\mathbf{A})$, slab thickness $(\tau),\langle$ Si-O $>$ and $\langle\mathrm{O}-\mathrm{H}\rangle$ bonds, the surface $\mathrm{OH}$ density $(\rho)$ and surface energy $\left.\Delta \mathrm{E}_{\text {surf }}\right)$

\begin{tabular}{|c|c|c|c|c|c|c|c|c|c|c|c|c|}
\hline & $Q(001)$ & $Q(010)$ & $Q(100)$ & $Q(011)$ & $Q(101)$ & $\mathbf{C}(\mathbf{0 0 1})$ & $\mathrm{C}(\mathbf{1 0 0})$ & $\mathrm{C}(101)$ & $\mathrm{C}(110)$ & $\mathbf{T}(001)$ & $\mathbf{T}(\mathbf{1 0 0})$ & $\mathbf{T}(\mathbf{1 1 0})$ \\
\hline $\mathbf{b}(\AA)$ & 4.963 & 5.565 & 5.361 & 7.290 & 4.983 & 5.002 & 6.873 & 5.014 & 6.733 & 5.076 & 7.923 & 7.951 \\
\hline$\gamma\left({ }^{\circ}\right)$ & 119.1 & 90.0 & 90.0 & 110.1 & 137.3 & 90.0 & 89.5 & 90.0 & 90.0 & 121.5 & 89.9 & 90.1 \\
\hline$A\left(\AA^{2}\right)$ & 21.1 & 26.9 & 26.7 & 34.3 & 34.5 & 24.9 & 34.5 & 41.4 & 46.2 & 22.0 & 40.3 & 63.9 \\
\hline$<$ Si-O $>(\AA)$ & 1.633 & 1.635 & 1.632 & 1.636 & 1.636 & 1.631 & 1.631 & 1.633 & 1.637 & 1.629 & 1.630 & 1.632 \\
\hline$<\mathrm{O}-\mathrm{H}>(\AA)$ & 0.980 & 0.970 & 0.980 & 0.968 & 0.972 & 0.973 & 0.965 & 0.974 & 0.979 & 0.964 & 0.971 & 0.979 \\
\hline$\rho\left(\mathrm{OH} / \mathbf{n m}^{2}\right)$ & 9.5 & 7.4 & 7.5 & 5.8 & 5.8 & 8.0 & 5.8 & 4.8 & 8.7 & 4.5 & 5.0 & 6.3 \\
\hline$\Delta \mathbf{E}_{\text {surf }}\left(\mathbf{J ~ m}^{-2}\right)$ & -0.35 & -0.12 & -0.27 & -0.01 & -0.06 & -0.21 & 0.00 & -0.06 & -0.28 & 0.01 & -0.08 & -0.24 \\
\hline
\end{tabular}




\section{Amorphous silica surfaces}

As we have seen in Section 4, the most common way to deal with silica simulations is to adopt the periodic boundary condition method. Still, the problem of modeling the surface of amorphous silica is an open one, as no reference to experimental structural data can be made due to the lack of information about the fine details of the bulk and even more seriously of the surface itself. As we have already mentioned, a proper combination of different hydroxylated surfaces derived from crystalline silica can be used to mimic the complexity of the amorphous silica surface. In this section we only focus on models of amorphous silica surface exclusively derived from a true amorphous bulk within the PBC and which have been characterized in detail in terms of their surface physico-chemical features. We are also referring here to surfaces which have healed their main structural and electronic defects (see Section 2), i.e. surfaces only exhibiting Si-O-Si bridges and $\mathrm{Si}-\mathrm{OH}$ groups, the main functionalities present in water environment. We have singled out two models obtained using quantum mechanical methods based on DFT: the first one from Tielens et $\mathrm{al}^{510}$ and the second one from Ugliengo et al, ${ }^{509}$ respectively. 


\subsection{The Tielens model}

This model derives from an amorphous silica bulk originally proposed by Garofalini ${ }^{464}$ who adopted molecular dynamics based on a specifically $\mathrm{SiO}_{2}$ derived empirical potential for the simulation (see Section 5.6.3). The usual procedure based on PBC, as described in Section 4, was adopted by the authors who carefully avoided unstable defects at the surface by transforming threecoordinated $\mathrm{Si}$ atoms and non-bridging oxygen atoms, derived from the geometrical cut of the $\mathrm{SiO}_{2}$ bulk in $\mathrm{SiOH}$ groups, by manual addition of $\mathrm{H}_{2} \mathrm{O}$ molecules. To be able to adopt DFT based methods the starting slab model was about $10 \AA$ thick, with an in plane cell size of $13 \AA$ x $17.5 \AA$, which resulted in $\mathrm{Si}_{26} \mathrm{O}_{65} \mathrm{H}_{27}$ composition (118 atoms as a whole). Ab initio molecular dynamics based on plane waves basis set was carried out at $400 \mathrm{~K}$ using PBE functional at $\Gamma$ point for 5 ps. Spontaneous dehydroxylation was observed, resulting in the formation of small silica rings. Subsequent optimization at $0 \mathrm{~K}$ with higher accuracy in the number of $\mathrm{k}$ points and the kinetic cutoff was carried out. Results shows that the surface is very thin (about three-layer thick) with thickness varying between 5 and $8 \AA$, respectively (see Figure 33 for details).
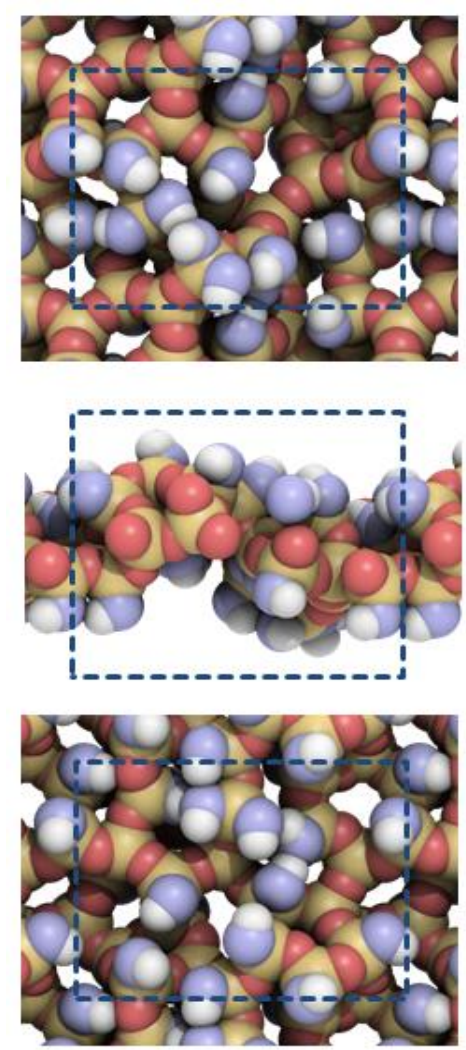

Figure 33. The top, lateral and bottom views of the Tielens model. Oxygen atoms of the surface $\mathrm{OH}$ groups are colored in blue. Molecular graphics rendered by Qutemol. ${ }^{73}$

The film thickness is not uniform and at the thinnest point the upper side is separated by the lower one by just one HO-Si-O-Si-OH link. The full set of harmonic frequencies have been computed as well as the NMR shielding constants by means of the GIPAW ${ }^{511}$ approach which ensures good 
quality of the results. Ring nuclearity peaked around 4, 5 and 6-membered rings, with 7, 8, 9 and 10-membered rings being only a minor feature. Interestingly, the model does not show either twoor three-membered rings, the former being unstable with respect to reaction with $\mathrm{H}_{2} \mathrm{O}$. The Si-O-Si angle distribution is very broad, covering a 130-180 degrees range, without a distinct maximum, in agreement with the high pliability of this bond (see Section 2 on this point). The $\mathrm{OH}$ density of 5.8 $\mathrm{OH} / \mathrm{nm}^{2}$ is the same for the upper and the lower face and it is higher than the value of 4.5/4.9 $\mathrm{OH} / \mathrm{nm}^{2}$ reported by Zhuravlev. ${ }^{78}$ A relatively high population of geminal silanols has been found, with an uneven distribution between the two surfaces (23\% and $46 \%$ for the upper/lower face, respectively). Due to the high $\mathrm{OH}$ density, the population of $\mathrm{H}$-bonded $\mathrm{SiOH}$ groups is more than $1 / 3$ of the total $(38 \%$ and $31 \%$ for the upper/lower face, respectively). Table 5 shows the distribution of the various kinds of $\mathrm{SiOH}$ groups. The absolute deprotonation energy of the $\mathrm{OH}$ groups has been computed, showing a higher acidity of the $\mathrm{Q}^{2}$ sites compared to the $\mathrm{Q}^{3}$ ones, resulting in a theoretical difference of 2.5 units of $\mathrm{pK}_{\mathrm{a}}$ in favour of the geminal sites. The NMR $\delta\left({ }^{29} \mathrm{Si}\right)$ chemical shift has been found in nice linear correlation with the Si-O-Si angle for values belonging to the three $\mathrm{Q}^{2}, \mathrm{Q}^{3}$ and $\mathrm{Q}^{4}$ families in agreement with experimental data. ${ }^{512,513}$ In light of the relevance of the $\mathrm{OH}$ vibrational features for silica surfaces (see Section 2 and 3), the ab initio harmonic $\mathrm{OH}$ stretching frequency values have been computed and shown to linearly correlate with the $\mathrm{OH}$ bond length. Calculations report a rather high difference between the two internal $\mathrm{OH}$ stretching frequency values for a $\mathrm{Q}^{2}$ site, about $36 \mathrm{~cm}^{-1}$, large enough to be distinguishable experimentally. This is in disagreement with both experimental evidences which shows that the $\mathrm{OH}$ frequency values for $\mathrm{Q}^{2}$ and $\mathrm{Q}^{3}$ sites are almost indistinguishable (Ref. ${ }^{118}$ and Section 3.1.4) and quantum mechanical calculations on the (001) hydroxylated surfaces of edingtonite for which the B3LYP (scaled) harmonic frequencies were 3737,3733 and $3735 \mathrm{~cm}^{-1}$ for the $\mathrm{Q}^{3}$ and $\mathrm{Q}^{2}$ sites, respectively. The discrepancy between the amorphous model and the one derived from a crystalline bulk is probably due to the high $\mathrm{OH}$ density of the amorphous model which forces almost all $\mathrm{SiOH}$ groups to "feel" their neighboring groups with a consequent perturbation of the $\mathrm{OH}$ stretching frequency, known to be very sensitive to local electric fields. In light of this result, it has to be established whether the acidity difference between the $Q^{3}$ and $Q^{2}$ sites is not the consequence of different intermolecular interactions rather than being an intrinsic electronic effect of the $Q^{2}$ moiety (see also Section 10 on this question).

In conclusion, the Tielens model, due to the addressed limitations, should be used with some caution to mimic the behavior of a fully hydroxylated amorphous silica surface $\left(4.5 / 4.9 \mathrm{OH} / \mathrm{nm}^{2}\right)$. The number of atoms still allows the adoption of normal computational resources to run plane waves based DFT calculations. Its main weakness is the exceedingly small thickness, which may render the model too flexible when adopted to simulate adsorption phenomena. This model has 
been used to study the adsorption of water and glycine and these studies are reviewed in sections 8 and 9 , respectively.

\subsection{The Ugliengo Model}

This model was derived starting from a bulk cristobalite with 192 atoms in the unit cell. Classical empirical partial charge rigid ion model potential ${ }^{514}$ has been used to run molecular dynamics at very high $\mathrm{T}$ and then cooled down to room temperature. This process ensured an amorphization of the starting material without introducing defects (two-membered rings, $>\mathrm{Si}=\mathrm{O}$ and others) in the final structure. The bulk was then cut along one of the cell axes and the surface unfilled valencies (above/below) were automatically saturated by an algorithm encoded in the graphical program MOLDRAW. ${ }^{515}$ Basically, a double-bonded Si is transformed into a $\mathrm{Q}^{2}$ site by attaching two $\mathrm{OH}$ groups, whereas a single-bonded $\mathrm{Si}$ is removed, leaving the only bonded oxygen atoms singly-bonded to the underneath slab. This is to avoid the creation of $\mathrm{Q}^{1}$ sites (by attaching three $\mathrm{OH}$ groups to the singly-bonded $\mathrm{Si}$ atom) as $\mathrm{Q}^{1}$ are not found at the silica surface (see Sections 3.1.4 and 3.1.5). A triple-bonded Si atom is transformed into a $\mathrm{Q}^{3}$ site by attaching a single $\mathrm{OH}$ group. All singly-bonded oxygen atoms are transformed into $\mathrm{OH}$ groups. As these transformation are operated in a geometrical fashion they do not require any quantum mechanical calculations. The healed structure showed a silanol density of $7.2 \mathrm{OH} / \mathrm{nm}^{2}$, typical of values for a sol-gel amorphous silica, was $14.5 \AA$ thick and consisted of 222 atoms. It was fully relaxed (internal and cell size) at B3LYP/6-31G(d,p) level using a massive parallel version of CRYSTAL06 code. ${ }^{291,516}$ The final structure is shown in Figure 34. 


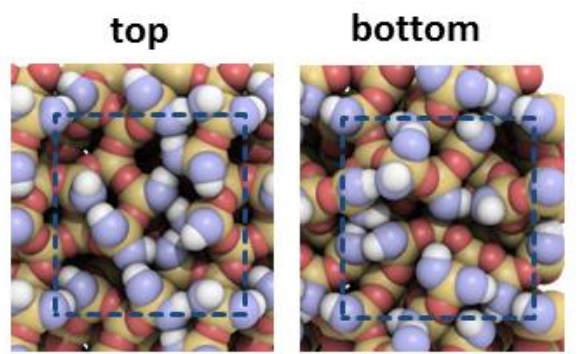

$7.2 \mathrm{OH} / \mathrm{nm}^{2}$

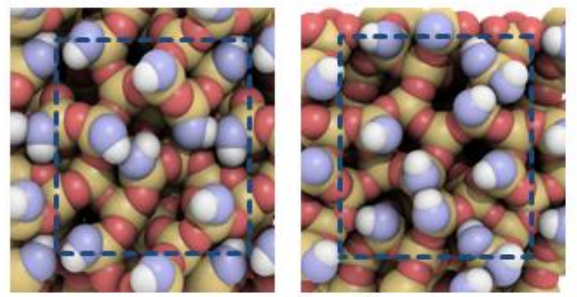

$4.5 \mathrm{OH} / \mathrm{nm}^{2}$

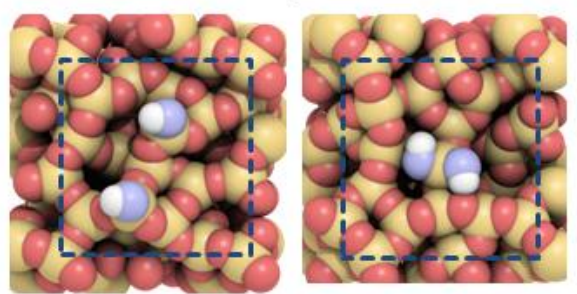

$1.5 \mathrm{OH} / \mathrm{nm}^{2}$

Figure 34. Top and bottom views of the Ugliengo model with variable $\mathrm{OH}$ densities. Oxygen atoms of the surface $\mathrm{OH}$ groups are colored in blue. Unit cell as dashed lines. Molecular graphics rendering by Qutemol. ${ }^{73}$

The number of geminal silanols amounts to $29 \%$ of the total $\mathrm{OH}$ population, not too far from the value for the Tielens model. Using this structure as a starting model, the dehydration process occurring by outgassing the silica at high $\mathrm{T}$ was simulated by geometrically removing one water molecule from the closest $\mathrm{OH}^{\cdots} \mathrm{OH}$ pairs and making a new Si-O-Si silanol bridge. The new resulting structure was fully relaxed and this process was repeated in order to get $\mathrm{OH}$ densities of 5.4, 4.5, 2.4 and $1.5 \mathrm{OH} / \mathrm{nm}^{2}$, respectively. This simulates different stages of the dehydroxylation process. Three representative structures are shown in Figure 34 and the corresponding data on the $\mathrm{SiOH}$ populations are reported in Table 5 together with the data for the Tielens model. 
Table 5. Properties of the Ugliengo amorphous silica surfaces. ${ }^{509}$ The column labeled as $\mathrm{OH}$ (geminals) reports the total number of $\mathrm{OH}$ groups (for the upper/lower surfaces) and, in parentheses, the number of geminal $\mathrm{SiOH}$ groups.

\begin{tabular}{cccccc}
\hline$\rho\left[\mathrm{OH} \mathrm{nm}^{-2}\right]$ & $\mathrm{OH}($ geminals $)$ & \multicolumn{2}{c}{$\mathrm{H}^{\text {-bond contacts }}{ }^{\mathrm{a}}$} & Free $\mathrm{OH}$ & $\%$ Free $\mathrm{OH}$ \\
\hline 7.2 & $28(8)$ & $\mathrm{OH}^{\cdots} \mathrm{OH}$ & $\mathrm{OH}^{\cdots} \mathrm{OSi}_{2}$ & & \\
$5.8^{\mathrm{b}}$ & $24(6)$ & 16 & 1 & 4 & 14 \\
5.4 & $20(3)$ & 7 & 1 & 4 & 17 \\
4.5 & $16(1)$ & 3 & 1 & 7 & 35 \\
2.4 & $8(1)$ & 2 & 3 & 7 & 43 \\
1.5 & $4(1)$ & 0 & 0 & 4 & 50 \\
\hline
\end{tabular}

${ }^{a}$ H-bond cutoff: $2.15 \AA .{ }^{b}$ Data for the Tielens surface. ${ }^{510}$

Data of Table 5 shows that the number of $\mathrm{OH}^{\cdots} \mathrm{OH} \mathrm{H}$-bonds decreases dramatically with the drop in $\mathrm{OH}$ density. For the most representative, $4.5 \mathrm{OH} / \mathrm{nm}^{2}$ case, it corresponds to $19 \%$ of the total $\mathrm{OH}$ groups. Interestingly, the same percentage resulted for the $\mathrm{H}$-bonds between $\mathrm{OH}$ and the siloxane $\mathrm{Si}-\mathrm{O}-\mathrm{Si}$ bridge. This is somehow surprising as the $\mathrm{Si}-\mathrm{O}-\mathrm{Si}$ is a very weak Lewis base. In the present case the distorsion introduced in the structure in the simulated dehydroxylation process brings about $\mathrm{Si}-\mathrm{O}-\mathrm{Si}$ angles much smaller than the normal $145^{\circ}$ value (see also Section 2 for the flexibility of the Si-O-Si bond) thereby increasing the associated basicity. B3LYP calculations of the dehydroxylation processes that bring the $7.2 \mathrm{OH} / \mathrm{nm}^{2}$ towards the $1.5 \mathrm{OH} / \mathrm{nm}^{2}$ model confirm the expected endothermic nature of the process as schematically shown in Figure 35. 


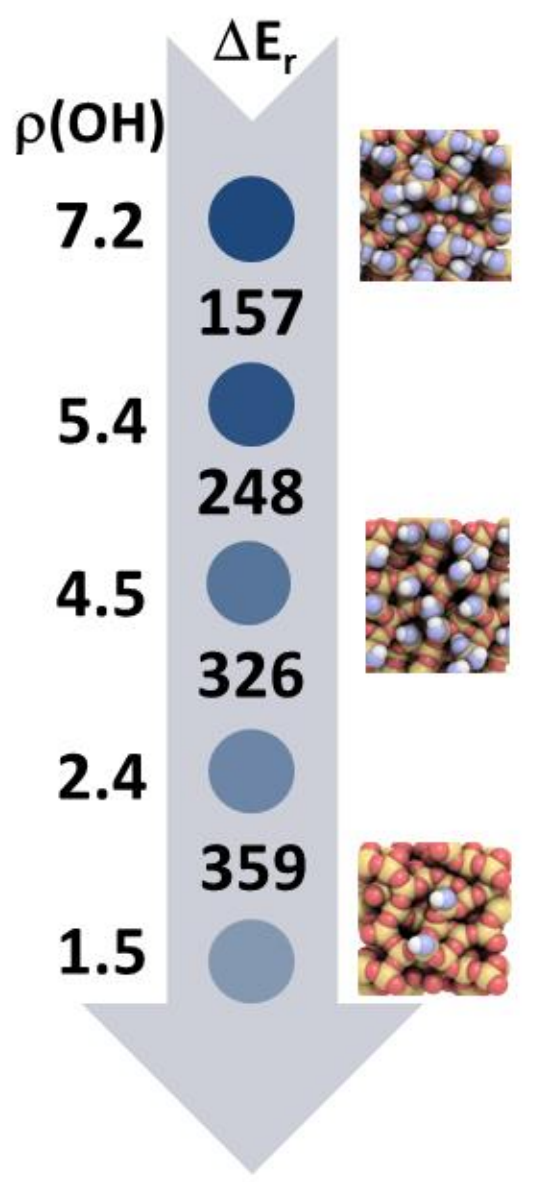

Figure 35. Left of the arrow: silica surface $\mathrm{OH}$ density $\rho\left(\mathrm{OH} / \mathrm{nm}^{2}\right)$; Within the arrow: dehydration reaction energies $(\mathrm{kJ} / \mathrm{mol})$ for reducing the $\mathrm{OH}$ density; Right of the arrow: cartoon of the silica surface for $\rho\left(\mathrm{OH} / \mathrm{nm}^{2}\right)=7.2,4.5$ and 1.5, respectively. Oxygen atoms of the surface $\mathrm{OH}$ groups are depicted in blue. Molecular graphics rendering by Qutemol. ${ }^{73}$

The values also confirm that the endothermicity dramatically increases with the degree of dehydroxylation up to $359 \mathrm{~kJ} / \mathrm{mol}$ to reach the $1.5 \mathrm{OH} / \mathrm{nm}^{2}$ case. The infrared spectrum in the $\mathrm{OH}$ region for a hypothetical surface exhibiting $4.5 \mathrm{OH} / \mathrm{nm}^{2}$ is given in Figure 36 (see original work ${ }^{509}$ for technical details). In order to account for the non-uniform $\mathrm{OH}$ density of a real silica surface, the spectral contributions from each of the considered models have been merged, with weights reflecting the expected experimental populations, obtaining a very good agreement with the experimental spectrum recorded for an Aerosil 300 from Degussa outgassed at $450 \mathrm{~K}$ in the $\mathrm{OH}$ stretching region (see Figure 36). 


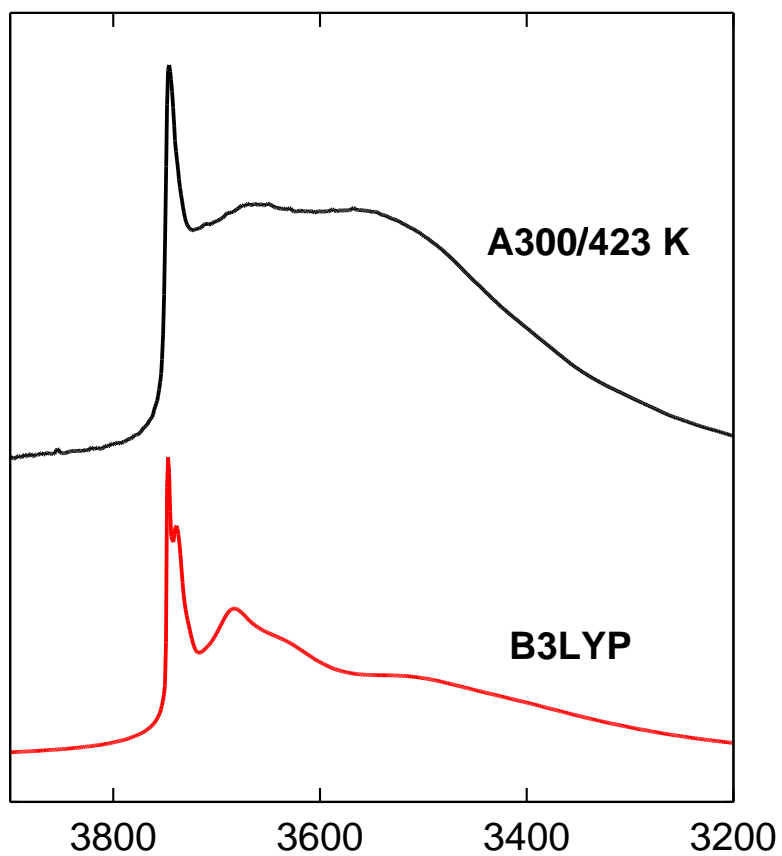

Figure 36. Top: experimental infrared spectrum of silica Aerosil 300 outgassed at 423K. Bottom: simulated B3LYP infrared spectrum for the Ugliengo models. The range of wavenumbers (units in $\mathrm{cm}^{-1}$ ) covers the $\mathrm{OH}$ stretching region.

The $1.5 \mathrm{OH} / \mathrm{nm}^{2}$ Ugliengo model (albeit with some reduction in the slab thickness to decrease the computational burden) has been recently chosen to simulate the energetic and vibrational features of adsorbed ammonia and compared with experimental results. ${ }^{169}$ The same approach was adopted to define a thinner, $4.5 \mathrm{OH} / \mathrm{nm}^{2}$ surface, and to study the interaction of aspirin and ibuprofen on both surfaces. ${ }^{517}$

\subsection{Other models}

A number of authors have provided models for the amorphous silica surface. Since many of them were focusing on the surface defects, we will not discuss all of them in details. Table 6 collects a brief summary of the considered models together with the methods for their simulations. 
Table 6. Different methods and target for the simulation of amorphous silica surfaces. S2R refers to the two-membered $(\mathrm{SiO})_{2}$ ring, $\mathrm{Si}(3)$ to the three-coordinated Silicon, NBO to the non-bridging oxygen of silanone.

\begin{tabular}{|c|c|c|}
\hline Method & Studied systems & Ref. \\
\hline $\begin{array}{l}\text { MD with an effective three-body } \\
\text { potential specific for } \mathrm{SiO}_{2}\end{array}$ & $\begin{array}{l}\text { Bulk silica and surfaces without water reconstruction. } \\
\text { Detection of S2R only at the surface. NBO defects } \\
\text { coherent with the experimental SiOH density. }\end{array}$ & \\
\hline $\begin{array}{l}\text { MD with modified TTAM } \\
\text { potential }{ }^{473} \text { for } \mathrm{SiO}_{2} \text { and } \mathrm{SPC}^{519} \\
\text { for } \mathrm{H}_{2} \mathrm{O}\end{array}$ & $\begin{array}{l}\text { Simulation of a fully hydrophobic silica surface. The MD } \\
\text { protocol was tweaked in order to minimize the formation } \\
\text { of } \mathrm{S} 2 \mathrm{R}, \mathrm{Si}(3) \text { and } \mathrm{NBO} \text {. Hydrophobicity was defined } \\
\text { with respect to the heat of liquefaction of } \mathrm{H}_{2} \mathrm{O} \text {. A silica } \\
\text { surface free from } \mathrm{S} 2 \mathrm{R}, \mathrm{Si}(3) \text { and } \mathrm{NBO} \text { was found } \\
\text { hydrophobic. }\end{array}$ & 520 \\
\hline
\end{tabular}

MD with van Beest-Kramer-van Santen $\left(\mathrm{BKS}^{457}\right)$ empirical Silica clusters $(14,19,27 \AA)$ and film $(29 \AA$ thick). potential

BLYP CPMD $^{442}$ on BKS $^{457}$ generated surface Comparison between MD BKS $\mathrm{BS}^{457}$ and BLYP CPMD ${ }^{442}$

MD with Born-Mayer-Huggins empirical potential ${ }^{523}$

MD with Buckingham, Coulomb and Morse, three-body term to $\mathrm{Si}$ $\mathrm{O}-\mathrm{H}$ BLYP CPMD $^{442}$ on $\mathrm{BKS}^{457}$ generated periodic $\mathrm{SiO}_{2}$ surfaces. MD with Cerius ${ }^{2}$ GLASSFF_2.01 and GLASSFF_1.01 ${ }^{526}$ force fields to silica and CHARMM ${ }^{527}$ developed parameters for water interaction.

MD with an extension of $\mathrm{BKS}^{457}$ and $\mathrm{SPC} / \mathrm{E}^{529}$ force fields to silica/ $\mathrm{H}_{2} \mathrm{O}$ interactions

MD with modified StillingerWeber potential ${ }^{530}$ for $\mathrm{Si}$ and Vashishta et al. ${ }^{531}$ for $\mathrm{SiO}_{2}$

MD with a water modified Guillot-Giussani model potential $^{478}$ merged with specific $\mathrm{SiO}_{2}$ potential to allow bond breaking/making.

MD with $\mathrm{SiO}_{2}$ reactive force field, SERIAL-REAX ${ }^{470}$

MD using specific $\mathrm{SiO}_{2} / \mathrm{H}_{2} \mathrm{O}$ force field $^{532,533}$.

$\mathrm{MD}$, ab initio and refinement of the Cole et $\mathrm{al}^{307}$ potential for $\mathrm{SiO}_{2} / \mathrm{H}_{2} \mathrm{O}$ system.
Simulation of very large $\mathrm{SiO}_{2}$ surface unit cells and their reaction with $\mathrm{H}_{2} \mathrm{O}$. Spontaneous reconstruction by $\mathrm{H}_{2} \mathrm{O}$ reaction in 250 ps.

Simulation of water adsorbed in Vicor glass nanopores with fully hydroxylated internal walls. No bulk $\mathrm{H}_{2} \mathrm{O}$ features for 1-4 nm pores. parameters as a function of the adopted method. CPMD disfavors the S2R formation over BKS

Silica surface without $\mathrm{SiOH}$ groups rich in $\mathrm{NBO}$ and $\mathrm{Si}(3)$. Electrostatic potential maps of silica surface to establish reactivity with $\mathrm{H}_{2} \mathrm{O}$.

Silica surface with $\mathrm{NBO}, \mathrm{Si}(3)$ and $\mathrm{S} 2 \mathrm{R}$ reacted with $\mathrm{H}_{2} \mathrm{O}$. Concentration of $4.5 \mathrm{OH} / \mathrm{nm}^{2}$. Surface reactivity: separated $\mathrm{NBO}$ and $\mathrm{Si}(3)>\mathrm{NBO}+\mathrm{Si}(3)>\mathrm{S} 2 \mathrm{R}>\mathrm{S} 3 \mathrm{R}$.

Exothermic reaction of $\mathrm{H}_{2} \mathrm{O}$ with $\mathrm{S} 2 \mathrm{R}$ at the silica surface.

Silica slabs and pores produced by annealing an amorphous bulk. Care to avoid $\mathrm{NBO}, \mathrm{Si}(3)$ and $\mathrm{S} 2 \mathrm{R}$ at the surface and to convert them to $\mathrm{SiOH}$ groups. $\mathrm{SiO}^{-}$also included in the simulation. Study of the wettability as a function of surface hydroxylation.

Hydrated and hydroxylated silica surfaces. Ab initio parametrization of the force field. Hydrophilic and density. Heat of immersion in good agreement with experiment.

Oxidized $\mathrm{Si}$ surfaces compared to full amorphous $\mathrm{SiO}_{2}$ model. Heat of immersion and water structuring higher for the oxidized $\mathrm{Si}$ surfaces than for the amorphous $\mathrm{SiO}_{2}$. Atomistic mechanism to the healing of $\mathrm{Si}(3)$ and $\mathrm{NBO}$. Silica surface models with $3.8 \mathrm{OH} / \mathrm{nm}^{2}$. Proton transfer allows penetration of water up to $7 \AA$ within the surface. treat $\mathrm{Si}-\mathrm{O}^{-} / \mathrm{H}_{2} \mathrm{O}$ interaction.
Study of the distribution of rings and geometrical hydrophobic patches resulted as a function of $\mathrm{SiOH}$ 


\subsection{The S2R surface defect}

As it was discussed in Sections 2 and 3, the defects remaining at the silica surface are, in principle, three-coordinated $\mathrm{Si}(3)$, non-bridging oxygen $\mathrm{NBO},>\mathrm{Si}=\mathrm{O}$ and strained rings $\mathrm{S} 2 \mathrm{R}$, or $(\mathrm{SiO})_{2}$. At ambient condition, due to the presence of moisture, the $\mathrm{Si}(3)$ and NBO defects can hardly survive the reaction with $\mathrm{H}_{2} \mathrm{O}$ (see also references in Table 6). The $\mathrm{S} 2 \mathrm{R}$ is an exception, because it naturally results from the vicinal sites condensation during the thermal treatment of silica at $\mathrm{T}>900$ K. As already reported in Section 2, 3.1.4 and 3.1.7, the S2R concentration is rather small (in the initial works ${ }^{137,535,536}$ it was reported to be around $0.1 \mathrm{~S} 2 \mathrm{R} / \mathrm{nm}^{2}$, although more recent data after severe activation steps suggests that it can reach up to $0.5 \mathrm{~S} 2 \mathrm{R} / \mathrm{nm}^{2}$ ). Interestingly, the formation of S2R surface defects is quite general as they have also been identified by their IR fingerprint in crystalline all-silica silicalite. ${ }^{363} \mathrm{~S} 2 \mathrm{R}$ defects are very reactive towards molecules with hydrogen attached to electronegative elements like oxygen and nitrogen (Section 3.1.7): $\mathrm{H}_{2} \mathrm{O}, \mathrm{MeOH}$ and $\mathrm{NH}_{3}$ react very fast with $\mathrm{S} 2 \mathrm{R}$ defects, forming new surface functionalities, i.e. two $\mathrm{SiOH}$ groups, $\mathrm{SiOH}$ and $\mathrm{Si}-\mathrm{O}-\mathrm{Me}$ and $\mathrm{SiOH}$ and $\mathrm{SiNH}_{2}$, respectively. Due to their relevance, a number of studies have been especially aimed at modeling S2R and they are summarized in Table 7.

Table 7. Different methods to model the reactivity of $\mathrm{S} 2 \mathrm{R}$ defects towards $\mathrm{H}_{2} \mathrm{O}$ and glycine. $\Delta_{\mathrm{r}} \mathrm{E}$ and $\Delta_{\mathrm{a}} \mathrm{E}$ are the reaction and activation energies for the opening of the $\mathrm{S} 2 \mathrm{R}$ defect, respectively. Units in $\mathrm{kJ} / \mathrm{mol}$.

\begin{tabular}{|c|c|c|c|}
\hline Model and methods & $\Delta_{\mathrm{r}} \mathrm{E}$ & $\Delta_{\mathrm{a}} \mathrm{E}$ & Ref. \\
\hline $\begin{array}{l}\text { BLYP/DZP on } \mathrm{H}_{2}(\mathrm{SiO})_{2} \mathrm{H}_{2} \text { as a cluster model of the } \\
\text { S2R defect. S2R IR prediction of D2 modes }\end{array}$ & -134 & 27 & 537 \\
\hline $\begin{array}{l}\text { BLYP } \beta \text {-cristobalite (111) reconstructed surface with } \\
\text { periodic formation of S2R defects. Amorphous silica } \\
\text { model from } B K S^{457} \text { simulation to simulate isolated } \\
\text { S2R defects. }\end{array}$ & -127 & l & 506 \\
\hline $\begin{array}{l}\text { ONIOM2[B3LYP/6-31G*:UHF/STO-3G] on clusters } \\
\text { containing the } \mathrm{S} 2 \mathrm{R} \text { defect cut out from } \mathrm{SiO}_{2} \text { silica } \\
\text { models from MD with polarizable ion model potential } \\
\text { based on extension of WAC potential }{ }^{538} \text {. }\end{array}$ & / & $\begin{array}{c}68 \\
\text { (gas-phase } \\
\text { molecule) } \\
107 \\
\text { (embedded in the } \\
\text { silica matrix) }\end{array}$ & 502,539 \\
\hline $\begin{array}{l}\text { BLYP CPMD } \\
\text { surfaces. }\end{array}$ & -168 & 34 & 507 \\
\hline PBE/plane waves mechanically embedded & / & 40 & 540 \\
\hline $\begin{array}{l}\text { MD with Buckingham, Coulomb and Morse, three- } \\
\text { body term to Si-O-H. Periodic surface models. }\end{array}$ & -458 & & 466 \\
\hline $\begin{array}{l}\text { ONIOM2[B3LYP/6-31+G(d,p):MNDO] on } \\
\mathrm{Si}_{10} \mathrm{O}_{16} \mathrm{H}_{12}(\mathrm{SiO})_{2} \text { cluster model. IR prediction of } \mathrm{S} 2 \mathrm{R} \\
\text { modes. }\end{array}$ & -122 & 82 & 75 \\
\hline $\begin{array}{l}\text { B3LYP/6-31+G(d,p)//ONIOM2[B3LYP/6- } \\
\text { 31+G(d,p):MNDO] on } \mathrm{Si}_{10} \mathrm{O}_{16} \mathrm{H}_{12}(\mathrm{SiO})_{2} \text { cluster model. } \\
\text { Interaction and reactivity with glycine. }\end{array}$ & -109 & 0.5 & 541 \\
\hline
\end{tabular}


The majority of these studies focused on the calculation of the reaction of $\mathrm{S} 2 \mathrm{R}$ with $\mathrm{H}_{2} \mathrm{O}$, for which both the reaction energy $\Delta_{\mathrm{r}} \mathrm{E}$ and the energy barrier $\Delta_{\mathrm{a}} \mathrm{E}$ have been computed. Data from Table 7 reveal that there is no general consensus on the values associated to the two quantities. In our opinion there are two main reasons for that: i) different procedures to define the amorphous silica surface; ii) different computational methods. The first issue is responsible for a variable degree of geometrical tension on the S2R defect, which does not only depend on the internal Si-O-Si angles (which are close to 90 degrees) but also on the distortion of $\left[\mathrm{SiO}_{4}\right]$ tetrahedra surrounding the $\mathrm{S} 2 \mathrm{R}$ defect. This, in turn, is determined by the model adopted (cluster or periodic, see Section 4) and by the procedure to arrive to the S2R defect starting from a given bulk silica model (see Section 4). For instance, adoption of small clusters like in Ref. ${ }^{75,537}$ decreases the tension outside the S2R zone as no constraints can be enforced as the rest of the surface is missing. Some further geometrical constraints imposed on the larger cluster adopted by Rimola et al. ${ }^{75}$ showed their relevance to the computed value of the $\Delta_{\mathrm{a}} \mathrm{E}$. When $\mathrm{PBC}$ are adopted to arrive at a dehydroxylated $\mathrm{SiO}_{2}$ surface, the heat-and-cool MD procedure to arrive at the S2R defect also matters as it is difficult to ensure that the internal geometrical tension has been properly equilibrated. Reproducibility of the calculations can also be critical. The second issue is even more critical as the level of adopted theory has a deep influence on both $\Delta_{\mathrm{r}} \mathrm{E}$ and $\Delta_{\mathrm{a}} \mathrm{E}$. Even considering DFT as more reliable than empirical force fields, the adopted functional is very important. For instance, data from Table 7 reveal that functionals based on Perdew exchange like PBE and PW91 tend to underestimate the value of $-\Delta_{\mathrm{a}} \mathrm{E}$ as compared to MP2 and to Becke based functional like B3LYP and BLYP ones. In summary, what is lacking is a systematic investigation of both aspects in order to provide a more robust picture of this important class of reactions.

The interaction between S2R defects and glycine has been modeled by Rimola et al. ${ }^{541}$ since it has been argued that they may be present at the surface of the silica grains which are the core of the interstellar dust particles. This means that glycine, eventually formed under prebiotic conditions within the icy mantle surrounding the core grain, can react with the S2R defect, thus remaining firmly attached to the grain dust. As comets are the results of accretion of dust grains this can be one mechanism to seed the primordial Earth with glycine by comets bombardment. Rimola et al. ${ }^{541}$ also showed that glycine can then be released from the grain by the action of at least four water molecules at room temperature.

\section{Adsorption of water on silica surfaces}

The interaction of silica with water occurs in a great number of situations, either natural, as in geochemistry, prebiotic chemistry or biomineralization, because water and silica are the two most 
common terrestrial compounds, or derived from human activity, as in the fields of sensors, biomedical devices or chromatography. Silicon is spontaneously covered with a $\mathrm{SiO}_{2}$ layer, so that $\mathrm{SiO}_{2}$-water interactions also occur on electronic devices. Water adsorption on hydrophilic surfaces induces large differences in the adhesion of species in solution at the nanoscale. Indeed, at the local scale, water organization (or lack of organization) at the surface has a major influence on the adsorption mode of species in solution. At a larger scale, it can provide surface and dissolved species charge screening in the form of a dielectric. ${ }^{542}$

Before entering into the details of the main contributions, it is worth mentioning the major experimental data that sheds light on the influence of the hard matter (silica) on the soft one, even at short length scales ( $1 \mathrm{~nm}$ or less): Shen et $\mathrm{al}^{543}$ reported that water at the interface with Q(0001) organizes in an ice-like structure in a certain $\mathrm{pH}$ range (Figure $37 \mathrm{a}$ ). At the opposite, Engemann et $\mathrm{al}^{544}$ evidenced that amorphous silica induces a liquid-like layer at the silica-ice interface (Figure $37 b)$. 

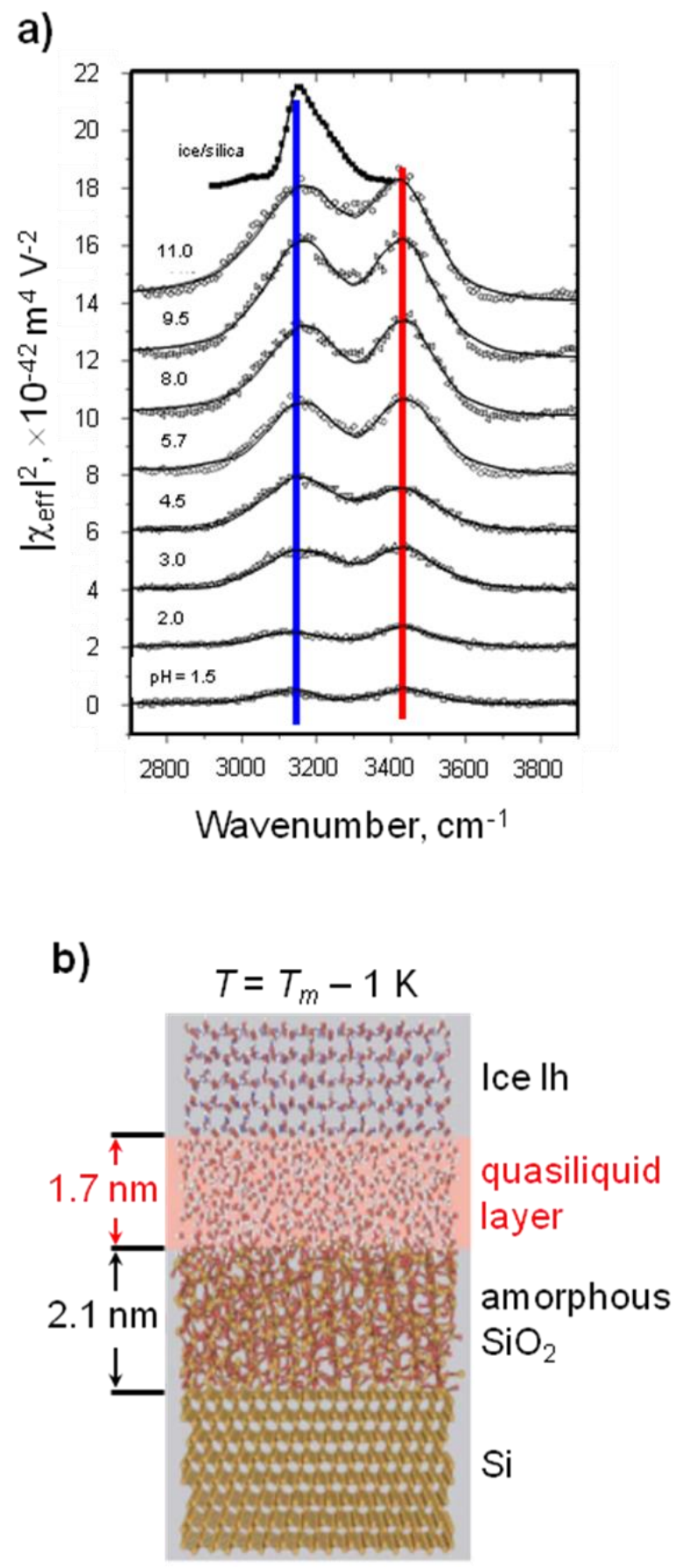

Figure 37. a) SF vibrational spectra of water/ $\alpha$-quartz (001) at various bulk $\mathrm{pH}$ values. The ice/fused-silica spectrum is shown for comparison; adapted with permission from Ref ${ }^{543}$ Copyright 2004 Elsevier. b) Real space model of the ice- $\mathrm{SiO}_{2}$ interface associated with $T=T_{m}-1 \mathrm{~K} . T_{m}=$ melting temperature of ice. The densities used in this representation correspond to the values deduced from the measurement. Reprinted with permission from Ref. ${ }^{544}$ (Engemann, S.; Reichert, H.; Dosch, H.; Bilgram, J.; Honkimaki, V.; Snigirev, A. Phys. Rev. Lett. 2004, 92, 205701). Copyright 2004 American Physical Society.

However, experiments do not -or not yet- give information on the local H-bond breaking/making between silanols and water. Theoretical works are needed to disentangle those local aspects and help interpreting spectroscopic data. 
Here we focus on the influence of the hydroxylation structure in the energetics and structure of adsorbed water, and on the interplay between the preexisting $\mathrm{H}$-bond network between silanol groups and the formation of a new network between silanols and water. In this chapter, only fully hydroxylated surfaces are considered in interaction with water. Theoretical works on the reaction of water with defects like S2R that are known to be healed in the presence of water ${ }^{507,75,140}$ are not considered (with the exception of the $\equiv \mathrm{SiO} \bullet$ species, which is a possible responsible of crystalline silicas toxicity), neither are works focusing on the first steps of silica dissolution (see refs. ${ }^{501,545-549}$ for a non-exhaustive list) that can occur for instance on rough surfaces. ${ }^{547,550,551}$ Complex interface processes such as dissolution/precipitation and polymerisation reactions in solution ${ }^{552,553}$ are not discussed here. In addition, whereas crystalline or amorphous plane surfaces are reviewed, confined spaces in nano- or mesoporous silicas (see eg Ref. ${ }^{534}$ ) are not treated.

The bulk of the literature available may be partitioned into several categories that are illustrated in Figure 38 and detailed in Tables 8-12. Two axes have been considered: they correspond to increasing complexity of the silica model, and increasing amount of water adsorbed, respectively. The zones of intensive research have been the clusters/isolated water/high level of theory, the crystalline and amorphous silica/water/classical molecular dynamics, and crystalline silica/isolated to bulk water/ab initio. One may note two facts: i) there are only two systems that have been covered integrally with ab initio methods from the isolated water molecule to bulk liquid interface, $\mathrm{Q}(001)$ and $\mathrm{C}(100)$, and ii) the amorphous silica/liquid water interface has not yet been investigated by means of ab initio techniques. Surprisingly, the reactivity of thin $\mathrm{SiO}_{2}$ films on $\mathrm{Si}$ with water (Section 3.1.8) has not been a subject of investigation, with a single exception. ${ }^{307}$

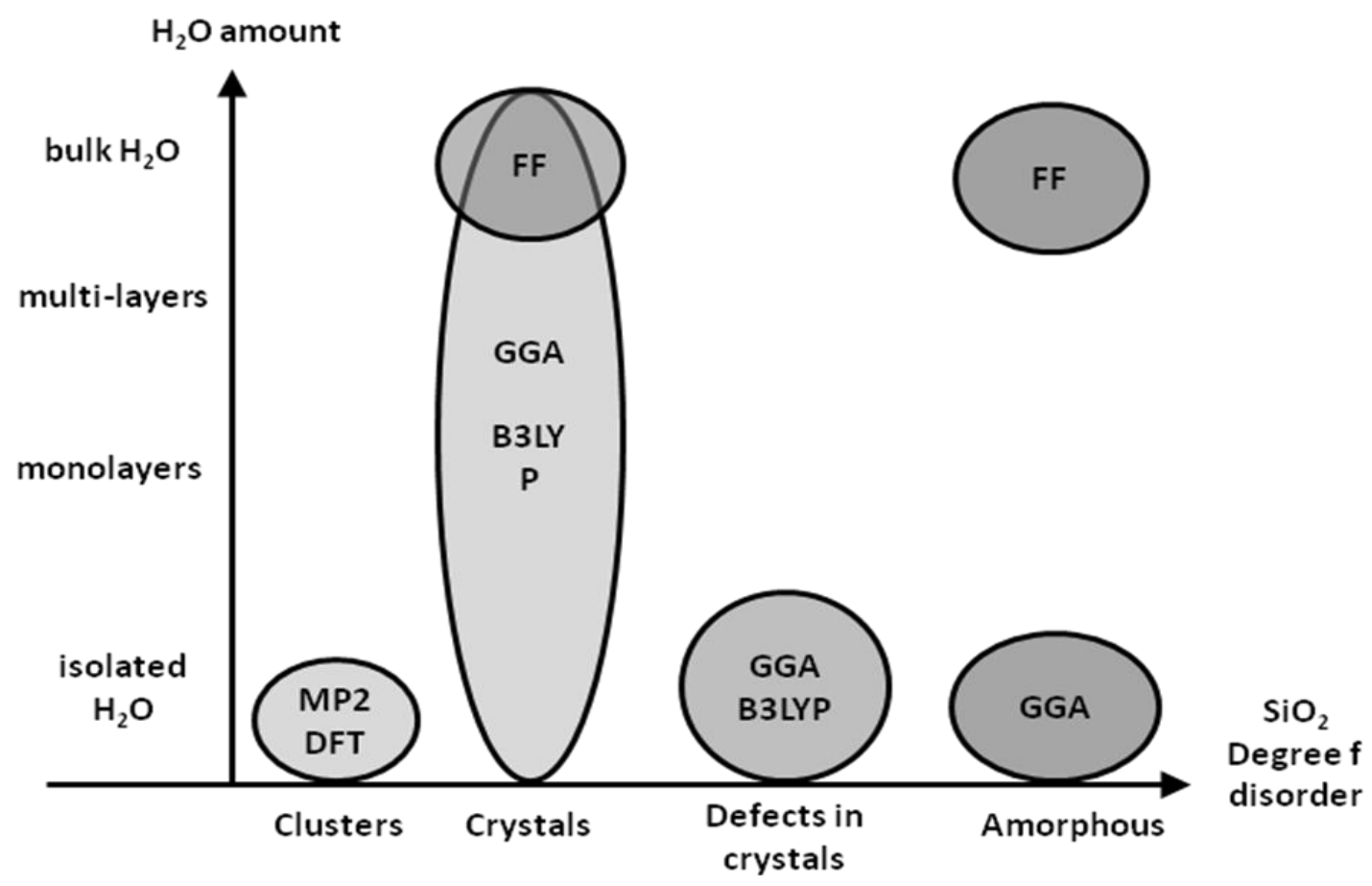

Figure 38. Main theoretical studies performed on the silica/water system. 
A strong evolution of the research topics and methods with time is noticed: many works have been performed in the last years of the XXth century using cluster calculations at a high level of theory. Indeed, the first attempts to study water interactions with silanols were performed using cluster calculations. Since 2000, more and more works have been devoted to crystalline surfaces with ab initio periodic calculations; meanwhile, force fields have been developed to mimic silica (crystalline and amorphous) properties. In the last few years, significant breakthroughs in the understanding of crystalline and amorphous silica water interactions, with discrete molecules and with bulk water, have been achieved with DFT based methods.

For obvious reasons, several periodic ab initio works have been devoted to the interaction of crystalline surfaces, mainly quartz surfaces, with water. In contrast, because several methods have been developed to generate bulk amorphous silica with force fields, works on amorphous silica surface are more numerous with classical methods.

$\mathrm{Ab}$ initio works on crystalline silica surfaces have been reviewed by Yang et Wang in $2006 .{ }^{554}$ Since then, a significant number of important studies based on electronic structure methods have appeared, including the comparison of several crystalline polymorphs and terminations, new crystalline surfaces analysis, new ab initio based amorphous silica models and the properties of silica-water interface such as pKa calculations (see Section 3.1.2).

\subsection{The early story: first principles cluster based calculations}

In the early days, water-silanol interactions were studied using a cluster approach. ${ }^{555}$ These cluster calculations were reviewed ${ }^{39}$ in 1994 as mentioned in Section 4.2 and continued by some authors until the end of the 90's and early $2000 .{ }^{223,294,338-340,556,557}$ Here we do not aim to perform an exhaustive presentation of the work done, but to describe the average trends. Generally the quantitative data (adsorption energies) are compared to experimental calorimetric data ${ }^{8,82,173,342,558-}$ 563 and gas chromatography. ${ }^{87}$ The measured heat of water adsorption has been reported around $60 \mathrm{~kJ} / \mathrm{mol}$, but heterogeneity of hydrophilic patches caused adsorption enthalpies ranking from -90 to $-44 \mathrm{~kJ} / \mathrm{mol}$. The calculated frequencies were also compared to vibrational spectroscopic data. ${ }^{564}$ As a general rule, water interacts with silanols more strongly as a H-bond acceptor than as a H-bond donor, due to the rather low basicity of surface $\mathrm{OH}$ and Si-O-Si groups. Most studies focus on two types of silanols: isolated ${ }^{565}$ and geminals, ${ }^{566}$ as the differences between these two types is easily modeled through cluster calculations. In particular, the reactivity of geminal silanols was addressed to understand the cytotoxic reactivity of quartz, a surface which exhibits a high density of geminal silanols. ${ }^{567}$ 
The configurations obtained for the adsorption of a single water molecule on different types of silanols with cluster calculations are reported in Figure 39. The isolated silanol acts as H-bond donor to water ${ }^{338,339}$ with a value of $\Delta \mathrm{E}_{\mathrm{ads}}=-40 \mathrm{~kJ} / \mathrm{mol}$, as compared to $-46.5 \mathrm{~kJ} / \mathrm{mol}$ on the geminal silanols, ${ }^{339}$ where $\mathrm{H}_{2} \mathrm{O}$ bridges the two species, as calculated at the B3LYP/DZP level. Other authors calculated room temperature enthalpies of -21.4 and $-23.2 \mathrm{~kJ} / \mathrm{mol}$ for water adsorption on the isolated and geminal species at the B3LYP/ 6-311++G(3df,2pd) level. ${ }^{568}$ Highly accurate calculations (MP2/aug-cc-pVTZ) on minimal clusters provide an adsorption energy of water on an isolated silanol of -27.3 and $-17.3 \mathrm{~kJ} / \mathrm{mol}$, without and with the counterpoise correction for BSSE included, respectively. ${ }^{338}$ Note that in the work ZPE correction was not accounted for. Note that the BSSE error cannot be neglected. Water adorption enthalpies at the MP2/6-31++G** level at $350 \mathrm{~K}$ were calculated to be $-45.7,-56.9$ and $-46.7 \mathrm{~kJ} / \mathrm{mol}$ on the isolated, vicinal and geminal silanols respectively, ${ }^{87}$ clearly showing the same $\mathrm{H}$-bond donor capability of $\mathrm{Q}^{3}$ and $\mathrm{Q}^{2}$ sites. Water adsorption on siloxane is athermic (calculations performed at the MP2/6-311++G** level). ${ }^{462}$ To the best of our knowledge, cluster calculations of water adsorption on associated silanols are rare, because of the lack of structural information. Cyprik ${ }^{569}$ studied the interaction of several silanol clusters with water, from the isolated silanol to H-bonded vicinal silanols. On those silanols, the adsorption of water (calculated at the MP4/6-31G* level) is twice more exothermic $(-60.2 \mathrm{~kJ} / \mathrm{mol}$ and $-30.3 \mathrm{~kJ} / \mathrm{mol}$ with BSSE correction) than that of water on isolated silanol (-30.1 and -19.7 $\mathrm{kJ} / \mathrm{mol})$.

a)

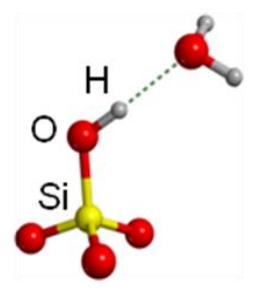

b)

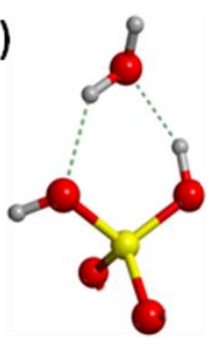

c)

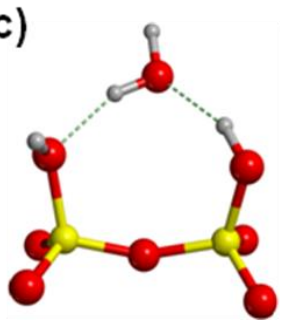


Figure 39. Synthetic view of the interaction of a single water molecule with silica clusters representing a) an isolated silanol b) a geminal pair c) vicinal silanols.

\subsection{Water on crystalline hydroxylated silicas}

Periodic studies of water at crystalline surfaces originate either from the chemists or the physicists communities; chemists are mostly interested in crystalline surfaces as potential models for amorphous silicas, whereas physicists are interested in the crystalline phases per se, for example on reactivity at defects. Another difference lies in the studied coverages: in the chemistry community, a strong attention has been put on the low water coverage, and frequency calculations, in order to assign IR spectra recorded on amorphous silica polymophs. ${ }^{317,503,566,568,570-572}$ In contrast, even if low coverages are also investigated in the physics community, a strong effort is devoted to characterize complete water layers. ${ }^{346,504,573-576}$ Whereas numerous IR data are available on amorphous silicas, ${ }^{82,577-581}$ calculations of vibration frequencies ${ }^{317,346,504,573,575,582}$ are more difficult to compare with experimental data recorded on crystalline samples ${ }^{543}$ because of the lack of data. It is interesting to notice that IR can help to estimate the amount of bulk water contained in a glassy silica. $^{581}$

Hydroxylated crystalline silica has a hydrophilic character. Water always interacts with silanol groups through H-bonding. Figure 40 shows the nomenclature generally adopted to describe water orientation versus the surface, which will also be used here.
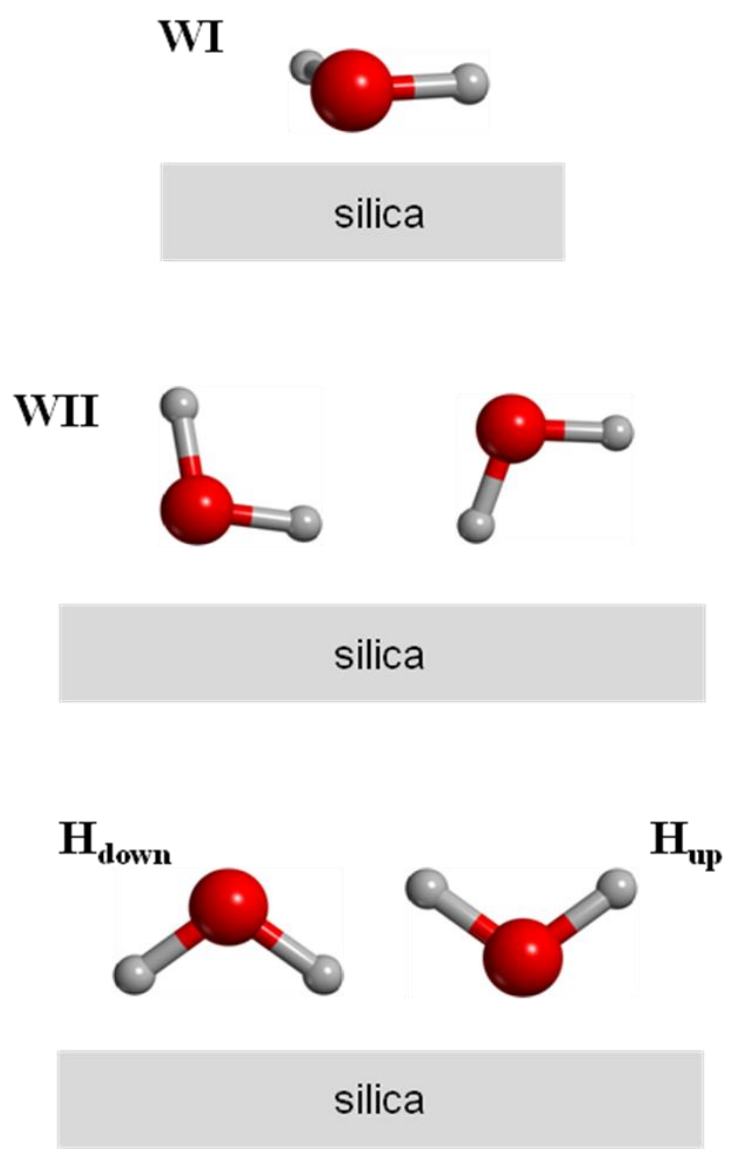
Figure 40. Nomenclature adopted for designing water orientation at silica surface. See text for description.

There are different possible orientations for water: In type I (WI, see Figure 40) water is adsorbed parallel to the surface. Consequently, water can only act as a H-bond acceptor from the surface. In type II (WII) water is not parallel to the surface; it may be placed either with one $\mathrm{H}$ pointing away from the surface (water then acts as a $\mathrm{H}$-bond acceptor) or with an $\mathrm{H}$ pointing towards the surface (water acts as a H-bond donor). In some particular cases water is oriented with both $\mathrm{H}$ pointing towards the surface, $\left(\mathrm{H}_{\text {down }}\right)$. In other cases water has the two protons directed away from the surface $\left(\mathrm{H}_{\mathrm{up}}\right)$.

Some first principles works have explored the adsorption of water molecules beyond the saturation (full monolayer), ${ }^{542,574,583}$ and of increasing numbers of water molecules on hydroxylated silica surfaces, from one isolated water to one monolayer, ${ }^{317,346,504,573,575}$ from one monolayer to several layers ${ }^{501,576}$ and finally at the interface with bulk water ${ }^{501,582,584-588}$ (see Tables 8-11). Calculations using force fields have been devoted to the interface with water thin films and bulk water, ${ }^{542,559,589-599}$ with only two exceptions where a single water layer was investigated. ${ }^{594,600}$

Table 8. Theoretical works based on electronic calculations, using periodic conditions, devoted to water at crystalline silicas: nature of the surface studied, method and water coverages investigated.

\begin{tabular}{|c|c|c|c|c|}
\hline Polymorph & Surface & Method & Water coverage & Ref. \\
\hline \multirow[t]{11}{*}{$\mathrm{Q}$} & \multirow[t]{7}{*}{$(001)$} & PW91 UPP $350 \mathrm{eV}$ & Isolated water to monolayer & 504 \\
\hline & & B3LYP TZ polarization & Isolated water & 574 \\
\hline & & PW91 PAW 400eV & One to five water layers & 576 \\
\hline & & B3LYP Lanl2dz & Isolated water & 542 \\
\hline & & PBE 400 eV, AIMD & Bulk water & 584 \\
\hline & & $\begin{array}{l}\text { BLYP TZVP2 }+ \text { AGTH, } \\
280 \text { Ryd, BOMD }\end{array}$ & Bulk water & 585 \\
\hline & & $\begin{array}{l}\text { CPMD PBE Vanderbilt } 25 \\
\text { Ry, } 1000 \mathrm{~K}\end{array}$ & Water film (2-3 water layers) & 501 \\
\hline & $(100)$ & B3LYP TZ polarization & Isolated water & 574 \\
\hline & $(011)$ & B3LYP TZ polarization & Isolated water & 574 \\
\hline & $(101)$ & PBE PAW $400 \mathrm{eV}$ & Isolated water to monolayer & 575 \\
\hline & $(0110)$ & PW91, 680 eV cutoff & Bulk water & 587 \\
\hline $\mathrm{C}$ & $(100)$ & PW91 UPP $350 \mathrm{eV}$ & Isolated water to monolayer & 346 \\
\hline
\end{tabular}




\begin{tabular}{|c|c|c|c|c|}
\hline \multirow{6}{*}{$\mathrm{E}$} & $(100)$ & rPBE and PW91 & One water monolayer & 600 \\
\hline & $(101)$ & B3LYP TZ polarization & Isolated water & 574 \\
\hline & (111) & PW91 UPP $350 \mathrm{eV}$ & $\begin{array}{l}\text { Isolated water to monolayer } \\
\text { and frequency analysis }\end{array}$ & 573 \\
\hline & $\begin{array}{l}(001) \\
(100)\end{array}$ & B3LYP GTO & $\begin{array}{l}\text { Isolated water and monolayer } \\
\text { and frequency calculations }\end{array}$ & 317 \\
\hline & $(001)$ & B3LYP 6-31G* & $\begin{array}{l}\text { One and two water molecules } \\
\text { in acid and basic media }\end{array}$ & 583 \\
\hline & $(100)$ & DFT-TB MD 15 ps & Bulk water & 323,586 \\
\hline $\mathrm{T}$ & 001 & B3LYP TZ polarization & Isolated water & 574 \\
\hline
\end{tabular}

Table 9. Theoretical works based on classical force fields, using periodic conditions, devoted to water at crystalline silicas: nature of the surface studied, method and water coverages investigated.

\begin{tabular}{|c|c|c|c|c|}
\hline Polymorph & Surface & Method & $\begin{array}{l}\text { Coverage and water layer } \\
\text { thickness and properties studied }\end{array}$ & Ref. \\
\hline $\mathrm{Q}$ & $(0001)$ & CLAYSFF & Isolated water to bulk liquid & 542 \\
\hline Q & $(0001)$ & $\begin{array}{l}\text { PW91 300eV } \\
\text { cutoff ultrasoft } \\
\text { pseudopotentials } \\
\text { and Sanders, } \\
\text { Baram and Parker } \\
\text { potentials }\end{array}$ & $\begin{array}{l}\text { Dissociative adsorption of water } \\
\text { on a dry surface }\end{array}$ & 589 \\
\hline Q & $(0001)$ & $\begin{array}{l}\text { Sanders, } \\
\text { Baram and Parker }\end{array}$ & $\begin{array}{l}\text { Bulk water at the non- } \\
\text { hydroxylated and fully } \\
\text { hydroxylated surface, and quartz } \\
\text { dissolution }\end{array}$ & 590 \\
\hline Q & $\begin{array}{l}(100) \\
(0001) \\
(011)\end{array}$ & CHARMM & Bulk water & 591 \\
\hline Q & $\begin{array}{l}(100),(001) \\
\text { and }(011)\end{array}$ & CHARMM & Bulk water & 595 \\
\hline Q & $(111)$ & COMPASS & $\begin{array}{l}\text { Water and oil mixture adsorption } \\
\text { competition }\end{array}$ & 597 \\
\hline Q & $(010)$ & Cerius2 & $\begin{array}{l}\text { Bulk water, diffusion coefficient } \\
\text { study in water near the surface }\end{array}$ & 599 \\
\hline $\mathrm{C}$ & $(100)$ & COMPASS & Water monolayer & 600 \\
\hline $\mathrm{C}$ & (111) & $\mathrm{SCP}$ and $\mathrm{LJ}$ & $\begin{array}{l}\text { Bulk water interaction with } \\
\text { surfaces of different } \mathrm{OH} \\
\text { coverages }\end{array}$ & 592 \\
\hline $\mathrm{C}$ & $\begin{array}{l}(100) \\
(111)\end{array}$ & SPC and PN-TrAZ & $\begin{array}{l}\text { Bulk water, simulated adsorption } \\
\text { isotherm }\end{array}$ & 559 \\
\hline Q & $\begin{array}{l}(100) \\
(011)\end{array}$ & CHARMM & Bulk water & 593 \\
\hline $\mathrm{C}$ & $(100)$ & CHARMM & Water monolayer & 594 \\
\hline
\end{tabular}




\begin{tabular}{l|llll}
\hline & & & & \\
$\mathrm{C}$ & $(111)$ & ClayFF & Bulk water & 596 \\
$\mathrm{C}$ & $(111)$ & ClayFF & Bulk water & 598 \\
\hline
\end{tabular}

\subsubsection{The low coverage case}

At low coverage, the water-hydroxyl interaction is predominant. The adsorption of one isolated water molecule has a very local effect.

- Few crystalline surfaces exhibit isolated silanols, or rather non-interacting ones, E(100) and $\mathrm{C}(111)$ being the main ones. In the most stable configuration of $\mathrm{E}(100)$, water acts as a single H-bond acceptor, and may be H-donor to a siloxane oxygen (Figure $41 \mathrm{a}$ ). ${ }^{574}$ The same was found using cluster models. ${ }^{340}$ In contrast, on the C(111) surface which exhibits non-interacting silanols (separated by $5 \AA$ ), water adsorbs in a hollow site and is double proton-donor and a protonacceptor. ${ }^{573}$ This example shows the limits of cluster models to mimick non-interacting silanols.

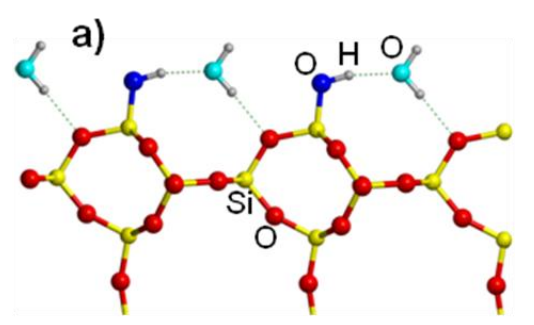

b)
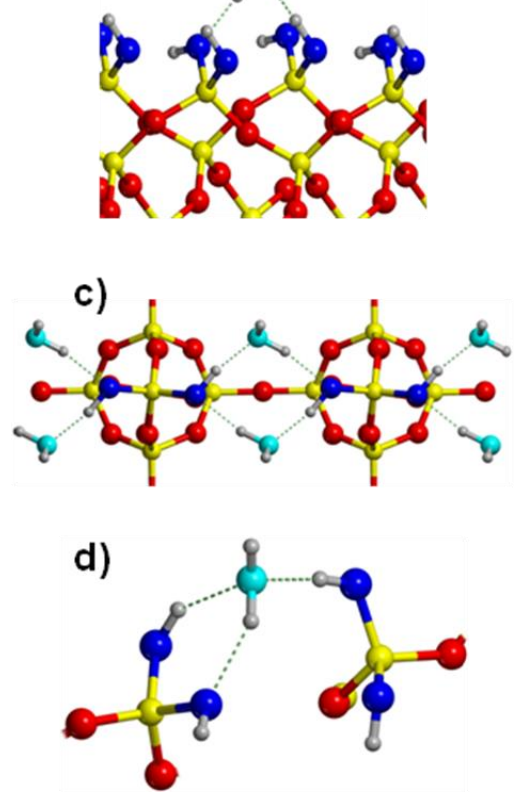

Figure 41. Water at low coverage on crystalline silicas (periodic DFT calculations): a) isolated silanols on edingtonite; adapted with permission from Ref. ${ }^{317}$. Copyright 2008 Institute of Physics b) water on geminal silanols of $\mathrm{Q}(001)$; adapted with permission from Ref. ${ }^{574}$. Copyright 2011 
American Chemical Society. c) water on geminal silanols of E(001); adapted with permission from Ref. $^{317}$. Copyright 2008 Institute of Physics. d) water on C(100); drawn with data from Ref. ${ }^{573}$.

Geminal silanols cover the $\mathrm{Q}(001),{ }^{295,499} \mathrm{Q}(010)^{499,599} \mathrm{C}(100)^{573}$ and $\mathrm{E}(001)^{317,601}$ surfaces. On $\mathrm{Q}(001)$ water does not adsorb above a geminal pair but rather in a bridging position between two geminal pairs (Figure $41 \mathrm{~b}$ and 41c). ${ }^{306,504}$ Among the two possible sites, a weakly and a strongly $\mathrm{H}$-bonded site, adsorption is more stable on the weakly $\mathrm{H}$-bonded site, as breaking this bond is less energetically expensive than breaking a strong $\mathrm{H}$-bond. A similar result is found on $\mathrm{E}(001)^{317}$ (Figure $41 \mathrm{~b}$ and $41 \mathrm{c}$ ). An even more complex pattern occurs on the $\mathrm{C}(100)$ surface with water bridging the geminal silanol pairs and accepting an additional H-bond from a silanol (Figure 41d). ${ }^{573}$ The complexity of these surface structures cannot be easily modeled by a cluster approach. To our knowledge, no ab initio study is available on the $\mathrm{Q}(010) /$ water interaction.

-The $\mathrm{Q}(101)^{575}$ surface exhibits vicinal silanols. Water interacts with a vicinal pair as $\mathrm{H}$ bond donor, and also as a H-bond acceptor from a surface silanol (Figure 42).

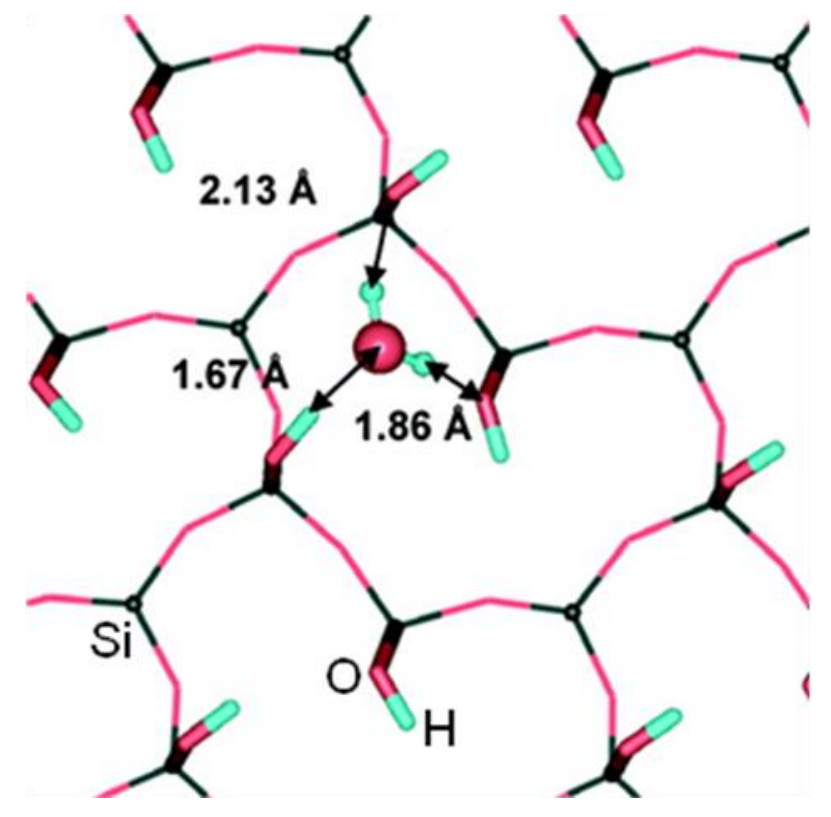

Figure 42. Snapshot of an isolated water adsorbed on vicinal silanols of Q(101) surface. Reprinted with permission from Ref. ${ }^{575}$. Copyright 2011 American Chemical Society.

-Finally, non-vicinal silanols able to bind to a water molecule are present on all but the $\mathrm{E}(100)$ surface. The situation is complex as the possible adsorption sites are not unique, and a preexisting H-bond network may be broken. Water always acts as $\mathrm{H}$-bond acceptor from a silanol and as H-bond donor to one or two silanols. Water binds to $\mathrm{Q}^{2}$ silanols belonging to different 
geminal pairs on $\mathrm{Q}(001), \mathrm{E}(001)$ and $\mathrm{C}(100)$, and $\mathrm{Q}^{3}$ silanols on the other surfaces, with the exception of $\mathrm{E}(100)$ (isolated silanol). Figure 43 illustrates the configuration obtained for the $\mathrm{Q}(011), \mathrm{Q}(100), \mathrm{C}(101)$ and $\mathrm{T}(001)$ surfaces. ${ }^{574}$ Remarkably, in all cases, rows of H-bonds involving silanols and water are formed at the surface. In other words, water on crystalline surface helps the formation of the H-bond network.

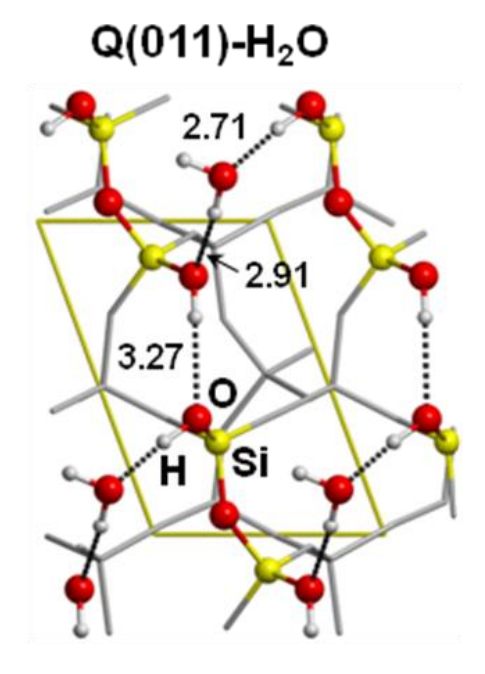

$$
Q(100)-\mathrm{H}_{2} \mathrm{O}
$$

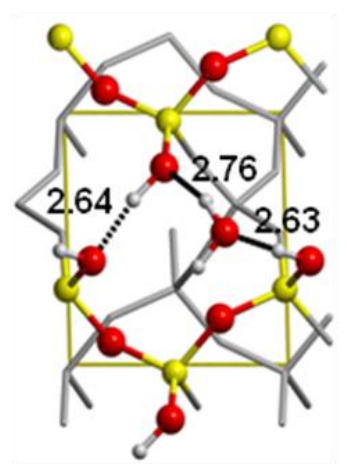

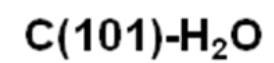

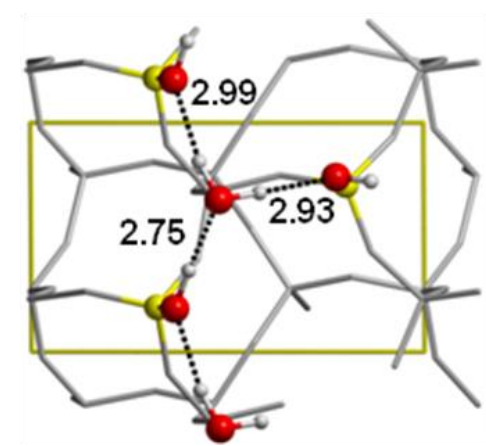

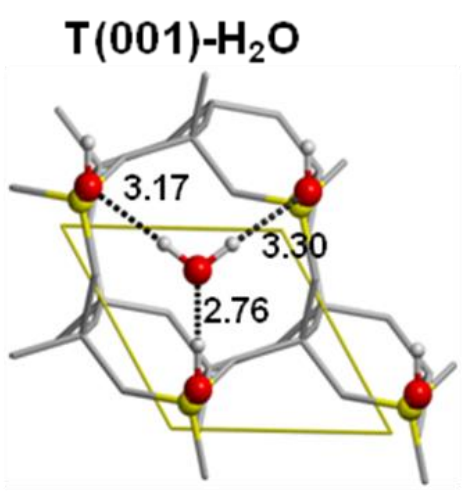

Figure 43. Adsorption of water at low coverage on adjacent silanols: a) $Q(011)$, b) $Q(100)$, c) $\mathrm{C}(101)$ and d) $\mathrm{T}(001)$. Reprinted with with permission from Ref. ${ }^{574}$. Copyright 2011 American Chemical Society.

The absolute adsorption energies recorded in the literature at low coverage (or isolated in the case of cluster calculations) are all within the $48-58 \mathrm{~kJ} / \mathrm{mol}$ range, when water forms one or two $\mathrm{H}$ bonds. A higher value of $77 \mathrm{~kJ} / \mathrm{mol}$ is obtained for water inside a cycle of silanols, as on the (111) cristobalite surface, with the formation of three H-bonds.

\subsubsection{Intermediate coverages}

The situation is also intricate at intermediate coverages because, on the one hand, stabilizing $\mathrm{H}$-bond structures between water molecules and between water and silanols are formed, and on the other hand, preexisting H-bonds between silanols must be broken in order to establish these 
interactions. Table 10 shows that there is no apparent coherence between the calculated water adsorption energy and either the water coverage or the number of $\mathrm{H}$-bonds formed per water molecule.

Table 10. DFT studies of water adsorption on hydroxylated crystalline silicas at low and intermediate coverages: energetics and H-bonding.

\begin{tabular}{|c|c|c|c|c|c|c|c|}
\hline Polymorph & Surface & $\begin{array}{l}\text { Hydroxyl } \\
\text { type }\end{array}$ & Method & $\begin{array}{l}\text { Adsorption } \\
\text { mode: water } \\
\text { is: }\end{array}$ & $\begin{array}{l}\text { Water density } \\
\text { Molecule/nm }\end{array}$ & $\begin{array}{l}\text { Absolute } \\
\text { adsorption } \\
\text { energy } \\
(\mathrm{kJ} / \mathrm{mol})\end{array}$ & Ref. \\
\hline \multirow[t]{5}{*}{ Q } & $(0001)$ & geminal & $\begin{array}{l}\text { PW91 UPP } \\
350 \mathrm{eV}\end{array}$ & $1 \mathrm{H}_{\mathrm{acc}}, 1 \mathrm{H}_{\mathrm{don}}$ & 1.2 & 52.1 & 504 \\
\hline & (0001) & geminal & $\begin{array}{l}\text { B3LYP } \\
\text { TZ basis + } \\
\text { polarization }\end{array}$ & $\begin{array}{l}1 \mathrm{H}_{\mathrm{acc}} \\
1 \mathrm{H}_{\mathrm{don}}\end{array}$ & 4.5 & 56.4 & 574 \\
\hline & (100) & vicinal & $\begin{array}{l}\text { B3LYP } \\
\text { TZ basis + } \\
\text { polarization }\end{array}$ & $\begin{array}{l}1 \mathrm{H}_{\mathrm{acc}} \\
1 \mathrm{H}_{\mathrm{don}}\end{array}$ & 3.5 & 64.9 & 574 \\
\hline & (011) & $\begin{array}{l}\text { Interacting } \\
\text { silanols }\end{array}$ & $\begin{array}{l}\text { B3LYP } \\
\text { TZ basis + } \\
\text { polarization }\end{array}$ & $\begin{array}{l}1 \mathrm{H}_{\mathrm{acc}} \\
1 \mathrm{H}_{\mathrm{don}}\end{array}$ & 2.8 & 77.5 & 574 \\
\hline & (101) & vicinal & $\begin{array}{l}\text { PBE PAW } \\
400 \mathrm{eV}\end{array}$ & $\begin{array}{l}1 \mathrm{H}_{\mathrm{acc}} \\
2 \mathrm{H}_{\mathrm{don}}\end{array}$ & 0.7 & 60.8 & 575 \\
\hline \multirow[t]{3}{*}{$\mathrm{C}$} & $(100)$ & geminal & $\begin{array}{l}\text { GGA, } \\
\text { Vanderbilt, } \\
350 \mathrm{eV}\end{array}$ & $\begin{array}{l}1 \mathrm{H}_{\mathrm{acc}}, \\
1 \mathrm{H}_{\mathrm{don}}\end{array}$ & 1.9 & 51.1 & 346,573 \\
\hline & (101) & & $\begin{array}{l}\text { B3LYP } \\
\text { TZ basis + } \\
\text { polarization }\end{array}$ & & 2.2 & 81.4 & 574 \\
\hline & (111) & isolated & $\begin{array}{l}\text { PW91 UPP } \\
350 \mathrm{eV}\end{array}$ & $\begin{array}{l}1 \mathrm{H}_{\mathrm{acc}} \\
2 \mathrm{H}_{\mathrm{don}}\end{array}$ & 3.7 & 74.3 & 573 \\
\hline \multirow[t]{2}{*}{$\mathrm{E}$} & $(001)$ & geminal & B3LYP & & 3.5 & 55.0 & 317 \\
\hline & $(100)$ & isolated & GTO & $\begin{array}{l}1 \mathrm{H}_{\mathrm{acc}}, \\
1 \mathrm{H}_{\text {don }} \text { to a } \mathrm{O}\end{array}$ & 2.2 & 46.0 & \\
\hline $\mathrm{T}$ & 001 & isolated & $\begin{array}{l}\text { B3LYP } \\
\text { GTO }\end{array}$ & $1 \mathrm{H}_{\mathrm{acc}}, 2 \mathrm{H}_{\mathrm{don}}$ & 4.3 & & 574 \\
\hline
\end{tabular}

It is not an easy task to disentangle this question. This was addressed in Ref., ${ }^{574}$ where it is shown that the water-surface binding energy shows an inverse correlation with the $\mathrm{OH}$ surface density (Figure 44). This interesting result somewhat contradicts, at least for low water coverages, the general view that surface hydrophilicity increases with the $\mathrm{OH}$ density. This study also demonstrates that the adsorption occurs at the cost of breaking the preexisting $\mathrm{H}$-bond network (Figure 44). For $\mathrm{T}(001)$ and $\mathrm{Q}(011)$, the adsorption process does not imply the rupture of preexisting H-bonds between silanols, which are absent in the former case and maintained on the silica/water molecule adduct in the latter, whereas in the case of $\mathrm{Q}(100)$ and $\mathrm{Q}(001)$, the adsorption process implies breaking pre-existing infinite chains of H-bonds. The $\mathrm{C}(101)$ surface exhibits an 
intermediate behavior with a weak H-bond (O---O distance is equal to $3.363 \AA$ ) being disrupted upon adsorption of water.
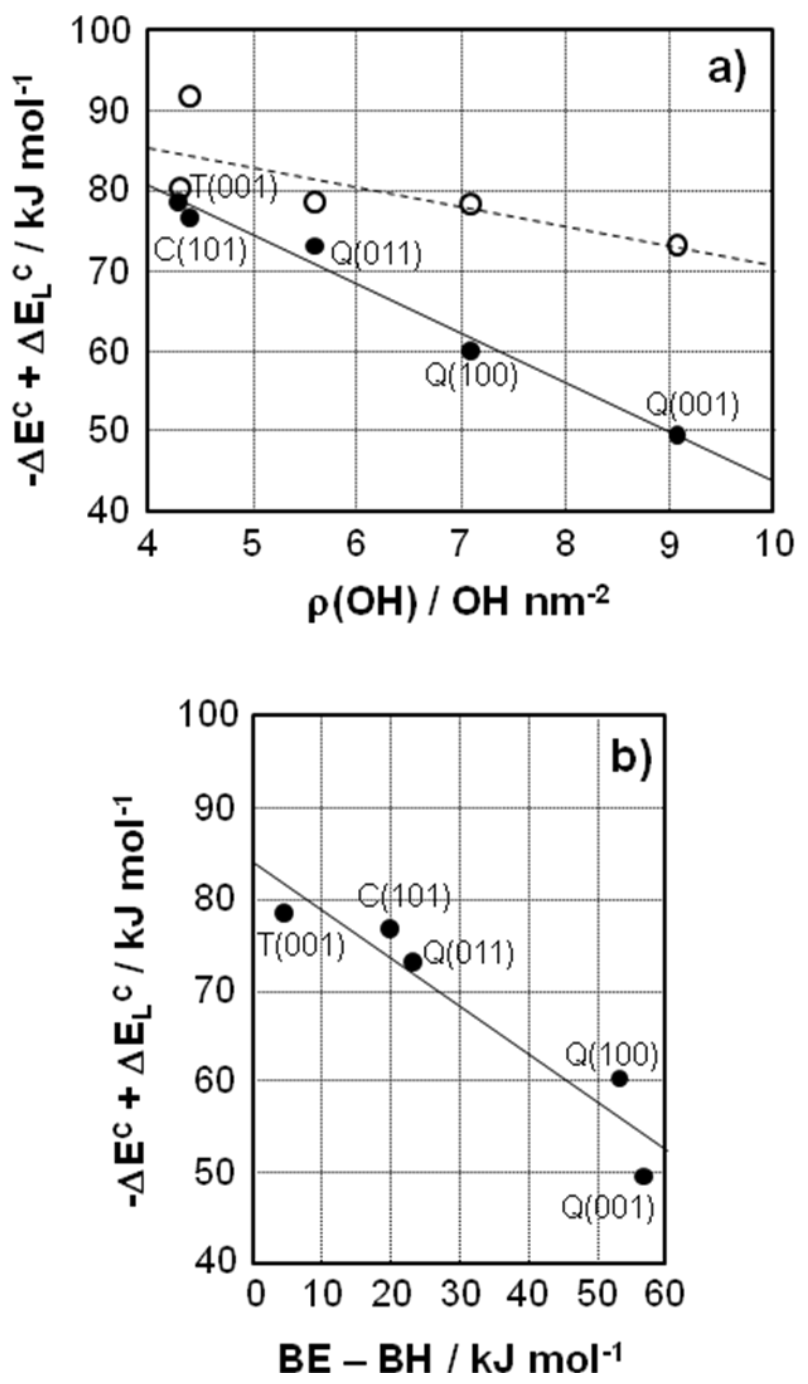

Figure 44. Water-Surfaces Binding energy $(\mathrm{kJ} / \mathrm{mol})$ (i.e. total interaction energy minus lateral interactions between adsorbates) reported for water on several crystalline surfaces, a) as a function of the $\mathrm{OH}$ surface density b) as a function of the initial H-bond binding energy between surface silanols. Reprinted with permission from Ref. ${ }^{574}$. Copyright 2011 American Chemical Society.

The computed adsorption enthalpy values for the quartz surfaces (at $\mathrm{T}=303 \mathrm{~K}$ ), which span the -51 to $-70 \mathrm{~kJ} / \mathrm{mol}$ range, are in reasonable agreement with experimental data. ${ }^{82}$ For cristobalite outgassed at $423 \mathrm{~K}$, an experimental value of $-55 \mathrm{~kJ} / \mathrm{mol}$ for $\theta\left(\mathrm{H}_{2} \mathrm{O}\right)=2.2$ has been measured, ${ }^{560}$ to be compared with the computed enthalpy value of $-73 \mathrm{~kJ} / \mathrm{mol}$ (at $0 \mathrm{~K}$ ) for the adsorption on the $\mathrm{C}(101)$ surface at the same $\mathrm{H}_{2} \mathrm{O}$ coverage. 
Some authors have studied the interesting case of the water dimer adsorption, as this is the simplest case where the lateral water-water interactions as well as $\mathrm{H}$-bond formation with the surface contribute in the adsorption process.

On $\mathrm{Q}(0001)^{504}$ the water dimer retains its configuration and adsorbs on top of two vicinal silanols, forming a bridge (Figure 45a). As for the monomer, adsorption is stronger on the weak-Hbond site. The adsorption is slightly less energetic than in the monomer case, $-45.3 \mathrm{~kJ} / \mathrm{mol}$, due to the decreased number of $\mathrm{H}$-bonds between water and the surface. In the adsorbed dimer, the two types of water molecules WI and WII are identified. WI, (see Figure 40), adsorbed parallel to the surface, shares H-bonds with neighboring water molecules and acts as H-bond acceptor from surface silanols, while WII is either $\mathrm{H}_{\text {up }}$ (H ponting out of the surface) or $\mathrm{H}_{\text {down }}$ (H pointing towards the surface) directed. The much reduced $\mathrm{O}-\mathrm{O}$ distance of $2.63 \AA$ in the adsorbed dimer, as compared to the computed value of $2.90 \AA$ for the free dimer clearly indicates that adsorption enhances $\mathrm{H}$ bonding of the water dimer.

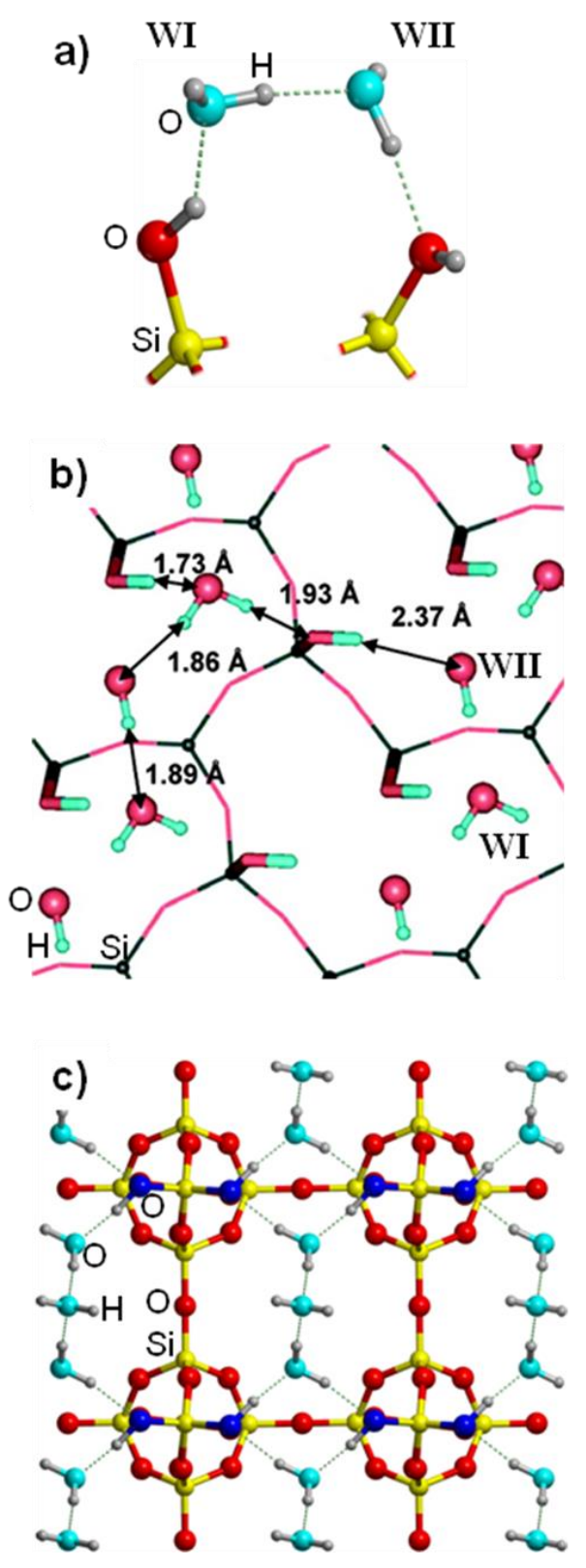


Figure 45. a) Scheme of water dimer adsorption on Q(0001), showing WI and WII types, b) water dimer on $\mathrm{Q}(101)$; reprinted with permission from Ref. ${ }^{575}$. Copyright 2011 American Chemical Society. c) water trimer on E, reprinted with permission from Ref. ${ }^{317}$. Copyright 2008 Institute of Physics.

Yang et $\mathrm{al}^{346,504}$ found that on $\beta$-cristobalite (100), the most stable state for water dimer adsorption (with an adsorption enthalpy of $-72 \mathrm{~kJ} / \mathrm{mol}$ ) is in a bridge configuration between two pairs of geminal silanols. Interestingly, the H-bond in the adsorbed dimer is enhanced as compared to the free, gas phase dimer. A similar result was found by Bandura et $\mathrm{l}^{575}$ on $\mathrm{Q}(101)$ for which the most stable water dimer configuration is found with an adsorption energy of $-55 \mathrm{~kJ} / \mathrm{mol}$ (Figure 45b).

In contrast, Tosoni et al report the adsorption of a water trimer on edingtonite that has no increased adsorption energy as compared to a single water molecule adsorption (-44 kJ/mol) (Figure $45 \mathrm{c}) .^{317}$

\subsubsection{Water monolayer}

At increasing coverage, a water monolayer may be formed on the surface. Electron density profiles for all measured interfaces are consistent with a single layer of adsorbed water on (001) and (101) quartz surfaces at ambient conditions. ${ }^{602}$ Etzler and Fagundus ${ }^{603}$ postulated that interfacial structuring of water would result in a decrease of the water density close to the silica surface. Schlegel et $\mathrm{al}^{602}$ measured by X-ray reflectivity that the number of adsorbed water molecules is consistent with the number of silanol groups per unit cell, suggesting that the driving force for the formation of this layer is likely to be the hydrogen bonding of water molecules to surface silanol groups. This conclusion fully agrees with calorimetry data ${ }^{604,605}$ recorded on amorphous and crystalline silicas, which conclude that the differences in immersional heats of silicas in water significantly depend on the interaction of hydrogen bonds between water molecules and surface hydroxyl groups. It was concluded that hydrogen-bonded hydroxyl groups interact with water molecules more strongly than free hydroxyl groups.

However, if morphologically flat crystal surfaces adsorb only a single layer of water (monolayer), it was also proposed that corrugated surfaces have a disordered and random distribution of adsorbed $\mathrm{H}_{2} \mathrm{O}$ molecules. ${ }^{606}$

The formation of a stable water monolayer on flat surfaces was later confirmed by ab initio works which characterized the water monolayer on crystalline surfaces as e.g. 
$\mathrm{Q}(0001)^{542,554,574,576,590}$ (Figure 46ab) and C (100) (Figure 47a). ${ }^{346,573}$ Thanks to a "smooth epitaxy" mechanism ${ }^{607}$ between water and silanols, ice-like structures are formed on the surfaces. The WI/WII ratio on quartz (0001) and cristobalite (100) is 50/50. WII is always found H-down orientated. This type of water monolayer adsorption will fully saturate the hydrogen bonds of water molecules and hydroxyl groups on the surface: each water molecule shares three H-bonds with neighboring waters and one H-bond with surface silanols. Accordingly, the cohesion in the water layer is more important than the water-surface interaction. A WI water molecule is twice H-bond donor to water neighbors and twice H-bond receiver, from one silanol and one neighboring water. WII is twice H-bond receiver from water, and twice H-bond donor, to silanol and water. However the crystalline structures of the layers formed on the different surfaces are reminiscent of the surface hydroxyl structure, as detailed hereafter.

a)

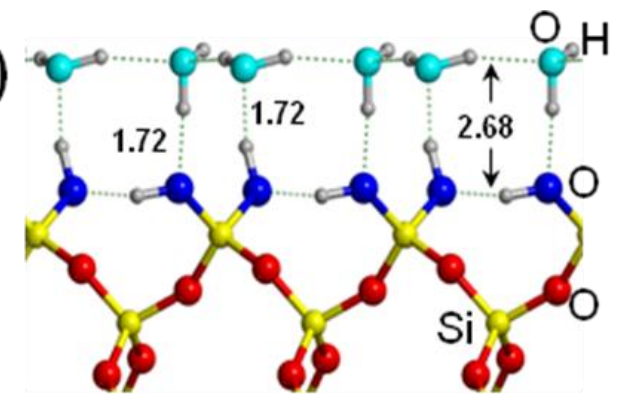

b)
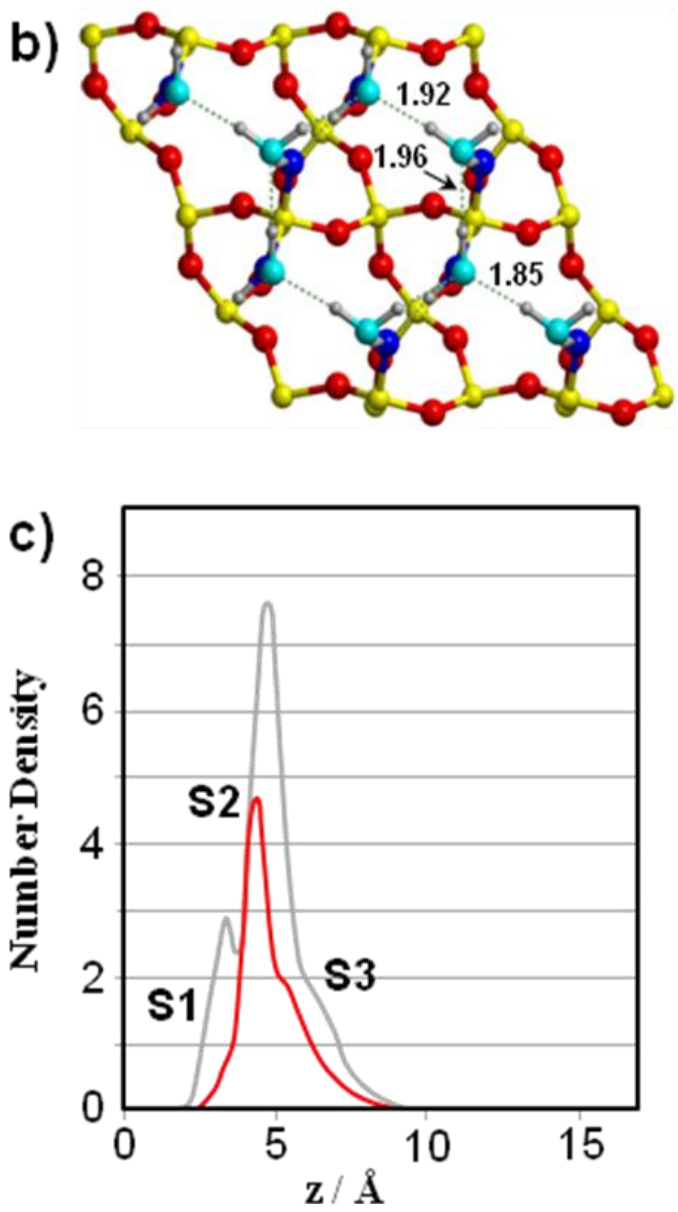
Figure 46. Water monolayer structure on $\mathrm{Q}(001)$; adapted with permission from Ref. ${ }^{576}$. Copyright 2011 American Physical Society. a) side view, and b) view from above, c) MD vertical water profiles for water/Q(0001) interfaces at coverage $1 \mathrm{H}_{2} \mathrm{O} / 10 \AA^{2}$; adapted with permission from Ref. $^{542}$. Copyright 2008 American Chemical Society.
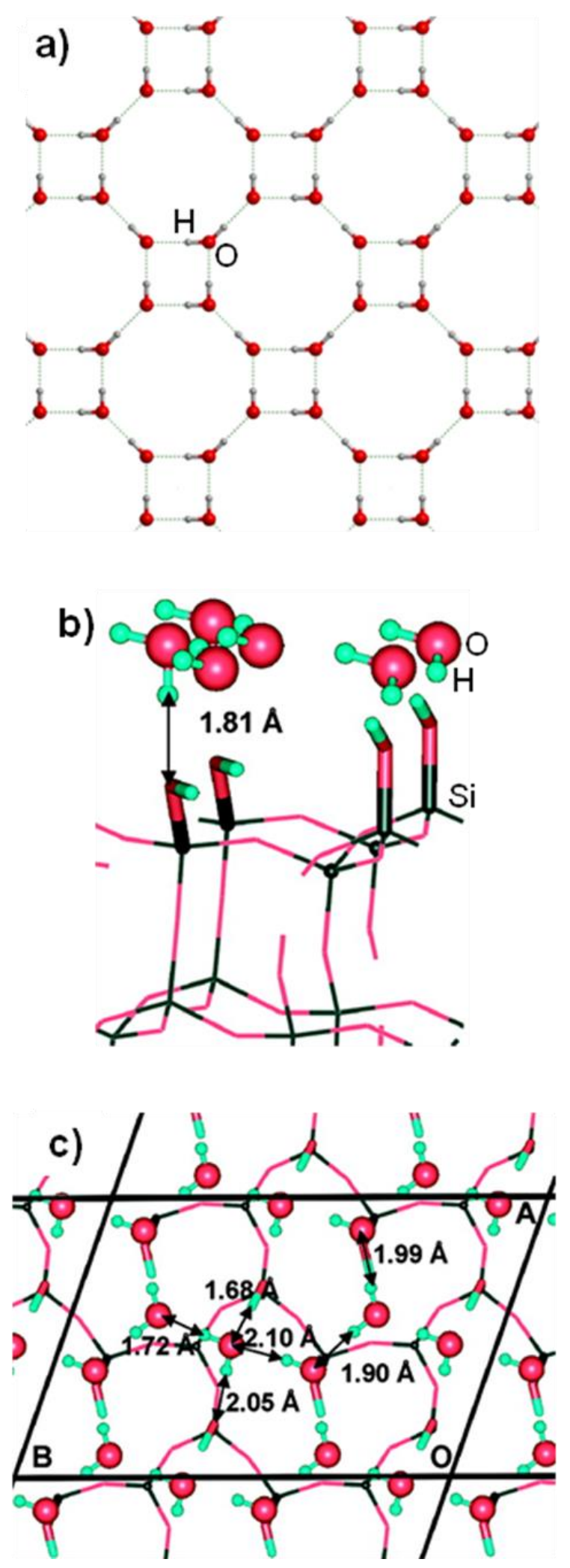
Figure 47. Water monolayer structures of $a-b) C(100)$, view from above and side view; adapted with permission from Ref. ${ }^{346}$. Copyright 2004 American Physical Society, c) Q(101); reprinted with permission from Ref. $^{575}$. Copyright 2011 American Chemical Society.

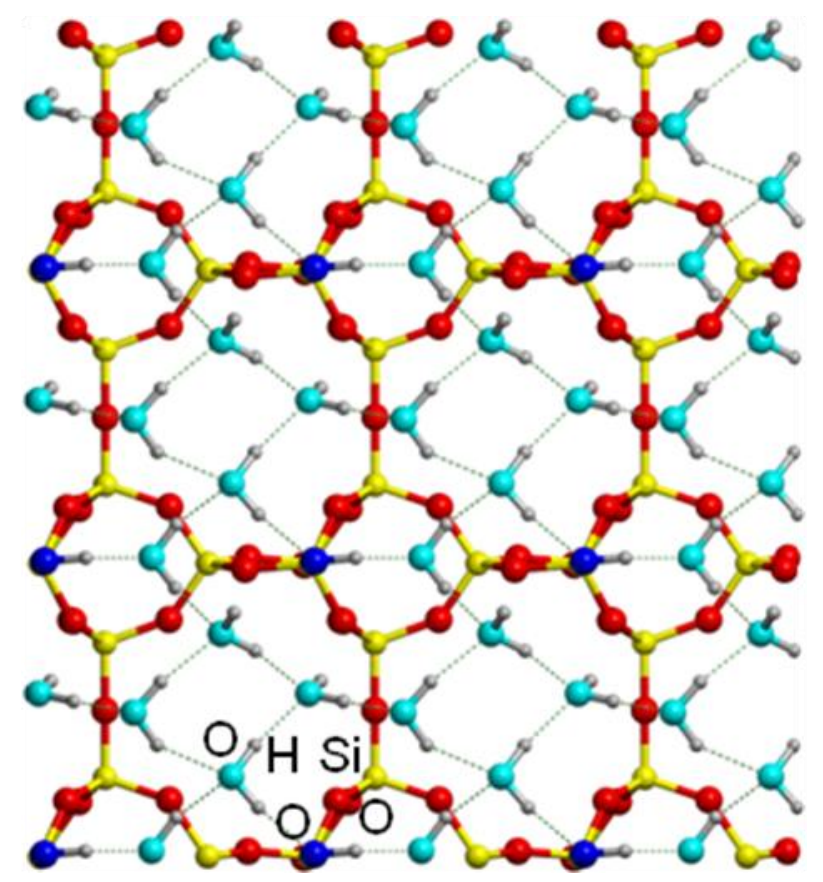

Figure 48. Water monolayer structure on E(001). Reprinted with permission from Ref. ${ }^{317}$. Copyright 2008 Institute of Physics.

Water forms an hexagonal ice layer on $\mathrm{Q}(001) .{ }^{504}$ The $\mathrm{Q}(001)$ surface has an hexagonal lattice constant of $4.91 \AA$, which is comparable with the periodicity in bulk ice Ih, $4.52 \AA$. The adsorption energy of this layer is $-62.7 \mathrm{~kJ} / \mathrm{mol}$, which is higher in absolute value than the value for adsorption as isolated molecules, namely $-55.6 \mathrm{~kJ} / \mathrm{mol}$. Most of the adsorption energy gain in the layer structure is due to the formation of lateral H-bonding interactions between molecules. Accordingly, the water-surface interaction is reduced with respect to the adsorption as single water molecules. An interesting combined DFT (geometry optimization at the B3LYP/Lanl2DZ) and classical MD (using the CLAYFF force field) study was performed for one water monolayer (10 $\mathrm{H}_{2} \mathrm{O} / \mathrm{nm}^{2}$ ) on hydroxylated (001) quartz. ${ }^{542}$ The authors studied the effects of temperature (through MD simulation) on this monolayer. The dynamic picture has only partial occupation of the structure obtained at $0 \mathrm{~K}$ (S1, Figure 46c). Another configuration (S2), less stable at 0K, with the two water hydrogens donating $\mathrm{H}$-bonds to the surface $\mathrm{SiOH}$ groups is clearly thermally accessible. Water molecules coalescence on the surface, to form a droplet of water that corresponds to multilayer adsorption is also evidenced. 
-On Q $(101)^{575}$ Bandura et al, using the PBE GGA with PAW pseudopotentials, and a cutoff of $400 \mathrm{eV}$, calculated that water adsorption at the monolayer is exothermic by $59.2 \mathrm{~kJ} / \mathrm{mol}$. Several stable structures were investigated, with differences in energy ranging from 5 to $20 \mathrm{~kJ} / \mathrm{mol}$ with respect to the most stable structure. On this surface, as there is no distinct adsorption site on the hydroxylated surface, the amount of water adsorbed may reach the density of bulk water. ${ }^{602}$ In this structure, a stable bilayer is found (Figure 47b). Again, WI and WII types are identified. Type WI sits nearly parallel to the surface by accepting a H-bond from one lifted surface hydroxyl group, and type WII points one of its protons down to the $\mathrm{O}$ atom of another surface hydroxyl. Each $\mathrm{H}_{2} \mathrm{O}$ molecule is H-bonded with its three neighbors and a surface hydroxyl.

-On cristobalite (100) and (111), a quadrangular/octagonal ice phase structure was evidenced by Yang et al. ${ }^{346,573}$ The elementary water dimer structure formed on geminal silanols, with one WI and one WII, is retained at high coverage. The adsorption energy in the water layer is $68.5 \mathrm{~kJ} / \mathrm{mol}$. A different adsorption energy for the same structure was calculated by Lu et al. ${ }^{600}$ This ice tessellation was calculated to be stable until $150 \mathrm{~K}$ by classical MD simulation (COMPASS), in contrast to a temperature desorption near $300 \mathrm{~K}$ calculated by Yang et al. in AIMD using the PW91 pure GGA. ${ }^{573}$ Lu et al ${ }^{600}$ attribute this discrepancy to the overestimation of the $\mathrm{H}$ bond interaction between water and the surface with PW91 (near $-70 \mathrm{~kJ} / \mathrm{mol}$ ), whereas the value calculated with $\mathrm{rPBE}(-48.7 \mathrm{~kJ} / \mathrm{mol})$ is more realistic. Indeed $\mathrm{rPBE}$ accounts better than PW91 for bulk water description. ${ }^{608}$

Table 11. DFT studies of water adsorption on crystalline surfaces at full coverage.

\begin{tabular}{|c|c|c|c|c|c|c|}
\hline $\begin{array}{l}\text { Poly } \\
\text { morp } \\
\text { h }\end{array}$ & Surface & $\begin{array}{l}\text { Nature of } \\
\text { hydroxyls }\end{array}$ & Method & $\begin{array}{l}\text { Water density } \\
\left(\mathrm{H}_{2} \mathrm{O} / \mathrm{nm}^{2}\right)\end{array}$ & Water Organisation & Ref. \\
\hline \multirow[t]{2}{*}{$\mathrm{Q}$} & $(0001)$ & $\begin{array}{l}\text { Geminal } \\
\text { silanols }\end{array}$ & & 9.1 & $\begin{array}{l}\text { Hexagonal, Planar, type I } \\
\text { parallel and type II Hdown }\end{array}$ & $\begin{array}{l}504,542,5 \\
74,576\end{array}$ \\
\hline & $(101)$ & $\begin{array}{l}\text { No strong } \\
\mathrm{H} \text { bonds }\end{array}$ & & $\begin{array}{l}2.8 \text { water/unit } \\
\text { cell = bulk } \\
\text { water }\end{array}$ & $\begin{array}{l}\text { Ice-like structure similar to } \\
(0001)\end{array}$ & 295,575 \\
\hline $\mathrm{C}$ & $(100)$ & $\begin{array}{l}\text { Zig-zag } \\
\text { with vicinal } \\
\mathrm{OH}\end{array}$ & & & $\begin{array}{l}\text { Quadrangular tessellation } \\
\text { structure }\end{array}$ & 346 \\
\hline \multirow[t]{2}{*}{$\mathrm{E}$} & $(100)$ & Isolated & B3LYP & 10 & Monolayer & 317 \\
\hline & & & GTO & & & \\
\hline
\end{tabular}


Few theoretical works using classical MD have been devoted to the adsorption of a single water monolayer on crystalline silica surfaces, (see Table 9). An interesting screening of 40 cristobalite surfaces was performed by Nangia et al ${ }^{594}$ who modeled different cristobalite surfaces with the multibody potential proposed by Feuston and Garofalini ${ }^{472}$ and $\mathrm{CHARMM}^{593}$ and the adsorption of water thereon. First, the results using empirical potentials are validated by comparison to ab initio results on the $\mathrm{C}(100)$ surface $\mathrm{e}^{346,554}$ and then, the work is extended to other, less explored, cristobalite surfaces. Indeed, quartz and cristobalite surfaces have no preferred crystallographic cleavage plane and therefore, grounded samples have a complex structure composed of the combination of low index Miller planes. The results indicate that the surfaces with ordered topology and hydroxyl groups in planes parallel to the surface, for example, the (100) and (111) hydroxylated surfaces of cristobalite, have accessible hydroxyl groups that can adsorb water molecules through $\mathrm{H}$-bonds. This result is in agreement with the X-ray reflectivity experimental data reporting that flat quartz surfaces adsorb a single water monolayer in a highly oriented way. ${ }^{602}$ In contrast, surfaces that are corrugated or have less ordered topology do not have the hydroxyls in one plane to adsorb water effectively and thus, to form a stable monolayer. Depending on the local topology of the corrugation, the surface can adsorb single water molecules with very different adsorption energies and stabilities.

To the best of our knowledge, only one study of water monolayer formation on isolated silanols is reported in literature, for the edingtonite (001) surface (Figure 48). ${ }^{317}$ Here, the waterwater interactions are still more important than in the Q and C cases, as the silanol density is lower. Adsorbed water molecules form tetramers H-bonded to the surface. The ratio WI/WII is 50/50 as for other surfaces, but, interestingly, type II molecules are now equally distributed in between $\mathrm{H}_{\text {up }}$ and $\mathrm{H}_{\text {down }}$ orientations. In other words, the lesser silanol density induces a lesser water layer-surface interaction, and the first water layer is bulk water-like, although with a more random orientation. The adsorption energy $(-43 \mathrm{~kJ} / \mathrm{mol})$ in the water layer is also less negative than on other surfaces, as the type I molecules do not act as H-bond acceptors from silanol groups.

\subsubsection{Water multilayers}

Wander and Clark, ${ }^{542}$ in a classical MD study of the quartz-water interface, found no drastic change of the interfacial water layer when a second water layer is present on the surface. Very recently, layer-by-layer water adsorption on fully hydroxylated Q(0001) was performed by DFT. ${ }^{576}$ The first water layer has a strong energy of adsorption $(-63.7 \mathrm{~kJ} / \mathrm{mol}$ in this work), as shown in the preceding paragraph. When the second water layer is deposited on the first water layer on HY$\mathrm{Q}(0001)$, the structure of the first water is almost unaffected, the hydrogen bonds between the first and second water layers being relatively long (about $2 \AA$ ) (Figure 49a). The adsorption of the 
second water layer is less exothermic than the first one, $-51.4 \mathrm{~kJ} / \mathrm{mol}$, and the bonding energy between the two water layers is only $0.093 \mathrm{~J} / \mathrm{m}^{2}$, indicating that the interaction between the two water layers is relatively weak and that the first water layer effectively shields the long-range surface-water interaction. When the third water layer is deposited onto the water bilayer, the out-ofplane protons in the second water layer reverse their orientations from pointing down to pointing up. As a result, the optimized structure consists of the first layer structure and a water bilayer structure (Figure 49b). Accordingly, the adsorption energy of the third layer is higher than that of the second layer, $-68.0 \mathrm{~kJ} / \mathrm{mol}$. When the fourth water layer is deposited a trilayer structure is obtained with an adsorption energy of $-48.9 \mathrm{~kJ} / \mathrm{mol}$ (Figure $49 \mathrm{c}$ ). Results of trilayer and quadlayer adsorptions confirm that the bilayer-like structure at the silica-water interface effectively shields the interaction between the hydroxyl groups on the silica surface and water layers beyond the first layer. 

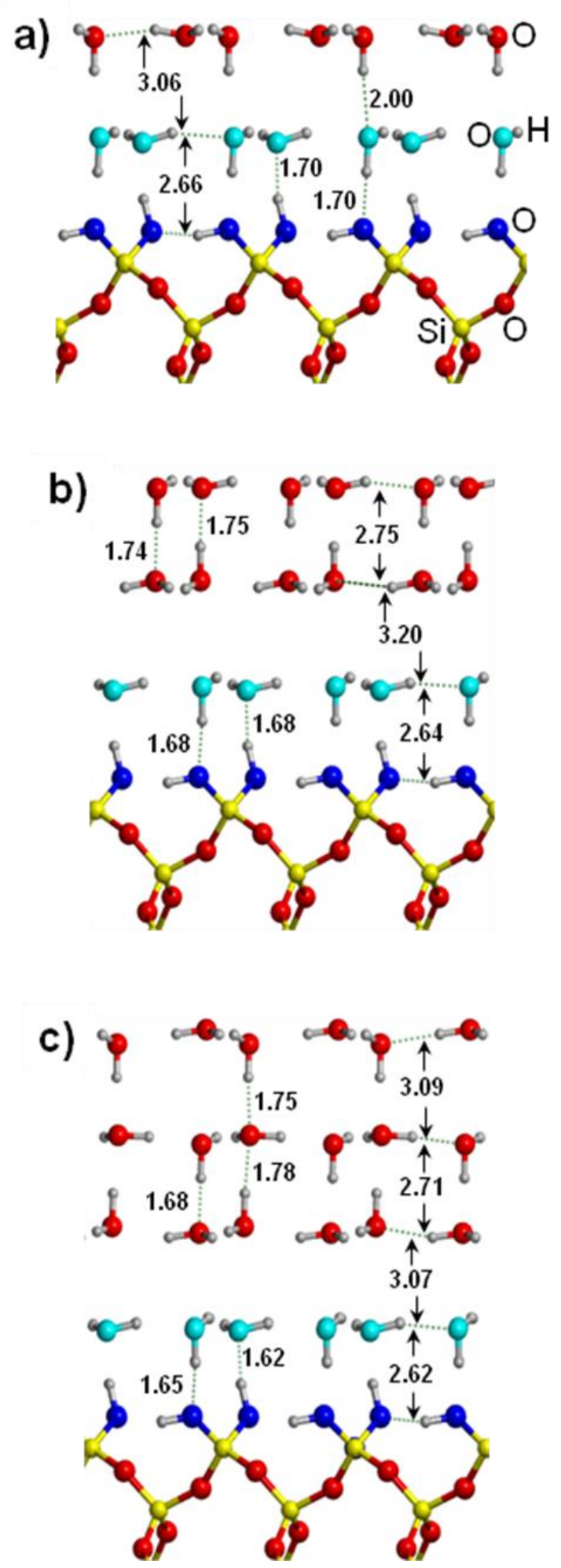

Figure 49. Water multilayers on $\mathrm{Q}(0001)$. Drawn with data from Ref. ${ }^{576}$

\subsubsection{Crystalline silicas-liquid water interface}

The quartz/water interface has been intensively studied experimentally. Quartz has a point of zero charge (PZC) of about 2 (Ref. $^{609}$ and Section 3.1.2), which implies that at $\mathrm{pH}>2$, the surface is negatively charged. ${ }^{91,102,609-612}$ A few experimental studies allow characterizing the quartz-water interface at the atomistic level. Measurements of interfacial pKa (defined as $-\log _{10} \mathrm{Ka}$, 
where $K \mathrm{a}$ is the acid dissociation constant) have been revolutionized by surface-sensitive secondharmonic generation (SHG) and sum frequency vibrational spectroscopy (SFVS, or SFG) techniques. $^{613-614}$ We may recall here results already mentioned in Section 3.1.2. (Ref. ${ }^{96}$ ) demonstrating that $19 \%$ of silanol groups on fused silica surfaces exhibit a $\mathrm{pKa}$ of 4.5 while the remaining $81 \%$ exhibit $\mathrm{pKa}$ of 8.5 . Other authors ${ }^{185}$ estimate the density of strong acid silanols at only $0.83 \mathrm{OH} \mathrm{nm}^{-2}$, only about $20 \%$ of the total silanol density. SFVS experiments on $\alpha$-quartz reached similar conclusions and further suggested that the low-acidity silanol groups reside in regions with strong water-water hydrogen bonds. ${ }^{97}$ As mentioned in Section 3.1.2, many titration curves are not precise enough to discriminate silanols of different acidities, yet at least one titration study on silica gel (amorphous silica) ${ }^{615}$ and X-ray photoelectron spectroscopy measurements on quartz $^{91}$ also independently suggested the existence of $\mathrm{SiOH}$ groups with $\mathrm{pKa}$ between 4 and 5.5. Such qualitative agreement on different forms of silica is expected because liquid water is known to react slowly with crystalline silica to form an amorphous layer. ${ }^{68}$ Indeed, in the presence of water even at $\mathrm{pH} \mathrm{7,} \mathrm{water} \mathrm{molecules} \mathrm{slowly} \mathrm{generate} \mathrm{an} \mathrm{amorphous} \mathrm{layer,} \mathrm{which} \mathrm{weakens} \mathrm{the} \mathrm{strong}$ hydrogen bond network of the near-surface water molecule.

SFG experiments on the neutral (low pH) quartz-water hydrophilic interface give two bands, respectively located at $\sim 3200$ and $\sim 3400 \mathrm{~cm}^{-1} .{ }^{97,543,564,616}$ The $3200 \mathrm{~cm}^{-1}$ band occurs at the same frequency as in the IR and Raman of ice, ${ }^{617-618}$ while the $3400 \mathrm{~cm}^{-1}$ band is found approximately at the same frequency as in the IR and Raman spectra of liquid water. Note that the $3200 \mathrm{~cm}^{-1}$ band gains in intensity at higher $\mathrm{pH}$. Assignment of these two SFG peaks is still debated, with two conflicting views in the literature: 1) The peak at $3200 \mathrm{~cm}^{-1}$ arises from a H-bond network of water molecules with an "ice-like" ordering, representative of $\mathrm{O}-\mathrm{H}$ stretch of tetrahedrally coordinated water molecules, while the peak at $3400 \mathrm{~cm}^{-1}$ comes from a "liquid-like" arrangement of the water molecules, i.e. more disordered H-bond network, 2) Another interpretation is that contributions to the $3400 \mathrm{~cm}^{-1}$ band mainly arise from water molecules directly adjacent to the surface while the $3200 \mathrm{~cm}^{-1}$ peak comes from water molecules in the more distant water layers.

Crystalline silicas-bulk water interactions have been studied by classical methods, and, very recently, with AIMD investigations. All the studies mentioned consider a neutral, uncharged surface. This is justified as, at $\mathrm{pH} 7$, the surface charge is expected to be $-1 \mu \mathrm{C} / \mathrm{cm}^{2}\left(\right.$ Ref. $\left.{ }^{89}\right)$, i.e. one negative $\mathrm{SiO}^{-}$group every $16 \mathrm{~nm}^{2}$, thus large areas of the surface have a neutral character.

Force field studies have focused on quartz, ${ }^{68,457,473,499,542,587,588,590-593,619,620}$ and cristobalite. ${ }^{559,594}$ De Leeuw et al studied the $\alpha-(0001)$ hydroxylated quartz-water interface. ${ }^{590}$ They found that interfacial water molecules losely associate with the surface via hydrogen bonding between the oxygen atoms of the interfacial water molecules and hydrogen atoms of the surface Si- 
$\mathrm{OH}$ groups. In terms of configuration and bonding, the surface $\mathrm{Si}-\mathrm{OH}$ groups resemble water molecules themselves and a relatively disordered 3-dimensional interfacial water region forms near the surface, similar to bulk liquid water. The authors conclude that this liquid-like behavior is in agreement with experimental findings on interfacial water near the quartz surface at low $\mathrm{pH} .{ }^{97,543}$ However, experimentally, at neutral $\mathrm{pH}$ water was observed to structuring near the surface. ${ }^{97}$

Wander and Clark ${ }^{542}$ found a loss of water specific orientation for interfacial water layers more distant than the first one, in which for the most part, the $\mathrm{H}_{\mathrm{up}}$-oriented waters are above the $\mathrm{SiOH}$ groups, and the H-down are in between. When structure is present in the second layer, it appears to be more diffuse than that observed in first one.

Lopes et al. developed a force-field for silica, which was applied to the (011) and (100) quartz-water interface. ${ }^{593} \mathrm{Q}(011)$ was modeled with single silanols (hydrophilic side) on one face and single silanes (hydrophobic side) on the other face. Q(100) was modeled similarly, having geminal silanols instead of single groups. The density profile of water between quartz slabs was described (Figure 50). On the (011) surface, there is an accumulation of water near the silanol groups. Water has some overlap with the surface silanols, and this is represented by the black area of Figure 50a, starting approximately at $0 \AA$. After this area of accumulation there is a smaller area of depletion followed by a very small area of accumulation before reaching the bulk density at about $7 \AA$ from the hydroxyl hydrogens. On Q(100) (Figure 50b), there is a significant penetration of water in the interstices formed by the surface hydroxyls. These waters form a distinct first layer although its density is about half of that of the bulk phase (black region on the bottom left of Figure 50b). Contrary to (011), the region of strong water accumulation is not contiguous to the hydroxyl hydrogen atoms layer but is located about $2 \AA$ away. The regions of accumulation and depletion are more extensive than those on (011) and water only reaches its bulk density $12 \AA$ from the hydroxyl hydrogen layer. These results were explained by considering the differences in surface groups densities between quartz (011) and quartz (100): on quartz (011) (Figure 50a) the density of silanol groups is much higher than on quartz (100). On quartz (011) there is a significant overlap between water and quartz slabs and this is due to the formation of $\mathrm{H}$ bonds between surface hydroxyls and water molecules. The situation on quartz (100) is reversed. The distribution of surface groups allows for sufficiently large areas into which water molecules can adsorb on the surface. This originates the distinct black peak in Figure 50b. Water is able to occupy the void spaces bounded by the protruding silanols. Interstitial water molecules are then stabilized by water-water and silanolwater $\mathrm{H}$ bonds. 

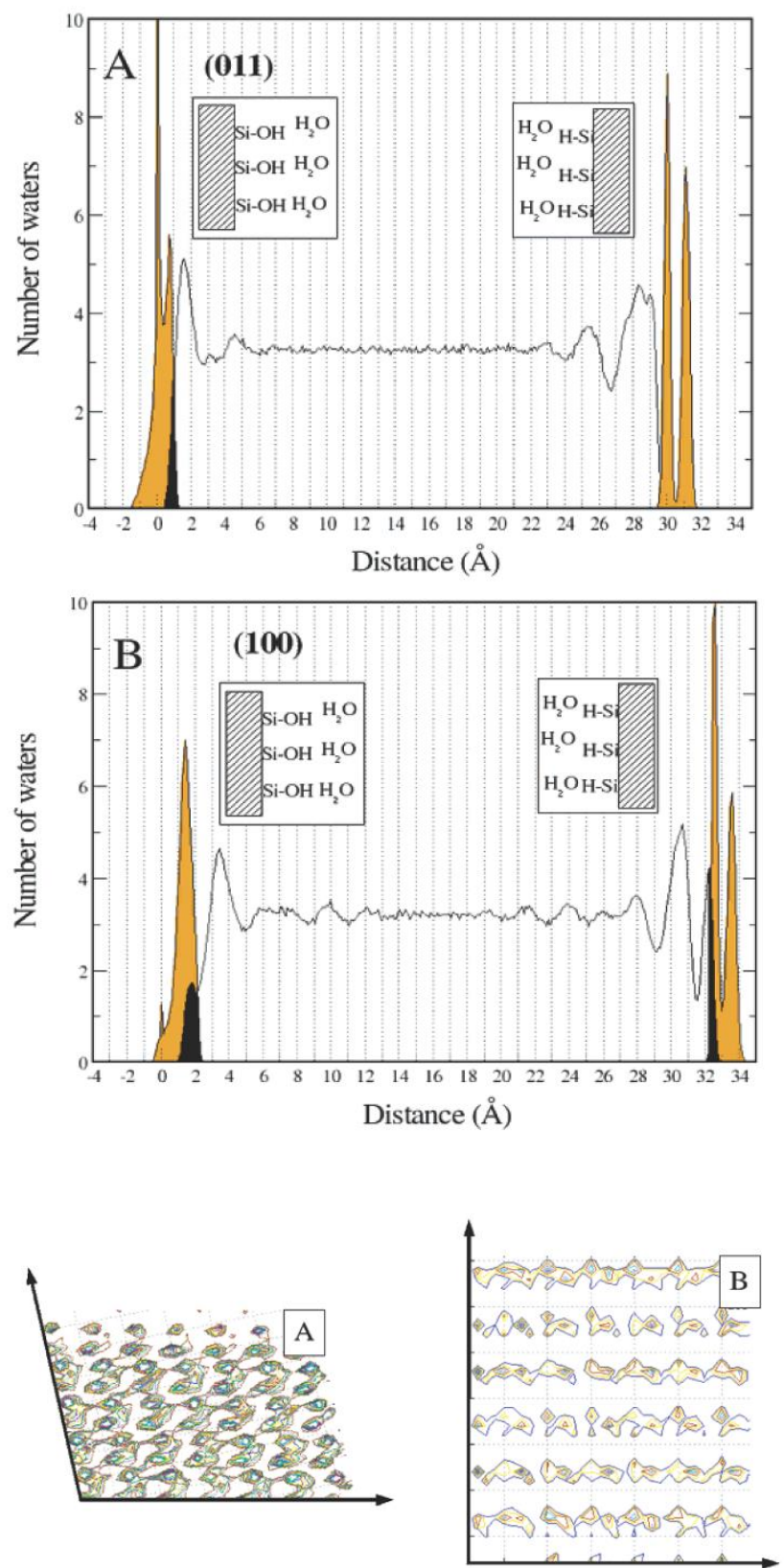

Figure 50. Top and middle: Density profiles for water adsorbed on quartz A) (011) and B) (100) surfaces. Orange represents the surface hydrogen atoms and the black areas mark the layers where surface hydrogen atoms coexist with water oxygens. Bottom: Probability density of silanols for $\mathrm{Q}(011)$ (single silanols) and Q(100) (geminal silanols) surfaces. Reprinted with permission from Ref. ${ }^{593}$ Copyright 2006 American Chemical Society.

The dynamic properties of confined water have been investigated. Water molecules close to the surface can travel smaller distances than bulk water molecules, as a result of the increased difficulty to break H-bonds with the surface or even leave the confinement areas.

The interfaces between several crystalline quartz surfaces (100), (001) and (011) and water has been investigated by Walsh et al. ${ }^{591,595}$ The water density and orientation near the surface were studied. It was found that water is rather weakly ordered and the orientation of water molecules 
relative to the plane of the bilayer is always close to random. Common features appear among the three crystalline planes: they include a favored water orientation WII, with one $\mathrm{H}$ pointing towards the surface in the interfacial water layer. This structural effect is lost at around $1.2 \mathrm{~nm}$ from the surface. Examining the interface in more detail, however, one can note differences between the three cases under study. Water penetrates into interstices between silanols on the (100) surface but not on the (001) one, due to a higher silanol density and thus, to the presence of a H-bond network on the latter. The lateral ordering of water on quartz was found to be strongly dependent on the surface, as the positions of water molecules are closely correlated to the positions of the surface silanol groups. On the (100) surface, water forms striations that run diagonally across the surface, along the direction of the [011] vector. Water on the (001) surface is arranged in rings that correspond to the underlying Si-O rings of the surface. The first water layer is denser on the $(011)$ than on the other surfaces. Accordingly, the most pronounced lateral ordering occurs in this surface. A contributing factor may be the interactions of water with the bridging $\mathrm{Si}-\mathrm{O}-\mathrm{Si}$ oxygen atoms, which are present in the surface plane. Lateral diffusion coefficients of the first layer of water were found lower than in bulk water, yet the water molecules still have some degree of mobility.

Puibasset at Pellenq ${ }^{559}$ studied four different cristobalite surfaces, the $(11-1)$ and (100) surfaces, and the (111) covered with a low (LD) and high (HD) silanol density. They found that the heat of adsorption of water at low coverage becomes less negative with increasing silanol content, with values of $-68,-61,-50$ and $-45 \mathrm{~kJ} / \mathrm{mol}$ for silanols densities of $13.5,7.8,5.5$ and $4.5 \mathrm{OH} / \mathrm{nm}^{2}$ (surfaces (111-HD), (11-1), (100) and (111-LD).

Argyris et al ${ }^{592,621}$ performed a study of the C (111) water interface using SPC model for water and a 12-6 Lennard-Jones potential for Si water interaction. Several degrees of hydroxylation were considered. In all cases, the perturbation of the water structure due to the surface interaction decreases as the distance from the solid substrate increases, bulk water properties being recovered for distances greater than $\sim 14 \AA$. The fully hydroxylated surface with $13.6 \mathrm{OH} / \mathrm{nm}^{2}$ is more hydrophilic, as evidenced by the first water layer-surface average distance $(2.15 \AA)$ than the partially hydroxylated and anhydrous surfaces (2.75 and $2.95 \AA$ respectively). On this surface, the first water layer is found massively $\mathrm{H}_{\text {down }}$ directed towards the surface. The second water layer has an opposite orientation. This is the signature of layering of water molecules with opposite orientation and is indicative of an attempt to maximize water-water hydrogen bonds. The solid substrate affects the structural properties of water, but also strongly alters its dynamic behavior: Interfacial water molecules at the fully hydroxylated surface remain longer on average in the interfacial region than water molecules at partially and nonhydroxylated surfaces. Zeitler et al $^{596}$ recently proposed a new method to calculate the accessible volume to water for complicated surfaces (e.g., those with kinks or hydroxyl groups). Zhao ${ }^{323,586}$ used a hybrid approach (DFTB) to 
model a (100) edingtonite surface in vacuum and at the interface with water. In vacuum, a significant reconstruction is observed, where silanols form H-bonds with each other. This is not the case in water, which prevents surface deformation.

More recently AIMD calculations were performed on the (0001) $\alpha$-quartz surface ${ }^{584,501,582}$ and cristobalite surfaces. ${ }^{584}$

Adeagbo et al studied the Q(001) water interface with PBE functional and Vanderbilt pseudopotentials. ${ }^{501}$ They showed that the originally clean quartz surfaces is rapidly hydroxylated by chemical reaction with the water layer and that geminal silanol groups $\left[\mathrm{Si}(\mathrm{OH})_{2}\right]$ are formed predominantly.

The bimodal acid-base behavior of the water-silica interface (exhibiting two sites of respective pKas of 8.5 and 4.5 with a ratio of $4: 1$ ) was investigated by Leung et al. ${ }^{54}$ To take into account the surface heterogeneity of amorphous silicas, as well as the lack of information of the crystalline silica-water structural modifications at the interface, six different silanol environnements were compared: hydroxylated cristobalite (100) (Figure 51a), hydroxylated cristobalite (100) with one $\mathrm{SiOH}$ removed (Figure 51b), reconstructed cristobalite (100) (Figure 51c), a molecular system (Figure 51e-d), and two distinct $\mathrm{SiOH}$ on reconstructed quartz (0001) (Figure 51f).

a)
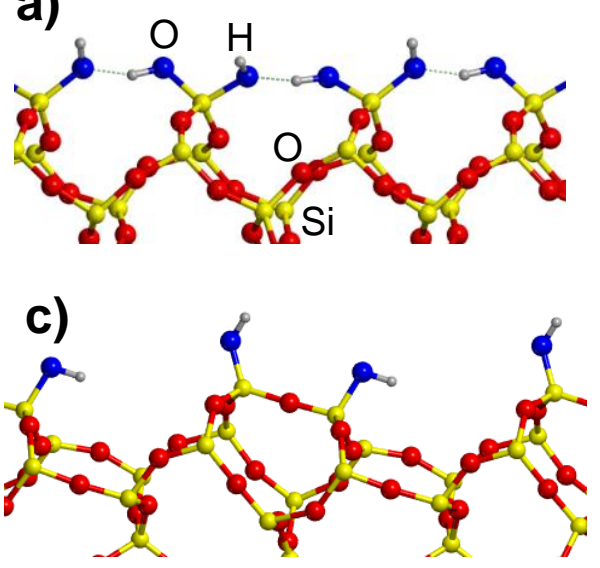

e)

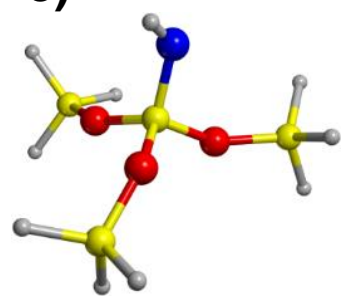

b)

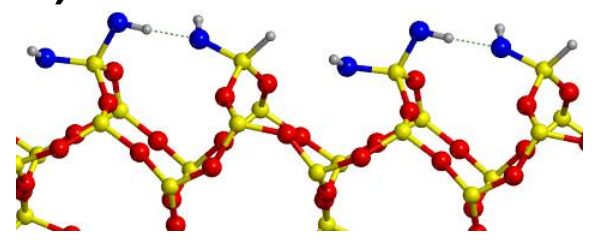

d)

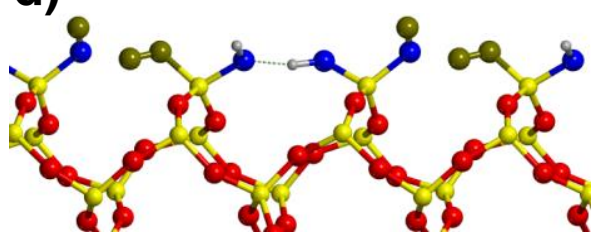

f)

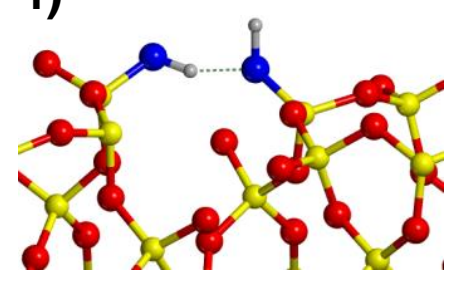

Figure 51. Systems studied in Ref. ${ }^{584}$ (a) Hydroxylated $\beta$-cristobalite (100) surface. The SiOH groups ( $\mathrm{O}$ tagged in deep blue) have $\rho(\mathrm{SiOH}) \sim 8 \mathrm{~nm}^{-2}$ and are all $\mathrm{Q}^{2}$ and $\mathrm{H}$ bonded. The calculated pKa is $7.6 \pm 0.3$. (b) Hydroxylated (100) $\beta$-cristobalite surface with one $\mathrm{OH}$ group replaced by a $\mathrm{H}$ to break the chain of hydrogen bonds. The $\mathrm{SiOH}$ near this defect $\left(\mathrm{Q}^{2}\right.$ and isolated) exhibits $\mathrm{pKa}=$ $8.9 \pm 0.3$. (c) Reconstructed (100) $\beta$-cristobalite surface, $\rho(\mathrm{SiOH}) \sim 4 \mathrm{~nm}^{-2}, \mathrm{Q}^{3}$, and isolated; $\mathrm{pKa}=$ $8.1 \pm 0.5$ (6 layers of water) and $7.0 \pm 0.4$ (4 layers). (d) The structure in panel c comes from 
removing atoms shown here in black and linking the resulting undercoordinated $\mathrm{Si}$ and $\mathrm{O}$ atoms. Panels a-d show the side view of $\sim 1.5$ simulation cells. (e) $\left(\mathrm{H}_{3} \mathrm{SiO}\right)_{3} \mathrm{SiOH}$, which is $\mathrm{Q}^{3}$ and isolated, exhibits $\mathrm{pKa}=7.9 \pm 0.5$. (f) Top half of a reconstructed (0001) quartz surface model containing cyclic silica trimers $(\mathrm{Si}-\mathrm{O})_{3}, \rho(\mathrm{SiOH}) \sim 2.3 \mathrm{~nm}^{-2}, \mathrm{Q}^{3}$, and $\mathrm{H}$ bonded; the $\mathrm{SiOH}$ on the trimer ring exhibits $\mathrm{pKa}=5.1 \pm 0.3$ while the other has $\mathrm{pKa}=3.8 \pm 0.4$ and $4.8 \pm 0.4$ depending on whether a nearby trimer ring breaks (see text). $\mathrm{Si}, \mathrm{O}$, and $\mathrm{H}$ atoms are in yellow, red, and white, respectively. ( $b$ and e) Finite-temperature AIMD snapshots with water molecules omitted for clarity; (a, c, and f) Shown at $T=0 \mathrm{~K}$.

On the $\mathrm{C}(100)$ surface, with a silanol density of $8 \mathrm{OH} / \mathrm{nm}^{2}$, the $\mathrm{SiOH}$ are all chemically equivalent. The zig zag surface $\mathrm{H}$-bonds features between silanols are preserved in the finitetemperature aqueous phase simulation. The umbrella sampling method was used to compute the $W(R)$ associated with $\mathrm{SiOH}$ deprotonation using a four-atom reaction coordinate $R$ (Figure 52a). 


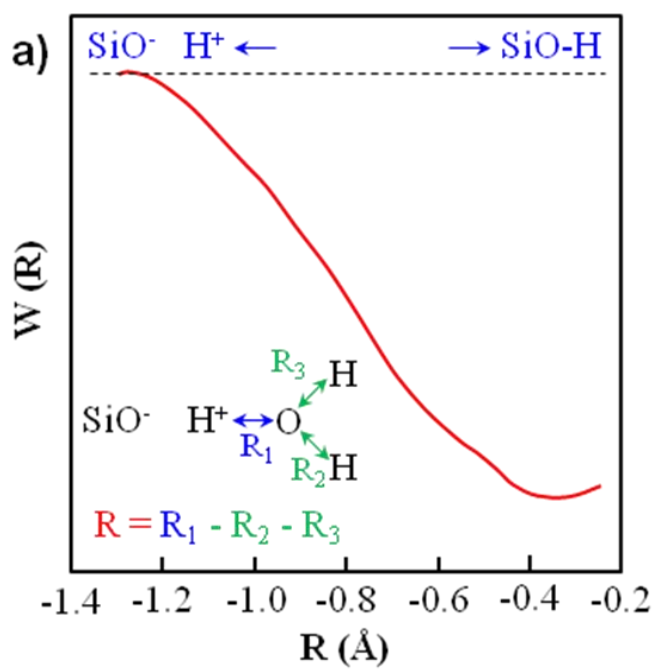

b)

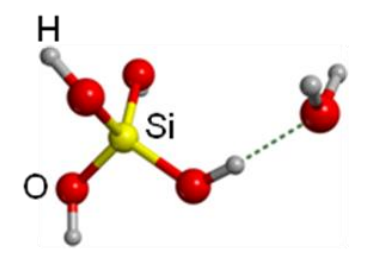

c)

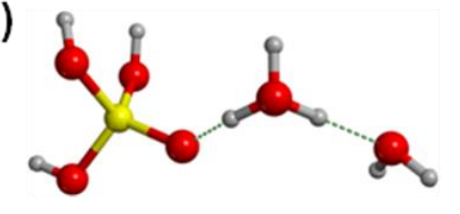

d)

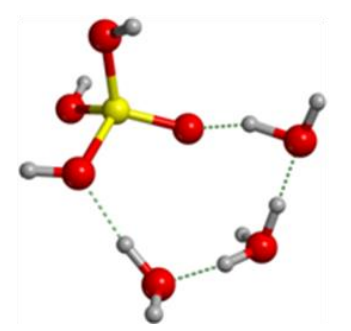

Figure 52. Umbrella method: Four-atom reaction coordinate R, illustrated for silicic acid in water but is similar for all silanol-containing species. (b-d) Snapshots from AIMD deprotonation simulations with outershell $\mathrm{H}_{2} \mathrm{O}$ molecules removed for clarity. As deprotonation proceeds, $\mathrm{R}$ progresses from intact $\mathrm{SiO}-\mathrm{H}$ ( $\mathrm{R}$ around $-0.4 \AA$, panel b), to the $\mathrm{SiO}-\mathrm{H}_{3} \mathrm{O}^{+}$contact ion pair ( $\mathrm{R}$ about -1.0 $\AA$, panel c), and then via a Grotthuss proton transfer to a solventseparated $\mathrm{SiO}-/ \mathrm{H}_{3} \mathrm{O}^{+}$ pair (R around -1.32 $\AA$, panel d). Yellow, red, white, and green spheres represent $\mathrm{Si}, \mathrm{O}, \mathrm{H}$, and the "O" atoms, respectively. The water $\mathrm{O}$ atoms which are second and third nearest neighbors to the $\mathrm{SiO}^{-}$oxygen are colored blue and pink, respectively. Adapted with permission from Ref. ${ }^{584}$. Copyright 2009 American Chemical Society.

On this surface, silanols exhibit pKa values of 7.5 and 7.7. The other surfaces, containing isolated, H-bonded, and geminal silanols all exhibit a $\mathrm{pKa}>$ 7.0. The isolated $\mathrm{SiOH}$ group on cristobalite (Figure 52b) has a pK of 8.9, and is less acidic by 1.2-1.4 pH units than when the $\mathrm{SiOH}$ 
hydrogen donor is present. In Figure 52c, a reconstructed cristobalite surface exhibits isolated silanols $\left(4 \mathrm{OH} / \mathrm{nm}^{2}\right)$, with $\mathrm{pKa}=8.1$. The authors deduced that in the range $4<\rho(\mathrm{OH})<8 \mathrm{OH} / \mathrm{nm}^{2}$, the $\mathrm{OH}$ density has no effect on pKa. All those silanol models may explain the experimental value of 8.5. To model a high acidity silanol, a reconstructed, quartz (0001) model, featuring (Si-O) $)_{3}$ (S3R), was used. It presents $2.3 \mathrm{SiOH} / \mathrm{nm}^{2}$ with two types of silanol groups which are hydrogen bonded to each other; one member of the pair resides on a cyclic trimer, while the other does not (Figure 51f). These $\mathrm{SiOH}$ groups on the strained surface exhibit $\mathrm{pKa} \sim 5.1$ and 3.8 respectively, close to the experimental value of 4.5. This study highlights the role of defective regions as the most promising candidate to explain the elusive bimodal acid-base behavior of silica surfaces.

The (001) quartz-water interface has also been recently investigated by Sulpizi et al ${ }^{582}$ using PBE-D, who confirm that the H-bond network on this surface is partially maintained: two types of silanols, "in plane" and "out of plane", are present on the surface (Figure 53). The "in plane" silanols are H-bond donor to a silanol (strong H-bond), and the "out of plane" ones are H-bond donor to water (Figure 53b). The strong in plane H-bond is maintained. Water in the interfacial layer is now only of type II, with half being strong $\mathrm{H}$-bond acceptor from silanols and half weaker $\mathrm{H}$-bond donor to silanols. In the local structure formed, a reminiscence of the dimer adsorption is observed, where, however, the H-bond between water molecules is lost. Water in the subsequent layer has a random orientation. From the ice bilayer found by Yang et al, ${ }^{504}$ only the water-silanol interaction is retained, while all water-water interactions are lost. Thus, strictly speaking, the interfacial layer cannot be referred as ice-like, as no water-water favorite lateral interactions are present. A noticeable difference between results from classical and ab initio calculations is that the former does not predict the structuring of the first water layer compared to the latter. Another AIMD study shows that the water orientation at the surface is lost at the increased temperature of $1000 \mathrm{~K}^{501}$ 

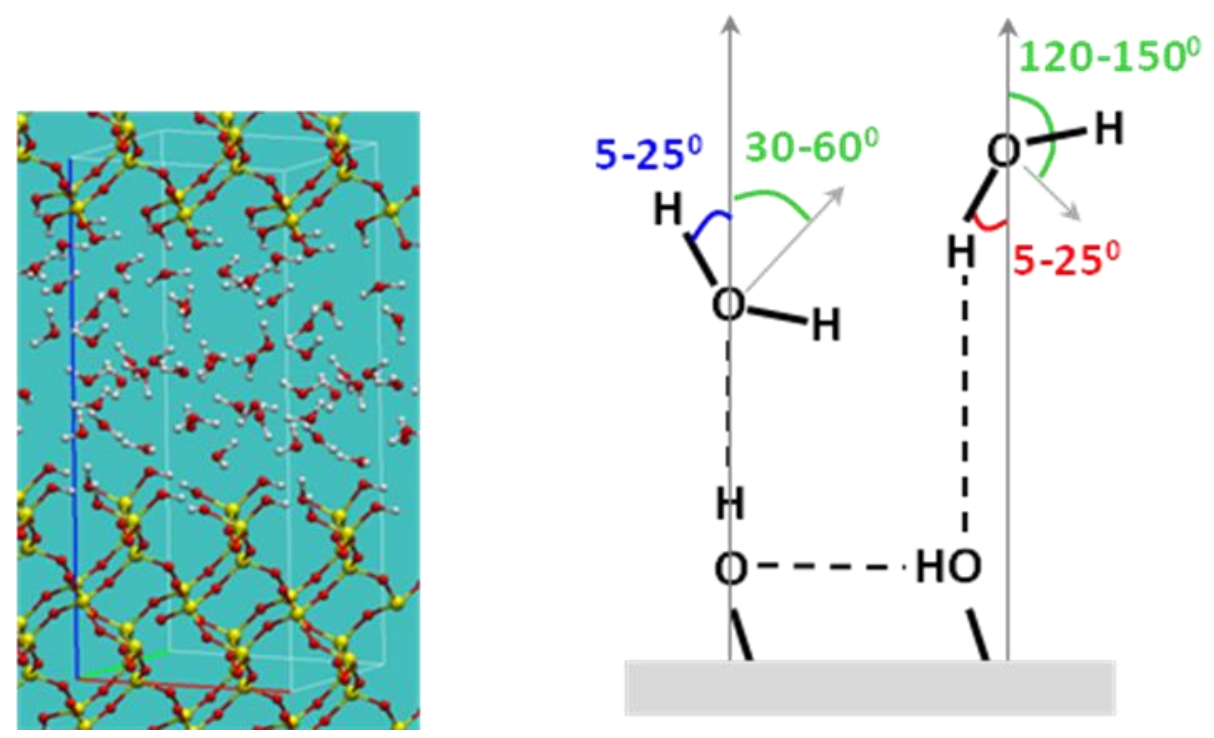

Figure 53: Quartz slab quartz-water interface and water orientation at the surface. Reprinted with permission from Ref. ${ }^{582}$. Copyright 2012 American Chemical Society.

The acidity constants of surface silanols and silicic acid are computed using the reversible proton insertion/deletion method. The most acidic silanol species, out of plane, has a calculated $\mathrm{pKa}$ of 5.6, while $\mathrm{pKa}=8.5$ for the in-plane silanol. The authors conclude that there is a bimodal distribution of silanols. However, the 1:1 ratio of the considered species does not fit the experimental value of $1: 4$.

To summarize, the final word on the nature of the acidic species on silica has not yet been written. Whereas Leung et al. open the route to defects of low silanol density and especially strained (S3R) rings on the surface, Sulpizi et al. results suggest that $\mathrm{H}$-bonds are responsible of the increase of acidity of otherwise equivalent silanols.

The "methodological gap" between AIMD and FF was bridged in the case of the (101) quartz surface. AIMD (PW91) was compared with X-ray reflectivity (XRR) experiments, and with three force fields, $\mathrm{LFF},{ }^{593}$ ClayFF, ${ }^{622}$ and $\mathrm{CWCA}^{528}$. Discrepancies between the force fields, but also between AIMD and experimental data were evidenced. ClayFF was found to better agree with AIMD, however AIMD did not allow to totally recover the XRR data. This is likely due to the overestimation of H-bonds by pure GGA, and by the absence of van der Waals forces in these AIMD calculation. One reason for the discrepancy between FF and ab initio methods is the absence of dispersion in the latter (usually included in FF) so that it is mandatory to address the above problems with a level of theory of DFT-D quality, at the least.

One can remember from this chapter that water and crystalline silicas modify each other at the interface. On the one hand, strong silanol-silanol H-bonds are not disrupted by water even at the 
quartz-liquid interface at room temperature. Indeed, a recent PBE AIMD study of water-crystalline silica interfaces ${ }^{623}$ shows that the interaction between crystalline silica surfaces and water does not necessarily cleave the pre-existent $\mathrm{H}$-bonds between the surface silanol groups. On the contrary, in some cases such as $\mathrm{Q}(100)$ and $\mathrm{C}(001)$, it is observed that $\mathrm{SiOH} \cdots \mathrm{OHSi} \mathrm{H}$-bonds are even strengthened, as the result of a mutual cooperative $\mathrm{H}$-donor/H-acceptor enhancement between silanols and water molecules. In consequence, on those crystalline surfaces, the interaction with water is highly dependent on the preexisting H-bond network. On the other hand, the surface tends to impose a preferred orientation to the first water layer, thus giving rise to water surface layers properties closer to those of solid ice than that of bulk liquid. Studies at the silica-liquid interface (generally quartz) all conclude that the effect of the surface is lost beyond three layers.

\subsubsection{Water on hydrophobic crystalline silicas}

It is well established that whereas fully hydroxylated surfaces (surface density of $4-5 \mathrm{Si}-\mathrm{OH} / \mathrm{nm}^{2}$ as measured by Zhuravlev ${ }^{78}$ ) have a hydrophilic character, highly dehydroxylated surfaces (around 1$2 \mathrm{Si}-\mathrm{OH} / \mathrm{nm}^{2}$ ) manifest hydrophobic character. ${ }^{82}$

Du et al. showed that the reconstructed dry $\alpha$-quartz (0001) surface is hydrophobic with respect to the interaction with a water molecule. ${ }^{589}$ Rignanese et al. ${ }^{500}$ showed with the PW91 GGA associated to a 50 Ry cutoff that the reconstructed dry $\alpha$-quartz (0001) surface is stable and hydroxylation of such a surface by a water molecule is energetically unfavorable. ${ }^{500}$

Tosoni et al, ${ }^{503}$ with B3LYP, used a completely dehydroxylated crystalline silica surface to model adsorption processes on highly dehydrated silica materials. The structure envisages only unstrained siloxane Si-O-Si bridges, a model not easy to devise. To do this, the authors have used a siloxane face of a 1:1 layered phyllosilicate as found in kaolinite. The surface exposes regular 6R rings (Figure 54). Its hydrophobic/hydrophilic character has been characterized by simulating the adsorption of water, from low to high water coverage. Three $\mathrm{H}_{2} \mathrm{O}$ loading has been simulated as adsorbates: (i) a single $\mathrm{H}_{2} \mathrm{O}$ molecule (one $\mathrm{H}_{2} \mathrm{O}$ /cell); (ii) a hexagonal C-ice layer derived from a proton order form of ice XI (two $\mathrm{H}_{2} \mathrm{O}$ / cell); (iii) a water monolayer (three $\mathrm{H}_{2} \mathrm{O} /$ cell). At low coverage, the oxygen atom of the adsorbed water molecule lies on the silicon atom of the slab, whereas the water protons are directed toward the siloxane oxygen atoms without a clear indication of hydrogen bonding. Adsorption is athermic. The interaction energy of $\mathrm{H}_{2} \mathrm{O}$ with the siloxane layer is indeed negligible at any coverage and for cases (ii) and (iii) the dominant factor is the lateral $\mathrm{H}_{2} \mathrm{O}-\mathrm{H}_{2} \mathrm{O}$ interaction. When dispersion is included at the B3LYP-D* level, a weak interaction is established between the siloxane slab and the adsorbed water layer. Water does indeed prefer to move away from the siloxane surface to maximize the H-bond interactions within the ice-like layer. The H-bond features resemble those of a pure ice-like layer although the mismatch between the 
siloxane unit cell and that of the free water layer causes a lengthening/weakening of the H-bonds and the interaction energy per $\mathrm{H}_{2} \mathrm{O}$ molecule is well below that in water ice (in absolute value). The free energy of adsorption at $\mathrm{P}=1 \mathrm{~atm}$, for cases ii) and iii), remains indicative of an exothermic process until the thermal energy of the molecules is high enough to break the order of the ice layer, at $\mathrm{T}=298 \mathrm{~K}$, according to the calculations. This is in qualitative agreement with IRAS and TPD measurements concerning water adsorbed on thin silica layers grown on $\operatorname{Mo}(112),{ }^{624}$ where at 140 $\mathrm{K}$ an ordered ice monolayer film was detected which becomes completely desorbed above $160 \mathrm{~K}$. 

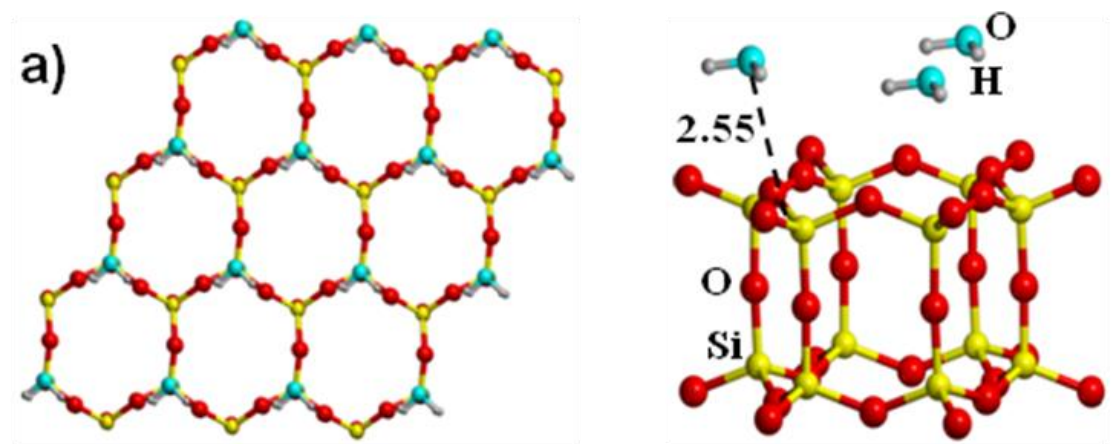

b)
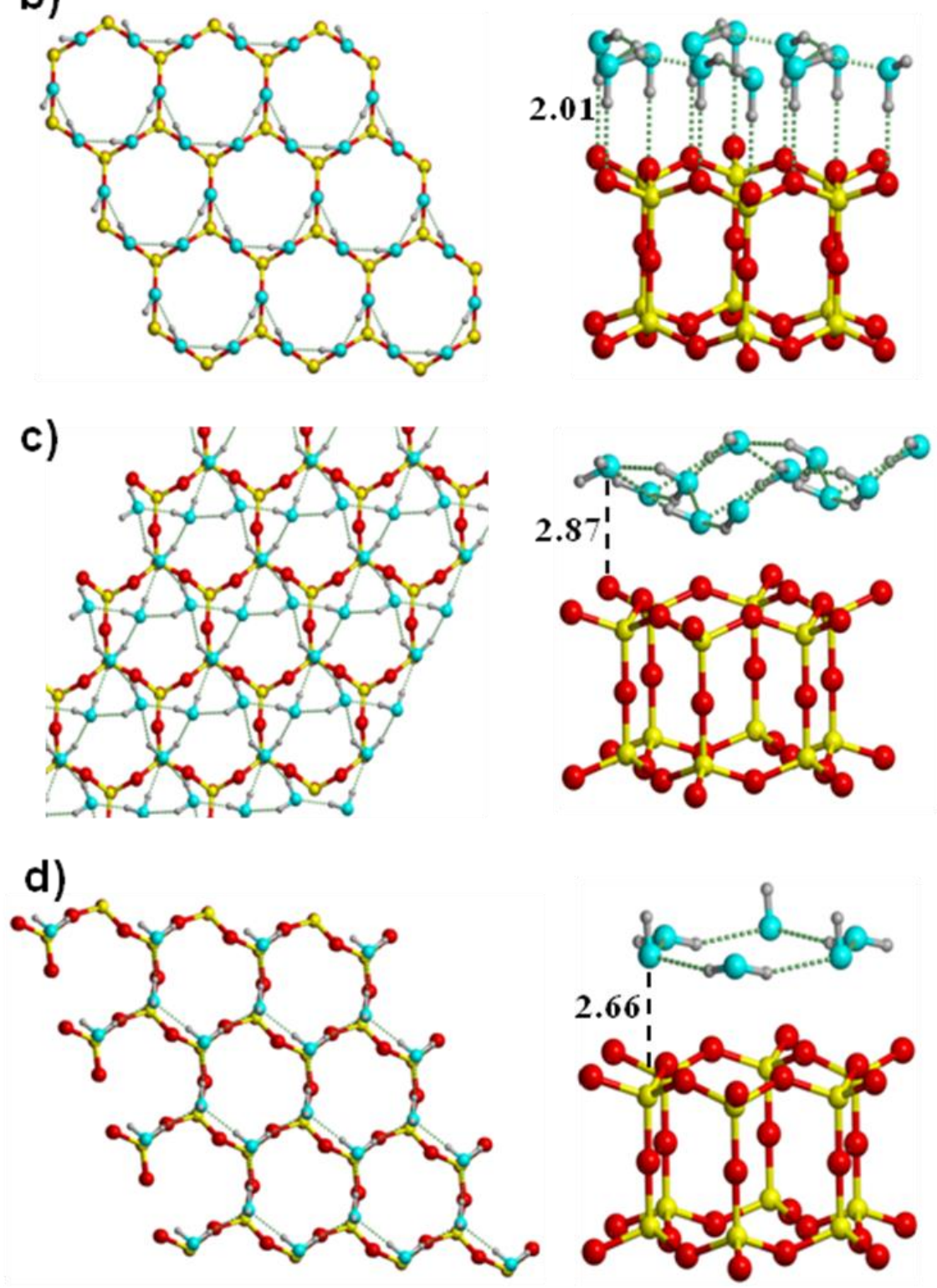

Figure 54. Water on hydrophobic surfaces. Reprinted with permission from Ref. ${ }^{503}$. Copyright 2010 American Chemical Society.

\subsubsection{Adsorption of water on crystalline defects}

As explained earlier, we will not consider in this chapter crystalline defects that are healed in reacting with water, as e.g. the two-membered ring, S2R. Theoretical ${ }^{75,625}$ studies converge with 
experimental ones (Sections 3.1.3 and 3.1.7, e.g. Ref. ${ }^{178}$ ) in suggesting the disappearance of the S2R upon reaction with water at room temperature (see Section 7.4 for a discussion of this question). However, some defects of crystalline silicas which are not healed by water adsorption, are mentioned here, as well as the reactivity with water of the $\equiv \mathrm{SiO} \bullet$ defect, which has been reported as the possible responsible for quartz toxicity.

Chen and Cheng ${ }^{626}$ have used DFT (PW91) method to study dry $(1 \times 1) \alpha$-quartz $(0001)$ surfaces that have Frenkel-like defects such as oxygen vacancies. Single water molecules, water clusters and water films were adsorbed on four defective surfaces.

-According to the calculated adsorption and bonding energies, the hydrogen bonds within water clusters or water films are stronger than the defect-water interaction. In cases of water cluster adsorption on surfaces, the defect sites do enhance the interaction between water clusters and surfaces compared to the perfect surface. In the case of a single molecule adsorption, the moleculesurface defect interaction is larger than on the perfect surface. The situation is opposite in water dimer-surface adsorption; adsorption energies on defective surfaces are smaller than on the perfect surface.

-When water films are deposited on surfaces, the energy contributions from the hydrogen bond network and from the water-silica interaction differ by an order of magnitude. Neither the single-point defect of oxygen vacancies nor the oxygen displacements alter the basic water adsorption geometry on a $(1 \times 1) \alpha$-quartz $(0001)$ surface. The calculations indicate that at low temperature and low defect density (oxygen vacancy and oxygen displacement), $\alpha$-quartz (0001) surfaces are basically hydrophobic. Therefore, a surface with a low number of strained $\mathrm{Si}-\mathrm{O}$ bonds and a low density of Frenkel defects is highly stable and not easily hydroxylated. The high stability of the water bilayer further reduces the water-defect interaction. Interestingly the dimer and the bilayer share a common feature, namely the fact that defect/surface interaction is weakened because of their intrinsic stable hydrogen bond patterns. In a review article, Yates ${ }^{627}$ made a similar conclusion for water adsorption on silica surfaces with low defect density.

Radicals on crystalline silicas were evidenced through EPR spectroscopy (see Section 3.1.7). Murashov et $\mathrm{al}^{495}$ demonstrated the facility of quartz cleavage into several faces exhibiting radicals. Musso et $\mathrm{al}^{345}$ have performed a periodic DFT study of the properties of the $\equiv \mathrm{SiO} \bullet$ radical defect on quartz, cristobalite, tridymite, and amorphous surface models. The radical defect is constructed by removing a hydrogen atom from a silanol of the surface while conserving the other features of the crystalline polymorph. Comparison between the hydroxylated and the radical slab shows that the radical defect at the surface does not induce significant tension at the crystalline structure, the Si-O bond distance associated to the radical defect being the only geometrical parameter that varies significantly $(\sim 0.04 \AA)$. The spin density analysis shows that, regardless of the 
surface, the unpaired electron is mainly localized on the radical defect and, thus, does not provide a clue to understand the different behavior between crystalline and amorphous silica regarding the $\equiv \mathrm{SiO} \bullet+\mathrm{H}_{2} \mathrm{O} \rightarrow \mathrm{SiOH}+\mathrm{OH} \bullet$ reaction. The enthalpy of this reaction is linearly dependant on the strength of the H-bond formed at the surface after the reaction (Figure 55). Finally, the enthalpy of reaction is twice less energetic on amorphous silica than on quartz, suggesting an explanation for the toxicity of quartz. However, the presence of water molecules smoothes the differences out to some extent.

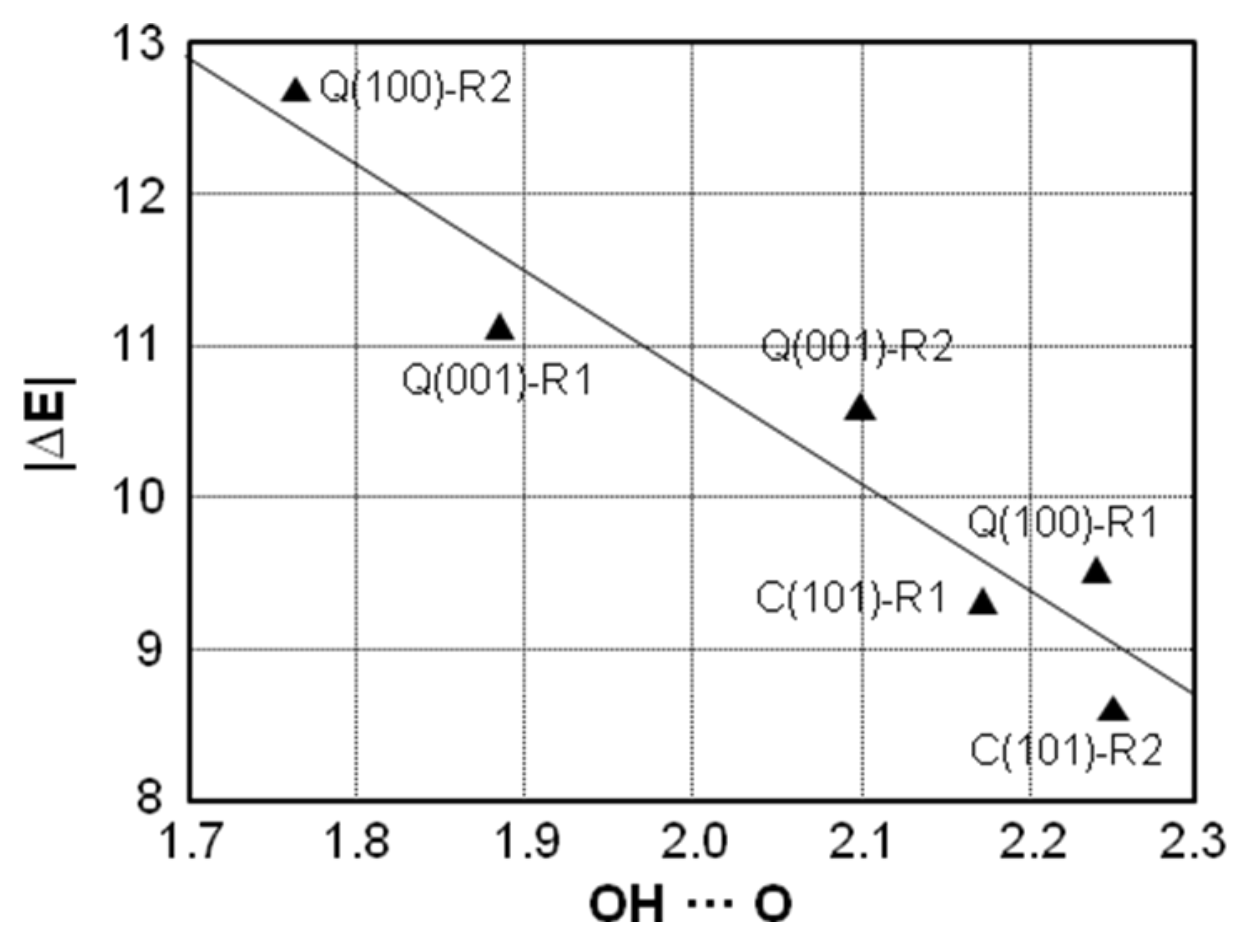

Figure 55. BHandHLYP correlation between $\Delta E$ of reaction $\mathrm{SiO} \bullet+\mathrm{H}_{2} \mathrm{O} \rightarrow \mathrm{SiOH}+\mathrm{OH} \bullet\left(\mathrm{kcalmol}^{-}\right.$ $\left.{ }^{1}\right)$ and the length $(\AA)$ of the $\mathrm{H}$-bond $(\mathrm{H}$. . O distance) formed as product in the case of $\mathrm{Q}(001)$, $\mathrm{Q}(100)$, and C(101) surfaces. Reprinted with permission from Ref. ${ }^{345}$. Copyright 2010 American Chemical Society.

\subsection{Water on amorphous hydroxylated silicas}

Remember that amorphous silicas are reported to have a hydroxyl group surface density of about 4.6 OH/nm² (Section 3.1.1). In recent years, a great amount of effort has been devoted to generate models of amorphous silica that are easy to handle with periodic ab initio methods. Chuang and Maciel $^{88}$ proposed a generalized $\beta$-cristobalite model of planes alternance where single silanols are situated on (111)-type surface segments and geminal silanols are situated on (100)-type surface segments. ${ }^{628}$ This allows to mimic a large panel of H-bonding situations, which is not possible on homogeneous crystalline surfaces. However to our knowledge, the reactivity towards water has not been envisaged on this model. 
In contrast to water/crystalline silica interface, the water/amorphous silica interface is not expected to induce an ordering of water in an ice-like structure. Actually, it has been shown that the ice-amorphous silica (native oxide film grown on $\mathrm{Si}$ ) interface already has a "quasiliquid structure" at a temperature close to the interfacial melting point. ${ }^{544}$ In other words, interfacial melting occurs at the ice-amorphous $\mathrm{SiO}_{2}$ contact, just the opposite of the phenomenon mentioned for crystalline silica interfaces. The thickness of the interfacial layer within which the chemical and physical properties of the solution are affected by the amorphous silica surface was estimated to be 0.5 $\mathrm{nm}^{629}$

For amorphous silica, the main challenge is -and remains- to describe to what extent and how the H-bond breaking may occur in the preexisting $\mathrm{H}$-bond surface network to form a new network with water. Another question concerns the well-known heterogeneity of amorphous silicas, exhibiting hydrophilic and hydrophobic zones, and finally, the link between nanoscopic reactivity and large scale measurements such as contact angles.

Table 12. Theoretical works based on classical force fields, devoted to bulk water at amorphous silicas: nature of the surface studied and methods.

\begin{tabular}{|c|c|c|}
\hline Silica surface Properties & Method & $\overline{\text { Ref. }}$ \\
\hline Hydrophobic and hydrophilic silica fully hydroxylated & LMcCR force field ${ }^{630}$ & 631 \\
\hline $\mathrm{SiO}$ and $\mathrm{SiOH}$ units with a $3: 1$ ratio & $\begin{array}{l}\text { Collins \& Catlow } \\
\text { force field }^{632,633}\end{array}$ & 634 \\
\hline $\begin{array}{l}\text { Silanol density } 3.33 \mathrm{OH} / \mathrm{nm}^{2} \text { and } \mathrm{SiO} \text { - deprotonation } \\
\text { sites }\end{array}$ & $\begin{array}{l}\text { Stillinger-Weber } \\
\text { potential }^{530} \text { for } \mathrm{Si} \text { and } \\
\mathrm{Vashista}^{53} \mathrm{al}^{531} \text { for } \\
\mathrm{SiO}_{2}\end{array}$ & 285,307 \\
\hline Surfaces of different silanol density & BKS (see Table 3) & 462,635 \\
\hline Surface with $1.3 \mathrm{OH} / \mathrm{nm}^{2}$ & BMH (see Table 3) & 524,636 \\
\hline Silanol density from 1 to $3.2 \mathrm{OH} / \mathrm{nm}^{2}$ & CC force field & 528 \\
\hline Silanol density $3.2 \mathrm{OH} / \mathrm{nm}^{2}$ & CC Force field & 637 \\
\hline Surface with $1.8 \mathrm{OH} / \mathrm{nm}^{2}$ & BMH (see Table 3) & 638 \\
\hline
\end{tabular}

\subsubsection{Hydroxylated amorphous silicas models obtained by classical MD}

The first works on water adsorption on amorphous silicas were performed by Garofalini et al. to study the adsorption mechanisms and the effect of water on the relaxation of surfaces ${ }^{464,639}$ (Table 12). However, no water adsorption on hydroxylated silica was considered. Later on, Lee and Rossky ${ }^{631}$ studied the structural and dynamic properties of water above two hydrophobic (rough and flat) and one hydrophilic silica surfaces. Warne et al. ${ }^{634}$ studied the effects of increasing the ionic strength of water confined between two amorphous silica surfaces. Unfortunately, in these works no details are given about the nature (terminal or geminal) of silanols, the $\mathrm{OH}$ density, the distribution 
of silica rings or the silica H-bond network. Cruz-Chu et al. ${ }^{528}$ simulated wetting angles of water adsorbed on silicas surfaces with 0,1 and $3.2 \mathrm{SiOH} / \mathrm{nm}^{2}$. They showed that the presence of silanols induces hydrophilicity. However, it was not possible to identify differences in wetting angles (that were equal to zero) between the surfaces with silanol densities 1 and $3.2 \mathrm{~nm}^{-2}$. Colombi Ciacchi et al. ${ }^{307}$ proposed two amorphous silica models, one obtained from bulk silica, and one ultrathin $\mathrm{SiO}_{2}$ layer on metal $\mathrm{Si}$, both having a $\mathrm{SiOH}$ density of $3.33 \mathrm{OH} / \mathrm{nm}^{2}$. The two surfaces, when immersed in water, induce a structuring of water in layers of higher and lower densities with respect to the bulk. Water penetration into the relatively open structure of the hydroxylated surfaces was also observed. The bulk water density is reached at 4-5 $\AA$ from the surfaces. Heat of immersion values of $166 \mathrm{~mJ} / \mathrm{m}^{2}$ and $203 \mathrm{~mJ} / \mathrm{m}^{2}$ for the amorphous silica and native oxide, respectively, were calculated. The first of the two values may be compared with experimentally measured values of 157 and $158 \mathrm{~mJ} / \mathrm{m}^{2}$, for surfaces with estimated densities of terminal $\mathrm{SiOH}$ groups of 2.3 and 3.4 $\mathrm{nm}^{-2} \cdot{ }^{604,605}$ Water in the first layer above amorphous silica form on average $2.54 \mathrm{H}$-bonds with water, $0.14 \mathrm{H}$-bonds with siloxane $\mathrm{O}, 0.3$ donor and 0.3 acceptor bonds with silanol groups. On the ultrathin film, the water-siloxane H-bond is increased to 0.24 . We note, as for crystalline surfaces, the occurrence of donor $\mathrm{H}$ bonds from water to siloxane that were not confirmed with ab initio studies on crystalline surfaces. However an ab initio study of an amorphous silica surface/water interface is still lacking. More recently, the same group proposed a theoretical study of water adsorption on a periodic model of amorphous silica (see Figure 56), from which a cluster containing a negatively charged $\mathrm{SiO}^{-}$group is cut. ${ }^{285} \mathrm{Ab}$ initio preliminary calculations show that water preferentially adsorbs on $\mathrm{S} \mathrm{SiO}^{-}$group with the protons pointing towards the negative charge. Force field parameters have been parametrized to fit an onset of ab initio MP2 benchmark. It is found that the heat of immersion of the negatively charged surface is highly dependent on the surface charges and of the chosen water model. 

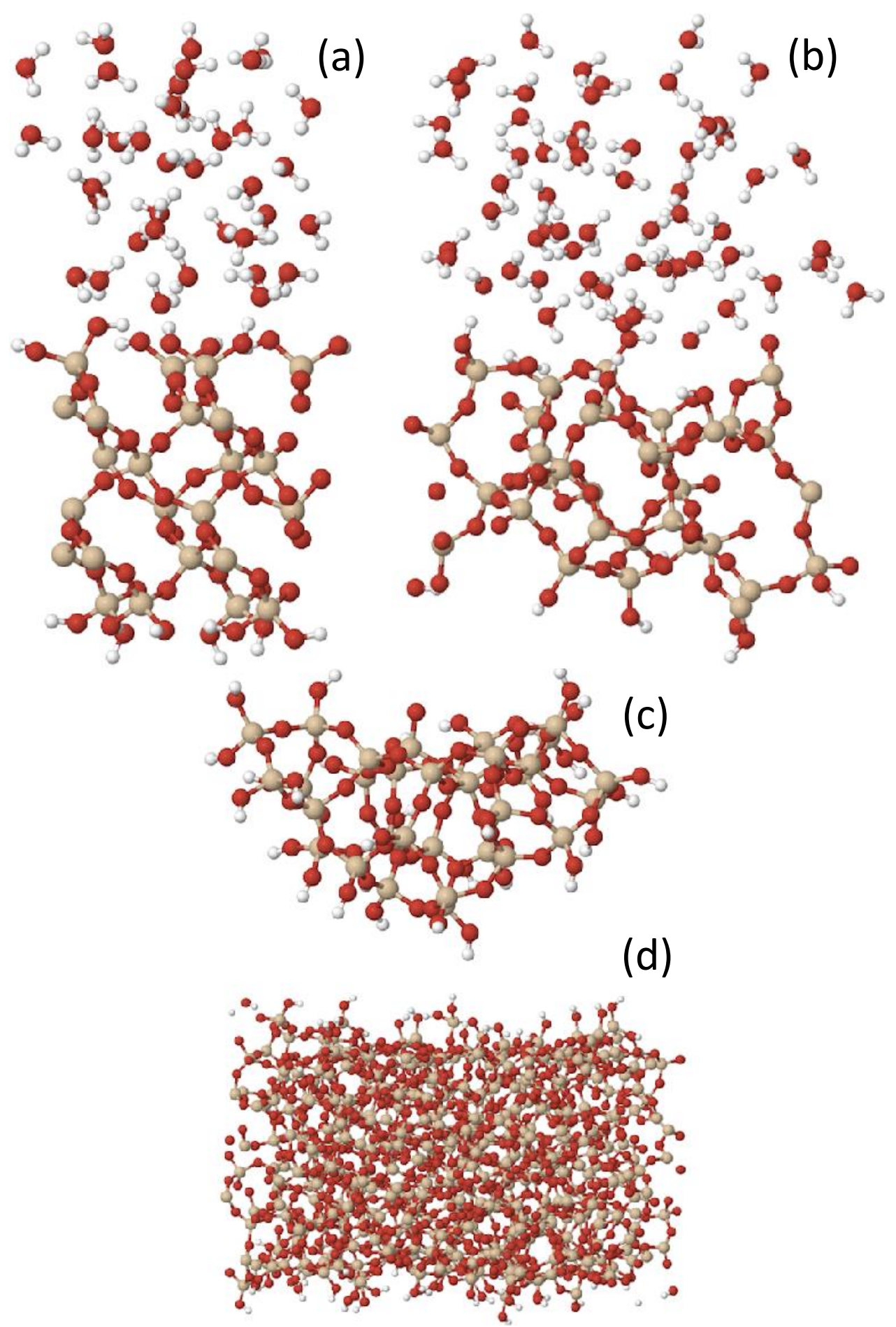

Figure 56. Model systems used for the calculation of atomic charge differences after the deprotonation of a terminal silanol group. (a) The (0001) surface of $\alpha$-quartz in contact with liquid water. (b) A small periodically repeated amorphous $\mathrm{SiO}_{2}$ slab in contact with liquid water. (c) A deprotonated amorphous $\mathrm{SiO}_{2}$ cluster carved out from the large amorphous $\mathrm{SiO}_{2}$ slab shown in (d) and terminated with $\mathrm{OH}$ groups to saturate the artificial dangling bonds. Reprinted with permission from Ref. ${ }^{285}$. Copyright 2011 John Wiley and Sons.

Hassanali and Singer ${ }^{462,463,635}$ generated an amorphous silica surface exhibiting a silanol density of $6.4 \mathrm{OH} / \mathrm{nm}^{2}$ with $70 \%$ of geminal silanols and $30 \%$ of vicinal groups, and a surface with 
4.0 OH/nm², 40\% geminal and 60\% vicinal silanols, which also had strained S2R rings. The calculated heats of immersion are 0.832 and $0.279 \mathrm{~J} / \mathrm{m}^{2}$ respectively, suggesting an increased hydrophilicity of the high $\mathrm{OH}$ density silica. This was confirmed by the analysis of the surface heterogeneity, in which patches of siloxane covered surfaces exhibit a hydrophobic character, whereas zones of high silanol densities have an increased hydrophilic character, a trend confirmed by the radial density analysis (Figure 57).
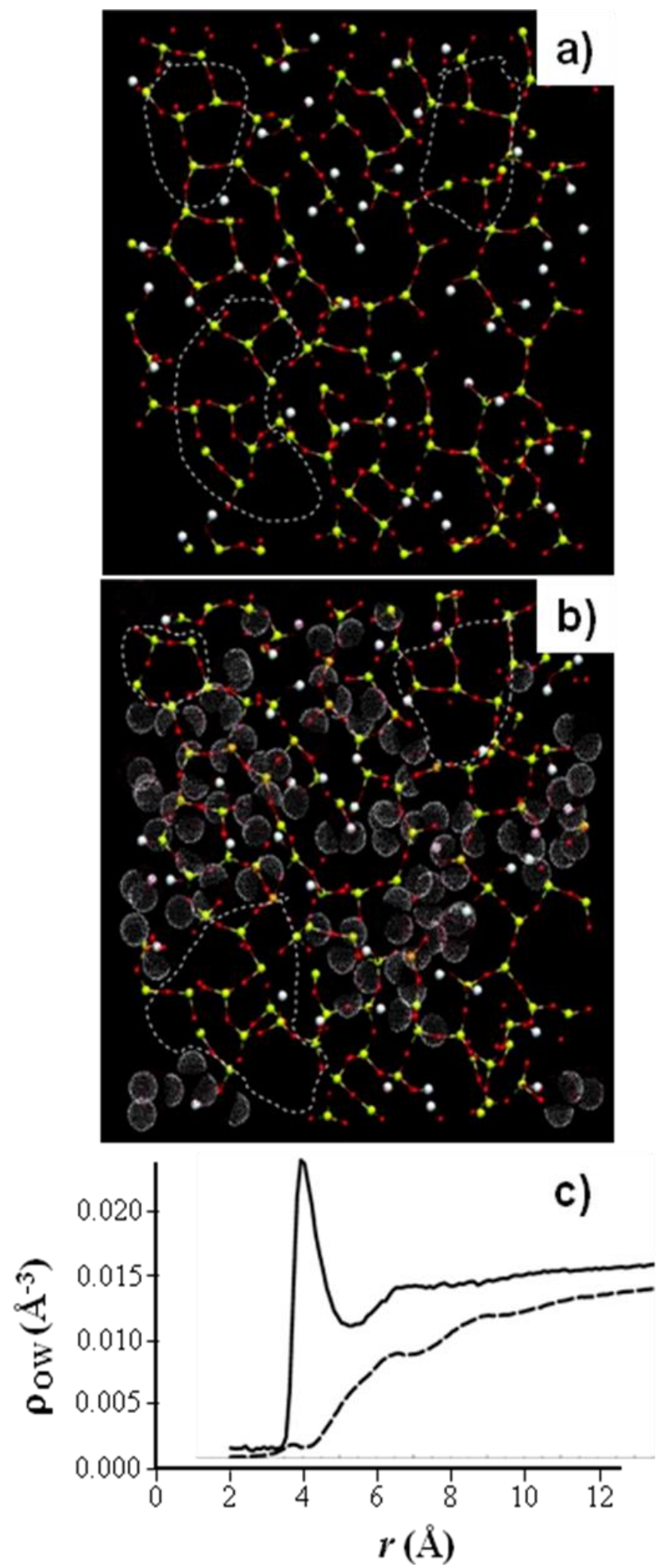
Figure 57. a) Hydrophobic and hydrophilic zones onamorphous silica, b) Radial density of water molecules surrounding silanol oxygens (solid curve) and surface siloxane (dashed curve). Reprinted with permission from Ref. ${ }^{462}$. Copyright 2007 American Chemical Society.

Floess and Murad ${ }^{637}$ studied the competitive adsorption of PDMS and water on amorphous silica. The generated amorphous silica surface features are unfortunately not described. The adsorption of water vapor is found stronger than the PDMS one, with penetration into the silica network. Water seems to clusterize on the surface rather than form a continuous film.

Leed and Pantano ${ }^{524,636}$ generated an amorphous silica surface containing a hydroxyl concentration of $1.3 \mathrm{OH} / \mathrm{nm}^{2}$, a rather low $\mathrm{OH}$ content. No local information on the $\mathrm{OH}$ distribution at the surface is provided. The heat of water adsorption on this surface ranged from -75 to -40 $\mathrm{kJ} / \mathrm{mol} .50 \%$ of the surface sites exhibit an interaction with water more negative than $-44 \mathrm{~kJ} / \mathrm{mol}$, the heat of water liquefaction. ${ }^{562}$

Chai and Yang ${ }^{638}$ modelled water/amorphous silica interaction and the wetting angle (Figure 58).

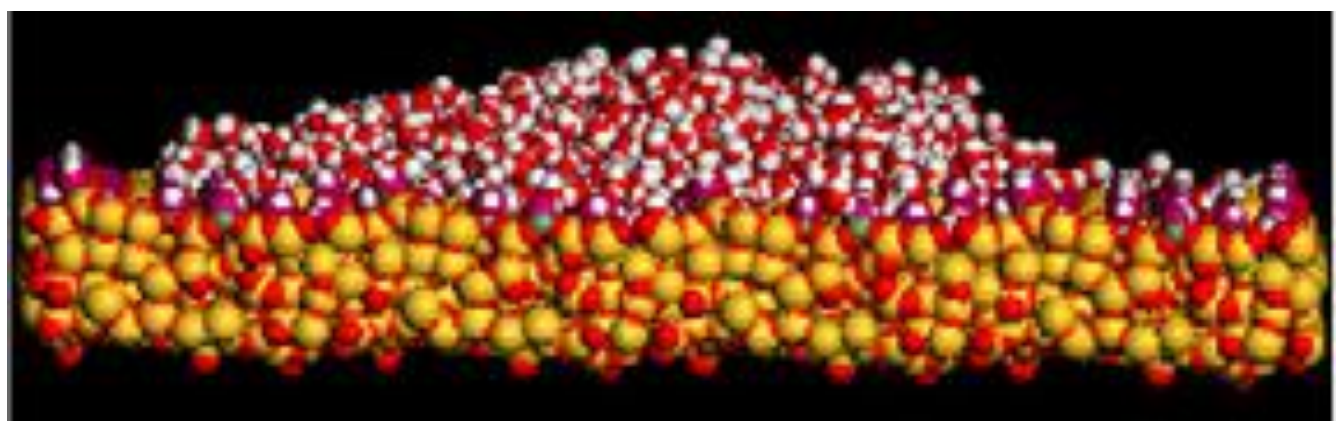

Figure 58: Wetting of amorphous silica by water. Reprinted with permission from Ref. ${ }^{638}$. Copyright 2009 Elsevier.

\subsubsection{Hydroxylated amorphous silicas models obtained by ab initio methods}

In the recent years, ab initio amorphous silica models have been proposed. Minibaev et al ${ }^{640}$ proposed a silica surface with a silanol density of $6.9 \mathrm{OH} / \mathrm{nm}^{2}$. Ab initio studies of adsorption of simple molecules on this silica gel surface allowed the most preferential adsorption positions to be found. The energies of adsorption of water with the silica gel surface ranged from $-19.3 \mathrm{~kJ} / \mathrm{mol}$ to $77.2 \mathrm{~kJ} / \mathrm{mol}$, in the most stable configuration, water is twice $\mathrm{H}$-bond acceptor from silanols and once H-bond donor.

Adsorption energies of isolated water molecules on the Tielens' model is -46 and $-50 \mathrm{~kJ}$ $\mathrm{mol}^{-1}$ on the terminal and geminal silanols respectively, showing no significant reactivity 
differences between terminal and germinal silanols. ${ }^{510}$ A more favorable energy of adsorption is calculated for water adsorption on silanol nests: a study of discrete water molecules adsorption on amorphous silica was performed by Costa et al. ${ }^{641}$ The amorphous silica surface model is inspired on the model of Masini and Bernasconi. ${ }^{507}$ This surface presents a high silanol density of $7.66 \mathrm{OH}$ per $\mathrm{nm}^{2}$, partitioned in $66 \%$ geminal silanols, and $34 \%$ terminal-associated ones. AIMD of water molecules evenly spread on the whole silica surface resulted in water clusterization and concentration on the parts of the surface richest silanols (Figure 59). The mean energy of adsorption of water on this surface is $-61 \mathrm{~kJ} \mathrm{~mol}^{-1}$, slightly more negative than that found on isolated silanols. Poly-H-bonded silanols have been reported to exhibit an increased acidic character by comparison with mono- and di-H-bonded silanols. ${ }^{200,642}$

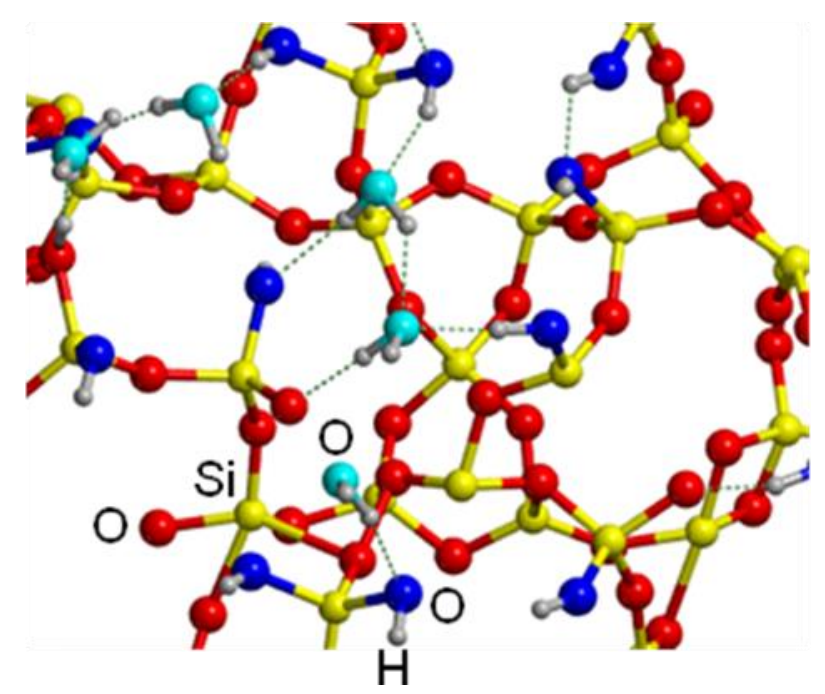

Figure 59. Water cluster adsorbed on amorphous silica. Drawn with data from Ref. ${ }^{641}$

In conclusion, silica-water interface represents a complex system to be studied because of the subtle interplay between complementary/competing forces (hydroxyl-hydroxyl network vs water-hydroxyls interactions) on one hand, and intrincate $\mathrm{H}$-bond patterns occurring at the amorphous silica surfaces, on the other hand. One clear point is that $\mathrm{Q}^{2}$ and $\mathrm{Q}^{3}$ develop the same attitude to $\mathrm{H}$-bond with adsorbed water molecules and can be considered to exhibit the same Brönsted acidity. Experimental data reveal that the silica interface has a structuring effect on the adsorbed water layer. Ab initio studies show that water adsorption is efficiently described by its interactions with the preexisting H-bond pattern: water may either break or cooperatively reinforce this pattern. The $\mathrm{OH}$ density and nature are two factors affecting the preexisting $\mathrm{H}$-bond pattern. $\mathrm{Ab}$ initio MD appears to be the most promising tool to reveal silica surface properties related to the acid/base character of its surface sites. Furthermore, results from AIMD MD allow developing and 
validating new force fields, which in turn permit to study the properties of more complex model systems and for a longer time evolution.

\section{Interaction with Biomolecules}

Understanding the interaction between biomolecules and inorganic materials is of extraordinary relevance due to its direct implications in several fields such as biotechnology, nanotechnology, biomaterials, biomineralization and drug delivery as mentioned in the Introduction. ${ }^{261}$ The practically relevant properties of, for instance, a protein/surface system, are mediated by protein adhesion at the surface, which in turn is dictated by molecular recognition. Deep knowledge of the biomolecule/surface interactions would indeed allow the design and/or the functionalization of biocompatible materials in a more rational fashion, since they could then be prepared with a particular target in mind. Silica surfaces are particularly suitable for these purposes. Furthermore, in a prebiotic chemistry context, it was suggested long ago and reviewed recently ${ }^{205}$ that mineral surfaces could have played a role in the polymerization of biomonomeric building blocks to form the first biopolymers. Although Si in the Earth's crust is primarily found in silicates that contain cations of crucial relevance in the condensation processes, studying the biomolecule/silica surface interactions constitutes the first step in understanding the role of such surfaces in concentrating, immobilizing, selecting and organizing the relevant raw biomolecules in the reactions. Additionally, peptide/silica surface interactions may induce conformational changes, which may stimulate the peptide to show a specific bioactivity lacking in its unfolded state. Such an activation of the potentially "hidden" bioactivity might have triggered the first biocatalytic reactions in a primordial Earth, hence giving rise to the emergence of metabolic cycles, a crucial aspect for the origin of life. ${ }^{643}$

Useful information for the above mentioned purposes can be provided by answering the following questions: i) which regions of the biomolecule are responsible for the biomolecule/silica surface interactions, and why? ii) is the biomolecule/surface contact direct or through solvent water molecules? iii) among the different surface sites present in a given biocompatible material, does the biomolecule show any preferential interaction and why? iv) does the biomolecule undergo significant conformational changes upon adsorption? Most of this information can be obtained by carefully analyzing the biomolecule/silica interface region.

From the modeling point of view, works purporting to study the interactions between full biomacromolecular systems and silica surfaces, which normally include the presence of water solvent, were carried out with classical molecular dynamics. Studying this kind of systems at an ab initio level, despite the fast progress in computer architecture and codes development, is not yet feasible because of the enormous complexity of the systems, which would render calculations 
extremely costly. However, useful information about biomolecule/silica surface systems at an ab initio level can be obtained by using drastically simplified biosystems; e.g., amino acids, small peptides, nucleobases, base-pairs, etc. Additionally, this procedure allows focusing the problem in a more oriented way, for instance, from a set of amino acids, determining those more prone to interact with a particular silica surface feature.

Among biomolecules, proteins are by far the most studied systems and thus, results involving proteinaceous-based systems have significant presence in this section. This section is organized as follows. First, a review of the results concerning amino acids interaction with silica surfaces will be provided, both at the gas phase-silica and solution-silica interfaces, paying particular attention to glycine, the simplest amino acid. Second, we will focus on peptides and proteins adsorbed on silica, and finally, we will address the interaction of other biomolecules (i.e., DNA-derivatives), drugs and natural products with silica surfaces.

\subsection{Interaction with amino acids}

\subsubsection{The glycine case}

\subsubsection{Gas-phase interaction}

Glycine (Gly, shown in Figure 60), is the simplest amino acid and is ideal to model AA/silica systems because the defining functionalities of an amino acid are not obscured by the lateral substituent. For years, Gly was the only known natural amino acid to sublimate before decomposing so that chemical vapor deposition (CVD) on silica (and on other solid surfaces as well) allowed to study experimentally the intrinsic Gly/silica surface features, free from the influence of water, which resulted in a fertile ground for a fruitful interplay between theory and experiments. Currently, the fast thermal heating techniques allow obtaining neutral amino acids in the gas phase. ${ }^{644,645}$ 


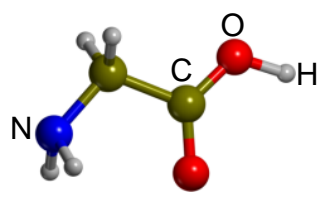

glycine

(Gly)

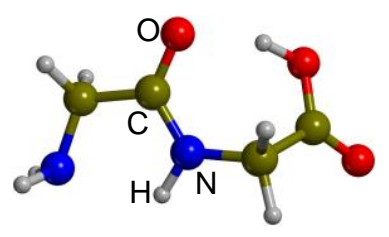

peptide bond

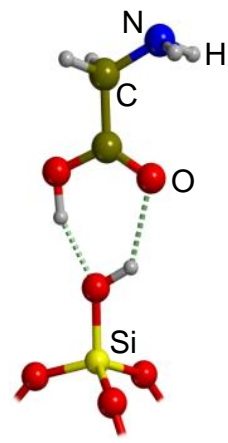

H-bonding

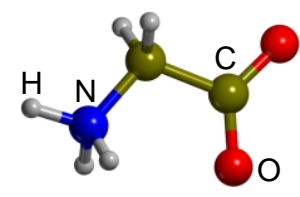

zwitterionic glycine

(Gly-zwitt)

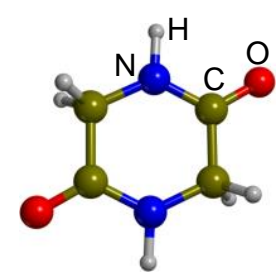

diketopiperazine

(DKP)

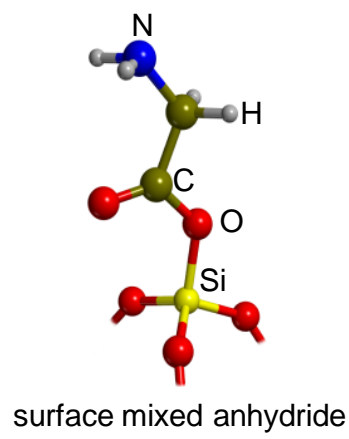

(SMA)

Figure 60. Chemical systems relevant for glycine adsorption mentioned in this review.

The works of Groenewegen et al. ${ }^{646}$ and Basiuk et al. ${ }^{647}$ are two seminal studies focused on the interaction of Gly vapors with silica surfaces outgassed at $650-700{ }^{\circ} \mathrm{C}$ using IR spectroscopy measurements. More recently, Lomenech et al. ${ }^{223}$ reported the FTIR spectrum of the adsorption of Gly by CVD on a silica sample pretreated at $100^{\circ} \mathrm{C}$. Because some of the Gly IR absorption modes strongly overlap with vibrational modes of silica or residual water (in particular, the $v(\mathrm{OH})_{\mathrm{COOH}}$ and the $\delta(\mathrm{HNH})$ Gly modes are obscured by the the $v(\mathrm{OH})$ of $\mathrm{Si}-\mathrm{OH}$ and the $\delta(\mathrm{HOH})$ of water, respectively), the $v(\mathrm{C}=\mathrm{O})$ mode is usually used as fingerprint to characterize Gly adsorption onto silica surfaces due to its sharp and intense band. The experimental $v(\mathrm{C}=\mathrm{O})$ vibrational mode for Gly in the gas-phase was measured by Stepanian et al. ${ }^{648}$ by entrapping Gly in a cold Ar matrix, resulting in a value of $1784 \mathrm{~cm}^{-1}$. Table 13 collects the $v(C=O)$ registered in these works and the assignments made by the authors. 
Table 13. Reported stretching $v(\mathrm{C}=\mathrm{O})$ values $\left(\right.$ in $\mathrm{cm}^{-1}$ ) and the assignments made by the authors. Figure 60 shows the chemical systems associated to these assignments.

\begin{tabular}{lll}
\hline Authors & $v(\mathrm{C}=\mathrm{O})$ & Assignment according to the authors \\
\hline Groenewegen et al. & 1740 & "Surface mixed anhydride" \\
& 1670 & H-bonding \\
\hline Basiuk et al. & 1760 & "Surface mixed anhydride" \\
& 1720 & H-bonding \\
& 1670 & Peptide bond \\
\hline Lomenech et al. & & \\
\hline
\end{tabular}

Both Groenewegen et al. ${ }^{646}$ and Basiuk et al. ${ }^{647}$ observed one band at 1720 and $1740 \mathrm{~cm}^{-1}$ region, respectively, which was associated to the formation of the "surface mixed anhydride", i.e., of grafted glycine $\equiv \mathrm{Si}_{\text {surf }} \mathrm{O}-\mathrm{C}(=\mathrm{O})-\mathrm{CH}_{2} \mathrm{NH}_{2}$ (SMA, see Figure 60). As mentioned in Section 3, this kind of surface feature should form if Gly condensates with a silanol surface group releasing water; i.e., $\equiv \mathrm{Si}-\mathrm{OH}+\mathrm{HOOC}-\mathrm{CH}_{2} \mathrm{NH}_{2} \rightarrow \equiv \mathrm{Si}_{\text {surf }} \mathrm{O}-\mathrm{C}(=\mathrm{O})-\mathrm{CH}_{2} \mathrm{NH}_{2}+\mathrm{H}_{2} \mathrm{O}$. In contrast, Lomenech et al. ${ }^{223}$ did not observe any band at this region. The three works assigned $v(\mathrm{C}=\mathrm{O})$ bands corresponding to Gly interacting with $\mathrm{SiOH}$ groups via H-bonding, although discrepancies concerning the position were also reported. Finally, a value of $1670 \mathrm{~cm}^{-1}$ was found only in the Basiuk's spectra, which was interpreted as the $\mathrm{C}=\mathrm{O}$ stretch corresponding to a peptide bond (see Figure 60 ), suggesting that silica surfaces catalyzed the peptide bond formation.

The FTIR spectra recorded by Lomenech et al., ${ }^{223}$ both for the naked silica powder and for the Gly/silica prepared by CVD, within the $2000-1300 \mathrm{~cm}^{-1}$ region are shown in Figure 61. In addition to the above mentioned $1699 \mathrm{~cm}^{-1}$ band, two sharper bands at 1423 and $1371 \mathrm{~cm}^{-1}$, probably resulting from the $\delta(\mathrm{HCH})$ and $\delta(\mathrm{CCH})$ modes, respectively, were attributed to Gly adsorption, as they were not present in the spectrum of bare silica. The other two bands present in the two spectra $\left(1861\right.$ and $\left.1630 \mathrm{~cm}^{-1}\right)$ were assigned to the $v_{\text {as }}(\mathrm{SiOSi})$ and $\delta(\mathrm{HOH})$ frequencies of adsorbed water, respectively, although in the latter a contribution from the $\delta(\mathrm{HNH})$ mode was also tentatively assigned. No bands associated to SMA were observed. 


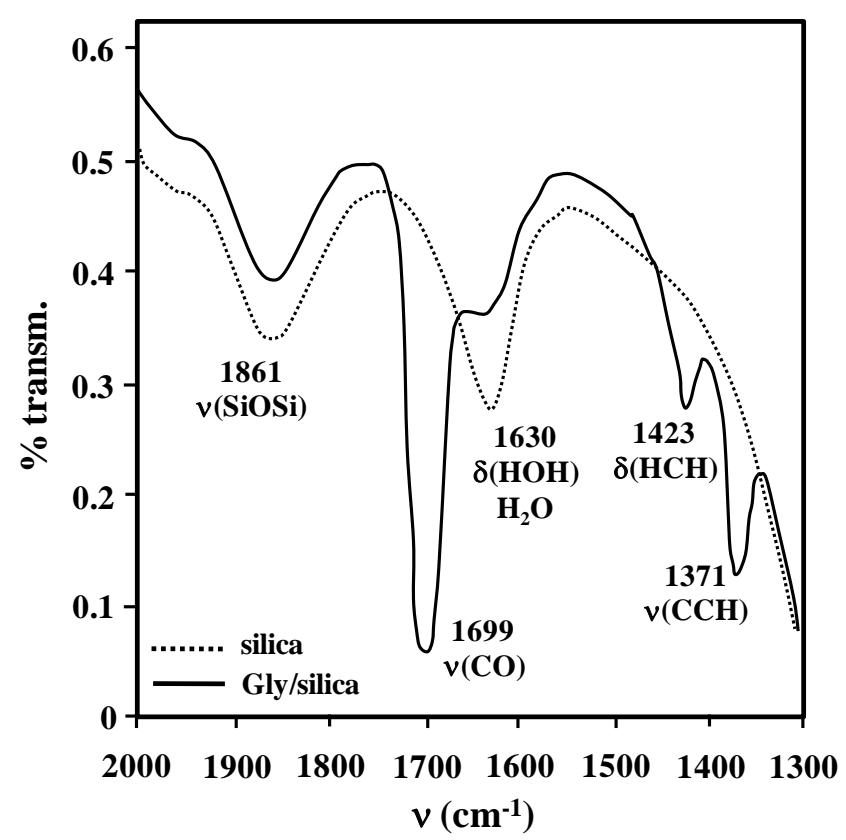

Figure 61. FTIR spectra of the naked silica powder (dotted line spectrum) and Gly/silica prepared by CVD (solid line spectrum). Adapted with permission from Ref. ${ }^{223}$. Copyright 2005 John Wiley and Sons.

It was suggested that fully hydroxylated silica surfaces may act as solid solvents, since the presence of a large amount of $\mathrm{Si}-\mathrm{OH}$ groups may have the same effect as a large number of water molecules. This can be checked by analyzing the presence of adsorbed Gly zwitterion, ${ }^{+} \mathrm{H}_{3} \mathrm{NCH}_{2} \mathrm{COO}^{-}$(Gly-zwitt, see Figure 60) in the Gly/silica spectrum of Figure 61. Results indicated no signs of adsorbed Gly-zwitt. Indeed, neither the bending modes of $\mathrm{NH}_{3}{ }^{+}$, at approximately 1617 and $1523 \mathrm{~cm}^{-1}$, nor the $\mathrm{COO}^{-}$stretching modes, which appear at lower values than those in $\mathrm{C}=\mathrm{O}(\mathrm{OH})$, were present in the spectrum. Thus, from the gas-phase, Gly adsorbs in its canonical form (Gly-can), indicating that the silanol groups present at the surface are not sufficient to make silica behave as a solid solvent and transform Gly-can into Gly-zwitt.

A final interesting point to analyze in Lomenech's spectra (Figure 61) is whether other transformed forms of Gly are present. The $v(\mathrm{C}=\mathrm{O})$ bands due to a peptide bond and the diketopiperazine (DKP, shown in Figure 60) cycle were not present. The formation of DKP, which takes place by cyclodimerization of two Gly molecules at $150-200{ }^{\circ} \mathrm{C}$ with the emission of two $\mathrm{H}_{2} \mathrm{O}$ molecules, could be ruled out as the ring stretching mode at $1471 \mathrm{~cm}^{-1}$, the most conspicuously sign for DKP, was not present. Both absences indicate that no Gly condensation proceeds on the surfaces in the conditions used.

Despite the very interesting results provided by these IR data, several questions still remain open, such as: i) what is the actual picture of Gly adsorbed on a silica surface at a molecular level? ii) why are there discrepancies in the $v(\mathrm{C}=\mathrm{O})$ value for the $\mathrm{H}$-bonding interacting form between the 
different works? iii) why is the surface mixed anhydride observed in some works and not in others? Answers to these questions have been provided in works based on quantum mechanics.

In the same work of Lomenech et al. ${ }^{223}$ B3YLP calculations on the Gly interaction with minimal clusters $\left(\mathrm{H}_{3} \mathrm{SiOH}, \mathrm{H}_{2} \mathrm{Si}(\mathrm{OH})_{2}\right.$ and $\mathrm{H}_{4} \mathrm{Si}_{2} \mathrm{O}(\mathrm{OH})_{2}$ simulating isolated, geminal and vicinal sites, respectively) were also reported. Calculations allowed performing a complete search for all possible H-bonded complexes between one single Gly and a variable number of silanol groups, representing the complex distribution of $\mathrm{OH}$ groups at the surface of amorphous silica. On the other hand, Rimola et al. ${ }^{74,309}$ simulated at the B3LYP level the adsorption of Gly on the (001) surface of edingtonite terminated with isolated hydroxyl groups, using both a cluster approach and a full periodic treatment. The structures that best match the experimental results as well as the computed adsorption energies $\left(\Delta \mathrm{E}_{\mathrm{ads}}\right)$ are shown in Figure 62. Table 14 summarizes the comparison between the computed and the experimental $v(\mathrm{C}=\mathrm{O})$ values.

Table 14. Comparison between the experimental and computed $v(\mathrm{C}=\mathrm{O})$ values $\left(\mathrm{in}_{\mathrm{cm}}{ }^{-1}\right)$ reported in the literature. The structural interpretation made by the authors is defined in the "Interaction pattern/Model" column and shown in Figure 62.

\begin{tabular}{|c|c|c|c|}
\hline Experimental & Computed & Interaction pattern/Model & Authors \\
\hline \multirow[t]{3}{*}{1699} & $1694^{a}$ & double H-bonding/periodic & Rimola et al. ${ }^{309}$ \\
\hline & $1701^{b}$ & double H-bonding/minimal cluster & Lomenech et \\
\hline & & & al. $^{223}$ \\
\hline \multirow[t]{4}{*}{1720} & $1717^{a}$ & Single H-bonding/Periodic & Rimola et al. ${ }^{309}$ \\
\hline & $1724^{c}$ & Single H-bonding/large cluster & Rimola et al. ${ }^{309}$ \\
\hline & $1723^{b}$ & Single H-bonding/minimal cluster & Lomenech et \\
\hline & & & $\mathrm{al}^{223}$ \\
\hline \multirow[t]{2}{*}{1760} & $1767^{c}$ & Surface mixed anhydride/large cluster & Rimola et al. $^{74}$ \\
\hline & $1769^{a}$ & Surface mixed anhydride/ periodic & Rimola et al. ${ }^{74}$ \\
\hline
\end{tabular}

Computational details for: ${ }^{a} \mathrm{~B} 3 \mathrm{LYP} / \mathrm{double}-\zeta$ and a scaling factor of $0.957 ;{ }^{b} \mathrm{~B} 3 \mathrm{LYP} / 6-31++\mathrm{G}(\mathrm{d}, \mathrm{p})$ and a scaling factor of $0.961 ;{ }^{c} \mathrm{~B} 3 \mathrm{LYP} / 6-31+\mathrm{G}(\mathrm{d}, \mathrm{p})$ and a scaling factor of 0.975 . 


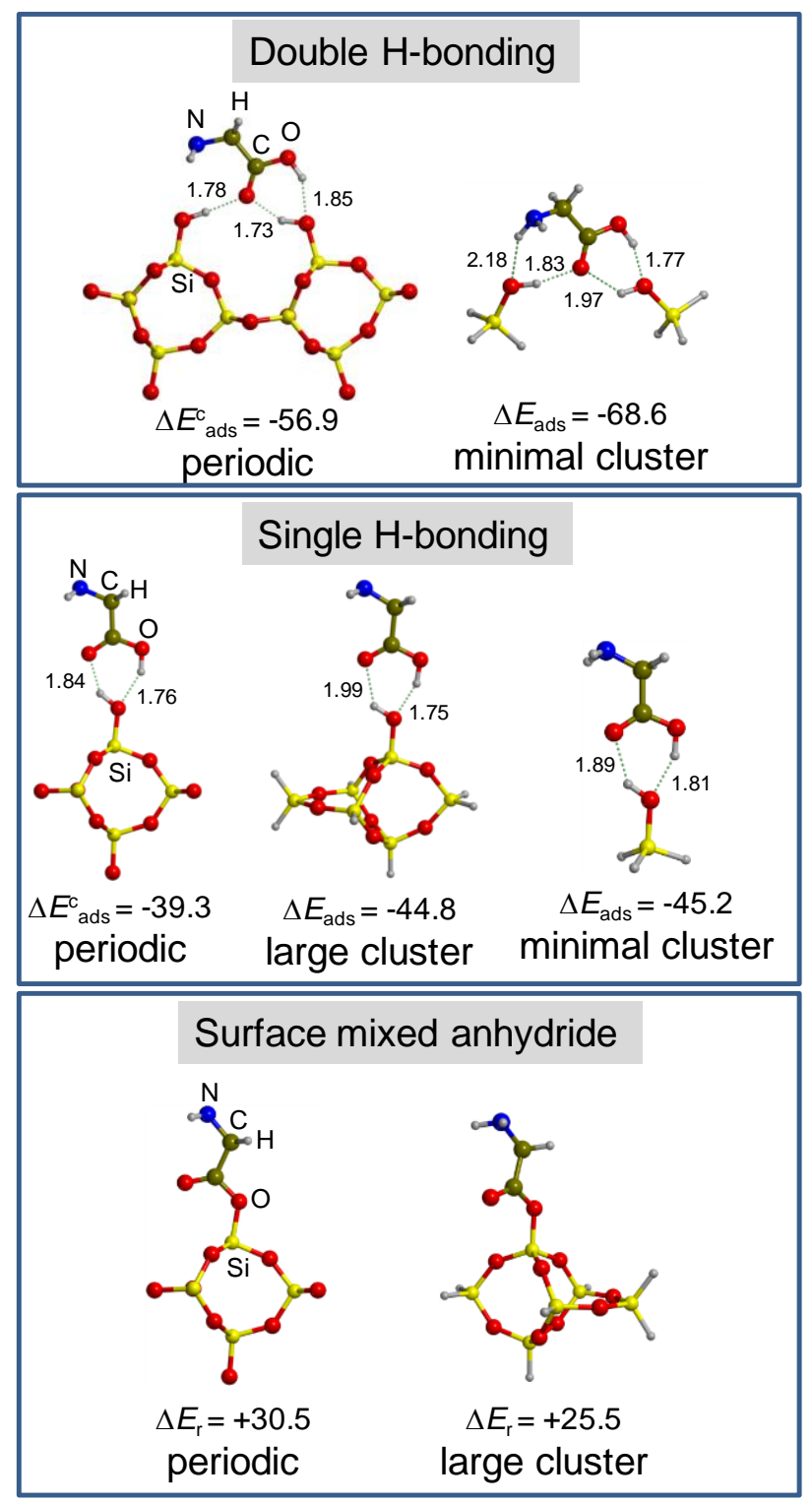

Figure 62. Different Gly/silica surface complexes obtained from ab initio calculations: "periodic" means calculations performed at the B3LYP/double- $\zeta$ level using a periodic silica surface model; "minimal cluster" means calculations performed at the B3LYP/6-31++G(d,p) level using minimal cluster models; "large cluster" means calculations performed at the B3LYP/6-31+G(d,p) level using a larger cluster model than the minimal ones. Computed adsorption energies (in $\mathrm{kJ} \mathrm{mol}^{-1}$ ) are also reported: $\Delta \mathrm{E}_{\mathrm{ads}}$ and $\Delta \mathrm{E}_{\text {ads }}^{\mathrm{C}}$ refer to adsorption energies without and with the basis set superposition error corrected, respectively. $\Delta \mathrm{E}_{\mathrm{r}}$ refers to the reaction energy (in $\mathrm{kJ} \mathrm{mol}^{-1}$ ) for the formation of the surface mixed anhydride ( $\left.\equiv \mathrm{Si}_{\text {surf }} \mathrm{OH}+\mathrm{HOOC}-\mathrm{CH}_{2}-\mathrm{NH}_{2} \rightarrow \equiv \mathrm{Si}_{\text {surf }} \mathrm{O}-\mathrm{C}(=\mathrm{O})-\mathrm{CH}_{2}-\mathrm{NH}_{2}+\mathrm{H}_{2} \mathrm{O}\right)$. The bond distances reported are in $\AA$. Drawn from data of Refs. ${ }^{74,223}$

The value of $1699 \mathrm{~cm}^{-1}$ represents the maximum bathochromic shift $\left(-85 \mathrm{~cm}^{-1}\right)$ of the $v(\mathrm{C}=\mathrm{O})$ fingerprint mode and results from the $\mathrm{H}$-bonding with two different terminal silanol groups (see "double H-bonding" interaction pattern in Figure 62). Theoretical results indicate that the interaction of Gly with silica surfaces may also take place with only one $\mathrm{SiOH}$ group through the "single H-bonding" interaction pattern (see Figure 62). The computed $v(\mathrm{C}=\mathrm{O})$ values for Gly adopting this adsorption mode $\left(1717,1724\right.$ and $1723 \mathrm{~cm}^{-1}$, for periodic, minimal and large cluster 
models, respectively) agree fairly well with the experimental value of $1720 \mathrm{~cm}^{-1}$. Noticeably, the "single H-bonding" interaction pattern corresponds to a cooperative $\mathrm{H}$-bond similar to that commonly found for carboxylic acid dimers; i.e., the $\mathrm{COOH}$ group is envisaged in two H-bonds with one silanol group, one in which the glycine $\mathrm{OH}$ group acts as a $\mathrm{H}$-bond donor and another one in which the $\mathrm{C}=\mathrm{O}$ group acts as a $\mathrm{H}$-bond acceptor. Such a cooperative effect is responsible for the significant bathochromic shift observed for $v(C=O)$ (experimentally, $-64 \mathrm{~cm}^{-1}$ ). Remarkably, the computed Gly/silica complexes that exhibit these two H-bond patterns were found to be the most stable adducts. Differences between the experimental results of Basiuk et al. ${ }^{647}$ and those of Lomenech et al. $^{223}$ can be related to the different pretreatment of the silica surfaces. The latter authors pretreated the silica sample at $100^{\circ} \mathrm{C}$ overnight, while the former ones outgassed the silica sample at about $700{ }^{\circ} \mathrm{C}$. Silanol population is smaller in the silica sample treated at $700{ }^{\circ} \mathrm{C}$ because at high temperatures silica surfaces undergo silanol condensations. Thus, isolated silanol groups are predominant in Basiuk's silica and the interaction with Gly takes place via the "single H-bonding" interaction pattern. In contrast, Lomenech's silica, richer in silanol groups (both in amount and diversity), is able to adsorb Gly via two contiguous surface silanol groups adopting the "double $\mathrm{H}$ bonding" interaction pattern.

Basiuk et al. ${ }^{647}$ also observed a band at $1760 \mathrm{~cm}^{-1}$, which was associated to the $v(\mathrm{C}=\mathrm{O})$ mode of the SMA bond. Calculations addressed to check whether this band could correspond to this surface functionality indicated that this is indeed the case. The computed $v(\mathrm{C}=\mathrm{O})$ values using both the "large cluster" and the "periodic" surface models were found at 1767 and $1769 \mathrm{~cm}^{-1}$, respectively (see Table 14). ${ }^{309}$ Nonetheless, theoretical calculations also revealed that SMA formation from reacting Gly with an isolated $\mathrm{SiOH}$ has a reaction free energy $(\mathrm{T}=298 \mathrm{~K})$ of +25.5 $\mathrm{kJ} \mathrm{mol}^{-1}$; that is, the reaction is thermodynamically disfavored. However, as already discussed in a previous section, a pretreatment of the silica sample at $700{ }^{\circ} \mathrm{C}$ causes silanol condensation processes, which as we have previously explained may result in the formation of some strained surface defects such as $(\mathrm{SiO})_{2}$ or $\mathrm{S} 2 \mathrm{R}$, and $(\mathrm{SiO})_{3}$ or S3R. Rimola et al. ${ }^{541}$ have theoretically studied the reactivity of S2R defects with Gly to give the SMA. Calculations indicated that this reaction is indeed feasible as the computed free energy barrier and reaction free energy were found to be about 54 and $-63 \mathrm{~kJ} \mathrm{~mol}^{-1}$, respectively. This is probably due to the opening of the very strained S2R ring. Moreover, these results provide a plausible explanation of why Basiuk et al. ${ }^{647}$ observed the formation of the silica SMA whereas Lomenech et al. ${ }^{223}$ did not. That is, in Basiuk's surfaces, after pretreatment at $700{ }^{\circ} \mathrm{C}$, some S2R defects were probably present, which reacted with vapors of Gly during the CVD experiments to form the SMA bond; in contrast, Lomenech's silica surface was pretreated at $100{ }^{\circ} \mathrm{C}$ so that such strained defects were probably not produced and consequently SMAs were not able to form. 
Noticeably, calculations agree with the experimental observations about the fact that the Gly/silica surface interactions do not induce the preferential adsorption of Gly-zwitt over Gly-can. Figure $63 \mathrm{a}$ and Figure 63b show that attempts to optimize Gly-zwitt with the presence of one $\mathrm{SiOH}$ group (structures on the left) evolved towards Gly-can (structures of the right). ${ }^{309,556}$ In fact, adsorption of Gly-zwitt is only determined to be favorable at large coverages ${ }^{309}$ due to stabilizing lateral interactions (Figure 63c), similarly to what occurs in the Gly molecular crystal. ${ }^{649,650}$ This brings about a rather strong $\mathrm{H}$-bond cooperativity between adsorbed Gly molecules that helps increasing the electrostatic interactions between them up to a limit in which adsorbed zwitterionic forms are stable. It is worthy to mention that this structure can only be found with a periodic treatment; otherwise the lateral interactions between adjacent Gly molecules, crucial for the stabilization of Gly-zwitt, cannot be accounted for.

a)
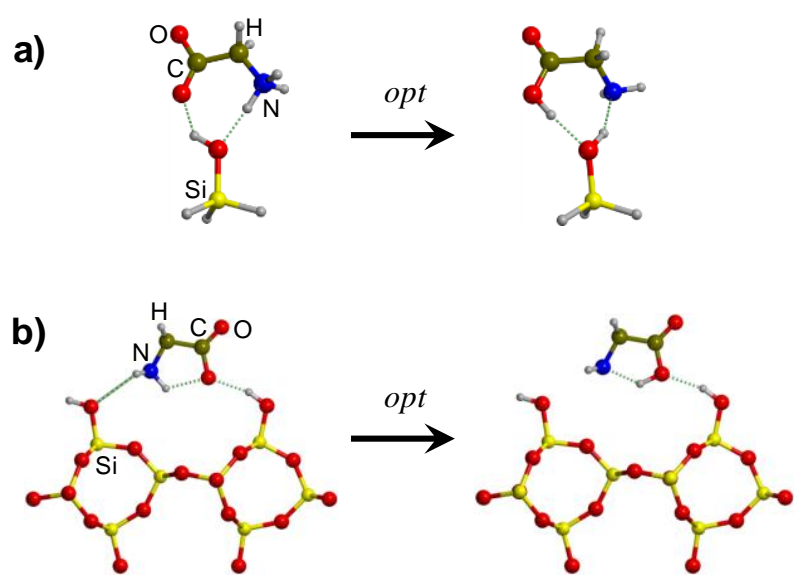

c)

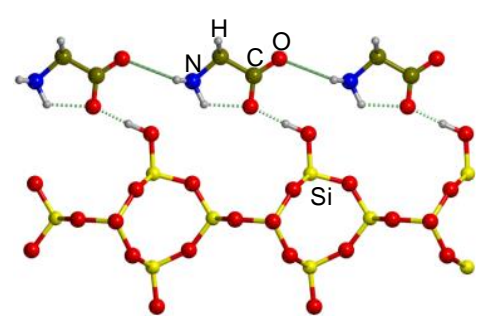

Figure 63. (a) and (b): attempts to optimize glycine in its zwiettrionic state when interacting with one $\mathrm{SiOH}$ group. Results indicated that glycine evolved towards its canonical state; (c): zwitterionic glycine found as a stable form only when considering a high loading adsorption. Drawn from data of Refs. ${ }^{309,556}$

Similarly, from theoretical results in Ref. ${ }^{223}$ and Ref. ${ }^{556}$ it is confirmed that an increment of the $\mathrm{SiOH}$ groups interacting with Gly induces a significant stabilization of Gly-zwitt over Gly-can (see Figure 64). It is expected that with four $\mathrm{SiOH}$ groups Gly-zwitt would become more stable than the canonical form. However, a glycine molecule interacting with four $\mathrm{SiOH}$ group is not physically sound, as it would mean a silica surface with an unreasonable high density of silanols. Along this line, Han and Sholl ${ }^{651}$ found that Gly-zwitt adsorbed on a periodic model of a fully hydroxylated $\alpha$-quartz (0001) surface, featured to exhibit $\mathrm{H}$-bonded geminal $\mathrm{SiOH}$ groups, was 
practically isoenergetic with the analogous canonical system, in both cases four $\mathrm{SiOH}$ groups were involed in the Gly binding.

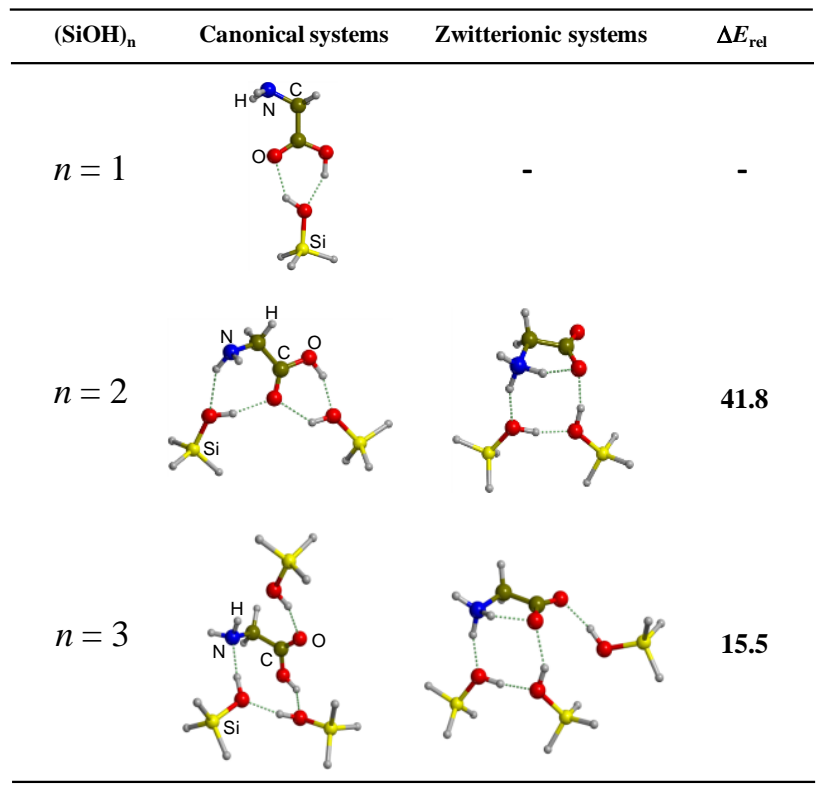

Figure 64. Relative energies $\left(\Delta E_{\mathrm{rel}}\right.$, in $\left.\mathrm{kJ} \mathrm{mol}^{-1}\right)$ between canonical glycine and zwitterionic glycine, as a function of the number of $\mathrm{SiOH}$ groups (Drawn from data of Ref. ${ }^{223}$ ). Computed at B3LYP/6$31++\mathrm{G}(\mathrm{d}, \mathrm{p})$.

All the structures shown above simulate the interaction of Gly with silica surfaces considering isolated, vicinal and interacting silanol groups. However, geminal silanols are also present on silica surfaces. Geminal silanol groups, although being present in a lesser degree than the others, have been reported to exhibit a slightly more acidic character (see Sections 3.1.2 and 8.2.6) and because of that the interaction of Gly with these silanol groups also deserves to be studied. Adopting the minimal $\mathrm{Si}(\mathrm{OH})_{4}$ cluster model, Lomenech et al. ${ }^{223}$ computed at B3LYP/6$31++\mathrm{G}(\mathrm{d}, \mathrm{p})$ level the complex shown in Figure $65 \mathrm{a}$, as a first attempt to picture the $\mathrm{Gly} / \mathrm{SiOH}$ geminal interface, in which the $\mathrm{COOH}$ establishes a cooperative $\mathrm{H}$-bond with two geminal $\mathrm{OH}$ groups. 


\section{a)}
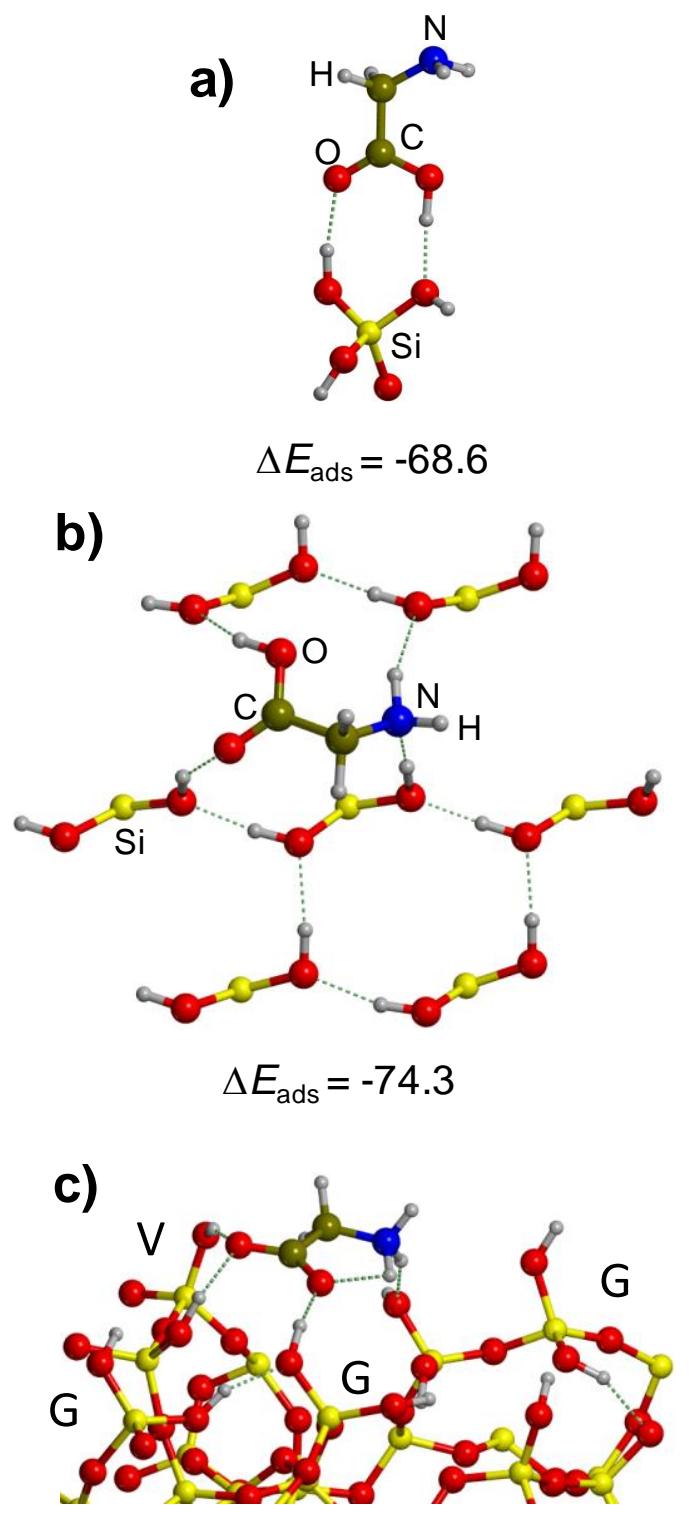

$$
\Delta E_{\mathrm{ads}}=-141.8
$$

Figure 65. (a): Optimized structure and computed adsorption energy $\left(\Delta \mathrm{E}_{\mathrm{ads}}\right.$, in $\left.\mathrm{kJ} \mathrm{mol}^{-1}\right)$ at B3LYP/6-31++G for Gly interacting with a minimal cluster $\left(\mathrm{Si}(\mathrm{OH})_{4}\right)$ model representing geminal silanol groups (drawn with data from Ref. ${ }^{223}$ ). (b): Optimized structure and computed adsorption energy at PBE for Gly interacting with the hydroxylated (0001) $\alpha$-quartz surface (drawn with data from Ref. $\left.{ }^{651}\right)$; (c): The dominant configuration obtained from several ab initio molecular dynamics simulations (PBE method and $\mathrm{T}=300 \mathrm{~K}$ ) for Gly interacting with a silica surface model rich in geminal silanol groups (drawn with data from Ref. $\left.{ }^{641}\right)$. The computed adsorption energy $\left(\Delta \mathrm{E}_{\mathrm{ads}}\right.$, in $\mathrm{kJ} \mathrm{mol}^{-1}$ ) was obtained by performing a geometry optimization onto this configuration at the B3LYP/6-311++G(d,p) level. V and G refers to vicinal and geminal silanol groups, respectively.

Han and Sholl ${ }^{651}$ also calculated at the PBE level the most stable structure of Gly adsorbed on the hydroxylated $\alpha$-quartz (0001) surface (see Figure 65b). For this case, two H-bonds present on the pristine surface were destroyed in order to form the four H-bonds that bind Gly onto the surface. In a more recent work, Costa et al. ${ }^{641}$ computed the interaction of Gly on a highly hydroxylated amorphous silica surface $\left(7.66 \mathrm{OH}\right.$ per $\mathrm{nm}^{2}$ ) particularly dominated by geminal $\mathrm{SiOH}$ groups ( 5.1 per $\mathrm{nm}^{2}$, with a ratio of about $66 \%$ of the total surface $\mathrm{SiOH}$ ). Calculations were based 
on ab initio molecular dynamics simulations carried out at $\mathrm{T}=300 \mathrm{~K}$ using the $\mathrm{PBE}$ density functional method. The most stable configuration obtained is represented in Figure 65c. As one can observe, Gly was found as Gly-zwitt, the carboxylate groups being engaged by the surface through two vicinal and one geminal $\mathrm{SiOH}$ groups (labeled as $\mathrm{V}$ and $\mathrm{G}$, respectively) and the ammonium being $\mathrm{H}$-bonded the $\mathrm{O}$ atom of a geminal $\mathrm{SiOH}$. The authors interpreted that the stabilization of the Gly-zwitt could be due to two factors: i) the large amount of silanol groups present on the surface, which facilitated the formation of several H-bonds with Gly (about 4); ii) the increased acidic character of the geminal $\mathrm{SiOH}$, which enabled to form more favorable electrostatic interactions with negative charge densities, as the one present in the carboxylate group. Therefore, according to these theoretical results, it seems that highly hydroxylated silica surfaces $\left(7-8 \mathrm{OH}\right.$ per $\mathrm{nm}^{2}$ ) may indeed act as solid solvents. However, this statement should be taken with caution considering that it is generally accepted that the silanol density for fumed silica is approximately $4-5$ per $\mathrm{nm}^{2}$ and the geminal silanol density is considerably lower than the one exhibited by the surface model used by Costa et al. ${ }^{641}$ In fact, no traces of Gly-zwitt were found in the experimental Gly/silica IR spectrum. Despite that, these results are of relevance for studies using silicas formed in conditions of high water activity such as the biogenic ones, in which the density of $6.2 \mathrm{OH} \mathrm{nm}^{-2}$ has been quoted. Additionally, precipitated silica, where $\mathrm{SiOH}$ densities between $7-8$ per $\mathrm{nm}^{2}$ have been reported, might be the most probably relevant materials present in the prebiotic earth so that these results gain significance in a prebiotic context.

\subsubsection{Interaction in presence of water.}

All the works commented so far dealt with the adsorption of Gly under strict gas phase conditions; namely, in absence of water. Nonetheless, water is ubiquitous in biological media so the adsorption of glycine in wet conditions is of great relevance. Lambert and coworkers have investigated in depth ${ }^{210,224,247,652}$ Gly adsorption on amorphous silica from aqueous solutions at different initial concentrations and pHs, by means of different spectroscopic techniques such as XRD, diffuse reflectance infrared Fourier transform spectroscopy (DRIFTS), solid state nuclear magnetic resonance (ssNMR) and thermoprogrammed desorption coupled to mass spectrometry (TPD-MS). From these works, three main conclusions can be drawn, which are exposed below:

i) A molecularly adsorbed Gly species coexists with the $\alpha$-and $\beta$-glycine crystalline forms.

Glycine is known to crystallize as three polymorphs: $\alpha$-, $\beta$ - and $\gamma$-glycine (those other forms found only in extreme conditions are not included). In these three polymorphs Gly is found in its zwitterionic state and the local environments of these zwitterions differ only by specific H-bonding arrangements. The ${ }^{13} \mathrm{C}$ NMR chemical shifts in the carboxylate region (170-178 ppm) for the $\alpha-, \beta$ - and $\gamma$-glycines are well differentiated: 176.4, 175.4 and 
174.5, respectively. Considering that the reproducibility of the chemical shifts is better than $0.05 \mathrm{ppm}$, ssNMR is therefore a suitable technique to discriminate between these and other Gly forms.

The obtained ${ }^{13} \mathrm{C}$ cross polarization magic angle spinning (CP-MAS) NMR spectra focused on the carboxylate region for Gly/silica with low and high Gly concentrations ( $\mathrm{pH} 6$ -7) are shown in Figure 66a.

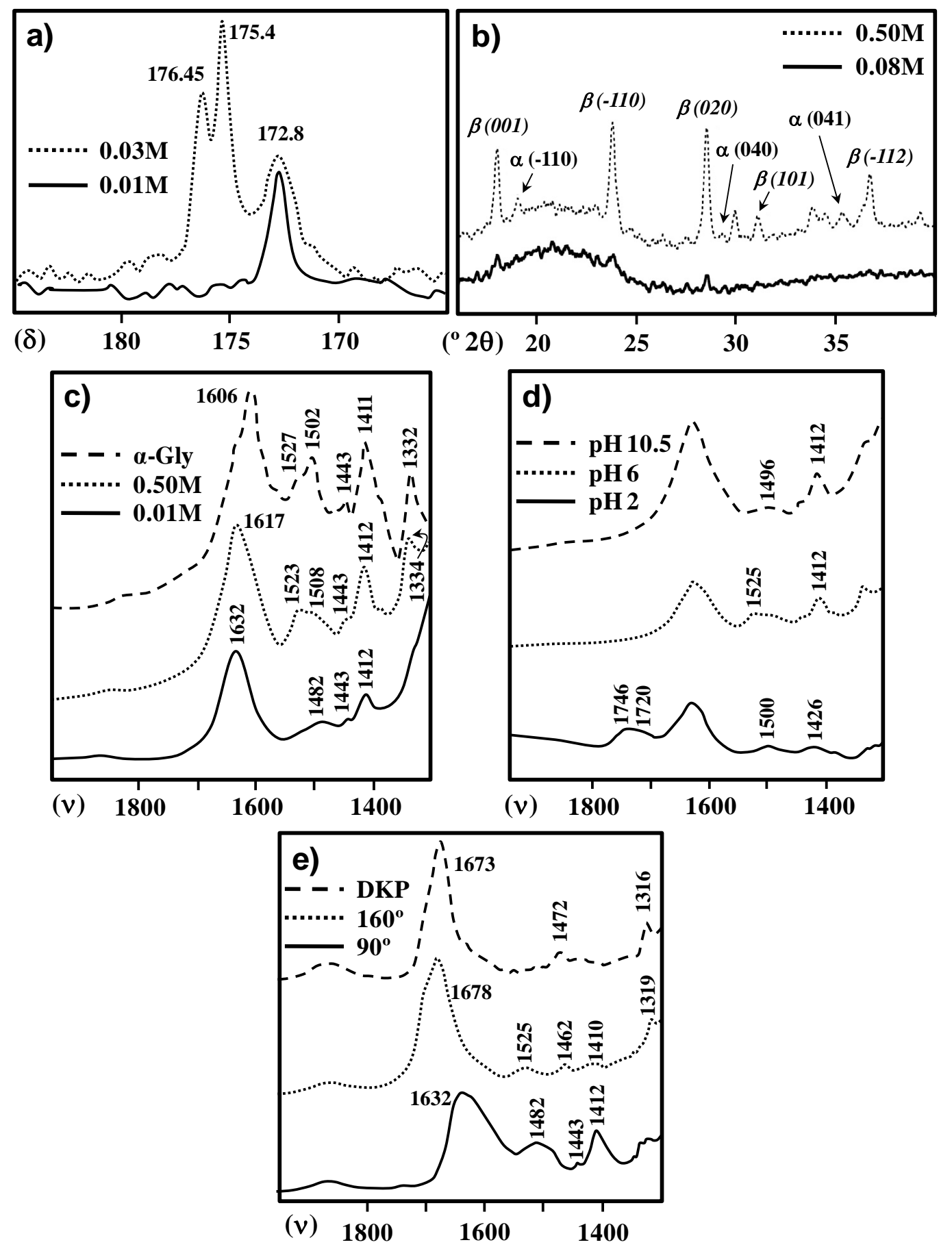

Figure 66. (a): ${ }^{13} \mathrm{C}$ CP-MAS NMR (in ppm) spectra in the carboxylate region for Gly/silica at low (solid line spectrum) and high (dotted line spectrum) Gly concentrations ( $\mathrm{pH} \mathrm{6-7).} \mathrm{(b):} \mathrm{XRD}$ diffractograms for Gly/silica at low (solid line spectrum) and high (dotted line spectrum) Gly concentrations. (c) FTIR spectra (in $\mathrm{cm}^{-1}$ ) for Gly/silica at low (solid line spectrum) and high (dotted line spectrum) Gly concentrations ( $\mathrm{pH} \mathrm{6)}$ and for the $\alpha$-glycine polymorph (broken line 
spectrum). (d) FTIR spectra for Gly/silica (in $\mathrm{cm}^{-1}$ ) at different $\mathrm{pH}$ values. (e): DRIFTS spectra (in $\mathrm{cm}^{-1}$ ) for Gly/silica at 90 and $160{ }^{\circ} \mathrm{C}$ (solid and dotted line spectra, respectively) and for DKP/silica (broken line spectrum). Adapted with permission from Ref. ${ }^{210,224,247}$. Copyright 2004, 2009 American Chemical Society, copyright 2007, Schweizerbart Science Publishers.

At high Gly concentrations three characteristic signals were observed, among which two were assigned to the $\alpha$ - and $\beta$-Gly forms (176.45 and $175.4 \mathrm{ppm}$, respectively). When the Gly loading was decreased, these two signals vanished while the one at $173 \mathrm{ppm}$ remained present with the same intensity, indicating that it does not belong to any bulk polymorph but to a form of Gly interacting with silica surface groups, hereafter called "molecularly adsorbed Gly". Additionally, it was noted that the change in the chemical shift of this form with respect to the bulk zwitterionic ones was of the same order as the difference in $\delta$ between the polymorphs, which suggested modifications by $\mathrm{H}$-bonding to the surface (formation of covalent bonds would result in at least twice larger shifts). Accordingly, the authors suggested that the "molecularly adsorbed Gly" was defined by the formation of H-bond networks with the silica surfaces in the line of a typical "surface adduct". The registered XRD diffractograms shown in Figure $66 \mathrm{~b}$ confirmed the precipitation of bulk Gly crystals at high Gly concentrations. Additionally, FTIR spectra (Figure 66c) clearly indicated that at high Gly concentration the observed bands matched perfectly well those for pure $\alpha$-Gly (assignments available in Table 15). Therefore, at low concentrations Gly was specifically adsorbed on silica surface sites, presumably interacting through H-bonds yielding a "molecularly adsorbed Gly" form, whereas at higher concentrations the $\alpha$ - and $\beta$-Gly bulk polymorphs were also present. 
Table 15. Assignments of the bands observed in the spectra shown in Figure 66c, 66d and 66e.

\begin{tabular}{|c|c|c|c|c|c|}
\hline \multicolumn{4}{|c|}{ Assignments for Figure $66 \mathrm{c}\left(\mathrm{cm}^{-1}\right)$} & \multirow[b]{2}{*}[\mathrm{Gly}]{$=0.50 \mathrm{M}$} & \multirow[b]{2}{*}{ pure $\alpha$-glycine } \\
\hline \multicolumn{2}{|c|}{ Vibrational mode } & \multicolumn{2}{|c|}{$[\mathrm{Gly}]=0.01 \mathrm{M}$} & & \\
\hline$\delta_{\mathrm{as}}\left(\mathrm{NH}_{3}^{+}\right)$ & & \multicolumn{2}{|l|}{1632} & 1617 & 1606 \\
\hline$\delta_{\mathrm{s}}\left(\mathrm{NH}_{3}{ }^{+}\right)$ & & \multicolumn{2}{|l|}{1482} & 1523,1508 & 1527,1502 \\
\hline$\delta_{\mathrm{s}}\left(\mathrm{CH}_{2}\right)$ & & 1443 & & 1443 & 1443 \\
\hline$v_{s}\left(\mathrm{COO}^{-}\right)$ & & \multicolumn{2}{|l|}{1412} & 1412 & 1411 \\
\hline$\rho_{\mathrm{w}}\left(\mathrm{CH}_{2}\right)$ & & \multicolumn{2}{|l|}{-} & 1334 & 1332 \\
\hline \multicolumn{6}{|c|}{ Assignments for Figure $66 \mathrm{~d}\left(\mathrm{~cm}^{-1}\right)$} \\
\hline species & & ational mode & $\mathrm{pH} 2$ & $\mathrm{pH} 6$ & $\mathrm{pH} 10.5$ \\
\hline Gly-can & & $=\mathrm{O})$ & 1746,17 & 20 & - \\
\hline \multirow[t]{2}{*}{ Gly-zwitt } & & $\left.\mathrm{H}_{3}^{+}\right)$ & 1500 & 1525 & 1496 \\
\hline & & $\left.\mathrm{OO}^{-}\right)$ & 1426 & 1412 & 1412 \\
\hline \multicolumn{6}{|c|}{ Assignments for Figure $66 \mathrm{e}\left(\mathrm{cm}^{-1}\right)$} \\
\hline species & $\mathrm{Vib}$ & ional mode & $90^{\circ} \mathrm{C}$ & $160^{\circ} \mathrm{C}$ & $\mathrm{DKP} /$ silica \\
\hline \multirow[t]{4}{*}{ Gly-zwitt } & $\delta_{\mathrm{as}}(1$ & $\left.I_{3}^{+}\right)$ & 1632 & & - \\
\hline & $\delta_{\mathrm{s}}(\mathrm{N}$ & & 1482 & 1525 & - \\
\hline & $\delta_{\mathrm{s}}(\mathrm{C}$ & & 1443 & & - \\
\hline & $v_{s}(C$ & $\left.\mathrm{O}^{-}\right)$ & 1412 & 1410 & - \\
\hline \multirow[t]{3}{*}{ DKP } & $v(\mathrm{C}$ & & - & 1678 & 1673 \\
\hline & DK & ing breathing & - & 1462 & 1472 \\
\hline & $\rho_{\mathrm{w}}(\mathrm{C}$ & $2)+v(C-C)$ & - & 1319 & 1316 \\
\hline
\end{tabular}

The authors highlighted the formation of the $\beta$ polymorph in addition to the $\alpha$ one (see the intensities of the XRD difractogram of the Figure 66b), an unexpected fact as the latter normally precipitates from saturated solutions. Based on the observations in which precipitation of different Gly allomorphs from a concentrated solution can be controlled by polarization effects using laser light, ${ }^{653}$ the authors proposed that the electric field gradients imposed by the negative charges present on the silica surface are strong enough to cause specific orientations of the molecules, although they also pointed out that other structuring effects such as H-bonding with surface groups could be operating as well.

Based on these results, the authors suggested that the most likely picture of glycine adsorption consists in an initial specific adsorption on a limited number of surface sites 
followed by surface precipitation in the vicinity of the silica surface, or possibly on the selectively adsorbed Gly molecules.

ii) At low pH glycinum cation and zwitterion species coexist, at higher $\mathrm{pH}$ only zwitterions are present.

Figure 66d shows the recorded FTIR spectra of the Gly/silica system as a function of adsorption $\mathrm{pH}$, while Table 15 summarizes the assignments of the bands. At $\mathrm{pH} 2$ the two bands at 1720 and $1746 \mathrm{~cm}^{-1}$ due to $v(\mathrm{C}=\mathrm{O})$ of protonated $\mathrm{COOH}$ and the band at $1500 \mathrm{~cm}^{-1}$ due to $\delta_{\mathrm{s}}\left(\mathrm{NH}_{3}{ }^{+}\right)$indicated that the glycinium cation was present in the sample. Moreover, Gly-zwitt also seemed to be present since the band for the $v_{\mathrm{s}}\left(\mathrm{COO}^{-}\right)$mode appeared at 1426 $\mathrm{cm}^{-1}$. With the solution $\mathrm{pH}$ increase (see the $\mathrm{pH} 6$ spectra) the $v(\mathrm{C}=\mathrm{O})$ modes totally disappeared while the $v_{\mathrm{s}}\left(\mathrm{COO}^{-}\right)$mode considerably increased, thus indicating that only Glyzwitt was present. The $v_{\mathrm{s}}\left(\mathrm{COO}^{-}\right)$and $\delta_{\mathrm{s}}\left(\mathrm{NH}_{3}{ }^{+}\right)$modes changed to 1412 and $1525 \mathrm{~cm}^{-1}$, respectively, almost the same as for the zwitterion in the $\alpha$-Gly polymorph. At $\mathrm{pH} 10.5$ the registered spectrum was very similar to the one at pH 6 so that Gly-zwitt was still present, and contrarily to what one would expect no evidences of the glycinate anion was observed. Interestingly, the band at $1525 \mathrm{~cm}^{-1}$ was shifted to $1496 \mathrm{~cm}^{-1}$, which closely resembled the one observed at $\mathrm{pH} 6$ for low coverage samples. Concerning the presence of only zwitterions instead of glycinate anions at $\mathrm{pH} 10.5$, the authors suggested that the speciation of Gly could be governed by local values of $\mathrm{pH}$ close to the surface rather than its bulk value. That is, at this $\mathrm{pH}$ the silica surface bears a strong negative charge and, accordingly, the solution neighboring the surface forms a diffuse layer of opposite charge, which automatically produces a local $\mathrm{pH}$ lower than the value in the solution bulk. Therefore, the speciation at the interface may be different than in the bulk and for this particular case the lower local pH might justify the observed higher percentages of zwitterions.

Interestingly, the authors also indicated that no covalent bonds between Gly and the silica surface occurred, a fact which allowed ruling out any "surface acylation". In other words, the SMA form was not present. This strengthened the thesis that the "molecularly adsorbed Gly" was formed by specific H-bonds with the surface, rather than by covalent bonds.

iii) Thermal treatments of adsorbed Gly molecules induce the formation of DKP.

Figure 66e shows the in-situ DRIFT spectra of the Gly/silica system as a function of heating temperature with a sample that mostly contained the "molecularly adsorbed Gly" form ( $\mathrm{pH} 6$ and concentration of $0.08 \mathrm{M}$ ). Table 15 collects the assignments of the bands 
observed. At $90{ }^{\circ} \mathrm{C}$ no changes were observed in comparison to FTIR spectra at room temperature (Figure 66c). At $160{ }^{\circ} \mathrm{C}$, the spectra underwent strong modifications and became quite similar to that of DKP/silica system. In particular, the transformation was evidenced by the presence of the intense absorption band at $1678 \mathrm{~cm}^{-1}$ due to the $v(C=O)$ of the adsorbed DKP, by the small sharp band at $1462 \mathrm{~cm}^{-1}$ corresponding to the DKP ringbreathing and by an increase in intensity of the $\rho_{\mathrm{w}}\left(\mathrm{CH}_{2}\right)$ mode due to the contribution of the C-C stretching vibrations at approximately the same frequency $\left(1316 \mathrm{~cm}^{-1}\right)$. The presence of small bands at 1410 and $1525 \mathrm{~cm}^{-1}\left(\nu_{\mathrm{s}}\left(\mathrm{COO}^{-}\right)\right.$and $\delta_{\mathrm{s}}\left(\mathrm{NH}_{3}{ }^{+}\right)$, respectively) also evidenced small amounts of residual adsorbed Gly-zwitt. Conversely, the bands associated to the linear Gly-Gly dimer (for instance, its strongest band at $1598 \mathrm{~cm}^{-1}$ ) were not observed. Additionally, TPD-MS experiments (not shown here) revealed $\mathrm{H}_{2} \mathrm{O}^{+}$signals (18 amu) between $160-200{ }^{\circ} \mathrm{C}$, in agreement with DKP formation, as the reaction is accompanied by the production of two water molecules. Therfore, the authors concluded that adsorption of Gly-zwitt on silica surfaces activates the formation of amide bonds, contrarily to Gly on $\operatorname{Pt}(111),{ }^{654}$ graphite $^{655}$ and $\mathrm{Cu}(110) .{ }^{219}$

In addition to this set of works, Ben Shir et al. ${ }^{228}$ also studied the binding of Gly on silica surfaces using multinuclear ssNMR-techniques (Section 3.2.5). For this case SBA-15, a mesoporous silica material with a high surface area (pore diameters of 5-30 nm), was used as silica surface model. The effect of the amount of water present in the system was also considered adopting both low and high hydration states. In low hydration conditions, the presence of a single population of surface-bound Gly species was observed. Despite the low $\mathrm{H}$ atom density $\left(<4 \mathrm{H} \mathrm{nm}^{-}\right.$ ${ }^{2}$ ), it was possible to determine, by means of ${ }^{15} \mathrm{~N}\left\{{ }^{1} \mathrm{H}\right\}$ separated local field (SLF) NMR experiments, that the Gly amine moiety was protonated (i.e., $-\mathrm{NH}_{3}{ }^{+}$). Cross polarization $(\mathrm{CP}){ }^{15} \mathrm{~N}$ $\left\{{ }^{29} \mathrm{Si}\right\}$ and ${ }^{29} \mathrm{Si}\left\{{ }^{15} \mathrm{~N}\right\}$ REDOR measurements allowed to establish the geometry, stoichiometry and specificity of Gly to the silica surface binding sites. In particular, it was found that the $-\mathrm{NH}_{3}{ }^{+}$ moiety was positioned above a plane formed by 3 or $4 \mathrm{Si}$ surface atoms, predominantly $\mathrm{Q}^{3} \mathrm{Si}$

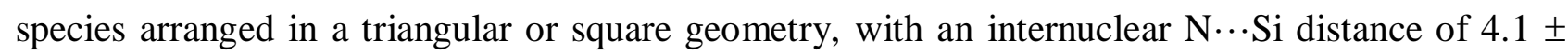
$0.3 \AA$. In addition to the high abundance of $\mathrm{Q}^{3}$ silanol sites, $\mathrm{Q}^{2}$ and $\mathrm{Q}^{4}$ Si species were also observed on the SBA-15 surface in lesser amounts. Moreover, ${ }^{15} \mathrm{~N}\left\{{ }^{13} \mathrm{C}\right\}$ REDOR data indicated that the $\mathrm{COO}^{-}$had a very restricted motion, which practically indicated no contribution to surface binding. For the high hydration state (estimated surface hydrogen density of 6-12 $\mathrm{H}$ atoms $/ \mathrm{nm}^{2}$ ) the presence of two different surface-bound Gly populations, plus a third population with "free" (non surfacebound) Gly molecules was determined. One interacting species was identified to be the same as the one present in the low hydration state; the other bound population was found to have the same 
binding stoichiometry and geometry, but the $\mathrm{COO}^{-}$group experienced a larger amplitude motion. The "free" population was interpreted as Gly being completely solvated by water molecules because Gly exhibited large libration motions. The physical picture suggested by the authors for the three populations detected, emerged from the accumulated data is shown in Figure 67.

a)

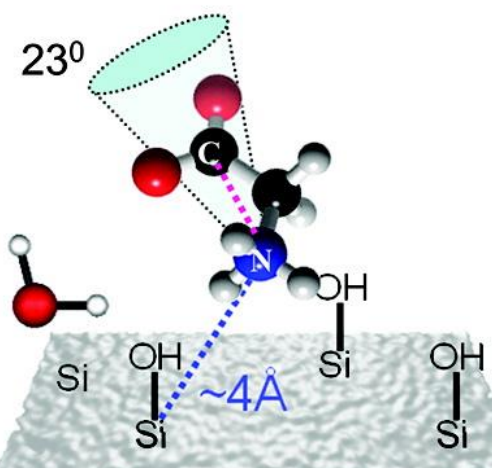

b)

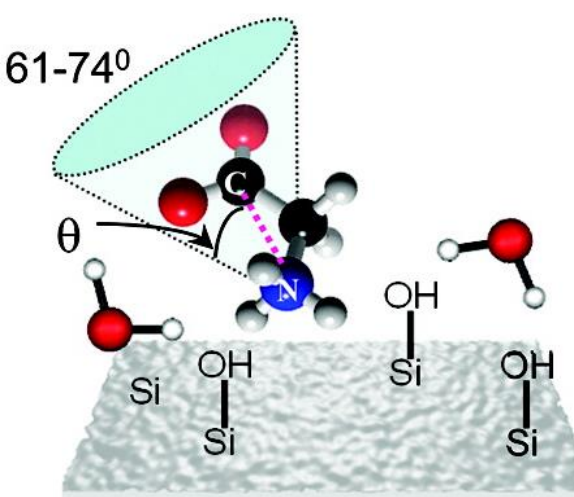

c)

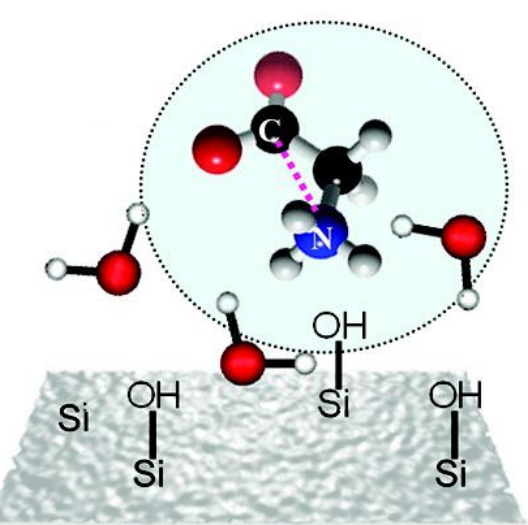

Figure 67. Picture of the three populations (bound, a and b, and free, c) for Gly/SBA-15 detected by multinuclear ssNMR experiments.Adapted with permission from Ref. ${ }^{228}$. Copyright 2012 American Chemical Society).

$\mathrm{H}$-bonding network between the ammonium $\mathrm{H}$ atoms and the lone pair electrons of the oxygens atoms of the $\mathrm{SiOH}$ and of $\mathrm{H}_{2} \mathrm{O}$ (for bound and non-bound populations) were postulated to be the chemical driving forces that underlie Gly/silica surface specific interactions. Confirming the 
results of Lambert and coworkers, ${ }^{210,224,247}$ surface-induced crystallization of $\alpha$ and $\beta$ polymorphs was also observed.

Despite these very interesting results, important aspects, however, still remain unclear. An important issue is to determine whether the interaction between Gly and the silica surface takes place through an actual direct contact or is mediated by solvent water molecules. Moreover, for the former case, it is also of interest to know which are the preferred groups $\left(\mathrm{COO}^{-}\right.$or $\left.\mathrm{NH}_{3}^{+}\right)$ responsible of the contact, although, as shown above, sophisticated ssNMR techniques can provide some clues. ${ }^{228}$ Additionally, it is relevant to establish the number of $\mathrm{OH}$ groups (both from $\mathrm{SiOH}$ and water) needed to stabilize the zwitterionic form over the canonical one. Although these points were not fully resolved by the previous spectroscopic works, some ab initio studies were devoted to provide information on these aspects.

Costa et al. ${ }^{556}$ and Rimola et al. ${ }^{322}$ simulated the interaction of Gly in its zwitterionic state with silica surfaces terminated by isolated $\mathrm{SiOH}$ groups in a microsolvated state, that is, accounting for a small number of water molecules. The former authors used the minimal $\mathrm{H}_{3} \mathrm{SiOH}$ clusters in the presence of 1 and 2 water molecules and the B3LYP/6-31++G(d,p) level of theory. The most stable complexes found are shown in Figure 68. Results indicated that with one water molecule the interaction of Gly with one isolated $\mathrm{SiOH}$ group can take place directly through the $\mathrm{NH}_{3}{ }^{+}$group, the $\mathrm{COO}^{-}$interaction being mediated by the water molecule (Figure 68a), or adopting the reverse situation (Figure 68b) as both complexes were found to be energetically almost degenerate. Additionally, with two $\mathrm{H}_{2} \mathrm{O}$ molecules and two isolated $\mathrm{SiOH}$ groups, the most stable adduct is the structure shown in Figure 68c, in which the $\mathrm{COO}^{-}$moiety interacts directly with the silanols whereas waters bridge the interaction with $\mathrm{NH}_{3}{ }^{+}$. Rimola et al. ${ }^{322}$ used a periodic model of the (001) edingtonite silica surface with two independent $\mathrm{SiOH}$ groups per unit cell to study at the B3LYP/triple- $\zeta$ level the successive addition of water molecules (from 1 to 5 ) at the Gly/silica interface. The most stable adducts found are shown in Figure 69. 


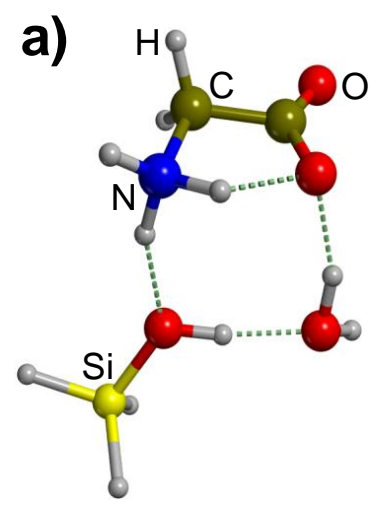

b)
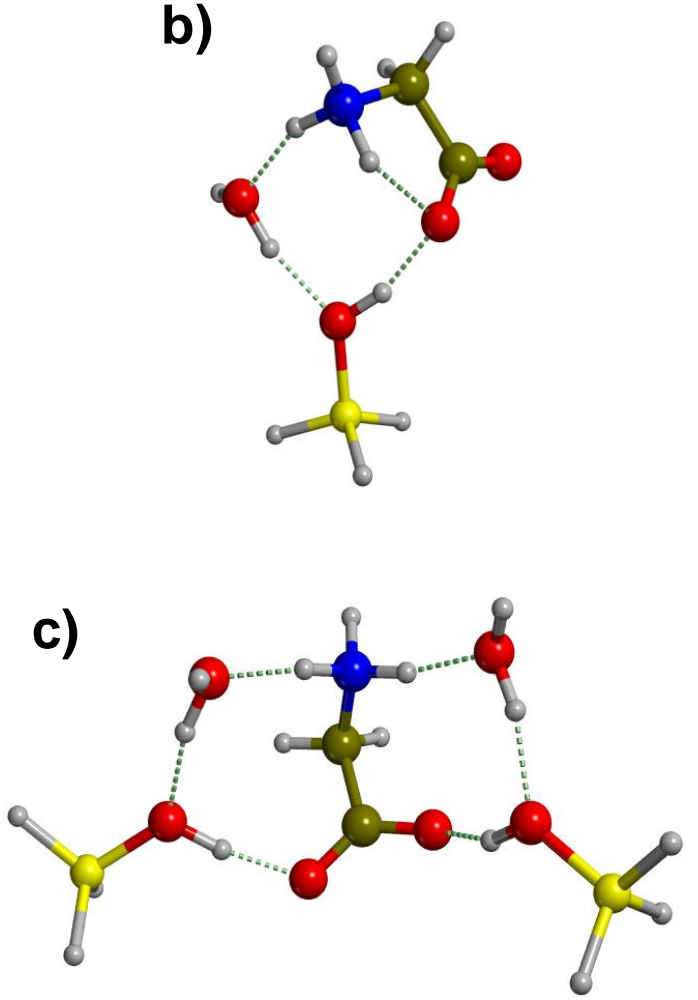

Figure 68. Stable structures found in Ref. ${ }^{556}$ for the interaction of zwitterionic glycine with minimal silanol clusters in the presence of 1 and 2 explicit water molecules. Optimized at the B3LYP/6$31++\mathrm{G}(\mathrm{d}, \mathrm{p})$ level. 

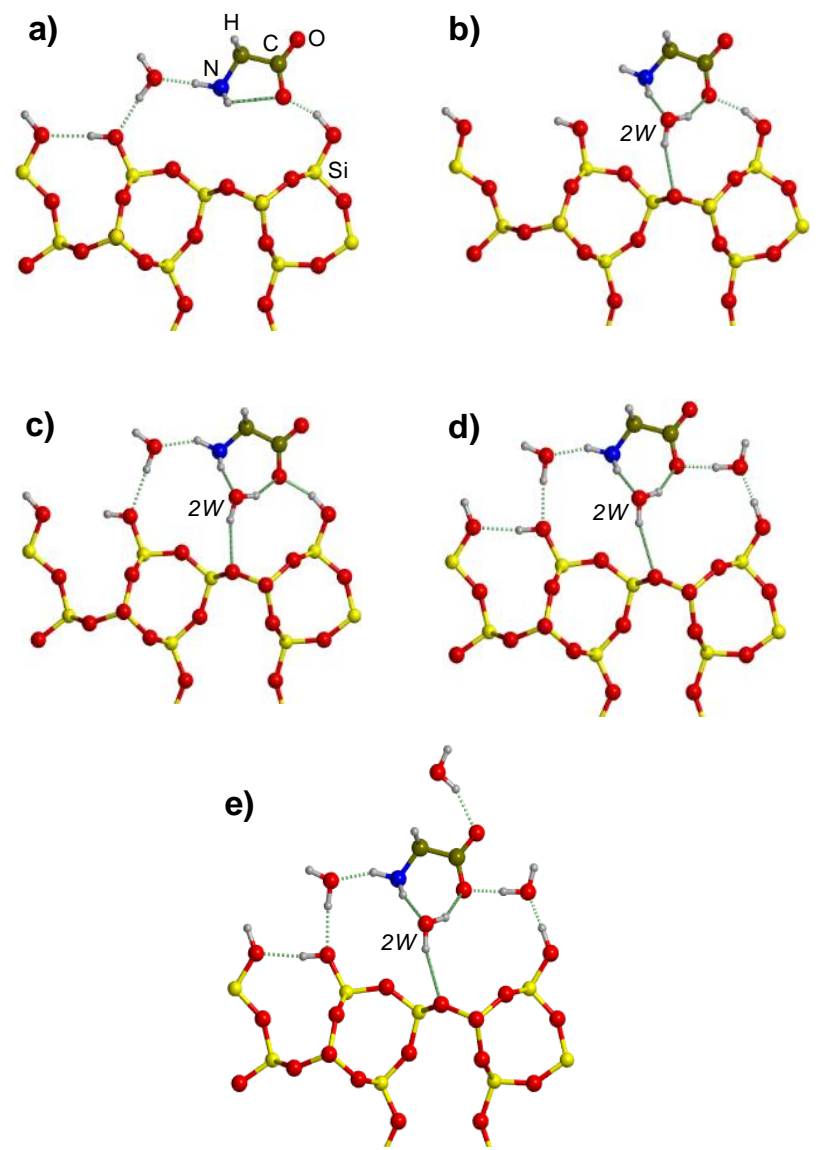

Figure 69. The most stable structures found in Ref. ${ }^{322}$ for the successive addition of water (from 1 to 5, a-e, respectively) at the Gly-zwitt/silica surface interface. Periodic systems optimized at the B3LYP/triple- $\zeta$ level. Adapted with permission from Ref. ${ }^{322}$. Copyright 2008 American Chemical Society.

Interestingly, with one water molecule (Figure 69a), the direct contact of Gly with the silica surface occurred through the $\mathrm{COO}^{-}$group, whereas water interacted with $\mathrm{NH}_{3}{ }^{+}$. With two and three water molecules (Figure 69b and Figure 69c, respectively), $\mathrm{COO}^{-}$still was found to be involved in the direct contact with $\mathrm{SiOH}$, while it interacted with two water molecules. However, for 4 and 5 water molecules (Figure 69d and Figure 69e, respectively) such a direct contact disappeared and the Gly/silica interaction was mediated by water. This progressive microsolvation procedure indicates that the direct contact between Gly and the silica surface becomes lost upon increasing the number of water molecules until reaching a situation in which the Gly/silica interface is completely dominated by water. It is worth mentioning that this situation was not observed by Costa et al. ${ }^{556}$ due to the limited number of water molecules considered. In the work of Rimola et al., ${ }^{322}$ the vibrational frequencies of the structure shown in Figure 69e were also computed and compared with the experimental IR results of Meng et al. ${ }^{210}$ (data shown in Table 16). In this case, the $v_{a s}\left(\mathrm{COO}^{-}\right.$) and $v\left(\mathrm{NH}_{3}{ }^{+}\right)$modes useful for comparison are hidden by the stretching and bending modes of solvent water, which cover the $3900-3500 \mathrm{~cm}^{-1}$ and $1700-1600 \mathrm{~cm}^{-1}$ regions. Because of that, the $\delta(\mathrm{CCH})+v_{\mathrm{s}}\left(\mathrm{COO}^{-}\right), \delta(\mathrm{HCH})$ and $\delta_{\mathrm{as}}\left(\mathrm{NH}_{3}{ }^{+}\right)$were used, as they do not interfere with water bands. 
The comparison between the experimental measurements and the B3LYP data is fairly good, with a maximum deviation of $30 \mathrm{~cm}^{-1}$. Indeed, the B3LYP $\delta_{\mathrm{as}}\left(\mathrm{NH}_{3}{ }^{+}\right)$and $\delta(\mathrm{CCH})+v_{\mathrm{s}}\left(\mathrm{COO}^{-}\right)$values are in very good agreement with the experimental ones, thus supporting the view that $\mathrm{NH}_{3}{ }^{+}$and $\mathrm{COO}^{-}$are completely solvated by $\mathrm{H}_{2} \mathrm{O}$.

Table 16. Comparison between the experimental and harmonic B $3 L Y P$ frequencies $\left(\mathrm{cm}^{-1}\right)$ for the Gly/silica system. The experimental values are at $\mathrm{pH} 6$ (taken from Figure 66c). The computed frequencies correspond to the structure shown in Figure 69e.

\begin{tabular}{lcc}
\hline Vibrational mode & Experiments $(\mathrm{pH} \mathrm{6)}$ & B3LYP \\
\hline$\delta(\mathrm{CCH})+v_{\mathrm{s}}\left(\mathrm{COO}^{-}\right)$ & 1412 & 1405 \\
$\delta(\mathrm{HCH})$ & 1443 & 1422 \\
$\delta_{\mathrm{as}}\left(\mathrm{NH}_{3}^{+}\right)$ & 1617 & 1619 \\
\hline
\end{tabular}

The analysis focused on the Gly-zwitt Gly-can relative stability as a function of the number of $\mathrm{OH}$ groups (shown in Figure 70) is also of interest. Results indicated that, as expected, the larger the number of $\mathrm{OH}$, the higher the stability of Gly-zwitt. Rameakers et al. ${ }^{656}$ found that, for the $\mathrm{Gly} /\left(\mathrm{H}_{2} \mathrm{O}\right)_{n}$ system, with $n=2$ Gly-zwitt was metastable (i.e., Gly-zwitt did not reverse to Gly-can) whereas Gly-can was the dominating form. With $n=4$ Gly-zwitt was found to be more stable than Gly-can. When silica surface is present, results show the same trend, even though in this case the $\mathrm{OH}$ groups belong to water and silanols. That is, with two $\mathrm{OH}$ groups in direct contact with Gly, Gly-zwitt becomes stable, although the canonical form is the preferred configuration, whereas when Gly is in contact with four $\mathrm{OH}$ groups Gly-zwitt becomes the most stable form. 


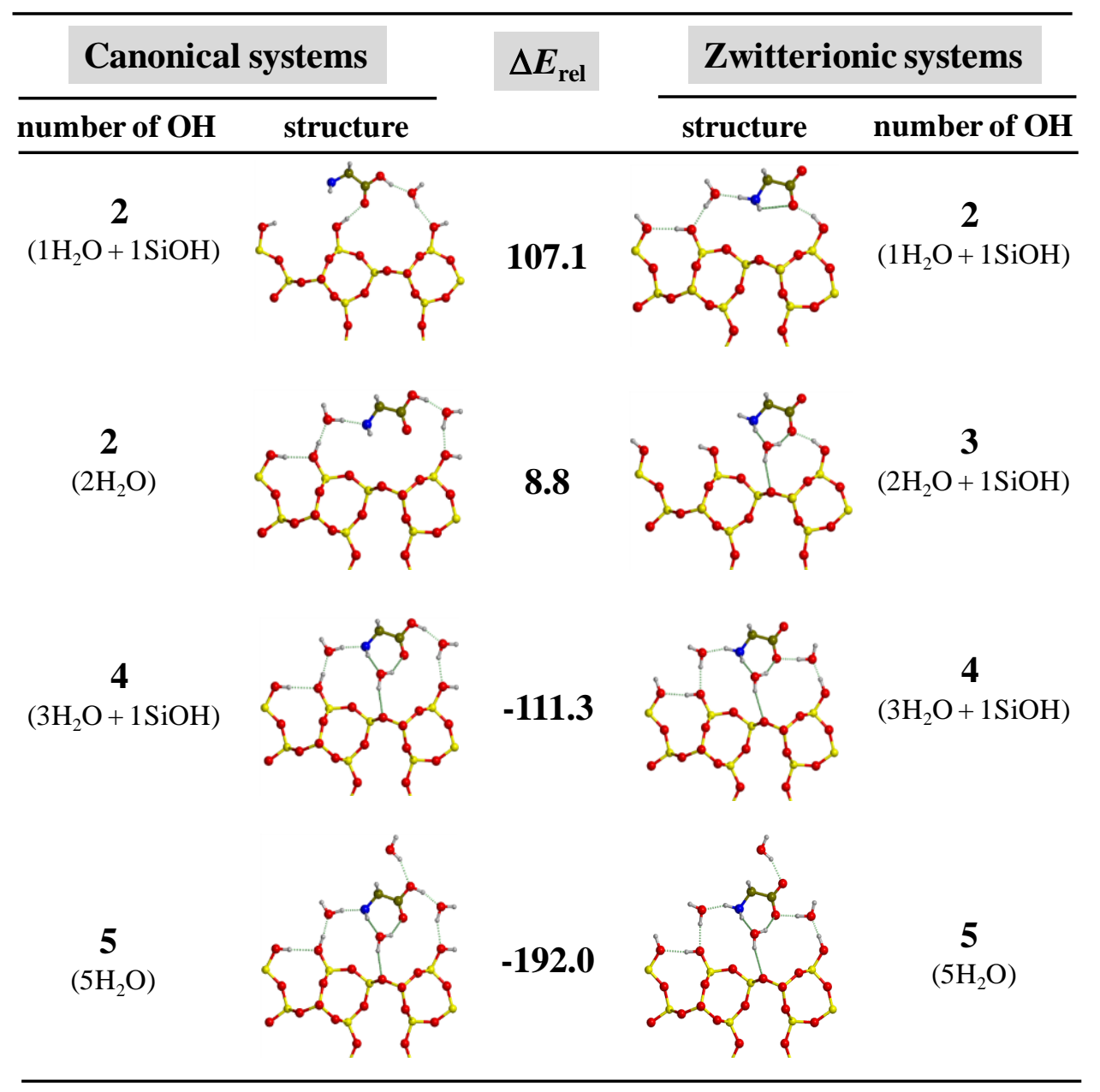

Figure 70. Relative electronic energies $\left(\Delta E_{\text {rel }}\right.$, in $\left.\mathrm{kJ} \mathrm{mol}^{-1}\right)$ of the zwitterionic systems with respect to the canonical ones as a function of the number of $\mathrm{OH}$ groups interacting with glycine, with different $\mathrm{H}_{2} \mathrm{O}$ loadings (1, 2, 3 and 5 water molecules). Adapted with permission from Ref. ${ }^{322}$. Copyright 2008 American Chemical Society.

As mentioned, these theoretical results only addressed the interaction of Gly with isolated $\mathrm{SiOH}$ groups and not with geminal ones, which due to their more acidic behavior are expected to be different. Because of that, Costa et al. ${ }^{641}$ and Zhao et al. ${ }^{323}$ studied the adsorption of Gly on silica surfaces rich in geminal silanol groups in the presence of water, both works using first-principles molecular dynamics simulations. In the work of Costa et al. ${ }^{641}$ the adsorption was simulated considering a microsolvated environment constituted by 5 water molecules (enough to ensure the stabilization of Gly-zwitt over Gly-can). Several MD runs at the PBE level and T $=300 \mathrm{~K}$ with evolution times of $1500 \mathrm{fs}$ indicated that in the dominant conformation (shown in Figure 71a) the $\mathrm{COO}^{-}$moiety was in direct contact with the surface through one geminal $\mathrm{SiOH}$ group, whereas $\mathrm{NH}_{3}{ }^{+}$was interacting with water molecules far from the surface. 

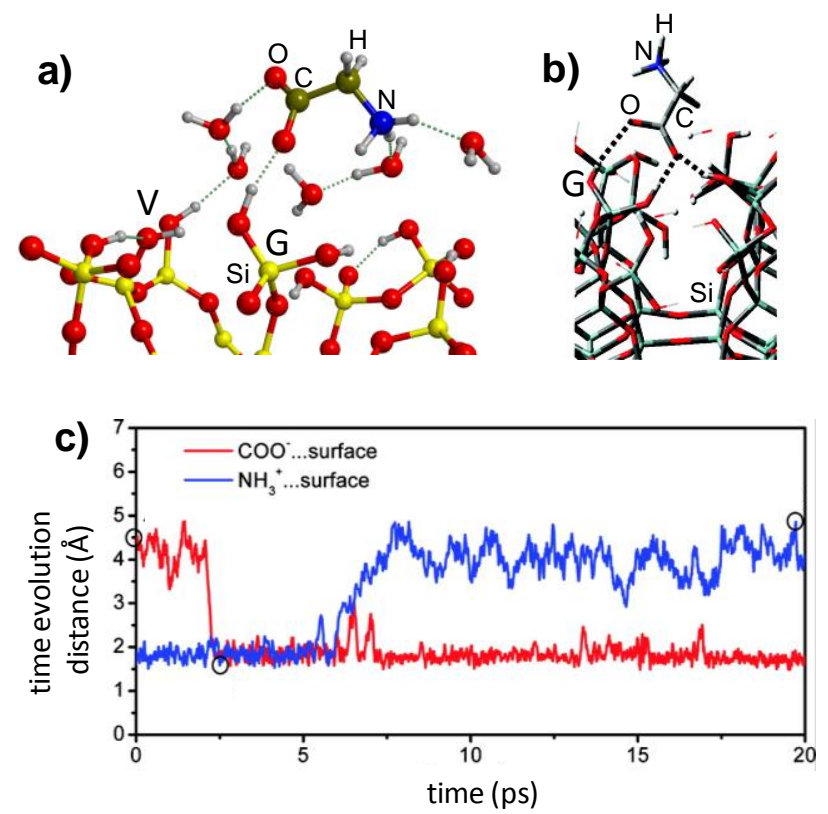

Figure 71. Interaction between glycine and a silica surface rich in geminal silanols with the presence of water: (a) Dominant configuration obtained by means of ab initio MD simulations with 5 explicit water molecules (PBE density functional method, $\mathrm{T}=300 \mathrm{~K}$ and evolution times of 1500 fs; drawn with data from Ref. ${ }^{641}$ ); (b): Dominant configuration obtained by means of SCC-DFTB simulations with 140 explicit water molecules ( $\mathrm{T}=300 \mathrm{~K}$ and evolution times of $20 \mathrm{ps}$;). The water molecules have been removed for the sake of clarity; (c) time evolution of the distance of the $\mathrm{COO}^{-}$ and $\mathrm{NH}_{3}{ }^{+}$moieties with the surface (red and blue lines, respectively) for the MD run associated to the configuration shown in (b). b) and c) reprinted with permission from Ref. ${ }^{323}$. Copyright 2011 American Chemical Society.

Zhao et al. ${ }^{323}$ also studied Gly adsorption, but unlike this previous work, they included 140 water molecules in order to simulate the bulk solvent effects and carried out MD simulations based on the self-consistent charge density functional tight binding (SCC-DFTB) method (see Section 5.6.2 for details of the method). After running several MD simulations at $\mathrm{T}=300 \mathrm{~K}$ with evolution times of 20 ps for different initial guess structures, the results obtained did not differ much from those found by Costa et al. ${ }^{641}$ Indeed, they found that the dominant configuration (shown in Figure 71b) exhibited the $\mathrm{COO}^{-}$group pointing towards the surface and establishing direct contact with geminal $\mathrm{SiOH}$ groups, while $\mathrm{NH}_{3}{ }^{+}$, though they were able to establish direct $\mathrm{H}$-bonds with geminal $\mathrm{SiOH}$ groups, preferred to interact with bulk water, away from the surface. These trends can be seen in the time evolution of the distance between the functional $\mathrm{COO}^{-}$and $\mathrm{NH}_{3}{ }^{+}$groups of Gly-zwitt and the surface silanols obtained for one MD run (see Figure 71c): $\mathrm{COO}^{-}$was close to the surface during all the MD simulation while $\mathrm{NH}_{3}{ }^{+}$only interacted with the surface for a short lapse of time, being far from the surface the rest of the time. Analysis of the Mulliken charges after the MD simulations showed that the interaction involved a considerable charge transfer, as the surface carried a negative charge (from -0.381 to -1.09 electrons) whereas Gly and water molecules hold a positive charge (from +0.0719 to +0.0124 electrons, for Gly). Moreover, the authors indicated that, as the surface exhibited a negative charge, the low affinity of $\mathrm{NH}_{3}{ }^{+}$to the surface could not be 
explained using the conventional acid-base (proton) theory, but considering that the large positive net charge on the geminal $\mathrm{H}$ must repel any incoming $\mathrm{NH}_{3}{ }^{+}$, which would explain the great affinity of the $\mathrm{COO}^{-}$group for the surface. Remarkably, these two works coincide in estimating the adsorption energy of Gly-zwitt on the geminal silanol groups to about $-140 /-150 \mathrm{~kJ} \mathrm{~mol}^{-1}$.

\subsubsection{Other Amino Acids}

\subsubsection{Alanine}

The side chain of alanine (Ala) is the $-\mathrm{CH}_{3}$ group and therefore, it is reasonable to consider that no significant changes will occur compared to Gly because the side chain cannot establish $\mathrm{H}$ bonds with free silanol groups, although dispersive interactions are larger for Ala than for Gly due to the presence of the $\mathrm{CH}_{3}$ group. In spite of that, there are some theoretical and experimental works addressed exclusively to the interaction of Ala with silica surfaces.

The gas-phase interaction of Ala with a large cluster model simulating the (001) edingtonite surface that contains 4 isolated $\mathrm{SiOH}$ groups has been reported by Rimola et al. ${ }^{321}$ Calculations were carried out using the ONIOM2 strategy with B3LYP/6-311++G(d,p) as high level and MNDO as low level. It was found that the interaction through the backbone chain followed the same features as for Gly; that is, adsorption in the canonical state and engaging at least two H-bonds between the $\mathrm{SiOH}$ groups and the $\mathrm{C}=\mathrm{O}$ Ala group. The optimized structure is shown in Figure 72a. 
a)
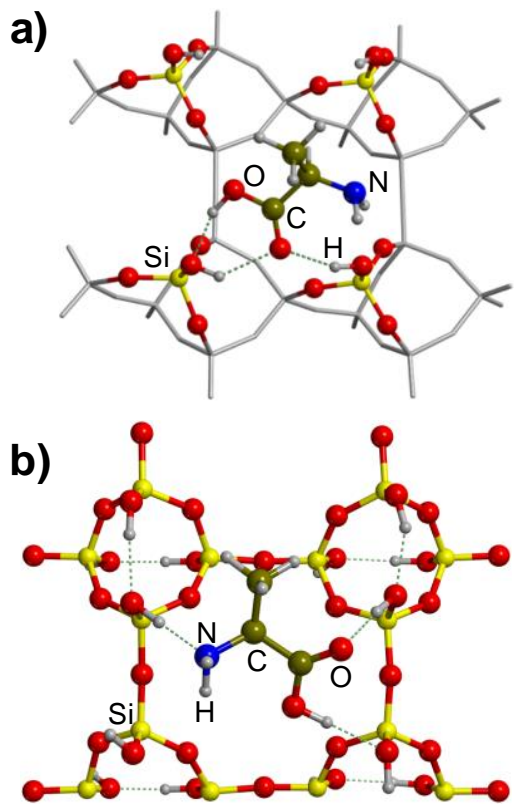

c)

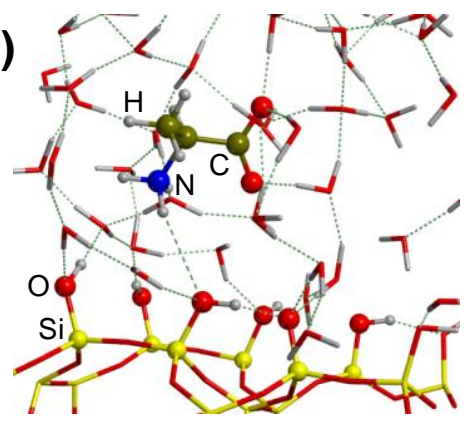

d)
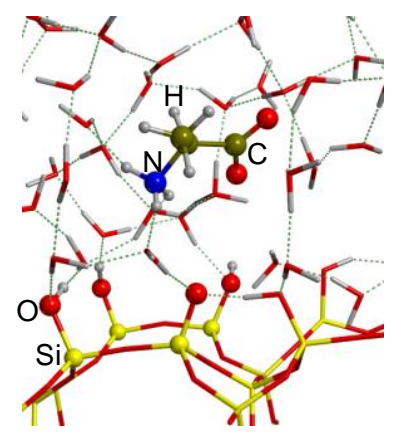

Figure 72. (a) Optimized structure for the Ala/silica system in gas-phase at the ONIOM2(B3LYP/6-311++G(d,p):MNDO) level (drawn with data from Ref. ${ }^{321}$ ); (b) dominant configuration for the Ala/silica system in gas phase by means of ab initio MD simulations (PBE method, $\mathrm{T}=100 \mathrm{~K}$ ); (c) dominant configuration for the Ala/silica system in aqueous solvent (63 water molecules) by means of ab initio MD simulations (PBE method, $\mathrm{T}=300 \mathrm{~K}$ ); (d) Eventual configuration observed during the same MD simulation that yielded (c). Sections b-d drawn from data of Ref. ${ }^{324}$

Nonella et al. ${ }^{324}$ also studied the Ala/silica interactions but in this case by means of first-principles MD simulations at the PBE level considering both vacuum conditions and the presence of explicit water molecules. In vacuo adsorption simulations, carried out at $\mathrm{T}=100 \mathrm{~K}$ and with evolution times of $2.5 \mathrm{ps}$, showed that the dominant configuration is the one given in Figure 72b. This is notably different from the one previously observed (Figure 72a) as in this case there is a rather strong $\mathrm{H}$-bond between the $\mathrm{NH}_{2}$ and one $\mathrm{SiOH}$ groups and the $\mathrm{H}$-bonds between $\mathrm{SiOH}$ and $\mathrm{COOH}$ 
adopt a different pattern. Although in both cases the silica surface model is based on edingtonite, these differences are probably due to the fact that Nonella's model is richer in SiOH surface groups than the Rimola's one (8 vs $4 \mathrm{SiOH}$ groups per unit cell, respectively). This higher $\mathrm{SiOH}$ density probably favors the formation of the additional interaction with the $\mathrm{NH}_{2}$ moiety and the different $\mathrm{H}$ bonding patterns in the $\mathrm{COOH}$ group. Furthermore, in Nonella's model the $\mathrm{SiOH}$ are interacting whereas in Rimola's one they are isolated. MD simulations carried out in the presence of 63 explicit water molecules at $\mathrm{T}=300 \mathrm{~K}$ converged into a picture in which Ala is adsorbed as a zwitterion on the neutral surface (i.e., with no evidences of negatively charged $\mathrm{SiO}^{-}$surface groups) through indirect $\mathrm{H}$-bonds mediated by water molecules (see Figure 72c). Noticeably, $\mathrm{NH}_{3}{ }^{+}$eventually established direct $\mathrm{H}$-bond interactions with $\mathrm{O}$ atoms of silanols (see Figure 72d). Additionally, the results indicated that the $\mathrm{NH}_{3}{ }^{+}$group interacted more strongly with the surface than the $\mathrm{COO}^{-}$one, which was reflected by the orientation of Ala $\left(\mathrm{NH}_{3}{ }^{+}\right.$pointing towards the surface).

From an experimental point of view, Ben Shir et al. ${ }^{227}$ have also reported ssNMR results focused on the Ala/SBA-15 interface, in a procedure similar to that used later for the Gly/SBA-15 system $^{228}$ (see above). The results obtained are, as expected, very similar between the two works. That is, the low hydration state was found to be dominated by a surface-bound Ala population, with the protonated $-\mathrm{NH}_{3}{ }^{+}$moiety close to $3-4 \mathrm{Q}^{3}$ silicon centers with an internuclear $\mathrm{Si} \cdots \mathrm{N}$ distance of 4.0-4.2 $\AA$ and the $\mathrm{COO}^{-}$moiety not showing contributions to surface binding, whereas in the high hydration state three populations were found, two of them surface-bound and one "free". The only significant difference found between the two works is that surface-induced crystallization was not observed for the Ala case. Remarkably, this view of transforming a surface immobilized Ala into a "free" Ala molecule solvated by water when the hydration conditions are increased is in full agreement with the results of Amitay-Rosen et al., ${ }^{225}$ in which they observed, by means of dynamic MAS NMR measurements, that a single water molecules was enough to remove the bound Ala molecules from the SBA-15 surface. This behavior is moreover consistent with the calculations of Nonella et al. ${ }^{324}$ (see above) where it was observed that the adsorption of Ala on a silica surface is not direct through the $\mathrm{NH}_{3}{ }^{+}$or $\mathrm{COO}^{-}$groups but bridged by solvent water molecules.

\subsubsection{Lysine and Glutamic acid}

The side chain of lysine (Lys) and glutamic acid (Glu) are the $-\left(\mathrm{CH}_{2}\right)_{4}-\mathrm{NH}_{2}$ and $-(\mathrm{CH})_{2}-$ $\mathrm{COOH}$ groups, respectively, and thus, these amino acids are of interest because they exhibit different protonated forms depending on the $\mathrm{pH}$. Figure 73 shows the different states as well as respective $\mathrm{p} K a$ values. 
a)

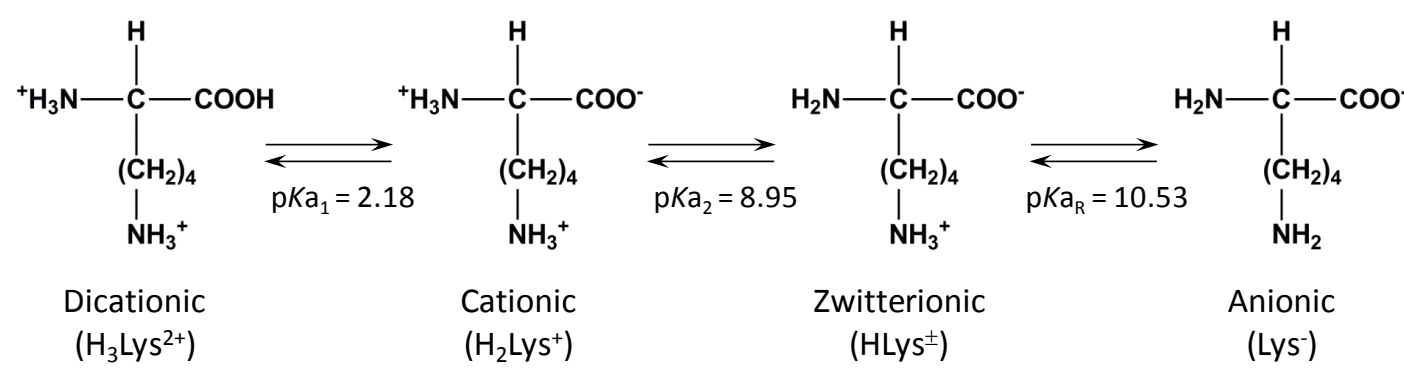

b)

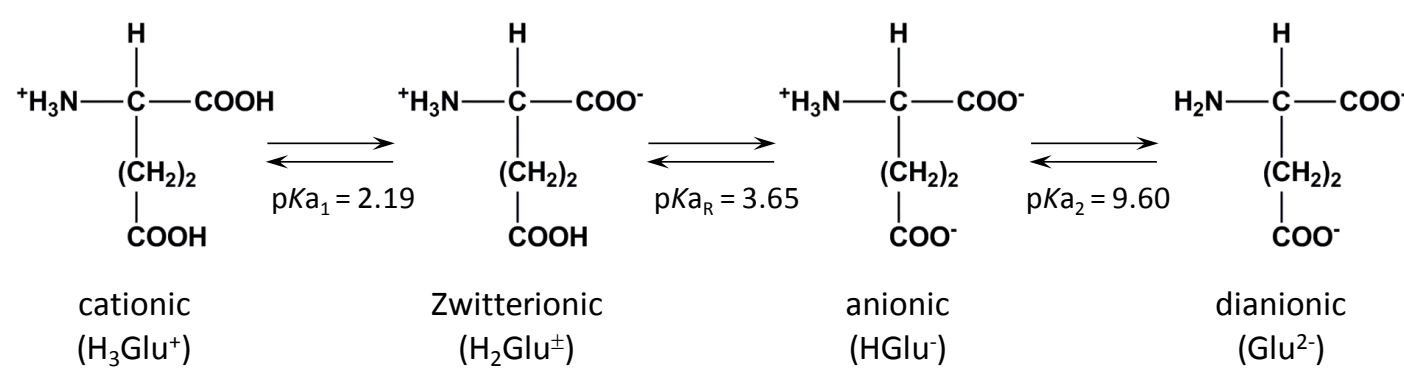

Figure 73. Different protonated states for lysine (Lys, (a)) and glutamic acid (Glu, (b)), including corresponding $\mathrm{pKa}$ values.

The adsorption of Lys on amorphous silica in the 7.1-9.8 $\mathrm{pH}$ range was measured by Kitadai et al. $^{217}$ by means of attenuated total reflectance infrared (ATR-IR) spectroscopy. At $\mathrm{pH}<7$, the absorbance of adsorbed Lys was found to be small because non-adsorbed Lys was predominant, and therefore at these values the ATR-IR spectra were not recorded. Figure 74 shows the spectra of Lys in solution (a) and when adsorbed on silica (b) over the whole range of $\mathrm{pH}$ values tested. 


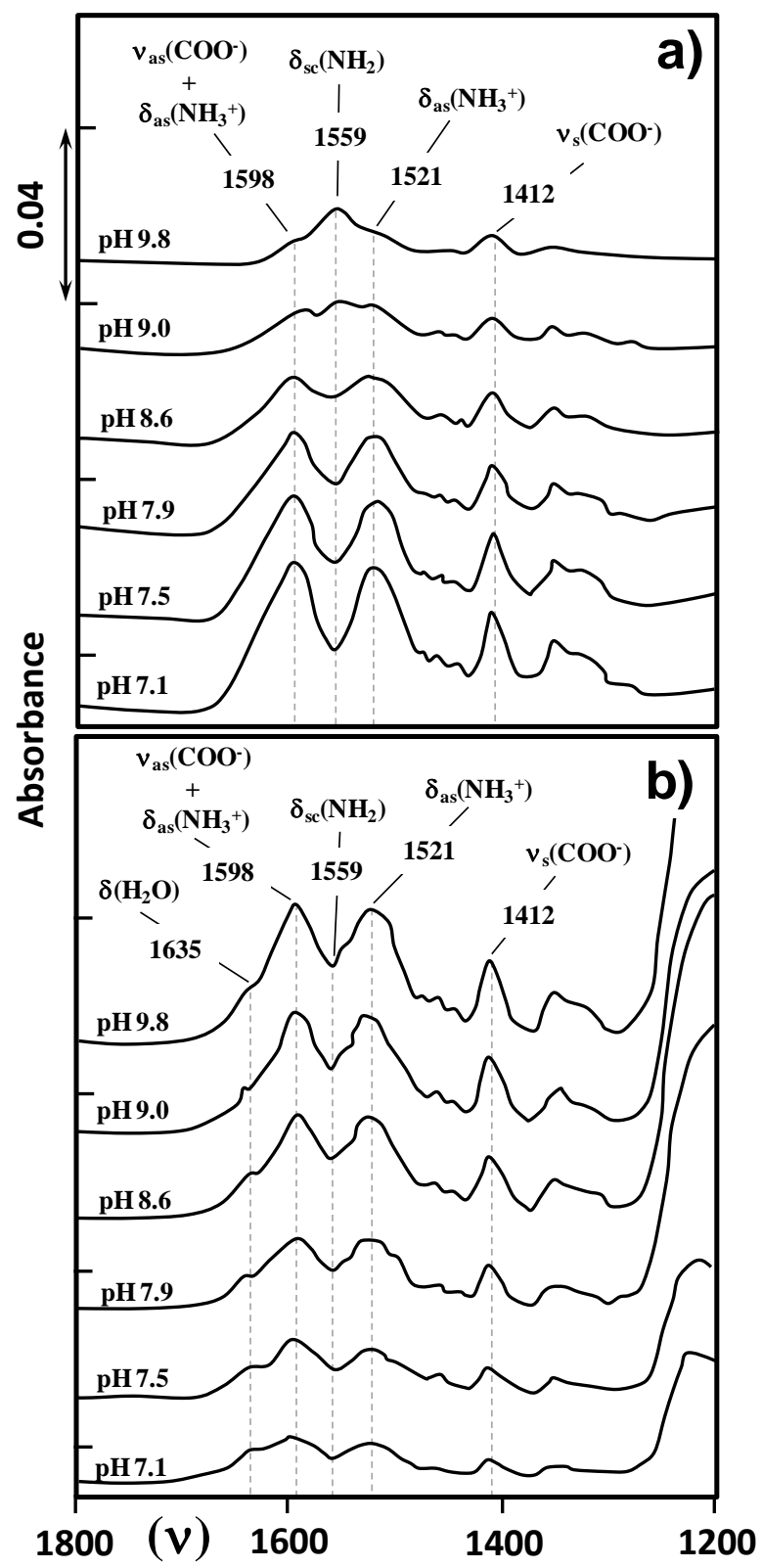

Figure 74. ATR-IR spectra (in $\mathrm{cm}^{-1}$ ) for Lys in solution (a) and when adsorbed on silica surface (b), respectively, as a function of $\mathrm{pH}$. Adapted with permission from Ref. ${ }^{217}$. Copyright 2009 Elsevier.

Spectra in Figure 74a indicated that, as expected, Lys changes its protonation state from the zwitterionic to the cationic state upon $\mathrm{pH}$ decrease. This was clearly evidenced by the disappearance of the $\mathrm{NH}_{2}$ scissoring $\left(\delta_{\mathrm{sc}}\left(\mathrm{NH}_{2}\right)\right)$ band at $1559 \mathrm{~cm}^{-1}$, only present in the zwitterionic state, and by the increase in intensity of those bands involving $\mathrm{NH}_{3}{ }^{+}$, due to the protonation of the $\alpha$-amino group when the $\mathrm{pH}$ decreased. In contrast, it was observed that the spectra for the adsorbed Lys on silica remained mostly invariable upon $\mathrm{pH}$ change (see Figure 74b). Quantitative spectral analysis based on calibration lines for Lys in solution indicated that, regardless of the $\mathrm{pH}, 81 \pm 5 \%$ of the adsorbed Lys was present in the cationic state and $19 \pm 5 \%$ in the zwitterionic state. With these results and considering that the point of zero charge of the used silica sample is about 2 , the authors interpreted that adsorption of Lys was mainly driven by electrostatic interactions between the 
negatively charged silica surface and the protonated $\mathrm{NH}_{3}{ }^{+}$groups, although hydrophobic interactions between Lys and siloxane bridges were not ruled out. Remarkably, this binding mechanism based on electrostatic forces agrees with the isothermal experiments of O'Connor et al. $^{215}$ on the adsorption of Lys on siliceous MCM-41 and is consistent with the experimental findings of Stievano et al., ${ }^{247}$ which showed a preferential adsorption of Lys from an equimolar mixture of Gly and Lys. Indeed, considering that at $\mathrm{pH} 7$ Gly is in its zwitterionic state, Lys predominantly in its cationic state, and silica bears a significant negative charge, Lys adsorption should certainly be more favorable than Gly adsorption.

As far as Glu is concerned, Bouchoucha et al. ${ }^{211}$ recorded the IR spectrum for the Glu/silica system, results showing that at $\mathrm{pH} 3$ the predominant species was the $\mathrm{H}_{2} \mathrm{Glu}^{ \pm}$zwitterionic state, as the dominant bands were those involving the backbone $\mathrm{NH}_{3}{ }^{+}$and $\mathrm{COO}^{-}$groups and the protonated side chain $\mathrm{COOH}$ (see Figure 75a).
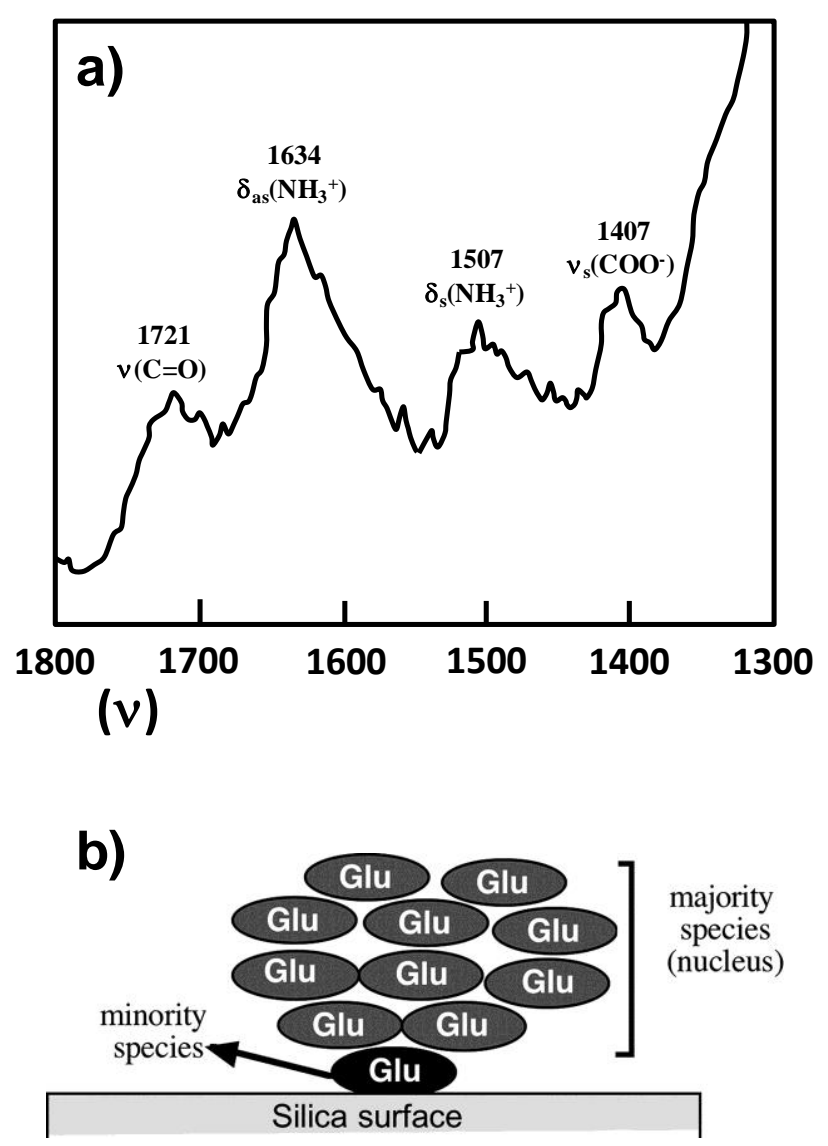

Figure 75. (a): IR spectrum (in $\mathrm{cm}^{-1}$ ) for Glu when adsorbed on silica at $\mathrm{pH} 3$ (adapted with permission from Ref. ${ }^{211}$. Copyright 2011 American Chemical Society). (b) Schematic representation of the Glu/silica interface where two forms of Glu (the molecularly adsorbed and the precipitated ones, as minority and majority species, respectively) were found to coexist.

As expected, at $\mathrm{pH} 8-9$ the $\mathrm{C}=\mathrm{O}$ band associated to $\mathrm{COOH}$ was absent indicating that the predominant species was the $\mathrm{HGlu}^{-}$anion. Similar IR features were obtained for free Glu in solution 
at these $\mathrm{pH}$ values, thereby confirming that the contact with silica does not significantly modify Glu speciation. Additionally, quantitative adsorption data from HPLC suggested a weak affinity between Glu species and the hydrated silica surfaces, and therefore that the interaction mainly takes place via H-bonding rather than electrostatic forces. On the other hand, the obtained XRD diffractograms showed the formation of bulk Glu crystallites at concentrations lower than saturation. This phenomenon was comparable to what geochemists sometimes call "surface precipitation" or better "surface-induced precipitation", i.e., the precipitation of a mineral exclusively on the surface adsorbent, under conditions where the bulk solution is undersaturated. Such a growth has also been reported for the $\mathrm{Gly} / \mathrm{TiO}_{2}(110)$ system" ${ }^{657}$ and this "surface-induced precipitation" has been even used in several procedures of preparation of supported heterogeneous catalysts. $^{658}$ Incidentally, the Glu crystallite formation is different compared to the Gly one. For Glu, the shape of the adsorption isotherm suggests that nucleation of a bulk Glu phase does indeed occur at the silica-solution interface whereas for Gly bulk crystallites can be formed from nonspecifically retained solution during the drying step. However, for Glu, the bulk phase that precipitated was the same that would be obtained from a concentrated aqueous solution of Glu, that is, the $\beta$-polymorph. Solid state NMR measurements confirmed the presence of these two Glu populations; i.e., the molecularly adsorbed form and the precipitated one, as minority and majority species, respectively, (see the schematic picture of Figure 75b) and also suggested that at the interface the adsorbed species compete with a few water molecules to directly interact with the silica surface.

From a theoretical point of view, Lys and Glu adsorptions considering strict gas-phase conditions were simulated by Rimola et al. ${ }^{321}$ using a (001) edingtonite-based surface cluster model that contains 4 isolated $\mathrm{SiOH}$ groups and the ONIOM2(B3LYP/6-311++G(d,p):MNDO) level of theory. As expected, the computed adduct exhibited a rather strong $\mathrm{H}$-bond between the proton of one $\mathrm{SiOH}$ and the $\mathrm{N}$ atom of the amine side chain (see structure of Figure 76a). A similar $\mathrm{H}$ bonding pattern was found by Han et al. ${ }^{659}$ when studying the adsorption of methylamine (considered as a model of the side chain of Lys) on the hydroxylated (0001) surface of $\alpha$-quartz, although in this case the $\mathrm{H}$ of the $\mathrm{NH}_{2}$ group also established a $\mathrm{H}$-bond with an $\mathrm{O}$ atom of one surface $\mathrm{SiOH}$ group. Glu holds a $\mathrm{COOH}$ group at the end of the side chain and thus the most stable adduct found for this amino acid exhibits the cooperative H-bonding pattern between the $\mathrm{COOH}$ and one $\mathrm{SiOH}$ groups, (see Figure 76b). To the best of our knowledge, calculations accounting for water solvent effects have only been carried out for the Lys case by Gambino et al., ${ }^{660}$ who modeled by means of classical MD simulations the adsorption of Lys on a hydroxylated quartz surface with the presence of 350 explicit water molecules. At variance with the ATR-IR results, they found HLys $^{ \pm}$as the most stable state, over the cationic one. Despite that, the results obtained are likewise 
of valuable interest as it was found that Lys interacts adopting an end-on orientation with the protonated $\mathrm{NH}_{3}{ }^{+}$side chain group pointing towards the surface (see schematic structure of Figure 76c). Moreover, it was observed that water provides an indirect cooperative contribution to the Lys/quartz interactions, decreasing the solvation shell around the charged $\mathrm{NH}_{3}{ }^{+}$and $\mathrm{SiO}^{-}$groups and in turn strengthening the Lys/quartz interactions.

a)

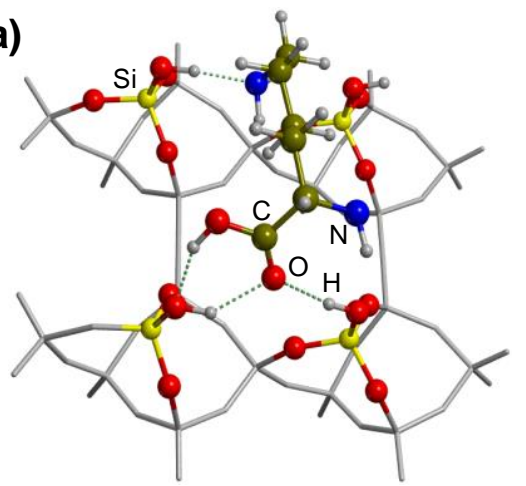

b)

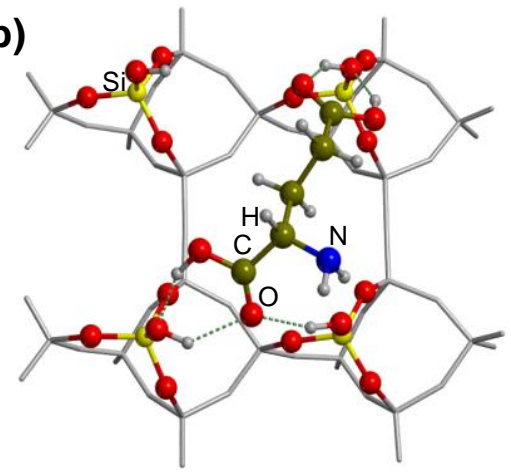

c)

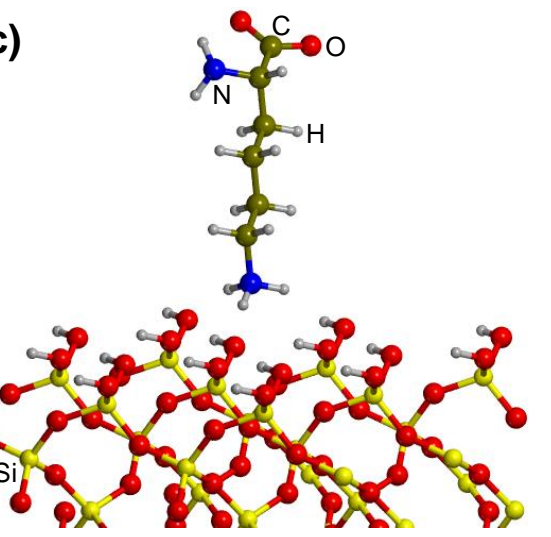

Figure 76. Interaction of Lys (a) and Glu (b) with a hydroxylated (001) edingtonite surface model (drawn from data of Ref. ${ }^{321}$ ) computed at the ONIOM2(B3LY/6-311++G(d,p):MNDO level). (c) Schematic representation of the dominant configuration obtained for zwitterionic Lys interacting with a hydroxylated $\alpha$-quartz surface model in aqueous solution using classical MD simulations (drawn from data of Ref. ${ }^{660}$ ). Water molecules have been omitted for the sake of clarity.

\subsubsection{General trends found in the amino acid adsorption on silica surfaces}

Static quantum chemical calculations have the advantage that they allow to compute binding adsorption energies straightforwardly and efficiently. This is important because by computing the 
adsorption energies of a set of AA on silica surfaces one may establish a scale of relative stability of the AA/silica adducts and hence obtain trends on the affinity of AA with silica. This was actually performed by Rimola et al., ${ }^{321}$ who presented a comprehensive computational study for the gasphase adsorption of 15 AA (Table 17 shows some selected cases) in their canonical state on a model cluster of hydroxylated silica surface based on the (001) edingtonite surface terminated by four isolated $\mathrm{SiOH}$ groups.

Table 17. BSSE-corrected adsorption energies $\left(\Delta E^{\mathrm{C}}{ }_{\mathrm{ads}}\right)$, free energies $\left(\Delta G_{\text {ads }}^{\mathrm{C}}, \mathrm{T}=298 \mathrm{~K}\right)$ and including dispersion (D) for several amino acids interacting with hydroxylated silica surface from Ref. $^{321}$. Units in $\mathrm{kJ} \mathrm{mol}^{-1}$.

\begin{tabular}{lllll}
\hline Amino acid & Side chain behavior & $\Delta E_{\text {ads }}^{\mathrm{C}}$ & $\Delta G_{\text {ads }}^{\mathrm{C}}$ & $\Delta G_{\text {ads }}^{\mathrm{C}}+D$ \\
\hline glycine (Gly) & non-polar & -48.5 & 18.4 & -13.0 \\
alanine (Ala) & non-polar & -46.4 & 18.8 & -7.5 \\
phenylalanine (Phe) & non-polar & -43.1 & 29.7 & -15.9 \\
serine (Ser) & polar/neutral & -56.1 & 25.1 & -20.5 \\
glutamic acid (Glu) & polar/acidic & -76.6 & 7.5 & -36.0 \\
glutamine (gln) & polar/amide function & -72.8 & 14.2 & -33.5 \\
lysine (Lys) & polar/basic & -73.6 & 7.1 & -37.2 \\
arginine (Arg) & polar/basic & -72.4 & 11.7 & -41.0 \\
\hline
\end{tabular}

Geometry optimizations were performed at the ONIOM2(B3LYP/6-311++G(d,p):MNDO) level and adsorption energies were determined after including Grimme's correction for dispersion. ${ }^{420}$ It is worth mentioning that in all cases the adsorbed complexes showed the same interactions observed for Gly (see Figure 62) plus those derived from the AA side chain with the available free $\mathrm{SiOH}$ groups. This ensured that differences in the adsorption energies were due to the AA side chain interactions with silica. The computed adsorption energies (Table 17) indicated that $\Delta E^{\mathrm{C}}$ ads become more favorable as the number of polar groups in the $\mathrm{AA}$ side chain increases due to the formation of stronger H-bonds with the surface. Interestingly, the computed $\Delta G^{\mathrm{C}}$ ads were found to be all positive due to the unfavorable entropic terms. However, since the dispersive forces play a fundamental role on the adsorption energy, when adding the correction for dispersion, final free adsorption energies $\left(\Delta G_{\text {ads }}^{\mathrm{C}}+D\right)$ become all negative. Overall, the authors pointed out that the adsorption processes were mainly dictated by both H-bonding and dispersion. That is, while the former interactions seemed to determine the relative silica affinity for the different AA, the latter ones mainly contributed by greatly lowering the adsorption energies. From the $\Delta G^{\mathrm{C}}{ }_{\text {ads }}+D$ values, the authors established the following relative scale for AA affinity towards silica surfaces of 
moderate hydroxylation degree: polar/basic > polar acidic $\sim$ polar/amidic > polar/neutral > nonpolar.

Sholl and coworkers ${ }^{651,661}$ have reported theoretical results addressed to the enantiospecific adsorption of AA. All natural $\alpha$-AA with the exception of Gly are intrinsically chiral and only one of the two possible enanatiomers is naturally found in proteins. The existence of this biological homochirality raises profound questions about the origins of life. Furthermore, in nature, not only biomolecules are chiral but also naturally occurring minerals may present chirality. Some authors have invoked the possibility that naturally occurring chiral surfaces may discriminate one enantiomer over the other, which in turn is controlled by the enantiospecific interactions between the two partners. Quartz is chiral and because of that, Sholl and coworkers calculated the adsorption energies of the two possible enantiomers of several AA on the chiral (0001) and (0-10) $\alpha$-quartz surfaces. The results obtained are summarized in Table 18. The authors highlighted that measureable energy differences were obtained between the two enantiomers of Ser and Cys, and for Ser, Asp and Asn when adsorbed on the (0001) and (0-10) surfaces, respectively, while for the other amino acids negligible energy differences were computed. They also mentioned, however, that the largest enantiospecific energy difference, given by Asp, was the only case comparable to the computed enantiospecific energy differences reported on metal chiral surfaces, which are indeed able to carry out chiral recognition. ${ }^{62,663}$

Table 18. Computed electronic adsorption energies $\left(\Delta \mathrm{E}_{\mathrm{ads}}\right)$ and including ZPE-corrections $\left(\Delta \mathrm{U}_{\mathrm{ads}}\right)$ for the adsorption of the R and $\mathrm{S}$ enantiomers of alanine (Ala), serine (Ser), cysteine (Cys), aspartic acid (Asp) and asparigine (Asn) on the (0001) and (0-10) $\alpha$-quartz surfaces. In $\mathrm{kJ} \mathrm{mol}^{-1}$. Data obtained from ref. ${ }^{651,661}$

\begin{tabular}{lllll}
\hline Chiral AA & $(0001)^{651}$ & \multicolumn{3}{c}{$(0-10)^{661}$} \\
\cline { 2 - 5 } & $\Delta \mathrm{E}_{\text {ads }}$ & $\Delta \mathrm{U}_{\text {ads }}$ & $\Delta \mathrm{E}_{\text {ads }}$ & $\Delta \mathrm{U}_{\text {ads }}$ \\
\hline$R$-Ala & -74.3 & -68.5 & -70.4 & -69.5 \\
$S$-Ala & -73.3 & -66.5 & -72.4 & -72.4 \\
$R$-Ser & -85.9 & -76.2 & -73.3 & -70.4 \\
$S$-Ser & -90.7 & -83.9 & -69.5 & -67.5 \\
$R$-Cys & $-83.2 /-83.0$ & $-75.0 /-75.5$ & -78.1 & -75.3 \\
$S$-Cys & $-79.2 /-78.7$ & $-71.6 /-70.9$ & -78.1 & -75.3 \\
$R$-Asp & & & -75.3 & -73.3 \\
$S$-Asp & & & -84.9 & -81.0 \\
$R$-Asn & & & -69.5 & -64.6 \\
$S$-Asn & & & -73.3 & -68.5 \\
\hline
\end{tabular}


To the best of our knowledge, systematic studies focused on the adsorption of AA on silica surfaces in solution to obtain an affinity scale have not been reported yet, which is unfortunate since experimental data exist for comparison (Ref. ${ }^{207}$; see Section 3.2.2). However, Walsh and coworkers $^{591,595}$ have reported the binding of several AA analogues (i.e., small molecules that resemble the side chain of AA) on the (001), (100) and (011) hydroxylated $\alpha$-quartz surfaces under aqueous conditions (Table 19 reports some selected cases). It is worth noting that, because of the aqueous environment, the butyl ammonium and ethanoate molecules (as analogues for Lys and Glu, respectively) were considered as charged forms; i.e., carrying $\mathrm{NH}_{3}^{+}$and $\mathrm{COO}^{-}$moieties, respectively. In this work, free energies of adsorption were obtained using the potential of mean force (PMF) method. This method allows running MD simulations for a fixed distance between the center of mass of the adsorbate and the surface. By modifying this distance over a certain range one can get the PMF free energy profile of adsorption and the binding free energy. For this case, the MD simulations were based on classical force fields. Results obtained (see Table 19) indicated that, in general, the PMF free energy of adsorption did not correlate with the surface silanol density (which ranges, from highest to lowest, as $(001)>(100)>(011))$, suggesting that the surface topology, and in particular the interstitial spaces between silanol groups, was important. Indeed, visual inspection of the typical configurations of the AA analogues adsorbed on the $\alpha$-quartz surfaces (see Figure 77) showed that molecules tended to be inserted within the interstitial grooves.

Table 19. PMF free adsorption energies (in $\mathrm{kJ} \mathrm{mol}^{-1}$ ) for some amino acid analogue molecules interacting with the (001), (100) and (011) $\alpha$-quartz surfaces from Ref. ${ }^{595}$

\begin{tabular}{lllll}
\hline molecule & AA-analogue for & $(001)$ & $(100)$ & $(011)$ \\
\hline $\mathrm{CH}_{4}$ & Ala & -4.6 & -5.0 & -6.7 \\
$\mathrm{CH}_{3} \mathrm{OH}$ & Ser & -4.2 & -3.8 & -5.9 \\
$\mathrm{CH}_{3}\left(\mathrm{CH}_{2}\right)_{3} \mathrm{NH}_{3}^{+}$ & Lys & -2.1 & -2.9 & -4.2 \\
$\mathrm{C}_{6} \mathrm{H}_{6}$ & Phe & -7.1 & -8.8 & -11.7 \\
$\mathrm{CH}_{3} \mathrm{COO}-$ & Glu & -6.7 & -5.4 & -12.1 \\
\hline
\end{tabular}



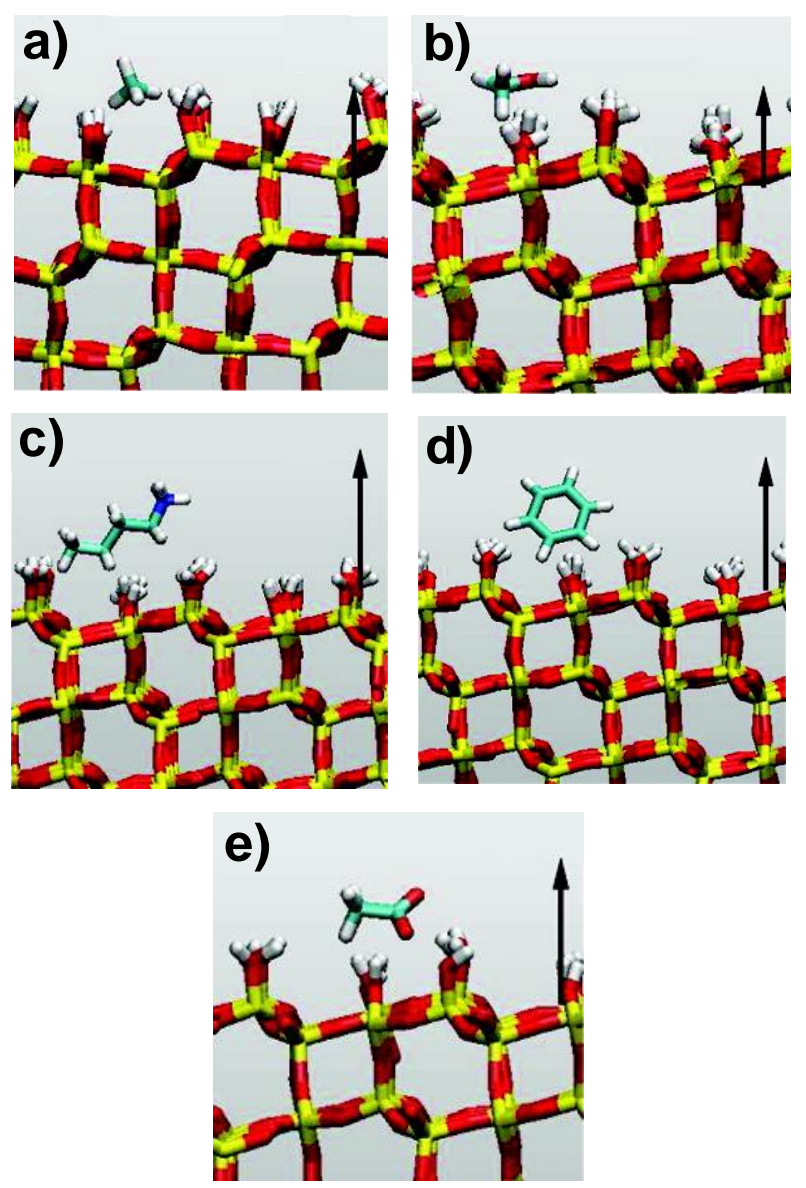

Figure 77. PMF typical configurations of methane (a), methanol (b), butyl ammonium (c), benzene (d) and ethanoate (e) interacting with the (011) $\alpha$-quartz surface in aqueous solution. Water molecules have been removed for the sake of clarity. Adapted with permission from Ref. ${ }^{595}$. Copyright 2012 American Chemical Society).

Accordingly, the authors interpreted that hydrophobic interactions between aliphatic chains and the surfaces contributed significantly to the surface binding. For those AA analogues that bear polar groups, however, it was observed that $\mathrm{H}$-bonding is also relevant for the binding. The obtained PMF adsorption free energies revealed the following trend in the binding: nonpolar/aromatic $>$ polar/negatively charged $>$ nonpolar/aliphatic $>$ polar/neutral $>$ polar/positively charged. The authors also mentioned the limitations of the biomolecule models used. That is, for most residues, the alkyl parts of their side chains will be restrained to point away from the surface (not within or in close proximity the interstitial spaces) by the backbone chains of amino acids and peptides, so their contribution to the binding energy is limited. This is, for instance, the case of methanol and ethanoate (to model Ser and Glu, respectively), in which the methyl groups were found to lie in an interstitial groove, which is not possible if the full amino acid residue is accounted for due to geometry constraints.

The previous results were obtained considering that the silica surfaces did not bear any surface charge; namely, they were neutral. However, silica surfaces may exhibit net electrical surface charges, the sign of which strongly depends on the $\mathrm{pH}$ of the solution when silica is 
immersed. As stated in Section 3.1.2, at solution $\mathrm{pH}$ values above/below the PZC the mineral respectively displays a negative/positive surface charge. Obviously, the higher the difference between the solution $\mathrm{pH}$ and the PZC, the more charges the surface exhibits. The PZC marks a changeover in the electrochemical properties of the silica surfaces; however, since it is quite low for silica (about 2), pH values below the PZC are not very relevant. Analogously, as shown for the Lys and Glu cases, AA can be found in different charged states depending on the $\mathrm{pH}$ of the medium. The isoelectric point $(\mathrm{pI})$ of one $\mathrm{AA}$ is the solution $\mathrm{pH}$ at which the AA carries equal amounts of positive and negative charges; that is, it is found as zwitterion. In fact, if there are no ionizable groups in the side chains, the $\mathrm{pI}$ is simply the mean value of the $\mathrm{pKa}$ 's that involve the carboxylic acid and the ammonium functions, $\mathrm{pKa}_{1}$ and $\mathrm{pKa}_{2}$, respectively. When there is an ionizable group in the side chain, its $\mathrm{pKa}$ value $\left(\mathrm{pKa}_{\mathrm{R}}\right)$ also intervenes. Accordingly, at $\mathrm{pH}$ values above/below the $\mathrm{pI}$, the AA is found to be anionic/cationic. Due to these $\mathrm{pH}$-dependent electrical properties shown by silica surfaces and AA, the charged states of the two partners in the adsorption processes may determine the strength of the AA/silica interactions, which in this case will be mainly dictated by electrostatic forces. As already mentioned in Section 3.1.2, Churchill et al. ${ }^{105}$ have studied the influence of the PZC of quartz surfaces and the $\mathrm{pI}$ of some amino acids in the adsorption processes. The PZC of the quartz surfaces was determined, using the atomic force microscope (AFM) technique to be $\approx 2.8$, whereas the amount of the adsorbed AA on quartz was analyzed with liquid chromatography techniques. Table 20 shows the results obtained, jointly with pKa and pI values of the AA tested.

Table 20. Relative adsorption of amino acids analyzed by high-performance liquid chromatography (HPLC) and reverse-phase liquid chromatography (RPLC) on quartz, and $\mathrm{pKa}$ 's and isoelectric points (pI) of the amino acids tested. $\mathrm{pKa} \mathrm{a}_{1}, \mathrm{pKa} \mathrm{a}_{2}$ and $\mathrm{pKa}$ refer to $\mathrm{pKa}$ values for backbone $\mathrm{COOH}$, $\mathrm{NH}_{3}{ }^{+}$and side chain $\mathrm{R}$ groups, respectively.

\begin{tabular}{lllllll}
\hline Amino acid & \multicolumn{2}{l}{ Relative adsorption } & $\mathrm{pKa}$ values & $\mathrm{pI}$ \\
\hline \multirow{2}{*}{ Lysine (Lys) } & HPLC $^{a}$ & RPLC $^{b}$ & $\mathrm{pKa} \mathrm{a}_{1}$ & $\mathrm{pK \textrm {a } _ { 2 }}$ & $\mathrm{pK \textrm {a } _ { \mathrm { R } }}$ & \\
\cline { 2 - 6 } Tyrosine (Tyr) & 1.00 & - & 2.18 & 8.95 & 10.53 & 9.74 \\
Alanine (Ala) & $<0.20$ & 1.00 & 2.20 & 9.11 & 10.07 & 5.66 \\
Glycine (Gly) & $<0.05$ & 0.81 & 2.34 & 9.69 & - & 6.01 \\
Glutamic acid (Glu) & $<0.05$ & 0.51 & 2.34 & 9.60 & - & 5.97 \\
Aspartic acid (Asp) & $<0.05$ & 0.12 & 1.88 & 9.60 & 3.65 & 2.77
\end{tabular}

${ }^{a}$ measurements normalized with respect to Lys. ${ }^{b}$ measurements normalized with respect to Tyr 
AA with pI values that greatly differ from the PZC of quartz adsorbed in greater abundance than AA for which pI values and the PZC are similar. That is, Lys was found to be the most adsorbed AA (it is indeed the amino acid that exhibits the largest difference between pI and PZC, 9.74 vs 2.8, respectively), while Asp and Glu are the least adsorbed amino acids (with pI values similar to PZC, 2.80 and 3.04 vs 2.8, respectively). This could be understood considering that at $\mathrm{pH}$ values above the PZC of quartz but below the pI of the AA means the quartz surfaces and AA have opposite charge and thus, electrostatic interaction is attractive and large. For instance, at $\mathrm{pH}$, quartz has a moderate negative charge density on the surfaces but Lys is mostly found in its cationic state, so that attractive electrostatic interactions occur between them, thus favoring adsorption. In contrast, Asp is mostly found as anion (like quartz) so that repulsive electrostatic interactions are established, thereby giving rise to poor adsorption of Asp. This can be visualized in Figure 78.

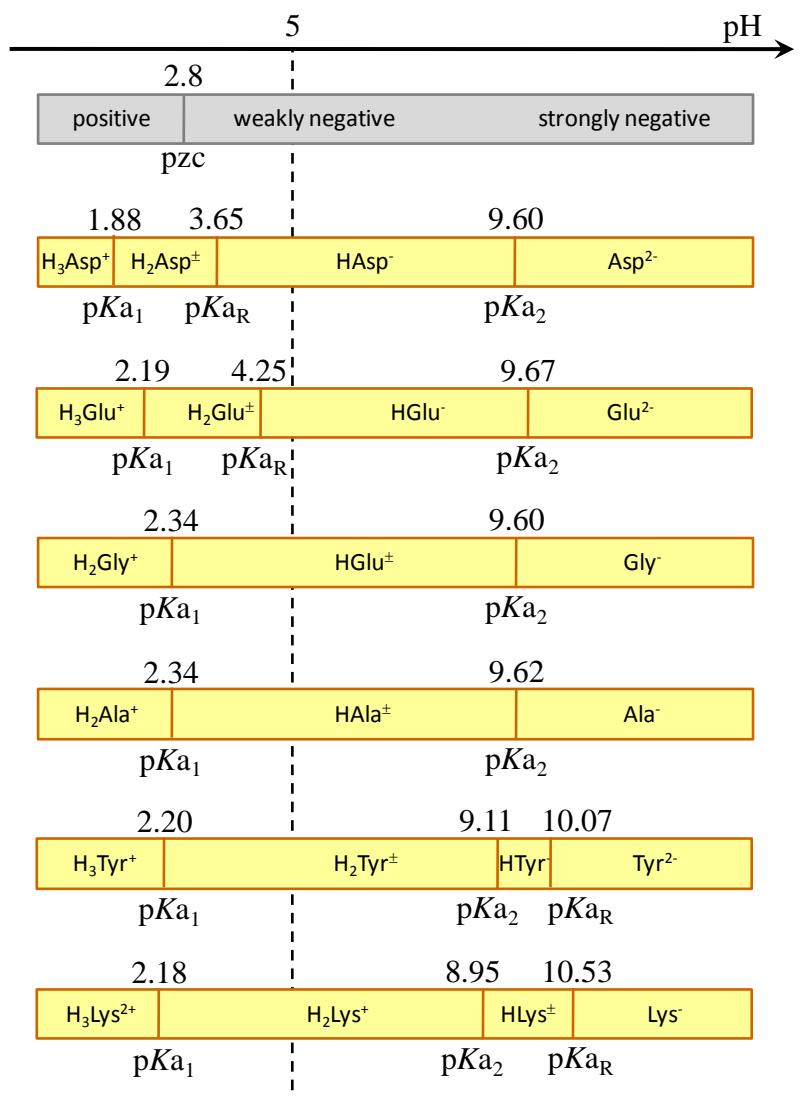

Figure 78. Theoretical acid-base speciation of the $\alpha$-quartz and the AA tested in Ref. ${ }^{105}$ as a function of $\mathrm{pH}$. $\mathrm{pKa}, \mathrm{pKa} \mathrm{a}_{2}$ and $\mathrm{pKa} \mathrm{a}_{\mathrm{R}}$ refer to $\mathrm{pKa}$ values for backbone $\mathrm{COOH}, \mathrm{NH}_{3}{ }^{+}$and side chain $\mathrm{R}$ groups, respectively.

Interestingly, the greater the separation between the PZC and the pI values, the greater the charge difference between the mineral surface and the amino acid and thus, the more favorable the electrostatic interaction. This $\mathrm{pH}$-dependent adsorption has been also observed by other authors via acid-base titration experiments. Bouchoucha et al. ${ }^{211}$ and Gao et al. ${ }^{253}$ also observed low amounts of Glu adsorption on amorphous silica and SBA-15, respectively, which was attributed to the negligible difference in $\mathrm{pH}$ between the $\mathrm{pI}$ of Glu and the PZC of these silica materials (around 3). 
In the same line, both Gao at al. ${ }^{253}$ and Vlasova et al. ${ }^{214}$ observed that adsorption of Arg (pI = 10.76) readily occurred on SBA-15 and on surfaces of highly dispersed silica particles, respectively, and that the adsorption amounts quickly increased from $\mathrm{pH} 3$ to 9 because, while the amount of positively charged Arg was kept almost constant within this range, the silica surfaces became more and more negatively charged.

All these facts evidence that electrostatic forces play an important role in the adsorption processes, especially in the $\mathrm{pH}$ regions which correspond to changes in the speciation of the AA and silica surfaces. In the work of Gao et al. ${ }^{253}$ the adsorption of AA with non-polar side chains (Phe, leucine (Leu) and Ala) was also studied. For the particular case of Phe, the mean charge per molecule was approximately zero in the $\mathrm{pH} 4-8$ range (namely, it was present as zwitterion) but Phe was intensively adsorbed on the surfaces of the SBA-15 and the amount adsorbed was insensitive to $\mathrm{pH}$ variation since the acido-basic speciation of Phe was hardly affected. Furthermore, these authors also found that the adsorption amounts of these three amino acids increased with the increase of the size of their side chains (i.e., Phe > Leu > Ala). These facts led them to interprete that the adsorption of Phe was mainly driven by hydrophobic interactions between the non-polar side chain and the siloxane Si-O-Si surface groups. Along this line, it has been recently demonstrated that hydrophobic interactions indeed play an essential role which is clearly revealed when electrostatic and H-bonding interactions can be considered as constant. ${ }^{426}$

In summary, it seems clear that the adsorption mechanism of AA on silica surfaces may be driven by three main kinds of contributions: i) electrostatic forces between AA ions and negatively charged $\equiv \mathrm{SiO}^{-}$surface groups, which become particularly favorable when the silica surfaces and AA exhibit a net charge of opposite sign achieved by modulating the $\mathrm{pH}$; ii) $\mathrm{H}$-bonding interactions between AA (either as neutral molecules or ions) and neutral $\equiv \mathrm{SiOH}$ groups; iii) hydrophobic interactions between the AA side chains and the siloxane $\equiv \mathrm{Si}-\mathrm{O}-\mathrm{Si} \equiv$ groups.

\subsubsection{Thermal-induced transformations of adsorbed amino acids on silica surfaces}

Upon adsorption amino acids may react to give DKP (vide supra). Indeed, early experiments of Basiuk et al. (ref. 241) developed a synthethic procedure for obtaining DKPs derivatives from simple aminoacids by means of their sublimation in the presence of silica gel. This was the starting point for further very extensive studies of silica catalyzed thermal transformations of aminoacids. Even though DKPs are usually major products, the catalytic activity of silica is so high that DKPs can be further singly or even doubly N-acylated to form imidazopyrazine systems. ${ }^{664-667}$ Formation of DKP derivatives via silica-catalyzed dehydrocyclization of linear dipeptides has also been reported. ${ }^{668}$ These examples show that thermal transformations of amino acids in the presence of silica is the gateway to a very rich chemistry that has not yet been fully investigated. 
Experiments in water solution with Gly, Ala, Lys and Glu, carried out by Lambert et al, ${ }^{652}$ have shown that upon thermal activation at moderate temperatures $\left(150-160 ;{ }^{\circ} \mathrm{C}\right)$, clean polymerization events occur, which can easily be observed by thermogravimetric analysis. Figure 79 shows the differential thermogravimetric (DTG) analysis for these AA/silica systems.

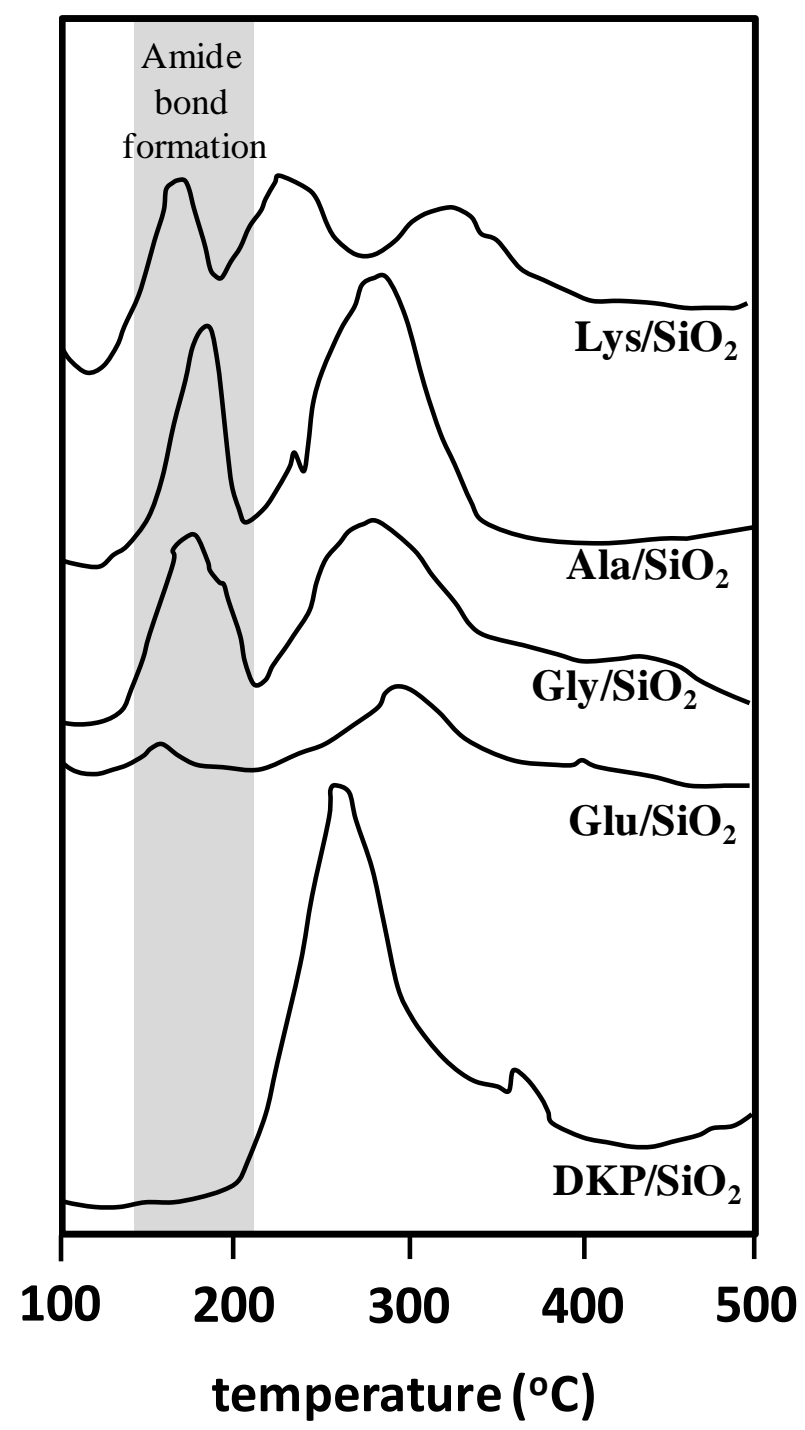

Figure 79. Differential thermogravimetric analysis of several AA and DKP on amorphous silica The temperature region where amide bond formation seems to occur is highlighted in grey. Adapted with permission from Ref. ${ }^{652}$. Copyright 2009 Elsevier.

At least two clear regions were differentiated: i) one around $150^{\circ} \mathrm{C}$, which was attributed to amide bond formation, and ii) another one around $300^{\circ} \mathrm{C}$, which corresponded to the degradation of the adsorbed organic matter. Noticeably, the peak at $150{ }^{\circ} \mathrm{C}$ was not present for the $\mathrm{DKP} /$ silica system as no amide bond formation could occur (there are no free amine or carboxylic groups in this molecule). In spite of these general similarities between AA, the case of Glu has been studied in more detail by Bouchoucha et al. ${ }^{211}$ because, in addition to the peak at $150-160{ }^{\circ} \mathrm{C}$, another well defined peak at $110-120^{\circ} \mathrm{C}$ was also detected in some conditions (see Figure 80a). 
a)
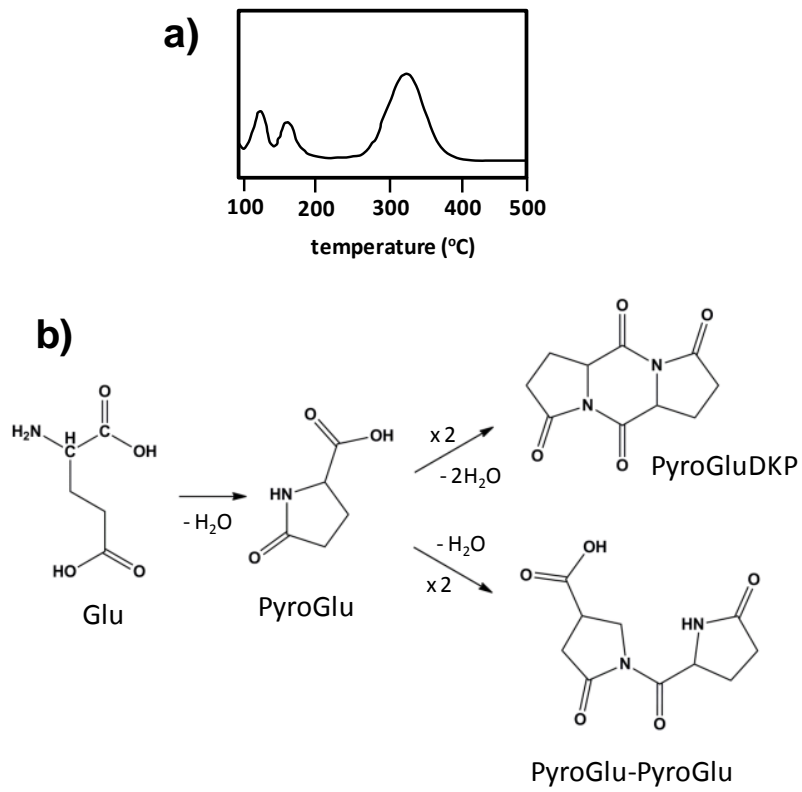

Figure 80. (a) Thermogram (DTG) of Glu on amorphous silica. (b) Schematic representation of the thermal events suggested in Ref. ${ }^{211}$

Experimental measurements performed with FTIR, ssNMR and ESI-MS (ElectroSpray Ionization-Mass Spectrometry) techniques clearly indicated that this first peak was due to an intramolecular lactam ring closure of Glu to yield pyroglutamic acid (PyroGlu, see Figure 80b). The evidence, however, was less clear for the second peak. Thermogravimetric results indicated that this second step could also be associated to the loss of one water molecule per Glu. Additionally, in situ IR showed that the product formed had some bands attributable to the imide functions. These two facts led the authors to deduce that the product formed was the PyroGluDKP (see Figure 80b), which was formed via cyclic condensation of two PyroGlu molecules with the subsequent release of two water molecules. ESI-MS experiments also showed the presence of a peptide constituted by two PyroGlu moieties; i.e., PyroGlu-PyroGlu, which also bears an imide bond (see Figure 80b). However, the peptide could itself be the result of a very quick hydrolysis of previously extant PyroGluDKP upon exposure to water in ESI conditions. Irrespective of that, these results evidenced the capability of silica surfaces to act as activators of condensation events. In water solutions the internal cyclization to give PyroGlu is known to be thermodynamically disfavored. In the absence of water the process becomes thermodynamically favorable but may be kinetically slow; in the form of bulk crystalline Glu, it does indeed occur but only at $197^{\circ} \mathrm{C}$, whereas when supported on silica the condensation was observed to take place at $118^{\circ} \mathrm{C}$. Therefore, the silica surface exerts a kinetic effect on the reaction by working as a catalyst.

\subsection{Interaction with peptides}


The works available in literature addressed to study the interaction of peptides with silica surfaces pursue common objectives (Section 3.2.7). Most of them focus on determining the binding forces that dictate the peptide/silica interactions; other works aim at unraveling the amino acidic residues most prone to interact with silica surfaces, and just a few evaluate the behavior of peptides upon adsorption. In this review, some details of these works will be provided. It is worth underlining that a great number of works concerning the immobilization of proteins in mesoporous silicates are also available in literature. These works are of great interest because of their potential applications in the areas of biotechnology and biocatalysis. They have been recently reviewed by Hudson et al. ${ }^{669}$ and therefore will not be addressed in this review.

As far as the first group of works is concerned, a very recent study of Patwardhan et al. ${ }^{273}$ emerges as a landmark on the chemistry of aqueous silica nanoparticle surfaces and the mechanism of peptide adsorption. In this work, three types of amorphous silica nanoparticles with 15, 82 and $450 \mathrm{~nm}$ diameter were synthesized and by means of base titrations and zeta potential measurements it was found that the acidity of the silica nanoparticles increased with the particle size. The authors stated that such a dependence of the acidity with the nanoparticle size was due to the adoption of a modified Stöber synthesis ${ }^{670,671}$ for the nanoparticle preparation. In this procedure the concentration of ammonia was varied to control the particle size, and higher ammonia concentration precipitated larger particles in such a way that more silanol groups became ionized. The PZC decreased from 4.2 to 2.4 as the size of the silica particles increased from 15 to $450 \mathrm{~nm}$ and by means of classical MD dynamics the authors estimated that between 5-20\% (corresponding to 15-450 nm size) of silanol groups were ionized in the form of $\equiv \mathrm{Si}^{-} \mathrm{O}^{-}$at $\mathrm{pH}$ 7. Peptide binding assays with seven different dodecapeptides were performed using different synthetic peptides by means of zeta potential measurements. It was shown that peptides with higher $\mathrm{pI}$ values (i.e. those that contained Lys and Arg residues) were attracted more strongly for those surfaces that exhibited larger surface density of negatively charged $\equiv \mathrm{Si}^{-} \mathrm{O}^{-}$groups, thus indicating electrostatic contribution to binding. This was also observed by Chen et al., ${ }^{233}$ who showed that two peptides enriched with basic amino acid residues had a great binding affinity to $\mathrm{SiO}_{2}$ supports, and this is also in line with the trend observed by Churchill et al. ${ }^{105}$ using AA instead of peptides (see Section 9.1.3). Contribution of electrostatic forces in the peptide/silica interaction was also demonstrated by the fact that at low initial concentration (less than $0.2 \mathrm{mg} / \mathrm{l}$ ) cationic peptides (namely, those with higher pI) were immediately attracted by silica nanoparticles, presumably via ion pairing, whereas for non-cationic peptides (namely, those with lower pI) weak or no adsorption occurred, since the electrostatic interaction was not favorable. However, these non-cationic peptides indeed adsorbed after overcoming a certain initial peptide concentration threshold value. Since these peptides contained polar amino acidic residues such as serine, threonine and tyrosine (with $\mathrm{R}=\mathrm{CH}_{2} \mathrm{OH}, \mathrm{CH}\left(\mathrm{CH}_{3}\right) \mathrm{OH}$ 
and $\mathrm{PhOH}$, respectively), the authors interpreted that $\mathrm{H}$-bonding directed the interaction of these peptides with silica nanoparticles. Additionally, at high concentrations, adsorption of cationic peptides seemed to level off due to saturation of $\equiv \mathrm{Si}^{-} \mathrm{O}^{-}$sites (Langmuir adsorption, monolayer), whereas for non-cationic peptides the adsorption increased even after further elevating the initial concentration, in support of the formation of $\mathrm{H}$-bonded peptide multilayers. The authors stated that these two binding mechanisms were consistent with the different initial concentrations of the two peptide groups needed to reach significant adsorption. That is, since H-bonds are weaker than electrostatic interactions, the adsorption of cationic peptides required less peptide concentration than for non-cationic peptides.

In this same work, ${ }^{273}$ classical MD simulations using polymer consistent force field augmented for silica (PCFF-SILICA) at $\mathrm{T}=300 \mathrm{~K}$ were also carried out. Simulations addressed the interaction of a cationic and a non-cationic peptide on neutral and partially ionized silica surfaces. Results showed that the former peptide was found within a distance $<3 \AA$ from the superficial $\mathrm{SiOH} / \mathrm{Si}^{-} \mathrm{O}^{-}$layer during $80 \%$ of simulation time while the non-cationic one remained separated from the same surface by several water layers. In the bound conformations $\mathrm{NH}_{3}{ }^{+}$moieties from Nterminal and side chains tended to be close to surface $\equiv \mathrm{Si}^{-} \mathrm{O}^{-}$, thereby confirming the formation of ion pairs. Despite that, it was also observed that polar groups established intermittent contacts with $\mathrm{SiOH}$ through $\mathrm{H}$-bonds. Figure 81 shows snapshots of the MD that reflect these situations. 


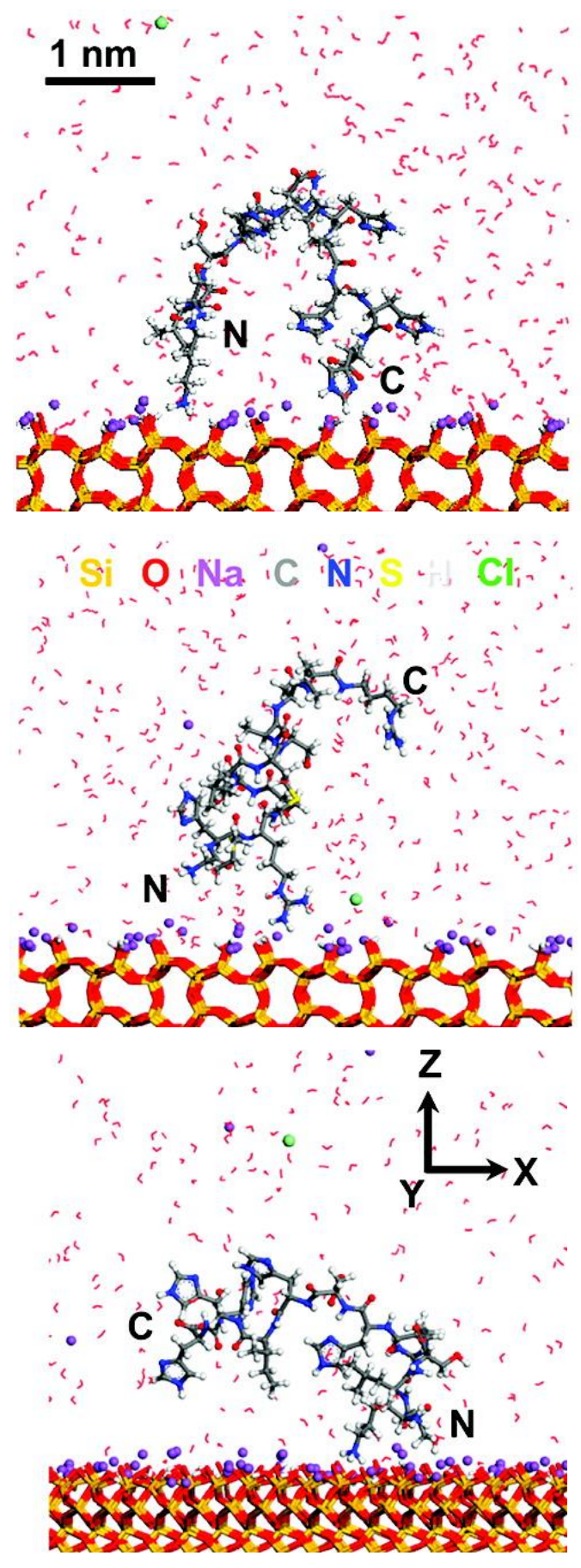

Figure 81. Snapshots of the peptides adsorbed on silica surfaces during classical MD simulations (PCFF-SILICA force field, $\mathrm{T}=300 \mathrm{~K}$ ). Interaction with a neutral silica surface (top and middle) and with a negatively charged silica surface (bottom) due to the presence of $\mathrm{SiO}^{-}$surface groups. The $\mathrm{N}$ and C-terminal have been labeled. Adapted with permission from Ref. ${ }^{273}$. Copyright 2012 American Chemical Society.

In view of these results, the authors concluded that the major contributions to binding are ion pairing via electrostatic forces and H-bonds, although they were aware that less energetic iondipole, dipole-dipole and van der Waals interactions must be also present, and that the balance of these two major contributions depends on the acidity of the silica and on the pI and conformational flexibility of the peptides. 
Results obtained from classical MD simulations carried out by Nonella et al., ${ }^{325}$ in this case using the CHARMM-modified force field, are also along this line. In this work the peptide model was the Ala-Lys-Lys-Lys-Ala sequence (AKKKA, charge of +3) and the silica surface was based on the edingtonite structure with some deprotonated silanol groups (charge of -19), the electroneutrality of the system being completed by the inclusion of $\mathrm{Cl}^{-}$or $\mathrm{Na}^{+}$ions. After several MD runs authors observed that the interaction occurred through direct peptide/surface and indirect peptide/water/surface H-bonds, which in turn were continuously formed and broken during the trajectories. Such a peptide/surface interaction variability caused the peptide to exhibit a certain mobility, both in terms of diffusion along the surface and of internal conformational changes, a fact that led the authors to interpret that the "adsorbate state" showed strong dynamical features and, accordingly, an ensemble of different adsorbed structures should exist. Figure 82 shows two MD snapshots in which the peptide interacts directly through $5 \mathrm{H}$-bonds with the surface (a) or indirectly bridged by water solvent (b).

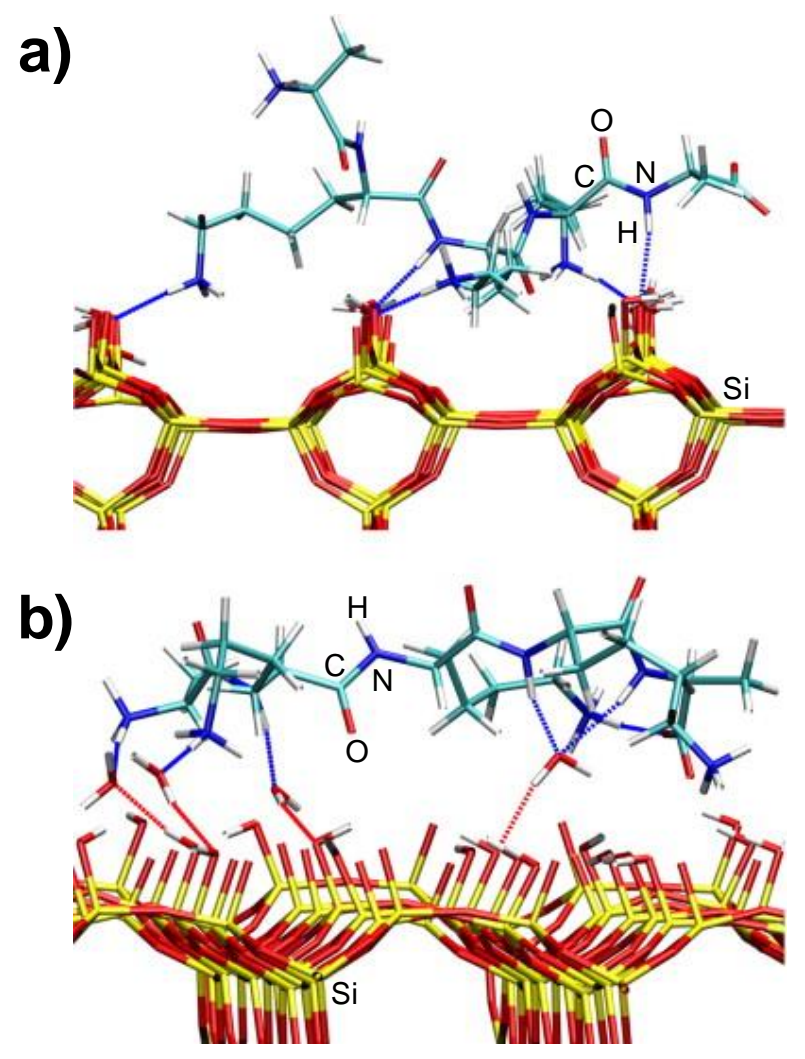

Figure 82. Snapshots of the peptide/silica system during classical MD simulations in aqueous solvent (CHARMM-type force field, $\mathrm{T}=300 \mathrm{~K}$ ). (a) direct $\mathrm{H}$-bonds between peptide and silica; (b) indirect $\mathrm{H}$-bonds between peptide and silica bridged by water. Only water molecules involved in $\mathrm{H}$ bonds are shown. Redraw with permission from data of Ref. ${ }^{325}$ Copyright 2010 Elsevier,

Remarkably, it was found that direct $\mathrm{H}$-bonds all adopted the $\mathrm{Lys}-\mathrm{NH}_{3}{ }^{+} \ldots{ }^{-} \mathrm{OSi} \equiv$ pattern, thus including a strong electrostatic component, whereas indirect H-bonds adopted either 


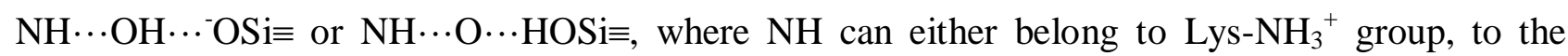
terminal $\mathrm{NH}_{3}{ }^{+}$group or to the backbone $\mathrm{NH}$ moiety and $\mathrm{O}$ or $\mathrm{OH}$ belong to a water molecule.

It seems clear that electrostatic interactions between charged peptides and silica surfaces play a significant role in peptide binding. This adsorption driving force has been exploited by Jonsson and coworkers ${ }^{672,673}$ in order to induce helical peptide structures when adsorbed on silica particles, as already mentioned in Section 3.2.6. Indeed, the authors beautifully showed that designed peptides, which were unconstructed in solution, adopted a well-defined helix structure upon silica adsorption due to the electrostatic interaction between the two partners (see Figure 83a).
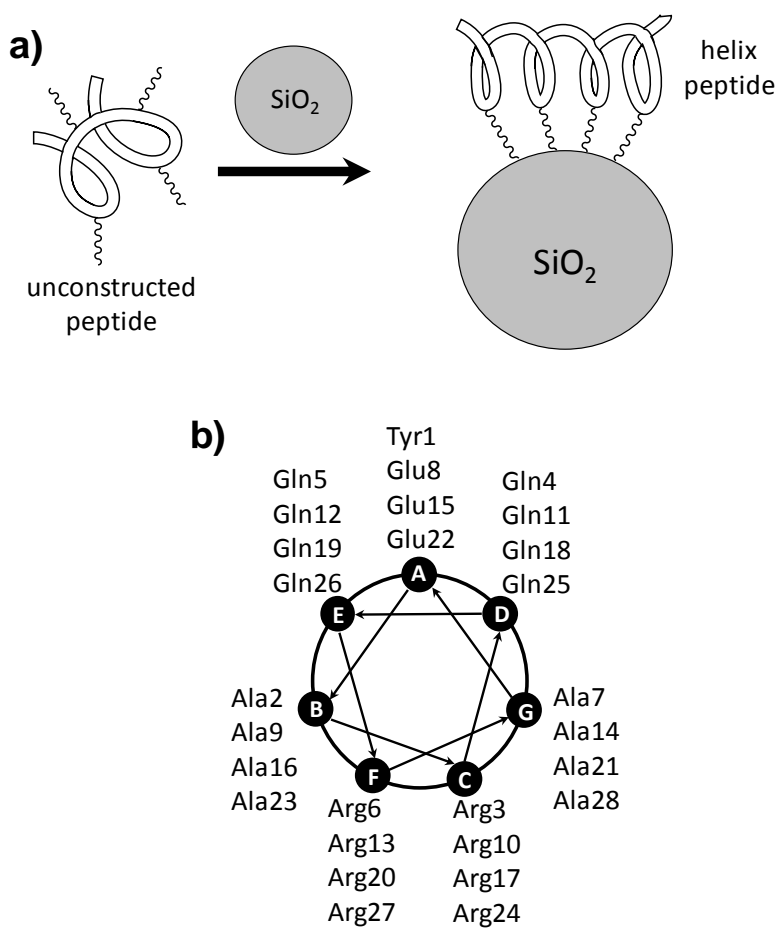

Figure 83. (a) Schematic representation of the silica surface-induced helix formation. (b) Amino acid sequence of a peptide presented in Ref. ${ }^{673}$ that folds as an $\alpha$-helix in the presence of silica surfaces, depicted as a helical wheel, where each point gives rise to a row of amino acids.

In these works, several peptides were synthesized with the particularity that basic amino acidic residues such as Arg were precisely placed at strategic positions in such a way that when adopting an $\alpha$-helix structure they were positioned on the same side of the helix (see Figure $83 \mathrm{~b}$ ). Since at $\mathrm{pH} \mathrm{8-10} \mathrm{the} \mathrm{basic} \mathrm{residues} \mathrm{and} \mathrm{the} \mathrm{surfaces} \mathrm{of} \mathrm{the} \mathrm{silica} \mathrm{nanoparticles} \mathrm{exhibited} \mathrm{net}$ positive and negative charges, respectively, the electrostatic complementarity induced the formation of helical structures upon adsorption. Additionally, the addition of acidic residues such as Glu, which were deprotonated at these $\mathrm{pH}$ values, at positions that pointed away the surface when adopting helix structure (i.e., facing the solution, see Figure $83 \mathrm{~b}$ ) resulted in a significant increase of the helical content of the peptide upon silica adsorption, probably due to both electrostatic repulsion with the surface and attraction to water solvent. Interestingly, Bolivar et al. ${ }^{209}$ have demonstrated that proteins that in solution exhibited helix conformations constituted by Arg 
residues, which in turn were exposed on one side of the helix, retained the very same conformation upon silica surface adsorption. This was due to the large affinity between the Arg-rich helix regions and the surface, hence opening up a potential way to immobilize enzymes on silica surfaces, which usually tends to denaturation.

Despite these findings, Manyar et al. $^{674}$ observed that during the enzyme pepsin immobilization in SBA-15 the maximum loading was achieved when both the enzyme and the matrix were negatively charged. Because of these surprising results, Giussani et al. ${ }^{675}$ have recently presented a theoretical study to understand this behavior. The work was carried out by means of classical MD simulations (CHARMM22 force field) where a complete solvated pepsin with a -6 charge interacted with a flat silica surface exhibiting negatively charged $\mathrm{SiO}^{-}$groups in the presence of either $\mathrm{Na}^{+}$and $\mathrm{K}^{+}$cation to insure electroneutrality. Results indicated that, irrespective of the counter ion, solvated pepsin adhered to the surface regions where the $\mathrm{SiO}^{-}$groups were located because of the mediation of the cations and solvent. It was found that the actual pepsin/silica interaction depended on the nature of the co-solvating cation. That is, $\mathrm{K}^{+}$was found to coordinate the $\mathrm{SiO}^{-}$oxygen atom dragging the solvated pepsin on top of the negative surface, whereas $\mathrm{Na}^{+}$ showed more affinity towards water than $\mathrm{SiO}^{-}$, forcing water molecules that surround pepsin to stick via H-bonding to silanolates, thereby approaching pepsin towards the surface.

Related to the question of which residues are more prone to interact with silica surfaces, Sarikaya and coworkers ${ }^{271,272,676}$ have reported results that provide clues to understand the connection between the amino acid content, the sequence and the molecular structure of peptides on the one hand, and their interaction and affinity to silica on the other hand. Remarkably, this has been achieved by combining bioinformatics, experiments and theoretical simulations. They first developed a bioinformatic approach ${ }^{271}$ that allowed to identify and select available peptides according to their inorganic-solid binding properties (Section 3.2.6), so that a classification from strong to weak-binding peptides sequences was established. Interestingly, the binding properties of these selected peptides towards quartz experimentally measured using surface plasmon resonance spectroscopy very well matched those predicted by bioinformatic tools. Among the different selected peptides, it was found that the strong binding ones contained hydrophobic regions characterized by the presence of proline (Pro) along with other hydrophobic residues such as tryptophan (Trp) and leucine (Leu), whereas the weak-binding sequences did not. In order to evaluate the role of these residues in the interaction, classical MD simulations (CHARMM force field) of three peptides (two strong-binders and one weak-binder) in interaction with quartz (100) surface were carried out. ${ }^{272,676}$ It was found that the strong-binding peptides exhibited the Pro-rich regions in close proximity to silica surface, and visual inspection revealed that these residues were 
buried at interstitial sites of quartz, whereas polar groups (Ser, His and C-terminus carboxylate) were involved in H-bonds with the surface (see Figure 84a and Figure 84b).
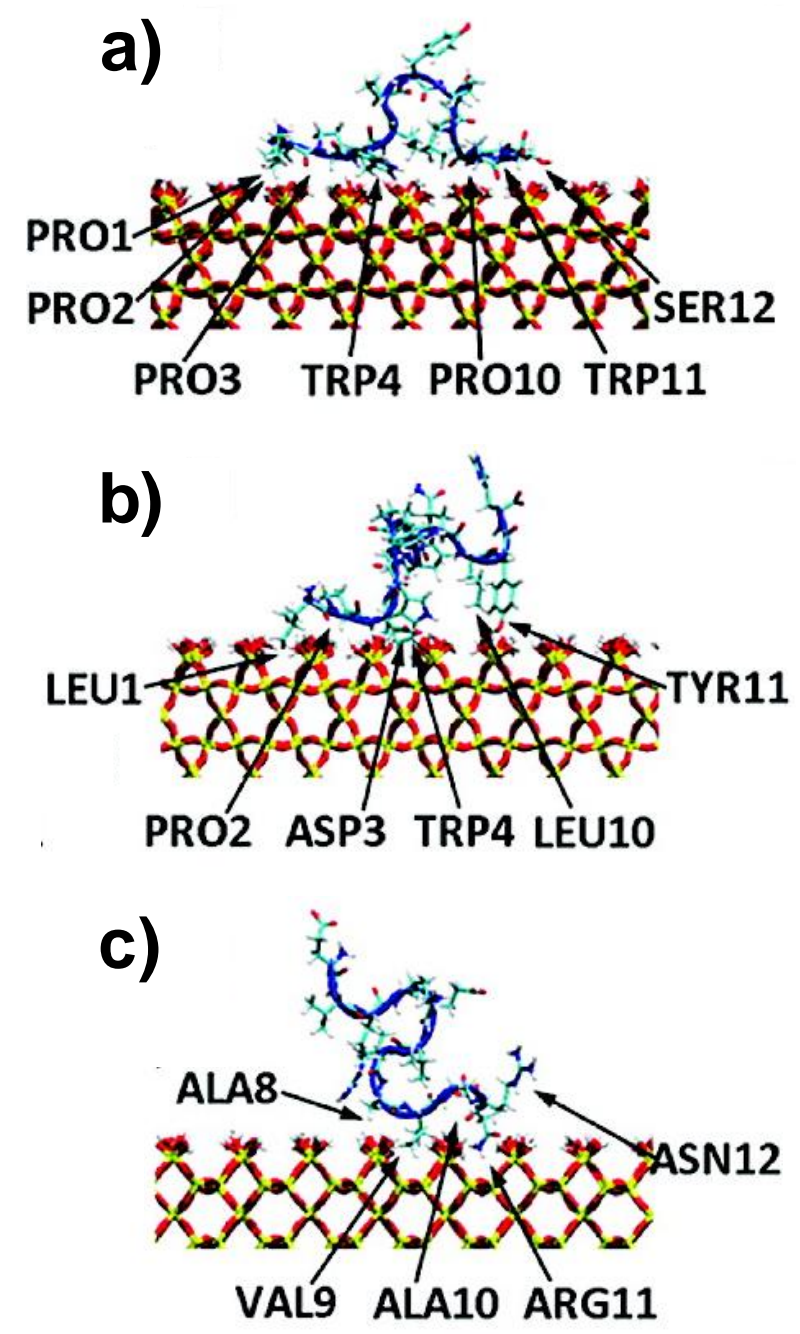

Figure 84. Snapshots of the peptide/silica systems during classical MD simulations in aqueous solvent (CHARMM-type force field, $\mathrm{T}=300 \mathrm{~K}$ ), of the for strong-binding peptides $(\mathrm{a}$ and $\mathrm{b}$ ) and the weak-binding peptide (c). Water molecules have been omitted for the sake of clarity. Adapted with permission from Ref. ${ }^{272}$. Copyright 2010 American Chemical Society.

For the weak-binding peptide, it was observed that the interaction occurred through $\mathrm{Val}(\mathrm{R}=$ $\mathrm{CH}\left(\mathrm{CH}_{3}\right)_{2}$ ), Ala and Arg (see Figure 84c), the former two residues being attracted to the hydrophobic quartz interstices while the Arg/quartz contact was attributed to a combination of electrostatic and $\mathrm{H}$-bonding. Therefore, it seems that hydrophobic regions are prone to interact with quartz surfaces due to the presence of hydrophobic cavities. This had already been suggested and shown using amino acid analogues (see above, Section 9.1.3). The authors also stated that the Prorich region play a second role to favor the interaction. That is, analysis of internal H-bonds (namely, intrapeptide and peptide-water $\mathrm{H}$-bonds) both in solution alone and in interaction with quartz revealed that the two strong-binding peptides do not change the number of internal H-bonds, while the weak-binding one loosens a certain number of its internal $\mathrm{H}$-bonds upon adsorption. This 
suggested that the Pro-rich regions might have hindered the formation of internal H-bonds in the sense that their internal stability remained constant between the solution and contact with the surface, whereas for the other peptide the internal stability decreased when adsorbed due to the loss of internal H-bonds, rendering the peptide/silica complex globally less stable.

According to these results, the propensity of certain residues to interact more or less favorably with silica surfaces, which ultimately leads to the specific recognition of a given silica surface by a given peptide sequence, arises from the complex interplay of interactions such as electrostatic interactions, directional H-bonding, and hydrophobic (dispersive) forces. However, a very recent work of Schneider et al. ${ }^{308}$ highlighted that the peptide-sequence/silica surface recognition may also be helped by the structuring of the water solvent when in contact with the silica surface features; that is, amino acid side chains are capable to "sense" the molecular solvent structure, and in particular the local solvent density variations, at the solid/liquid interface with atomic scale precision, similarly to the lock-and-key recognition mechanism typical in biological systems. The authors performed several classical MD simulations (AMBER force field) for the adsorption of Arg-Lys-Leu-Pro-Asp-Ala (AKLPDA) onto an oxidized silicon surface that contained $\mathrm{SiOH}$ and $\mathrm{SiO}^{-}$surface groups. Results indicated that the Arg and Lys residues remained close to the surface during most of the simulation time (see Figure 85a), the computed adsorption free energy being -15 and $-7 \mathrm{~kJ} \mathrm{~mol}^{-1}$ for Arg and Lys, respectively. 

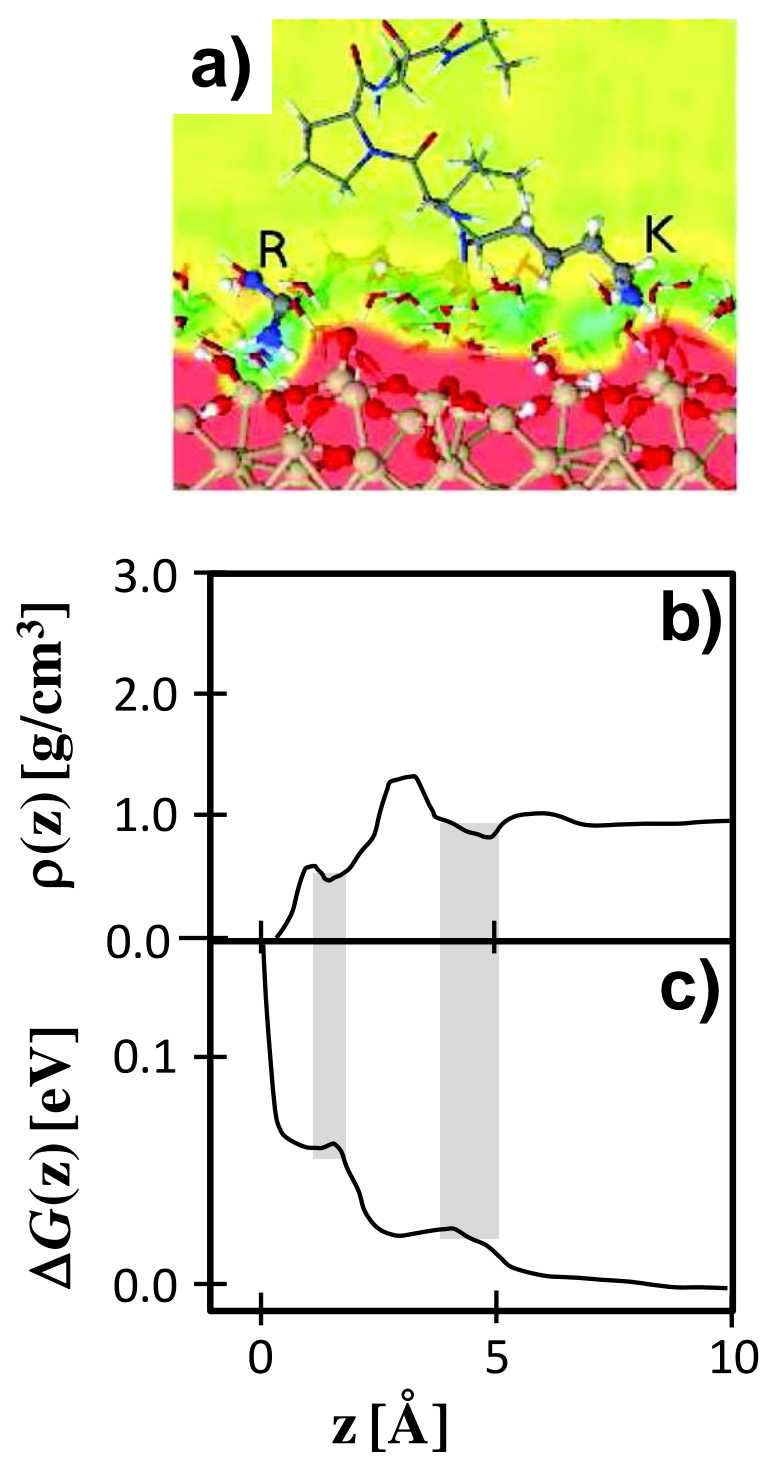

Figure 85. (a) Snapshots of the peptide/oxidized silicon surface system during classical MD simulations in aqueous solvent (AMBER force field, $\mathrm{T}=300 \mathrm{~K}$ ). Arginine $(\mathrm{R})$ and lysine $(\mathrm{K})$ residues have been labeled. (b) Density profile of water oxygen on the oxidized silicon surface. (c) Free energy profile of a spherical hydrophobic solute on the oxidized silicon surface. Grey regions indicate that positions with low water density on the surface match with maxima of $\Delta G(\mathrm{z})$. Adapted with permission from Ref. ${ }^{308}$. Copyright 2012 American Chemical Society.

The authors also analyzed the water structuring during the simulations by examining the density profiles of water oxygen atoms along the direction normal to the surfaces, and they observed the presence of a first broad water layer as well as patches of reduced water density (see Figure $85 \mathrm{~b}$ ). The most interesting point was that the aliphatic parts of the side chains spread almost flat within regions of low water density while the charged moieties occupied high-density positions (namely, the first broad water layer) seeking the proximity of $\mathrm{SiOH}$ and $\mathrm{SiO}^{-}$to establish $\mathrm{H}$-bonds. This was confirmed by computing the free energy profile of a spherical hydrophobic solute from the surface to the bulk water along the normal direction of the surface $(\Delta \mathrm{G}(\mathrm{z}))$, in which the maximal values of $\Delta \mathrm{G}(\mathrm{z})$ were correlated with those positions with low water density (see Figure $85 \mathrm{c}$ ). These findings pointed out that the local solvent density variations near heterogeneous rough surfaces, as 
the silica ones, may be sensed by the side chains in a way that bears many features characteristic of the specific recognition in biomolecular aggregates. In this particular study, the alternation between hydrophobic and hydrophilic residues optimized the matching with the solvent density oscillations.

Finally the experimental works carried out by Somorjai and coworkers ${ }^{235,677}$ and already mentioned in Section 3.2.6 are also of interest because they show the evolution of peptides once they are adsorbed on silica surfaces. In these works the adsorption of peptides with XY composition (X hydrophobic and Y cationic) on hydrophilic silica were studied in situ. QCM measurements indicated that all peptides tested adsorbed onto silica. However, the most interesting peptide was the 14-AA containing Leu (L) and Lys (K) (sequence of Ac-LKKLLKLLKKLLKL-NH ${ }_{2}$ ) since SFG results indicated that it was the only peptide that, when adsorbed on silica, showed an $\mathrm{NH}$ stretching band $\left(3300 \mathrm{~cm}^{-1}\right)$ due to an amide peptide and the absence of $\mathrm{CH}$ stretching modes in the spectrum, suggesting a different orientational distribution of the peptide at the solid/water interface compared to that in solution. For this peptide the results obtained by AFM are also of interest, since the authors monitored the changes of the adsorbed peptide on the silica with time of adsorption. In general (see Figure 86, left column), it was observed that the peptide underwent a gradual morphological transition on silica from small spherical islands ( $200 \mathrm{~nm}$ wide) to much larger laterally aggregated domains (up to $1.5 \mu \mathrm{m}$ ) between $\mathrm{t}<5 \mathrm{~min}$ to $>30 \mathrm{~min}$. Despite that, the authors observed that the lateral expansion was not accompanied by an incremental growth in feature height, since topographic heights of 2.5 and $5.0 \mathrm{~nm}$ remained constant (see Figure 86, right column). Interestingly, QCM results indicated that adsorption took place in a multistep fashion. These data, along with AFM observations, suggested that the process involved an initial rapid adsorption on top of several nucleation sites on the surface, followed by subsequent slower reorganization, which included island growth, and spreading over silica, to form large laterally coalesced domains. 

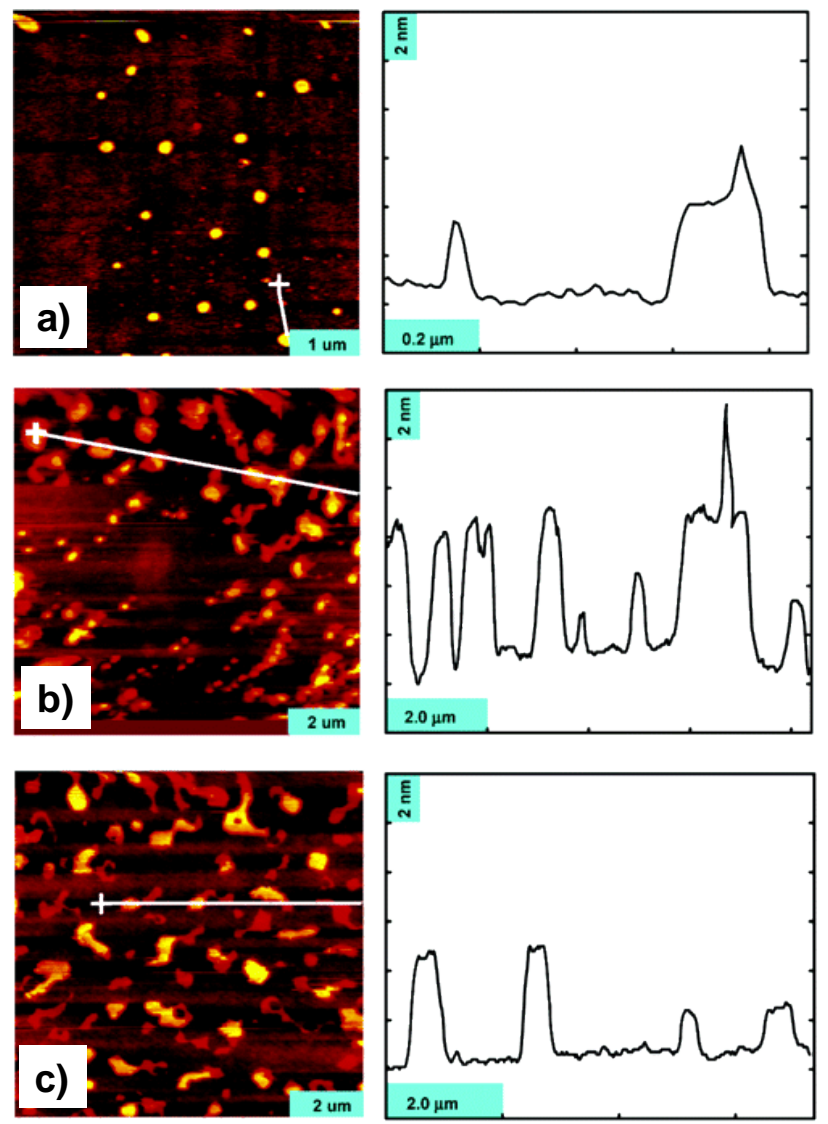

Figure 86. AFM topographical features of a peptide based on the Leu and Lys residues in interaction with silica imaged at $<5 \mathrm{~min}(\mathrm{a}), 5-10 \mathrm{~min}$ (b) and $>30 \mathrm{~min}$ (c). In the right column it is observed that the topographic heights do not dramatically change with adsorption time. Adapted with permission from Ref. ${ }^{235}$. Copyright 2006 American Chemical Society.

\subsection{Interaction with Nucleic acid-related biomolecules}

Understanding the adsorption of nucleic-acid derivatives (i.e., nucleobases, base-pairs, DNA and RNA sequences, etc.) to solid surfaces has important applications in DNA microarrays, ${ }^{678-680}$ gene therapy, ${ }^{681,682}$ DNA-based devices, ${ }^{683,684}$ high quality DNA purification by chromatographic techniques, ${ }^{685,686}$ and DNA hybridization. ${ }^{687,688}$ The initial stage of biofilm formation is facilitated by the attachment of extracellular DNA on the substrate surface ${ }^{689}$ so that a thorough understanding of DNA adsorption to surfaces is of great relevance to control the formation of bacterial biofilms. Additionally, it has been reported ${ }^{690}$ that adsorbed extracellular DNA on mineral surfaces may retain its ability to transform competent microorganisms (namely, microorganisms adapted for easier receiving of heterogeneous DNA) via horizontal gene transfer, thus giving rise to microbial diversity. In this context, the interaction with nucleic-acid derivatives becomes of particular relevance due to the capability of silica nanoparticles, especially those of mesoporous silica, to load and delivery genetic material, a fundamental process in gene therapy. ${ }^{691}$ Indeed, the prospect of gene therapy and genetic engineering as a new medical technique implies transferring custom genetic material into cellular environments to correct genetic diseases via loading and transport of the genetic material on carrier bodies. The most common and simplest method of gene delivery is 
the viral vector system, ${ }^{692}$ in which the genetic material is carried into nonpathogenic viruses. However, the risks involved in the expression of the viral genetic code and its nonspecificity have promoted the development of synthetic systems for these purposes, among which mesoporous silica nanoparticles are attractive candidates due to their ability to confine genetic material into their porous space, their high chemical resistance to microbial attack, low toxicity, thermal stability and ease of modifications.

Organizing this section adopting the "bottom up" scheme, in terms of size and complexity of the systems (similarly to what has been done for amino acids and peptides), has shown that much less data are available in the literature compared to AA and peptides. Indeed, to the best of our knowledge, only one work dealing with single nucleobase interacting with bare silica surfaces has been reported, while works involving functionalized silica surfaces can be found in the literature due to the relevance of these modified phases in chromatography. Even if we include modified silicas, all the works available are based on experimental measurements, while no computational studies have been found.

In this single work devoted to nucleobase/silica interactions, Plekan et al. ${ }^{258}$ studied the adsorption of adenine on $\mathrm{SiO}_{2}$ by means of photoemission and soft X-ray photoabsorption. The photoemission spectra was found to be quite similar to that of adenine multilayer but some striking differences were observed, which led to propose that adenine chemisorption had occurred.

As regards the polymeric nucleic acids, clear evidences of the fact that mesoporous silica can load DNA sequences into the pores were provided by Solberg et al. ${ }^{693}$ In this work, experiments of X-ray diffraction, nitrogen physisorption, UV-visible spectroscopy and fluorescence confocal microscopy performed before and after pore filling were used to demonstrate that double-stranded linear DNA sequences were certainly taken up into the pores of acid-prepared mesoporous silica (APMS). Powder XRD patterns (see Figure 87a) indicated that APMS alone adsorbed certain amount of DNA. 

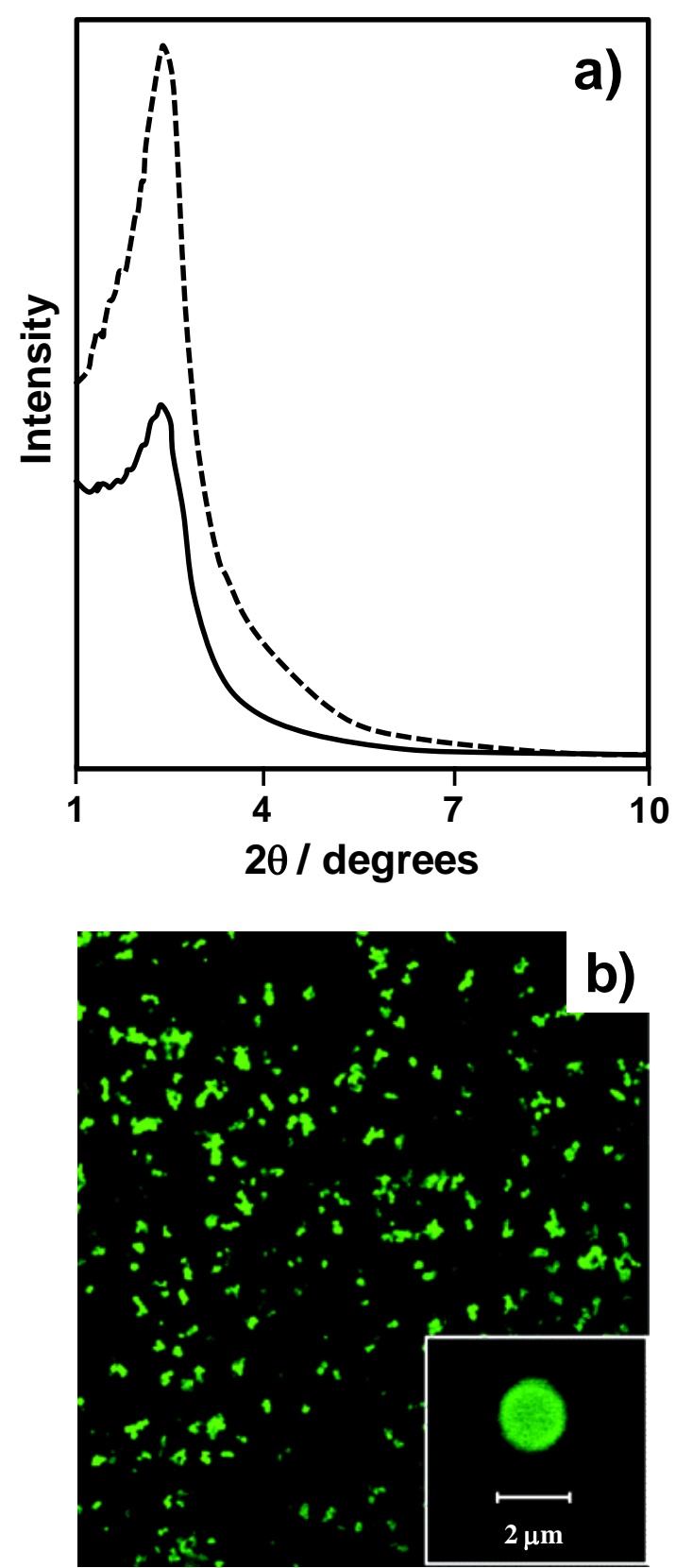

Figure 87. (a) Powder XRD patterns before (dashed-line) and after (solid line) DNA filling. (b) Fluorescence confocal scanning laser microscopy image of APMS after DNA adsorption. Adapted with permission from Ref. ${ }^{63}$. Copyright 2006 American Chemical Society.

The presence of a single broad diffraction peak of the parent material alone (dashed-line peak) is typical of ordered mesoporous silica due to the amorphous nature of the walls, while the decrease in intensity (solid-line peak) was claimed to be indicative of the presence of a guest molecule inside the pores. ${ }^{694}$ Clearer evidences of this is provided by the fluorescence confocal scanning laser microscopy images (see Figure 87b), which also indicated DNA penetration of the bulk of the micrometric particles.

This view that DNA can be loaded within the pores of mesoporous silica nanoparticles has been recently strengthened by $\mathrm{Li}$ et al. ${ }^{695,696}$ In these two works, the adsorption behavior of a short salmon DNA in the presence of either a mesoporous silica nanoparticle or a non-mesoporous silica 
nanoparticle was compared. DNA adsorption isotherms produced a typical Langmuir shape (see Figure 88) suggesting that monolayer adsorption at the DNA/silica interface occurred, but differences on the maximum adsorption capacity between the two materials were detected; i.e., 121.6 and $48.3 \mathrm{mg} \mathrm{DNA} / \mathrm{mg}$ nanoparticle for mesoporous and non-porous silica nanoparticles, respectively.

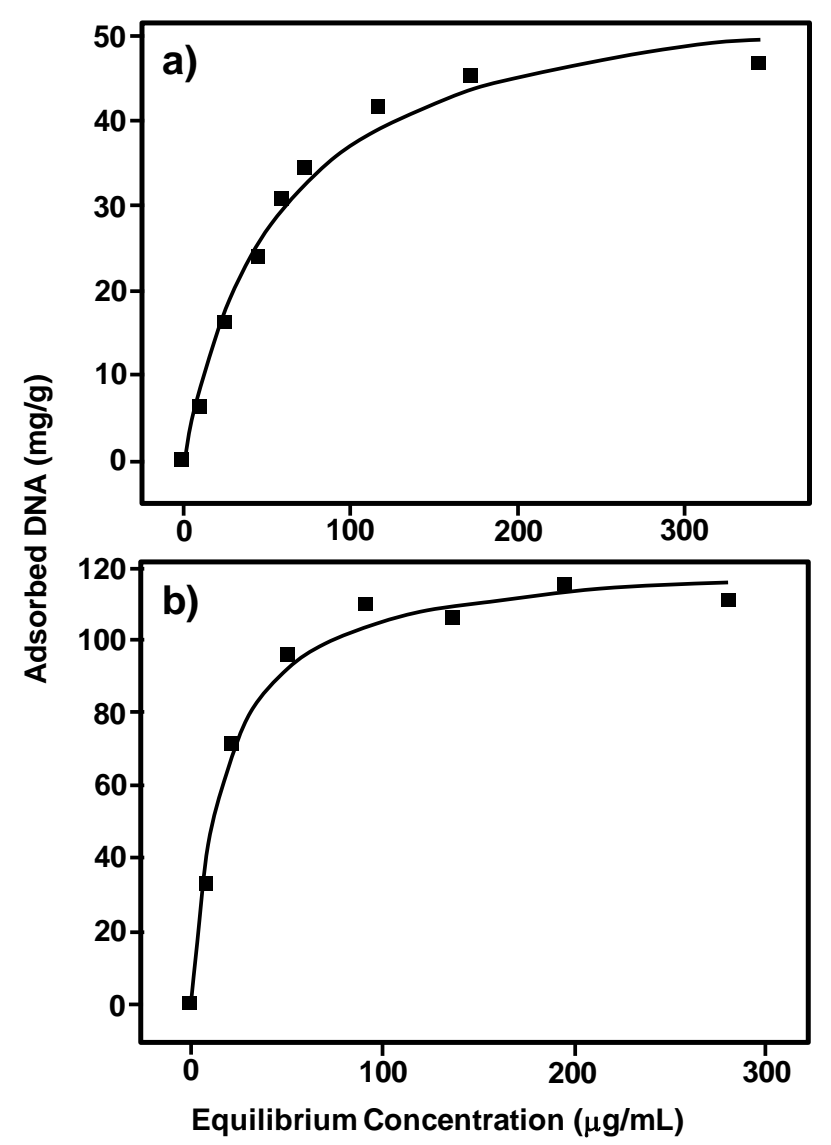

Figure 88. Ascending adsorption isotherms measured for salmon DNA with non-porous (a) and mesoporous (b) silica nanoparticles. Reprinted with permission from Ref. ${ }^{696}$. Copyright 2012 American Chemical Society.

The most remarkable finding of these works was that two desorption events were observed: one at $\mathrm{T}=0.5^{\circ} \mathrm{C}$, in which a very small fraction of adsorbed DNA was released and a second one at $\mathrm{T}=37^{\circ} \mathrm{C}$, in which a large amount of adsorbed DNA was released. Supported by dynamic light scattering experiments, the authors interpreted that the first DNA desorption was due to the release of DNA sequences adsorbed on the external surfaces of the mesoporous silica particles, whereas the second one corresponded to DNA located within the pores.

Thus, it seems clear that DNA indeed adsorbs on pure silica surfaces. However, an interesting question arises: considering that at physiological $\mathrm{pH}$, both silica surfaces and DNA sequences have a net negative charge because of the presence of $\mathrm{SiO}^{-}$and phosphate groups, respectively, what are the driving forces for DNA adsorption? The same question was formulated by several authors. ${ }^{600,696-699}$ Summarizing the results of these different works, one may conclude that the energetic contributions to DNA interaction with a silica surface can be categorized into 
three parts: i) the electrostatic effect (repulsive); ii) the dehydration effect; iii) DNA/silica Hbonding. As regards point i), it was observed that an increase of the salt concentration (namely, the ionic strength) led to an increase of adsorption, which was more pronounced for divalent than for monovalent cations. The authors interpreted that the increase of salt concentration shielded to a greater extent the negative charges on DNA and silica surfaces, thus reducing the electrostatic repulsion. Dehydration effects were also considered as a binding mechanism based on the fact that an increase in concentration of a chaotropic salt (that is, a salt that tends to disrupt the structure of macromolecules and that can intensively capture free water molecules) also yielded an increase in adsorption. ${ }^{696,697}$ This was explained by considering that the reaction of DNA adsorption actually involves the release of water: DNA(hydrated) + silica(hydrated) + conterions $\Leftrightarrow$ DNA/silica + water. The formation of H-bonds between DNA and silica was confirmed by two findings. The first one was that a $\mathrm{pH}$ decrease resulted in an increase of adsorption, which led the authors to interprete that at low $\mathrm{pH}$ some of the phosphate groups became protonated, hence enabling H-bonding. The second evidence was that isothermal titration calorimetry for DNA injected into binding solution with silica indicated that its adsorption was exothermic. ${ }^{696}$ The overall picture for the adsorption mechanism of DNA-derivatives onto silica must thus consider the presence of cations to shield the unfavorable electrostatic interactions between the negative charges present in silica and DNA, as well as the dehydration effects and the formation of intermolecular H-bonds.

An interesting point concerning DNA/silica interactions is the conformation changes undergone by DNA due to adsorption. This was addressed by Scholes et al., ${ }^{700}$ who explored, by means of resonance energy transfer-based techniques, the conformation changes occurred within the helical structure of a well-defined oligonucleotide upon silica adsorption from aqueous solution. Results obtained via evanescent wave-induced time-resolved Förster resonance energy-transfer (EW-TRFRET) measurements, showed a dependence of the nucleotide conformational state on the electrolyte concentration. That is, at high electrolyte concentrations, an increase in the flexibility of the oligonucleotide was observed, which led to an unwinding of the helix and to a separation of the two strands, allowing the oligonucleotide to bind directly to the silica surfaces. In contrast, at low electrolyte concentrations, the rigidity of the oligonucletide prevented an unwinding of the double helix upon adsorption, which resulted in only small segments directly contacting with silica. These two situations are depicted in Figure 89. 


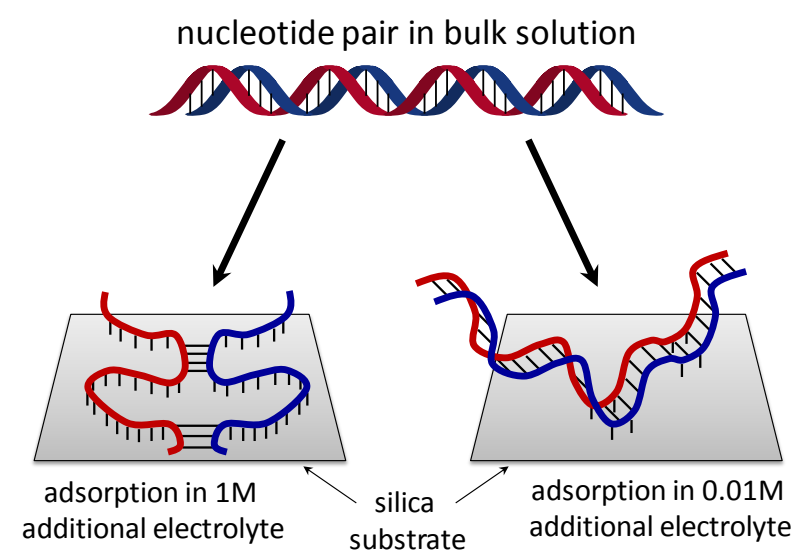

Figure 89. Schematic representation of the conformational changes of oligonucleotides upon silica adsorption depending in the electrolyte concentration. Reprinted with permission from Ref. ${ }^{700}$. Copyright 2011 American Chemical Society.

\subsection{Interaction with drugs and natural products}

A thorough characterization of the interface between silica surfaces and drugs or natural products is of prime relevance due to the growing interest in the field of drug delivery systems. Indeed, ordered mesoporous materials such as the ones based on silica (e.g., MCM-41, SBA-15) can be loaded with different organic molecules that would be released afterwards in a controlled fashion inside a living body. This potential route for drug intake is motivated by the lack of longterm efficiency for certain therapies when the drug is administrated orally or via injection, or their negative effects when conventionally administered. Because of the their lack of toxicity, their furtivity (they easily become invisible to the immune system) and porosity, silica-based ordered mesoporous materials have shown to be excellent candidates to act as drug delivery systems, since they are able to incorporate high dosages of drugs into the mesopores. ${ }^{17}$ Moreover, the silanolcontaining walls can be functionalized allowing a better control over drug release. ${ }^{701}$ In the last ten years, great efforts have been devoted to the confinement and storing of drugs in mesoporous silicas, as well as to the kinetics of the drug release. Along this line works devoted to several drug/mesoporous silica systems are available in literature: ibuprofen in MCM-41, ${ }^{17,702} \mathrm{MCM}-48^{703}$ and SBA-15, ${ }^{702}$ gentamicin in SBA- $15,{ }^{704}$ erythromycin in MCM-41, ${ }^{705} \mathrm{MCM}-48^{703}$ and SBA$15,{ }^{705}$ alendronate in SBA-15, ${ }^{706}$ amoxicillin in SBA-15. ${ }^{707}$ Moreover, Vallet-Regí and coworkers have recently authored two excellent reviews focused on this topic. ${ }^{15,708}$ For conciseness, in this section we will focus on studies that aim to provide a detailed picture at a molecular scale (either with spectroscopic methods or via theoretical calculations) of the actual drug/silica interface. A proper understanding on the drug/silica interactions is fundamental to understand the loading/delivery capability of a given system, since this ultimately depends on the chemical nature of the functionalities of the two partners. 
Within the present context, it is worth mentioning the works of Azaïs et al. ${ }^{709,710}$ who studied, by means of sophisticated ss-NMR techniques, the behavior of ibuprofen (IB) ${ }^{709}$ and benzoic acid $(\mathrm{BA})^{710}$ when encapsulated within different MCM-41 silica matrices, which differed by the pore diameters (for IB, 35 and $116 \AA$, hereafter referred as MCM-41 35 and MCM-41 116 , respectively; for BA, 30 and $100 \AA$, hereafter referred as MCM- $41_{30}$ and MCM-41 ${ }_{100}$, respectively). The ${ }^{1} \mathrm{H}$ MAS NMR spectra at ambient temperature of IB and BA were recorded both in their crystalline bulk forms and when confined in the MCM-41 matrices (shown in Figure 90a and Figure 90b).

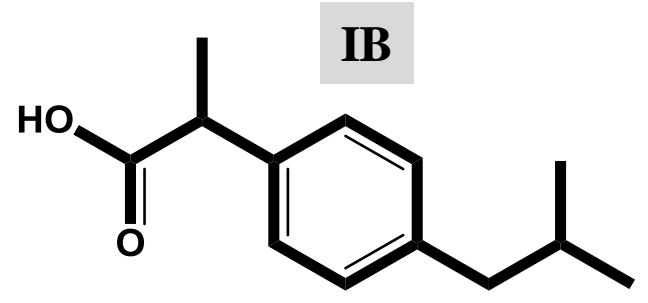

BA
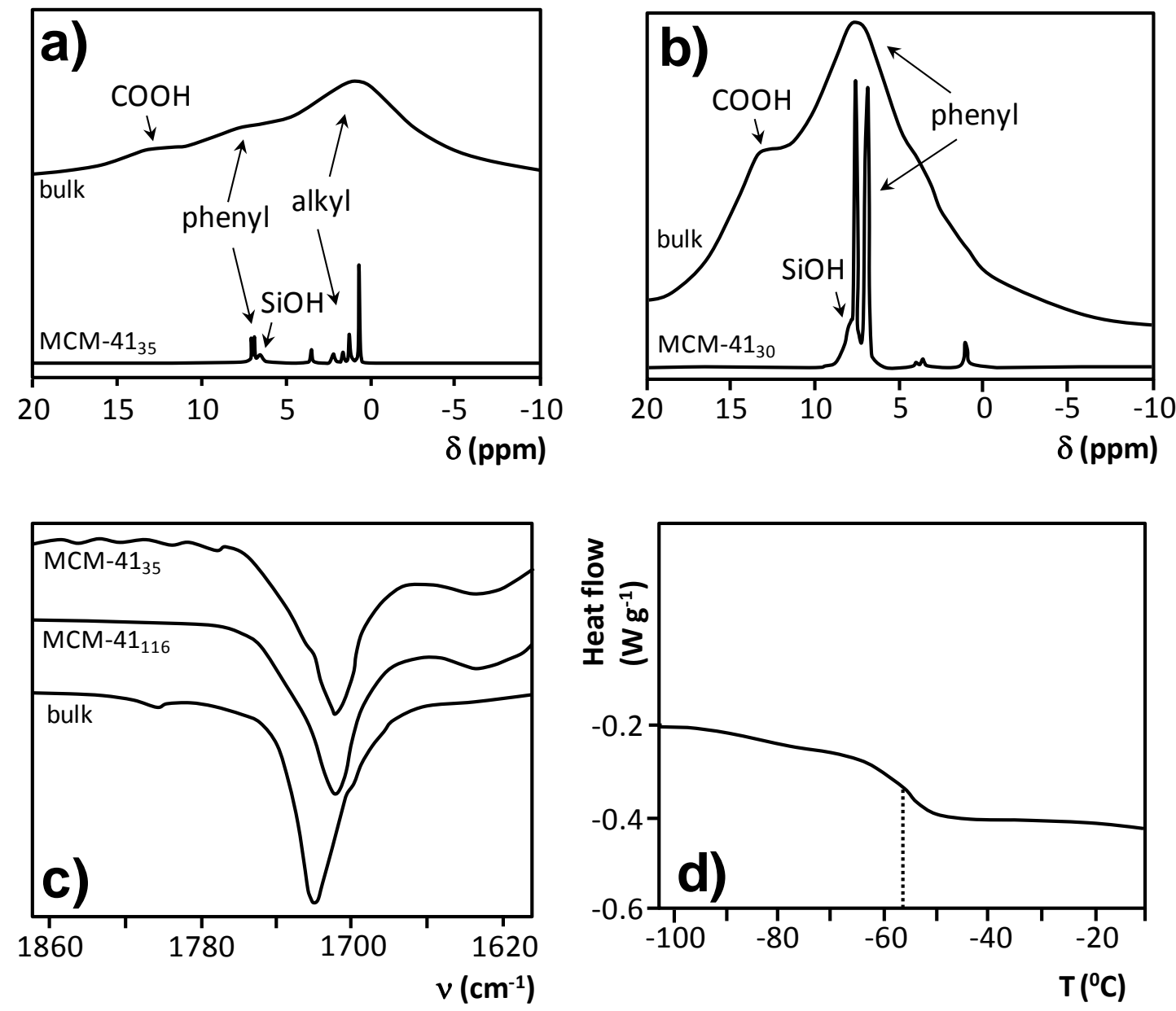

Figure 90. Recorded spectra for confined ibuprofen (IB) and benzoic acid (BA). (a) and (b): ${ }^{1} \mathrm{H}$ MAS NMR spectra of IB and BA, respectively, in their bulk states and in MCM-41 matrices; (c): FTIR spectra of IB in the $v(\mathrm{C}=\mathrm{O})$ region in its bulk state and in MCM-41 matrices; (d): DSC measurements of BA within MCM-41. Adapted with permission from Ref. ${ }^{709,710}$. Copyright 2006, 2010 American Chemical Society. 
Contrarily to the bulk crystalline forms, the spectra for the confined molecules were found to be extremely sharp, a fact which confirmed the high mobility behavior of IB and BA within the pores, in fact the NMR spectra look more like liquid phase than solid-phase. For both molecules the signals of the protons corresponding to alkyl and phenyl groups as well as of $\mathrm{SiOH}$ were unambiguously assigned (labeled in Figure 90a and Figure 90b). The proton signals coming from the $\mathrm{COOH}$ group of IB and $\mathrm{BA}$ were not visible, contrarily to the bulk forms, whereas they appeared in the spectra recorded at low temperatures. These findings suggested that at ambient temperature the proton of the $\mathrm{COOH}$ groups was in fast exchange with other environmental protons, namely $\mathrm{SiOH}$ and/or $\mathrm{H}_{2} \mathrm{O}$. Additional evidences for the involvement of the $\mathrm{COOH}$ group in the interaction with the silica walls were provided by FTIR measurements (only for IB, see Figure 90c), which observed an important bathochromic shift of the $v(\mathrm{C}=\mathrm{O})$ (from 1721 to $1709 \mathrm{~cm}^{-1}$ ). Measurements of apparent transverse relaxation times $\mathrm{T}_{2}{ }^{\prime}\left({ }^{1} \mathrm{H}\right)$ were carried out through solid echo sequence experiments using monoexponential time decay in order to determine the mobility of IB and BA within the pores (the higher the mobility, the longer the $\mathrm{T}_{2}{ }^{\prime}\left({ }^{1} \mathrm{H}\right)$ value). Unusually long values were obtained (2.4-16.6 and 14.7-20.2 $\mathrm{ms}$ for IB and BA, respectively) compared to crystalline forms (for bulk BA $\mathrm{T}_{2}{ }^{\prime}\left({ }^{1} \mathrm{H}\right) \sim 150 \mu \mathrm{s}$ ) although shorter compared to, for instance, BA in $\mathrm{CDCl}_{3}$ (around $600-700 \mathrm{~ms}$ ), suggesting a viscous (i.e. liquid-like) behavior of the confined molecules. For both IB and BA, ${ }^{1} \mathrm{H}$ MAS NMR experiments indicated that a liquid-solid phase transition took place when decreasing the temperature (at -50 and $-55{ }^{\circ} \mathrm{C}$, respectively), which intriguingly occurred at significantly lower temperatures than respective bulk melting points (76 and $125^{\circ} \mathrm{C}$, respectively). In order to get a deeper insight onto this phase transition, differential scanning calorimetry (DSC) measurements were carried out since it allows elucidating the physical state of confined molecules. The occurrence of phase transitions permits differentiation of glassy from crystalline states, while absence is indicative of molecular deposition onto the surfaces. DSC measurements (shown in Figure 90d) for BA in MCM-41 30 clearly evidenced a variation of the heat flow at $-55^{\circ} \mathrm{C}$, which was not detected on unloaded MCM- $41_{30}$ and on pure BA. Such depression of the phase transition temperatures has been reported in literature to be characteristic of confined molecules in porous materials. ${ }^{711-716}$ Remarkably, IB solidified in a glassy state and in a crystalline form when confined in MCM-41 35 and MCM-41 116 , respectively. Authors attributed these two radically different behaviors to steric effects; that is, crystalline ibuprofen is a dimer with a total length of $\sim 26 \AA$ so that pores of $116 \AA$ diameters were large enough to allow nucleation of crystallites, whereas pores of $35 \AA$ diameters, even if the dimer fitted inside, were exceedingly constrained, thus inducing a vitrification process. In contrast, BA solidified in a glassy state when confined in both MCM-41 silicas (no entrapped crystallites of BA were observed), a fact which led 
the authors to suggest that the minimal critical size needed for crystallization of BA in MCM-41 might be superior to $100 \AA$.

The studies of Mellaerts et al. ${ }^{717,718}$ are focused on investigating the loading and the molecular interactions of the itraconazole (ITR) drug into SBA-15 using a combination of DSC, XPS, DRS UV-vis, FTIR and ${ }^{1} \mathrm{H}$ MAS NMR spectroscopic techniques. DSC measurements allowed the authors to elucidate the phase transition of ITR occurring within the SBA-15 pores. Bulk crystalline ITR melts at $168{ }^{\circ} \mathrm{C}$ while it undergoes a glass transition at $60{ }^{\circ} \mathrm{C}$. These phase transitions were also observed (see Figure 91a) for ITR within the pores, which revealed the formation of an ITR bulk phase.
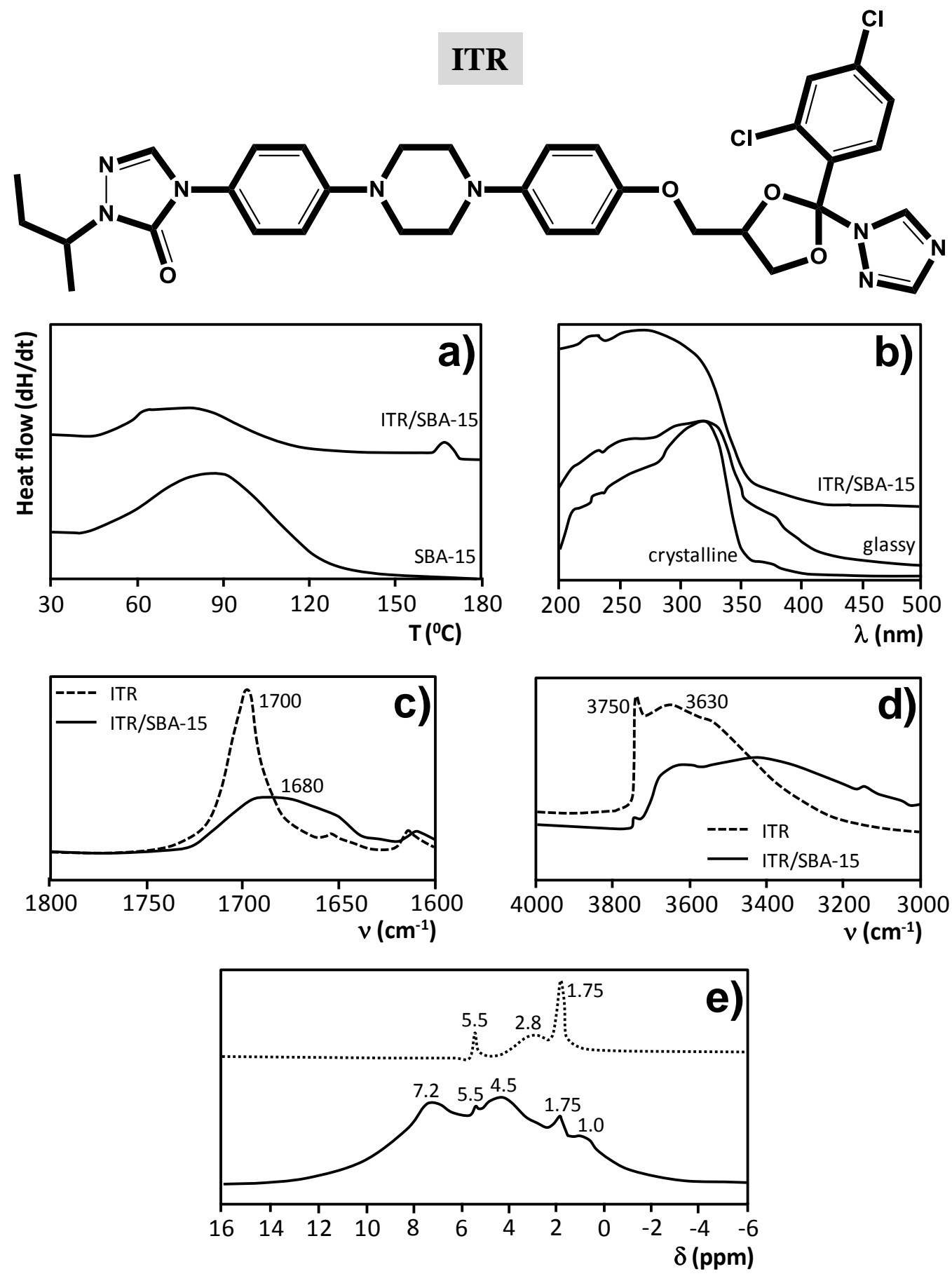
Figure 91. Recorded spectra for confined itraconazole (ITR). (a) DSC measurements of pure SBA15 and loaded with ITR; (b) DRS-UV spectra of crystalline and glassy ITR, and in SBA-15; (c) and (d): FTIR spectra of free ITR and in SBA-15 in the $\mathrm{C}=\mathrm{O}$ and $\mathrm{OH}$ stretching regions, respectively; (e): ${ }^{1}$ H MAS NMR spectra of confined ITR in SBA-15 (solid line and dashed-line spectra belong to single pulse excitation and spin echo spectra, respectively). Adapted with permission from Ref. $^{717,718}$. Copyright 2008 American Chemical Society and 2011 Royal Society of Chemistry.

XPS techniques allowed determining that ITR was deposited as a film or layer-wise along the pore walls. In order to preliminary define the molecular environment of the confined ITR a set of DRS UV-vis experiments were performed. Figure 91b shows the recorded spectra for glassy, crystalline and confined ITR. The band at $320 \mathrm{~nm}$ (also present in the pure bulk forms) and the shoulder between $350-400 \mathrm{~nm}$ revealed an aggregation of ITR molecules. Additionally, the band featured at 200-245 nm, according to the authors, was indicative of the contact of glassy ITR particles with the silica carrier. In order to have a deeper insight onto the actual contact between ITR and SBA-15, FTIR and ${ }^{1}$ H MAS NMR measurements were also carried out. Concerning FTIR, it was observed that in the carbonyl stretching region (see Figure 91c) the band at $1700 \mathrm{~cm}^{-1}$ of the isolated ITR underwent a bathochromic shift to $1680 \mathrm{~cm}^{-1}$ upon adsorption, indicating a H-bonding between the $\mathrm{C}=\mathrm{O}$ and the $\mathrm{SiOH}$ groups. Noticeably, similar IR shifts were also observed by Qu et al. for Captopril confined in the MCM-41 pores. ${ }^{719}$ Consistently, the absorption bands of isolated SBA-15 at 3750 and $3630 \mathrm{~cm}^{-1}$ due to isolated and H-bonded silanol groups, respectively, became red-shifted in the ITR/SBA-15 system (see Figure 91d). However, according to the authors, such shifts were not only caused by the interaction with the $\mathrm{C}=\mathrm{O}$ group but also by the interaction with the $\mathrm{O}$ ether and the $\mathrm{N}$ piperazine atoms, since significant spectral shifts in the $\mathrm{CH}$ stretching region of the neighboring ether and piperazine groups were also detected. Concerning ${ }^{1} \mathrm{H}$ MAS NMR (spectra shown in Figure 91e), the broad signals at 4.5 and $7.2 \mathrm{ppm}$ were assigned to aliphatic and aromatic protons of ITR. The sharp signal at $1.75 \mathrm{ppm}$ was due to protons of isolated $\mathrm{SiOH}$, while the signal around $1 \mathrm{ppm}$ to silanolic hydroxyl protons but with different acid strength and/or location to the isolated ones. The authors highlighted the considerable intensity of the $1.75 \mathrm{ppm}$ signal, despite the high ITR loading and the ITR/SiOH contact evidenced by FTIR. Due to that, authors interpreted that large part of the $\mathrm{SiOH}$ groups did not find a binding partner in the ITR monolayer due to the inaccessibility of the bulky adsorbed ITR molecules, probably because of the roughness of the SBA-15 walls. The authors assigned the signal at $5.5 \mathrm{ppm}$ to H-bonded silanols which were not bonded to $\mathrm{H}_{2} \mathrm{O}$ molecules (the $\mathrm{SiOH} \cdots \mathrm{H}_{2} \mathrm{O}$ bonding gave rise the signal at $\sim 2.8$ ppm due to the chemical exchange process between the chemically equivalent protons ${ }^{720}$ ), in particular to the interactions with adsorbed ITR. Since $\mathrm{SiOH} \cdots \mathrm{H}_{2} \mathrm{O}$ clustering was also observed, the picture the authors proposed was ITR in contact with the SBA-15 surfaces with some $\mathrm{H}_{2} \mathrm{O}$ molecules positioned between the two partners. 
As far as theoretical works on this subject are concerned, to the best of our knowledge, only one theoretical work focused on the interaction of drugs with silica surfaces has been published so far. This work $^{721}$ addresses the adsorption of aspirin (ASP) on a periodic model of the fully hydroxylated (001) $\alpha$-quartz surface, using the PBE density functional method and a linear combination of numerical atomic orbitals as implemented in the SIESTA code. The most stable adsorbed geometry found by the authors is shown in Figure 92.
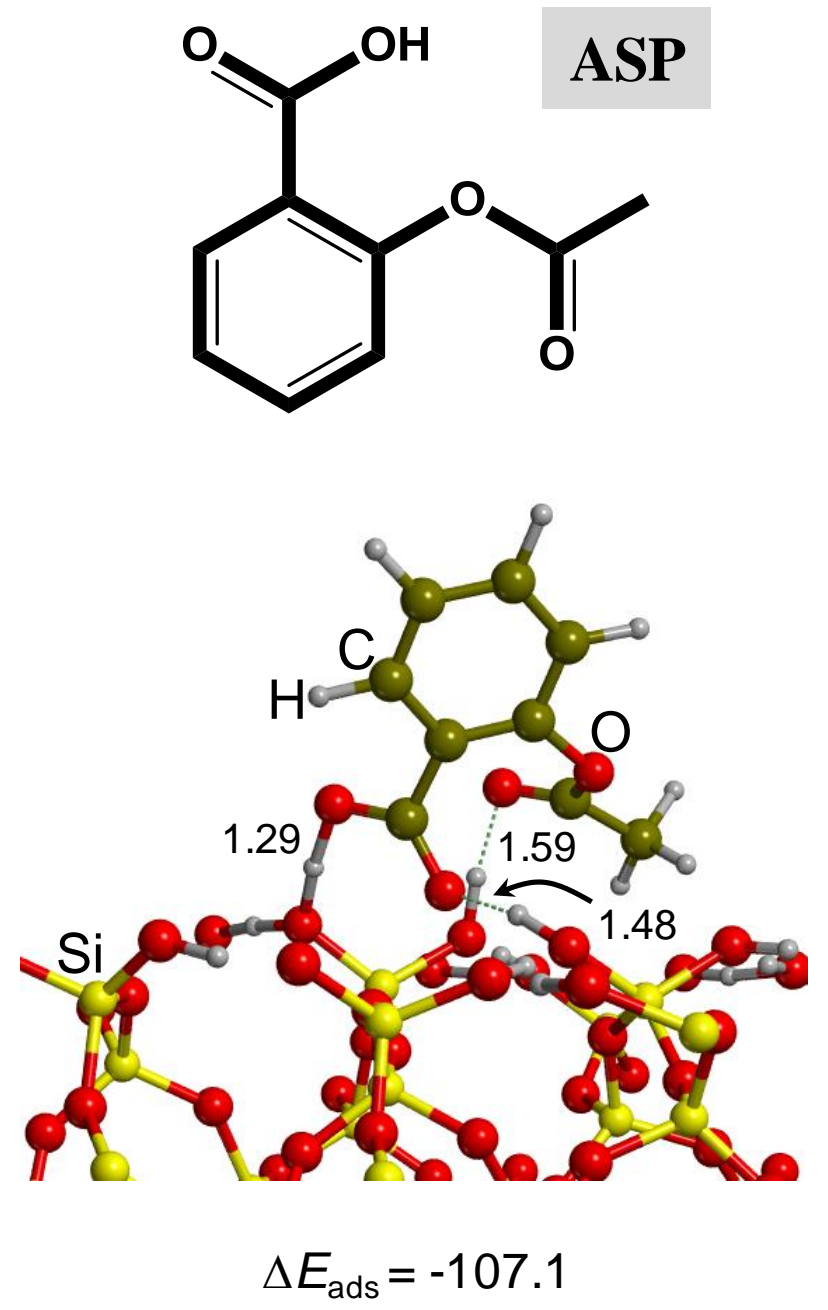

Figure 92. Structure of aspirin (ASP) and the optimized most stable structure of the ASP adsorbed on the (001) $\alpha$-quartz surface in gas-phase at the PBE level. The computed adsorption energies $\left(\Delta \mathrm{E}_{\mathrm{ads}}\right.$, in $\left.\mathrm{kJ} \mathrm{mol}^{-1}\right)$ is also reported. Bond distances in $\AA$. Drawn from data of Ref. ${ }^{721}$

The interaction between ASP and the surface was found to be dictated by three H-bonds. In two of them two surface $\mathrm{SiOH}$ groups acted as donor $\mathrm{H}$-bonding groups, the $\mathrm{C}=\mathrm{O}$ groups of ASP being the acceptor, while the third $\mathrm{H}$-bond involved the proton of the $\mathrm{COOH}$ group and the $\mathrm{O}$ atom of another $\mathrm{SiOH}$ group. As for Gly interacting with the same surface (see Section 9.1.1.1 and Figure $65 b$ ), the H-bonding network exhibited by the pristine $\alpha$-quartz surface was broken in order to form the ASP/silica adduct, suggesting a competition between the SiOH $\cdots$ ASP and $\mathrm{SiOH} \cdots \mathrm{SiOH} \mathrm{H}$ bond interactions. The adsorption energy was calculated to be $-107 \mathrm{~kJ} \mathrm{~mol}^{-1}$, similar to the values obtained for some amino acids. 
Concerning theoretical works on the interaction of natural products with silica surfaces, several works devoted to the adsorption of benzenediol compounds are available in the literature, in particular for catechol (1,2-benzendiol) ${ }^{722,723}$ and hydroquinone (1,4-benzendiol). ${ }^{426}$ Small amounts of catechol occur naturally in fruits, vegetables and in some mushrooms. Moreover, the catechol moiety is present in proteins of marine mussels, which have an unusual high content of 3,4dihydroxy-L-phenylalanine (the catechol functionality). These residues have been suggested to be the responsible for the strong adhesion of mussels in wet surfaces such as mineral oxides. Hydroquinone may be found as an active toxin in the agaricus mushrooms family and is one of the primary reagents in the defensive glands of bombardier beetles.

Mian et al. ${ }^{722}$ have theoretically studied, at the PBE level, the gas-phase adsorption of catechol onto periodic models of the (001) $\alpha$-cristobalite and (111) $\beta$-cristobalite surfaces, which only present geminal and isolated $\mathrm{SiOH}$ groups, respectively. The most stable adducts found are shown in Figure 93.
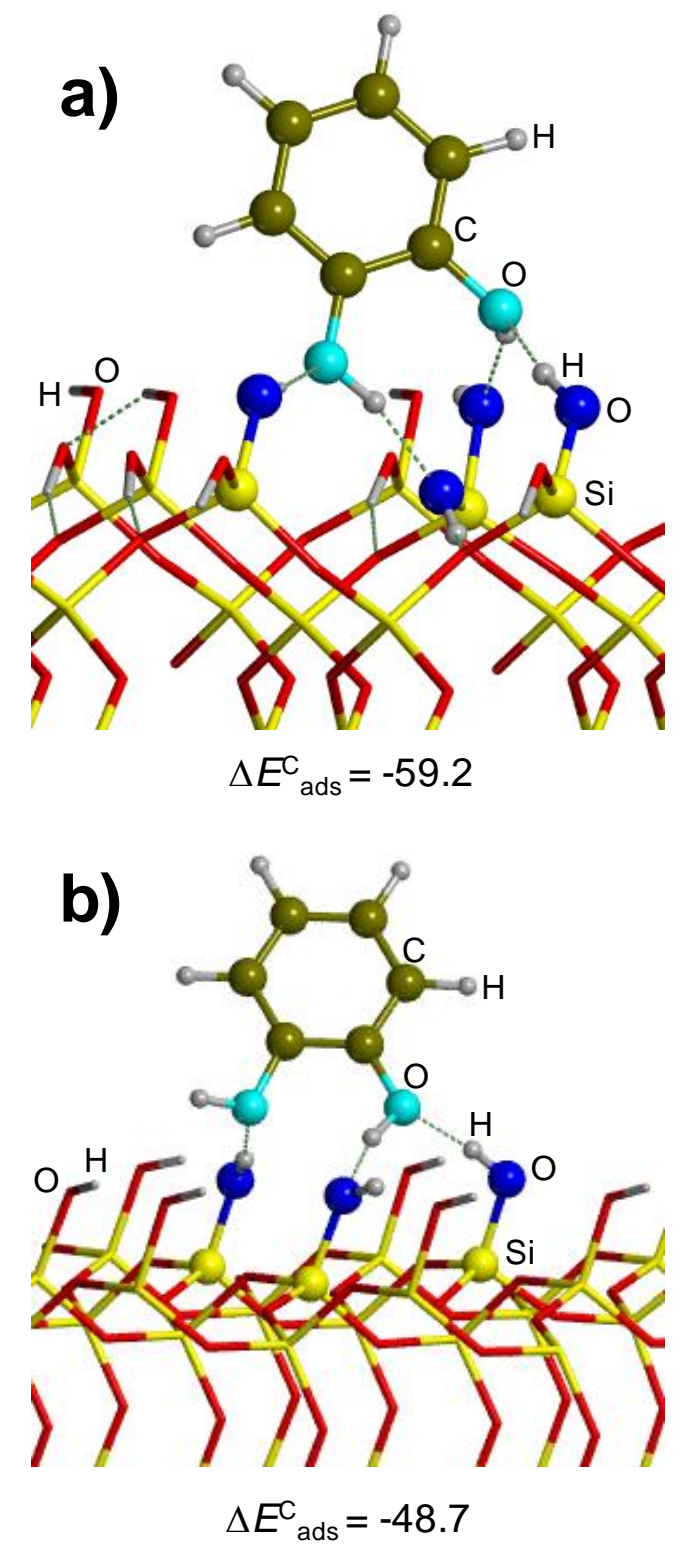
Figure 93. Optimized most stable structures of the catechol adsorbed on the (001) $\alpha$-cristobalite (a) (111) $\beta$-cristobalite surfaces in gas-phase at the PBE level. The computed BSSE-corrected adsorption energies $\left(\Delta \mathrm{E}^{\mathrm{C}}\right.$ ads, in $\left.\mathrm{kJ} \mathrm{mol}^{-1}\right)$ are also reported. Draw from data of Ref. ${ }^{722}$

On (001) $\alpha$-cristobalite, catechol forms four $\mathrm{H}$-bonds with the geminal $\mathrm{SiOH}$ groups, the $\mathrm{OH}$ catechol functions acting both as donor and acceptor H-bonding groups (see Figure 93a). Once again these $\mathrm{H}$-bonds were formed at the expenses of partial destruction of the $\mathrm{H}$-bonding network of the pristine surface. Catechol on (111) $\beta$-cristobalite was found to form three H-bonds (see Figure 93b), in which one catechol $\mathrm{OH}$ acts as $\mathrm{H}$-bond acceptor solely whereas the other one acts both as $\mathrm{H}$-bond donor and acceptor. Consistently with the number of $\mathrm{H}$-bonds formed, the BSSE-corrected adsorption energies $\left(\Delta \mathrm{E}_{\text {ads }}^{\mathrm{C}}\right.$ ) for catechol on the (001) $\alpha$-cristobalite and (111) $\beta$-cristobalite surfaces were computed to be -59 and $-49 \mathrm{~kJ} \mathrm{~mol}^{-1}$, respectively. Rimola et al. ${ }^{426}$ have studied the gas-phase adsorption of hydroquinone on periodic surface models based on the sanidine feldspar framework with hydrophobic and hydrophilic surface features at the B3LYP-D level. The most stable structures found considering low surface loading (i.e., with no lateral interactions between adsorbed molecules belonging to adjacent unit cells) are shown in Figure 94.

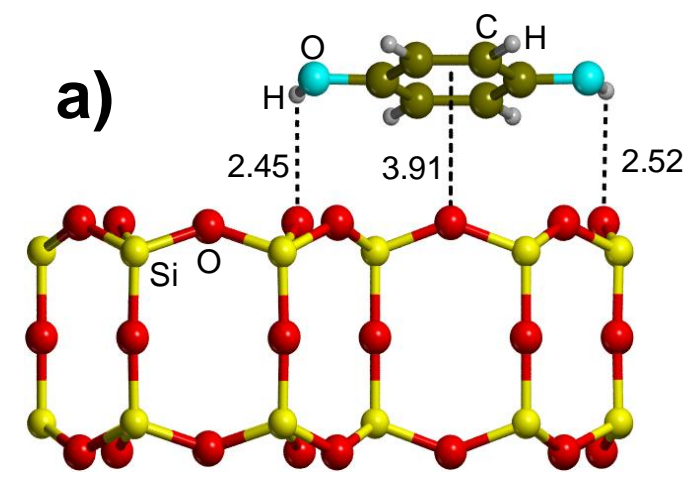

$\Delta E^{\mathrm{c}}{ }_{\mathrm{ads}}=-42.3$

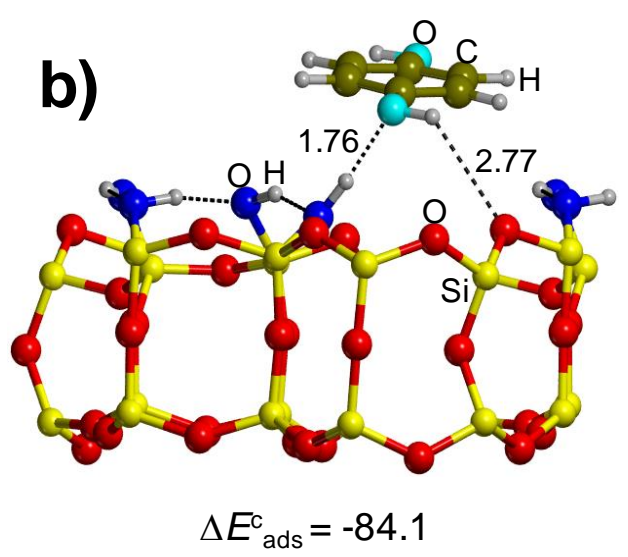

Figure 94. Optimized most stable structures of the hydroquinone adsorbed on models of hydrophobic (a) and hydrophilic (b) silica surfaces based on the sanidine feldspar framework at the B3LYP-D level. The computed BSSE-corrected adsorption energies $\left(\Delta \mathrm{E}^{\mathrm{C}}{ }_{\text {ads }}\right.$, in $\left.\mathrm{kJ} \mathrm{mol}^{-1}\right)$ are also reported. Bond distances in $\AA$. Drawn from data of Ref. ${ }^{426}$ 
Hydroquinone in interaction with the hydrophobic surface shows weak H-bonds between the hydroquinone $\mathrm{OH}$ functionalities and the $\mathrm{O}$ atoms of siloxane groups (see Figure 94a). The computed $\Delta \mathrm{E}_{\text {ads }}^{\mathrm{C}}$ value at B3LYP-D is $-42 \mathrm{~kJ} \mathrm{~mol}^{-1}$. The role of dispersive forces in the adsorption was found to be crucial since $\Delta \mathrm{E}_{\text {ads }}^{\mathrm{C}}$ computed at the pure B3LYP level was $-2 \mathrm{~kJ} \mathrm{~mol}^{-1}$. In the most stable adduct found for hydroquinone interacting with the hydrophilic surface (see Figure 94b), only one of the hydroquinone $\mathrm{OH}$ groups was engaged in $\mathrm{H}$-bonds with the surface. Interestingly, the $\mathrm{SiOH}$ group that interacts with hydroquinone is the one that exhibits the largest acidity among the different $\mathrm{SiOH}$ groups available in the unit cell, as indicated by the potential electrostatic maps of the naked surface. The $\Delta \mathrm{E}_{\text {ads }}^{\mathrm{C}}$ was computed to be $-84 \mathrm{~kJ} \mathrm{~mol}^{-1}$ at $\mathrm{B} 3 \mathrm{LYP}-\mathrm{D}$ level, twice more negative than for hydroquinone adsorbed on the hydrophobic surface. Remarkably, although the adduct was significantly $\mathrm{H}$-bonded, dispersion also played a major role since the computed $\Delta \mathrm{E}_{\text {ads }}^{\mathrm{C}}$ value at pure B3LYP was only $-30 \mathrm{~kJ} \mathrm{~mol}^{-1}$.

As noted, these works dealt with the adsorption of benzenediol compounds under strict gasphase conditions, so that no effects from the bulk water were accounted for. Aware of this limitation, Mian et al., ${ }^{723}$ in a subsequent work, studied catechol adsorption onto the (001) $\alpha$ cristobalite surface model in the presence of 25 explicit water molecules and ran a set of static calculations and ab initio MD simulations at the PBE level. The static calculations were used to proceed with a progressive microsolvation of the catechol/silica interface (i.e., progressive addition of water molecules arising from the wet environment) in a similar fashion to what has been already reported in the literature. ${ }^{724}$ By means of these calculations, the authors determined that the more water molecules at the interface bridging catechol and silica, the more unstable the system was, suggesting that catechol and the silica surface preferred to establish direct contact. In order to further confirm this finding, ab initio MD simulations were carried out, results showing that, starting from a configuration in which water molecules were fully occupying the catechol/silica interface region, after 10 ps of MD evolution, direct interactions between catechol and the surface were already established (see see Figure 95). 

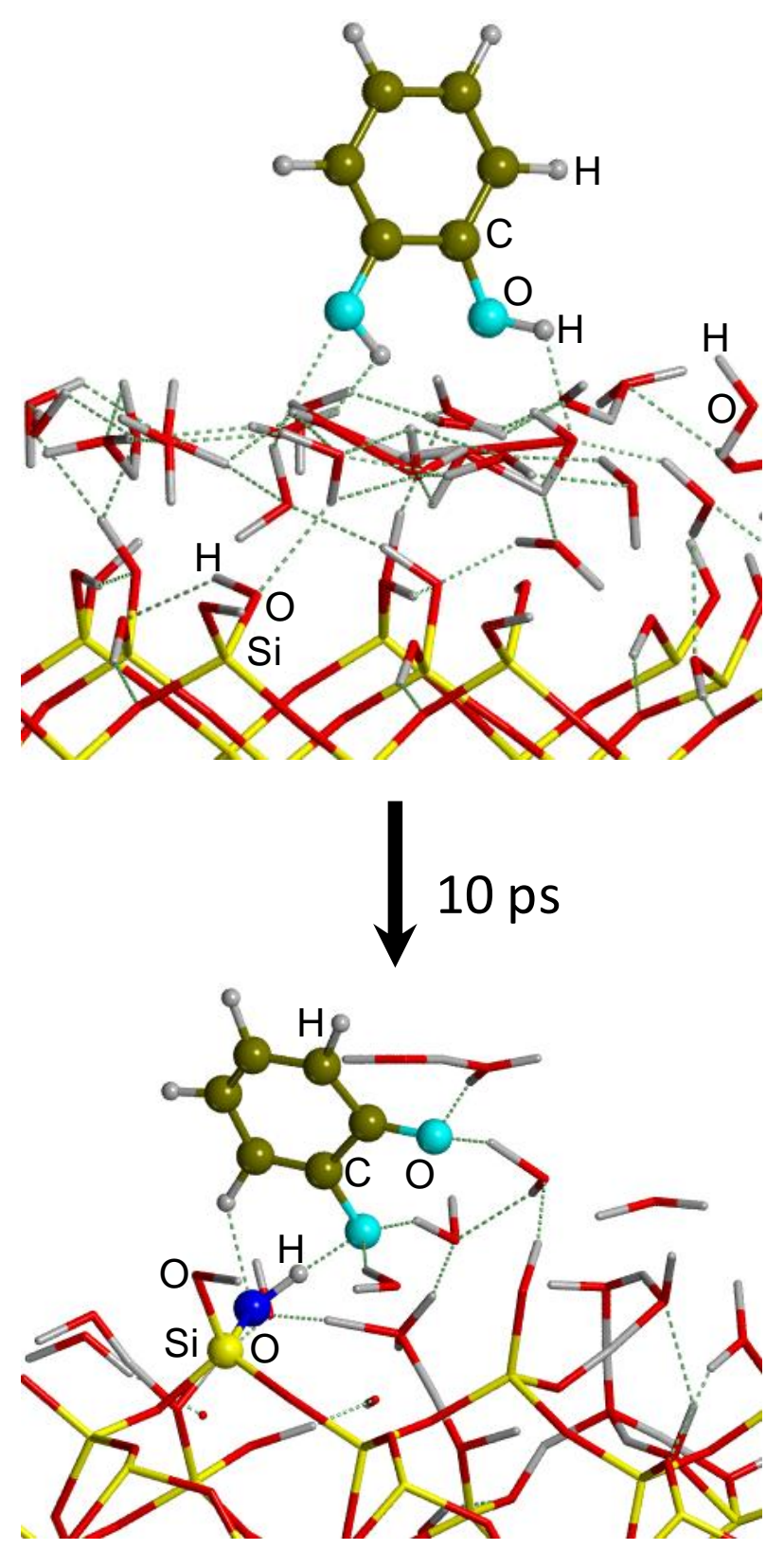

Figure 95. Initial configuration (top) and snapshot taken after 10 ps of evolution (bottom) of an ab initio MD simulations (PBE level) for catechol adsorption on the (001) $\alpha$-cristobalite surface with 25 explicit waters. Drawn from data of Ref. ${ }^{723}$

\section{Summary and perspectives}

Hopefully this review has highlighted the complexity and subtleties of silica in its crystalline and amorphous forms already as a bare material. The rather subtle nature of the different functionalities present at the silica surface is discussed in Section 2 and their characterization by experimental techniques reviewed in Section 3. In essence, for silica exposed to atmospheric moisture the dominant surface features are silanol $(\mathrm{SiOH})$ groups and siloxane bridges $(\mathrm{Si}-\mathrm{O}-\mathrm{Si})$, the relative ratio between them being dictated by the thermal history suffered by the silica sample. Siloxanes are rather unreactive unless highly strained, but silanols show diverse types of reactivity. 
They are weakly acidic and can be deprotonated in the presence of a solution. They readily engage in H-bonding, either between themselves or with Lewis basic molecules from their environment. Further structural distinctions can be made that have consequences on silanols reactivity. First, on the basis of bond connectivity, geminal silanols $\mathrm{Q}^{2}$ are different from terminal ones, $\mathrm{Q}^{3}$. Some experimental data suggest that the acidity of these two types of silanols may be different but, in opposition, quantum mechanical calculations reveal that, when isolated, $\mathrm{Q}^{2}$ and $\mathrm{Q}^{3}$ sites have almost the same acidity. In other words, it is difficult to conceive differences in reactivity with respect to the interaction of biolomolecules to $\mathrm{Q}^{2}$ sites compared to $\mathrm{Q}^{3}$ ones and, for instance, there is no definite indication that a $\mathrm{Q}^{2}$ rich surface could be connected to toxicity of crystalline silica. Second, on the basis of spatial proximity, silanols that are close enough to be associated by H-bonding exhibit a different reactivity from isolated ones. The terminal $\mathrm{SiOH}$ group belonging to a chain of mutually $\mathrm{H}$-bonded $\mathrm{SiOH}$ groups is much more acidic than isolated $\mathrm{Q}^{3}$ or $\mathrm{Q}^{2}$ groups. The acidity is modulated by the H-bond strength which, in turn, results from the topology and chain length of the interacting $\mathrm{SiOH}$. This is perhaps the real reason why in some cases the presence of $\mathrm{Q}^{2}$ which induce a local increase of silanol density, has been put in relation with a specific reactivity. On surfaces derived from crystalline silica polymorphs the H-bond cooperativity extends to infinity, whereas for amorphous silica surfaces only short chains are possible due to the uneven distribution of the $\mathrm{OH}$ groups. This is, in our opinion, the reason which brings about the existence of two acidic sites on amorphous silica surfaces ( $\mathrm{pKa}=8.5$ and 4.5 , respectively) as resulted from experimental measurements: the first acidity would be associated to isolated silanol species $\left(\mathrm{Q}^{2}\right.$ or $\left.\mathrm{Q}^{3}\right)$, the second one being due to the terminal silanols of H-bonded chains. Longer-range distinctions may be significant but this is still hypothetical. As regards to siloxanes, it is important to know what kind of $(-\mathrm{Si}-\mathrm{O}-)_{\mathrm{n}}$ rings (also called $\mathrm{SnR}$ ) they form. Experimental data are not precise so far, but it is estimated that S4R, and smaller rings are unstable. Two-silicon rings, $\mathrm{S} 2 \mathrm{R}$ or $(\mathrm{SiO})_{2}$, may be present in samples treated at high $\mathrm{T}$ as a minor surface feature, as their concentration is typically about one order of magnitude smaller than that of isolated $\mathrm{SiOH}$.

Through these surface functionalities silica interacts with adsorbed (bio)molecules and ultimately, with living organisms. The nature of the sites implies that $\mathrm{H}$-bonds (with the $\mathrm{SiOH}$ ) and dispersive interactions (with $\mathrm{Si}-\mathrm{O}-\mathrm{Si}$ ) are the key forces behind localized adsorption of (bio)molecules while chemical reactions involving these groups are less common. Therefore, experimental methods capable to track the changes in the $\mathrm{SiOH}$ spectroscopic features which are sensitive to H-bonding interactions are particularly useful (infrared, Raman and NMR). Unfortunately, the amorphous nature of most silica materials adopted in technological applications renders the elucidation of the atomic details difficult because diffraction-based techniques, such as LEED and electron diffraction microscopy, are not applicable. Therefore, although amorphous 
silicas have been studied experimentally for at least 50 years, progress in site identification has been excedingly slow and many features of the silica surfaces are still debated. This is also why $\mathrm{SiO}_{2}$ surface chemistry is a field where the contribution of theoretical chemistry methods has always been highly valued. Only the advent of powerful computers, together with highly efficient programs, allowed to overcome the intrinsic limits of experimental techniques, as the primary objective of computer modeling is to provide atomistic details of the system under investigation.

Strategies to efficiently model silica surfaces have been covered in Section 4 and the computational methods apt to describe with different level of accuracy their physico-chemical features and the adsorption of (bio)molecules have been briefly reviewed in Section 5. Clearly, techniques based on solving the electronic Schrödinger equation (either based on the wave function or the electron density) can be adopted if high accuracy and generality are sought, provided that density functional methods based on standard exchange-correlation functionals are supplemented with dispersion interaction correction. While these methods are, in principle, the best approach one can use, the complexity of the systems of interest (size, amorphous nature, role of water, etc.) and the need to account for temperature effect by molecular dynamics simulations, forces adopting more approximated methods based on classical force fields in a molecular mechanics approach.

Section 6 and 7 provide an overview of the properties of bare silica surfaces determined by the above computational methods. In general, we realize that there is no specific force field capable to "accurately" mimic all the features of both bulk and surfaces of silica. The best attempt to date is $\mathrm{FFSiOH}^{467}$ which is definitely capable to reproduce structure, dynamic properties and H-bond features of silica based materials, not restricted to dense silica ones. Much work needs, however, to be done to extend this promising force field toward the interaction with molecules, water being the most urgent one. Force fields were proved essential to allow for a multiscale approach, using a specific method according to a given system size, in order to arrive to a number of definite and realistic models of the amorphous silica surface, as described in Section 7. The complexity of the possible cases of interest when (bio)molecules interact with silica surfaces has been divided in logical pieces of increasing complexity, namely the case of $\mathrm{H}_{2} \mathrm{O}$ adsorption (Section 8), as water is the most ubiquitous molecule in a biological environment, followed by the cases of monomers (mainly amino acids), and then of the corresponding polymers, peptides and nucleic acid related biomolecules (Section 9). The adsorption of other molecules, which are not rigorously biomolecules, has also been addressed in Section 9, as their behaviors mimic that of residues to be found in real proteins. From the methodological point of view, only methods capable to properly handle relatively weak intermolecular interactions should be adopted. As the interaction of biomolecules with silica surfaces is driven by $\mathrm{H}$-bond and dispersion forces, this excludes DFT methods based on standard GGA functionals as they completely miss the dispersion component of 
the interaction. Also, care should be exterted when choosing a specific functional as those based on Perdew formulation of the exchange (PBE, PW91) tend to give too long $\mathrm{OH}$ bond length. Functionals based on the Becke formulation of the exchange (BLYP, BP86) or even hybrid ones (B3LYP) when coupled to empirical corrections to recover dispersion interactions, as suggested recently, have been shown to provide a more balanced behaviour. As a perspective, semiempirical methods based on the new PM7 method which includes H-bond and dispersion corrections, appear to be very promising. As semiempirical methods favourably scales with the system size, we look forward to seeing new applications in the silica/biomolecules context. The main "take home message" from the results of Section 9 is that computer simulation can provide excellent support to experimental interpretation providing that adsorption is carried out at the gas/solid interface. In the particular case of amino acids, this is indeed feasible, as they sublimate without decomposing and can be condensed on well-defined silica surfaces - few teams have studied such experimental setups until now, however. The situation is much more intricate when experiments envisage adsorption from water solution. In this case, the adsorption is strongly $\mathrm{pH}$-dependent as both the silica surface and the amino acids change their protonation state accordingly, greatly affecting the sign of the electrostatic interaction. More precisely, $\mathrm{pH}$ values higher than the point of zero charge of silica surfaces will cause deprotonation of $\mathrm{SiOH}$ groups with dramatic consequences for the adsorption of amino acids. The latter also exhibit different charge speciations (between zwitterionic, anionic, and cationic forms) as a function of their pKa values; according to this speciation, they undergo strong electrostatic interactions (attraction, or repulsion, as the case may be) with the silica surface, which often dominate the adsorption energetics. Carrying out adsorption from water solutions is experimentally less complex than from the gas phase: the opposite is true for the modeling approach. There, the need to account for the presence of significant amounts of water in order to bring the system to be stabilized in a different protonation state compared to the canonical forms in gas-phase renders the simulation a daunting task in terms of the requested computational resources. Resorting to micro solvation (few water molecules located at strategic points) is a clever idea but the applicability of this approach should not be overstated as many of the sought properties result from the presence of a large quantity of water (entropic factors, structuring of the second solvation shell, etc). In that respect, one of the key issue is to understand whether the (bio)molecule is directly attached to the surface functionalities or its interaction is mediated by the presence of pre-adsorbed water. This is not easily addressed by experiments or by simulations. Experimentally, it is hard to detect spectral features which can be unambiguously attributed to a "first layer of adsorbed water" and discriminate them from the features for bulk water, although vibrational sum frequency spectroscopy ${ }^{725}$ can indeed help in obtaining such data. For the latter, the relatively long residence time of a protein residue or a water molecule attached to the surface requests rather sophisticated 
and expensive metadynamics procedure ${ }^{726}$ to bring the time scale of molecular dynamics simulations down to acceptable values.

More generally, there are not many techniques that allow characterizing in situ molecules at "buried interfaces", including the silica/solution interface. An extensive review of these techniques, concerning solid/solution interfaces in general, has recently appeared; a significant number of the examples provided concern biomolecules adsorption, but mostly their application is not yet routine. $^{727}$

Gathering precise experimental data on even a single biomolecule/silica system is a long, protracted endeavor. It can be seen from Section 9 that only for the the simplest amino acid has the amount of experimental data reached a "critical mass" where systematic comparisons between experiment and theory become fruitful, and even in the case of glycine/silica many experimental data are still missing. On the other hand, the progress of spectroscopic techniques (especially vibrational and NMR) now allows to reach an unprecedented level of detail in the molecular characterization of adsorbed biomolecules. If close cooperations of experimentalists and theoreticians on well-focused systems can be undertaken, future progress could be fast.

During the search for papers focusing on the present topic we discovered that nothing has been done to model the interaction of DNA bases with silica while few works have appeared addressing their interactions with montmorillonites. ${ }^{412,728}$ The same is also true for studies dealing with phospholipids as model of cell membranes in which only one paper has been published. ${ }^{729}$ Both fields should be explored in depth, among other reasons because of the importance of DNA in developing nano-devices within a bottom-up approach. ${ }^{730}$ For instance, in a way similar to nanolithography, one could pattern ordered DNA to the desired positions on a substrate that includes hydrophilicity and covalent attachment as provided by silica surfaces. ${ }^{731}$ On the other hand, cell membranes are the main "contact surfaces" between a living organism and a biomaterial and, as a perspective, we believe that understanding the process of membrane lipids adhesion is key to ultimately improve the design on biocompatible materials. ${ }^{732}$ Despite the difficulties described above we are confident that new technological improvements in the high performance computing machines as well as on experimental methods will widen our possibility to address problems in the "biological surface science" $" 733$ context, like understanding how conformational changes are induced in peptides by adsorption at oxides surface, or better drug protection and delivery using mesoporous materials, to mention just two of them. Hopefully, these basic research advances will provide the grounds and the driving force needed for rapid developments in a wide range of applications including tissue engineering, biosensors, drug delivery or medical diagnosis. 


\section{Acknowledgments}

Part of the Introduction and Section 2 have been derived from the $\mathrm{PhD}$ thesis by Federico Musso, "Theoretical Study of the surface properties of crystalline and amorphous silica polymorphs", 2011, Universitat Autònoma de Barcelona and University of Torino. PU is grateful to the Ministerio de Educacíon Cultura y Deporte del Gobierno de Espana for supporting his stage (SAB2011-0033) at the UAB during the writing of this review and to Progetti di Ricerca di Ateneo-Compagnia di San Paolo-2011-Linea 1A, progetto ORTO11RRT5 for funding. Fruitful discussion and support with Dr. M. Corno and M. Delle Piane of the Dipartimento di Chimica, Università di Torino are also acknowledged. PU acknowledges the invaluable and continuous scientific support provided by Prof. Vera Bolis on silica topic. Many of the results reported in this review would have not been possible without allowance of the massive parallel version of the CRYSTAL09 code provided by the Theoretical Chemistry group at Dipartimento di Chimica, Università di Torino. MS gratefully acknowledges support through 2011 ICREA Academia award. Financial support from MICINN and the DIUE of the Genneralitat de Catalunya through the CTQ2011-24847/BQU and 2009SGR638 projects, respectively, is also acknowledged. AR is indebted to Ministerio de Economía y Competitividad for a Juan de la Cierva contract. The authors are also grateful to Prof. Lucio Colombi Ciacchi (University of Bremen) for providing the coordinates of the amorphous silica surface from which Figure 20 has been made. DC thanks B. Diawara for graciously providing the Modelview software which has allowed the construction and analyses of some data reported here. DC and JFL thank Frederik Tielens for discussion on many aspects of amorphous silica models. 
Art Cover

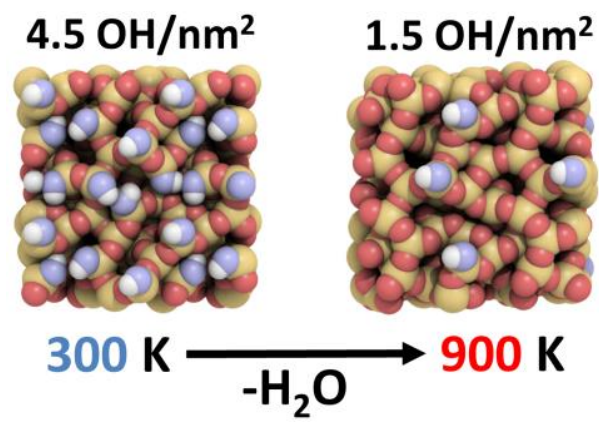




\section{References}

(1) Brown, G. E.; Henrich, V. E.; Casey, W. H.; Clark, D. L.; Eggleston, C.; Felmy, A.; Goodman, D. W.; Grätzel, M.; Maciel, G.; McCarthy, M. I.; Nealson, K. H.; Sverjensky, D. A.; Toney, M. F.; Zachara, J. M. Chem. Rev. 1998, 99, 77-174.

(2) Clayton, D. C. Principles of Stellar Evolution and Nucleosynthesis; The University of Chicago Press: Chicago, 1983.

(3) Iler, R. K. The Chemistry of Silica; 2nd ed.; John Wiley \& Sons, Inc.: New York, Chichester, Brisbane, Toronto, 1979.

(4) The Surface Properties of Silica; Legrand, A. P., Ed.; John Wiley \& Sons: Chichester, 1998.

(5) Adsorption on Silica Surfaces; Papirer, E., Ed.; Marcel Dekker Inc.: New York, 2000; Vol. 90.

(6) El Shafei, G. M. S. In Adsorption on Silica Surfaces; Papirer, E., Ed.; CRC Press, Taylor \& Francis Group: Santa Barbara, California, 2000; Vol. 90, p 35.

(7) Morrow, B. A.; Gay, I. D. In Adsorption on Silica Surfaces; Papirer, E., Ed.; CRC Press, Taylor \& Francis Group: Santa Barbara, California, 2000; Vol. 90, p 9.

(8) Davydov, V. Y. In Adsorption on Silica Surfaces; Papirer, E., Ed.; CRC Press, Taylor \& Francis Group: Santa Barbara, California, 2000; Vol. 90, p 63.

(9) Characterization and Chemical Modification of the Silica Surface; Vansant, E. F.; van der Voort, P.; Vrancken, K. C., Eds.; Elsevier Science B.V.: Amsterdam, 1995; Vol. 93.

(10) Vallee, A.; Humblot, V.; Pradier, C. M. Acc. Chem. Res. 2010, 43, 1297-1306.

(11) Nawrocki, J. J. Chromatogr. A 1997, 779, 29-71.

(12) Hench, L. L.; Splinter, R. J.; Allen, W. C.; Greenlee, T. K. J. Biomed. Mater. Res. Symp. 1971, 2, 117-141.

(13) Hench, L. L. Sol-gel silica. Properties, processing and technology transfer; Noyes Publications: Westwood, New Jersey 07675, 1998. 639-656.

(14) Colilla, M.; Izquierdo-Barba, I.; Vallet-Regí, M. Expert Opin. Ther. Pat. 2008, 18,

(15) Vallet-Regí, M.; Balas, F.; Arcos, D. Angew. Chem. Int. Ed. 2007, 46, 7548-7558.

(16) Barbe, C.; Bartlett, J.; Kong, L. G.; Finnie, K.; Lin, H. Q.; Larkin, M.; Calleja, S.; Bush, A.; Calleja, G. Adv. Mater. 2004, 16, 1959-1966.

(17) Vallet-Regí, M.; Rámila, A.; del Real, R. P.; Pérez-Pariente, J. Chem. Mater. 2001, $13,308-311$.

(18) Castner, D. G.; Ratner, B. D. Surf. Sci. 2002, 500, 28-60.

(19) Lai, C. Y.; Trewyn, B. G.; Jeftinija, D. M.; Jeftinija, K.; Xu, S.; Jeftinija, S.; Lin, V. S.-Y. J. Am. Chem. Soc. 2003, 125, 4451.

(20) Slowing, I.; Trewyn, B. G.; Lin, V. S.-Y. J. Am. Chem. Soc. 2006, 128, 1479214793.

(21) Fubini, B. In The Surface Properties of Silicas; Legrand, A. P., Ed.; John Wiley \& Sons: West Sussex, 1998, p 415-458.

(22) Fubini, B. Ann. Occup. Hyg. 1998, 42, 521-530.

(23) Fubini, B.; Otero Areán, C. Chem. Soc. Rev. 1999, 28, 373-381.

(24) Merget, R.; Bauer, T.; Kupper, H. U.; Philippou, S.; Bauer, H. D.; Breitstadt, R.; Bruening, T. Arch. Toxicol. 2002, 75, 625-634.

(25) Groppo, E.; Lamberti, C.; Bordiga, S. S., G.; Zecchina, A. Chem. Rev. 2005, 105, 115-184.

(26) Shahriari, M. R. In Optical Fiber Sensors Technology; Grattan, K. T. V., Meggitt, B. T., Eds.; Kluwer Academic: Dordrecht, 1999; Vol. 4.

(27) Cho, E. J.; Bright, F. V. Anal. Chem. 2001, 73, 3289-3293.

(28) Shughart, E. L.; Ahsan, K.; Detty, M. R.; Bright, F. V. Anal. Chem. 2006, 78, $3165-$ 
(29) Lichtenstein, L.; Buchner, C.; Yang, B.; Shaikhutdinov, S.; Heyde, M.; Sierka, M.; Wlodarczyk, R.; Sauer, J.; Freund, H. J. Angew. Chem. Int. Ed. 2012, 51, 404-407.

(30) Berman, H. M.; Westbrook, J.; Feng, Z.; Gilliland, G.; Bhat, T. N.; Weissig, H.; Shindyalov, I. N.; Bourne, P. E. Nucleic Acids Res. 2000, 28, 235-242.

(31) Water in biomaterials surface science; Morra, M., Ed.; John Wiley \& Sons Ltd: Baffin Lane, Chichester, 2001.

(32) Young, G. J. J. Colloid Sci. 1958, 13, 67-85.

(33) Morrow, B. A.; Cody, I. A.; Lee, L. S. M. J. Phys. Chem. 1976, 80, 2761-2767.

(34) Blum, J.; Avnir, D. In Handbook of Sol-Gel Science and Technology; Sakka, S., Ed.; Springer: New York, 2003; Vol. III.

(35) Pagliaro, M. Silica-Based Materials for Advanced Chemical Applications; RSC Publishing: Cambridge, 2009.

(36) Corno, M.; Rimola, A.; Bolis, V.; Ugliengo, P. Phys. Chem. Chem. Phys. 2010, 12, 6309-6329.

(37) Tilocca, A. Proc. - R. Soc. Edinburgh, Sect. A: Math. Phys. Sci. 2009, 465, 10031027.

(38) Knözinger, H. In The Hydrogen Bond. Recent Developments in Theory and Experiments; Schuster, P., Zundel, G., Sandorfy, C., Eds. North-Holland: Amsterdam, 1976; Vol. 3, p 1263.

(39) Sauer, J.; Ugliengo, P.; Garrone, E.; Saunders, V. R. Chem. Rev. 1994, 94, 20952160.

(40) Burneau, A.; Gallas, J. P. In The Surface Properties of Silicas; Legrand, A. P., Ed.; John Wiley \& Sons: Chichester, 1998, p 145-234.

(41) Zachariasen, W. H. J. Am. Chem. Soc. 1932, 54, 3841-3851.

(42) Bruckner, R. Encyclopedia of Applied Physics 1997, 18, 101-131.

(43) Catlow, C. R. A.; Cormack, A. N. Int. Rev. Phys. Chem. 1987, 6, 227-250.

(44) Chao, E. C. T.; Shoemaker, E. M.; Madsen, B. M. Science 1960, 132, 220-222.

(45) Stishov, S. M.; Popova, S. V. Geokhimiya 1961, $10,837$.

(46) Ross, N. L.; Shu, J.-F.; Hazen, R. M. Am. Mineral. 1990, 75, 739-747.

(47) Nicholas, J. B.; Winans, R. E.; Harrison, R. J.; Iton, L. E.; Curtiss, L. A.; Hopfinger, A. J. J. Phys. Chem. 1992, 96, 7958-7965.

(48) Bär, M. R.; Sauer, J. Chem. Phys. Lett. 1994, 226, 405-412.

(49) Luke, B. T. J. Phys. Chem. 1993, 97, 7505-7510.

(50) Tielens, F.; De Proft, F.; Geerlings, P. J. Mol. Struct. THEOCHEM 2001, 542, $227-$

237.

(51) West, R.; Whatley, L. S.; Lake, K. J. J. Am. Chem. Soc. 1961, 83, 761-764.

(52) Baerlocher, C.; McCusker, L. B. Database of Zeolite Structures.

(53) Diffusion Mechanisms in Crystalline Materials; Catlow, C. R. A.; Cowern, N. E. B.; Farkas, D.; Mishin, Y., Eds.; Material Research Society, 1999; Vol. 527.

(54) Computer Modelling of Microporous Materials; Catlow, C. R. A.; van Santen, R. A.; Smit, B., Eds.; Elsevier Ltd.: London, 2004.

(55) Weiss, A.; Weiss, A. Z. Anorg. Allg. Chem. 1954, 276, 95-112.

(56) Hamann, D. R. Phys. Rev. B 1997, 55, 14784-14793.

(57) Sing, K. S. W. Pure Appl. Chem. 1985, 57, 603-619.

(58) Brunauer, S.; Emmett, P. H.; Teller, E. J. Am. Chem. Soc. 1938, 60, 309-319.

(59) Avnir, D.; Farin, D. Nature 1984, 308, 261-263.

(60) Hench, L. L.; Ulrich, D. B. Ultrastructure Processing of Ceramics Glasses and Composites; Wiley: New York, 1984.

(61) Depradel, A. C.; Imelik, B. C. R. Hebd. Seances Acad. Sci. 1956, 242, 122-124.

(62) Kresge, C. T.; Leonowicz, M. E.; Roth, W. J.; Vartuli, J. C.; Beck, J. S. Nature 1992, $359,710$. 
(63) Beck, J. S.; Vartuli, J. C.; Roth, W. J.; Leonowicz, M. E.; Kresge, C. T.; Schmitt, K. D.; Chu, C. T.-W.; Olson, D. H.; Sheppard, E. W.; McCullen, S. B.; Higgins, J. B.; Schlenker, J. L. J. Am. Chem. Soc. 1992, 114, 10834-10843.

(64) Hatton, B.; Landskron, K.; Whitnall, W.; Perovic, D.; Ozin, G. A. Acc. Chem. Res. 2005, 38, 305-312.

(65) Ehrlich, H.; Demadis, K. D.; Pokrovsky, O. S.; Koutsoukos, P. G. Chem. Rev. 2010, $110,4656-4689$.

(66) Dickerson, M. B.; Sandhage, K. H.; Naik, R. R. Chem. Rev. 2008, 108, 4935-4978.

(67) D'Souza, A. S.; Pantano, C. G. J. Am. Ceram. Soc. 1999, 82, 1289-1293.

(68) Li, I.; Bandara, J.; Shultz, M. J. Langmuir 2004, 20, 10474-10480.

(69) Zhou, F.; Head, J. D. J. Phys. Chem. B 2000, 104, 9981-9986.

(70) Mondal, B.; Ghosh, D.; Das, A. K. Chem. Phys. Lett. 2009, 478, 115-119.

(71) Eyre, R. J.; Goss, J. P.; Macleod, R. M.; Briddon, P. R. Phys. Chem. Chem. Phys. 2008, 10, 4495-4502.

(72) Maciel, G. E.; Sindorf, D. W. J. Am. Chem. Soc. 1980, 102, 7606-7607.

(73) Tarini, M.; Cignoni, P.; Montani, C. IEEE Trans. Vis. Comput. Graph. 2006, 12, $1237-1244$.

(74) Rimola, A.; Tosoni, S.; Sodupe, M.; Ugliengo, P. ChemPhysChem 2006, 7, 157-163.

(75) Rimola, A.; Ugliengo, P. J. Chem. Phys. 2008, 128, 204702.

(76) Mathias, J.; Wannemacher, G. J. Colloid Interface Sci. 1988, 125, 61-68.

(77) Zhuravlev, L. T.; Potapov, V. V. Russ. J. Phys. Chem. 2006, 80, 1119-1128.

(78) Zhuravlev, L. T. Colloids Surf., A 2000, 173, 1-38.

(79) Zhuravlev, L. T. Colloids Surf., A 1993, 74, 71-90.

(80) Zhuravlev, L. T. Langmuir 1987, 3, 316-318.

(81) Bolis, V.; Cavenago, A.; Fubini, B. Langmuir 1997, 13, 895-902.

(82) Bolis, V.; Fubini, B.; Marchese, L.; Martra, G.; Costa, D. J. Chem. Soc. Faraday Trans. 1991, 87, 497-505.

(83) Trebosc, J.; Wiench, J. W.; Huh, S.; Lin, V. S. Y.; Pruski, M. J. Am. Chem. Soc. 2005, 127, 3057-3068.

(84) Landmesser, H.; Kosshck, H.; Storek, W.; Fricke, R. Solid State Ionics 1997, 101 $103,271-277$.

(85) Jal, P. K.; Sudarshan, M.; Saha, A.; Patel, S.; Mishra, B. K. Colloids Surf., A 2004, $240,173-178$.

(86) Lesnichin, S. B.; Kamdem, N.; Mauder, D.; Denisov, G. S.; Shenderovich, I. G. Rus. J. Gen. Chem. 2010, 80, 2027-2031.

(87) Taylor, D. E.; Runge, K.; Cory, M. G.; Burns, D. S.; Vasey, J. L.; Hearn, J. D.; Henley, M. V. J. Phys. Chem. C 2011, 115, 24734-24742.

(88) Chuang, I. S.; Maciel, G. E. J. Phys. Chem. B 1997, 101, 3052-3064.

(89) Bolt, G. H. J. Phys. Chem. 1957, 61, 1166-1169.

(90) Abendroth, R. P. J. Colloid Interface Sci. 1970, 34, 591-598.

(91) Duval, Y.; Mielczarski, J. A.; Pokrovsky, O. S.; Mielczarski, E.; Ehrhardt, J. J. J. Phys. Chem. B 2002, 106, 2937-2945.

(92) Sverjensky, D. A.; Sahai, N. Geochim. Cosmochim. Acta 1998, 62, 3703-3716.

(93) Dixit, S.; Van Cappellen, P. Geochem. Cosmochem. Acta 2002, 66, 2559-2568.

(94) Hiemstra, T.; DeWit, J. C. M.; VanRiemsdijk, W. H. J. Colloid Interface Sci. 1989, 133, 91-104.

(95) Schwarz, J. A. J. Colloid Interface Sci. 1999, 218, 1-12.

(96) Ong, S. W.; Zhao, X. L.; Eisenthal, K. B. Chem. Phys. Lett. 1992, 191, 327-335.

(97) Ostroverkhov, V.; Waychunas, G. A.; Shen, Y. R. Phys. Rev. Lett. 2005, 94, 046102.

(98) Schnippering, M.; Neil, S. R. T.; Mackenzie, S. R.; Unwin, P. R. Chem. Soc. Rev. 2011, 40, 207-220.

(99) O’Reilly, J. P.; Butts, C. P.; I'Anson, I. A.; Shaw, A. M. J. Am. Chem. Soc. 2005, 127, 1632-1633. 
(100) Fisk, J. D.; Batten, R.; Jones, G.; O'Reilly, J. P.; Shaw, A. M. J. Phys. Chem. B 2005, $109,14475-14480$.

(101) Fan, H.-F.; Li, F.; Zare, R. N.; Lin, K.-C. Anal. Chem. 2007, 79, 3654-3661.

680-692.

(102) Hiemstra, T.; Venema, P.; VanRiemsdijk, W. H. J. Colloid Interface Sci. 1996, 184,

(103) Bickmore, B. R.; Tadanier, C. J.; Rosso, K. M.; Monn, W. D.; Eggett, D. L. Geochem. Cosmochem. Acta 2004, 68, 2025-2042.

(104) Machesky, M. L.; Predota, M.; Wesolowski, D. J.; Vlcek, L.; Cummings, P. T.; Rosenqvist, J.; Ridley, M. K.; Kubicki, J. D.; Bandura, A. V.; Kumar, N.; Sofo, J. O. Langmuir 2008, 24, 12331-12339.

(105) Churchill, H.; Teng, H.; Hazen, R. M. Amer. Mineral. 2004, 89, 1048-1055.

(106) Alexander, G. B.; Iler, R. K. J. Phys. Chem. 1953, 57, 932-934.

(107) Sonnefeld, J. J. Colloid Interface Sci. 1996, 183, 597-599.

(108) Behrens, S. H.; Grier, D. G. J. Chem. Phys. 2001, 115, 6716-6721.

(109) Charlet, L.; Schindler, P. W.; Spadini, L.; Furrer, G.; Zysset, M. Aquatic Sci. 1993,

55, 291-303.

(110) Sahai, N.; Sverjensky, D. A. Geochem. Cosmochem. Acta 1997, 61, 2801-2826.

(111) Foissy, A.; Persello, J. In The surface properties of silicas; Legrand, A. P., Ed.; J. Wiley \& Sons: Chichester, 1998, p 147-234.

(112) Marmier, N.; Delisée, A.; Fromage, F. J. Colloid Interface Sci. 1999, 212, 228-233.

(113) Turov, V. V.; Leboda, R.; Bogillo, V. I.; Skubiszewska-Zieba, J. Langmuir 1997, 13, 1237-1244.

(114) Turov, V. V.; Mironyuk, I. F. Colloids Surf., A 1998, 134, 257-263.

(115) Morrow, B. A.; McFarlan, A. J. Langmuir 1991, 7, 1695-1701.

(116) Kamiya, H.; Mitsui, M.; Takano, H.; Miyazawa, S. J. Am. Ceram. Soc. 2000, 83, 287-293.

(117) Bolis, V.; Busco, C.; Bordiga, S.; Ugliengo, P.; Lamberti, C.; Zecchina, A. Appl. Surf. Sci. 2002, 196, 56-70.

(118) Hoffmann, P.; Knozinger, E. Surf. Sci. 1987, 188, 181-198.

(119) McFarlan, A. J.; Morrow, B. A. J. Phys. Chem. 1991, 95, 5388-5390.

(120) Takei, T.; Kato, K.; Meguro, A.; Chikazawa, M. Colloids Surf., A 1999, 150, 77-84.

(121) Boccuzzi, F.; Coluccla, S.; Ghlottl, G.; Morterra, C.; Zecchina, A. J. Phys. Chem. 1978, 22, 1298-1303.

(122) Queeney, K. T.; Weldon, M. K.; Chang, J. P.; Chabal, Y. J.; Gurevich, A. B.; Sapjeta, J.; Opila, R. L. J. Appl. Phys. 2000, 87, 1322-1330.

(123) Queeney, K. T.; Herbots, N.; Shaw, J. M.; Atluri, V.; Chabal, Y. J. Appl. Phys. Lett. 2004, 84, 493-495.

(124) Olsen, J. E.; Shimura, F. Appl. Phys. Lett. 1988, 53, 1934-1936.

(125) Nagai, N.; Hashimoto, H. Appl. Surf. Sci. 2001, 172, 307-311.

(126) Pasternack, R. M.; Rivillon Amy, S.; Chabal, Y. J. Langmuir 2008, 24, 12963-12971.

(127) Kim, J.; Seidler, P.; Fill, C.; Wan, L. S. Surf. Sci. 2008, 602, 3323-3330.

(128) Kim, J.; Seidler, P.; Wan, L. S.; Fill, C. J. Colloid Interface Sci. 2009, 329, 114-119.

(129) Kim, J.; Cho, J.; Seidler, P. M.; Kurland, N. E.; Yadavalli, V. K. Langmuir 2010, 26, 2599-2608.

(130) Aissaoui, N.; Bergaoui, L.; Landoulsi, J.; Lambert, J.-F.; Boujday, S. Langmuir 2012, 28, 656-665.

(131) Brinker, C. J.; Brow, R. K.; Tallant, D. R. J. Non-Cryst. Sol. 1990, 120, 26-33.

(132) Maniar, P. D.; Navrotsky, A. J. Non-Cryst. Sol. 1990, 120, 20-25.

(133) Humbert, B.; Burneau, A.; Gallas, J. P.; Lavalley, J. C. J. Non-Cryst. Sol. 1992, 143, $75-83$.

(134) Riegel, J. o. N.-C. S.; Hartmann, I.; Kiefer, W.; Gross, J.; Fricke, J. J. Non-Cryst. Solids 1997, 211, 294-298.

(135) Wallace, S.; West, J. K.; Hench, L. L. J. Non-Cryst. Sol. 1993, 152, 101-108. 
(136) Chakoumakos, B. C.; Hill, R. J.; Gibbs, G. V. Am. Miner. 1981, 66, 1237-1249.

(137) Morrow, B. A.; Cody, I. A. J. Phys. Chem. 1976, 80, 1995-1998.

(138) Michalske, T. A.; Bunker, B. C. J. Appl. Phys. 1984, 56, 2686-2693.

(139) Bunker, B. C.; Haaland, D. M.; Ward, K. J.; Michalske, T. A.; Smith, W. L.; Binkley, J. S.; Melius, C. F.; Balfe, C. A. Surf. Sci. 1989, 210, 406-428.

(140) Grabbe, A.; Michalske, T. A.; Smith, W. L. J. Phys. Chem. 1995, 99, 4648-4654.

(141) Inaki, Y.; Yoshida, H.; Yoshida, T.; Hattori, T. J. Phys. Chem. B 2002, 106, 90989106.

(142) Chiang, C.-M.; Zegarski, B. R.; Dubois, L. H. J. Phys. Chem. 1993, 97, 6948-6950.

(143) Zarubin, D. P. J. Non-Cryst. Sol. 2001, 286, 80-88.

(144) West, J. K.; LaTorre, G.; Hench, L. L. J. Non-Cryst. Sol. 1996, 195, 45-53.

(145) Engelhardt, G.; Michel, D. High Resolution Solid State NMR of Silicates and Zeolites; Wiley \& Sons: New-York, 1987.

(146) Boujday, S. PhD Thesis, UPMC Paris 6, 2002.

(147) Zhao, H.; Chen, Q.; Zhang, S. Microporous Mesoporous Mater. 2012, 155, 240-244.

(148) Rossini, A. J.; Zagdoun, A.; Lelli, M.; Gajan, D.; Rascon, F.; Rosay, M.; Maas, W. E.; Coperet, C.; Lesage, A.; Emsley, L. Chem. Sci. 2012, 3, 108-115.

(149) Spearing, D. R.; Farnan, I.; Stebbins, J. F. Phys. Chem. Miner. 1992, 19, 307-321.

(150) van Eck, E. R. H.; Smith, M. E.; Kohn, S. C. Sol. State Nucl. Mag. Res. 1999, 15, $181-188$.

(151) Hu, J. Z.; Kwak, J. H.; Herrera, J. E.; Wang, Y.; Peden, C. H. F. Solid State Nucl. Magn. Reson. 2005, 27, 200-205.

(152) d'Espinose de la Caillerie, J.-B.; Aimeur, M. R.; Kortobi, Y. E.; Legrand, A. P. J. Colloid Interface Sci. 1997, 197, 434-439.

(153) Liu, C. H. C.; Maciel, G. E. J. Am. Chem. Soc. 1996, 118, 5103-5119.

(154) Hughes, C. E. Progr. Nucl. Magn. Res. Spectr. 2004, 45, 301-313.

(155) Baum, J.; Munowitz, M.; Garroway, A. N.; Pines, A. J. Chem. Phys. 1985, 83, 20152025.

(156) Hwang, S.-J.; Uner, D. O.; King, T. S.; Pruski, M.; Gerstein, B. C. J. Phys. Chem. 1996, 99, 3697-3703.

(157) Shantz, D. F.; Schmedt auf der Günne, J.; Koller, H.; Lobo, R. F. J. Am. Chem. Soc. 2000, 122, 6659-6663.

(158) Fubini, B.; Giamello, E.; Volante, M.; Bolis, V. Toxicol. Ind. Health 1990, 6, 571598.

(159) Stathis, J. H.; Kastner, M. A. Phys. Rev. B 1984, 29, 7079-7081.

(160) Pacchioni, G.; Vitiello, M. J. Non-Cryst. Solids 1999, 245, 175-182.

(161) Mashkovtsev, R. I.; Pan, Y. M. Phys. Chem. Miner. 2012, 39, 79-85.

(162) Buscarino, G.; Vaccaro, G.; Agnello, S.; Gelardi, F. M. J. Non-Cryst. Solids 2009, 355, 1092-1094.

(163) Feng, P. B.; Wang, Y.; Rong, X.; Su, J. H.; Ju, C. Y.; Du, J. F. Phys. Lett. A 2012, $376,2195-2199$.

(164) Romanelli, M.; Di Benedetto, F.; Bartali, L.; Innocenti, M.; Fornaciai, G.; Montegrossi, G.; Pardi, L. A.; Zoleo, A.; Capacci, F. Phys. Chem. Miner. 2012, 39, 479-490.

(165) Fenoglio, I.; Fubini, B.; Ghibaudi, E. M.; Turci, F. Adv. Drug Deliv. Rev. 2011, 63, $1186-1209$.

(166) Zelenak, V.; Zelenakova, A.; Kovac, J. Colloids Surf., A 2010, 357, 97-104.

(167) Lercher, J. A.; Grundling, C.; EderMirth, G. Catal. Today 1996, 27, 353-376.

(168) Marchese, L.; Gianotti, E.; Maschmeyer, T.; Martra, G.; Coluccia, S.; Thomas, J. Il Nuovo Cimento D 1997, 19, 1707-1718.

(169) Armandi, M.; Bolis, V.; Bonelli, B.; Arean, C. O.; Ugliengo, P.; Garrone, E. J. Phys. Chem. C 2011, 115, 23344-23353.

(170) Bonelli, B.; Cozzolino, M.; Tesser, R.; Di Serio, M.; Piumetti, M.; Garrone, E.; Santacesaria, E. J. Catal. 2007, 246, 293-300. 
(171) Parry, E. P. J. Catal. 1963, 2, 371-379.

(172) Trombetta, M.; Busca, G.; Rossini, S.; Piccoli, V.; Cornaro, U.; Guercio, A.; Catani, R.; Willey, R. J. Catal. 1998, 179, 581-596.

(173) Fubini, B.; Bolis, V.; Cavenago, A.; Garrone, E.; Ugliengo, P. Langmuir 1993, 9, 2712-2720.

(174) Borello, E.; Zecchina, A.; Morterra, C. J. Phys. Chem. 1967, 71, 2938-2945.

(175) Borello, E.; Zecchina, A.; Morterra, C. J. Phys. Chem. 1967, 71, 2945-2951.

(176) Natal-Santiago, M. A.; Dumesic, J. A. J. Catal. 1998, 175, 252-268.

(177) Luts, T.; Katz, A. Top. Catal. 2012, 55, 84-92.

(178) Bunker, B. C.; Haaland, D. M.; Michalske, T. A.; Smith, W. L. Surf. Sci. 1989, 222, 95-118.

(179) Natal-Santiago, M. A.; Hill, J. M.; Dumesic, J. A. J. Mol. Catal. A 1999, 140, 199214.

(180) Brunel, D.; Cauvel, A.; Di Renzo, F.; Fajula, F.; Fubini, B.; Onida, B.; Garrone, E. New J. Chem. 2000, 24, 807-813.

(181) Srinivasan, S.; Datye, A. K.; Smith, M. H.; Peden, C. H. F. J. Catal. 1994, 145, 565573.

(182) Sindorf, D. W.; Maciel, G. E. J. Phys. Chem 1982, 86, 5208-5219.

(183) Koltsov, S. I.; Malygin, A. A.; Volkova, A. N.; Aleskovskii, V. B. Russ. J. Inorg. Chem. 1973, 47, 988-991.

(184) Fleischman, S. D.; Scott, S. L. J. Am. Chem. Soc. 2011, 133, 4847-4855

(185) Dong, Y.; Pappu, S. V.; Xu, Z. Anal. Chem. 1998, 70, 4730-4735.

(186) Boujday, S.; Lambert, J.-F.; Che, M. J. Phys. Chem. B 2003, 107, 651-654.

(187) Boujday, S.; Lambert, J.-F.; Che, M. ChemPhysChem 2004, 5, 1003-1013.

(188) Schroeder, T.; Adelt, M.; Richter, B.; Naschitzki, M.; Bäumer, M.; Freund, H.-J. Surf. Rev. Lett. 2000, 7, 7-14.

(189) Wendt, S.; Frerichs, M.; Wei, T.; Chen, M. S.; Kempter, V.; Goodman, D. W. Surf. Sci. 2004, 565, 107-120.

(190) Chen, M. S.; Santra, A. K.; Goodman, D. W. Phys. Rev. B 2004, 69, 155404.

(191) Yu, X.; Yang, B.; Boscoboinik, J. A.; Shaikhutdinov, S.; Freund, H.-J. Appl. Phys. Lett. 2012, 100, 151608.

(192) Mirji, S. A.; Halligudi, S. B.; Mathew, N.; Ravi, V.; Jacob, N. E.; Patil, K. R. Colloids Surf., A 2006, 287, 51-58.

(193) Takahashi, T.; Fukatsu, S.; Itoh, K. M.; Uematsu, M.; Fujiwara, A.; Kageshima, H.; Takahashi, Y.; Shiraishi, K. J. Appl. Phys. 2003, 93, 3674-3676.

(194) Chang, C.-C.; Shu, M.-C. J. Phys. Chem. B 2003, 107, 7076-7087.

(195) Wu, W. L.; Wallace, W. E.; Lin, E. K.; Lynn, G. W.; Glinka, C. J.; Ryan, E. T.; Ho, H. M. J. Appl. Phys. 2000, 87, 1193-1200.

(196) Chiba, K.; Takenaka, Y. Appl. Surf. Sci. 2008, 254, 2534-2539.

(197) Chandrasekhar, V.; Boomishankar, R.; Nagendran, S. Chem. Rev. 2004, 104, 58475910.

(198) Feher, F. J.; Newman, D. A.; Walzer, J. F. J. Am. Chem. Soc. 1989, 111, 1741-1748.

(199) Abbenhuis, H. C. L.; Burrows, A. D.; Kooijman, H.; Lutz, M.; Palmer, M. T.; vanSanten, R. A.; Spek, A. L. Chem. Commun. 1998, 2627-2628.

(200) Dijkstra, T. W.; Duchateau, R.; van Santen, R. A.; Meetsma, A.; Yap, G. P. A. J. Am. Chem. Soc. 2002, 124, 9856-9864.

(201) Gun'ko, V. M.; Turov, V. V.; Zarko, V. I.; Voronin, E. F.; Tischenko, V. A.; Dudnik, V. V.; Pakhlov, E. M.; Chuiko, A. A. Langmuir 1997, 13, 1529-1544.

(202) Totland, C.; Steinkopf, S.; Blokhus, A. M.; Nerdal, W. Langmuir 2011, 27, 46904699.

(203) Basiuk, V. A. In Encyclopedia of Surface and Colloid Science; Marcel Dekker: 2002, p 277-293. 
(204) Parida, S. K.; Dash, S.; Patel, S.; Mishra, B. K. Adv. Coll. Interf. Sci. 2006, 121, 77110.

(205) Lambert, J.-F. Origins Life Evol. Biospheres 2008, 38, 211-242.

(206) Popat, A.; Hartono, S. B.; Stahr, F.; Liu, J.; Qiao, S. Z.; Lu, G. Q. Nanoscale 2011, 3, 2801-2818.

(207) Basiuk, V. A. Surfact. Sci. Ser. 1998, 75, 55-87.

(208) Hartmann, M. Chem. Mater. 2005, 17, 4577-4593.

(209) Bolivar, J. M.; Nidetzky, B. Langmuir 2012, 26, 10040-10049.

(210) Meng, M.; Stievano, L.; Lambert, J.-F. Langmuir 2004, 20, 914-923.

(211) Bouchoucha, M.; Jaber, M.; Onfroy, T.; Lambert, J.-F.; Xue, B. J. Phys. Chem. C 2011, 115, 21813-21825.

(212) Janossy, I.; Menyhard, M. Surf. Sci. 1974, 25, 647-649.

(213) Clegg, M. L.; Morales de la Garza, L.; Karakatsani, S.; King, D. A.; Driver, S. M. Top. Catal. 2012, 19-20, 1429-1444.

(214) Vlasova, N. N.; Golovkova, L. P. Colloid J. 2004, 66, 657-662.

(215) O’Connor, A. J.; Hokura, A.; Kisler, J. M.; Shimazu, S.; Stevens, G. W.; Komatsu, Y. Sep. Purif. Technol. 2006, 48, 197-201.

(216) Basiuk, V. A.; Gromovoy, T. Y. Colloids Surf., A 1996, 118, 127-140.

(217) Kitadai, N.; Yokoyama, T.; Nakashima, S. J. Colloid Interface Sci. 2009, 329, 31-37.

(218) Paszti, Z.; Keszthelyi, T.; Hakkel, O.; Guczi, L. J. Phys. Cond. Matt. 2008, 20, 224014.

(219) Barlow, S. M.; Kitching, K. J.; Haq, S.; Richardson, N. V. Surf. Sci. 1998, 401, 322335.

(220) Holinga, G. J.; York, R. L.; Onorato, R. M.; Thompson, C. M.; Webb, N. E.; Yoon, A. P.; Somorjai, G. A. J. Am. Chem. Soc. 2011, 133, 6243-6253

(221) Macklin, J. W.; White, D. H. Spectrochim. Acta A 1985, 41A, 851-859.

(222) Basiuk, V. A.; Gromovoy, T. Y. React. Kinet. Catal. Lett. 1993, 50, 297-303.

(223) Lomenech, C.; Bery, G.; Costa, D.; Stievano, L.; Lambert, J.-F. ChemPhysChem 2005, 6, 1061-1070.

18172.

(224) Lopes, I.; Piao, L.; Stievano, L.; Lambert, J.-F. J. Phys. Chem. C 2009, 113, 18163-

(225) Amitay-Rosen, T.; Kababya, S.; Vega, S. J. Phys. Chem. B 2009, 113, 6267-6282.

(226) Amitay-Rosen, T.; Vega, S. Phys. Chem. Chem. Phys. 2010, 12, 6763-6773.

(227) Ben Shir, I.; Kababya, S.; Amitay-Rosen, T.; Balazs, Y. S.; Schmidt, A. J. Phys. Chem. B 2010, 114, 5989-5996.

(228) Ben Shir, I.; Kababya, S.; Schmidt, A. J. Phys. Chem. C 2012, 116, 9691-9702.

(229) Stievano, L.; Tielens, F.; Lopes, I.; Folliet, N.; Gervais, C.; Costa, D.; Lambert, J.-F. Cryst. Growth Design 2010, 10, 3657-3667.

(230) Mirau, P. A.; Naik, R. R.; Gehring, P. J. Am. Chem. Soc. 2011, 133, 18243-18248.

(231) Wolny, P. M.; Spatz, J. P.; Richter, R. P. Langmuir 2010, 26, 1029-1034.

(232) Satriano, C.; Fragal a, M. E.; Forte, G.; Santoro, A. M.; La Mendola, D.; Kasemo, B. Soft Matt. 2012, 8, 53-56.

(233) Chen, H.; Su, X.; Neoh, K.-G.; Choe, W.-S. Anal. Chem. 2006, 78, 4872-4879.

(234) Phillips, D. C.; York, R. L.; Mermut, O.; McCrea, K. R.; Ward, R. S.; Somorjai, G. A. J. Phys. Chem. C 2007, 111, 255-261.

(235) Mermut, O.; Phillips, D. C.; York, R. L.; McCrea, K. R.; Ward, R. S.; Somorjai, G. A. J. Am. Chem. Soc. 2006, 128, 3598-3607.

(236) York, R. L.; Holinga, G. J.; Somorjai, G. A. Langmuir 2009, 25, 9369-9374.

(237) Wei, Y.; Latour, R. A. Langmuir 2008, 24, 6721-6729.

(238) Thyparambil, A. A.; Wei, Y.; Latour, R. A. Langmuir 2012, 28, 5687-5694.

(239) Landoulsi, J.; Dupres, V. ChemPhysChem 2011, 12, 1310-1316.

(240) Cha, P.; Krishnan, A.; Fiore, V. F.; Vogler, E. A. Langmuir 2008, 24, 2553-2563.

(241) Basiuk, V. A. Russ. J. Theor. Exp. Chem. 1990, 26, 89-93 (97-102 original russian). 
(242) Basiuk, V. A.; Gromovoy, T. Y.; Glukhoy, A. M.; Golovaty, V. G. Origins Life Evol. Biospheres 1991, 21, 129-144.

(243) Basiuk, V. A.; Gromovoy, T. Y.; Chuiko, A. A.; Soloshonok, V. A.; Kukhar, V. P. Synthesis 1992, 5, 449-451.

(244) Basiuk, V. A.; Gromovoy, T. Y.; Khil'chevskaya, E. G. Origins Life Evol. Biospheres 1995, 25, 375-393.

(245) Bujdák, J.; Rode, B. M. React. Kinet. Catal. Lett. 1997, 62, 281-286.

(246) Bujdák, J.; Rode, B. M. Origins Life Evol. Biospheres 1999, 29, 451-461.

(247) Stievano, L.; Yu Piao, L.; Lopes, I.; Meng, M.; Costa, D.; Lambert, J.-F. Eur. J. Mineral. 2007, 19, 321-331.

(248) Kunota, L. T.; Gambero, A.; Santana Santos, A.; Granjeiro, J. M. J. Colloid Interface Sci. 1996, 185, 453-457.

(249) Alaeddine, S.; Nygren, H. Colloids Surf., B 1996, 6, 71-79.

(250) Vlasova, N. N. Colloid J. 2005, 67, 537-541.

(251) Palit, D.; Moulik, S. P. J. Colloid Interface Sci. 2001, 239, 20-26.

(252) Pizzanelli, S.; Kababya, S.; Frydman, V.; Landau, M.; Vega, S. J. Phys. Chem. B 2005, 109, 8029-8039.

(253) Gao, Q.; Xu, W.; Xu, Y.; Wu, D.; Sun, Y.; Deng, F.; Shen, W. J. Phys. Chem. B 2008, 112, 2261-2267.

(254) Goering, J.; Sah, S.; Burghaus, U.; Street Jr., K. W. Surf. Interf. Anal. 2008, 40, $1423-1429$.

(255) Casado, C.; Castán, J.; Gracia, I.; Yus, M.; Mayoral, A. 1.; Sebastián, V.; LópezRam-de-Viu, P.; Uriel, S.; Coronas, J. Langmuir 2012, 28, 6638-6644.

(256) Shin, J. H.; Park, S. S.; Selvaraj, M.; Ha, C.-S. J. Porous Mat. 2012, 19, 29-35.

(257) Kim, J.; Desch, R. J.; Thiel, S. W.; Guliants, V. V.; Pinto, N. G. Micr. Mes. Mat. 2012, 149, 60-68.

(258) Plekan, O.; Feyer, V.; Sutara, F.; Skála, T.; Svec, M.; Cháb, V.; Matolín, V.; Prince, K. C. Surf. Sci. 2007, 603, 1973-1980.

(259) Poeckh, T.; Lopez, S.; Fuller, A. O.; Solomon, M. J.; Larson, R. G. Anal. Biochem. 2008, 373, 253-262.

(260) Nakanishi, K.; Sakiyama, T.; Imamura, K. J. Biosci. Bioeng. 2001, 91, 233-244.

(261) Gray, J. J. Curr. Op. Struct. Biol. 2004, 14, 110-115.

(262) Lundqvist, M.; Sethson, I.; Jonsson, B.-H. Langmuir 2005, 21, 5974-5979.

(263) Greenfield, N. J. Anal. Biochem. 1996, 235, 1-10.

(264) Giacomelli, C. E.; Bremer, M. G. E. G.; Norde, W. J. Colloid Interface Sci. 1999, 220, 13-23.

(265) Xia, N.; May, C. J.; McArthur, S. L.; Castner, D. G. Langmuir 2002, 18, 4090-4097.

(266) Han, S.; Xu, W.; Meiwen, C.; Jiqian, W.; Xia, D.; Xu, H.; Zhao, X.; Lu, J. R. Soft Matt. 2012, 8, 645-652.

(267) Burkett, S. L.; Read, M. J. Langmuir 2001, 17, 5059-5065.

(268) Read, M. J.; Burkett, S. L. J. Colloid Interface Sci. 2003, 261, 255-263.

(269) Sethuraman, A.; Vedantham, G.; Imoto, T.; Przybycien, T.; Belfort, G. Proteins:

Struct. Funct. Bioinform. 2004, 56, 669-678.

(270) Felsovalyi, F.; Mangiagalli, P.; Bureau, C.; Kumar, S. K.; Banta, S. Langmuir 2011, 27, 11873-11882.

(271) Oren, E. E.; Tamerler, C.; Sahin, D.; Hnilova, M.; Seker, U. O. S.; Sarikaya, M.; Samudral, R. Bioinform. 2007, 23, 2816-2822.

(272) Oren, E. E.; Notman, R.; Kim, I. W.; Evans, J. S.; Walsh, T. R.; Samudrala, R.;

Tamerler, C.; Sarikaya, M. Langmuir 2010, 26, 11003-11009.

(273) Patwardhan, S. V.; Emami, F. S.; Berry, R. J.; Jones, S. E.; Naik, R. R.; Deschaume,

O.; Heinz, H.; Perry, C. C. J. Am. Chem. Soc. 2012, 134, 6244-6256.

(274) Ikeda, T.; Kuroda, A. Colloids Surf., B 2011, 85, 359-363. 
(275) Dovesi, R. In Quantum-Mechanical Ab-initio Calculation of the Properties of Crystalline Materials; Pisani, C., Ed.; Springer: Berlin, 1996; Vol. 67, p 31.

(276) Pisani, C. In Quantum-Mechanical Ab-initio Calculation of the Properties of Crystalline Materials; Pisani, C., Ed.; Springer: Berlin, 1996; Vol. 67, p 47.

(277) Pisani, C.; Dovesi, R.; Roetti, C. In Lecture notes in Chemistry; Springer-Verlag: Berlin, 1988; Vol. 48, p 193.

(278) Bergerhoff, G.; Hundt, R.; Sievers, R.; Brown, I. D. J. Chem. Inf. Comput. Sci. 1983, $23,66-69$.

(279) Giacovazzo, C.; Monaco, H. L.; Artioli, G.; Viterbo, D.; Ferraris, G.; Gilli, G.; Zanotti, G.; Catti, M. Fundamentals of Crystallography; 2nd ed.; Oxford University Press: New York, 2002.

(280) Bakos, T.; Rashkeev, S. N.; Pantelides, S. T. Phys. Rev. Lett. 2002, 88, 055508.

(281) Malavasi, G.; Menziani, M. C.; Pedone, A.; Segre, U. J. Non-Cryst. Solids 2006, 352, 285-296.

(282) Pedone, A.; Malavasi, G.; Menziani, M. C.; Segre, U.; Cormack, A. N. Chem. Mater. 2008, 20, 4356-4366.

(283) Pedone, A. J. Phys. Chem. C 2009, 113, 20773-20784.

(284) Colombi Ciacchi, L.; Payne, M. C. Phys. Rev. Lett. 2005, 95, 196101.

(285) Butenuth, A.; Moras, G.; Schneider, J.; Koleini, M.; Koppen, S.; Meissner, R.;

Wright, L. B.; Walsh, T. R.; Colombi Ciacchi, L. Phys. Status Solidi B 2012, 249, 292-305.

(286) Sarnthein, J.; Pasquarello, A.; Car, R. Phys. Rev. B 1995, 52, 12690-12695.

(287) Sarnthein, J.; Pasquarello, A.; Car, R. Phys. Rev. Lett. 1995, 74, 4682-4685.

(288) Haworth, R.; Mountjoy, G.; Corno, M.; Ugliengo, P.; Newport, R. J. Phys. Rev. B 2010, $81,060301$.

(289) Corno, M.; Pedone, A.; Dovesi, R.; Ugliengo, P. Chem. Mater. 2008, 20, 5610-5621.

(290) Dracinsky, M.; Benda, L.; Bour, P. Chem. Phys. Lett. 2011, 512, 54-59.

(291) Dovesi, R.; Civalleri, B.; Orlando, R.; Roetti, C.; Saunders, V. R. In Reviews in Computational Chemistry, Vol 21; Lipkowitz, K. B., Larter, R., Cundari, T. R., Eds.; Wiley-Vch, Inc: New York, 2005; Vol. 21, p 1-125.

(292) Tasker, P. W. J. Phys. C Solid State Phys. 1979, 12, 4977-4984.

(293) Meyer, B.; Marx, D. Phys. Rev. B 2003, 67, 0035403.

(294) Murashov, V. J. Mol. Struct. 2003, 650, 141-157.

(295) Murashov, V. V. J. Phys. Chem. B 2005, 109, 4144-4151.

(296) Radzig, V. A. In Defects in SiO2 and Related Dielectrics: Science and Technology;

Pacchioni, G., Skuja, L., Griscom, D. L., Eds. 2000; Vol. 2, p 339-370.

(297) Radzig, V. A.; Berestetskaya, I. V.; Kostritsa, S. N. Kinet. Catal. 1998, 39, 863-867.

(298) Pacchioni, G.; Ferrari, A. M.; Ierano, G. Faraday Discuss. 1997, 106, 155-172.

(299) Pacchioni, G.; Ierano, G. Phys. Rev. Lett. 1997, 79, 753-756.

(300) Pacchioni, G.; Ierano, G. Phys. Rev. B 1998, 57, 818-832.

(301) Pacchioni, G.; Ierano, G.; Marquez, A. M. Phys. Rev. Lett. 1998, 81, 377-380.

(302) Zwijnenburg, M. A.; Sokol, A. A.; Sousa, C.; Bromley, S. T. J. Chem. Phys. 2009, $131,034705-034711$.

(303) Boero, M.; Pasquarello, A.; Sarnthein, J.; Car, R. Phys. Rev. Lett. 1997, 78, 887-890.

(304) Rudra, J. K.; Fowler, W. B. Phys. Rev. B 1987, 35, 8223-8230.

(305) Vaccaro, G.; Buscarino, G.; Agnello, S.; Sporea, A.; Oproiu, C.; Sporea, D. G.; Gelardi, F. M. J. Phys. Chem. C 2012, 116, 144-149.

17884.

(306) Musso, F.; Sodupe, M.; Corno, M.; Ugliengo, P. J. Phys. Chem. C 2009, 113, 17876-

(307) Cole, D. J.; Payne, M. C.; Csanyi, G.; Spearing, S. M.; Colombi Ciacchi, L. J. Chem. Phys. 2007, 127, 204704.

(308) Schneider, J.; Colombi Ciacchi, L. J. Am. Chem. Soc. 2012, 134, 2407-2413.

(309) Rimola, A.; Sodupe, M.; Tosoni, S.; Civalleri, B.; Ugliengo, P. Langmuir 2006, 22, 6593-6604. 
(310) Corno, M.; Busco, C.; Bolis, V.; Tosoni, S.; Ugliengo, P. Langmuir 2009, 25, 21882198.

(311) Rimola, A.; Corno, M.; Garza, J.; Ugliengo, P. Philos. Trans. R. Soc., A 2012, 370, 1478-1498.

(312) Rimola, A.; Corno, M.; Zicovich-Wilson, C. M.; Ugliengo, P. J. Am. Chem. Soc. 2008, 130, 16181-16183. 14940.

(313) Rozanska, X.; Delbecq, F.; Sautet, P. Phys. Chem. Chem. Phys. 2010, 12, 14930-

(314) Peri, J. B.; Hensley, J. A. L. J. Phys. Chem. 1968, 72, 2926-2933.

(315) Vigne-Maeder, F.; Sautet, P. J. Phys. Chem. B 1997, 101, 8197-8203.

(316) Civalleri, B.; Casassa, S.; Garrone, E.; Pisani, C.; Ugliengo, P. J. Phys. Chem. B. 1999, 103, 2165-2171.

(317) Tosoni, S.; Civalleri, B.; Pascale, F.; Ugliengo, P. J. Phys.: Conference Series 2008, 117, 012026-012033.

(318) Civalleri, B.; Ugliengo, P. J. Phys. Chem. B. 2000, 104, 9491-9499. 134709.

(319) Goumans, T. P. M.; Catlow, C. R. A.; Brown, W. A. J. Chem. Phys. 2008, 128,

(320) Goumans, T. P. M.; Wander, A.; Catlow, C. R. A.; Brown, W. A. Mon. Not. R. Astron. Soc. 2007, 382, 1829-1832.

(321) Rimola, A.; Sodupe, M.; Ugliengo, P. J. Phys. Chem. C 2009, 113, 5741-5750.

(322) Rimola, A.; Civalleri, B.; Ugliengo, P. Langmuir 2008, 24, 14027-14034.

(323) Zhao, Y. L.; Koppen, S.; Frauenheim, T. J. Phys. Chem. C 2011, 115, 9615-9621.

(324) Nonella, M.; Seeger, S. ChemPhysChem 2008, 9, 414-421.

(325) Nonella, M.; Seeger, S. Chem. Phys. 2010, 378, 73-81.

(326) Orlando, R.; Delle Piane, M.; Bush, I. J.; Ugliengo, P.; Ferrabone, M.; Dovesi, R. J. Comput. Chem. 2012, 33, 2276-2284.

(327) Pisani, C.; Maschio, L.; Casassa, S.; Halo, M.; Schutz, M.; Usvyat, D. J. Comput. Chem. 2008, 29, 2113-2124.

(328) Pisani, C.; Schutz, M.; Casassa, S.; Usvyat, D.; Maschio, L.; Lorenz, M.; Erba, A. Phys. Chem. Chem. Phys. 2012, 14, 7615-7628.

(329) Paier, J.; Ren, X.; Rinke, P.; Scuseria, G. E.; Gruneis, A.; Kresse, G.; Scheffler, M.

New J. Phys. 2012, 14, 043002.

(330) Hafner, J. J. Comput. Chem. 2008, 29, 2044-2078.

(331) Maschio, L.; Civalleri, B.; Ugliengo, P.; Gavezzotti, A. J. Phys. Chem. A 2011, 115, 11179-11186.

(332) Maschio, L. J. Chem. Theory Comput. 2011, 7, 2818-2830.

(333) Sherrill, C. D. J. Chem. Phys. 2010, 132, 110902-110906.

(334) Tomasi, J.; Persico, M. Chem. Rev. 1994, 94, 2027-2094.

(335) Sauer, J. Chem. Rev. 1989, 89, 199-255.

(336) Meier, W. M.; Olson, D. H.; Baerlocher, C. Zeolites 1996, 17, 1-229.

(337) Sauer, J.; Hill, J. R. Chem. Phys. Lett. 1994, 218, 333-337.

(338) Civalleri, B.; Garrone, E.; Ugliengo, P. Chem. Phys. Lett. 1998, 294, 103-108.

(339) Civalleri, B.; Garrone, E.; Ugliengo, P. Chem. Phys. Lett. 1999, 299, 443-450.

(340) Civalleri, B.; Garrone, E.; Ugliengo, P. Langmuir 1999, 15, 5829-5835.

(341) Bordiga, S.; Roggero, I.; Ugliengo, P.; Zecchina, A.; Bolis, V.; Artioli, G.; Buzzoni,

R.; Marra, G.; Rivetti, F.; Spano, G.; Lamberti, C. J. Chem. Soc., Dalton Trans. 2000, 3921-3929.

(342) Bolis, V.; Busco, C.; Ugliengo, P. J. Phys. Chem. B 2006, 110, 14849-14859.

(343) Bolis, V.; Busco, C.; Aina, V.; Morterra, C.; Ugliengo, P. J. Phys. Chem. C 2008, $112,16879-16892$.

(344) Koch, W.; Holthausen, M. C. A Chemist's Guide to Density Functional Theory; 2nd ed.; Wiley-VCH Verlag GmbH: Weinheim, 2002.

(345) Musso, F.; Ugliengo, P.; Solans-Monfort, X.; Sodupe, M. J. Phys. Chem. C 2010, $114,16430-16438$. 
(346) Yang, J. J.; Meng, S.; Xu, L. F.; Wang, E. G. Phys. Rev. Lett. 2004, 92, 146102.

(347) Bakowies, D.; Thiel, W. J. Phys. Chem. 1996, 100, 10580-10594.

(348) Zhang, Y.; Lee, T.-S.; Yang, W. J. Chem. Phys. 1999, 110, 46-54.

(349) Poteau, R.; Alary, F.; Abou El Makarim, H.; Heully, J. L.; Barthelat, J. C.; Daudey, J. P. J. Phys. Chem. A 2001, 105, 206-214.

(350) Poteau, R.; Ortega, I.; Alary, F.; Solis, A. R.; Barthelat, J. C.; Daudey, J. P. J. Phys. Chem. A 2001, 105, 198-205.

(351) Taylor, C. E.; Cory, M. G.; Bartlett, R. J.; Thiel, W. Comput. Mater. Sci. 2003, 27, 204-211.

(352) Taylor, D. E.; Bunte, S. W.; Runge, K. J. Phys. Chem. A 2006, 110, 6279-6284.

(353) Mallik, A.; Taylor, D. E.; Runge, K.; Dufty, J. W. Int. J. Quantum Chem. 2004, 100, $1019-1025$.

(354) Maseras, F.; Morokuma, K. J. Comput. Chem. 1995, 16, 1170-1179.

(355) Frisch, M. J.; Trucks, G. W.; Schlegel, H. B.; Scuseria, G. E.; Robb, M. S.; Cheeseman, J. R.; Zakrzewski, V. G.; Montgomery Jr., J. A.; Stratmann, R. E.; Burant, J. C.; Dapprich, S.; Millam, J. M.; Daniels, A. D.; Kudin, K. N.; Strain, M. C.; Farkas, O.; Tomasi, J.; Barone, V.; Cossi, M.; Cammi, R.; Mennucci, B.; Pomelli, C.; Adamo, C.; Clifford, S.; Ochterski, J.; Petersson, G. A.; Ayala, P. Y.; Cui, Q.; Morokuma, K.; Salvador, P.; Dannenberg, J. J.; Malick, D. K.; Rabuck, A. D.; Raghavachari, K.; Foresman, J. B.; Cioslowski, J.; Ortiz, J. V.; Baboul, A. G.; Stefanov, B. B.; Liu, G.; Liashenko, A.; Piskorz, P.; Komaromi, I.; Gomperts, R.; Martin, R. L.; Fox, D. J.; Keith, T.; Al-Laham, M. A.; Peng, C. Y.; Nanayakkara, A.; Challacombe, M.; Gill, P. M. W.; Johnson, B.; Chen, W.; Wong, M. W.; Andres, J. L.; Gonzalez, C.; Head-Gordon, M.; Replogle, E. S.; Pople, J. A.; Gaussian 98, Gaussian, Inc.: Pittsburgh, PA, 1998.

(356) Svensson, M.; Humbel, S.; Froese, R. D. J.; Matsubara, T.; Sieber, S.; Morokuma, K. J. Phys. Chem. 1996, 100, 19357-19363.

(357) Humbel, S.; Sieber, S.; Morokuma, K. J. Chem. Phys. 1996, 105, 1959-1967.

(358) Dapprich, S.; Komaromi, I.; Byun, K. S.; Morokuma, K.; Frisch, M. J. J. Mol. Struct. THEOCHEM 1999, 461, 1-21.

(359) Eichler, U.; Kolmel, C. M.; Sauer, J. J. Comput. Chem. 1997, 18, 463-477.

(360) Vreven, T.; Morokuma, K. J. Comput. Chem. 2000, 21, 1419-1432.

(361) Roggero, I.; Civalleri, B.; Ugliengo, P. Chem. Phys. Lett. 2001, 341, 625-632.

(362) Bolis, V.; Barbaglia, A.; Broyer, M.; Busco, C.; Civalleri, B.; Ugliengo, P. Origins Life Evol. Biosphere 2004, 34, 69-77.

(363) Bordiga, S.; Ugliengo, P.; Damin, A.; Lamberti, C.; Spoto, G.; Zecchina, A.; Spano, G.; Buzzoni, R.; Dalloro, L.; Rivetti, F. Top. Catal. 2001, 15, 43-52.

(364) Sauer, J.; Sierka, M. J. Comput. Chem. 2000, 21, 1470-1493.

(365) Brandle, M.; Sauer, J. J. Am. Chem. Soc. 1998, 120, 1556-1570.

(366) Nachtigallova, D.; Nachtigall, P.; Sierka, M.; Sauer, J. Phys. Chem. Chem. Phys. 1999, 1, 2019-2026.

(367) Tuma, C.; Sauer, J. Phys. Chem. Chem. Phys. 2006, 8, 3955-3965.

(368) Sillar, K.; Hofmann, A.; Sauer, J. J. Am. Chem. Soc. 2009, 131, 4143-4150.

(369) Svelle, S.; Tuma, C.; Rozanska, X.; Kerber, T.; Sauer, J. J. Am. Chem. Soc. 2009, $131,816-825$.

(370) Vreven, T.; Byun, K. S.; Komaromi, I.; Dapprich, S.; Montgomery, J. A.; Morokuma, K.; Frisch, M. J. J. Chem. Theory Comput. 2006, 2, 815-826.

(371) Cramer, C. Essentials of Computational Chemistry: Theories and Models; 2nd ed.;

Wiley, 2004.

(372) Jensen, F. Introduction to Computational Chemistry; 2on ed.; Wiley, 2006.

(373) Szabo, A.; Ostlund, N. S. Modern Quantum Chemistry: Introduction to Advanced Electronic Structure Theory Dover, 1996.

(374) Pyykkö, P.; Stanton, J. F. Chem. Rev. 2012, 112, 1-3.

(375) Crawford, T. D.; King, R. A. Chem. Phys. Lett. 2002, 366, 611-622.

(376) Lee, M. S.; Maslen, P. E.; Head-Gordon, M. J. Chem. Phys. 2000, 112, 3592-3601. 
(377) Maslen, P. E.; Head-Gordon, M. Chem. Phys. Lett. 1998, 283, 102-108.

(378) Saebo, S.; Pulay, P. Chem. Phys. Lett. 1985, 113, 13-18.

(379) Saebo, S.; Pulay, P. J. Chem. Phys. 1988, 88, 1884-1890.

(380) Saebo, S.; Pulay, P. Annu. Rev. Phys. Chem. 1993, 44, 213-236.

(381) Schutz, M. J. Chem. Phys. 2002, 116, 8772-8785.

(382) Scuseria, G. E.; Ayala, P. Y. J. Chem. Phys. 1999, 111, 8330-8343.

(383) Werner, H. J.; Manby, F. R.; Knowles, P. J. J. Chem. Phys. 2003, 118, 8149-8160.

(384) Pisani, C.; Busso, M.; Capecchi, G.; Casassa, S.; Dovesi, R.; Maschio, L.; ZicovichWilson, C.; Schutz, M. J. Chem. Phys. 2005, 122, 094113.

10452.

(385) Sousa, S. F.; Fernandes, P. A.; Ramos, M. J. J. Phys. Chem. A 2007, 111, 10439-

(386) Musso, F. PhD Thesis, Universitat Autònoma de Barcelona and Università di Torino, 2011.

(387) Tucker, M. G.; Keen, D. A.; Dove, M. T. Mineral. Mag. 2001, 65, 489-507.

(388) Schmahl, W. W.; Swainson, I. P.; Dove, M. T.; Graeme-Barber, A. Z. Kristallogr. 1992, 201, 125-145.

(389) Kihara, K.; Matsumoto, T.; Imamura, M. Z. Kristallogr. 1986, 177, 27-38.

(390) Saxena, S. K.; Chatterjee, N.; Fei, Y.; Shen, G. Thermodynamic Data on Oxides and Silicates; Springer-Verlag: Berlin, 1993.

(391) Goumans, T. P. M.; Wander, A.; Brown, W. A.; Catlow, C. R. A. Phys. Chem. Chem. Phys. 2007, 9, 2146-2152.

(392) Zicovich-Wilson, C. M.; Pascale, F.; Roetti, C.; Saunders, V. R.; Orlando, R.; Dovesi, R. J. Comput. Chem. 2004, 25, 1873-1881.

(393) Sicolo, S.; Palma, G.; Di Valentin, C.; Pacchioni, G. Phys. Rev. B 2007, 76, 075121.

(394) Giordano, L.; Sushko, P. V.; Pacchioni, G.; Shluger, A. L. Phys. Rev. Lett. 2007, 99, 136801. 024109.

(395) Giordano, L.; Sushko, P. V.; Pacchioni, G.; Shluger, A. L. Phys. Rev. B 2007, 75,

(396) Raghavachari, K.; Ricci, D.; Pacchioni, G. J. Chem. Phys. 2002, 116, 825-831.

(397) Sodupe, M.; Bertran, J.; Rodriguez-Santiago, L.; Baerends, E. J. J. Phys. Chem. A 1999, 103, 166-170.

(398) Braida, B.; Hiberty, P. C.; Savin, A. J. Phys. Chem. A 1998, 102, 7872-7877.

(399) Gilli, G.; Gilli, P. J. Mol. Struct. 2000, 552, 1-15.

(400) Kim, K.; Jordan, K. D. J. Phys. Chem. 1994, 98, 10089-10094.

(401) Tuma, C.; Boese, A. D.; Handy, N. C. Phys. Chem. Chem. Phys. 1999, 1, 3939-3947.

(402) Novoa, J. J.; Sosa, C. J. Phys. Chem. 1995, 99, 15837-15845.

(403) Civalleri, B.; Garrone, E.; Ugliengo, P. J. Mol. Struct. THEOCHEM 1997, 419, 227 -

238.

(404) Ugliengo, P.; Civalleri, B.; Garrone, E. Nuovo Cimento D 1997, 19, 1765-1771.

(405) Thanthiriwatte, K. S.; Hohenstein, E. G.; Burns, L. A.; Sherrill, C. D. J. Chem. Theory Comput. 2011, 7, 88-96.

(406) Ireta, J.; Neugebauer, J.; Scheffler, M. J. Phys. Chem. A 2004, 108, 5692-5698.

(407) Ugliengo, P.; Pascale, F.; Merawa, M.; Labeguerie, P.; Tosoni, S.; Dovesi, R. J. Phys. Chem. B 2004, 108, 13632-13637.

(408) Hobza, P.; Sponer, J.; Reschel, T. J. Comput. Chem. 1995, 16, 1315-1325.

(409) Allen, M. J.; Tozer, D. J. J. Chem. Phys. 2002, 117, 11113-11120.

(410) Kristyan, S.; Pulay, P. Chem. Phys. Lett. 1994, 229, 175-180.

(411) Perezjorda, J. M.; Becke, A. D. Chem. Phys. Lett. 1995, 233, 134-137.

(412) Mignon, P.; Ugliengo, P.; Sodupe, M. J. Phys. Chem. C 2009, 113, 13741-13749.

(413) Mignon, P.; Ugliengo, P.; Sodupe, M.; Hernandez, E. R. Phys. Chem. Chem. Phys. 2010, 12, 688-697.

(414) Lin, I. C.; Seitsonen, A. P.; Coutinho-Neto, M. D.; Tavernelli, I.; Rothlisberger, U. J. Phys. Chem. B 2009, 113, 1127-1131. 
(415) Grossman, J. C.; Schwegles, E.; Draeger, E. W.; Gygi, F.; Galli, G. J. Chem. Phys. 2004, 120, 300-311.

(416) Sit, P. H. L.; Marzari, N. J. Chem. Phys. 2005, 122, 204510.

(417) Todorova, T.; Seitsonen, A. P.; Hutter, J.; Kuo, I. F. W.; Mundy, C. J. J. Phys. Chem. B 2006, 110, 3685-3691.

(418) Elstner, M.; Hobza, P.; Frauenheim, T.; Suhai, S.; Kaxiras, E. J. Chem. Phys. 2001, $114,5149-5155$.

(419) Grimme, S. J. Comput. Chem. 2004, 25, 1463-1473.

(420) Grimme, S. J. Comput. Chem. 2006, 27, 1787-1799.

(421) Grimme, S.; Antony, J.; Ehrlich, S.; Krieg, H. J. Chem. Phys. 2010, 132, 154104.

(422) Civalleri, B.; Zicovich-Wilson, C. M.; Valenzano, L.; Ugliengo, P. CrystEngComm 2008, 10, 405-410.

(423) Jurecka, P.; Cerny, J.; Hobza, P.; Salahub, D. R. J. Comput. Chem. 2007, 28, 555569.

(424) Kabelac, M.; Valdes, H.; Sherer, E. C.; Cramer, C. J.; Hobza, P. Phys. Chem. Chem. Phys. 2007, 9, 5000-5008.

(425) Morgado, C.; Vincent, M. A.; Hillier, I. H.; Shan, X. Phys. Chem. Chem. Phys. 2007, $9,448-451$.

(426) Rimola, A.; Civalleri, B.; Ugliengo, P. Phys. Chem. Chem. Phys. 2010, 12, 63576366.

(427) Tkatchenko, A.; Scheffler, M. Phys. Rev. Lett. 2009, 102, 073005.

(428) von Lilienfeld, O. A.; Tavernelli, I.; Rothlisberger, U.; Sebastiani, D. Phys. Rev. Lett. 2004, 93, 153004.

(429) von Lilienfeld, O. A.; Tavernelli, I.; Rothlisberger, U.; Sebastiani, D. Phys. Rev. B 2005, 71, 195119.

(430) Torres, E.; DiLabio, G. A. J. Phys. Chem. Lett. 2012, 3, 1738-1744.

(431) Dion, M.; Rydberg, H.; Schroder, E.; Langreth, D. C.; Lundqvist, B. I. Phys. Rev. Lett. 2004, 92, 246401.

(432) Zhao, Y.; Truhlar, D. G. J. Chem. Theory Comput. 2007, 3, 289-300.

(433) Zhao, Y.; Truhlar, D. G. Acc. Chem. Res. 2008, 41, 157-167.

(434) Boys, S.; Bernardi, F. Mol. Phys. 1970, 19, 553-566.

(435) Goltl, F.; Hafner, J. J. Chem. Phys. 2012, 136.

(436) Civalleri, B.; Zicovich-Wilson, C. M.; Ugliengo, P.; Saunders, V. R.; Dovesi, R. Chem. Phys. Lett. 1998, 292, 394-402. 154102 .

(437) Tosoni, S.; Tuma, C.; Sauer, J.; Civalleri, B.; Ugliengo, P. J. Chem. Phys. 2007, 127,

(438) Paier, J.; Hirschl, R.; Marsman, M.; Kresse, G. J. Chem. Phys. 2005, 122, 234102.

(439) Leach, A. R. Molecular Modelling. Principles and Applications; 2 ed.; Pearson Education Limited: Dorchester (UK), 2001.

(440) Alder, B. J.; Wainwright, T. E. J. Chem. Phys. 1959, 31, 459-466.

(441) Rahman, A. Phys. Rev. 1964, 136, A405-A411.

(442) Car, R.; Parrinello, M. Phys. Rev. Lett. 1985, 55, 2471-2474.

(443) Marx, D.; Hutter, J. Ab initio Molecular Dynamics: Basic Theory and Advanced Methods; Cambridge University Press: Cambridge, 2009.

(444) Pople, J. A.; Santry, D. P.; Segal, G. A. J. Chem. Phys. 1965, 43, S129-S135.

(445) Dewar, M. J. S.; Thiel, W. J. Am. Chem. Soc. 1977, 99, 4899-4907.

(446) Stewart, J. P.; MOPAC2012, Ed.; http://OpenMOPAC.net: Colorado Springs (CO) USA, 2012.

(447) Korth, M.; Pitonák, M.; Rezác, J. J. Chem. Theory Comp. 2010, 6, 344-352.

(448) Řezáč, J.; Hobza, P. J. Chem. Theory Comput. 2011, 8, 141-151.

(449) Slater, J. C.; Koster, G. F. Phys. Rev. 1954, 94, 1498-1524.

(450) Elstner, M.; Frauenheim, T.; Kaxiras, E.; Seifert, G.; Suhai, S. Phys. Status Solidi B 2000, 217, 357-376. 
(451) Elstner, M.; Frauenheim, T.; Suhai, S. J. Mol. Struct. THEOCHEM 2003, 632, 29-41.

(452) Elstner, M.; Porezag, D.; Jungnickel, G.; Elsner, J.; Haugk, M.; Frauenheim, T.; Suhai, S.; Seifert, G. Phys. Rev. B 1998, 58, 7260-7268.

(453) Frauenheim, T.; Seifert, G.; Elsterner, M.; Hajnal, Z.; Jungnickel, G.; Porezag, D.; Suhai, S.; Scholz, R. Phys. Status Solidi B 2000, 217, 41-62.

(454) Thomas, F.; Gotthard, S.; Marcus, E.; Thomas, N.; Christof, K.; Marc, A.; Michael,

S.; Zoltán, H.; Aldo Di, C.; Sándor, S. J. Phys.: Condens. Matter 2002, 14, 3015-3047.

(455) Elstner, M. Theor. Chem. Acc. 2006, 116, 316-325.

(456) Allinger, N. L. J. Am. Chem. Soc. 1977, 99, 8127-8134.

(457) van Beest, B. W. H.; Kramer, G. J.; van Santen, R. A. Phys. Rev. Lett. 1990, 64, 1955-1958.

(458) Dick, B. G.; Overhauser, A. W. Phys. Rev. 1958, 112, 90-103.

(459) Jackson, R. A.; Catlow, C. R. A. Mol. Simul. 1988, 1, 207-224.

(460) Gale, J. D. Z. Kristallogr. 2005, 220, 552-554.

(461) Schaible, M. Crit. Rev. Solid State Mater. Sci. 1999, 24, 265-323.

(462) Hassanali, A. A.; Singer, S. J. J. Phys. Chem. B 2007, 111, 11181-11193.

(463) Hassanali, A. A.; Singer, S. J. J. Comput. Aided Mater. Des. 2007, 14, 53-63.

(464) Garofalini, S. H. J. Non-Cryst. Solids 1990, 120, 1-12.

(465) Mahadevan, T. S.; Garofalini, S. H. J. Phys. Chem. C 2008, 112, 1507-1515.

(466) Du, J.; Cormack, A. N. J. Am. Ceram. Soc. 2005, 88, 2532-2539.

(467) Pedone, A.; Malavasi, G.; Menziani, M. C.; Segre, U.; Musso, F.; Corno, M.;

Civalleri, B.; Ugliengo, P. Chem. Mater. 2008, 20, 2522-2531.

(468) van Duin, A. C. T.; Dasgupta, S.; Lorant, F.; Goddard, W. A. J. Phys. Chem. A 2001, 105, 9396-9409.

(469) Pauling, L. J. Am. Chem. Soc. 1947, 69, 542-553.

(470) van Duin, A. C. T.; Strachan, A.; Stewman, S.; Zhang, Q. S.; Xu, X.; Goddard, W. A. J. Phys. Chem. A 2003, 107, 3803-3811.

(471) Fogarty, J. C.; Aktulga, H. M.; Grama, A. Y.; van Duin, A. C. T.; Pandit, S. A. J. Chem. Phys. 2010, 132, 174704.

(472) Feuston, B. P.; Garofalini, S. H. J. Chem. Phys. 1988, 89, 5818-5824.

(473) Tsuneyuki, S.; Tsukada, M.; Aoki, H.; Matsui, Y. Phys. Rev. Lett. 1988, 61, 869-872.

(474) Schröder, K.-P.; Sauer, J.; Leslie, M.; Richard, C.; Catlow, A.; Thomas, J. M. Chem. Phys. Lett. 1992, 188, 320-325.

(475) Hill, J. R.; Sauer, J. J. Phys. Chem. 1994, 98, 1238-1244.

(476) Schroder, K. P.; Sauer, J. J. Phys. Chem. 1996, 100, 11043-11049.

(477) Sierka, M.; Sauer, J. Faraday Discuss. 1997, 106, 41-62.

(478) Guillot, B.; Guissani, Y. J. Chem. Phys. 2001, 114, 6720-6733.

(479) Skuja, L. N.; Silin, A. R. Phys. Status Solidi A 1979, 56, K11-K13.

(480) Griscom, D. L. J. Non-Cryst. Solids 1985, 73, 51-77.

(481) Skuja, L. J. Non-Cryst. Solids 1994, 179, 51-69.

(482) Skuja, L.; Suzuki, T.; Tanimura, K. Phys. Rev. B 1995, 52, 15208-15216.

(483) Skuja, L.; Tanimura, T.; Itoh, N. J. Appl. Phys. 1996, 80, 3518-3525.

(484) Skuja, L.; Naber, A. In Proceedings of the 13th International Conference on Defects in Insulating Materials - Icdim 96; Matthews, G. E., Williams, R. T., Eds. 1997; Vol. 239-, p 25-28.

(485) Vaccaro, L.; Cannas, M.; Radzig, V.; Boscaino, R. Phys. Rev. B 2008, 78, 075421.

(486) Vaccaro, L.; Cannas, M.; Radzig, V. J. Non-Cryst. Solids 2009, 355, 1020-1023.

(487) Zyubin, A. S.; Mebel, A. M.; Lin, S. H.; Glinka, Y. D. J. Chem. Phys. 2002, 116, 9889-9896.

(488) Griscom, D. L. J. Non-Cryst. Solids 1978, 31, 241-266.

(489) Stapelbroek, M.; Griscom, D. L.; Friebele, E. J.; Sigel, G. H. J. Non-Cryst. Solids 1979, 32, 313-326.

(490) Griscom, D. L.; Friebele, E. J. Phys. Rev. B 1981, 24, 4896-4898.

(491) Griscom, D. L. J. Non-Cryst. Solids 2011, 357, 1945-1962. 
(492) Glinka, Y. D.; Lin, S. H.; Chen, Y. T. Phys. Rev. B 2002, 66, 035404.

(493) Raghavachari, K.; Pacchioni, G. J. Chem. Phys. 2001, 114, 4657-4662.

(494) Murashov, V. V.; Demchuk, E. Surf. Sci. 2005, 595, 6-19.

(495) Murashov, V. V.; Demchuk, E. J. Phys. Chem. B 2005, 109, 10835-10841.

(496) Koudriachova, M. V.; Beckers, J. V. L.; de Leeuw, S. W. Comput. Mater. Sci. 2001, 20, 381-386.

(497) Rignanese, G. M.; De Vita, A.; Charlier, J. C.; Gonze, X.; Car, R. Phys. Rev. B 2000, $61,13250-13255$.

(498) Lopes, P. E. M.; Demchuk, E.; Mackerell, A. D., Jr. Int. J. Quantum Chem. 2009, $109,50-64$.

(499) de Leeuw, N. H.; Higgins, F. M.; Parker, S. C. J. Phys. Chem. B 1999, 103, 12701277.

(500) Rignanese, G. M.; Charlier, J. C.; Gonze, X. Phys. Chem. Chem. Phys. 2004, 6, 1920-1925.

(501) Adeagbo, W. A.; Doltsinis, N. L.; Klevakina, K.; Renner, J. ChemPhysChem 2008, 9, 994-1002.

(502) Walsh, T. R.; Wilson, M.; Sutton, A. P. J. Chem. Phys. 2000, 113, 9191-9201.

(503) Tosoni, S.; Civalleri, B.; Ugliengo, P. J. Phys. Chem. C 2010, 114, 19984-19992.

(504) Yang, J. J.; Wang, E. G. Phys. Rev. B 2006, 73, 035406.

(505) Zyubin, A. S.; Glinka, Y. D.; Mebel, A. M.; Lin, S. H.; Hwang, L. P.; Chen, Y. T. J. Chem. Phys. 2002, 116, 281-294.

(506) Ceresoli, D.; Bernasconi, M.; Iarlori, S.; Parrinello, M.; Tosatti, E. Phys. Rev. Lett. 2000, 84, 3887-3890.

(507) Masini, P.; Bernasconi, M. J. Phys.: Condens. Matter 2002, 14, 4133-4144.

(508) Mishler, C.; Horbach, J.; Kob, W.; Binder, K. J. Phys.: Condens. Matter 2005, 17, 4005-4013.

(509) Ugliengo, P.; Sodupe, M.; Musso, F.; Bush, I. J.; Orlando, R.; Dovesi, R. Adv. Mater. 2008, 20, 4579-4583.

(510) Tielens, F.; Gervais, C.; Lambert, J. F.; Mauri, F.; Costa, D. Chem. Mater. 2008, 20, 3336-3344.

(511) Pickard, C. J.; Mauri, F. Phys. Rev. B 2001, 63, 245101.

(512) Thomas, J. M.; Klinowski, J.; Ramdas, S.; Hunter, B. K.; Tennakoon, D. T. B. Chem. Phys. Lett. 1983, 102, 158-162.

(513) Engelhardt, G.; Radeglia, R. Chem. Phys. Lett. 1984, 108, 271-274.

(514) Pedone, A.; Malavasi, G.; Menziani, M. C.; Cormack, A. N.; Segre, U. J. Phys. Chem. B 2006, 110, 11780-11795.

(515) Ugliengo, P.; Viterbo, D.; Chiari, G. Z. Kristallogr. 1993, 207, 9-23.

(516) Dovesi, R.; Saunders, V. R.; Roetti, C.; Orlando, R.; Zicovich-Wilson, C. M.; Pascale, F.; Civalleri, B.; Doll, K.; Harrison, N. M.; Bush, I. J.; D'Arco, P.; Llunell, M.; Università di Torino: Torino, 2006.

(517) Delle Piane, M.; Corno, M.; Ugliengo, P. In Satellite Meeting of the World Association of Theoretical and Computational Chemists, WATOC 2011: Theoretical Modeling of Materials Barcelona, 13-15 July 2011.

(518) Feuston, B. P.; Garofalini, S. H. J. Chem. Phys. 1989, 91, 564-570.

(519) Berendsen, H. J. C.; Postma, J. P. M.; van Gunsteren, W. F.; Hermans, J. In Intermolecular Forces; Pullmann, B., Ed. 1981, p 331.

(520) Bakaev, V. A.; Steele, W. A. J. Chem. Phys. 1999, 111, 9803-9812.

(521) Roder, A.; Kob, W.; Binder, K. J. Chem. Phys. 2001, 114, 7602-7614.

(522) Mischler, C.; Kob, W.; Binder, K. Comput. Phys. Commun. 2002, 147, 222-225.

(523) Okuno, M.; Kawamura, K. J. Non-Cryst. Solids 1995, 191, 249-259.

(524) Leed, E. A.; Pantano, C. G. J. Non-Cryst. Solids 2003, 325, 48-60.

(525) Mischler, C.; Horbach, J.; Kob, W.; Binder, K. J. Phys.: Condens. Matter 2005, 17, 4005-4013. 
(526) Cerius2 v.4.9 2003, Accelrys, Inc.

(527) Brooks, B. R.; III, C. L. B.; Jr, A. D. M.; Nilsson, L.; Petrella, R. J.; Roux, B.; Won, Y.; Archontis, G.; Bartels, C.; Boresch, S.; Caflisch, A.; Caves, L.; Cui, Q.; Dinner, A. R.; Feig, M.; Fischer, S.; Gao, J.; Hodoscek, M.; Im, W.; Kuczera, K.; Lazaridis, T.; Ma, J.; Ovchinnikov, V.; Paci, E.; Pastor, R. W.; Post, C. B.; Pu, J. Z.; Schaefer, M.; Tidor, B.; Venable, R. M.; Woodcock, H. L.; Wu, X.; Yang, W.; York, D. M.; Karplus, M. J. Comput. Chem. 2009, 30, 1545-1614.

(528) Cruz-Chu, E. R.; Aksimentiev, A.; Schulten, K. J. Phys. Chem. B 2006, 110, $21497-$ 21508.

(529) Berendsen, H. J. C.; Grigera, J. R.; Straatsma, T. P. J. Phys. Chem. 1987, 91, 62696271.

(530) Stillinger, F. H.; Weber, T. A. Phys. Rev. B 1985, 31, 5262-5271. 12209.

(531) Vashishta, P.; Kalia, R. K.; Rino, J. P.; Ebbsjö, I. Phys. Rev. B 1990, 41, $12197-$

(532) Lerbret, A.; Lelong, G.; Mason, P. E.; Saboungi, M. L.; Brady, J. W. Food Biophysics 2011, 6, 233-240.

(533) Rustad, J. R.; Hay, B. P. Geochim. Cosmochim. Acta 1995, 59, 1251-1257.

(534) Bourg, I. C.; Steefel, C. I. J. Phys. Chem. C 2012, 116, 11556-11564.

(535) Morrow, B. A.; Cody, I. A. J. Phys. Chem. 1975, 79, 761-762.

(536) Morrow, B. A.; Cody, I. A. J. Phys. Chem. 1976, 80, 1998-2004.

(537) Ferrari, A. M.; Garrone, E.; Spoto, G.; Ugliengo, P.; Zecchina, A. Surf. Sci. 1995, $323,151-162$.

(538) Woodcock, L. V.; Angell, C. A.; Cheeseman, P. J. Chem. Phys. 1976, 65, 15651578.

(539) Wilson, M.; Walsh, T. R. J. Chem. Phys. 2000, 113, 9180-9190.

(540) Du, M.-H.; Kolchin, A.; Cheng, H.-P. J. Chem. Phys. 2004, 120, 1044-1054.

(541) Rimola, A.; Ugliengo, P. Phys. Chem. Chem. Phys. 2009, 11, 2497-2506.

(542) Wander, M. C. F.; Clark, A. E. J. Phys. Chem. C 2008, 112, 19986-19994.

(543) Ostroverkhov, V.; Waychunas, G. A.; Shen, Y. R. Chem. Phys. Lett. 2004, 386, 144148.

(544) Engemann, S.; Reichert, H.; Dosch, H.; Bilgram, J.; Honkimaki, V.; Snigirev, A. Phys. Rev. Lett. 2004, 92, 205701.

(545) Pelmenschikov, A.; Leszczynski, J.; Pettersson, L. G. M. J. Phys. Chem. A 2001, $105,9528-9532$.

(546) Criscenti, L. J.; Kubicki, J. D.; Brantley, S. L. J. Phys. Chem. A 2006, 110, 198-206.

(547) Gautier, J. M.; Oelkers, E. H.; Schott, J. Geochim. Cosmochim. Acta 2001, 65, 10591070.

(548) Nangia, S.; Garrison, B. J. J. Phys. Chem. A 2008, 112, 2027-2033.

(549) Nangia, S.; Garrison, B. J. Mol. Phys. 2009, 107, 831-843.

(550) Gratz, A. J.; Bird, P. Geochim. Cosmochim. Acta 1993, 57, 977-989.

(551) Castagliola, M.; Tellier, C. R.; Vaterkowski, J. L. J. Mater. Sci. 1986, 21, 3551-3560.

(552) Zeitler, T. R.; Cormack, A. N. J. Cryst. Growth 2006, 294, 96-102.

(553) Sjoberg, S. J. Non-Cryst. Solids 1996, 196, 51-57.

(554) Yang, J. J.; Wang, E. G. Curr. Opin. Solid State Mater. Sci. 2006, 10, 33-39.

(555) Sauer, J.; Schroder, K. P. Z. Phys. Chem. (Leipzig) 1985, 266, 379-387.

(556) Costa, D.; Lomenech, C.; Meng, M.; Stievano, L.; Lambert, J. F. J. Mol. Struct. THEOCHEM 2007, 806, 253-259.

(557) Chashchikhin, V.; Rykova, E.; Bagaturyants, A. Phys. Chem. Chem. Phys. 2011, 13, $1440-1447$.

(558) Fubini, B.; Bolis, V.; Bailes, M.; Stone, F. S. Solid State Ionics 1989, 32-3, 258-272.

(559) Puibasset, J.; Pellenq, R. J. M. Phys. Chem. Chem. Phys. 2004, 6, 1933-1937.

(560) Fubini, B.; Bolis, V.; Cavenago, A.; Ugliengo, P. J. Chem. Soc., Faraday Trans. 1992, 88, 277-290. 
(561) Douillard, J. M.; Elwafir, M.; Partyka, S. J. Colloid Interface Sci. 1994, 164, 238244.

(562) Fubini, B. Thermochim. Acta 1988, 135, 19-29.

(563) Klier, K.; Shen, J. H.; Zettlemoyer, A. C. J. Phys. Chem. 1973, 77, 1458-1465.

(564) Du, Q.; Freysz, E.; Shen, Y. R. Phys. Rev. Lett. 1994, 72, 238-241.

(565) Garrone, E.; Kazansky, V. B.; Kustov, L. M.; Sauer, J.; Senchenya, I. N.; Ugliengo, P. J. Phys. Chem. 1992, 96, 1040-1045.

(566) Ferrari, A. M.; Ugliengo, P.; Garrone, E. J. Phys. Chem. 1993, 97, 2671-2676.

(567) Murashov, V.; Harper, M.; Demchuk, E. J. Occup. Environ. Hyg. 2006, 3, 718-723.

(568) Thompson, K. C.; Margey, P. Phys. Chem. Chem. Phys. 2003, 5, 2970-2975.

(569) Cypryk, M. J. Organomet. Chem. 1997, 545, 483-493.

(570) Pelmenschikov, A. G.; Morosi, G.; Gamba, A. J. Phys. Chem. A 1997, 101, 11781187.

(571) Ignatyev, I. S.; Partal, F.; Gonzalez, J. J. L.; Sundius, T. Spectrochim. Acta, Part A 2004, 60, 1169-1178.

(572) Pelmenschikov, A. G.; Morosi, G.; Gamba, A. J. Phys. Chem. 1992, 96, 7422-7424.

(573) Yang, J. J.; Meng, S.; Xu, L. F.; Wang, E. G. Phys. Rev. B 2005, 71, 035413.

(574) Musso, F.; Ugliengo, P.; Sodupe, M. J. Phys. Chem. A 2011, 115, 11221-11228.

(575) Bandura, A. V.; Kubicki, J. D.; Sofo, J. O. J. Phys. Chem. C 2011, 115, 5756-5766.

(576) Chen, Y. W.; Chu, I. H.; Wang, Y.; Cheng, H. P. Phys. Rev. B 2011, 84, 155444.

(577) Casarin, M.; Falcomer, D.; Glisenti, A.; Natile, M. M.; Poli, F.; Vittadini, A. Chem. Phys. Lett. 2005, 405, 459-464.

(578) Takeuchi, M.; Bertinetti, L.; Martra, G.; Coluccia, S.; Anpo, M. Appl. Catal., A 2006, $307,13-20$.

(579) Gallas, J.-P.; Goupil, J.-M.; Vimont, A.; Lavalley, J.-C.; Gil, B.; Gilson, J.-P.; Miserque, O. Langmuir 2009, 25, 5825-5834.

(580) Lutz, W.; Weber, M.; Bertram, R.; Kurzhals, R.; Kryukova, G. Z. Anorg. Allg. Chem. 2011, 637, 421-425.

(581) Behrens, H.; Stuke, A. Glass Sci. Technol. 2003, 76, 176-189.

(582) Sulpizi, M.; Gaigeot, M.-P.; Sprik, M. J. Chem. Theory Comput. 2012, 8, 1037-1047.

(583) Fernandez-Cata, G.; Perez-Gramatges, A.; Alvarez, L. J.; Comas-Rojas, H.; Zicovich-Wilson, C. M. J. Phys. Chem. C 2009, 113, 13309-13316.

18365.

(584) Leung, K.; Nielsen, I. M. B.; Criscenti, L. J. J. Am. Chem. Soc. 2009, 131, 18358-

(585) Gaigeot, M.-P.; Sprik, M.; Sulpizi, M. J. Phys.: Condens. Matter 2012, 24, 124106.

(586) Zhao, Y. L. J. Theor. Comput. Chem. 2012, 11, 155-162.

(587) Skelton, A. A.; Fenter, P.; Kubicki, J. D.; Wesolowski, D. J.; Cummings, P. T. J. Phys. Chem. C 2011, 115, 2076-2088.

(588) Skelton, A. A.; Wesolowski, D. J.; Cummings, P. T. Langmuir 2011, 27, 8700-8709.

(589) Du, Z. M.; de Leeuw, N. H. Surf. Sci. 2004, 554, 193-210.

(590) Du, Z. M.; de Leeuw, N. H. Dalton Trans. 2006, 2623-2634.

(591) Notman, R.; Walsh, T. R. Langmuir 2009, 25, 1638-1644.

(592) Argyris, D.; Cole, D. R.; Striolo, A. J. Phys. Chem. C 2009, 113, 19591-19600.

(593) Lopes, P. E. M.; Murashov, V.; Tazi, M.; Demchuk, E.; MacKerell, A. D. J. Phys.

Chem. B 2006, 110, 2782-2792.

(594) Nangia, S.; Washton, N. M.; Mueller, K. T.; Kubicki, J. D.; Garrison, B. J. J. Phys.

Chem. C 2007, 111, 5169-5177.

(595) Wright, L. B.; Walsh, T. R. J. Phys. Chem. C 2012, 116, 2933-2945.

(596) Zeitler, T. R.; Greathouse, J. A.; Cygan, R. T. Phys. Chem. Chem. Phys. 2012, 14, 1728-1734.

(597) Xuefen, Z.; Guiwu, L.; Xiaoming, W.; Hong, Y. Appl. Surf. Sci. 2009, 255, 64936498. 

15973.

(598) Phan, A.; Ho, T. A.; Cole, D. R.; Striolo, A. J. Phys. Chem. C 2012, 116, $15962-$

(599) Greathouse, J. A.; O'Brien, R. J.; Bemis, G.; Pabalan, R. T. J. Phys. Chem. B 2002, $106,1646-1655$.

(600) Lu, Z. Y.; Sun, Z. Y.; Li, Z. S.; An, L. J. J. Phys. Chem. B 2005, 109, 5678-5683.

(601) Tosoni, S.; Pascale, F.; Ugliengo, P.; Orlando, R.; Saunders, V. R.; Dovesi, R. Mol. Phys. 2005, 103, 2549-2558.

(602) Schlegel, M. L.; Nagy, K. L.; Fenter, P.; Sturchio, N. C. Geochim. Cosmochim. Acta 2002, 66, 3037-3054.

(603) Etzler, F. M.; Fagundus, D. M. J. Colloid Interface Sci. 1987, 115, 513-519.

(604) Takei, T.; Chikazawa, M. J. Colloid Interface Sci. 1998, 208, 570-574.

(605) Takei, T.; Eriguchi, E.; Fuji, M.; Watanabe, T.; Chikazawa, M. Thermochim. Acta 1998, 308, 139-145.

(606) Fubini, B.; Zanetti, G.; Altilia, S.; Tiozzo, R.; Lison, D.; Saffiotti, U. Chem. Res. Toxicol. 1999, 12, 737-745.

(607) Heinz, H.; Farmer, B. L.; Pandey, R. B.; Slocik, J. M.; Patnaik, S. S.; Pachter, R.; Naik, R. R. J. Am. Chem. Soc. 2009, 131, 9704-9714.

(608) Perdew, J. P.; Burke, K.; Ernzerhof, M. Phys. Rev. Lett. 1998, 80, 891-891.

(609) Fuerstenau, D. W.; Pradip Adv. Colloid Interface Sci. 2005, 114, 9-26.

(610) Ahmed, S. M.; Vancleav.Ab Can. J. Chem. Eng. 1965, 43, 23-26.

(611) Li, H. C.; Debruyn, P. L. Surf. Sci. 1966, 5, 203-220.

(612) Pokrovsky, O. S.; Golubev, S. V.; Mielczarski, J. A. J. Colloid Interface Sci. 2006, 296, 189-194.

(613) Eisenthal, K. B. Chem. Rev. 2006, 106, 1462-1477.

(614) Shen, Y. R.; Ostroverkhov, V. Chem. Rev. 2006, 106, 1140-1154.

(615) Allen, L. H.; Matijevi.E; Meties, L. J. Inorg. Nucl. Chem. 1971, 33, 1293-1299.

(616) Gurau, M. C.; Kim, G.; Lim, S. M.; Albertorio, F.; Fleisher, H. C.; Cremer, P. S. ChemPhysChem 2003, 4, 1231-1233.

(617) Bertie, J. E.; Labbe, H. J.; Whally, E. J. Chem. Phys. 1969, 50, 4501-4520.

(618) Scherer, J. R.; Snyder, R. G. J. Chem. Phys. 1977, 67, 4794-4811. 061202.

(619) Lorenz, C. D.; Travesset, A. Phys. Rev. E: Stat. Phys., Plasmas, Fluids, 2007, 75,

(620) Skelton, A. A.; Kubicki, J. D.; Fenter, P.; Van Duin, A. C. T.; Wesolowski, D. J.; Cummings, P. T. Geochim. Cosmochim. Acta 2010, 74, A969-A969.

(621) Argyris, D.; Cole, D. R.; Striolo, A. Langmuir 2009, 25, 8025-8035.

(622) Cygan, R. T.; Liang, J. J.; Kalinichev, A. G. J. Phys. Chem. B 2004, 108, 1255-1266.

(623) Musso, F.; Mignon, P.; Ugliengo, P.; Sodupe, M. Phys. Chem. Chem. Phys. 2012, 14, 10507-10514.

(624) Kaya, S.; Weissenrieder, J.; Stacchiola, D.; Shaikhutdinov, S.; Freund, H. J. J. Phys. Chem. C 2007, 111, 759-764.

(625) Du, M. H.; Kolchin, A.; Cheng, H. P. J. Chem. Phys. 2003, 119, 6418-6422.

(626) Chen, Y. W.; Cheng, H. P. J. Chem. Phys. 2011, 134, 114703.

(627) Yates, J. T. Surf. Sci. 2004, 565, 103-106.

(628) Branda, M. M.; Montani, R. A.; Castellani, N. J. Surf. Sci. 2000, 446, L89-L94.

(629) Shchukarev, A.; Sjoberg, S. Surf. Sci. 2005, 584, 106-112.

(630) Lee, C. Y.; McCammon, J. A.; Rossky, P. J. J. Chem. Phys. 1984, 80, 4448-4455.

(631) Lee, S. H.; Rossky, P. J. J. Chem. Phys. 1994, 100, 3334-3345.

(632) Collins, D. R.; Catlow, C. R. A. Am. Mineral. 1992, 77, 1172-1181.

(633) deLeeuw, N. H.; Watson, G. W.; Parker, S. C. J. Chem. Soc.-Faraday Trans. 1996, 92, 2081-2091.

(634) Warne, M. R.; Allan, N. L.; Cosgrove, T. Phys. Chem. Chem. Phys. 2000, 2, 36633668 . 
(635) Hassanali, A. A.; Zhang, H.; Knight, C.; Shin, Y. K.; Singer, S. J. J. Chem. Theory Comput. 2010, 6, 3456-3471.

(636) Leed, E. A.; Sofo, J. O.; Pantano, C. G. Phys. Rev. B 2005, 72, 155427.

(637) Floess, J. K.; Murad, S. Chem. Phys. Lett. 2011, 516, 216-219.

(638) Chai, J. C.; Liu, S. Y.; Yang, X. N. Appl. Surf. Sci. 2009, 255, 9078-9084.

(639) Feuston, B. P.; Garofalini, S. H. J. Appl. Phys. 1990, 68, 4830-4836.

(640) Minibaev, R. F.; Zhuravlev, N. A.; Bagatur'yantz, A. A.; Alfimov, M. V. Russ. Phys. J. 2009, 52, 1164-1169.

(641) Costa, D.; Tougerti, A.; Tielens, F.; Gervais, C.; Stievano, L.; Lambert, J. F. Phys. Chem. Chem. Phys. 2008, 10, 6360-6368.

(642) Duchateau, R.; Dijkstra, T. W.; van Santen, R. A.; Yap, G. P. A. Chem. --Eur. J. 2004, 10, 3979-3990.

(643) Wächtershäuser, G. Proc. Natl. Acad. Sci. USA 1990, 87, 200-204.

260-264.

(644) Linder, R.; Nispel, M.; Häber, T.; Kleinermanns, K. Chem. Phys. Lett. 2005, 409,

(645) Linder, R.; Seefeld, K.; Vavra, A.; Kleinermanns, K. Chem. Phys. Lett. 2008, 453, 1-

6.

(646) Groenewegen, J. A.; Sachtler, W. M. H. J. Catal. 1974, 33, 176-183.

(647) Basiuk, V. A.; Gromovoy, T. Y.; Golovaty, V. G.; Glukhoy, A. M. Origins Life Evol. Biospheres 1990, 20, 483-498.

(648) Stepanian, S. G.; Reva, I. D.; Radchenko, E. D.; Rosado, M. T. S.; Duarte, M. L. T.

S.; Fausto, R.; Adamowicz, L. J. Phys. Chem. A 1998, 102, 1041-1054.

(649) Marsh, R. E. Acta Cryst. 1958, 11, 654-663.

(650) Chowdhry, B. Z.; Dines, T. J.; Jabeen, S.; Withnall, R. J. Phys. Chem. A 2008, 112, 10333-10347.

(651) Han, J. W.; Sholl, D. S. Langmuir 2009, 25, 10737-10745.

(652) Lambert, J.-F.; Stievano, L.; Lopes, I.; Gharsallah, M.; Piao, L. Planet. Space Sci. 2009, 57, 460-467.

(653) Garetz, B. A.; Matic, J.; Myerson, A. S. Phys. Rev. Lett. 2002, 89, 175501.

(654) Löfgren, P.; Krozer, A.; Lausmaa, J.; Kasemo, B. Surf. Sci. 1997, 370, 277-292.

(655) Löfgren, P.; Krozer, A.; Chakarov, D. V.; Kasemo, B. J. Vac. Sci. Technol., A 1998, 16, 2961-2966.

(656) Ramaekers, R.; Pajak, J.; Lambie, B.; Maes, G. J. Chem. Phys. 2004, 120, 41824193. 543-548.

(657) Soria, E.; Román, E.; Williams, E. M.; de Segovia, J. L. Surf. Sci. 1999, 433-435,

(658) Charlet, L.; Manceau, A. A. J. Colloid Interface Sci. 1992, 148, 443-458.

(659) Han, J. W.; James, J. N.; Sholl, D. S. Surf. Sci. 2008, 602, 2478-2485.

(660) Gambino, G. L.; Lombardo, G. M.; Grassi, A.; Marletta, G. J. Phys. Chem. B 2004, $108,2600-2607$.

(661) Han, J. W.; Sholl, D. S. Phys. Chem. Chem. Phys. 2010, 12, 8024-8032.

(662) Greber, T.; Šljivančanin, Ž.; Schillinger, R.; Wider, J.; Hammer, B. Phys. Rev. Lett. 2006, 96, 056103.

(663) Bhatia, B.; Sholl, D. S. Angew. Chem. Int. Ed. 2005, 44, 7761-7764.

(664) Basiuk, V. A.; Navarro-Gonzalez, R.; Basiuk, E. V. Origins Life Evol. Biosphere 1998, 28, 167-193.

(665) Basiuk, V. A.; Navarro-Gonzalez, R. J. Chromatogr. A 1997, 776, 255-273.

(666) Basiuk, V. A.; Navarro-Gonzales, R.; Basiuk, E. V. Bioorg. Khim 1998, 24, 842-847.

(667) Basiuk, V. A. Origins Life Evol. Biosphere 1992, 22, 333-348.

(668) Basiuk, V. A.; Gromovoy, T. Y. Collect. Czech. Chem. Commun. 1994, 59, 461-466.

(669) Hudson, S.; Cooney, J.; Magner, E. Angew. Chem. Int. Ed. 2008, 47, 8582-8594.

(670) Stöber, W.; Fink, A.; Bohn, E. J. Colloid Interface Sci. 1968, 26, 62-69.

(671) Roach, P.; Farrar, D.; Perry, C. C. J. Am. Chem. Soc. 2006, 128, 3939-3945. 
(672) Lundqvist, M.; Nygren, P.; Jonsson, B.-H.; Broo, K. Angew. Chem. Int. Ed. 2006, 45, 8169-8173.

(673) Nygren, P.; Lundqvist, M.; Broo, K.; Jonsson, B.-H. Nano Lett. 2008, 8, 1844-1852.

(674) Manyar, H. G.; Gianotti, E.; Sakamoto, Y.; Terasaki, O.; Coluccia, S.; Tumbiolo, S. J. Phys. Chem. C 2008, 112, 18110-18116.

(675) Giussani, L.; Tabacchi, G.; Gianotti, E.; Coluccia, S.; Fois, E. Philos. Trans. R. Soc., A 2012, 370, 1463-1477.

(676) Notman, R.; Oren, E. E.; Tamerler, C.; Sarikaya, M.; Samudrala, R.; Walsh, T. R. Biomacromolecules 2010, 11, 3266-3274.

(677) Phillips, D. C.; York, R. L.; Mermut, O.; McCrea, K. R.; Ward, R. S.; Somorjai, G. A. J. Phys. Chem. C 2006, 111, 255-261.

(678) Fodor, S. P. A.; Rava, R. P.; Huang, X. C.; Pease, A. C.; Holmes, C. P.; Adams, C. L. Nature 1993, 364, 555-556.

(679) Pease, A. C.; Solas, D.; Sullivan, E. J.; Cronin, M. T.; Holmes, C. P.; Fodor, S. P. Proc. Natl. Acad. Sci. USA 1994, 91, 5022-5026.

(680) Schena, M.; Shalon, D.; Heller, R.; Chai, A.; Brown, P. O.; Davis, R. W. Proc. Natl. Acad. Sci. USA 1996, 93, 10614-10619.

(681) Cheng, L.; Ziegelhoffer, P. R.; Yang, N. S. Proc. Natl. Acad. Sci. USA 1993, 90, 4455-4459.

(682) Rädler, J. O.; Koltover, I.; Salditt, T.; Safinya, C. R. Science 1997, 275, 810-814.

(683) Sen, T.; Sebastianelli, A.; Bruce, I. J. J. Am. Chem. Soc. 2006, 128, 7130-7131.

(684) Mannelli, I.; Minunni, M.; Tombelli, S.; Wang, R.; Michela Spiriti, M.; Mascini, M. Bioelectrochemistry 2005, 66, 129-138.

(685) Ferreira, G. N. M. Chem. Eng. Technol. 2005, 28, 1285-1294.

(686) Stellwagen, N. C.; Stellwagen, E. J. Chromatogr. A 2009, 1216, 1917-1929.

(687) Hook, F.; Ray, A.; Nordén, B.; Kasemo, B. Langmuir 2001, 17, 8305-8312.

(688) Larsson, C.; Rodahl, M.; Höök, F. Anal. Chem. 2003, 75, 5080-5087.

(689) Branda, S. S.; Vik, Å.; Friedman, L.; Kolter, R. Trends Microbiol. 2005, 13, 20-26.

(690) Romanowski, G.; Lorenz, M. G.; Wackernagel, W. Appl. Environ. Microbiol. 1991, 57, 1057-1061.

(691) He, X.-X.; Wang, K.; Tan, W.; Liu, B.; Lin, X.; He, C.; Li, D.; Huang, S.; Li, J. J. Am. Chem. Soc. 2003, 125, 7168-7169.

(692) Verma, I. M.; Somia, N. Nature 1997, 389, 239-242.

(693) Solberg, S. M.; Landry, C. C. J. Phys. Chem. B 2006, 110, 15261-15268. $375-383$.

(694) Marler, B.; Oberhagemann, U.; Vortmann, S.; Gies, H. Microporous Mater. 1996, 6,

(695) Li, X.; Zhang, J.; Gu, H. Langmuir 2011, 27, 6099-6106.

(696) Li, X.; Zhang, J.; Gu, H. Langmuir 2012, 28, 2827-2834.

(697) Melzak, K. A.; Sherwood, C. S.; Turner, R. F. B.; Haynes, C. A. J. Colloid Interface Sci. 1996, 181, 635-644.

(698) Gani, S. A.; Mukherjee, D. C.; Chattoraj, D. K. Langmuir 1999, 15, 7130-7138.

(699) Nguyen, T. H.; Elimelech, M. Biomacromolecules 2006, 8, 24-32.

(700) Scholes, C. A.; Millar, D. P.; Gee, M. L.; Smith, T. A. J. Phys. Chem. B 2011, 115, 6329-6339.

(701) Muñoz, B.; Rámila, A.; Pérez-Pariente, J.; Díaz, I.; Vallet-Regí, M. Chem. Mater. 2002, 15, 500-503.

(702) Izquierdo-Barba, I.; Sousa, E.; Doadrio, J.; Doadrio, A.; Pariente, J.; Martínez, A.; Babonneau, F.; Vallet-Regí, M. J. Sol-Gel Sci. Technol. 2009, 50, 421-429.

(703) Izquierdo-Barba, I.; Martinez, Á.; Doadrio, A. L.; Pérez-Pariente, J.; Vallet-Regí, M. Eur. J. Pharm. Sci. 2005, 26, 365-373.

(704) Doadrio, A. L.; Sousa, E. M. B.; Doadrio, J. C.; Pérez Pariente, J.; Izquierdo-Barba, I.; Vallet-Regí, M. J. Controlled Release 2004, 97, 125-132. 
(705) Doadrio, J. C.; Sousa, E. M. B.; Izquierdo-Barba, I.; Doadrio, A. L.; Perez-Pariente, J.; Vallet-Regi, M. J. Mater. Chem. 2006, 16, 462-466.

(706) Balas, F.; Manzano, M.; Horcajada, P.; Vallet-Regí, M. J. Am. Chem. Soc. 2006, 128, 8116-8117.

(707) Vallet-Regí, M.; Doadrio, J. C.; Doadrio, A. L.; Izquierdo-Barba, I.; Pérez-Pariente, J. Solid State Ionics 2004, 172, 435-439.

(708) Vallet-Regí, M. Chemistry - A European Journal 2006, 12, 5934-5943.

(709) Azaïs, T.; Tourné-Péteilh, C.; Aussenac, F.; Baccile, N.; Coelho, C.; Devoisselle, J.M.; Babonneau, F. Chem. Mater. 2006, 18, 6382-6390.

(710) Azaïs, T.; Hartmeyer, G.; Quignard, S.; Laurent, G.; Babonneau, F. J. Phys. Chem. C 2010, 114, 8884-8891.

(711) Alcoutlabi, M.; McKenna, G. B. J. Phys.: Condens. Matter 2005, 17, R461-R524.

(712) Morishige, K.; Kawano, K. J. Chem. Phys. 2000, 112, 11023-11029.

(713) Morineau, D.; Guegan, R.; Xia, Y.; Alba-Simionesco, C. J. Chem. Phys. 2004, 121, 1466-1473.

(714) Takei, T.; Konishi, T.; Fuji, M.; Watanabe, T.; Chikazawa, M. Thermochim. Acta 1995, 267, 159-167.

(715) Gedat, E.; Schreiber, A.; Albrecht, J.; Emmler, T.; Shenderovich, I.; Findenegg, G.

H.; Limbach, H. H.; Buntkowsky, G. J. Phys. Chem. B 2002, 106, 1977-1984.

(716) Dosseh, G.; Xia, Y.; Alba-Simionesco, C. J. Phys. Chem. B 2003, 107, 6445-6453.

(717) Mellaerts, R.; Jammaer, J. A. G.; Van Speybroeck, M.; Chen, H.; Humbeeck, J. V.; Augustijns, P.; Van den Mooter, G.; Martens, J. A. Langmuir 2008, 24, 8651-8659.

(718) Mellaerts, R.; Roeffaers, M. B. J.; Houthoofd, K.; Van Speybroeck, M.; De Cremer, G.; Jammaer, J. A. G.; Van den Mooter, G.; Augustijns, P.; Hofkens, J.; Martens, J. A. Phys. Chem. Chem. Phys. 2011, 13, 2706-2713.

(719) Qu, F.; Zhu, G.; Huang, S.; Li, S.; Sun, J.; Zhang, D.; Qiu, S. Microporous Mesoporous Mater. 2006, 92, 1-9.

(720) Grünberg, B.; Emmler, T.; Gedat, E.; Shenderovich, I.; Findenegg, G. H.; Limbach, H.-H.; Buntkowsky, G. Chemistry - A European Journal 2004, 10, 5689-5696.

(721) Abbasi, A.; Nadimi, E.; Plänitz, P.; Radehaus, C. Surf. Sci. 2009, 603, 2502-2506.

(722) Mian, S. A.; Saha, L. C.; Jang, J.; Wang, L.; Gao, X.; Nagase, S. J. Phys. Chem. C 2010, 114, 20793-20800.

(723) Mian, S.; Gao, X.; Nagase, S.; Jang, J. Theor. Chem. Acc. 2011, 130, 333-339.

(724) Rimola, A.; Corno, M.; Zicovich-Wilson, C. M.; Ugliengo, P. Phys. Chem. Chem. Phys. 2009, 11, 9005-9007.

(725) Richmond, G. L. Chem. Rev. 2002, 102, 2693-2724.

(726) Barducci, A.; Bonomi, M.; Parrinello, M. Wiley Interdiscip. Rev.-Comput. Mol. Sci. 2011, $1,826-843$.

(727) Zaera, F. Chem. Rev. 2012, 112, 2920-2986.

(728) Mignon, P.; Sodupe, M. Phys. Chem. Chem. Phys. 2012, 14, 945-954.

(729) Murashov, V.; Leszcynski, J. J. Phys. Chem. A 1999, 103, 1228-1238.

(730) Biswas, A.; Bayer, I. S.; Biris, A. S.; Wang, T.; Dervishi, E.; Faupel, F. Adv. Colloid Interface Sci. 2012, 170, 2-27.

(731) Kershner, R. J.; Bozano, L. D.; Micheel, C. M.; Hung, A. M.; Fornof, A. R.; Cha, J. N.; Rettner, C. T.; Bersani, M.; Frommer, J.; Rothemund, P. W. K.; Wallraff, G. M. Nat. Nanotechnol. 2009, 4, 557-561.

(732) Kasemo, B. Curr. Opin. Solid State Mat. Sci. 1998, 3, 451-459.

(733) Hook, F.; Kasemo, B.; Grunze, M.; Zauscher, S. ACS Nano 2008, 2, 2428-2436. 